\title{
Article
}

Mycosphere

Doi 10.5943/mycosphere/11/1/8

\section{Outline of Fungi and fungus-like taxa}

Wijayawardene $\mathrm{NN}^{1}$, Hyde $\mathrm{KD}^{2}$, Al-Ani $\mathrm{LKT}^{3,4}$, Tedersoo $\mathrm{L}^{5}$, Haelewaters $\mathrm{D}^{6,7,8,9}$, Rajeshkumar KC ${ }^{10,11}$, Zhao $\mathrm{RL}^{12,13}$, Aptroot $\mathrm{A}^{14}$, Leontyev $\mathrm{DV}^{15}$, Saxena RK $^{16}$, Tokarev YS ${ }^{17}$, Dai DQ ${ }^{1, *}$, Letcher PM ${ }^{18}$, Stephenson SL ${ }^{19}$, Ertz $D^{20,21}$, Lumbsch HT $^{22}$, Kukwa $\mathbf{M}^{23}$, Issi $\mathbf{I V}^{17}$, Madrid $\mathbf{H}^{24}$, Phillips AJL ${ }^{25}$, Selbmann $\mathbf{L}^{26,27}$, Pfliegler $\mathbf{W P}^{28}$, Horváth $\mathbf{E}^{29}$, Bensch $\mathbf{K}^{30}$, Kirk $\mathbf{P M}^{31}$, Kolař́́ková $\mathbf{K}^{32}$, Raja $\mathbf{H A}^{33}$, Radek $\mathrm{R}^{34}$, Papp $\mathrm{V}^{35}$, Dima $\mathrm{B}^{36}$, Ma $\mathbf{J}^{37}$, Malosso $\mathrm{E}^{38}$, Takamatsu $S^{39,40}$, Rambold $G^{41}$, Gannibal $P_{B}{ }^{42}$, Triebel $D^{43}$, Gautam $\mathrm{AK}^{44}$, Avasthi $\mathrm{S}^{45}$, Suetrong $\mathrm{S}^{46,47}$, Timdal $\mathrm{E}^{48}$, Fryar $\mathrm{SC}^{49}$, Delgado $\mathbf{G}^{50}$, Réblová $\mathbf{M}^{51}$, Doilom $\mathbf{M}^{52,71,72,73}$, Dolatabadi $\mathrm{S}^{53}$, Pawłowska $\mathrm{JZ}^{54}$, Humber $\mathbf{R A}^{55}$, Kodsueb $\mathbf{R}^{56}$, Sánchez-Castro $I^{57}$, Goto BT ${ }^{58}$, Silva DKA ${ }^{59}$, de Souza FA ${ }^{60}$, Oehl $F^{61}$, da Silva $\mathrm{GA}^{62}$, Silva $\mathrm{IR}^{62}$, Blaszkowski $\mathrm{J}^{63}$, Jobim $\mathrm{K}^{64}$, Maia $\mathrm{LC}^{62}$, Barbosa $\mathrm{FR}^{65}$, Fiuza PO $^{66}$, Divakar $\mathrm{PK}^{67}$, Shenoy BD $^{68}$, Castañeda-Ruiz $\mathbf{R F}^{69}$, Somrithipol $\mathrm{S}^{47}$, Lateef $\mathbf{A A}^{70}$, Karunarathna $\mathbf{S C}^{71,72,73}$, Tibpromma $\mathbf{S}^{71,72,73}$, Mortimer $\mathbf{P E}^{71,72,73}$, Wanasinghe DN ${ }^{71,72,73}$, Phookamsak $R^{2,71,72,73,74}$, Xu $\mathbf{J}^{71,72,73,74}$, Wang $\mathrm{Y}^{75}$, Tian $F^{75}$, Alvarado $P^{76}$, Li DW ${ }^{77}$, Kušan $I^{78}$, Matočec $N^{78}$, Mešić $A^{78}$, Tkalčec $Z^{78}$, Maharachchikumbura $\mathrm{SSN}^{79}$, Papizadeh $\mathrm{M}^{80}$, Heredia $\mathrm{G}^{81}$, Wartchow $\mathrm{F}^{82}$, Bakhshi $\mathrm{M}^{83}$, Boehm $\mathrm{E}^{84}$, Youssef $\mathrm{N}^{85}$, Hustad $\mathrm{VP}^{86}$, Lawrey $\mathrm{JD}^{87}$, Santiago ALCMA $^{88}$, Bezerra JDP ${ }^{89}$, Souza-Motta $\mathbf{C M}^{89}$, Firmino $\mathrm{AL}^{90}$, Tian $\mathbf{Q}^{2}$, Houbraken $\mathrm{J}^{91}$, Hongsanan $\mathrm{S}^{92}$, Tanaka $\mathrm{K}^{93}$, Dissanayake $\mathbf{A J}^{79}$, Monteiro $\mathrm{JS}^{94}$, Grossart $\mathrm{HP}^{95,96}$, Suija $\mathrm{A}^{97}$, Weerakoon $\mathbf{G}^{98}$, Etayo $\mathrm{J}^{99}$, Tsurykau $\mathrm{A}^{100,101}$, Vázquez $V^{102}$, ${ }^{103}$, Mungai $P^{104}$, Damm $U^{105}$, Li QR ${ }^{106}$, Zhang $H^{107}$, Boonmee $S^{2}$, Lu YZ ${ }^{108,109}$, Becerra AG ${ }^{110}$, Kendrick $B^{111}$, Brearley $F Q^{112}$, Motiejūnaitė $J^{113}$, Sharma $B^{11}$, Khare $R^{11}$, Gaikwad $S^{11}$, Wijesundara $\operatorname{DSA}^{114}$, Tang $L^{1}{ }^{1 *}$, He $M^{12,13}$, Flakus $A^{115}$, Rodriguez-Flakus $\mathbf{P}^{116}$, Zhurbenko $\mathbf{M P}^{117}$, McKenzie EHC ${ }^{118}$, Stadler $\mathbf{M}^{119,120}$, Bhat $\mathbf{D J}^{121}$, Liu $\mathbf{J K}^{79}$, Raza $\mathbf{M}^{12}$, Jeewon $\mathbf{R}^{122}$, Nassonova $\mathbf{E S}^{123}$, Prieto $M^{124}$, Jayalal RGU ${ }^{125}$, Erdoğdu $M^{126}$, Yurkov $A^{127}$, Schnittler $\mathbf{M}^{128}$, Shchepin $\mathbf{O N}^{129}$, Novozhilov $\mathbf{Y K}^{129}$, Silva-Filho AGS $^{130}$, Gentekaki $E^{2}$, Liu $P^{131}$, Cavender $J^{132}$, Kang $Y^{133}$, Mohammad $S^{134}$, Zhang LF $^{135}$, Xu RF ${ }^{135}$, Li YM ${ }^{135}$, Dayarathne $\mathrm{MC}^{75}$, Ekanayaka $\mathrm{AH}^{2}$, Wen TC ${ }^{136,137}$, Deng CY ${ }^{138}$, Pereira $O L^{139}$, Navathe $S^{140}$, Hawksworth $D L^{141,142}$, Fan $X^{143}$, Dissanayake LS $^{137}$, Kuhnert $E^{144}$, Grossart HP ${ }^{145,}{ }^{146}$, Thines $M^{147,148}$

\footnotetext{
${ }^{1}$ Center for Yunnan Plateau Biological Resources Protection and Utilization, College of Biological Resource and Food Engineering, Qujing Normal University, Qujing, Yunnan 655011, P.R. China.

${ }^{2}$ Center of Excellence in Fungal Research, Mae Fah Luang University, Chiang Rai 57100, Thailand

${ }^{3}$ Department of Plant Protection, College of Agriculture, University of Baghdad, Baghdad, Iraq
} 
${ }^{4}$ School of Biology Science, Universiti Sains Malaysia, Minden Malaysia

${ }^{5}$ Natural History Museum, University of Tartu, 14 a Ravila, 50411 Tartu, Estonia

${ }^{6}$ Faculty of Science, University of South Bohemia, Branišovská 31, 37005 České Budéjovice, Czech Republic

${ }^{7}$ Department of Botany and Plant Pathology, Purdue University, 915 W. State Street, West Lafayette, Indiana 47907, USA

${ }^{8}$ Herbario UCH, Universidad Autónoma de Chiriquí, Apartado Postal 0427, David, Panama

${ }^{9}$ Department of Biology, Research Group Mycology, Ghent University, K.L. Ledeganckstraat 35, 9000 Ghent, Belgium

${ }^{10}$ National Fungal Culture Collection of India (NFCCI), Biodiversity and Palaeobiology (Fungi) Group, Agharkar Research Institute, Pune, Maharashtra 411 004, India

${ }^{11}$ Biodiversity and Palaeobiology (Lichens) Group, Agharkar Research Institute, Pune, Maharashtra 411004, India

${ }^{12}$ State Key Laboratory of Mycology, Institute of Microbiology Chinese Academy of Sciences, Chaoyang District, Beijing 100101, P.R. China

${ }^{13}$ College of Life Sciences, University of Chinese Academy of Sciences, Huairou District, Beijing 100408, P.R. China

${ }^{14}$ Laboratório de Botânica / Liquenologia, Instituto de Biociências, Universidade Federal de Mato Grosso do Sul, Avenida Costa e Silva s/n, Bairro Universitário, CEP 79070-900, Campo Grande, Mato Grosso do Sul, Brazil

${ }^{15}$ Department of Botany, H.S. Skovoroda Kharkiv National Pedagogical University, Valentynivs'ka 2, Kharkiv 61168 Ukraine

${ }^{16}$ Birbal Sahni Institute of Palaeosciences, 53 University Road, Lucknow-226007, India

${ }^{17}$ Laboratory of Microbiological Control, All-Russian Institute of Plant Protection, Shosse Podbelskogo 3, Pushkin, St. Petersburg, 196608, Russia

${ }^{18}$ Department of Biological Sciences, The University of Alabama, Tuscaloosa, Alabama 35487, USA

${ }^{19}$ Department of Biological Sciences, University of Arkansas, Fayetteville, Arkansas 72701, USA

${ }^{20}$ Botanic Garden Meise, Department of Research, Nieuwelaan 38, BE-1860 Meise, Belgium

21 Fédération Wallonie-Bruxelles, Direction générale de l'Enseignement non obligatoire et de la Recherche scientifique, Rue A. Lavallée 1, BE-1080 Bruxelles, Belgium

${ }^{22}$ Science \& Education, The Field Museum, 1400 S. Lake Shore Drive, Chicago, IL 60605, USA

${ }^{23}$ Department of Plant Taxonomy and Nature Conservation, Faculty of Biology, University of Gdańsk, Wita Stwosza 59, PL-80-308 Gdańsk, Poland

${ }^{24}$ Centro de Genómica y Bioinformática, Facultad de Ciencias, Universidad Mayor, Camino La Pirámide 5750, Huechuraba, Santiago, Chile

${ }^{25}$ Universidade de Lisboa, Faculdade de Ciências, Biosystems and Integrative Sciences Institute (BioISI), Campo Grande, 1749-016 Lisbon, Portugal

${ }^{26}$ Department of Ecological and Biological Sciences (DEB) University of Tuscia, Viterbo, Italy

${ }^{27}$ Italian Antarctic National Museum (MNA), Mycological Section, Genoa, Italy

${ }^{28}$ Department of Molecular Biotechnology and Microbiology, University of Debrecen, Debrecen, Egyetem tér 1. , 4032, Hungary

${ }^{29}$ Department of Genetics and Applied Microbiology, University of Debrecen, Debrecen, Egyetem tér $1 ., 4032$ Hungary

${ }^{30}$ SNSB IT-Center, Botanische Staatssammlung München, Menzinger Straße 67, 80638 München, Germany / Westerdijk Fungal Biodiversity Institute, Uppsalalaan 8, 3534 CT Utrecht, The Netherlands

${ }^{31}$ Royal Botanic Gardens, Kew, Richmond, Surrey TW9 3DS, UK

${ }^{32}$ Institute of Botany, Czech Academy of Sciences Prìhonice 252 43, Czech Republic

${ }^{33}$ Department of Chemistry and Biochemistry, University of North Carolina at Greensboro, Greensboro, NC 27402, USA

${ }^{34}$ Evolutionary Biology, Institute for Biology/Zoology, Freie Universität Berlin, 14195 Berlin, Germany

${ }^{35}$ Department of Botany, Szent István University, Ménesi st. 44, 1118 Budapest, Hungary

${ }^{36}$ Institute of Biology, Eötvös Loránd University, Pázmány Péter sétány 1/c, 1117 Budapest, Hungary

${ }^{37}$ College of Agronomy, Jiangxi Agricultural University, Nanchang, 330045, Jiangxi, P.R. China

${ }^{38}$ Laboratório de Hifomicetos de Folhedo, Departamento de Micologia, Centro de Biociências, Universidade Federal de Pernambuco, 50740-600, Brazil

${ }^{39}$ Graduate School of Bioresources, Mie University, 1577 Kurima-Machiya, Tsu, Mie 514-8507, Japan

${ }^{40}$ University of Southern Queensland, Centre of Crop Protection, Toowoomba, Queensland, 4350, Australia

${ }^{41}$ Department of Mykologie, Universität of Bayreuth, Universitätsstraße 30, 95440 Bayreuth, Germany

${ }^{42}$ All-Russian Institute of Plant Protection, Saint Petersburg, Russia

43 Staatliche Naturwissenschaftliche Sammlungen Bayerns, IT Center, Menzinger Straße 67, 80638 München, Germany

${ }^{44}$ School of Agriculture, Faculty of Agriculture, Abhilashi University, Mandi, Himachal Pradesh, India

${ }^{45}$ School of Studies in Botany, Jiwaji University, Gwalior, Madhya Pradesh, India

${ }^{46}$ National Biobank of Thailand (NBT), National Science and Technology Development Agency (NSTDA), 113 Thailand Science Park, Thanon Phahonyothin, Tambon Khlong Nueng, Amphoe Khlong Luang, Pathum Thani 12120 , Thailand 
${ }^{47}$ BIOTEC, National Science and Technology Development Agency (NSTDA), 113 Thailand Science Park, Thanon Phahonyothin, Tambon Khlong Nueng, Amphoe Khlong Luang, Pathum Thani 12120, Thailand

${ }^{48}$ Natural History Museum, University of Oslo, Blindern, P.O. Box 1172, 0318 Oslo, Norway

${ }^{49}$ College of Science and Engineering, Flinders University, G.P.O. Box 2100, Adelaide 5001 SA, Australia

${ }^{50}$ EMLab P\&K Houston, 10900 Brittmoore Park Drive Suite G Houston, Texas 77041, USA

${ }^{51}$ Institute of Botany, Academy of Sciences, Prühonice 252 43, Czech Republic

${ }^{52}$ Department of Biology, Faculty of Science, Chiang Mai University, Chiang Mai 50200, Thailand

${ }^{53}$ Faculty of Engineering, Sabzevar University of New Technology, Sabzevar, Iran

${ }^{54}$ Department of Molecular Phylogenetics and Evolution, Faculty of Biology, Biological and Chemical Research Center, University of Warsaw, ul. Żwirki i Wigury 101, 02-089 Warsaw, Poland

${ }^{55}$ USDA-ARS Emerging Pests and Pathogens Research, Robert W. Holley Center for Agriculture and Health, Tower Road, Ithaca, NY 14853-2901, USA

${ }^{56}$ Microbiology Program, Faculty of Science and Technology, Pibulsongkram Rajabhat University, Phitsanulok 65000, Thailand

${ }^{57}$ Departamento de Microbiología, Campus de Fuentenueva, Universidad de Granada, 18071, Granada, Spain

${ }^{58}$ Departamento de Botânica e Zoologia, Universidade Federal do Rio Grande do Norte, Campus Universitário, 59072-970, Natal, RN, Brazil

${ }^{59}$ Universidade Federal da Paraíba, Programa de Pós-Graduação em Ecologia e Monitoramento Ambiental, Centro de Ciências Aplicadas e Educação, Campus IV, Litoral Norte, Rio Tinto, PB 58297-000, Brazil

${ }^{60}$ Embrapa Milho \& Sorgo, BR-35702098 Sete Lagoas, MG, Brazil

${ }^{61}$ Agroscope, Competence Div Plants \& Plant Prod, Ecotoxicol, Schloss 1, CH-8820 Wadenswil, Switzerland

${ }^{62}$ Universidade Federal de Pernambuco, Programa de Pós-Graduação em Biologia de Fungos, Av. da Engenharia, s/n, Cidade Universitária, CEP 50740-600, Recife, PE, Brazil

${ }^{63}$ Department of Ecology, Protection and Shaping of Environment, West Pomeranian University of Technology, Stowackiego 17, PL-71434 Szczecin, Poland

${ }^{64}$ Departamento de Botânica e Zoologia, Universidade Federal do Rio Grande do Norte, Campus Universitário, 59072-970, Natal, RN, Brazil

${ }^{65}$ Acervo Biológico da Amazonia Meridional, Universidade Federal de Mato Grosso, Sinop 78557267, Brazil

${ }^{66}$ Universidade Federal do Rio Grande do Norte (UFRN), Programa de Pós-graduação em Sistemática e Evolução, Centro de Biociências, Campus Universitário, Av. Senador Salgado Filho, 3000, Lagoa Nova, Natal-RN, 59078-970, Brazil

${ }^{67}$ Departamento de Farmacología, Farmacognosia y Botánica, Facultad de Farmacia, Universidad Complutense de Madrid, Madrid 28040, Spain

${ }^{68}$ CSIR-National Institute of Oceanography Regional Centre, 176, Lawson's Bay Colony, Visakhapatnam - 530017, Andhra Pradesh, India

${ }^{69}$ Instituto de Investigaciones Fundamentales en AgriculturaTropical, 'Alejandro de Humboldt' (INIFAT), Santiago deLas Vegas, Habana, Cuba

${ }^{70}$ Department of Plant Biology, Faculty of Life Sciences, University of Ilorin, Nigeria

${ }^{71}$ Key Laboratory for Plant Diversity and Biogeography of East Asia, Kunming Institute of Botany, Chinese Academy of Science, Kunming 650201, Yunnan, P.R. China

${ }^{72}$ World Agroforestry Centre, East and Central Asia, Kunming 650201, Yunnan, P.R. China

${ }^{73}$ Centre for Mountain Futures (CMF), Kunming Institute of Botany, Kunming, Yunnan, 650201, P.R. China

${ }^{74}$ Institute of Animal Science, State Key Laboratory of Animal Nutrition, Chinese Academy of Agricultural Sciences, Beijing, 100193, P.R. China

${ }^{75}$ Department of Plant Pathology, Agriculture College, Guizhou University, Guiyang 550025, P.R. China

${ }^{76}$ ALVALAB, Dr. Fernando Bongera st., Severo Ochoa Bldg. S1.04, Oviedo, 33006, Spain

77 The Connecticut Agricultural Experiment Station, Valley Laboratory, 153 Cook Hill Road, Windsor, CT 06095, USA

${ }^{78}$ Ruđer Bošković Institute, Bijenička 54, HR-10000 Zagreb, Croatia

79 School of Life Science and Technology, University of Electronic Science and Technology of China, Chengdu 611731, P.R. China

${ }^{80}$ Department of Microbiology, Pasteur Institute of Iran, Tehran, Iran

${ }^{81}$ Laboratory of Microfungi, Instituto de Ecologı 'a A. C., Xalapa, Ver., Mexico

${ }^{82}$ Universidade Federal da Paraíba, Departamento de Sistemática e Ecologia, CEP 58051-970, João Pessoa, PB, Brazil

83 Department of Botany, Iranian Research Institute of Plant Protection, P.O. Box 19395-1454, Agricultural Research, Education and Extension Organization (AREEO), Tehran, Iran

${ }^{84} 42$ Longacre Drive Livingston, NJ, 07039, USA

${ }^{85}$ Oklahoma State University, Department of Microbiology and Molecular Genetics, Stillwater OK, USA

${ }^{86}$ Department of Natural Sciences, Northwest Missouri State University, Maryville, Missouri, 64468, USA

${ }^{87}$ Department of Environmental Science and Policy, George Mason University, 4400 University Drive, Fairfax, VA 22030-4444, USA 
${ }^{88}$ Post Graduate Program in Biology of Fungi, Department of Mycology, Federal University of Pernambuco, Av. Nelson Chaves, s/ n, 50670-420 Recife, PE, Brazil

${ }^{89}$ Departamento de Micologia Prof. Chaves Batista, Universidade Federal de Pernambuco, Av. Prof. Moraes Rego, s/n, Centro de Biociências, Cidade Universitária, CEP: 50670-901, Recife, PE, Brazil

${ }^{90}$ Instituto de Ciências Agrárias, Universidade Federal de Uberlândia, Monte Carmelo 38500-000, Brazil

${ }^{91}$ Westerdijk Fungal Biodiversity Institute, Uppsalalaan 8, 3584 CT Utrecht, the Netherlands

${ }^{2}$ Shenzhen Key Laboratory of Laser Engineering, College of Optoelectronic Engineering, Shenzhen University, Shenzhen, P.R. China

${ }^{93}$ Faculty of Agriculture and Life Science, Hirosaki University, 3 Bunkyo-cho, Hirosaki, Aomori 036-8561, Japan

${ }^{94}$ Coordination of Botany, Museu Paraense Emílio Goeldi, 66077830, Belém, PA, Brazil

${ }^{95}$ Leibnitz Institute of Freshwater Ecology and Inland Fisheries (IGB), Department Experimental Limnology, Alte Fischerhuette 2, D-16775 Stechlin, Germany

${ }^{96}$ Institute for Biochemistry and Biology, Potsdam University, Maulbeerallee 2, 14469 Potsdam, Germany

${ }^{97}$ Institute of Ecology and Earth Sciences, University of Tartu, 40 Lai st., EE-51005 Tartu, Estonia

${ }^{98}$ Algae, Fungi and Plants Division, Department of Life Sciences, The Natural History Museum, Cromwell Road, London, SW7 5BD, UK

${ }^{99}$ Department of Biology, IES Zizur, Ronda S. Cristo bal 196, 31180 Zizur Mayor, Navarra, Spain

${ }^{100}$ F. Skorina Gomel State University, Department of Biology, Sovetskaja Str. 104, Gomel 246019, Belarus

${ }^{101}$ Samara National Research University, Institute of Natural Sciences, Department of Ecology, Botany and Nature Protection, Moskovskoye shosse 34, Samara 443086, Russia

102 Department of Ecology, Faculty of Sciences, University of Málaga, Boulevard Louis Pasteur s/n, 29071-Málaga, Spain

${ }^{103}$ Department of Research and Development, Coccosphere Environmental Analysis, Málaga, Spain

${ }^{104}$ Ecological Monitoring Unit, Biodiversity Research and Monitoring Division, Kenya Wildlife Service, P.O. Box 40241, Nairobi 00100, Kenya

105 Senckenberg Museum of Natural History Görlitz, PF 300 154, 02806 Görlitz, Germany

106 Department of Pharmacology of Materia Medica, School of Pharmaceutical Sciences, Guizhou Medical University, University Town, Guian New District, Guizhou 550025, P.R. China

107 Yunnan Institute of Food Safety, Kunming University of Science and Technology, Kunming 650500, P.R. China

${ }^{108}$ Engineering and Research Center for Southwest Bio-Pharmaceutical Resources of National Education Ministry of China, Guizhou University, Guiyang 550025, Guizhou, P.R. China

${ }^{109}$ School of Pharmaceutical Engineering, Guizhou Institute of Technology, Guiyang, Guizhou, 550003, P.R. China

110 Laboratorio de Micología, Instituto Multidisciplinario de Biología Vegetal (IMBIV), CONICET, Universidad Nacional de Córdoba, Casilla de Correo 495, 5000 Córdoba, Argentina.

${ }^{111}$ Mycologue, 8727 Lochside Drive, Sidney, BC V8L 1M8, Canada

112 Department of Natural Sciences, Manchester Metropolitan University, Chester Street, Manchester, M1 5GD, UK.

${ }^{113}$ Laboratory of Mycology, Nature Research Centre, Žaliuju ežeru Str. 49, 08406 Vilnius, Lithuania

${ }^{114}$ National Institute of Fundamental Studies, Hantane Road, Kandy, Sri Lanka

115 Department of Lichenology, W. Szafer Institute of Botany, Polish Academy of Sciences, Lubicz 46, PL-31-512 Krakow, Poland

${ }^{116}$ Laboratory of Molecular Analyses, W. Szafer Institute of Botany, Polish Academy of Sciences, Lubicz 46, PL-31512 Krakow, Poland

117 Laboratory of the Systematics and Geography of Fungi, Komarov Botanical Institute, Russian Academy of Sciences, Professor Popov 2, St.-Petersburg, 197376, Russia

${ }_{118}$ Manaaki Whenua-Landcare Research, Private Bag No 92170, Auckland, New Zealand

119 Department of Microbial Drugs, Helmholtz-Zentrum für Infektionsforschung GmbH, Inhoffenstrasse 7, 38124 Braunschweig, Germany

120 German Centre for Infection Research (DZIF), partner site Hannover-Braunschweig, 38124 Braunschweig, Germany

${ }^{121}$ No. 128/1-J, Azad Co-Op Housing Society, Curca, P.O. Goa Velha-403108, India

${ }^{122}$ Dept Health Sciences, Faculty of Science, University of Mauritius, Reduit, Mauritius

${ }^{123}$ Laboratory of Cytology of Unicellular Organisms, Institute of Cytology of the Russian Academy of Sciences, Tikhoretsky ave. 4, St. Petersburg, 194064, Russia

${ }^{124}$ Departamento de Biología y Geología, Física y Química Inorgánica. Universidad Rey Juan Carlos. C/Tulipan sn, 28933, Móstoles, Madrid, Spain

${ }^{125}$ Department of Natural Resources, Sabaragamuwa University of Sri Lanka, Belihuloya, 70140, Sri Lanka

${ }^{126}$ Department of Landscape Architects, Faculty of Agriculture, Kirşehir Ahi Evran University, Kirşehir, Turkey

${ }^{127}$ Leibniz. Institute DSMZ-German Collection of Microorganisms and Cell Cultures GmbH, Inhoffenstrasse 7 B, 38124 Braunschweig, Germany

128 Institute of Botany and Landscape Ecology, Ernst Moritz Arndt University Greifswald, Soldmannstr. 15, Greifswald 17487, Germany

${ }^{129}$ The Komarov Botanical Institute of the Russian Academy of Sciences, Laboratory of Systematics and Geography of Fungi, Prof. Popov Street 2, 197376 St. Petersburg, Russia 
${ }^{130}$ Universidade Federal do Rio Grande do Norte, Programa de Pós Graduação em Sistemática e Evolução, CEP 59064-741, Natal, RN, Brazil

${ }^{131}$ Engineering Research Center of Chinese Ministry of Education for Edible and Medicinal Fungi, Jilin Agricultural University, Changchun, 130118, P.R. China

${ }_{132}$ Department of Environmental and Plant Biology, Ohio University, Athens, Ohio 45701

${ }^{133}$ Key Laboratory of Environmental Pollution Monitoring and Disease Control, Ministry of Education of Guizhou \& Guizhou Talent Base for Microbiology and Human Health, Key Laboratory of Medical Microbiology and Parasitology of Education Department of Guizhou, School of Basic Medical Sciences, Guizhou Medical University, Guiyang, P.R. China

${ }^{134}$ Department of Biotechnology, Iranian Research Organization for Science and Technology (IROST), Tehran, Iran

${ }^{135}$ College of Biological Resource and Food Engineering, Qujing Normal University, Qujing, Yunnan 655011, P.R. China

${ }^{136}$ State Key Laboratory Breeding Base of Green Pesticide and Agricultural Bioengineering, Key Laboratory of Green Pesticide and Agricultural Bioengineering, Ministry of Education, Guizhou University, Guiyang, 550025, P.R. China

${ }^{137}$ The Engineering Research Center of Southwest Bio-Pharmaceutical Resource, Ministry of Education, Guizhou University, Guiyang, 550025, P.R. China

${ }^{138}$ Guizhou institute of biology, Guizhou academy of science, Guiyang, 550009, P.R. China

${ }^{139}$ Universidade Federal de Viçosa, Departamento de Fitopatologia, Viçosa - Minas Gerais, Brazil

${ }^{140}$ Agharkar Research Institute, G. G. Agarkar Road, Pune 411004, India

${ }^{141}$ Department of Life Sciences, The Natural History Museum, London SW7 5BD, United Kingdom, and Comparative Plant and Fungal Biology, Royal Botanic Gardens, Kew, Richmond, Surrey TW9 3DS, UK

142 Jilin Agricultural University, Changchun, Jilin Province, 130118, P.R. China

${ }^{143}$ The Key Laboratory for Silviculture and Conservation of Ministry of Education, Beijing Forestry University, Beijing 100083, P.R. China

144 Leibniz University Hannover, Institute for Organic Chemistry and MWZ, Hannover 30167, Germany

145 Leibnitz. Institute of Freshwater Ecology and Inland Fisheries (IGB), Dept. Experimental Limnology, Alte Fischerhuette 2, D-16775 Stechlin, Germany

${ }^{146}$ Institute for Biochemistry and Biology, Potsdam University, Maulbeerallee 2, 14469 Potsdam, Germany

147 Goethe University, Department for Biological Sciences, Institute of Ecology, Evolution and Diversity, Max-vonLaue-Str. 13, D-60486 Frankfurt am Main, Germany

148 Senckenberg Biodiversity and Climate Research Centre, Senckenberganlage 25, D-60325 Frankfurt am Main, Germany

Wijayawardene NN, Hyde KD, Al-Ani LKT, Tedersoo L, Haelewaters D, Rajeshkumar KC, Zhao RL, Aptroot A, Leontyev DV, Saxena RK, Tokarev YS, Dai DQ, Letcher PM, Stephenson SL, Ertz D, Lumbsch HT, Kukwa M, Issi IV, Madrid H, Phillips AJL, Selbmann L, Pfliegler WP, Horváth E, Bensch K, Kirk PM, Kolaříková K, Raja HA, Radek R, Papp V, Dima B, Ma J, Malosso E, Takamatsu S, Rambold G, Gannibal PB, Triebel D, Gautam AK, Avasthi S, Suetrong S, Timdal E, Fryar SC, Delgado G, Réblová M, Doilom M, Dolatabadi S, Pawłowska J, Humber RA, Kodsueb R, Sánchez-Castro I, Goto BT, Silva DKA, de Souza FA, Oehl F, da Silva GA, Silva IR, Błaszkowski J, Jobim K, Maia LC, Barbosa FR, Fiuza PO, Divakar PK, Shenoy BD, Castañeda-Ruiz RF, Somrithipol S, Lateef AA, Karunarathna SC, Tibpromma S, Mortimer PE, Wanasinghe DN, Phookamsak R, Xu J, Wang Y, Tian F, Alvarado P, Li DW, Kušan I, Matočec N, Maharachchikumbura SSN, Papizadeh M, Heredia G, Wartchow F, Bakhshi M, Boehm E, Youssef N, Hustad VP, Lawrey JD, Santiago ALCMA, Bezerra JDP, Souza-Motta CM, Firmino AL, Tian Q, Houbraken J, Hongsanan S, Tanaka K, Dissanayake AJ, Monteiro JS, Grossart HP, Suija A, Weerakoon G, Etayo J, Tsurykau A, Vázquez V, Mungai P, Damm U, Li QR, Zhang H, Boonmee S, Lu YZ, Becerra AG, Kendrick B, Brearley FQ, Motiejūnaitė J, Sharma B, Khare R, Gaikwad S, Wijesundara DSA, Tang LZ, He MQ, Flakus A, Rodriguez-Flakus P, Zhurbenko MP, McKenzie EHC, Stadler M, Bhat DJ, Liu JK, Raza M, Jeewon R, Nassonova ES, Prieto M, Jayalal RGU, Erdoğdu M, Yurkov A, Schnittler M, Shchepin ON, Novozhilov YK, Silva-Filho AGS, Liu P, Cavender JC, Kang Y, Mohammad S, Zhang LF, Xu RF, Li YM, Dayarathne MC, Ekanayaka AH, Wen TC, Deng CY, Pereira OL, Navathe S, Hawksworth DL, Fan XL, Dissanayake LS, Kuhnert E, Grossart HP, Thines M 2020 - Outline of Fungi and fungus-like taxa. Mycosphere 11(1), 1060-1456, Doi 10.5943/mycosphere/11/1/8 


\begin{abstract}
This article provides an outline of the classification of the kingdom Fungi (including fossil fungi. i.e. dispersed spores, mycelia, sporophores, mycorrhizas). We treat 19 phyla of fungi. These are Aphelidiomycota, Ascomycota, Basidiobolomycota, Basidiomycota, Blastocladiomycota, Calcarisporiellomycota, Caulochytriomycota, Chytridiomycota, Entomophthoromycota, Entorrhizomycota, Glomeromycota, Kickxellomycota, Monoblepharomycota, Mortierellomycota, Mucoromycota, Neocallimastigomycota, Olpidiomycota, Rozellomycota and Zoopagomycota. The placement of all fungal genera is provided at the class-, order- and family-level. The described number of species per genus is also given. Notes are provided of taxa for which recent changes or disagreements have been presented. Fungus-like taxa that were traditionally treated as fungi are also incorporated in this outline (i.e. Eumycetozoa, Dictyosteliomycetes, Ceratiomyxomycetes and Myxomycetes). Four new taxa are introduced: Amblyosporida ord. nov. Neopereziida ord. nov. and Ovavesiculida ord. nov. in Rozellomycota, and Protosporangiaceae fam. nov. in Dictyosteliomycetes. Two different classifications (in outline section and in discussion) are provided for Glomeromycota and Leotiomycetes based on recent studies. The phylogenetic reconstruction of a four-gene dataset (18S and 28S rRNA, RPB1, RPB2) of 433 taxa is presented, including all currently described orders of fungi.
\end{abstract}

Keywords - Four new taxa - Ascomycota - Amblyosporida ord. nov. - Basal clades Basidiomycota - Classification - Emendation - Microsporidia - Neopereziida ord. nov. Ovavesiculida ord. nov. - Protosporangiaceae fam. nov. - Redonographaceae stat nov.

\title{
Introduction
}

Classification of the kingdom Fungi has been updated continuously, with the frequent inclusion of data from DNA sequences in recent studies. Re-collecting historic taxa and neo- or epitypifying them by using both fresh material and cultures is also an increasingly common practice among mycologists, although yet not easily accomplished in some groups. Utilization of environmental sequences for recognizing taxa that are not observed directly and naming them with only a sequence as a holotype is a controversial topic that remains to be addressed (Hongsanan et al. 2018, Lücking \& Hawksworth 2018, Lücking et al. 2018, Thines et al. 2018, Zamora et al. 2018).

Tedersoo et al. (2018) proposed a novel classification for the kingdom Fungi that was based on phylogenies and the divergence time of particular taxa. Using these criteria, they accepted 18 phyla: Aphelidiomycota, Ascomycota, Basidiobolomycota, Basidiomycota, Blastocladiomycota, Calcarisporiellomycota, Caulochytriomycota, Chytridiomycota, Entomophthoromycota, Glomeromycota, Kickxellomycota, Monoblepharomycota, Mortierellomycota, Mucoromycota, Neocallimastigomycota, Olpidiomycota, Rozellomycota and Zoopagomycota. This study was, however, based on only 111 taxa and it remains to be seen how widely it will be accepted and stand up as more taxa are analyzed. Outlines for the Ascomycota (and notes for genera in the Ascomycota) and the basal clades of fungi (Wijayawardene et al. 2017, 2018a, b) have been published, with the participation of experts in particular groups. Jaklitsch et al. (2016a) provided a synopsis of accepted Ascomycota families with descriptions and lists of included genera (and their synonyms), and Begerow et al. (2018) prepared a parallel treatment for the families of Basidiomycota and Entorrhizomycota, including brief diagnoses and indications of ecology and distributions for all genera (though without listing synonyms of genera and with some genera that are still debated). A separate outline, with notes and divergence times of Basidiomycota was also published by He et al. (2019).

\section{Classification of basal clades}

The higher level classification of basal clades has been subjected to drastic changes as in Tedersoo et al. (2018), who took up Rozellomycota to include Cryptomycota and Microsporidia and also accepted Aphelidiomycota in a fungal clade as did Letcher \& Powell (2019) in a synopsis 
of that group. Moreover, classes and orders of respective phyla were also provided in Tedersoo et al. (2018). Wijayawardene et al. (2018b) provided a detailed classification system (from phyla to genera) for basal clades of Fungi, agreeing with Tedersoo et al. (2018). Hence, Wijayawardene et al. (2018b) accepted 16 phyla viz. Aphelidiomycota, Basidiobolomycota, Blastocladiomycota, Calcarisporiellomycota, Caulochytriomycota, Chytridiomycota, Entomophthoromycota, Glomeromycota, Kickxellomycota, Monoblepharomycota, Mortierellomycota, Mucoromycota, Neocallimastigomycota, Olpidiomycota, Rozellomycota and Zoopagomycota.

\section{Classification of Glomeromycota}

Classification of Glomeromycota (which includes arbuscular mycorrhizal fungi [AMF]) has been a subject of debate. We provide two different classifications (phyla to genera) which are commonly used by taxonomists (see outline and discussion). In the outline section, we provide the classification which is supported by Tedersoo et al. (2018) and our analyses (Fig. 1). The classification provided in Wijayawardene et al. (2018b) is included in the discussion.

\section{Placement of the Rozellomycota in the tree of life}

The position of Microsporidia in the Eukaryotic Tree of Life has been a subject of discussion. Primarily identified as yeast-like fungi in Schizomycetes (Nägeli 1857), they were further recognized as protists, while drastic reconsiderations of taxonomy of unicellular eukaryotes resulted in placing to Sporozoa (Balbiani 1882), and particularly Cnidosporidia (Labbé 1899); Sarcodina in Protozoa (Cavalier-Smith 1981); Archezoa (Cavalier-Smith 1983) and Protista (Puytorac et al. 1987); as well as to different classes of Fungi (Keeling et al. 2000, Gill \& Fast 2006, James et al. 2006, 2013). The mycological community has widely accepted the affiliation of Microsporidia with the early diverging clades of Fungi. The Microsporidia, Cryptomycota and Aphelidea, have also been considered to represent a monophyletic lineage with shared ecological and structural features, defined as superphylum Opisthosporidia belonging to supergroup Opisthokontha and separated from Fungi (Karpov et al. 2014). In another system, however, it was proposed that Cryptomycota (also known as Rozellida, Rozellomycota, or Rozellosporidia) and Microsporidia join the phylum Rozellomycota, while Aphelidea were considered as a separate, though related phylum and all these groups were considered basal lineages of the kingdom Fungi (Tedersoo et al. 2018). The taking up of the name Rozellomycota in such a broad sense appears premature, especially as the structure and biological features of a larger part of these organisms are unclear as they are known only from environmental sequences. The borders between Fungi and Protista are therefore unstable and final delimitation of taxa is problematic due to poor coverage of molecular data for the representatives of the most basal groups. However, whatever the conclusion is on placement, it has been decided that the nomenclature of the names in Microsporidia will continue to follow the International Code of Zoological Nomenclature even if they are treated as fungi (Turland et al. 2018).

\section{Classification of Ascomycota}

Periodic outlines of the Ascomycota have been issued since 1982, with notes in the journal Systema Ascomycetum which was devoted to this project, and later by Lumbsch \& Huhndorf (2010) who accepted three subphyla: Pezizomycotina with eleven classes, the Saccharomycotina with one class, and Taphrinomycotina with four classes. The taxonomy of the phylum has been rapidly updated over the last few years (Hyde et al. 2013, 2017, 2020, Jaklitsch et al. 2016a, Ekanayaka et al. 2017, Hongsanan et al. 2017, Liu et al. 2017).

Recently, two studies were published on the classification of Leotiomycetes. These are Ekanayaka et al. (2019) based on a five-locus phylogeny, Johnston et al. (2019) based on genomic-scale and 15-gene phylogenies. We provide two outlines; i) based on Johnston et al. 
(2019), Karakehian et al (2019) and Quijada et al. (2020); and ii) based on Ekanayaka et al. (2019). These classifications are placed in the general outline and discussion, respectively.

Moreover, the concept of One fungus-One name, which ended the use of different names for morphs of the same fungus in July 2011, has resulted in several name changes in pleomorphic genera. Wijayawardene et al. (2018a) provided an updated outline of Ascomycota with three subphyla - Pezizomycotina (including the 13 classes Arthoniomycetes, Coniocybomycetes, Dothideomycetes, Eurotiomycetes, Geoglossomycetes, Laboulbeniomycetes, Lecanoromycetes, Leotiomycetes, Lichinomycetes, Orbiliomycetes, Pezizomycetes, Sordariomycetes, Xylonomycetes and Xylobotryomycetes), Saccharomycotina (with only class Saccharomycetes) and Taphrinomycotina (with five classes Archaeorhizomycetes, Neolectomycetes, Pneumocystidomycetes, Schizosaccharomycetes and Taphrinomycetes). These taxa along with a summary of other taxonomic ranks are summarized in Table 1.

\section{Classification of Basidiomycota}

Basidiomycota constitute a major phylum of the kingdom Fungi and is second in numbers of described species, to Ascomycota (Wijayawardene et al. 2017, 2018a). Since the last edition of Ainsworth \& Bisby's Dictionary of the Fungi (Kirk et al. 2008), numerous sequenced-based studies have enabled the introduction of a vast array of new taxa, which has greatly enriched the known diversity of Basidiomycota. It has also become clear that several basidiome forms can be found in the same order, family, or even genus (Hibbett et al. 2007). At the same time, related new taxonomic categories have been proposed. For example, in phylogenetic studies of basidiomycetous yeasts, three new classes Malasseziomycetes, Monilielliomycetes, and Spiculogloeomycetes, were introduced and three new orders, 16 new families, and 47 new genera were also introduced (Nasr et al. 2014, Wang et al. 2014a, 2015a, b, Liu et al. 2015, Riess et al. 2016). On the other hand, many new changes have also occurred in the Agaricomycotina. Approximately 60 new genera have been recognized for agarics, 40 for boletes, and 50 for bracket fungi (Desjardin et al. 2009, Hjortstam \& Ryvarden 2010, Petersen \& Hughes 2010, Cui et al. 2011, Vellinga et al. 2011, Vizzini et al. 2011, Hao et al. 2014, Hofstetter et al. 2014, Smith et al. 2015, Castellano et al. 2016, Henkel et al. 2016, Wu et al. 2016, Buyck et al. 2017, Orihara \& Smith 2017). Attention has already been drawn to the valuable syntheses of accepted genera, with diagnosis, provided by Begerow et al. (2018).

The phylogeny and divergence time ranges for higher level Basidiomycota, with the phylum originating ca. 530 Mya, the subphyla 406-490 Mya, most classes 245-393 Mya and orders 120290 Mya were inferred by Zhao et al. (2017). The outline includes 1928 genera with 1263 synonyms within Basidiomycota (He et al. 2019). The latest version of Ainsworth \& Bisby's Dictionary of the Fungi (Kirk et al. 2008), contains three subphyla, 16 classes, 52 orders, 177 families, 1589 genera and 31515 species in Basidiomycota. The updated outline of Basidiomycota has updated the numbers in Kirk et al. (2008) to four subphyla, 18 classes, 68 orders, 241 families, 1928 genera and 41270 species in Basidiomycota (He et al. 2019). Agaricomycotina embrace most of the species in Basidiomycota (30788 species) which includes three classes, 29 orders, 150 families and 1514 genera. Pucciniomycotina was estimated to comprise 8653 species including 10 classes, 22 orders, 49 families and 270 genera. Ustilaginomycetes with 1185 species is the largest group in Ustilaginomycotina. Malasseziomycetes and Moniliellomycetes, are the only two new classes recognized in the Basidiomycota since 2008, include 32 estimated species. Wallemiomycotina is a recently recognized subphylum (Zhao et al. 2017) with 12 species estimated in a single class, two orders and two families. Another early diverging group in the Agaricomycotina has recently identified by a phylogenomic study, the Bartheletiomycetes, including just a single species associated with Gingko biloba as a 'living fossil' (Mishra et al. 2018). Accepted taxa of Basidiomycota are summarized in Table 1. 
Table 1 Phyla, classes, orders and families of kingdom Fungi. The number of accepted genera in each family is indicated in brackets after the family name.

\begin{tabular}{|c|c|c|c|}
\hline Phylum & Class* & Order* & Family* \\
\hline Aphelidiomycota & Aphelidiomycetes & Aphelidiales & Aphelidiaceae (4) \\
\hline \multirow[t]{48}{*}{ Ascomycota } & Archaeorhizomycetes & Archaeorhizomycetales & Archaeorhizomycetaceae (1) \\
\hline & Arthoniomycetes & Arthoniales & Andreiomycetaceae (1) \\
\hline & & & Arthoniaceae (23) \\
\hline & & & Chrysotrichaceae (3) \\
\hline & & & Lecanographaceae (7) \\
\hline & & & Opegraphaceae (15) \\
\hline & & & Roccellaceae (41) \\
\hline & & & Roccellographaceae (3) \\
\hline & & $\begin{array}{l}\text { Arthoniales genera } \\
\text { incertae sedis }(23)\end{array}$ & \\
\hline & & Lichenostigmatales & Phaeococcomycetaceae (3) \\
\hline & Candelariomycetes & Candelariales & Candelariaceae (4) \\
\hline & & & Pycnoraceae (1) \\
\hline & Coniocybomycetes & Coniocybales & Coniocybaceae (2) \\
\hline & Dothideomycetes & Abrothallales & Abrothallaceae (2) \\
\hline & & Acrospermales & Acrospermaceae (3) \\
\hline & & $\begin{array}{l}\text { Acrospermales genus } \\
\text { incertae sedis (1) }\end{array}$ & \\
\hline & & Asterinales & Asterinaceae (19) \\
\hline & & & Asterotexaceae (1) \\
\hline & & & Hemigraphaceae (1) \\
\hline & & & Lembosiaceae (1) \\
\hline & & & Melaspileellaceae (1) \\
\hline & & & Neobueliellaceae (1) \\
\hline & & & Stictographaceae (5) \\
\hline & & $\begin{array}{l}\text { Asterinales genera } \\
\text { incertae sedis (8) }\end{array}$ & \\
\hline & & Botryosphaeriales & Aplosporellaceae (2) \\
\hline & & & Botryosphaeriaceae (22) \\
\hline & & & Melanopsaceae (1) \\
\hline & & & Phyllostictaceae (2) \\
\hline & & & Planistromellaceae (4) \\
\hline & & & Saccharataceae (4) \\
\hline & & $\begin{array}{l}\text { Botryosphaeriales } \\
\text { genera incertae sedis (8) }\end{array}$ & \\
\hline & & Capnodiales & Aeminiaceae (1) \\
\hline & & & Antennulariellaceae (4) \\
\hline & & & Capnodiaceae (9) \\
\hline & & & Cladosporiaceae (8) \\
\hline & & & Cystocoleaceae (1) \\
\hline & & & Dissoconiaceae (5) \\
\hline & & & Euantennariaceae (7) \\
\hline & & & Extremaceae (8) \\
\hline & & & Johansoniaceae (2) \\
\hline & & & Metacapnodiaceae (3) \\
\hline & & & Mycosphaerellaceae (111) \\
\hline & & & Neodevriesiaceae (2) \\
\hline & & & Phaeothecaceae (1) \\
\hline & & & Phaeothecoidiellaceae (8) \\
\hline & & & Piedraiaceae (1) \\
\hline & & & Racodiaceae (1) \\
\hline & & & Schizothyriaceae (10) \\
\hline
\end{tabular}


Table 1 Continued.

\begin{tabular}{|c|c|c|c|}
\hline Phylum & Class* & Order* & Family* \\
\hline & & & Teratosphaeriaceae (60) \\
\hline & & & Xenodevriesiaceae (1) \\
\hline & & $\begin{array}{l}\text { Capnodiales genera } \\
\text { incertae sedis (14) }\end{array}$ & \\
\hline & & Catinellales & Catinellaceae (1) \\
\hline & & Cladoriellales & Cladoriellaceae (1) \\
\hline & & Collemopsidiales & Xanthopyreniaceae (6) \\
\hline & & Dothideales & Dothideaceae (13) \\
\hline & & & Neocelosporiaceae (3) \\
\hline & & & Saccotheciaceae (7) \\
\hline & & & Zalariaceae (1) \\
\hline & & $\begin{array}{l}\text { Dothideales genera } \\
\text { incertae sedis (6) }\end{array}$ & \\
\hline & & Dyfrolomycetales & Pleurotremataceae (3) \\
\hline & & Eremithallales & Melaspileaceae (2) \\
\hline & & Eremomycetales & Eremomycetaceae (2) \\
\hline & & $\begin{array}{l}\text { Eremomycetales genus } \\
\text { incertae sedis (1) }\end{array}$ & \\
\hline & & Gloniales & Gloniaceae (3) \\
\hline & & Hysteriales & Hysteriaceae (13) \\
\hline & & $\begin{array}{l}\text { Hysteriales genus } \\
\text { incertae sedis (1) }\end{array}$ & \\
\hline & & Jahnulales & Aliquandostipitaceae (7) \\
\hline & & & Manglicolaceae (1) \\
\hline & & Kirschsteiniotheliales & Kirschsteiniotheliaceae (1) \\
\hline & & $\begin{array}{l}\text { Kirschsteiniotheliales } \\
\text { genera incertae sedis (2) }\end{array}$ & \\
\hline & & Lembosinales & Lembosinaceae (1) \\
\hline & & Lichenotheliales & Lichenotheliaceae (2) \\
\hline & & Microthyriales & Microthyriaceae (11) \\
\hline & & $\begin{array}{l}\text { Microthyriales genera } \\
\text { incertae sedis }(6)\end{array}$ & \\
\hline & & Minutisphaerales & Acrogenosporaceae (1) \\
\hline & & & Minutisphaeraceae (1) \\
\hline & & Monoblastiales & Monoblastiaceae (6) \\
\hline & & Murramarangomycetales & $\begin{array}{l}\text { Murramarangomycetaceae } \\
\text { (1) }\end{array}$ \\
\hline & & Muyocopronales & Muyocopronaceae (9) \\
\hline & & Myriangiales & Elsinoaceae (2) \\
\hline & & & Myriangiaceae (11) \\
\hline & & $\begin{array}{l}\text { Myriangiales genus } \\
\text { incertae sedis (1) }\end{array}$ & \\
\hline & & Mytilinidiales & Mytilinidiaceae (9) \\
\hline & & Natipusillales & Natipusillaceae (1) \\
\hline & & Parmulariales & Parmulariaceae (35) \\
\hline & & Patellariales & Patellariaceae (21) \\
\hline & & Phaeotrichales & Phaeotrichaceae (3) \\
\hline & & Pleosporales & Acrocalymmaceae (1) \\
\hline & & & Aigialaceae (6) \\
\hline & & & Amniculicolaceae (6) \\
\hline & & & Amorosiaceae (4) \\
\hline & & & Anteagloniaceae (2) \\
\hline & & & Aquasubmersaceae (1) \\
\hline & & & Arthopyreniaceae (2) \\
\hline
\end{tabular}


Table 1 Continued.

\begin{tabular}{|c|c|c|}
\hline Phylum & 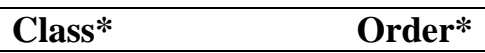 & Family* \\
\hline & & Ascocylindricaceae (1) \\
\hline & & Astrosphaeriellaceae (7) \\
\hline & & Bambusicolaceae (3) \\
\hline & & Biatriosporaceae (1) \\
\hline & & Camarosporiaceae (2) \\
\hline & & Camarosporidiellaceae (1) \\
\hline & & Caryosporaceae (1) \\
\hline & & Coniothyriaceae (5) \\
\hline & & Corynesporascaceae (2) \\
\hline & & Cryptocoryneaceae (1) \\
\hline & & Cucurbitariaceae (13) \\
\hline & & Cyclothyriellaceae (2) \\
\hline & & Dacampiaceae (6) \\
\hline & & Delitschiaceae (3) \\
\hline & & Diademaceae (2) \\
\hline & & Dictyosporiaceae (15) \\
\hline & & Didymellaceae (33) \\
\hline & & Didymosphaeriaceae (32) \\
\hline & & Dothidotthiaceae (7) \\
\hline & & Fuscostagonosporaceae (1) \\
\hline & & Fusculinaceae (2) \\
\hline & & Halojulellaceae (1) \\
\hline & & Halotthiaceae (6) \\
\hline & & Hermatomycetaceae (1) \\
\hline & & Hypsostromataceae (1) \\
\hline & & Latoruaceae (4) \\
\hline & & Lentimurisporaceae (2) \\
\hline & & Lentitheciaceae (13) \\
\hline & & Leptosphaeriaceae (13) \\
\hline & & Libertasomycetaceae (2) \\
\hline & & Ligninsphaeriaceae (2) \\
\hline & & Lindgomycetaceae (7) \\
\hline & & Lizoniaceae (1) \\
\hline & & Longipedicellataceae (3) \\
\hline & & Longiostiolaceae (1) \\
\hline & & Lophiostomataceae (28) \\
\hline & & Lophiotremataceae (7) \\
\hline & & Macrodiplodiopsidaceae (2) \\
\hline & & Massariaceae (3) \\
\hline & & Massarinaceae (8) \\
\hline & & Melanommataceae (35) \\
\hline & & Morosphaeriaceae (6) \\
\hline & & Mycoporaceae (1) \\
\hline & & Neocamarosporiaceae (2) \\
\hline & & Neohendersoniaceae (5) \\
\hline & & Neomassariaceae (1) \\
\hline & & Neomassarinaceae (2) \\
\hline & & Neophaeosphaeriaceae (1) \\
\hline & & Neopyrenochaetaceae (1) \\
\hline & & Nigrogranaceae (1) \\
\hline & & Occultibambusaceae (5) \\
\hline & & Ohleriaceae (1) \\
\hline & & Parabambusicolaceae (9) \\
\hline & & Paradictyoarthriniaceae (2) \\
\hline
\end{tabular}


Table 1 Continued.

\begin{tabular}{|c|c|c|c|}
\hline Phylum & Class* & Order* & Family* \\
\hline & & & Paralophiostomataceae (1) \\
\hline & & & Parapyrenochaetaceae (2) \\
\hline & & & Periconiaceae (4) \\
\hline & & & Phaeoseptaceae (2) \\
\hline & & & Phaeosphaeriaceae (82) \\
\hline & & & Pleomassariaceae (7) \\
\hline & & & Pleomonodictydaceae (2) \\
\hline & & & Pleosporaceae (23) \\
\hline & & & $\begin{array}{l}\text { Pseudoastrosphaeriellaceae } \\
\text { (3) }\end{array}$ \\
\hline & & & Pseudoberkleasmiaceae (1) \\
\hline & & & $\begin{array}{l}\text { Pseudocoleodictyosporaceae } \\
\text { (2) }\end{array}$ \\
\hline & & & Pseudolophiotremataceae (2) \\
\hline & & & Pseudomassarinaceae (1) \\
\hline & & & Pseudopyrenochaetaceae (1) \\
\hline & & & Pyrenochaetopsidaceae (3) \\
\hline & & & Roussoellaceae (12) \\
\hline & & & Salsugineaceae (2) \\
\hline & & & Shiraiaceae (3) \\
\hline & & & Sporormiaceae (9) \\
\hline & & & Striatiguttulaceae (2) \\
\hline & & & Sulcatisporaceae (6) \\
\hline & & & Teichosporaceae (13) \\
\hline & & & Testudinaceae (9) \\
\hline & & & Tetraplosphaeriaceae (8) \\
\hline & & & Thyridariaceae (7) \\
\hline & & & Torulaceae (6) \\
\hline & & & Trematosphaeriaceae (6) \\
\hline & & & Tzeananiaceae (1) \\
\hline & & & Wicklowiaceae (1) \\
\hline & & & Zopfiaceae (6) \\
\hline & & $\begin{array}{l}\text { Pleosporales genera } \\
\text { incertae sedis (48) }\end{array}$ & \\
\hline & & Stigmatodiscales & Stigmatodiscaceae (1) \\
\hline & & Strigulales & Strigulaceae (13) \\
\hline & & & Tenuitholiascaceae (1) \\
\hline & & Superstratomycetales & Superstratomycetaceae (1) \\
\hline & & Trypetheliales & Polycoccaceae (2) \\
\hline & & & Trypetheliaceae (19) \\
\hline & & Tubeufiales & Bezerromycetaceae (3) \\
\hline & & & Tubeufiaceae (46) \\
\hline & & & Wiesneriomycetaceae (6) \\
\hline & & Valsariales & Valsariaceae (3) \\
\hline & & Venturiales & Sympoventuriaceae (9) \\
\hline & & & Venturiaceae (14) \\
\hline & & $\begin{array}{l}\text { Venturiales genera } \\
\text { incertae sedis (3) }\end{array}$ & \\
\hline & & Zeloasperisporiales & Zeloasperisporiaceae (1) \\
\hline & & Incertae sedis $*$ & Alinaceae (1) \\
\hline & & & Argynnaceae (2) \\
\hline & & & Ascoporiaceae (1) \\
\hline & & & Aulographaceae (4) \\
\hline & & & Balladynaceae (3) \\
\hline
\end{tabular}


Table 1 Continued.

\begin{tabular}{|c|c|c|}
\hline Phylum & Order* & Family* \\
\hline & & Cleistosphaeraceae (1) \\
\hline & & Coccoideaceae (3) \\
\hline & & Cookellaceae (2) \\
\hline & & Dimeriaceae (1) \\
\hline & & Dubujianaceae (1) \\
\hline & & Dysrhynchisceae (1) \\
\hline & & Endosporiaceae (1) \\
\hline & & Englerulaceae (8) \\
\hline & & Eremomycetaceae (3) \\
\hline & & Eriomycetaceae (5) \\
\hline & & Homortomycetaceae (1) \\
\hline & & Hyalomeliolinaceae (1) \\
\hline & & Leptopeltidaceae (4) \\
\hline & & Macrovalsariaceae (1) \\
\hline & & Meliolinaceae (2) \\
\hline & & Mesnieraceae (3) \\
\hline & & Naetrocymbaceae (5) \\
\hline & & Nematotheciaceae (3) \\
\hline & & Neoparodiaceae (1) \\
\hline & & Palawaniaceae (1) \\
\hline & & Paranectriellaceae (2) \\
\hline & & Parodiellaceae (1) \\
\hline & & Perisporiopsidaceae (5) \\
\hline & & Phaeodimeriellaceae (1) \\
\hline & & Pododimeriaceae (2) \\
\hline & & Polyclypeolinaceae (1) \\
\hline & & Polystomellaceae (4) \\
\hline & & Protoscyphaceae (1) \\
\hline & & Pseudoperisporiaceae (4) \\
\hline & & Pseudorobillardaceae (1) \\
\hline & & Pyrenidiaceae (1) \\
\hline & & Seynesiopeltidaceae (1) \\
\hline & & Stomatogeneceae (1) \\
\hline & & Thyrinulaceae (3) \\
\hline & & Toroaceae (1) \\
\hline & & Trichopeltinaceae (7) \\
\hline & & Trichothyriaceae (4) \\
\hline & & Vizellaceae (3) \\
\hline
\end{tabular}

Dothideomycetes genera incertae sedis (278)

\begin{tabular}{|c|c|c|}
\hline Eurotiomycetes & Arachnomycetales & Arachnomycetaceae (2) \\
\hline & Chaetothyriales & Chaetothyriaceae (20) \\
\hline & & Coccodiniaceae (3) \\
\hline & & Cyphellophoraceae (2) \\
\hline & & Epibryaceae (1) \\
\hline & & Herpotrichiellaceae (16) \\
\hline & & Lyrommataceae (1) \\
\hline & & Microtheliopsidaceae (1) \\
\hline & & $\begin{array}{l}\text { Paracladophialophoraceae } \\
\text { (1) }\end{array}$ \\
\hline & & Pyrenotrichaceae (2) \\
\hline & & Trichomeriaceae (8) \\
\hline
\end{tabular}


Table 1 Continued.

\begin{tabular}{|c|c|c|c|}
\hline Phylum & Class* & Order* & Family* \\
\hline & & $\begin{array}{l}\text { Chaetothyriales genera } \\
\text { incertae sedis }(10)\end{array}$ & \\
\hline & & Coryneliales & Coryneliaceae (7) \\
\hline & & & Eremascaceae (1) \\
\hline & & Eurotiales & Aspergillaceae (15) \\
\hline & & & Elaphomycetaceae (2) \\
\hline & & & Thermoascaceae (2) \\
\hline & & & Trichocomaceae (7) \\
\hline & & Mycocaliciales & Mycocaliciaceae (7) \\
\hline & & Onygenales & Ajellomycetaceae (6) \\
\hline & & & Arthrodermataceae (10) \\
\hline & & & Ascosphaeraceae (3) \\
\hline & & & Gymnoascaceae (10) \\
\hline & & & Nannizziopsidaceae (1) \\
\hline & & & Onygenaceae (31) \\
\hline & & & Spiromastigaceae (4) \\
\hline & & $\begin{array}{l}\text { Onygenales genera } \\
\text { incertae sedis (3) }\end{array}$ & \\
\hline & & Phaeomoniellales & Celotheliaceae (10) \\
\hline & & Pyrenulales & Pyrenulaceae (12) \\
\hline & & $\begin{array}{l}\text { Pyrenulales genera } \\
\text { incertae sedis (2) }\end{array}$ & \\
\hline & & Sclerococcales & Dactylosporaceae (5) \\
\hline & & Verrucariales & Adelococcaceae (3) \\
\hline & & & Sarcopyreniaceae (1) \\
\hline & & & Verrucariaceae (52) \\
\hline & & $\begin{array}{l}\text { Verrucariales genera } \\
\text { incertae sedis (5) }\end{array}$ & \\
\hline & & Incertae sedis & Rhynchostomataceae (2) \\
\hline & $\begin{array}{l}\text { Eurotiomycetes } \\
\text { genus incertae sedis } \\
\text { (1) }\end{array}$ & & \\
\hline & Geoglossomycetes & Geoglossales & Geoglossaceae (7) \\
\hline & $\begin{array}{l}\text { Geoglossomycetes } \\
\text { genera incertae } \\
\text { sedis }(2)\end{array}$ & & \\
\hline & Laboulbeniomycetes & Herpomycetales & Herpomycetaceae (1) \\
\hline & & Laboulbeniales & Ceratomycetaceae (12) \\
\hline & & & Euceratomycetaceae (5) \\
\hline & & & Laboulbeniaceae (125) \\
\hline & & & Teratomyceteae (1) \\
\hline & & $\begin{array}{l}\text { Laboulbeniales genera } \\
\text { incertae sedis (3) }\end{array}$ & \\
\hline & & Pyxidiophorales & Pyxidiophoraceae (3) \\
\hline & $\begin{array}{l}\text { Laboulbeniomycetes } \\
\text { genus incertae sedis } \\
\text { (1) }\end{array}$ & & \\
\hline & Lecanoromycetes & Acarosporales & Acarosporaceae (11) \\
\hline & & & Eigleraceae (1) \\
\hline & & Baeomycetales & Arctomiaceae (4) \\
\hline & & & Arthrorhaphidaceae (1) \\
\hline & & & Baeomycetaceae (5) \\
\hline & & & Cameroniaceae (1) \\
\hline & & & Hymeneliaceae (3) \\
\hline & & & Protothelenellaceae (3) \\
\hline
\end{tabular}


Table 1 Continued.

\begin{tabular}{|c|c|c|c|}
\hline Phylum & Class* & Order* & Family* \\
\hline & & & Trapeliaceae (12) \\
\hline & & & Xylographaceae (4) \\
\hline & & Caliciales & Caliciaceae (36) \\
\hline & & & Physciaceae (18) \\
\hline & & Graphidales & Diploschistaceae(35) \\
\hline & & & Fissurinaceae (6) \\
\hline & & & Gomphillaceae (26) \\
\hline & & & Graphidaceae (31) \\
\hline & & & Redonographaceae (2) \\
\hline & & & Thelotremataceae (7) \\
\hline & & Gyalectales & Coenogoniaceae (1) \\
\hline & & & Gyalectaceae (3) \\
\hline & & & Phlyctidaceae (2) \\
\hline & & & Sagiolechiaceae (2) \\
\hline & & & Trichotheliaceae (7) \\
\hline & & Lecanorales & Bruceomycetaceae (2) \\
\hline & & & Catillariaceae (5) \\
\hline & & & Cladoniaceae (22) \\
\hline & & & Gypsoplacaceae (1) \\
\hline & & & Haematommataceae (1) \\
\hline & & & Lecanoraceae (28) \\
\hline & & & Malmideaceae (7) \\
\hline & & & Megalariaceae (2) \\
\hline & & & Parmeliaceae (71) \\
\hline & & & Pilocarpaceae (32) \\
\hline & & & Psilolechiaceae (1) \\
\hline & & & Psoraceae (6) \\
\hline & & & Ramalinaceae (37) \\
\hline & & & Ramboldiaceae (1) \\
\hline & & & Scoliciosporaceae (1) \\
\hline & & & Sphaerophoraceae (6) \\
\hline & & & Tephromelataceae (4) \\
\hline & & $\begin{array}{l}\text { Lecanorales genera } \\
\text { incertae sedis (14) }\end{array}$ & \\
\hline & & Lecideales & Lecideaceae (29) \\
\hline & & & Lopadiaceae (1) \\
\hline & & Leprocaulales & Leprocaulaceae (3) \\
\hline & & Micropeltidales & Micropeltidaceae (12) \\
\hline & & Ostropales & Odontotremataceae (10) \\
\hline & & & Phaneromycetaceae (1) \\
\hline & & & Spirographaceae (1) \\
\hline & & & Stictidaceae (28) \\
\hline & & $\begin{array}{l}\text { Ostropales genera } \\
\text { incertae sedis (6) }\end{array}$ & \\
\hline & & Peltigerales & Coccocarpiaceae (3) \\
\hline & & & Collemataceae (8) \\
\hline & & & Koerberiaceae (3) \\
\hline & & & Massalongiaceae (3) \\
\hline & & & Pannariaceae (27) \\
\hline & & & Peltigeraceae (15) \\
\hline & & & Placynthiaceae (3) \\
\hline & & & Vahliellaceae (1) \\
\hline & & Pertusariales & Agyriaceae (2) \\
\hline & & & Coccotremataceae (3) \\
\hline
\end{tabular}


Table 1 Continued.

\begin{tabular}{|c|c|c|c|}
\hline Phylum & Class* & Order* & Family* \\
\hline & & & Icmadophilaceae (7) \\
\hline & & & Megasporaceae (6) \\
\hline & & & Microcaliciaceae (1) \\
\hline & & & Ochrolechiaceae (1) \\
\hline & & & Pertusariaceae (3) \\
\hline & & & Varicellariaceae (1) \\
\hline & & & Variolariaceae (1) \\
\hline & & Rhizocarpales & Rhizocarpaceae (4) \\
\hline & & Sarrameanales & Sarrameanaceae (2) \\
\hline & & Schaereriales & Schaereriaceae (1) \\
\hline & & Sporastatiales & Sporastatiaceae (2) \\
\hline & & Teloschistales & Brigantiaeaceae (2) \\
\hline & & & Megalosporaceae (3) \\
\hline & & & Teloschistaceae (63) \\
\hline & & $\begin{array}{l}\text { Teloschistales genus } \\
\text { incertae sedis (1) }\end{array}$ & \\
\hline & & Thelenellales & Thelenellaceae (3) \\
\hline & & Turquoiseomycetales & Turquoiseomycetaceae (1) \\
\hline & & Umbilicariales & Elixiaceae (2) \\
\hline & & & Fuscideaceae (4) \\
\hline & & & Ophioparmaceae (3) \\
\hline & & & Ropalosporaceae (1) \\
\hline & & & Umbilicariaceae (3) \\
\hline & & Incertae sedis & Epigloeaceae (1) \\
\hline & $\begin{array}{l}\text { Lecanoromycetes } \\
\text { genera incertae } \\
\text { sedis }(15)\end{array}$ & & \\
\hline & Leotiomycetes & Chaetomellales & Chaetomellaceae (4) \\
\hline & & Cyttariales & Cyttariaceae (1) \\
\hline & & Helotiales & Amicodiscaceae (1) \\
\hline & & & Arachnopezizaceae (5) \\
\hline & & & Ascocorticiaceae (1) \\
\hline & & & Ascodichaenaceae (2) \\
\hline & & & Bloxamiaceae (1) \\
\hline & & & Bryoglossaceae (3) \\
\hline & & & Calloriaceae (14) \\
\hline & & & Cenangiaceae (11) \\
\hline & & & Chlorociboriaceae (1) \\
\hline & & & Chlorospleniaceae (1) \\
\hline & & & Chrysodiscaceae (1) \\
\hline & & & Cordieritidaceae (18) \\
\hline & & & Dermateaceae (14) \\
\hline & & & Discinellaceae (12) \\
\hline & & & Drepanopezizaceae (8) \\
\hline & & & Erysiphaceae (20) \\
\hline & & & Gelatinodiscaceae (9) \\
\hline & & & Godroniaceae (5) \\
\hline & & & Helotiaceae (33) \\
\hline & & & Heterosphaeriaceae (1) \\
\hline & & & Hyaloscyphaceae (37) \\
\hline & & & Lachnaceae (18) \\
\hline & & & Leptodontidiaceae (1) \\
\hline & & & Loramycetaceae (2) \\
\hline & & & Mitrulaceae (1) \\
\hline
\end{tabular}


Table 1 Continued.

\begin{tabular}{|c|c|c|c|}
\hline Phylum & Class* & Order* & Family* \\
\hline & & & Mollisiaceae (18) \\
\hline & & & Myxotrichaceae (3) \\
\hline & & & Neolauriomycetaceae (3) \\
\hline & & & Pezizellaceae (23) \\
\hline & & & Ploettnerulaceae (12) \\
\hline & & & Rutstroemiaceae (7) \\
\hline & & & Sclerotiniaceae (31) \\
\hline & & & Vibrisseaceae (5) \\
\hline & & $\begin{array}{l}\text { Helotiales genera } \\
\text { incertae sedis }(144)\end{array}$ & \\
\hline & & Lahmiales & Lahmiaceae (1) \\
\hline & & Lauriomycetales & Lauriomycetaceae (1) \\
\hline & & Leotiales & Cochlearomycetaceae (2) \\
\hline & & & Leotiaceae (4) \\
\hline & & & Mniaeciaceae (2) \\
\hline & & & Tympanidaceae (7) \\
\hline & & $\begin{array}{l}\text { Leotiales genera } \\
\text { incertae sedis (3) }\end{array}$ & \\
\hline & & Lichinodiales & Lichinodiaceae (1) \\
\hline & & Marthamycetales & Marthamycetaceae (9) \\
\hline & & Medeolariales & Medeolariaceae (1) \\
\hline & & Micraspidales & Micraspidaceae (1) \\
\hline & & Phacidiales & Helicogoniaceae (7) \\
\hline & & & Phacidiaceae (9) \\
\hline & & $\begin{array}{l}\text { Phacidiales genus } \\
\text { incertae sedis (1) }\end{array}$ & \\
\hline & & Rhytismatales & Cudoniaceae (2) \\
\hline & & & Rhytismataceae (52) \\
\hline & & & Triblidiaceae (2) \\
\hline & & $\begin{array}{l}\text { Rhytismatales genera } \\
\text { incertae sedis (9) }\end{array}$ & \\
\hline & & Thelebolales & Pseudeurotiaceae (8) \\
\hline & & & Thelebolaceae (10) \\
\hline & & $\begin{array}{l}\text { Thelebolales genera } \\
\text { incertae sedis (3) }\end{array}$ & \\
\hline & $\begin{array}{l}\text { Leotiomycetes } \\
\text { genera incertae } \\
\text { sedis }(20)\end{array}$ & & \\
\hline & Lichinomycetes & Lichinales & Gloeoheppiaceae (3) \\
\hline & & & Lichinaceae (43) \\
\hline & & & Peltulaceae (1) \\
\hline & Neolectomycetes & Neolectales & Neolectaceae (1) \\
\hline & Orbiliomycetes & Orbiliales & Orbiliaceae (12) \\
\hline & & $\begin{array}{l}\text { Orbiliales genus } \\
\text { incertae sedis }(1)\end{array}$ & \\
\hline & $\begin{array}{l}\text { Orbiliomycetes } \\
\text { genus incertae sedis } \\
\text { (1) }\end{array}$ & & \\
\hline & Pezizomycetes & Pezizales & Ascobolaceae (5) \\
\hline & & & Ascodesmidaceae (10) \\
\hline & & & Caloscyphaceae (1) \\
\hline & & & Chorioactidaceae (6) \\
\hline & & & Discinaceae (5) \\
\hline & & & Glaziellaceae (1) \\
\hline
\end{tabular}


Table 1 Continued.

\begin{tabular}{|c|c|c|c|}
\hline Phylum & Class* & Order* & Family* \\
\hline & & & Helvellaceae (5) \\
\hline & & & Kallistoskyphaceae (1) \\
\hline & & & Karstenellaceae (1) \\
\hline & & & Morchellaceae (7) \\
\hline & & & Pezizaceae (38) \\
\hline & & & Pseudombrophilaceae (4) \\
\hline & & & Pulvinulaceae (3) \\
\hline & & & Pyronemataceae (61) \\
\hline & & & Rhizinaceae (3) \\
\hline & & & Sarcoscyphaceae (12) \\
\hline & & & Sarcosomataceae (9) \\
\hline & & & Strobiloscyphaceae (1) \\
\hline & & & Tarzettaceae (6) \\
\hline & & & Tuberaceae (7) \\
\hline & & $\begin{array}{l}\text { Pezizales genera incertae } \\
\text { sedis (18) }\end{array}$ & \\
\hline & Pneumocystomycetes & Pneumocystidales & Pneumocystidaceae (1) \\
\hline & Saccharomycetes & Saccharomycetales & Alloascoideaceae (1) \\
\hline & & & Ascoideaceae (1) \\
\hline & & & Cephaloascaceae (1) \\
\hline & & & Debaryomycetaceae (13) \\
\hline & & & Dipodascaceae (5) \\
\hline & & & Lipomycetaceae (5) \\
\hline & & & Metschnikowiaceae (3) \\
\hline & & & Phaffomycetaceae (5) \\
\hline & & & Pichiaceae (10) \\
\hline & & & Saccharomycetaceae (16) \\
\hline & & & Saccharomycodaceae (2) \\
\hline & & & Saccharomycopsidaceae (2) \\
\hline & & & Trichomonascaceae (9) \\
\hline & & & Trigonopsidaceae (3) \\
\hline & & $\begin{array}{l}\text { Saccharomycetales } \\
\text { genera incertae sedis (21) }\end{array}$ & \\
\hline & Schizosaccharomycetes & Schizosaccharomycetales & $\begin{array}{l}\text { Schizosaccharomycetaceae } \\
\text { (1) }\end{array}$ \\
\hline & Sordariomycetes & Amphisphaeriales & Amphisphaeriaceae (3) \\
\hline & & & Apiosporaceae (5) \\
\hline & & & Beltraniaceae $(9)$ \\
\hline & & & $\begin{array}{l}\text { Clypeophysalosporaceae } \\
\text { (4) }\end{array}$ \\
\hline & & & Cylindriaceae (1) \\
\hline & & & Hansfordiaceae (1) \\
\hline & & & Hyponectriaceae (17) \\
\hline & & & Iodosphaeriaceae (1) \\
\hline & & & Melogrammataceae (1) \\
\hline & & & Phlogicylindriaceae (3) \\
\hline & & & Pseudomassariaceae (4) \\
\hline & & & Pseudotruncatellaceae (1) \\
\hline & & & Sporocadaceae (33) \\
\hline & & & Vialaeaceae (1) \\
\hline & & $\begin{array}{l}\text { Amphisphaeriales genus } \\
\text { incertae sedis (1) }\end{array}$ & \\
\hline & & Amplistromatales & Amplistromataceae (3) \\
\hline
\end{tabular}


Table 1 Continued.

\begin{tabular}{|c|c|c|c|}
\hline Phylum & Class* & Order* & Family* \\
\hline & & & Catabotryaceae (1) \\
\hline & & Annulatascales & Annulatascaceae (10) \\
\hline & & $\begin{array}{l}\text { Annulatascales genus } \\
\text { incertae sedis (1) }\end{array}$ & \\
\hline & & Atractosporales & Atractosporaceae (2) \\
\hline & & & Conlariaceae (2) \\
\hline & & & Pseudoproboscisporaceae (2) \\
\hline & & Boliniales & Boliniaceae $(9)$ \\
\hline & & Calosphaeriales & Calosphaeriaceae (4) \\
\hline & & & Jobellisiaceae (1) \\
\hline & & & Pleurostomataceae (1) \\
\hline & & $\begin{array}{l}\text { Calosphaeriales genera } \\
\text { incertae sedis (4) }\end{array}$ & \\
\hline & & Cephalothecales & Cephalothecaceae (5) \\
\hline & & Chaetosphaeriales & Chaetosphaeriaceae (44) \\
\hline & & & Helminthosphaeriaceae (7) \\
\hline & & & Leptosporellaceae (1) \\
\hline & & & Leptosporellaceae (1) \\
\hline & & & Linocarpaceae (2) \\
\hline & & $\begin{array}{l}\text { Chaetosphaeriales } \\
\text { genera incertae sedis } \\
\text { (7) }\end{array}$ & \\
\hline & & Coniochaetales & Coniochaetaceae (2) \\
\hline & & & Cordanaceae (1) \\
\hline & & $\begin{array}{l}\text { Coniochaetales genera } \\
\text { incertae sedis (2) }\end{array}$ & \\
\hline & & Conioscyphales & Conioscyphaceae (1) \\
\hline & & Coronophorales & Bertiaceae (2) \\
\hline & & & Ceratostomataceae (14) \\
\hline & & & Chaetosphaerellaceae (3) \\
\hline & & & Coronophoraceae (1) \\
\hline & & & Nitschkiaceae (14) \\
\hline & & & Scortechiniaceae (10) \\
\hline & & $\begin{array}{l}\text { Coronophorales genera } \\
\text { incertae sedis (2) }\end{array}$ & \\
\hline & & Delonicicolales & Delonicicolaceae (2) \\
\hline & & Diaporthales & Apiosporopsidaceae (1) \\
\hline & & & Apoharknessiaceae (2) \\
\hline & & & Asterosporiaceae (1) \\
\hline & & & Auratiopycnidiellaceae (1) \\
\hline & & & Coryneaceae (1) \\
\hline & & & Cryphonectriaceae (28) \\
\hline & & & Cytosporaceae (6) \\
\hline & & & Diaporthaceae (15) \\
\hline & & & Diaporthosporellaceae (1) \\
\hline & & & Diaporthostomataceae (1) \\
\hline & & & Dwiroopaceae (1) \\
\hline & & & Erythrogloeaceae (4) \\
\hline & & & Gnomoniaceae (36) \\
\hline & & & Harknessiaceae (2) \\
\hline & & & Juglanconidaceae (1) \\
\hline & & & Lamproconiaceae (2) \\
\hline
\end{tabular}


Table 1 Continued.

\begin{tabular}{|c|c|c|c|}
\hline Phylum & Class* & Order* & Family* \\
\hline & & & Macrohilaceae (1) \\
\hline & & & Melanconidaceae (1) \\
\hline & & & Melanconiellaceae (7) \\
\hline & & & Neomelanconiellaceae (1) \\
\hline & & & $\begin{array}{l}\text { Phaeoappendicosporaceae } \\
\text { (2) }\end{array}$ \\
\hline & & & Prosopidicolaceae (1) \\
\hline & & & Pseudomelanconidaceae (2) \\
\hline & & & Pseudoplagiostomataceae (1) \\
\hline & & & Schizoparmaceae (1) \\
\hline & & & Stilbosporaceae (4) \\
\hline & & & Sydowiellaceae (16) \\
\hline & & & Synnemasporellaceae (1) \\
\hline & & & Tubakiaceae (8) \\
\hline & & $\begin{array}{l}\text { Diaporthales genera } \\
\text { incertae sedis (36) }\end{array}$ & \\
\hline & & Distoseptisporales & Distoseptisporaceae (1) \\
\hline & & Falcocladiales & Falcocladiaceae (1) \\
\hline & & Fuscosporellales & Fuscosporellaceae (6) \\
\hline & & Glomerellales & Australiascaceae (1) \\
\hline & & & Glomerellaceae (1) \\
\hline & & & Malaysiascaceae (1) \\
\hline & & & Plectosphaerellaceae (24) \\
\hline & & & Reticulascaceae (4) \\
\hline & & $\begin{array}{l}\text { Glomerellales genus } \\
\text { incertae sedis (1) }\end{array}$ & \\
\hline & & Hypocreales & Bionectriaceae (47) \\
\hline & & & Calcarisporiaceae (1) \\
\hline & & & Clavicipitaceae (42) \\
\hline & & & Cocoonihabitaceae (1) \\
\hline & & & Cordycipitaceae (17) \\
\hline & & & Flammocladiellaceae (1) \\
\hline & & & Hypocreaceae (17) \\
\hline & & & Myrotheciomycetaceae (4) \\
\hline & & & Nectriaceae (69) \\
\hline & & & Niessliaceae (21) \\
\hline & & & Ophiocordycipitaceae (10) \\
\hline & & & Sarocladiaceae (2) \\
\hline & & & Stachybotryaceae (39) \\
\hline & & & Tilachlidiaceae (3) \\
\hline & & $\begin{array}{l}\text { Hypocreales genera } \\
\text { incertae sedis (29) }\end{array}$ & \\
\hline & & Jobellisiales & Jobellisiaceae (1) \\
\hline & & Koralionastetales & Koralionastetaceae (2) \\
\hline & & Lulworthiales & Lulworthiaceae (15) \\
\hline & & Magnaporthales & Ceratosphaeriaceae (1) \\
\hline & & & Magnaporthaceae (22) \\
\hline & & & Ophioceraceae (1) \\
\hline & & & Pseudohalonectriaceae (1) \\
\hline & & & Pyriculariaceae (11) \\
\hline & & Meliolales & Armatellaceae (1) \\
\hline & & & Meliolaceae (8) \\
\hline & & Microascales & Ceratocystidaceae (11) \\
\hline
\end{tabular}


Table 1 Continued.

\begin{tabular}{|c|c|c|c|}
\hline Phylum & Class* & Order* & Family* \\
\hline & & & Chadefaudiellaceae (2) \\
\hline & & & Gondwanamycetaceae (2) \\
\hline & & & Graphiaceae (1) \\
\hline & & & Halosphaeriaceae (66) \\
\hline & & & Microascaceae (23) \\
\hline & & & Triadelphiaceae (2) \\
\hline & & $\begin{array}{l}\text { Microascales genera } \\
\text { incertae sedis }(6)\end{array}$ & \\
\hline & & Myrmecridiales & Myrmecridiaceae (2) \\
\hline & & & Xenodactylariaceae (1) \\
\hline & & Ophiostomatales & Kathistaceae (2) \\
\hline & & & Ophiostomataceae (13) \\
\hline & & Pararamichloridiales & Pararamichloridiaceae (1) \\
\hline & & Parasympodiellales & Parasympodiellaceae (1) \\
\hline & & Phomatosporales & Phomatosporaceae (3) \\
\hline & & Phyllachorales & Phaeochoraceae (4) \\
\hline & & & Phaeochorellaceae (1) \\
\hline & & & Phyllachoraceae (54) \\
\hline & & & Telimenaceae (1) \\
\hline & & $\begin{array}{l}\text { Phyllachorales genus } \\
\text { incertae sedis (1) }\end{array}$ & \\
\hline & & Pisorisporiales & Pisorisporiaceae (2) \\
\hline & & Pleurotheciales & Pleurotheciaceae (11) \\
\hline & & Pseudodactylariales & Pseudodactylariaceae (1) \\
\hline & & Savoryellales & Savoryellaceae (4) \\
\hline & & Sordariales & Chaetomiaceae (37) \\
\hline & & & Lasiosphaeriaceae (32) \\
\hline & & & Podosporaceae (3) \\
\hline & & & Sordariaceae (7) \\
\hline & & $\begin{array}{l}\text { Sordariales genera } \\
\text { incertae sedis }(22)\end{array}$ & \\
\hline & & Spathulosporales & Hispidicarpomycetaceae (1) \\
\hline & & & Spathulosporaceae (2) \\
\hline & & Sporidesmiales & Sporidesmiaceae (1) \\
\hline & & Tirisporellales & Tirisporellaceae (3) \\
\hline & & Togniniales & Togniniaceae (2) \\
\hline & & Torpedosporales & Etheirophoraceae (2) \\
\hline & & & Juncigenaceae (5) \\
\hline & & & Torpedosporaceae (1) \\
\hline & & Tracyllalales & Tracyllaceae (1) \\
\hline & & Vermiculariopsiellales & Vermiculariopsiellaceae (1) \\
\hline & & Xenospadicoidales & Xenospadicoidaceae (5) \\
\hline & & Xylariales & Anungitiomycetaceae (1) \\
\hline & & & Barrmaeliaceae (2) \\
\hline & & & Castanediellaceae (1) \\
\hline & & & Clypeosphaeriaceae (6) \\
\hline & & & Coniocessiaceae (2) \\
\hline & & & Diatrypaceae (20) \\
\hline & & & Fasciatisporaceae (1) \\
\hline & & & Graphostromataceae (5) \\
\hline & & & Hypoxylaceae (19) \\
\hline & & & Induratiaceae (2) \\
\hline & & & Leptosilliaceae (1) \\
\hline & & & Lopadostomataceae (4) \\
\hline
\end{tabular}


Table 1 Continued.

\begin{tabular}{|c|c|c|c|}
\hline Phylum & Class* & Order* & Family* \\
\hline & & & Microdochiaceae (3) \\
\hline & & & Myelospermataceae (1) \\
\hline & & & Nothodactylariaceae (1) \\
\hline & & & Oxydothidaceae (1) \\
\hline & & & Polystigmataceae (1) \\
\hline & & & Pseudosporidesmiaceae (1) \\
\hline & & & Requienellaceae (4) \\
\hline & & & Xyladictyochaetaceae (1) \\
\hline & & & Xylariaceae (32) \\
\hline & & & Zygosporiaceae (1) \\
\hline & & $\begin{array}{l}\text { Xylariales genera } \\
\text { incertae sedis }(56)\end{array}$ & \\
\hline & & Incertae sedis & Acrodictyaceae (1) \\
\hline & & & Barbatosphaeriaceae (3) \\
\hline & & & Batistiaceae (1) \\
\hline & & & Cainiaceae (6) \\
\hline & & & Junewangiaceae (2) \\
\hline & & & Lautosporaceae (1) \\
\hline & & & Obryzaceae (1) \\
\hline & & & Papulosaceae (4) \\
\hline & & & Rhamphoriaceae (4) \\
\hline & & & Thyridiaceae (2) \\
\hline & & & Trichosphaeriaceae (10) \\
\hline & & & Woswasiaceae (3) \\
\hline & $\begin{array}{l}\text { Sordariomycetes } \\
\text { genera incertae } \\
\text { sedis }(131)\end{array}$ & & \\
\hline & Taphrinomycetes & Taphrinales & Protomycetaceae (6) \\
\hline & & & Taphrinaceae (1) \\
\hline & Xylobotryomycetes & Xylobotryales & Cirrosporiaceae (1) \\
\hline & & & Xylobotryaceae (1) \\
\hline & Xylonomycetes & Symbiotaphrinales & Symbiotaphrinaceae (1) \\
\hline & & Xylonales & Xylonaceae (2) \\
\hline & Incertae sedis & Thelocarpales & Thelocarpaceae (2) \\
\hline & & Vezdaeales & Vezdaeaceae (1) \\
\hline & Incertae sedis & Incertae sedis & Aphanopsidaceae (2) \\
\hline & & & Diporothecaceae (1) \\
\hline & & & Eoterfeziaceae (2) \\
\hline & & & Harpidiaceae (2) \\
\hline & & & Mucomassariaceae (1) \\
\hline & & & Saccardiaceae (7) \\
\hline & & & Seuratiaceae (2) \\
\hline & & & Strangosporaceae (1) \\
\hline $\begin{array}{l}\text { Ascomycota genera } \\
\text { incertae sedis } \\
(1485)\end{array}$ & & & \\
\hline Basidiobolomycota & Basidiobolomycetes & Basidiobolales & Basidiobolaceae (2) \\
\hline Basidiomycota & Agaricomycetes & Agaricales & Agaricaceae (59) \\
\hline & & & Amanitaceae (5) \\
\hline & & & Biannulariaceae (7) \\
\hline & & & Bolbitiaceae (15) \\
\hline & & & Broomeiaceae (1) \\
\hline & & & Chromocyphellaceae (1) \\
\hline & & & Clavariaceae (10) \\
\hline
\end{tabular}


Table 1 Continued.

\begin{tabular}{|c|c|c|c|}
\hline Phylum & Class* & Order* & Family* \\
\hline & & & Cortinariaceae (5) \\
\hline & & & Crassisporiaceae (2) \\
\hline & & & Crepidotaceae (6) \\
\hline & & & Cyphellaceae (16) \\
\hline & & & Cystostereaceae (7) \\
\hline & & & Entolomataceae (7) \\
\hline & & & Hemigasteraceae (1) \\
\hline & & & Hydnangiaceae (4) \\
\hline & & & Hygrophoraceae (26) \\
\hline & & & Hymenogastraceae (10) \\
\hline & & & Inocybaceae (3) \\
\hline & & & Limnoperdaceae (1) \\
\hline & & & Lycoperdaceae (7) \\
\hline & & & Lyophyllaceae (18) \\
\hline & & & Macrocystidiaceae (1) \\
\hline & & & Marasmiaceae (10) \\
\hline & & & Mycenaceae (16) \\
\hline & & & Mythicomycetaceae (2) \\
\hline & & & Niaceae (9) \\
\hline & & & Omphalotaceae (14) \\
\hline & & & Physalacriaceae (28) \\
\hline & & & Pleurotaceae (5) \\
\hline & & & Pluteaceae (3) \\
\hline & & & Porotheleaceae (2) \\
\hline & & & Psathyrellaceae (13) \\
\hline & & & Pseudoclitocybaceae (7) \\
\hline & & & Pterulaceae (13) \\
\hline & & & Schizophyllaceae (3) \\
\hline & & & Stephanosporaceae (5) \\
\hline & & & Strophariaceae (11) \\
\hline & & & Tricholomataceae (10) \\
\hline & & & Tubariaceae (7) \\
\hline & & & Typhulaceae (4) \\
\hline & & $\begin{array}{l}\text { Agaricales genera } \\
\text { incertae sedis (134) }\end{array}$ & \\
\hline & & Amylocorticiales & Amylocorticiaceae (11) \\
\hline & & Atheliales & Atheliaceae (20) \\
\hline & & Auriculariales & Auriculariaceae (12) \\
\hline & & & Hyaloriaceae (3) \\
\hline & & $\begin{array}{l}\text { Auriculariales genera } \\
\text { incertae sedis (31) }\end{array}$ & \\
\hline & & Boletales & Boletaceae (92) \\
\hline & & & Boletinellaceae (2) \\
\hline & & & Calostomataceae (1) \\
\hline & & & Coniophoraceae (5) \\
\hline & & & Diplocystidiaceae (4) \\
\hline & & & Gasterellaceae (1) \\
\hline & & & Gomphidiaceae (4) \\
\hline & & & Gyroporaceae (1) \\
\hline & & & Hygrophoropsidaceae (2) \\
\hline & & & Paxillaceae (10) \\
\hline & & & Protogastraceae (1) \\
\hline & & & Rhizopogonaceae (3) \\
\hline & & & Sclerodermataceae (5) \\
\hline
\end{tabular}


Table 1 Continued.

\begin{tabular}{|c|c|c|c|}
\hline \multirow[t]{4}{*}{ Phylum } & Class* & Order* & Family* \\
\hline & & & Serpulaceae (3) \\
\hline & & & Suillaceae (2) \\
\hline & & & Tapinellaceae (3) \\
\hline & & $\begin{array}{l}\text { Boletales genera } \\
\text { incertae sedis (4) }\end{array}$ & \\
\hline & & Cantharellales & Aphelariaceae (3) \\
\hline & & & Botryobasidiaceae (5) \\
\hline & & & Ceratobasidiaceae (6) \\
\hline & & & Hydnaceae (21) \\
\hline & & & Oliveoniaceae (1) \\
\hline & & & Tulasnellaceae (2) \\
\hline & Bartheletiomycetes & Bartheletiales & Bartheletiaceae (1) \\
\hline & & $\begin{array}{l}\text { Cantharellales genera } \\
\text { incertae sedis }(8)\end{array}$ & \\
\hline & & Corticiales & Corticiaceae (12) \\
\hline & & & Dendrominiaceae (1) \\
\hline & & & Punctulariaceae (3) \\
\hline & & & Vuilleminiaceae (3) \\
\hline & & $\begin{array}{l}\text { Corticiales genera } \\
\text { incertae sedis }(7)\end{array}$ & \\
\hline & & Geastrales & Geastraceae (7) \\
\hline & & & Sclerogastraceae (1) \\
\hline & & $\begin{array}{l}\text { Geastrales genus } \\
\text { incertae sedis }(1)\end{array}$ & \\
\hline & & Gloeophyllales & Gloeophyllaceae (12) \\
\hline & & $\begin{array}{l}\text { Gloeophyllales genus } \\
\text { incertae sedis }(1)\end{array}$ & \\
\hline & & Gomphales & Clavariadelphaceae (2) \\
\hline & & & Gomphaceae (14) \\
\hline & & & Lentariaceae (3) \\
\hline & & Hymenochaetales & Hymenochaetaceae (40) \\
\hline & & & Neoantrodiellaceae (1) \\
\hline & & & Nigrofomitaceae (1) \\
\hline & & & Oxyporaceae (1) \\
\hline & & & Rickenellaceae (8) \\
\hline & & & Schizoporaceae (13) \\
\hline & & $\begin{array}{l}\text { Hymenochaetales } \\
\text { genera incertae sedis } \\
\text { (15) }\end{array}$ & \\
\hline & & Hysterangiales & Gallaceaceae (3) \\
\hline & & & Hysterangiaceae (4) \\
\hline & & & Mesophelliaceae (8) \\
\hline & & & Phallogastraceae (2) \\
\hline & & & Trappeaceae (3) \\
\hline & & Jaapiales & Jaapiaceae (1) \\
\hline & & Lepidostromatales & Lepidostromataceae (3) \\
\hline & & Phallales & Claustulaceae (5) \\
\hline & & & Gastrosporiaceae (1) \\
\hline & & & Phallaceae (26) \\
\hline & & $\begin{array}{l}\text { Phallales genera } \\
\text { incertae sedis ( } 2 \text { ) }\end{array}$ & \\
\hline & & Polyporales & Cerrenaceae (4) \\
\hline & & & Dacryobolaceae (7) \\
\hline & & & Fomitopsidaceae (25) \\
\hline
\end{tabular}


Table 1 Continued.

\begin{tabular}{|c|c|c|c|}
\hline Phylum & Class* & Order* & Family* \\
\hline & & & Fragiliporiaceae (1) \\
\hline & & & Gelatoporiaceae (4) \\
\hline & & & Grifolaceae (2) \\
\hline & & & Hyphodermataceae (1) \\
\hline & & & Incrustoporiaceae (5) \\
\hline & & & Irpicaceae (14) \\
\hline & & & Ischnodermataceae (1) \\
\hline & & & Laetiporaceae (3) \\
\hline & & & Meripilaceae (3) \\
\hline & & & Meruliaceae (22) \\
\hline & & & Panaceae (2) \\
\hline & & & Phanerochaetaceae (18) \\
\hline & & & Podoscyphaceae (3) \\
\hline & & & Polyporaceae (85) \\
\hline & & & Sparassidaceae (3) \\
\hline & & & Steccherinaceae (22) \\
\hline & & $\begin{array}{l}\text { Polyporales genera } \\
\text { incertae sedis }(67)\end{array}$ & \\
\hline & & Russulales & Albatrellaceae (8) \\
\hline & & & Auriscalpiaceae (6) \\
\hline & & & Bondarzewiaceae (9) \\
\hline & & & Echinodontiaceae (3) \\
\hline & & & Hericiaceae (6) \\
\hline & & & Hybogasteraceae (1) \\
\hline & & & Peniophoraceae (16) \\
\hline & & & Russulaceae (7) \\
\hline & & & Stereaceae (22) \\
\hline & & & Xenasmataceae (3) \\
\hline & & $\begin{array}{l}\text { Russulales genera } \\
\text { incertae sedis (15) }\end{array}$ & \\
\hline & & Sebacinales & Sebacinaceae (8) \\
\hline & & & Serendipitaceae (1) \\
\hline & & Stereopsidales & Stereopsidaceae (1) \\
\hline & & Thelephorales & Bankeraceae (5) \\
\hline & & & Thelephoraceae (9) \\
\hline & & $\begin{array}{l}\text { Thelephorales genus } \\
\text { incertae sedis (1) }\end{array}$ & \\
\hline & & Trechisporales & Hydnodontaceae (13) \\
\hline & & Tremellodendropsidales & $\begin{array}{l}\text { Tremellodendropsidaceae } \\
\text { (1) }\end{array}$ \\
\hline & $\begin{array}{l}\text { Agaricomycetes } \\
\text { genera incertae } \\
\text { sedis }(40)\end{array}$ & & \\
\hline & $\begin{array}{l}\text { Agaricostilbomycete } \\
s\end{array}$ & Agaricostilbales & Agaricostilbaceae (3) \\
\hline & & & Chionosphaeraceae (5) \\
\hline & & & Kondoaceae (2) \\
\hline & & & Ruineniaceae (1) \\
\hline & & $\begin{array}{l}\text { Agaricostilbales genera } \\
\text { incertae sedis (2) }\end{array}$ & \\
\hline & Atractiellomycetes & Atractiellales & Atractogloeaceae (1) \\
\hline & & & Hoehnelomycetaceae (2) \\
\hline & & & Phleogenaceae (7) \\
\hline & Classiculomycetes & Classiculales & Classiculaceae (2) \\
\hline
\end{tabular}


Table 1 Continued.

\begin{tabular}{|c|c|c|c|}
\hline Phylum & Class* & Order* & Family* \\
\hline & Cryptomycocolacomycetes & Cryptomycocolacales & Cryptomycocolacaceae (2) \\
\hline & Cystobasidiomycetes & Buckleyzymales & Buckleyzymaceae (1) \\
\hline & & Cystobasidiales & Cystobasidiaceae (3) \\
\hline & & Erythrobasidiales & Erythrobasidiaceae (2) \\
\hline & & $\begin{array}{l}\text { Erythrobasidiales } \\
\text { genera incertae sedis } \\
\text { (3) }\end{array}$ & \\
\hline & & Naohideales & Naohideaceae (1) \\
\hline & & Sakaguchiales & Sakaguchiaceae (1) \\
\hline & & Incertae sedis & Microsporomycetaceae (1) \\
\hline & & Incertae sedid & Symmetrosporaceae $(1)$ \\
\hline & $\begin{array}{l}\text { Cystobasidiomycetes genus } \\
\text { incertae sedis (1) }\end{array}$ & & \\
\hline & Dacrymycetes & Dacrymycetales & Cerinomycetaceae (1) \\
\hline & & & Dacrymycetaceae (10) \\
\hline & & Unilacrymales & Unilacrymaceae (1) \\
\hline & Exobasidiomycetes & Ceraceosorales & Ceraceosoraceae (1) \\
\hline & & Doassansiales & Doassansiaceae (11) \\
\hline & & & Melaniellaceae (1) \\
\hline & & & Rhamphosporaceae (1) \\
\hline & & Entylomatales & Entylomataceae (2) \\
\hline & & Exobasidiales & Brachybasidiaceae (5) \\
\hline & & & Cryptobasidiaceae (6) \\
\hline & & & Exobasidiaceae (4) \\
\hline & & & Graphiolaceae (2) \\
\hline & & & Laurobasidiaceae (1) \\
\hline & & Georgefischeriales & Eballistraceae (1) \\
\hline & & & Georgefischeriaceae (2) \\
\hline & & & Gjaerumiaceae (1) \\
\hline & & & Tilletiariaceae (3) \\
\hline & & Golubeviales & Golubeviaceae (1) \\
\hline & & Microstromatales & Microstromataceae (1) \\
\hline & & & Quambalariaceae (1) \\
\hline & & & Volvocisporiaceae (1) \\
\hline
\end{tabular}

Microstromatales

genera incertae sedis

(4)

\begin{tabular}{|c|c|c|}
\hline & Robbauerales & Robbaueraceae (1) \\
\hline & Tilletiales & Erratomycetaceae (1) \\
\hline & & Tilletiaceae (6) \\
\hline Malasseziomycetes & Malasseziales & Malasseziaceae (1) \\
\hline Microbotryomycetes & Heterogastridiales & Heterogastridiaceae (3) \\
\hline & Kriegeriales & Camptobasidiaceae (2) \\
\hline & & Kriegeriaceae (4) \\
\hline & Leucosporidiales & Leucosporidiaceae (1) \\
\hline & Microbotryales & Microbotryaceae (4) \\
\hline & & Ustilentylomataceae (4) \\
\hline & Sporidiobolales & Sporidiobolaceae (3) \\
\hline & Incertae sedis & Chrysozymaceae (4) \\
\hline & & Colacogloeaceae (1) \\
\hline $\begin{array}{l}\text { Microbotryomycetes } \\
\text { genera incertae sedis (15) }\end{array}$ & & \\
\hline Mixiomycetes & Mixiales & Mixiaceae (1) \\
\hline Moniliellomycetes & Moniliellales & Moniliellaceae (1) \\
\hline
\end{tabular}


Table 1 Continued.

\begin{tabular}{|c|c|c|c|}
\hline Phylum & Class* & Order* & Family* \\
\hline & Pucciniomycetes & Helicobasidiales & Helicobasidiaceae (2) \\
\hline & & Pachnocybales & Pachnocybaceae (1) \\
\hline & & Platygloeales & Eocronartiaceae (5) \\
\hline & & & Platygloeaceae (4) \\
\hline & & Pucciniales & Chaconiaceae (9) \\
\hline & & & Coleosporiaceae (5) \\
\hline & & & Cronartiaceae (3) \\
\hline & & & Melampsoraceae (1) \\
\hline & & & Mikronegeriaceae (3) \\
\hline & & & Phakopsoraceae (15) \\
\hline & & & Phragmidiaceae (13) \\
\hline & & & Pileolariaceae (4) \\
\hline & & & Pucciniaceae (21) \\
\hline & & & Pucciniastraceae (10) \\
\hline & & & Pucciniosiraceae (10) \\
\hline & & & Raveneliaceae (24) \\
\hline & & & Sphaerophragmiaceae (2) \\
\hline & & & Uncolaceae $(2)$ \\
\hline & & & Uropyxidaceae (16) \\
\hline & & $\begin{array}{l}\text { Pucciniales genera } \\
\text { incertae sedis (24) }\end{array}$ & \\
\hline & & Septobasidiales & Septobasidiaceae (6) \\
\hline & Spiculogloeomycetes & Spiculogloeales & Spiculogloeaceae (2) \\
\hline & Tremellomycetes & Cystofilobasidiales & Cystofilobasidiaceae (1) \\
\hline & & & Mrakiaceae $(7)$ \\
\hline & & Filobasidiales & Filobasidiaceae (5) \\
\hline & & & Piskurozymaceae (2) \\
\hline & & Holtermanniales & Holtermanniaceae (2) \\
\hline & & Tremellales & Bulleraceae (4) \\
\hline & & & Bulleribasidiaceae (6) \\
\hline & & & Carcinomycetaceae (1) \\
\hline & & & Cryptococcaceae (2) \\
\hline & & & Cuniculitremaceae (3) \\
\hline & & & Naemateliaceae (2) \\
\hline & & & Phaeotremellaceae (2) \\
\hline & & & Phragmoxenidiaceae (1) \\
\hline & & & Rhynchogastremaceae (3) \\
\hline & & & Sirobasidiaceae (1) \\
\hline & & & Tremellaceae (3) \\
\hline & & & Trimorphomycetaceae (4) \\
\hline & & $\begin{array}{l}\text { Tremellales genera } \\
\text { incertae sedis }(8)\end{array}$ & \\
\hline & & Trichosporonales & Tetragoniomycetaceae (3) \\
\hline & & & Trichosporonaceae (8) \\
\hline
\end{tabular}

Tremellomycetes

genera incertae sedis

(3)

$\begin{array}{lll}\text { Tritirachiomycetes } & \text { Tritirachiales } & \text { Tritirachiaceae (2) } \\ \text { Ustilaginomycetes } & \text { Uleiellales } & \text { Uleiellaceae }(1)\end{array}$


Table 1 Continued.

\begin{tabular}{|c|c|c|c|}
\hline Phylum & Class* & Order* & Family* \\
\hline & & Urocystidales & Doassansiopsidaceae (1) \\
\hline & & & Fereydouniaceae (1) \\
\hline & & & Floromycetaceae (2) \\
\hline & & & Glomosporiaceae (1) \\
\hline & & & Mycosyringaceae (1) \\
\hline & & & Urocystidaceae (7) \\
\hline & & Ustilaginales & Anthracoideaceae (19) \\
\hline & & & Cintractiellaceae (1) \\
\hline & & & Clintamraceae (1) \\
\hline & & & Geminaginaceae (1) \\
\hline & & & Melanotaeniaceae (3) \\
\hline & & & Pericladiaceae (1) \\
\hline & & & Ustilaginaceae (6) \\
\hline & & & Websdaneaceae (2) \\
\hline & & $\begin{array}{l}\text { Ustilaginales genera } \\
\text { incertae sedis }(20)\end{array}$ & \\
\hline & & Violaceomycetales & Violaceomycetaceae (1) \\
\hline & $\begin{array}{l}\text { Ustilaginomycetes } \\
\text { genus incertae sedis } \\
\text { (1) }\end{array}$ & & \\
\hline & Wallemiomycetes & Geminibasidiales & Geminibasidiaceae (2) \\
\hline & & Wallemiales & Wallemiaceae (1) \\
\hline & $\begin{array}{l}\text { Wallemiomycetes } \\
\text { genus incertae sedis } \\
\text { (1) }\end{array}$ & & \\
\hline \multicolumn{4}{|l|}{$\begin{array}{l}\text { Basidiomycota } \\
\text { genera incertae } \\
\text { sedis (11) }\end{array}$} \\
\hline \multirow[t]{10}{*}{$\begin{array}{l}\text { Blastocladiomycot } \\
a\end{array}$} & Blastocladiomycetes & Blastocladiales & Blastocladiaceae (3) \\
\hline & & & Catenariaceae (2) \\
\hline & & & Paraphysodermataceae (1) \\
\hline & & & Sorochytriaceae (1) \\
\hline & & $\begin{array}{l}\text { Blastocladiales genus } \\
\text { incertae sedis (1) }\end{array}$ & \\
\hline & & Callimastigales & Callimastigaceae (1) \\
\hline & & & Coelomomycetaceae (2) \\
\hline & & Catenomycetales & Catenomycetaceae (1) \\
\hline & $\begin{array}{l}\text { Blastocladiomycetes } \\
\text { genus incertae sedis } \\
\text { (1) }\end{array}$ & & \\
\hline & $\begin{array}{l}\text { Physodermatomycet } \\
\text { es }\end{array}$ & Physodermatales & Physodermataceae (1) \\
\hline $\begin{array}{l}\text { Calcarisporiellom } \\
\text { ycota }\end{array}$ & $\begin{array}{l}\text { Calcarisporiellomyc } \\
\text { etes }\end{array}$ & Calcarisporiellales & Calcarisporiellaceae (2) \\
\hline $\begin{array}{l}\text { Caulochytriomyco } \\
\text { ta }\end{array}$ & $\begin{array}{l}\text { Caulochytriomycete } \\
s\end{array}$ & Caulochytriales & Caulochytriaceae (1) \\
\hline \multirow[t]{8}{*}{ Chytridiomycota } & Chytridiomycetes & Chytridiales & Asterophlyctaceae (2) \\
\hline & & & Chytridiaceae (6) \\
\hline & & & Chytriomycetaceae (11) \\
\hline & & & Phlyctochytriaceae (1) \\
\hline & & & Phlyctorhizaceae (1) \\
\hline & & & Pseudorhizidiaceae (1) \\
\hline & & & Scherffeliomycetaceae (1) \\
\hline & & & Zygorhizidiaceae (1) \\
\hline
\end{tabular}


Table 1 Continued.

\begin{tabular}{|c|c|c|c|}
\hline Phylum & Class* & Order* & Family* \\
\hline & & $\begin{array}{l}\text { Chytridiales genus } \\
\text { incertae sedis (1) }\end{array}$ & \\
\hline & & Nephridiophagales & Nephridiophagaceae (4) \\
\hline & & Polyphagales & Polyphagaceae (1) \\
\hline & & Saccopodiales & Saccopodiaceae (1) \\
\hline & & Incertae sedis & Amoebochytriaceae (1) \\
\hline & & & Sparrowiaceae $(1)$ \\
\hline & & & Sphaeromonadaceae (1) \\
\hline & & & Tetrachytriaceae (1) \\
\hline & & & Thalassochytriaceae (1) \\
\hline
\end{tabular}

Chytridiomycetes

genera incertae sedis

(39)

Cladochytriomycetes Cladochytriales Catenochytridiaceae (1)

Cladochytriaceae (1)

Endochytriaceae (2)

Nowakowskiellaceae (1)

Septochytriaceae (1)

Cladochytriales

genera incertae sedis

(3)

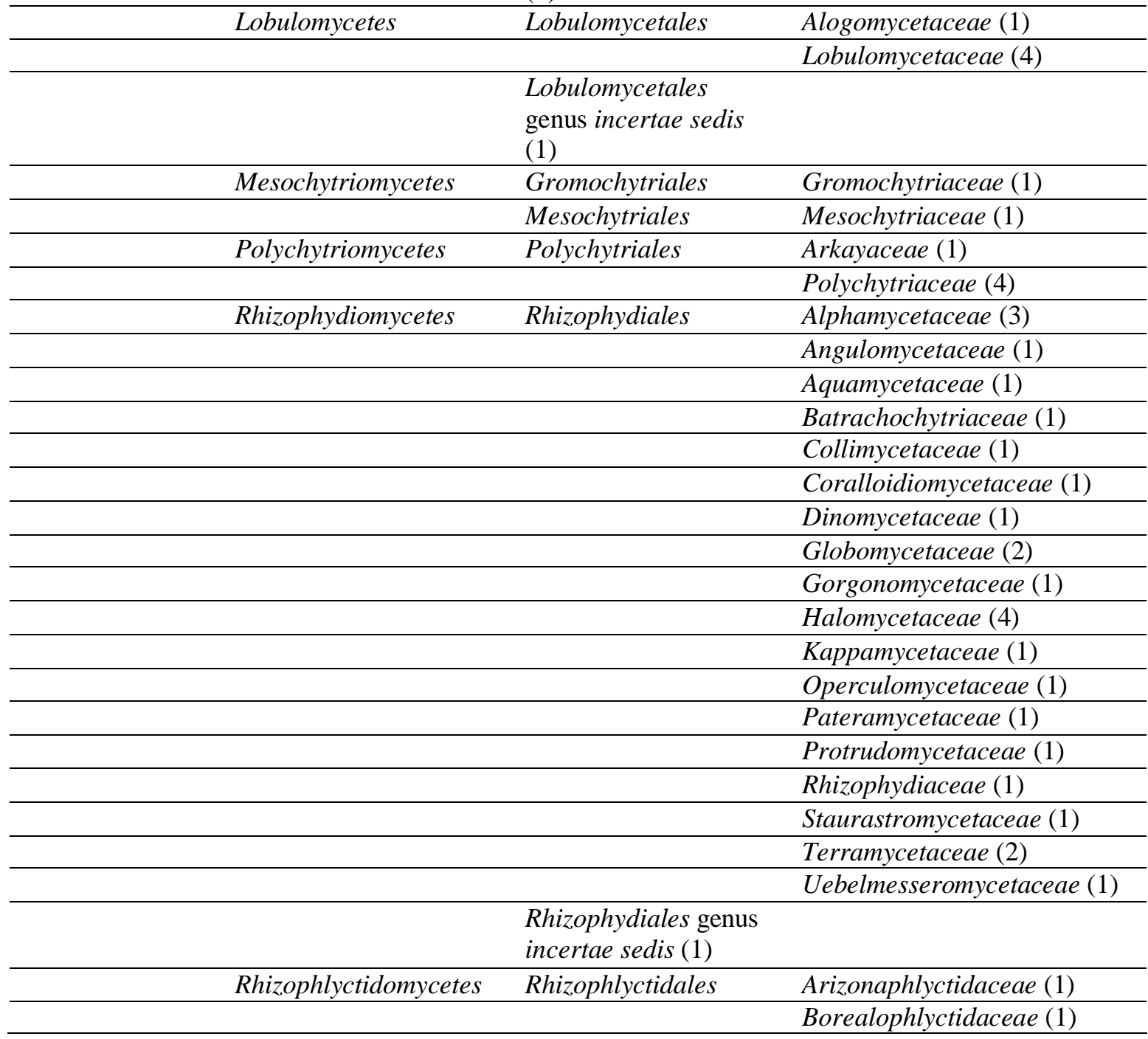


Table 1 Continued.

\begin{tabular}{|c|c|c|c|}
\hline Phylum & Class* & Order* & Family* \\
\hline & & & Rhizophlyctidaceae (1) \\
\hline & & & Sonoraphlyctidaceae (1) \\
\hline & Spizellomycetes & Spizellomycetales & Powellomycetaceae (4) \\
\hline & & & Spizellomycetaceae (8) \\
\hline & Synchytriomycetes & Synchytriales & Synchytriaceae (4) \\
\hline & & $\begin{array}{l}\text { Synchytriales genus } \\
\text { incertae sedis (1) }\end{array}$ & \\
\hline
\end{tabular}

Chytridiomycota

genera incertae sedis

(3)

\begin{tabular}{|c|c|c|c|}
\hline $\begin{array}{l}\text { Entomophthoromycot } \\
a\end{array}$ & Entomophthoromycetes & Entomophthorales & Ancylistaceae (3) \\
\hline & & & Completoriaceae (1) \\
\hline & & & Entomophthoraceae (11) \\
\hline & & & Meristacraceae (1) \\
\hline & Neozygitomycetes & Neozygitales & Neozygitaceae (4) \\
\hline Entorrhizomycota & Entorrhizomycetes & Entorrhizales & Entorrhizaceae (1) \\
\hline & & Talbotiomycetales & Talbotiomycetaceae (1) \\
\hline Glomeromycota & Archaeosporomycetes & Archaeosporales & Ambisporaceae (1) \\
\hline & & & Archaeosporaceae (3) \\
\hline & & & Geosiphonaceae (1) \\
\hline & Glomeromycetes & Diversisporales & Acaulosporaceae (1) \\
\hline & & & Diversisporaceae (7) \\
\hline & & & Pacisporaceae (1) \\
\hline & & & Sacculosporaceae (1) \\
\hline & & Gigasporales & Dentiscutataceae (3) \\
\hline & & & Gigasporaceae (1) \\
\hline & & & $\begin{array}{l}\text { Intraornatosporaceae } \\
\text { (2) }\end{array}$ \\
\hline & & & Racocetraceae (2) \\
\hline & & & Scutellosporaceae (3) \\
\hline & & Glomerales & Entrophosporaceae (3) \\
\hline & & & Glomeraceae (17) \\
\hline & Paraglomeromycetes & Paraglomerales & Paraglomeraceae (2) \\
\hline & & & Pervetustaceae (1) \\
\hline Kickxellomycota & Asellariomycetes & Asellarialles & Asellariaceae (1) \\
\hline & & $\begin{array}{l}\text { Asellariales genus } \\
\text { incertae sedis (1) }\end{array}$ & \\
\hline & Barbatosporomycetes & Barbatosporales & Barbatosporaceae (1) \\
\hline & Dimargaritomycetes & Dimargaritales & Dimargaritaceae (3) \\
\hline & & $\begin{array}{l}\text { Dimargaritales } \\
\text { genus incertae sedis } \\
\text { (1) }\end{array}$ & \\
\hline & Harpellomycetes & Harpellales & Harpellaceae (6) \\
\hline & & & Legeriomycetaceae (38) \\
\hline & & $\begin{array}{l}\text { Harpellales genus } \\
\text { incertae sedis (1) }\end{array}$ & \\
\hline & Kickxellomycetes & Kickxellales & Kickxellaceae (11) \\
\hline & Ramicandelaberomycetes & Ramicandelaberales & $\begin{array}{l}\text { Ramicandelaberaceae } \\
\text { (1) }\end{array}$ \\
\hline Monoblepharomycota & Hyaloraphidiomycetes & Hyaloraphidiales & Hyaloraphidiaceae (1) \\
\hline & Monoblepharidomycetes & Monoblepharidales & Gonapodyaceae (2) \\
\hline & & & $\begin{array}{l}\text { Harpochytriaceae (1) } \\
\text { Monoblepharidaceae (1) }\end{array}$ \\
\hline
\end{tabular}


Table 1 Continued.

\begin{tabular}{|c|c|c|c|}
\hline Phylum & Class* & Order* & Family* \\
\hline & & & $\begin{array}{l}\text { Oedogoniomycetaceae } \\
\text { (1) }\end{array}$ \\
\hline & & & Telasphaerulaceae (1) \\
\hline & Sanchytriomycetes & Sanchytriales & Sanchytriaceae (2) \\
\hline Mortierellomycota & Mortierellomycetes & Mortierellales & Mortierellaceae (6) \\
\hline \multirow[t]{17}{*}{ Mucoromycota } & Endogonomycetes & Endogonales & Densosporaceae (1) \\
\hline & & & Endogonaceae (5) \\
\hline & Mucoromycetes & Mucorales & Backusellaceae (1) \\
\hline & & & Choanephoraceae (4) \\
\hline & & & Cunninghamellaceae (6) \\
\hline & & & Lentamycetaceae (1) \\
\hline & & & Lichtheimiaceae (9) \\
\hline & & & Mucoraceae (20) \\
\hline & & & Mycocladaceae (1) \\
\hline & & & Mycotyphaceae (1) \\
\hline & & & Phycomycetaceae (2) \\
\hline & & & Pilobolaceae (2) \\
\hline & & & Radiomycetaceae (1) \\
\hline & & & Rhizopodaceae (3) \\
\hline & & & Saksenaeaceae (2) \\
\hline & & & Syncephalastraceae (2) \\
\hline & Umbelopsidomycetes & Umbelopsidales & Umbelopsidaceae (1) \\
\hline \multicolumn{4}{|l|}{$\begin{array}{l}\text { Mucoromycota genus } \\
\text { incertae sedis (1) }\end{array}$} \\
\hline Neocallimastigomycota & Neocallimastigomycetes & Neocallimastigales & $\begin{array}{l}\text { Neocallimastigaceae } \\
\text { (11) }\end{array}$ \\
\hline Olpidiomycota & Olpidiomycetes & Olpidiales & Olpidiaceae (4) \\
\hline \multirow[t]{20}{*}{ Rozellomycota } & Rudimicrosporea & Metchnikovellida & Amphiacanthidae (1) \\
\hline & & & Metchnikovellidae (4) \\
\hline & Microsporidea & Amblyosporida & Amblyosporidae (17) \\
\hline & & & Caudosporidae (10) \\
\hline & & & Gurleyidae (13) \\
\hline & & $\begin{array}{l}\text { Amblyosporida } \\
\text { genera incertae } \\
\text { sedis (5) }\end{array}$ & \\
\hline & & Neopereziida & Berwaldiidae (2) \\
\hline & & & Neopereziidae (6) \\
\hline & & & Tubulinosematidae (3) \\
\hline & & $\begin{array}{l}\text { Neopereziida } \\
\text { genera incertae } \\
\text { sedis (2) }\end{array}$ & \\
\hline & & Ovavesiculida & Ovavesiculidae (3) \\
\hline & & $\begin{array}{l}\text { Ovavesiculida } \\
\text { genus incertae } \\
\text { sedis }(1)\end{array}$ & \\
\hline & & Glugeida & Facilisporidae (1) \\
\hline & & & Glugeidae (8) \\
\hline & & & Myosporidae (1) \\
\hline & & & Pereziidae (4) \\
\hline & & & Pleistophoridae (7) \\
\hline & & & Spragueidae (7) \\
\hline & & & Thelohaniidae (15) \\
\hline & & & Unikaryonidae (4) \\
\hline
\end{tabular}


Table 1 Continued.

\begin{tabular}{|c|c|c|c|}
\hline \multirow[t]{2}{*}{ Phylum } & Class* & Order* & Family* \\
\hline & & \multicolumn{2}{|l|}{$\begin{array}{l}\text { Glugeida genus } \\
\text { incertae sedis (1) }\end{array}$} \\
\hline & & \multirow[t]{6}{*}{ Nosematida } & Encephalitozoonidae (2) \\
\hline & & & Enterocytozoonidae (6) \\
\hline & & & Heterovesiculidae (1) \\
\hline & & & Mrazekiidae (8) \\
\hline & & & Nosematidae (2) \\
\hline & & & Ordosporidae (1) \\
\hline & & \multicolumn{2}{|l|}{$\begin{array}{l}\text { Nosematida genera } \\
\text { incertae sedis }(15)\end{array}$} \\
\hline & & Incertae sedis & Abelsporidae (1) \\
\hline & & & Areosporiidae (1) \\
\hline & & & Burenellidae (3) \\
\hline & & & Cougourdellidae (1) \\
\hline & & & Cylindrosporidae (1) \\
\hline & & & Duboscqiidae (5) \\
\hline & & & Golbergiidae (3) \\
\hline & & & Microfilidae (1) \\
\hline & & & Neonosemoidiidae (1) \\
\hline & & & Pleistosporidiidae (1) \\
\hline & & & $\begin{array}{l}\text { Pseudopleistophoridae } \\
\text { (2) }\end{array}$ \\
\hline & & & Striatosporidae (1) \\
\hline & & & Telomyxidae (1) \\
\hline & & & Toxoglugeidae (2) \\
\hline & & & Tuzetiidae (4) \\
\hline & Incertae sedis & Chytridiopsidea & Buxtehudidae (2) \\
\hline & & & Chytridiopsidae (5) \\
\hline & & & Hesseidae (1) \\
\hline \multicolumn{4}{|c|}{$\begin{array}{l}\text { Rozellomycota genera } \\
\text { incertae sedis }(5)\end{array}$} \\
\hline \multirow[t]{6}{*}{ Zoopagomycota } & Zoopagomycetes & Zoopagales & Cochlonemataceae (7) \\
\hline & & & Helicocephalidaceae (4) \\
\hline & & & Piptocephalidaceae (3) \\
\hline & & & $\begin{array}{l}\text { Sigmoideomycetaceae } \\
(4)\end{array}$ \\
\hline & & & Zoopagaceae (7) \\
\hline & & $\begin{array}{l}\text { Zoopagales genus } \\
\text { incertae sedis (1) }\end{array}$ & \\
\hline
\end{tabular}

* Orders/families could be listed under different subclasses in this outline. In this table, we do not indicate auxiliary (intermediate) taxonomic ranks.

\section{Fossil Fungi}

Fossil fungi are reported in the form of dispersed spores, mycelia, sporophores, mycorrhizae, and are commonly observed in macerated residues prepared for palynological studies. Although fungal remains are encountered in the sediments of all ages, their frequency increases remarkably in the Tertiary Period. This clearly suggests that their proliferation is linked with diversification of angiosperms. Being fragmentary in nature, fossil fungi lack characteristic features that are diagnostic of extant taxa, hampering their classification with extant fungi. These are, therefore, described on the basis of morphological characters only. For example, spore taxa are based on their shape, size, symmetry, number and nature of apertures, septa and spore wall characters. On the other hand, fossil fungi (other than spores) can be assigned to their extant counterpart (up to order/family level). 
Artificial classification systems for fungal spores have been proposed by Van der Hammen (1954), Clarke (1965) and Elsik (1968). Pirozynski \& Weresub (1979) suggested the use of the 'Saccardoan System' for classifying fungal spore types. Kendrick \& Nag Raj (1979) modified the Saccardoan System to eliminate some of its inconsistencies. This scheme is based on shape and number of cells and accordingly fungal spores are recognized under Amerosporae, Didymosporae, Phragmosporae, Dictyosporae, Helicosporae, Staurosporae and Scolecosporae. This system is followed here.

Fungal sporophores of various kinds commonly occur on the surface of leaves, stems and flowers of vascular plants and have been extensively recorded over the world (Cookson 1947, Dilcher 1965, Elsik 1968, Kalgutkar \& Jansonius 2000, van Geel \& Aptroot 2006). Some are catathecia provided with radiating rows of mycelial cells giving an appearance of tissues arranged in a radial fashion. Ascomata contain asci that are surrounded by or enclosed within protective tissues and may be globose, flask-shaped or saucer-shaped open bodies. These may or may not possess an ostiolar opening. Fossil sporophores are also placed under artificial genera. Several workers have attempted to classify and formally describe the fossil structures (Edwards 1922, Rosendahl 1943, Cookson 1947, Rao 1959, Dilcher 1965, Venkatachala \& Kar 1969, Jain \& Gupta 1970, Elsik 1978, Pirozynski 1978). Fossil sporophores are classified on the basis of dehiscence mode (through irregular or regular cracking pattern or by a central pore or ostiole). Other characteristic features considered for their classification are shape and margin of the sporophores, presence or absence of pores in individual cells and nature of the central part of the sporophores.

\section{Slime molds}

The terms 'slime molds' or 'mycetozoans' have traditionally been used to describe motile, unicellular terrestrial predatory phagotrophs, which are capable of forming minute to relatively large spore-producing structures, referred to as fruiting bodies. The slime molds in which unicellular units aggregate to form first a pseudoplasmodium and then a sporocarp are referred to as cellular slime molds. Those organisms in which the cells remain solitary but undergo a dramatic increase in size and the number of nuclei to form a plasmodium and then a sporocarp are referred to as plasmodial slime molds (Martin \& Alexopoulos 1969). Both groups are polyphyletic. They represent two peculiar life strategies, which have appeared several times in different groups of Eukaryotes (Shadwick et al. 2009, Brown \& Silberman 2013). A strategy similar to what is found in cellular slime molds also occurs in the prokaryotic myxobacteria, which are sometimes predatory but never phagotrophic (Keane \& Berlemann 2016). The major groups of slime molds are listed in Table 2.

The slime molds were once considered as fungi, due to the presence of spore-producing structures in their life cycle. However, as it was pointed out long ago by de Bary (1887), these similarities relate only the dispersal biology of these groups. Slime molds do not have a fungal form of life, being predominately phagotrophic, demonstrating active motility and lacking a cell wall during their trophic stages. Consequently, the slime molds cannot be assigned to the kingdom Fungi in a taxonomic sense, but their nomenclature remains governed by the International Code of Nomenclature for algae, fungi, and plants. However, an exception is Fonticula alba (Brown et al. 2009) which belongs to the true Fungi (Nucletmycea sensu Adl et al. 2019), being a solitary slime mold that can be considered as a fungus in the taxonomic sense.

\section{Eumycetozoa}

Among the groups of slime molds, the Eumycetozoa (the "true" slime molds) are the most diverse and most complex in terms of morphology. All available phylogenies support the placement of these organisms in the supergroup Amoebozoa (Shadwick et al. 2009, Tice et al. 2016, Kang et al. 2017) (Table 2).

The name Eumycetozoa was initially proposed for three groups of slime molds - the myxomycetes, dictyostelids and protostelids (Olive \& Stoianovitch 1975). However, the latter 
taxon appears to be polyphyletic and includes spore-forming members of several different branches of the Amoebozoa, including the Protosporangiida, Protostelida sensu stricto, Fractovitellida, Cavosteliida, Centramoebia and Flabellinea (see Table 2). This has led to the conclusion that if all the protostelids with all their non-fruiting relatives are included in the Eumycetozoa, the latter becomes nearly synonymous with the Amoebozoa (Shadwick et al. 2009). To preserve this widely used name, Kang et al. (2017) proposed including in the Eumycetozoa only one group of protostelids, the Protosporangiida, which forms a monophyletic unit with myxomycetes and dictyostelids.

In terms of botanical nomenclature, the Protosporangiida may be considered as the class Ceratiomyxomycota (Leontyev et al. 2019). Therefore, in the classification given below, we recognize three classes within the Eumycetozoa. These are the Dictyosteliomycetes, Ceratiomyxomycetes and Myxomycetes, as outlined by Leontyev et al. (2019).

Table 2 Position of the cellular (C) and plasmodial (P) slime mold taxa in the classification of Eukaryotes, according to Adl et al. (2019)

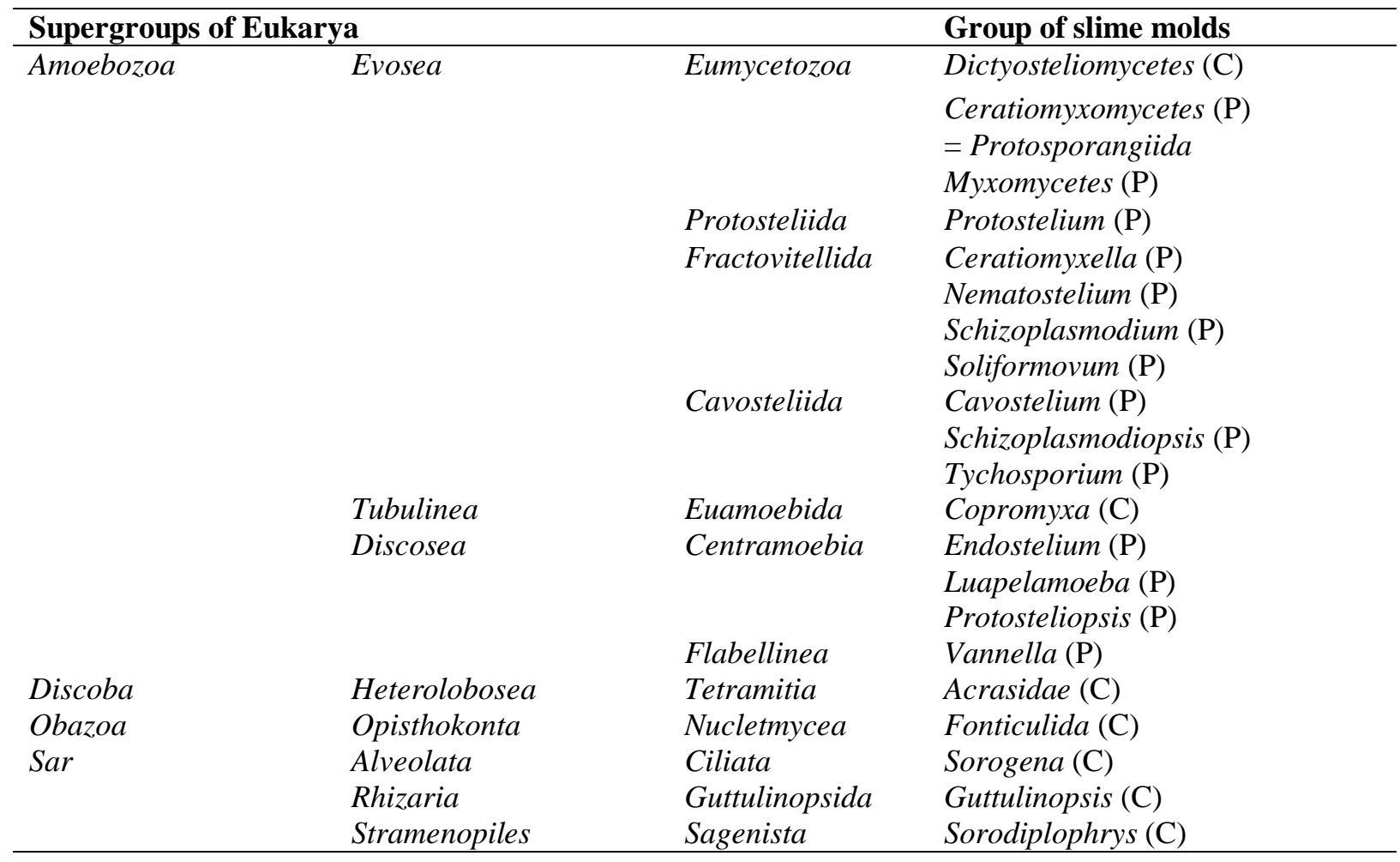

\section{Dictyosteliomycetes}

The dictyostelid cellular slime molds (also called dictyostelids) are common to sometimes abundant inhabitants of forest soil and leaf litter (Romeralo et al. 2013), grassland soil (Rollins et al. 2010), canopy soil (Stephenson \& Landolt 1998, 2011), the soil of agricultural fields (Stephenson \& Rajguru 2010) and animal dung (Stephenson \& Landolt 1992), where they feed primarily on bacteria (Singh 1947, Cavender \& Raper 1965a, b). Raper \& Smith (1939) and Sanders et al. (2017) reported that dictyostelids can feed on pathogenic bacteria, including biofilm enmeshed bacteria produced by human and plant pathogens. Interestingly, migratory birds have been demonstrated to serve as vectors for dictyostelids (Suthers 1985), which greatly increases their potential for long-distance dispersal.

The first species of dictyostelid, Dictyostelium mucoroides, was isolated from horse dung and rabbit dung by Brefeld (1869). Later, a number of additional species and three additional genera (Acytostelium, Coenonia and Polysphondylium) were described, although one of these (Coenonia) has not been isolated since it was first described by van Tieghem in 1884. It is possible that this organism is not a dictyostelid. Traditionally, these four genera (if Coenonia is 
retained), two families, and one order were classified on the basis of the morphology of the sorophore and the pattern of branching (Raper 1984, Hagiwara 1989). This type of traditional morphology-based classification was used by everyone working with dictyostelids until a phylogenetic analysis based on 18S rRNA and $\alpha$-tubulin gene markers indicated that the group needed a complete revision (Schaap et al. 2006, Romeralo et al. 2011, 2012), and the traditional genera did not hold together. More recently, a new classification of the class was proposed by Sheikh et al. (2018), using a single 18S rRNA gene marker. This new classification provided additional insight into the phylogeny of dictyostelids, with 12 genera, four families, and two orders currently being recognized (Table 3 ).

\section{Ceratiomyxomycetes}

This class unites protosteloid eumycetozoans, in which individual sporocarps may arise separately on a substrate (Protosporangium and Clastostelium), or form on a common layer of extracellular slime, which may be smooth, poroid or dissected into variously branched pillars (Ceratiomyxa) (Shadwick et al. 2009). This group was initially described under the zoological name Protosporangiida (Kang et al. 2017). The botanical name Ceratiomyxomycetes was proposed by Hawksworth et al. (1983) as nom. inval. (ICN, Art. 39.1), and later validated by Leontyev et al. (2019).

\section{Myxomycetes}

The myxomycetes (or myxogastrids, Myxogastrea) differ from the rest of the slime molds by their capability to form large fruiting bodies with a complicated structure, which may contain millions of spores. The traditional classification, first proposed by Massee (1892) and later developed by Martin \& Alexopoulos (1969), recognized within the myxomycetes four or five orders (Echinosteliales, Liceales, Physarales, Stemonitales, and Trichiales) based on a number of criteria, including the presence or absence of a capillitium and lime deposits in the fruiting bodies. This classification received worldwide recognition and was applied even in the most recent monographs (e.g., Poulain et al. 2011). However, the results obtained from studies of the molecular phylogeny of myxomycetes have shown that this classification does not properly reflect evolutionary relationships within the group (Fiore-Donno et al. 2012, 2013). Based on a comprehensive review of all published phylogenies of myxomycete subgroups and the full-length $18 \mathrm{~S}$ rDNA phylogeny of the entire group, a new classification of the class was recently proposed (Leontyev et al. 2019). In this classification, myxomycetes are divided into 13 families, nine orders, four superorders and two subclasses, the Lucisporomycetidae and the Columellomycetidae (Table 3). An additional order for the group is proposed below.

For a very long time, scientists studying myxomycetes have had a consensus about the use of botanical nomenclature (in its mycological version) for the myxomycetes. This nomenclature has been used in practically all published monographs of the group, from the late 19th century (Lister 1894) to the most recent efforts (Poulain et al. 2011). This agrees with the statement in Preamble 8 of the International Code of Nomenclature for algae, fungi, and plants, that the 'slime molds' are among the organisms for which the Code is applied (Turland et al. 2018). In contrast, the International Code of Zoological Nomenclature does not mention slime molds, eumycetozoans or myxomycetes (Ride et al. 1999). As indicated by Ronikier \& Halamski (2018), a transfer of myxomycetes to zoological nomenclature would cause nomenclatural chaos due to the existence of numerous homonyms and the difference between nomenclatural starting points of the two Codes. Such a transfer is as well not required by theoretical reasons, since myxomycetes are neither plants or fungi, nor animals, thus none of the two existing codes can reflect their proper position in the contemporary classification of living organisms. In order to preserve nomenclatural stability we use herein botanical names for members of the Eumycetozoa, corresponding to the rules of ICN. The botanical name for the family Protosporangiaceae, which had not yet been proposed, is published below according to the requirements of ICN. 


\section{Oomycota}

The Oomycota are a phylum of the kingdom Straminipila which evolved fungal characterstics - such as an osmotrophic mode of nutrition and hyphal growth - convergently to the fungal groups of the Mycota (Beakes \& Thines 2017). Thus, they are traditionally studied by mycologists and also covered by ICN. Since the last comprehensive monographic treatment by Dick (2001) their classification underwent significant revision, with the latest classification before the current article being that of Beakes \& Thines (2017). Based on more recent discoveries, especially regarding the early diverging oomycete lineages, this classification is updated here.

\section{Aims of the study}

The main aim of this study is to compile all outlines of fungi and fungus-like groups, updated with recent findings and published data. We believe that this type of compilation will be important for scientists to have a better understanding of the limitations and the definitions of the fungal clades. For example, the classification of basal clades of fungi is debatable without a broad agreement (e.g. Humber 2016 vs. Spatafora et al. 2016 on Entomophthoromycota and Glomeromycota; Karpov et al. 2014, 2017 vs Bass et al. 2018 vs Tedersoo et al. 2018 vs Adl et al. 2019 on aphelids, rozellids and microsporidia). Moreover, recent proposals of classification in Tedersoo et al. (2018) (such as elevating lower ranks to higher ranks and demoting higher ranks to lower ranks) might also cause disagreement and thus, need to be discussed.

Fossil fungi, which is another important area of fungal taxonomy is also included in this study. We also include fungus-like organisms to emphasize the reasons why they are excluded from the fungal clade. We will launch a new web page, outlineoffungi.org, which will provide an outline down to the level of genus for true Fungi, fossil fungi, and fungus-like organisms. This data will be important for many scientific disciplines such as genomics, medicine, plant pathology, novel compound discovery and biotechnology (Hyde et al. 2019).

It must always be borne in mind that the classifications being proposed now are based on only perhaps 3-8 \% of the fungal species present on Earth today (Hawksworth \& Lücking 2017). Any system proposed is therefore likely to be unstable and subject to change in the light of newly discovered Fungi or fungus-like organisms. For example, sequencing of the type species of a genus of Gyalectaceae for the first time supported the treatment of four genera as synonyms of Gyalecta (Lücking et al. 2019), while a recent re-analysis of Dothideomycetes following the discovery of the new genus Tenuitholiascus (which forms foliicolous lichens in China) found that five currently accepted orders formed a single well-supported clade (Jiang et al. 2020). The discovery of novel fungi and the sequencing of hitherto unsequenced genera can be expected to continually yield unexpected results which prompt a re-evaluation of which taxa merit recognition at particular ranks.

This outline is therefore not to be treated as a definitive, but a statement of the current situation as a basis for further discussion and in some cases future consensus. In particular, now the IBC permits lists of names to be proposed for protected status, we hope that it will be of value in working towards a protected list of generic names for fungi, updated from that of Kirk et al. (2013), which can be reviewed and in due course approved through the provisions of the Code.

\section{Materials \& methods}

\section{True Fungi}

To list genera and other higher taxonomic ranks into a single outline, we used Kirk et al. (2008, 2013) Lumbsch \& Huhndorf (2010), Humber (2012, 2016), Wijayawardene et al. (2012, 2017a, b, 2018a, b), Hyde et al. (2013, 2020), Benny et al. (2016), Jaklitsch et al. (2016a), Spatafora et al. (2016), Desirò et al. (2017), Lücking et al. (2017), Begerow et al. (2018), Kraichak et al. (2018a), Tedersoo et al. (2018), Haelewaters et al. (2019b), Species Fungorum (2019), Mapook et al. (2020) and Catalogue of Life (http://www.catalogueoflife.org/). Index Fungorum (2019), LIAS names (http://liasnames.lias.net/) and MycoBank 
(http://www.mycobank.org/) were consulted concerning supplementary information on synonyms. We generally followed He et al. (2019) for Basidiomycota classification.

Table 3 Classes, subclasses, orders and families of the Eumycetozoa with number of genera (in brackets)

\begin{tabular}{|c|c|c|c|}
\hline Class & Subclass & Order & Family \\
\hline \multirow[t]{6}{*}{ Dictyosteliomycetes } & & \multirow[t]{2}{*}{ Acytosteliales } & Acytosteliaceae (3) \\
\hline & & & Cavenderiaceae (1) \\
\hline & & \multirow[t]{3}{*}{ Dictyosteliales } & Dictyosteliaceae (2) \\
\hline & & & Raperosteliaceae (4) \\
\hline & & & Incertae sedis (1) \\
\hline & & \multicolumn{2}{|l|}{ Incertae sedis (1) } \\
\hline \multirow[t]{2}{*}{ Ceratiomyxomycetes } & & \multirow[t]{2}{*}{ Ceratiomyxales } & Ceratiomyxaceae (1) \\
\hline & & & Protosporangiidae (2) \\
\hline \multirow[t]{15}{*}{ Myxomycetes } & \multirow[t]{6}{*}{ Lucisporomycetidae } & Cribrariales & Cribrariaceae (3) \\
\hline & & Reticulariales & Reticulariaceae (6) \\
\hline & & Liceales & Liceaceae (2) \\
\hline & & \multirow{2}{*}{ Trichiales } & Dianemataceae (4) \\
\hline & & & Trichiaceae (8) \\
\hline & & Incertae sedis (4) & \\
\hline & \multirow[t]{9}{*}{ Columellomycetidae } & Echinosteliopsidales & $\begin{array}{l}\text { Echinosteliopsidaceae } \\
\text { (1) }\end{array}$ \\
\hline & & Echinosteliales & Echinosteliaceae (3) \\
\hline & & Clastodermatales & Clastodermataceae (1) \\
\hline & & Meridermatales & Meridermataceae (1) \\
\hline & & Stemonitidales & $\begin{array}{l}\text { Amaurochaetaceae (7) } \\
\text { Stemonitidaceae (3) }\end{array}$ \\
\hline & & \multirow[t]{3}{*}{ Physarales } & Lamprodermataceae (5) \\
\hline & & & Didymiaceae (4) \\
\hline & & & Physaraceae (9) \\
\hline & & Incertae sedis (5) & \\
\hline
\end{tabular}

The subdivision of Rozellomycota at the order and family levels is redefined according to the phylogenetic relationships of the respective type genera representatives. The list of genera is updated in accordance with the acknowledged checklists (Becnel et al. 2014, Cali et al. 2017, Sokolova et al. 2018) and recent studies. In particular, genus Kabatana is suppressed as it was shown to be the synonym of Inodosporus (Stentiford et al. 2018). Genera allocation to families and higher rank taxa is modified after Wijayawardene et al. (2018a, b) using molecular phylogenetic data when available. Polyphyletic higher rank taxa are suppressed. The major clades of Microsporidia tree of life established by Vossbrinck and co-authors (2014) are redefined as the order-rank taxa using previously published or novel names depending upon availability of information on the type taxa.

For the classification of Leotiomycetes, Johnston et al. (2019) and Quijada et al. (2020) are followed in the outline because the phylogenies in these papers are based on 15 loci. Ekanayaka et al. (2019) provided an alternative classification based on less genes, but more taxa and is included in the discussion to encourage positive dialogue.

\section{Fossil fungi}

For the sake of clarity and convenience, the fossil fungal genera are split here into three parts, e.g. 1. Fossil fungal spores (according to Saccardoan System); 2. Fossil fungal sporophores, mycelia and other fungal remains; and 3. Modern fungal genera to which fossil species have been assigned. The genera are listed in three separate tables after the outline of fungi.

The data presented here have been obtained from the literature on fossil fungi published during last seven decades or so, briefly mentioned below. In order to include all records of fossil fungal remains from the Indian Tertiary sediments, published till 2005, three catalogues were 
published (Lakhanpal et al. 1976, Saxena 1991, 2006). Besides, a monographic study was carried out by Saxena \& Tripathi (2011) with the objective to synthesize the available information on Indian fossil fungi. This incorporates description of 152 genera and 388 species, including 15 new species and 12 new combinations, with comments wherever required. Kalgutkar \& Jansonius (2000) published a synopsis of fossil fungi and tried to streamline taxonomic status of many fossil fungal genera and species. They described about 950 validly published species, attributed to approximately 300 genera. They proposed twelve new genera and about 350 new combinations. Transfers of species to more appropriate genera resulted in 31 junior homonyms, for which they provided new names. They also validated one genus and several species. In addition to the above monographic studies, data have been gathered from scores of publications, containing information on fossil fungi from all parts of the globe, published in various journals and conference proceedings.

\section{Fungus-like organisms}

The classification systems used for the Dictyosteliomycetes and Myxomycetes as presented herein are based on the critical revisions of Sheikh et al. (2018) and Leontyev et al. (2019), respectively. In each paper, the taxonomy of the particular group was strongly revised on the basis of original $18 \mathrm{~S}$ rDNA phylogenies and analyses of morphological synapomorphies.

The classification of the Oomycota follows the outline presented by Beakes \& Thines (2017), with some modifications in accordance to recent studies (Bennett et al. 2019, Buaya et al. 2017, 2019, Buaya \& Thines 2020).

In this classification, we have included all genera of the Eumycetozoa accepted as valid in the nomenclatural database of Lado (2005-2019), although some of the smaller myxomycete genera will probably be incorporated into larger ones on the basis of phylogenetic data (Leontyev et al. 2019). This is likely to be the case for such genera as Arcyodes, Badhamia, Collaria, Colloderma, Cornuvia, Elaeomyxa, Metatrichia, Diacheopsis, Listerella, Oligonema and Semimorula.

A resurrection of the forgotten order Echinosteliopsidales is proposed herein, based on the $18 \mathrm{~S}$ rDNA phylogeny of Columellomycetidae, which includes Echinosteliopsis oligospora together with a number of environmental sequences, obtained in three different studies (Shchepin et al. 2019).

All authors listed contributed information and comments to this work, but the inclusion of their names does not imply that all necessarily support all details of the outline presented. Notes are provided for recently introduced genera as well as changes in classification (marked with an asterisk in the outline). The authors of each note are indicated in brackets, after the notes.

\section{Phylogenetic analyses}

To build a reference phylogeny, we utilized information from four genes, 18S rRNA, 28S rRNA, RPB1 and RPB2. We used the initial set of $18 \mathrm{~S}$ and $28 \mathrm{~S}$ reference sequences from 1) James et al. (2006) and supplemented by Tedersoo et al. (2017); 2) and at least one representative sequence of each recognized order or order-level taxon, or orphan taxon as based on Tedersoo et al. (2018) and classification of this study. Order and family representatives were selected based on their type status, presence of all four genes, and length of the genes. The nucleotide sequences of all genes were aligned separately using MAFFT 7 (Katoh \& Standley 2013), followed by manual checking and editing where necessary. We took advantage of the protein alignment to remove codon-switching indels and make decision about the gaps and removal of introns. We checked for severe conflicts in the phylogenies of all genes and replaced or removed 10 sequences that were obviously obtained from contaminant or misidentified taxa. The initial alignment included 441 terminal taxa, which we reduced to 433 taxa to exclude putative contaminants and taxa with ultralong branches such as Oedogonomyces spp. 


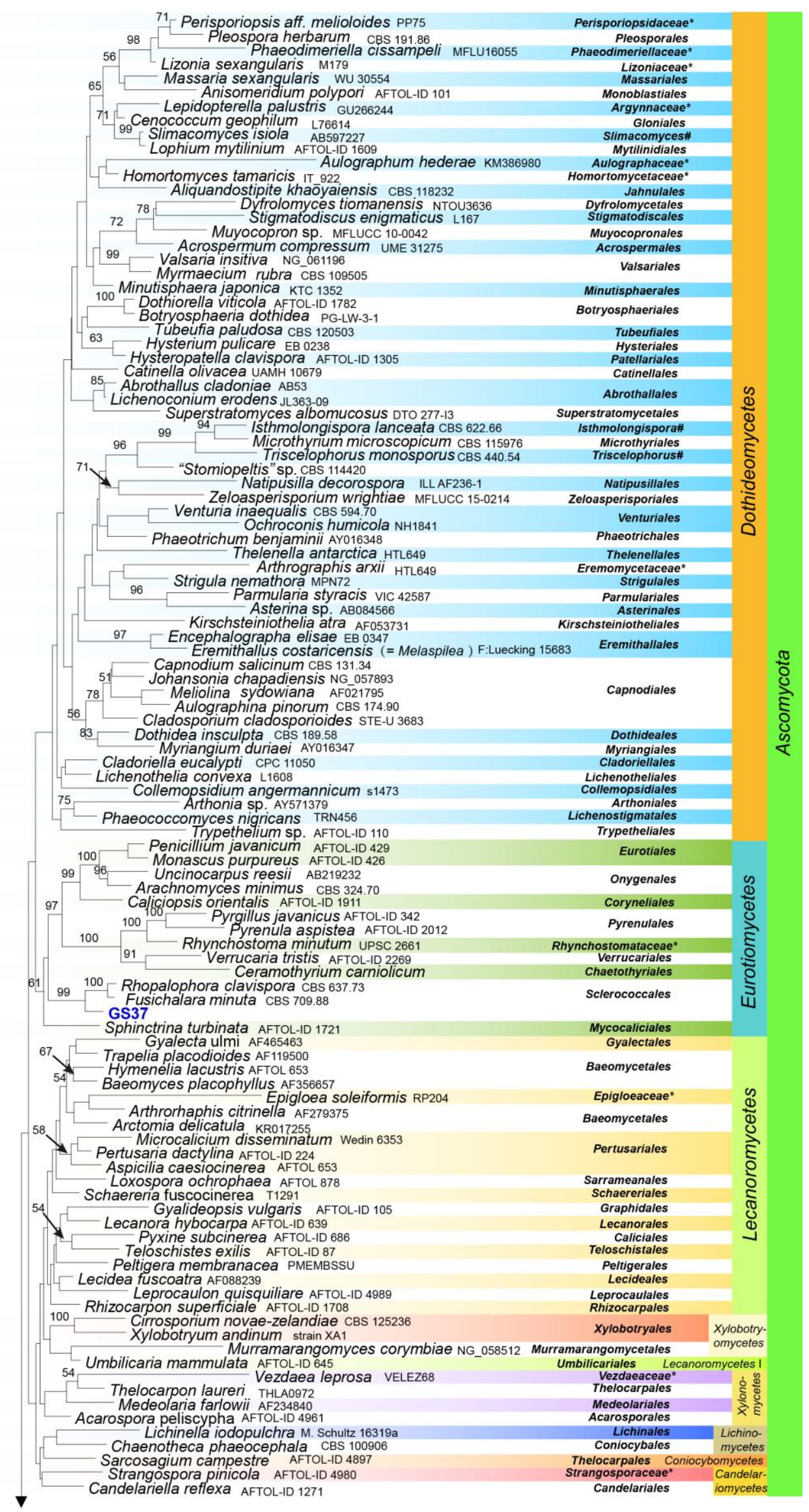

Figure 1 - Maximum likelihood phylogeny of the kingdom Fungi based on LSU, SSU, RPB1 and RPB2 combined sequence data. Numbers above branches indicate bootstrap support. Accession numbers of terminal taxa are indicated in appendant table. 


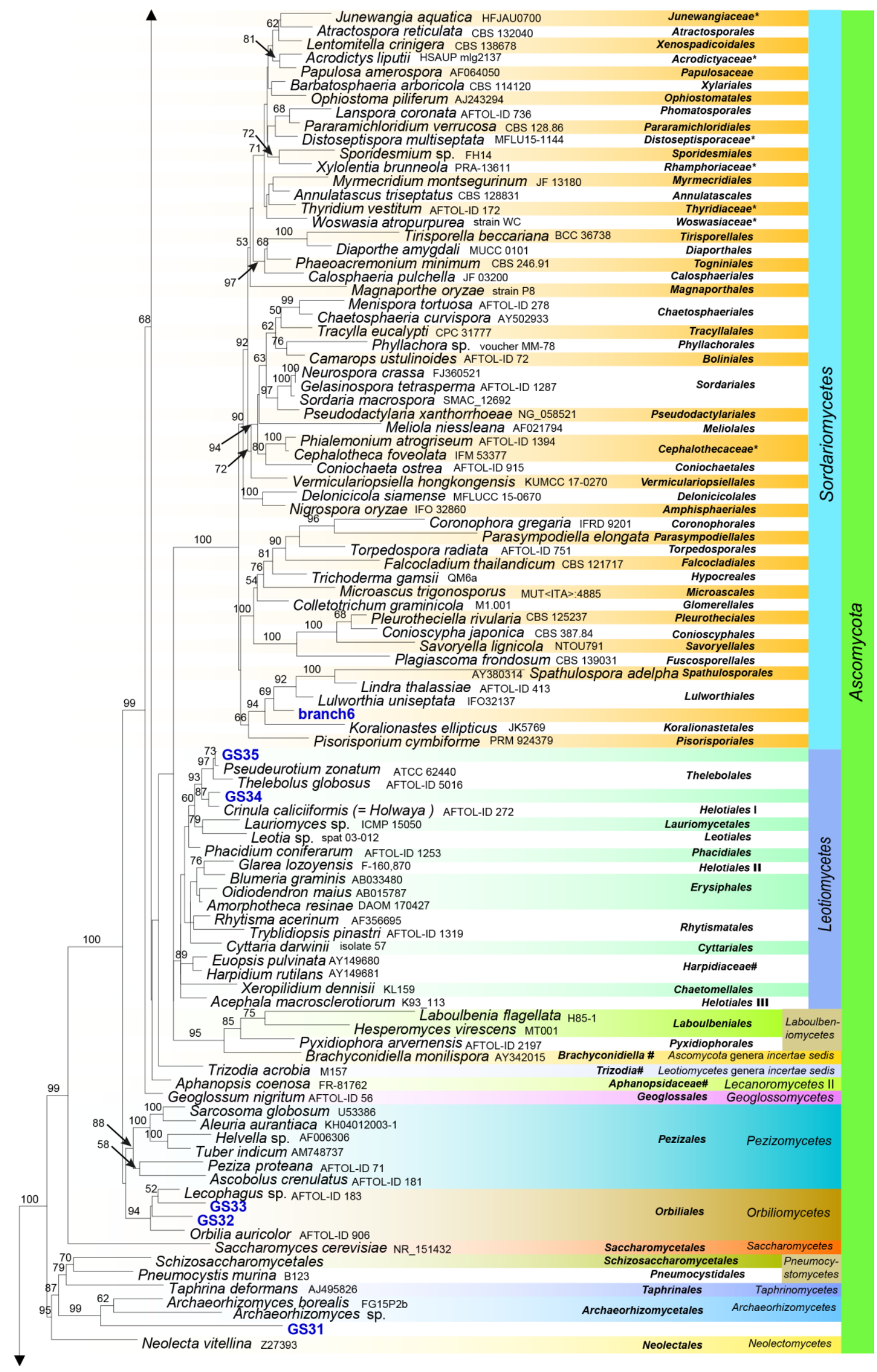

Figure 1 - Continued. 


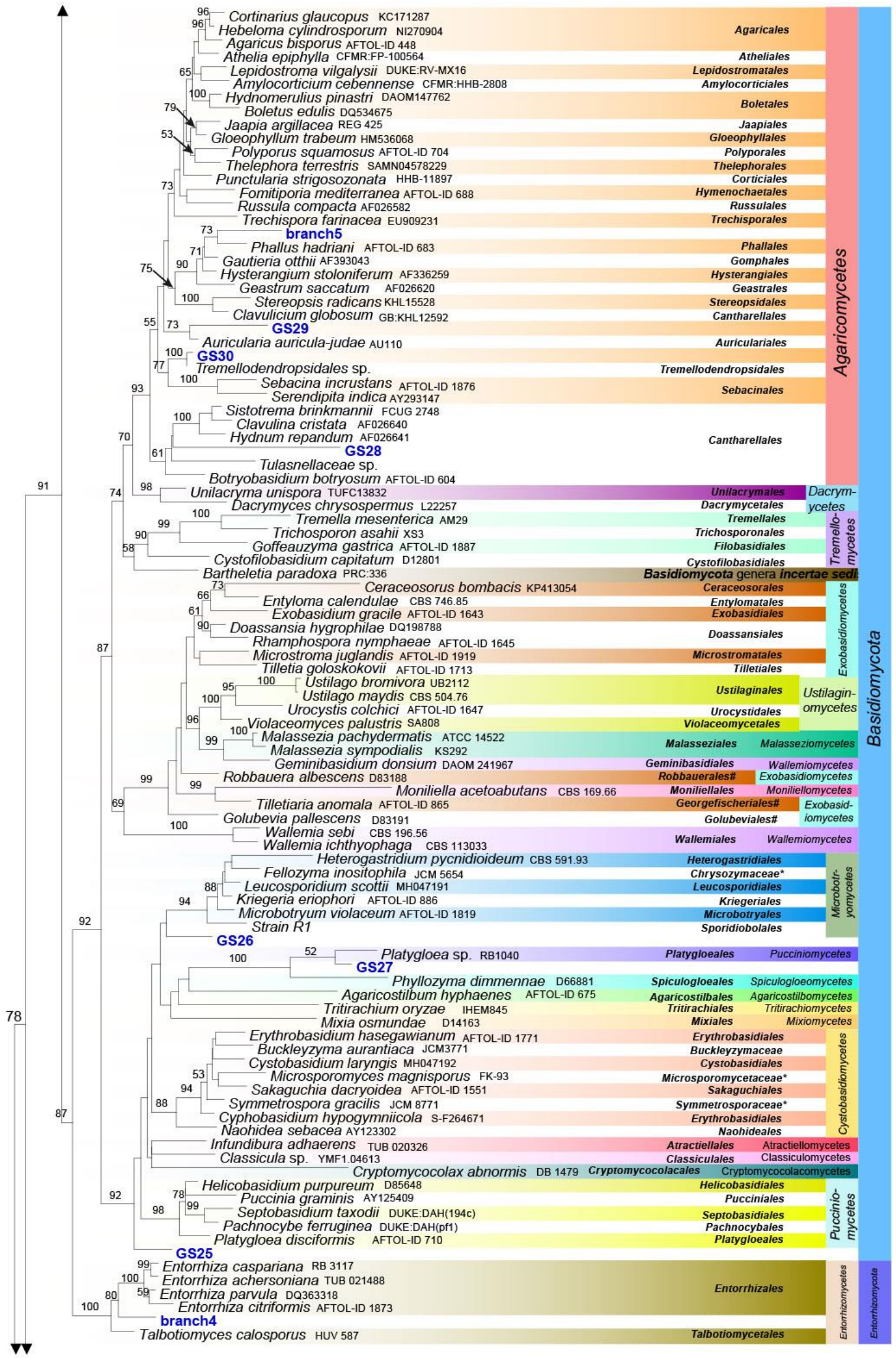

Figure 1 - Continued. 


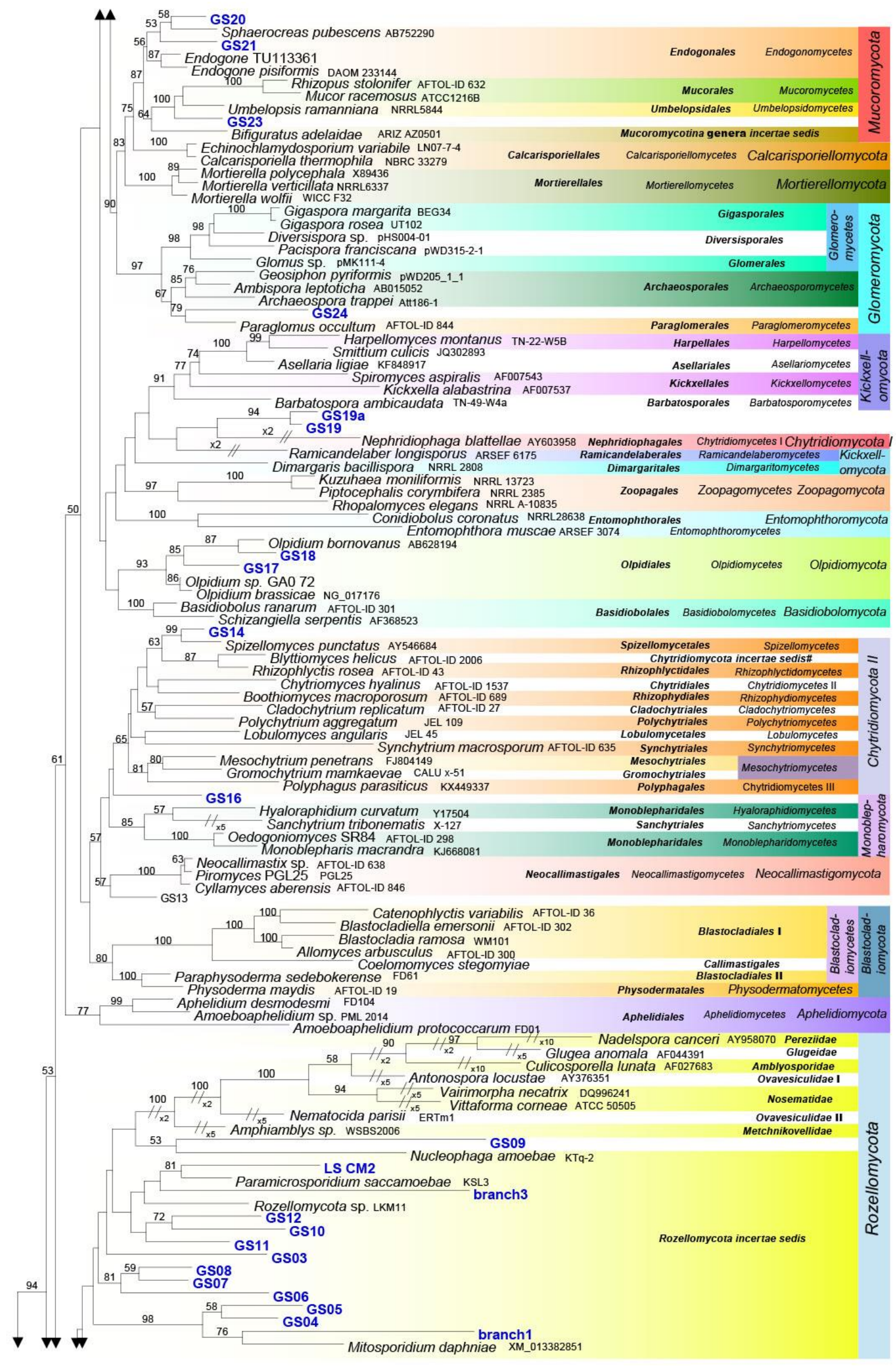

Figure 1 - Continued. 


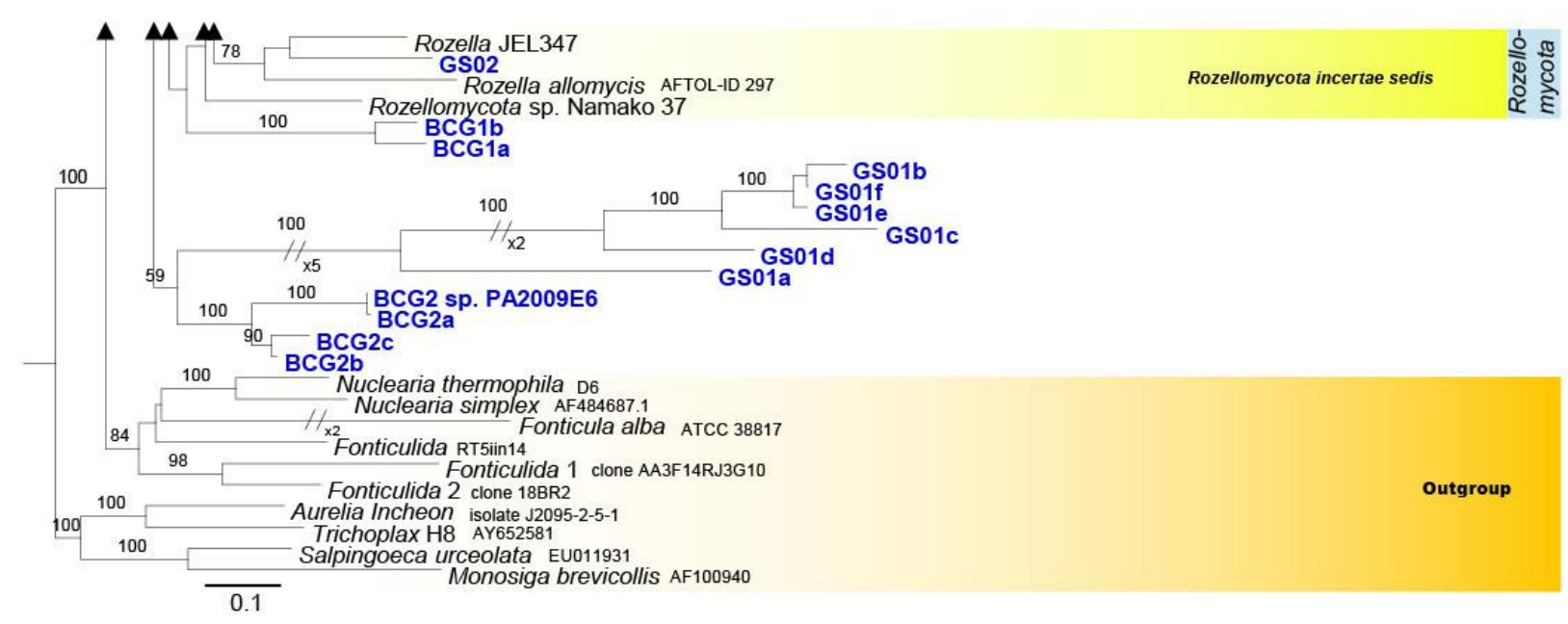

Figure 1 - Continued.

\section{Results}

\section{Taxonomy}

Helicobolomyces cinnabarinula (Müll. Arg.) Wijayaw. \& Ertz, comb. nov. Bas. Arthonia cinnabarinula Müll. Arg., Flora, Regensburg 64: 234 (1881).

Syn. nov. Helicobolomyces lichenicola Matzer, in Grube, Matzer \& Hafellner, Lichenologist 27: 28 (1995).

IF Registration Identifier: 555393

Description: Grube et al. (1995).

Redonographaceae (Lücking, Tehler \& Lumbsch) Lumbsch, stat nov.

Bas.: Redonographoideae Lücking, Tehler \& Lumbsch, Am. J. Bot. 100: 846 (2013)

IF Registration Identifier: 555399

Description: Lücking et al. (2013)

Amblyosporida Tokarev \& Issi, ord. nov.

IF Registration Identifier: 555592

Monophyletic group represented by Amblyosporidae (type genus Amblyospora) and related taxa, based on SSU rRNA gene phylogeny, corresponding to Clade 1 (Vossbrinck et al. 2014). Life cycles are diverse, either monomorphic (one type of sporogony) or dimorphic (two types of sporogony within the same or different hosts). Additional sporogonial sequences may also be present. Parasites of aquatic insects and crustaceans with rare exceptions of terrestrial insects (Multilamina in termites).

Order type: Amblyosporidae Weiser emend. Tokarev \& Issi

Neopereziida Tokarev \& Issi, ord. nov.

IF Registration Identifier: 555594

Monophyletic group represented by Neopereziidae (type genus Neoperezia) and related taxa, based on SSU rRNA gene phylogeny, corresponding to Clade 3 (Vossbrinck et al. 2014). Life cycles are diverse, either monomorphic (one type of sporogony) or dimorphic (two types of sporogony within the same or different hosts). Additional sporogonial sequences may also be present. Parasites of bryozoans, insects, crustaceans and human (Anncaliia, Tubulinosema).

Order type: Neopereziidae Voronin emend. Issi, Tokarev, Seliverstova \& Voronin 
Ovavesiculida Tokarev \& Issi, ord. nov.

IF Registration Identifier: 555610

Monophyletic group represented by Ovavesiculidae (type genus Ovavesicula) and related taxa, based on SSU rRNA gene phylogeny, corresponding to Clade 2 (Vossbrinck et al. 2014). Life cycles are diverse, either monomorphic (one type of sporogony) or dimorphic (two types of sporogony within the same or different hosts). Parasites of aquatic and terrestrial insects.

Order type: Ovavesiculidae Tokarev \& Issi

Protosporangiaceae Leontyev, Stephenson, Schnittler, Shchepin, Novozhilov, fam. nov. MycoBank number: MB 833618

Typus: Protosporangium L.S. Olive \& Stoian., J. Protozool. 19(4): 563 (1972)

Sporocarps stalked, arise separately on a substrate with no common structures, formed by extracellular slime. Stalk long, delicate, strait, flexuous or bent at one or several articulations. Apical portion of the stalk thin (Protosporangium) or inflated, banana-shaped (Clastostelium). Spores in clusters from two, four or eight units, spherical, hemispherical or compressed to each other to form quarter-spheres. Plasmodium colorless.

\section{Outline of fungi}

Aphelidiomycota Tedersoo, Sanchez-Ramirez, Kõljalg, Bahram, M. Döring, Schigel, T.W. May, M. Ryberg \& Abarenkov

Aphelidiomycetes Tedersoo, Sanchez-Ramirez, Kõljalg, Bahram, M. Döring, Schigel, T.W. May, M. Ryberg \& Abarenkov

Aphelidiales Tedersoo, Sanchez-Ramirez, Kõljalg, Bahram, M. Döring, Schigel, T.W. May, M. Ryberg \& Abarenkov

Aphelidiaceae Tedersoo, Sanchez-Ramirez, Kõljalg, Bahram, M. Döring, Schigel, T.W. May, M. Ryberg \& Abarenkov

Amoeboaphelidium Scherff. (5)

Aphelidium Zopf (7)

Paraphelidium Karpov, Moreira, López-García (2)

Pseudaphelidium Schweikert \& Schnepf (1)

Ascomycota Caval.-Sm.

Pezizomycotina O.E. Erikss. \& Winka

Arthoniomycetes O.E. Erikss. \& Winka

Arthoniales Henssen ex D. Hawksw. \& O.E. Erikss.

Andreiomycetaceae B.P. Hodk. \& Lendemer

Andreiomyces B.P. Hodk. \& Lendemer (2)

Arthoniaceae Reichenb. ex Reichenb.

Amazonomyces Bat. (2)

Arthonia Ach. (ca. $50+$ c. 300 orphaned)

Arthothelium A. Massal. (10 + ca. 100 orphaned)

Briancoppinsia Diederich, Ertz, Lawrey \& van den Boom (1)

Coniangium Fr. (4)

Coniarthonia Grube (12)

Coniocarpon DC. (6)

Crypthonia Frisch \& G. Thor (16)

Cryptophaea Van den Broeck \& Ertz (1)

Cryptothecia Stirt. (ca. 65)

Eremothecella Syd. \& P. Syd. (8) 
Glomerulophoron Frisch, Ertz \& G. Thor (2)

Herpothallon Tobler (ca. 50)

Inoderma (Ach.) Gray (4)

Leprantha Dufour ex Körb. (1)

Myriostigma Kremp. (7)

Pachnolepia A. Massal. (1)

Reichlingia Diederich \& Scheid. (4)

Snippocia Ertz, Kukwa \& Sanderson (1)*

Sporodophoron Frisch (4)

Staurospora Grube (1)

Stirtonia A.L. Sm. (ca. 25)

Tylophoron Nyl. ex Stizenb. (8 + 3 orphaned in Sporodochiolichen Aptroot \& Sipman)

Chrysotrichaceae Zahlbr.

Chrysothrix Mont. (ca. 18)

Galbinothrix Frisch, G. Thor, K.H. Moon \& Y. Ohmura (1)*

Melarthonis Frisch \& G. Thor (1)

Lecanographaceae Ertz, Tehler, G. Thor \& Frisch

Alyxoria Ach. (12)

Heterocyphelium Vain. (2)

Lecanographa Egea \& Torrente (ca. 40)

Mixtoconidium Etayo (2)

Phacographa Hafellner (3)

Plectocarpon Fée (ca. 40)

Zwackhia Körb. (6)

Opegraphaceae Körb. ex Stizenb.

Combea De Not. (2)

Dictyographa Müll. Arg. (2)

Dolichocarpus R. Sant. (2)

Fouragea Trevis. (4)

Ingaderia Darb. (3)

Llimonaea Egea \& Torrente (4)

Nyungwea Sérus., Eb. Fisch. \& Killmann (3)

Opegrapha Ach. (= Kalaallia Alstrup \& D. Hawksw.) (ca. $100+$ ca. 200 orphaned)

Paraingaderia Ertz \& Tehler (1)

Paralecanographa Ertz \& \& Tehler (1)

Paraschismatomma Ertz \& Tehler (1)

Pentagenella Darb. (5)

Schizopelte Th. Fr. (4)

Sclerophyton Eschw. (ca. 15)

Sparria Ertz \& Tehler (2)

Roccellaceae Chevall.

Ancistrosporella G. Thor (3)

Austrographa Sparrius, Elix \& A.W. Archer (3)

Austroroccella Tehler \& Ertz (1)

Chiodecton Ach. (ca. 22)

Cresponea Egea \& Torrente (21)

Crocellina Tehler \& Ertz (1)

Dendrographa Darb. (7) 
Dichosporidium Pat. (8)

Dirina Fr. (13)

Diromma Ertz \& Tehler (1)

Enterodictyon Müll. Arg. (2)*

Enterographa Fée (ca. 30 and 25 orphaned)

Erythrodecton G. Thor (3)

Follmanniella Peine \& Werner (1)

Gorgadesia Tav. (1)

Graphidastra (Redinger) G. Thor (4)

Gyrographa Ertz \& Tehler (3)

Gyronactis Ertz \& Tehler (2)

Halographis Kohlm. \& Volkm.-Kohlm. (1)

Haplodina Zahlbr. (3)

Isalonactis Ertz, Tehler, Eb. Fisch., Killmann, Razafindr. \& Sérus. (1)

Lecanactis Körb. (ca. 30)

Mazosia A. Massal. (27)

Neosergipea M. Cáceres, Ertz \& Aptroot (3)

Ocellomma Ertz \& Tehler (1)

Protoroccella Follmann ex Follmann (2)

Pseudolecanactis Zahlbr. (1)

Pseudoschismatomma Ertz \& Tehler (1)

Psoronactis Ertz \& Tehler (1)

Pulvinodecton Henssen \& G. Thor (2)

Roccella DC. (32)

Roccellina Darb. $(29+5$ orphaned in Sigridea $)$

Sagenidiopsis R.W. Rogers \& Hafellner (4)

Schismatomma Flot. \& Körb. ex A. Massal. (10)

Sigridea Tehler (6)

Simonyella J. Steiner (1)

Sipmania Egea \& Torrente (1)

Streimannia G. Thor (1)

Syncesia Taylor (ca. 25)

Tania Egea, Torrente \& Sipman (2)

Vigneronia Ertz (3)

Roccellographaceae Ertz \& Tehler

Dimidiographa Ertz \& Tehler (3)

Fulvophyton Ertz \& Tehler (6)

Roccellographa J. Steiner (4)

Arthoniales genera incertae sedis

Angiactis Aptroot \& Sparrius (3)

Arthophacopsis Hafellner (1)

Bactrospora A. Massal. (35)

Bryostigma Poelt \& Döbbeler (3)

Catarraphia A. Massal. (1)

Felipes Frisch \& G. Thor (1)

Glyphopsis Aptroot (1)

Gossypiothallon Aptroot (1)

Helminthocarpon Fee (3)

Hormosphaeria Lév. (1)

Minksia Müll. Arg. (2) 
Nipholepis Syd. (1)

Paradoxomyces Matzer (1)

Perigrapha Hafellner (5)

Phacothecium Trevis. (1)

Phoebus R.C. Harris \& Ladd (1)

Sporostigma Grube (1)

Synarthonia Müll. Arg. (5)

Synarthothelium Sparrius (2)

Tarbertia Dennis (1)

Trichophyma Rehm (2)

Tylophorella Vain. (1)

Wegea Aptroot \& Tibell (1)

Lichenostigmatales Ertz, Diederich \& Lawrey

Phaeococcomycetaceae McGinnis \& Schell

Etayoa Diederich \& Ertz (1)

Lichenostigma Hafellner (= Phaeosporobolus D. Hawksw. \& Hafellner) (5 and 26 orphaned species)

Phaeococcomyces de Hoog (5)

Candelariomycetes Voglmayr \& Jaklitsch

Candelariomycetidae Timdal \& M. Westb.

Candelariales Miądl., Lutzoni \& Lumbsch

Candelariaceae Hakul.

Candelaria A. Massal. (7)

Candelariella Müll. Arg. (ca. 50)

Candelina Poelt (3)

Placomaronea Räsänen (6)

Pycnoraceae Bendiksby \& Timdal

Pycnora Hafellner (3)

Coniocybomycetes M. Prieto \& Wedin

Coniocybales M. Prieto \& Wedin

Coniocybaceae Rchb.

Chaenotheca (Th. Fr.) Th. Fr. (ca. 25)

Sclerophora Chevall. (6)

Dothideomycetes sensu O.E. Erikss \& Winka

Dothideomycetidae P.M. Kirk, P.F. Cannon, J.C. David \& Stalpers ex C.L. Schoch, Spatafora, Crous \& Shoemaker

Capnodiales Woron.

Aeminiaceae J. Trovão, I. Tiago \& A. Portugal Aeminium J. Trovão, I. Tiago \& A. Portugal (1)

Antennulariellaceae Woron.

Achaetobotrys Bat. \& Cif. (1)

Antennulariella Woron. (1)

Eumela Syd. (4)

Capnodiaceae (Sacc.) Höhn. ex Theiss.

Capnodium Mont. (83) 
Chaetocapnodium Hongsanan \& K.D. Hyde (1)

Conidiocarpus Woron. (= Phragmocapnias Theiss. \& Syd.) (ca. 10)

Fumiglobus D.R. Reynolds \& G.S. Gilbert (10)

Leptoxyphium Speg. (19)

Limaciniaseta D.R. Reynolds (1)

Readerielliopsis Crous \& Decock (2)

Scoriadopsis Mend. (1)

Scorias Fr. (11)

Cladosporiaceae Chalm. \& R.G. Archibald

Acroconidiella J.C. Lindq. \& Alippi (5)

Cladosporium Link (237 accepted species, 631 legitimate names at species level)

Davidiellomyces Crous (2)

Graphiopsis Trail (11)

Neocladosporium J.D.P. Bezerra, Sandoval-Denis, C.M. Souza-Motta \& Crous (1)

Rachicladosporium Crous, U. Braun \& C.F. Hill (14)

Toxicocladosporium Crous \& U. Braun (14)

Verrucocladosporium K. Schub., Aptroot \& Crous (2)

Cystocoleaceae Locq. ex Lücking, B.P. Hodk. \& S.D. Leav.

Cystocoleus A. Massal. (1)

Dissoconiaceae Crous \& de Hoog

Dissoconium de Hoog, Oorschot \& Hijwegen (5)

Globoramichloridium Y. Marín \& Crous (1)

Pseudoveronaea Crous \& Batzer (2)

Ramichloridium Stahel ex de Hoog (35)

Uwebraunia Crous \& M.J. Wingf. (7)

Euantennariaceae Hughes \& Corlett

Capnokyma S. Hughes (2)

Euantennaria Speg. (9)

Hormisciomyces Bat. \& Nascim. (3)

Plokamidomyces Bat., C.A.A. Costa \& Cif. (1)

Rasutoria M.E. Barr (2)

Strigopodia Bat. (2)

Trichothallus F. Stevens (2)

Extremaceae Quaedvl. \& Crous (= Paradevriesiaceae Crous)*

Castanedospora G. Delgado \& A.N. Mill. (1)*

Extremus Quaedvl. \& Crous (2)

Paradevriesia Crous (3)

Petrophila de Hoog \& Quaedvl. (1)*

Pseudoramichloridium Cheew. \& Crous (3)

Saxophila Selbmann \& de Hoog (1)*

Staninwardia B. Sutton (2)

Vermiconidia Egidi \& Onofri (= Vermiconia Egidi \& Onofri) (4)*

Johansoniaceae Doilom, Phook. \& K.D. Hyde

Johansonia Sacc. (13)

Orthobellus Silva \& Cavalc. (3) 
Metacapnodiaceae Hughes \& Corlett

Capnobotrys S. Hughes (10)

Hyphosoma Syd. (6)

Metacapnodium Speg. (14)

\section{Mycosphaerellaceae Lindau}

Acervuloseptoria Crous \& Jol. Roux (2)

Amycosphaerella Quaedvl. \& Crous (2)

Annellosympodiella Crous \& Assefa (1)

Apseudocercosporella Videira \& Crous (1)

Asperisporium Maubl. (24)

Australosphaerella Videira \& Crous (1)

Brunneosphaerella Crous (3)

Brunswickiella Videira \& Crous (1)

Camptomeriphila Crous \& M.J. Wingf. (1)

Caryophylloseptoria Verkley, Quaedvl. \& Crous (4)

Catenulocercospora C. Nakash., Videira \& Crous (1)

Cercoramularia Videira, H.D. Shin, C. Nakash. \& Crous (1)

Cercospora Fresen. (ca. 1125)

Cercosporella Sacc. (ca. 100)

Cercosporidium Earle (ca. 10)

Chuppomyces Videira \& Crous (1)

Clarohilum Videira \& Crous (1)

Clypeosphaerella Guatim., R.W. Barreto \& Crous (3)

Collapsimycopappus A. Hashim., Y. Harada \& Kaz. Tanaka (1)

Collarispora Videira \& Crous (1)

Coremiopassalora U. Braun, C. Nakash., Videira \& Crous (2)

Cytostagonospora Bubák (5)

Deightonomyces Videira \& Crous (1)

Devonomyces Videira \& Crous (1)

Dictyosporina L.M. Abreu, R.F. Castañeda \& O.L. Pereira (1)

Distocercospora N. Pons \& B. Sutton (4)

Distocercosporaster Videira, H.D. Shin, C. Nakash. \& Crous (1)

Distomycovellosiella U. Braun, C. Nakash., Videira \& Crous (1)

Dothistroma Hulbary (5)

Epicoleosporium Videira \& Crous (1)

Exopassalora Videira \& Crous (1)

Exosporium Link (123)

Exutisphaerella Videira \& Crous (1)

Filiella Videira \& Crous (1)

Fulvia Cif. (2)*

Fusoidiella Videira \& Crous (2)

Graminopassalora U. Braun, C. Nakash., Videira \& Crous (1)

Hyalocercosporidium Videira \& Crous (1)

Hyalozasmidium U. Braun, C. Nakash., Videira \& Crous (2)

Janetia M.B. Ellis (22)

Lecanosticta Syd. (8)

Madagascaromyces U. Braun, C. Nakash., Videira \& Crous (1)

Microcyclosporella J. Frank, Schroers \& Crous (1)

Micronematomyces U. Braun, C. Nakash., Videira \& Crous (2)

Miuraea Hara (1)

Mycodiella Crous (3) 
Mycosphaerelloides Videira \& Crous (1)

Mycovellosiella Rangel (ca. 34)

Neoceratosperma Crous \& Cheew. (6)

Neocercospora Bakhshi, Arzanlou, Babai-ahari \& Crous (1)

Neocercosporidium Videira \& Crous (1)

Neodeightoniella Crous \& W.J. Swart (1)

Neomycosphaerella Crous (1)

Neopenidiella Quaedvl. \& Crous (1)

Neophloeospora U. Braun, C. Nakash., Videira \& Crous (1)

Neopseudocercospora Crous (2)

Neopseudocercosporella Videira \& Crous (2)

Neoramichloridium Phook., Thambug. \& K.D. Hyde (1)

Neoseptoria Quaedvl., Verkley \& Crous (1)

Nothopassalora U. Braun, C. Nakash., Videira \& Crous (1)

Nothopericoniella Videira \& Crous (1)

Nothophaeocryptopus Videira, C. Nakash. \& Crous (1)

Pachyramichloridium Videira \& Crous (1)

Pallidocercospora Crous (9)

Pantospora Cif. (1)

Paracercospora Deighton (5)

Paracercosporidium Videira \& Crous (2)

Paramycosphaerella Crous \& Jol. Roux (17)

Paramycovellosiella Videira, H.D. Shin \& Crous (1)

Parapallidocercospora Videira, Crous, U. Braun \& C. Nakash. (2)

Passalora Fr. (ca. 250)

Phaeocercospora Crous (2)

Phaeophleospora Rangel (31)

Phaeoramularia Munt.-Cvetk. (ca. 10)

Phloeospora Wallr. (141)

Piricauda Bubák (31)*

Pleopassalora Videira \& Crous (2)

Pleuropassalora U. Braun, C. Nakash., Videira \& Crous (1)

Pluripassalora Videira \& Crous (1)

Plurivorosphaerella O. Hassan \& T.H. Chang (1)

Polyphialoseptoria Quaedvl., R.W. Barreto, Verkley \& Crous (2)

Polythrincium Kunze (5)

Protostegia Cooke (2)

Pseudocercospora Speg. (ca. 1000)

Pseudocercosporella Deighton (127)

Pseudopericoniella Videira \& Crous (1)

Pseudophaeophleospora C. Nakash., Videira \& Crous (2)

Pseudozasmidium Videira \& Crous (4)

Ragnhildiana Solheim (18)

Ramularia Unger $(100<)$

Ramulariopsis Speg. (4)

Ramulispora Miura (18)

Rhachisphaerella U. Braun, C. Nakash., Videira \& Crous (1)

Rosisphaerella Videira \& Crous (1)

Ruptoseptoria Quaedvl., Verkley \& Crous (1)

Scolecostigmina U. Braun (23)

Septoria Sacc. (= Septocyta Petr. fide Quaedvlieg et al. 2013) (200<)

Sonderhenia H.J. Swart \& J. Walker (2) 
Sphaerulina Sacc. (65)

Stromatoseptoria Quaedvl., Verkley \& Crous (1)

Sultanimyces Videira \& Crous (1)

Trochophora R.T. Moore (2)

Uwemyces Hern.-Restr., Sarria \& Crous (1)

Virosphaerella Videira \& Crous (3)

Xenomycosphaerella Quaedvl. \& Crous (3)

Xenopassalora Crous (1)

Xenoramularia Videira, H.D. Shin \& Crous (3)

Xenosonderhenia Crous (2)

Xenosonderhenioides Videira \& Crous (1)

Zasmidium Fr. (=Periconiella Sacc. fide Quaedvlieg et al. 2013) (ca. 150)

Zymoseptoria Quaedvl. \& Crous (8)

Neodevriesiaceae Quaedvl. \& Crous

Neodevriesia Quaedvl. \& Crous (21)

Tripospermum Speg. (27)

Phaeothecaceae Darveaux

Phaeotheca Sigler, Tsuneda \& J.W. Carmich. (4)

Phaeothecoidiellaceae K.D. Hyde \& Hongsanan (= Nowamycetaceae Crous)

Chaetothyrina Theiss. (6)

Exopassalora Videira \& Crous (1)

Houjia G.Y. Sun \& Crous (2)

Nowamyces Crous (2)

Phaeothecoidiella Batzer \& Crous (2)

Rivilata Kohlm., Volkm.-Kohlm. \& O.E. Erikss. (1)

Sporidesmajora Batzer \& Crous (1)

Translucidithyrium X.Y. Zeng \& K.D. Hyde (1)

Piedraiaceae Viégas ex Cif., Bat. \& S. Camposa

Piedraia Fons. \& Leao (2)

Racodiaceae Link

Racodium Fr. (5)

Schizothyriaceae Höhn. ex Trotter, Sacc., D. Sacc. \& Traverso

Amazonotheca Bat. \& H. Maia (2)*

Hexagonella F. Stevens \& Guba ex F. Stevens (1)

Kerniomyces Toro (1)

Lecideopsella Höhn. (10)

Metathyriella Syd. (3)

Mycerema Bat., J.L. Bezerra \& Cavalc. (1)

Myriangiella Zimm. (5)

Plochmopeltis Theiss. (5)

Schizothyrium Desm. (40)

Vonarxella Bat., J.L. Bezerra \& Peres (1)

Teratosphaeriaceae Crous \& U. Braun

Acidiella Hujslová \& M. Kolařík (3) 
Acidomyces B.J. Baker, M.A. Lutz, S.C. Dawson, P.L. Bond \& Banfield ex Selbmann, de Hoog \& De Leo (2)

Acrodontium de Hoog (17)

Apenidiella Quaedvl. \& Crous (1)

Araucasphaeria Crous \& M.J. Wingf. (1)

Aulographina Arx \& E. Müll. (2)

Austroafricana Quaedvl. \& Crous (3)

Austrostigmidium Pérez-Ort. \& Garrido-Benavent (1)

Batcheloromyces Marasas, P.S. van Wyk \& Knox-Dav. (5)

Baudoinia J.A. Scott \& Unter. (5)

Bryochiton Döbbeler \& Poelt (5)

Camarosporula Petr. (1)

Capnobotryella Sugiy. (6)

Catenulostroma Crous \& U. Braun (7)

Constantinomyces Egidi \& Onofri (4)

Davisoniella H.J. Swart (1)

Devriesia Seifert \& N.L. Nick. (11)

Elasticomyces Zucconi \& Selbmann (1)

Eupenidiella Quaedvl. \& Crous (1)

Euteratosphaeria Quaedvl. \& Crous (1)

Friedmanniomyces Onofri (2)

Hispidoconidioma Tsuneda \& Davey (2)

Hortaea Nishim. \& Miyaji (2)*

Hyweljonesia R.G. Shivas, Y.P. Tan, Marney \& Abell (2)

Incertomyces Egidi \& Zucconi (2)

Lapidomyces de Hoog \& Stielow (1)

Leptomelanconium Petr. (7)

Meristemomyces Isola \& Onofri (2)

Microcyclospora J. Frank, Schroers \& Crous (5)

Monticola Selbmann \& Egidi (1)

Myrtapenidiella Quaedvl. \& Crous (8)

Neocatenulostroma Quaedvl. \& Crous (3)

Neophaeothecoidea Quaedvl. \& Crous (1)

Neotrimmatostroma Quaedvl. \& Crous (3)

Oleoguttula Selbmann \& de Hoog (1)

Pachysacca Syd. (3)

Parapenidiella Crous \& Summerell (2)

Parateratosphaeria Quaedvl. \& Crous (6)

Penidiella Crous \& U. Braun (4)

Penidiellomyces Crous, Attili-Angelis, A.P.M. Duarte, Pagnocca \& J.Z. Groenew. (2)

Penidiellopsis Sand.-Den., Gené, Deanna A. Sutton \& Guarro (2)

Phaeothecoidea Crous (5)

Placocrea Syd. (1)*

Pseudotaeniolina J.L. Crane \& Schokn. (2)

Pseudoteratosphaeria Quaedvl. \& Crous (6)

Queenslandipenidiella Quaedvl. \& Crous (1)

Readeriella Syd. \& P. Syd. (ca. 23)

Recurvomyces Selbmann \& de Hoog (1)

Simplicidiella Crous, Attili-Angelis, A.P.M. Duarte, Pagnocca \& J.Z. Groenew. (1)

Stenella Syd. (ca. 45)

Suberoteratosphaeria Quaedvl. \& Crous (3)

Teratoramularia Videira, H.D. Shin \& Crous (4) 
Teratosphaeria Syd. \& P. Syd. (58)

Teratosphaericola Quaedvl. \& Crous (1)

Teratosphaeriopsis Quaedvl. \& Crous (1)

Xanthoriicola D. Hawksw. (1)

Xenoconiothyrium Crous \& Marinc. (1)

Xenopenidiella Quaedvl. \& Crous (7)

Xenophacidiella Crous (1)

Xenoteratosphaeria Quaedvl. \& Crous (1)

Xenodevriesiaceae Crous

Xenodevriesia Crous (1)

Capnodiales genera incertae sedis

Arthrocatena Egidi \& Selbmann (1)

Catenulomyces Egidi \& de Hoog (1)

Eriosporella Höhn. (2)

Hyphoconis Egidi \& Quaedvl. (1)

Mucomycosphaerella Quaedvl. \& Crous (1)

Mycophycias Kohlm. \& Volkm.-Kohlm

Neohortaea Quaedvl. \& Crous (1)

Perusta Egidi \& Stielow (1)

Plurispermiopsis Pereira-Carv., Inácio \& Dianese (1)

Pseudoepicoccum M.B. Ellis (4)

Racoleus R. Sant. \& D. Hawksw. (1)

Ramimonilia Stielow \& Quaedvl. (1)

Ramopenidiella Crous \& R.G. Shivas (1)

Rosaria N. Carter (2)

Dothideales Lindau (= Neocelosporiales Crous)

Dothideaceae Chevall.

Delphinella (Sacc.) Kuntze (7)

Dictyodothis Theiss. \& Syd. (8)

Dothidea Fr. (ca. 20)

Dothiora Fr. $(50<)$

Endoconidioma Tsuneda (2)

Endodothiora Petr. (1)

Kabatina R. Schneid. \& Arx (5)

Neocylindroseptoria Thambug. \& K.D. Hyde (1)

Phaeocryptopus Naumov (6)

Plowrightia Sacc. (50)

Stylodothis Arx \& E. Müll. (2)

Sydowia Bres. (11)

Uleodothis Theiss. \& Syd. (4)

Neocelosporiaceae Crous

Celosporium Tsuneda \& M.L. Davey (1)

Muellerites L. Holm (1)

Neocelosporium Crous (1)*

Saccotheciaceae Bonord.

Aureobasidium Viala \& G. Boyer (23)

Columnosphaeria Munk (4) 
Kabatiella Bubák (19)

Pseudoseptoria Speg. (8)

Pseudosydowia Thambug. \& K.D. Hyde (1)

Saccothecium Fr. (9)

Selenophoma Maire (ca. 13)

Zalariaceae Visagie, Z. Humphries \& Seifert

Zalaria Visagie, Z. Humphries \& Seifert (2)

Dothideales genera incertae sedis

Asteromellopsis H.E. Hess \& E. Müll. (1)

Botryochora Torrend (1)

Coniozyma Crous (1)

Hormonema Lagerb. \& Melin (7)

Pringsheimia Schulzer (17)

Rhizosphaera L. Mangin \& Har. (8)

Myriangiales Starbäck

Elsinoaceae Höhn. ex Sacc. \& Trotter

Elsinoë Racib. (ca. 40)

Molleriella G. Winter (4)

\section{Myriangiaceae Nyl.}

Anhellia Racib. (9)

Ascostratum Syd. \& P. Syd. (2)

Butleria Sacc. (1)

Dictyocyclus Sivan., W.H. Hsieh \& Chi Y. Chen (1)

Eurytheca De Seynes (3)

Hemimyriangium J. Reid \& Piroz (1)

Mendogia Racib. (7)

Micularia Boedijn (2)

Myriangium Mont. \& Berk. (ca. 10)

Uleomyces P. Henn. (12)

Zukaliopsis Henn. (2)

Myriangiales genus incertae sedis

Dictyonella Höhn. (7)

Pleosporomycetidae C.L. Schoch, Spatafora, Crous \& Shoemaker

Gloniales Jayasiri \& K.D. Hyde*

Gloniaceae (Corda) E. Boehm, C.L. Schoch \& Spatafora

Cenococcum Moug. \& Fr. (5)

Glonium Mühl. (ca. 13)

Purpurepithecium Jayasiri \& K.D. Hyde (2)

Hysteriales Lindau

Hysteriaceae Chevall.

Actidiographium Lar. N. Vassiljeva (1)

Gloniella Sacc. (12)

Gloniopsis De Not. (ca. 17)

Hysterium Pers. (14)

Hysterobrevium E. Boehm \& C.L. Schoch (6) 
Hysterocarina Zogg (1)

Hysterodifractum D.A.C. Almeida, Gusmão \& A.N. Mill. (1)

Hysteroglonium Rehm ex Lindau (2)

Oedohysterium E. Boehm \& C.L. Schoch (3)

Ostreichnion Duby (4)

Pseudoscypha J. Reid \& Piroz. (1)

Psiloglonium Höhn. (ca. 15)

Rhytidhysteron Speg. (21)

Hysteriales genus incertae sedis

Graphyllium Clem. (11)

Mytilinidiales E. Boehm, C.L. Schoch \& Spatafora

Mytilinidiaceae Kirschst.

Actidium Fr. (ca. 6)

Lophium Fr. (ca. 6)

Mytilinidion Duby (12)

Ostreola Darker (8)

Peyronelia Cif. \& Gonz. Frag. (6 or 7)

Pseudocamaropycnis Crous (1)

Quasiconcha M.E. Barr \& M. Blackw. (1)

Septonema Corda (ca. 15)

Zoggium Lar.N. Vassiljeva (1)

Pleosporales Luttrell ex M.E. Barr

Acrocalymmaceae Crous \& Trakun.

Acrocalymma Alcorn \& J.A.G. Irwin (6)

Aigialaceae Suetrong, Sakay., E.B.G. Jones, Kohlm., Volkm.-Kohlm. \& C.L. Schoch Aigialus S. Schatz \& Kohlm. (5)

Ascocratera Kohlm. (1)

Fissuroma Jian K. Liu, Phook., E.B.G. Jones \& K.D. Hyde (11)

Neoastrosphaeriella Jian K. Liu, E.B.G. Jones \& K.D. Hyde (3)

Posidoniomyces Vohník \& Réblová (1)

Rimora Kohlm., Volkm.-Kohlm., Suetrong, Sakay. \& E.B.G. Jones (1)

Amniculicolaceae Y. Zhang ter, C.L. Schoch, J. Fourn., Crous \& K.D. Hyde

Amniculicola Y. Zhang ter \& K.D. Hyde (4)

Fusiformispora Phukhams. \& K.D. Hyde (1)

Murispora Y. Zhang ter, J. Fourn. \& K.D. Hyde (7)

Neomassariosphaeria Y. Zhang ter, J. Fourn. \& K.D. Hyde (1)

Pseudomassariosphaeria Phukhams., Ariyaw., Camporesi \& K.D. Hyde (2)

Vargamyces Tóth (1)

Amorosiaceae Thambug. \& K.D. Hyde

Alfoldia D.G. Knapp, Imrefi \& Kovács (1)

Amorosia Mantle \& D. Hawksw. (1)

Amorocoelophoma Jayasiri, E.B.G. Jones \& K.D. Hyde (1)

Angustimassarina Thambug., Kaz. Tanaka \& K.D. Hyde (11)

Anteagloniaceae K.D. Hyde, Jian K. Liu \& A. Mapook

Anteaglonium Mugambi \& Huhndorf (7) 
Flammeascoma Phook. \& K.D. Hyde (2)

Purplema W. Dong, H. Zhang \& K.D. Hyde (1)

Aquasubmersaceae A. Hashim. \& Kaz. Tanaka

Aquasubmersa K.D. Hyde \& Huang Zhang (2)

Arthopyreniaceae W. Watson

Arthopyrenia A. Massal. (= Arthopyreniomyces Cif. \& Tomas.) (5 + ca. 100 orphaned)

Mycomicrothelia Keissl. (ca. 10)*

Ascocylindricaceae Abdel-Wahab, Bahkali, E.B.G. Jones, Ariyaw. \& K.D. Hyde Ascocylindrica Abdel-Wahab, Bahkali \& E.B.G. Jones (1)

Astrosphaeriellaceae Phook. \& K.D. Hyde

Astrosphaeriella Syd. \& P. Syd. (ca. 10)

Astrosphaeriellopsis Phook., Jian K. Liu \& K.D. Hyde (2)

Javaria Boise (2)

Mycopepon Boise (5)

Pithomyces Berk. \& Broome (ca. 40)

Pteridiospora Penz. \& Sacc. (8)

Quercicola Jayasiri, E.B.G. Jones \& K.D. Hyde (2)

Xenoastrosphaeriella Jayasiri, E.B.G. Jones \& K.D. Hyde (1)

Bambusicolaceae D.Q. Dai \& K.D. Hyde

Bambusicola D.Q. Dai \& K.D. Hyde (10)

Leucaenicola Jayasiri, E.B.G. Jones \& K.D. Hyde (2)

Palmiascoma Phook. \& K.D. Hyde (1)

Biatriosporaceae K.D. Hyde

Biatriospora K.D. Hyde \& Borse (6)

Camarosporiaceae Wanas., Wijayaw., K.D. Hyde \& Crous

Camarosporium Schulzer (100+)

Camarosporomyces Crous (1)

Camarosporidiellaceae Wanas., Wijayaw., Crous \& K.D. Hyde

Camarosporidiella Wanas., Wijayaw., K.D. Hyde (22)

Caryosporaceae Huang Zhang, K.D. Hyde \& Ariyaw.

Caryospora De Not. (19)

Coniothyriaceae W.B. Cooke

Coniothyrium Corda (ca. 50)

Foliophoma Crous (2)

Neoconiothyrium Crous (3)

Ochrocladosporium Crous \& U. Braun (3)

Staurosphaeria Rabenh. (= Hazslinszkyomyces Crous \& R.K. Schumach.) (12)

\section{Corynesporascaceae Sivan.}

Corynespora Güssow (ca. 130)

Corynesporasca Sivan. (1) 
Cryptocoryneaceae A. Hashim. \& Kaz. Tanaka

Cryptocoryneum Fuckel (ca. 20)

Cucurbitariaceae G. Winter (= Fenestellaceae M.E. Barr)

Allocucurbitaria Valenz.-Lopez, Stchigel, Guarro \& Cano (1)

Astragalicola Jaklitsch \& Voglmayr (2)*

Cucitella Jaklitsch \& Voglmayr (1)*

Cucurbitaria Gray (= Pleurostromella Petr.) (ca. 40)

Fenestella Tul. \& C. Tul. (ca. 4)

Neocucurbitaria Wanas., E.B.G. Jones \& K.D. Hyde (21)

Paracucurbitaria Valenz.-Lopez, Stchigel, Guarro \& Cano (2)

Parafenestella Jaklitsch \& Voglmayr (3)*

Protofenestella Jaklitsch \& Voglmayr (1)*

Rhytidiella Zalasky (4)

Seltsamia Jaklitsch \& Voglmayr (1)*

Syncarpella Theiss. \& Syd. (ca. 6)

Synfenestella Jaklitsch \& Voglmayr (2)

Cyclothyriellaceae Jaklitsch \& Voglmayr

Cyclothyriella Jaklitsch \& Voglmayr (1)

Massariosphaeria (E. Müll.) Crivelli (25)*

\section{Dacampiaceae Körb.}

Aaosphaeria Aptroot (1)

Dacampia A. Massal. (15)

Eopyrenula R.C. Harris (6)

Leptocucurthis Aptroot (1)

Pseudonitschkia Coppins \& S.Y. Kondr. (1)

Weddellomyces D. Hawksw. (12)

Delitschiaceae M.E. Barr

Delitschia Auersw. (ca. 50)

Ohleriella Earle (1)

Semidelitschia Cain \& Luck-Allen (3)

Diademaceae Shoemaker \& C.E. Babc.

Diadema Shoemaker \& C.E. Babc. (8)

Dictyosporiaceae Boonmee \& K.D. Hyde

Aquadictyospora Z.L. Luo, K.D. Hyde \& H.Y. Su (1)

Aquaticheirospora Kodsueb \& W.H. Ho (1)

Cheirosporium L. Cai \& K.D. Hyde (2)

Dendryphiella Bubák \& Ranoj. (12)

Dictyocheirospora M.J. D'souza, Boonmee \& K.D. Hyde (16)

Dictyopalmispora Pinruan, Boonmee \& K.D. Hyde (1)

Dictyosporium Corda (59)

Digitodesmium P.M. Kirk (6)

Gregarithecium Kaz. Tanaka \& K. Hiray. (1)

Jalapriya M.J. D'souza, Hong Y. Su, Z.L. Luo \& K.D. Hyde (3)

Neodendryphiella Iturrieta-González, Dania García \& Gené (3)*

Pseudocoleophoma Kaz. Tanaka \& K. Hiray. (3)

Pseudoconiothyrium Crous \& R.K. Schumach. (1) 
Pseudodictyosporium Matsush. (4)

Vikalpa M.J. D'souza, Boonmee, Bhat \& K.D. Hyde (4)

Didymellaceae Gruyter, Aveskamp \& Verkley (=Microsphaeropsidaceae Qian Chen, L. Cai \& Crous fide Hongsanan et al. 2020)

Allophoma Q. Chen \& L. Cai (9)

Anthodidymella Phukhams., Camporesi \& K.D. Hyde (3)

Ascochyta Lib. (= Heracleicola Tibpromma, Camporesi \& K.D. Hyde) (ca. 400)

Boeremia Aveskamp, Gruyter \& Verkley (22)

Briansuttonomyces Crous (1)

Calophoma Q. Chen \& L. Cai (8)

Chaetasbolisia Speg. (7)

Cumuliphoma Valenz.-Lopez, Stchigel, Crous, Guarro \& Cano (3)

Didymella Sacc. ex D. Sacc. (ca. 100)

Didysimulans Tibpromma, Camporesi \& K.D. Hyde (2)

Ectophoma Valenz.-Lopez, Cano, Crous, Guarro \& Stchigel (2)

Epicoccum Link (16)

Heterophoma Q. Chen \& L. Cai (6)

Juxtiphoma Valenzuela-Lopez, Cano, Crous, Guarro \& Stchigel (1)

Leptosphaerulina McAlpine (30)

Macroventuria Aa (2)

Microsphaeropsis Höhn. (37)

Mixtura O.E. Erikss. \& J.Z. Yue (1)

Monascostroma Höhn. (ca. 5)

Neoascochyta Q. Chen \& L. Cai (12)

Neodidymella Phook., R.H. Perera \& K.D. Hyde (1)

Neodidymelliopsis Q. Chen \& L. Cai (10)

Neomicrosphaeropsis Thambug., Camporesi \& K.D. Hyde (10)

Nothophoma Q. Chen \& L. Cai (9)

Paraboeremia Q. Chen \& L. Cai (6)

Phoma Sacc. (= Endophoma Tsuneda \& M.L. Davey) (100)

Phomatodes Q. Chen \& L. Cai (2)

Platychora Petr. (1)

Remotididymella Valenz.-Lopez (2)

Similiphoma Valenz.-Lopez, Crous, Cano, Guarro \& Stchigel (1)

Stagonosporopsis Died. (22)

Vacuiphoma Valenz.-Lopez, Cano, Crous, Guarro \& Stchigel (2)

Xenodidymella Q. Chen \& L. Cai (6)

\section{Didymosphaeriaceae Munk}

Alloconiothyrium Verkley \& Stielow (1)

Austropleospora R.G. Shivas \& L. Morin (1)

Barria Z.Q. Yuan (1)

Bimuria D. Hawksw., Chea \& Sheridan (1)

Chromolaenicola Mapook \& K.D. Hyde (5)

Curreya Sacc. (2)

Cylindroaseptospora Jayasiri, E.B.G. Jones \& K.D. Hyde (2)

Deniquelata Ariyaw. \& K.D. Hyde (2)

Didymocrea Kowalski (1)

Didymosphaeria Fuckel (ca. 25)

Julella Fabre (ca. 20)

Kalmusia Niessl (16) 
Kalmusibambusa Phook., Tennakoon, Thambug. \& K.D. Hyde (1)*

Karstenula Speg. (16)

Laburnicola Wanas., Camporesi, E.B.G. Jones \& K.D. Hyde (4)

Letendraea Sacc. (ca. 3)

Lineostroma H.J. Swart (1)

Montagnula Berl. (ca. 30)*

Neokalmusia Ariyaw. \& K.D. Hyde (6)

Neptunomyces M. Gonçalves, T. Vicente \& A. Alves (1)*

Paracamarosporium Wijayaw. \& K.D. Hyde (7)

Paraconiothyrium Verkley (19)

Paramassariosphaeria Wanas., E.B.G. Jones \& K.D. Hyde (2)

Paraphaeosphaeria O.E. Erikss. (33)

Phaeodothis Syd. \& P. Syd. (5)

Pseudocamarosporium Wijayaw. \& K.D. Hyde (14)

Pseudopithomyces Ariyaw. \& K.D. Hyde (10)

Spegazzinia Sacc. (11)*

Tremateia Kohlm., Volkm.-Kohlm. \& O.E. Erikss. (5)

Verrucoconiothyrium Crous (4)

Vicosamyces Firmino, Machado \& Pereira (1)

Xenocamarosporium Crous \& M.J. Wingf. (1)

Dothidotthiaceae Crous \& A.J.L. Phillips

Belizeana Kohlm. \& Volkm. (1)

Dothidotthia Höhn. (= Neodothidotthia Crous) (ca. 10)

Mycocentrospora Deighton (4)

Phaeomycocentrospora Crous, H.D. Shin \& U. Braun (1)

Pleiochaeta (Sacc.) S. Hughes (4)

Thyrostroma Höhn. (ca. 45)

Wilsonomyces Adask., J.M. Ogawa \& E.E. Butler (1)

Fuscostagonosporaceae Jayasiri, Camporesi \& K.D. Hyde

Fuscostagonospora Kaz. Tanaka \& K. Hiray. (3)

Fusculinaceae Crous

Fusculina Crous \& Summerell (3)

Gordonomyces Crous \& Marinc. (1)

Halojulellaceae Suetrong, K.D. Hyde \& E.B.G. Jones

Halojulella Suetrong, K.D. Hyde \& E.B.G. Jones (1)

Halotthiaceae Ying Zhang, J. Fourn. \& K.D. Hyde

Brunneoclavispora Phook. \& K.D. Hyde (1)

Halotthia Kohlm. (1)

Mauritiana Poonyth, K.D. Hyde, Aptroot \& Peerally (1)

Neolophiostoma S. Boonmee \& K.D. Hyde (1)*

Pontoporeia Kohlm. (1)

Sulcosporium Phook. \& K.D. Hyde (1)

Hermatomycetaceae Locq.

Hermatomyces Speg. (ca. 20) 
Hypsostromataceae Huhndorf

Hypsostroma Huhndorf (2)

Latoruaceae Crous

Latorua Crous (1)

Matsushimamyces Rahul Sharma \& Rohit Sharma (2)

Polyschema H.P. Upadhyay (22)

Pseudoasteromassaria M. Matsum. \& Kaz. Tanaka (2)

Lentimurisporaceae N.G. Liu, J.K Liu \& K.D. Hyde

Bahusandhika Subram. (9)*

Lentimurispora N.G. Liu, Bhat \& K.D. Hyde (1)*

Lentitheciaceae Y. Zhang ter, C.L. Schoch, J. Fourn., Crous \& K.D. Hyde

Darksidea D.G. Knapp, Kovács, J.Z. Groenew. \& Crous (6)

Halobyssothecium Dayar., E.B.G. Jones \& K.D. Hyde (1)

Katumotoa Kaz. Tanaka \& Y. Harada (1)

Keissleriella Höhn. (ca. 36)

Lentithecium K.D. Hyde, J. Fourn. \& Ying Zhang (9)*

Murilentithecium Wanas., Camporesi, E.B.G. Jones \& K.D. Hyde (3)

Neoophiosphaerella Kaz. Tanaka \& K. Hiray. (1)

Phragmocamarosporium Wijayaw., Yong Wang bis \& K.D. Hyde (2)

Pleurophoma Höhn. (ca. 9)

Poaceascoma Phook. \& K.D. Hyde (4)

Pseudomurilentithecium Mapook \& K.D. Hyde (1)

Setoseptoria Quaedvl., Verkley \& Crous (7)

Tingoldiago K. Hiray. \& Kaz. Tanaka (1)

Towyspora Wanas., E.B.G. Jones \& K.D. Hyde (1)

Leptosphaeriaceae M.E. Barr

Alloleptosphaeria Ariyaw., Wanas. \& K.D. Hyde (1)

Alternariaster E.G. Simmons (4)

Chaetoplea (Sacc.) Clem. (ca. 20)

Heterosporicola Crous (2)

Leptosphaeria Ces. \& De Not. (151)

Neoleptosphaeria Ariyaw. \& K.D. Hyde (2)

Paraleptosphaeria Gruyter, Aveskamp \& Verkley (7)

Plenodomus Preuss (19)

Pseudoleptosphaeria Ariyaw. \& K.D. Hyde (1)

Querciphoma Crous (2)

Sclerenchymomyces Phukhams. \& K.D. Hyde (2)

Sphaerellopsis Cooke (6)

Subplenodomus Gruyter, Aveskamp \& Verkley (6)

\section{Libertasomycetaceae Crous}

Libertasomyces Crous \& Roets (3)

Neoplatysporoides Crous \& M.J. Wingf. (1)

Ligninsphaeriaceae K.D. Hyde \& Ariyaw. (Nom. inval., Art. 38.1(a) (Melbourne) fide Index Fungorum 2020)

Ligninsphaeria Jin F. Zhang, Jian K. Liu, K.D. Hyde \& Zi Y. Liu (1)

Ligninsphaeriopsis Phukh., Feng \& K.D. Hyde (1) 
Lindgomycetaceae K. Hiray., Kaz. Tanaka \& Shearer

Arundellina Wanas., E.B.G. Jones \& K.D. Hyde (1)

Clohesyomyces K.D. Hyde (1)

Hongkongmyces C.C.C. Tsang, J.F.W. Chan, Trend.-Sm., A.H.Y. Ngan, I.W.H. Ling,

S.K.P. Lau \& P.C.Y. Woo (3)

Lindgomassariosphaeria W. Dong, H. Zhang \& K.D. Hyde (1)

Lindgomyces K. Hiray., Kaz. Tanaka \& Shearer (13)

Lolia Abdel-Aziz \& Abdel-Wahab (1)

Neolindgomyces Jayasiri, E.B.G. Jones \& K.D. Hyde (4)

Lizoniaceae Boonmee \& K.D. Hyde

Lizonia (Ces. \& De Not.) De Not. (22)

Longipedicellataceae Phukhams., Bhat \& K.D. Hyde

Longipedicellata H. Zhang, K.D. Hyde \& Jian K. Liu (1)

Pseudoxylomyces Kaz. Tanaka \& K. Hiray. (1)

Submerspora W. Dong, H. Zhang \& K.D. Hyde (1)

Longiostiolaceae Phukhams., Doilom \& K.D. Hyde

Longiostiolum Doilom, Ariyaw. \& K.D. Hyde (1)

Lophiostomataceae Sacc.

Alpestrisphaeria Thambug. \& K.D. Hyde (2)

Biappendiculispora Thambug., Kaz. Tanaka \& K.D. Hyde (1)

Capulatispora Thambug. \& K.D. Hyde (1)

Coelodictyosporium Thambug. \& K.D. Hyde (3)

Crassiclypeus A. Hashim., K. Hiray. \& Kaz. Tanaka (1)*

Decaisnella Fabre (13)

Dimorphiopsis Crous (1)

Flabellascoma A. Hashim., K. Hiray. \& Kaz. Tanaka (2)*

Guttulispora Thambug., Qing Tian \& K.D. Hyde (1)

Kiskunsagia D.G. Knapp, Imrefi \& Kovács (1)

Lentistoma A. Hashim., K. Hiray. \& Kaz. Tanaka (1)

Leptoparies A. Hashim., K. Hiray. \& Kaz. Tanaka (1)*

Lophiohelichrysum Dayar., Camporesi \& K.D. Hyde (1)

Lophiopoacea Ariyaw., Thambug. \& K.D. Hyde (2)

Lophiostoma Ces. \& De Not. (ca. 100)

Neopaucispora Wanas., Gafforov \& K.D. Hyde (1)

Neotrematosphaeria Thambug., Kaz. Tanaka \& K.D. Hyde (1)

Neovaginatispora A. Hashim., K. Hiray. \& Kaz. Tanaka (1)

Parapaucispora A. Hashim., K. Hiray. \& Kaz. Tanaka (1)

Paucispora Thambug., Kaz. Tanaka \& K.D. Hyde (3)

Platystomum Trevis. (ca. 20)

Pseudocapulatispora Mapook \& K.D. Hyde (inpress)

Pseudolophiostoma Thambug., Kaz. Tanaka \& K.D. Hyde (5)

Pseudopaucispora A. Hashim., K. Hiray. \& Kaz. Tanaka (1)*

Pseudoplatystomum Thambug. \& K.D. Hyde (1)

Quintaria Kohlm. \& Volkm.-Kohlm (3)

Sigarispora Thambug. \& K.D. Hyde (14)

Vaginatispora K.D. Hyde (8) 
Lophiotremataceae K. Hiray. \& Kaz.

Atrocalyx A. Hashim. \& Kaz. Tanaka (6)

Crassimassarina A. Hashim. \& Kaz. Tanaka (1)

Cryptoclypeus A. Hashim. \& Kaz. Tanaka (2)

Galeaticarpa A. Hashim. \& Kaz. Tanaka (1)

Koordersiella Höhn. (6)

Lophiotrema Sacc. (17)

Pseudocryptoclypeus A. Hashim. \& Kaz. Tanaka (1)

Macrodiplodiopsidaceae Voglmayr, Jaklitsch \& Crous

Macrodiplodiopsis Petr. (2)

Pseudochaetosphaeronema Punith. (4)

Massariaceae Nitschke

Massaria De Not. (31)

Massarioramusculicola Huanral., Thambug. \& K.D. Hyde (1)

Paramassaria Samarak. \& K.D. Hyde (1)

\section{Massarinaceae Munk}

Byssothecium Fuckel (8)

Helminthosporium Link (= Helminthosporiella Hern.-Restr., G.A. Sarria \& Crous) (ca. 416)

Massarina Sacc. (ca. 100)

Pseudodidymosphaeria Thambug. \& K.D. Hyde (2)

Pseudosplanchnonema Chethana \& K.D. Hyde (1)

Semifissispora H.J. Swart (5)

Stagonospora (Sacc.) Sacc. (220)

Suttonomyces Wijayaw., Camporesi \& K.D. Hyde (2)

Melanommataceae G. Winter (= Pseudodidymellaceae A. Hashim. \& Kaz. Tanaka)

Alpinaria Jaklitsch \& Voglmayr (1)

Aposphaeria Sacc. (189)

Asymmetricospora J. Fröhl. \& K.D. Hyde (1)

Bertiella (Sacc.) Sacc. \& P. Syd. (2)

Bicrouania Kohlm. \& Volkm.-Kohlm. (1)

Byssosphaeria Cooke (27)

Calyptronectria Speg. (3)

Camposporium Harkn. (21)*

Exosporiella P. Karst. (1)

Fusiconidium Jun F. Li, Phook. \& K.D. Hyde (2)

Herpotrichia Fuckel (101)

Mamillisphaeria K.D. Hyde, S.W. Wong \& E.B.G. Jones (1)

Marjia Wanas., Gafforov \& K.D. Hyde (1)

Melanocamarosporioides D. Pem, R. Jeewon, Gafforov \& K.D. Hyde (1)

Melanocamarosporium Wijayaw., Camporesi, Bhat \& K.D. Hyde (2)

Melanocucurbitaria Wanas., Gafforov \& K.D. Hyde (1)

Melanodiplodia Wanas., Gafforov \& K.D. Hyde (1)

Melanomma Nitschke ex Fuckel (ca. 30)

Monoseptella Wanas., Gafforov \& K.D. Hyde (1)

Muriformistrickeria Q. Tian, Wanas., Camporesi \& K.D. Hyde (2)

Navicella Fabre (5)

Neobyssosphaeria Wanas., Jones \& K.D. Hyde (1)

Petrakia Syd. \& P. Syd. (6) 
Phragmocephala E.W. Mason \& S. Hughes (10)

Phragmotrichum Kunze (4)

Pleotrichocladium Hern.-Restr., R.F. Castañeda \& Gené (1)

Praetumpfia Jaklitsch \& Voglmayr (1)

Pseudobyssosphaeria H.B. Jiang \& K.D. Hyde (1)

Pseudodidymella C.Z. Wei, Y. Harada \& Katum. (2)

Pseudostrickeria Q. Tian, Wanas., Camporesi \& K.D. Hyde (3)

Sarimanas M. Matsum., K. Hiray. \& Kaz. Tanaka (2)

Seifertia Partr. \& Morgan-Jones (2)

Tumularia Descals \& Marvanová (2)

Uzbekistanica Wanas., Gafforov \& K.D. Hyde (3)

Xenostigmina Crous (2)

Morosphaeriaceae Suetrong, Sakay., E.B.G. Jones \& C.L. Schoch

Aquihelicascus W. Dong, H. Zhang \& Doilom (3)

Aquilomyces D.G. Knapp, Kovács, J.Z. Groenew. \& Crous (2)

Clypeoloculus Kaz. Tanaka \& K. Hiray. (4)

Helicascus Kohlm. (12)

Morosphaeria Suetrong, Sakay., E.B.G. Jones \& C.L. Schoch (4)

Neohelicascus W. Dong, H. Zhang \& Doilom (8)

Mycoporaceae Zahlbr.

Mycoporum Flot. ex Nyl. (ca. 5 + c. 35 orphaned, partly in Mycoporellum Müll. Arg.)

Neocamarosporiaceae Wanas., Wijayaw., Crous \& K.D. Hyde

Dimorphosporicola Crous (1)

Neocamarosporium Crous \& M.J. Wingf. (15)

Neohendersoniaceae Giraldo \& Crous

Brevicollum Kaz. Tanaka (2)

Crassiparies M. Matsum., K. Hiray. \& Kaz. Tanaka (1)

Medicopsis Gruyter, Verkley \& Crous (2)

Neohendersonia Petr. (4)

Neomedicopsis Crous \& Akulov (1)

Neomassariaceae H.A. Ariyaw., Jaklitsch \& Voglmayr

Neomassaria Mapook, Camporesi \& K.D. Hyde (2)

Neomassarinaceae Mapook \& K.D. Hyde

Neomassarina Phook., Jayasiri \& K.D. Hyde (2)

Pseudohelminthosporium Phukhams. \& K.D. Hyde (1)

Neophaeosphaeriaceae Ariyaw. \& K.D. Hyde

Neophaeosphaeria M.P.S. Câmara, M.E. Palm \& A.W. Ramaley (6)

Neopyrenochaetaceae Valenz.-Lopez, Crous, Cano, Guarro \& Stchigel

Neopyrenochaeta Valenz.-Lopez, Crous, Stchigel, Guarro \& Cano (5)

Nigrogranaceae Jaklitsch \& Voglmayr

Nigrograna Gruyter, Verkley \& Crous (12) 
Occultibambusaceae D.Q. Dai \& K.D. Hyde

Brunneofusispora S.K. Huang \& K.D. Hyde (1)*

Neooccultibambusa Doilom \& K.D. Hyde (4)

Occultibambusa D.Q. Dai \& K.D. Hyde (7)

Seriascoma Phook., D.Q. Dai \& K.D. Hyde (2)

Versicolorisporium Sat. Hatak., Kaz. Tanaka \& Y. Harada (1)

Ohleriaceae Jaklitsch \& Voglmayr

Ohleria Fuckel (13)

Parabambusicolaceae Kaz. Tanaka \& K. Hiray.

Aquastroma Kaz. Tanaka \& K. Hiray. (1)

Lonicericola Phook., Jayasiri \& K.D. Hyde (1)*

Multilocularia Phook. (1)

Multiseptospora Phook. \& K.D. Hyde (2)

Neoaquastroma Wanas., E.B.G. Jones \& K.D. Hyde (3)

Parabambusicola Kaz. Tanaka \& K. Hiray. (2)

Paramonodictys N.G. Liu, K.D. Hyde \& J.K. Liu (1)

Paratrimmatostroma Jayasiri, Phook., D.J. Bhat \& K.D. Hyde (1)*

Pseudomonodictys Doilom, Ariyaw., Bhat \& K.D. Hyde (1)

Paradictyoarthriniaceae Doilom, Ariyaw., Bhat \& K.D. Hyde

Paradictyoarthrinium Matsush. (4)

Xenomassariosphaeria Jayasiri, Wanas. \& K.D. Hyde (1)

Paralophiostomataceae V.V. Sarma \& M. Niranjan.

Paralophiostoma V.V. Sarma \& M. Niranjan. (1)

Parapyrenochaetaceae Valenz.-Lopez, Crous, Stchigel, Guarro \& Cano

Parapyrenochaeta Valenz.-Lopez, Crous, Stchigel, Guarro \& Cano (2)

Quixadomyces Cantillo \& Gusmão (1)

\section{Periconiaceae Nann.}

Bambusistroma D.Q. Dai \& K.D. Hyde (1)

Flavomyces D.G. Knapp, Kovács, J.Z. Groenew. \& Crous (1)

Noosia Crous, R.G. Shivas \& McTaggart, Persoonia (1)*

Periconia Tode (46)

Phaeoseptaceae S. Boonmee, Thambug. \& K.D. Hyde

Phaeoseptum Ying Zhang, J. Fourn. \& K.D. Hyde (2)

Pleopunctum N.G. Liu, K.D. Hyde \& J.K. Liu (2)

Phaeosphaeriaceae M.E. Barr

Acericola Wanas., Camporesi, E.B.G. Jones \& K.D. Hyde (1)

Allophaeosphaeria Ariyaw., Camporesi \& K.D. Hyde (3)

Amarenographium O.E. Erikss. (4)

Amarenomyces O.E. Erikss. (2)*

Ampelomyces Ces. ex Schltdl. (ca. 5)

Aphanostigme Syd. (21)

Arezzomyces Y. Marín \& Crous (1)

Banksiophoma Crous (1)

Bhagirathimyces S.M. Singh \& S.K. Singh (1) 
Bhatiellae Wanas., Camporesi \& K.D. Hyde (1)

Bricookea M.E. Barr (1)

Brunneomurispora Phook., Wanas. \& K.D. Hyde (1)*

Camarosporioides W.J. Li \& K.D. Hyde (1)

Chaetosphaeronema Moesz (12)

Dactylidina Wanas., Camporesi \& K.D. Hyde (2)

Dematiopleospora Wanas., Camporesi, E.B.G. Jones \& K.D. Hyde (8)

Didymocyrtis Vain. (21)

Dlhawksworthia Wanas., Camporesi \& K.D. Hyde (3)

Edenia M.C. González, A.L. Anaya, Glenn, Saucedo \& Hanlin (2)

Embarria Wanas., Camporesi \& K.D. Hyde (1)

Equiseticola Abdelsalam, Tibpromma, Wanas. \& K.D. Hyde (1)

Eudarluca Speg. (8)*

Galiicola Tibpromma, Camporesi \& K.D. Hyde (3)

Hydeomyces Maharachch., H.A. Ariyaw., Wanas. \& Al-Sadi (2)

Hydeopsis J.F. Zhang, J.K. Liu \& Z.Y. Liu (1)

Italica Wanas., Camporesi \& K.D. Hyde (2)

Jeremyomyces Crous \& R.K. Schumach. (1)

Juncaceicola Tennakoon, Camporesi, Phook. \& K.D. Hyde (8)

Kwanghwana A. Karunarathna, C. H. Kuo \& K. D. Hyde (1)

Leptospora Rabenh. (15)

Longispora Phukhams. \& K.D. Hyde (1)

Loratospora Kohlm. \& Volkm.-Kohlm. (2)

Mauginiella Cavara (1)

Melnikia Wijayaw., Goonas., Bhat \& K.D. Hyde (1)

Murichromolaenicola Mapook \& K.D. Hyde (2)

Muriphaeosphaeria Phukhams., Bulgakov \& K.D. Hyde (3)

Neoophiobolus Mapook \& K.D. Hyde (1)

Neosetophoma Gruyter, Aveskamp \& Verkley (15)

Neosphaerellopsis Crous \& Trakun. (10)

Neostagonospora Quaedvl., Verkley \& Crous (6)

Neostagonosporella C.L. Yang, X.L. Xu \& K.D. Hyde (1)*

Neosulcatispora Crous \& M.J. Wingf. (2)

Nodulosphaeria Rabenh. (ca. 52)

Ophiobolopsis Phook., Wanas. \& K.D. Hyde (1)*

Ophiobolus Riess (350)

Ophiosimulans Tibpromma, Camporesi \& K.D. Hyde (1)

Ophiosphaerella Speg. (10)

Paraleptospora Mapook \& K.D. Hyde (2)

Paraloratospora Bundhun, Tennakoon, Phookamsak \& K.D. Hyde (2)

Paraophiobolus Phook., Wanas. \& K.D. Hyde (2)*

Paraphoma Morgan-Jones \& J.F. White (8)

Parastagonospora Quaedvl., Verkley \& Crous (ca. 19)

Parastagonosporella M. Bakhshi, Arzanlou \& Crous (1)

Phaeopoacea Thambug., Dissan. \& K.D. Hyde (3)*

Phaeoseptoriella Crous (1)

Phaeosphaeria I. Miyake (ca. 95)

Phaeosphaeriopsis M.P.S. Câmara, M.E. Palm \& A.W. Ramaley (12)

Phaeostagonospora A.W. Ramaley (1)

Piniphoma Crous \& R.K. Schumach. (1)

Poaceicola W.J. Li, Camporesi, Bhat \& K.D. Hyde (10)

Populocrescentia Wanas., E.B.G. Jones \& K.D. Hyde (3) 
Pseudoophiobolus Phook., Wanas. \& K.D. Hyde (8)*

Pseudoophiosphaerella J.F. Zhang, J.K. Liu \& Z.Y. Liu (1)

Pseudophaeosphaeria Jayasiri, Camporesi \& K.D. Hyde (1)

Pseudostaurosphaeria Mapook \& K.D. Hyde (2)

Sclerostagonospora Höhn. (ca. 15)

Scolicosporium Lib. ex Roum. (13)

Septoriella Oudem. (=Wojnowicia Sacc.) (22)*

Setomelanomma M. Morelet (1)

Setophoma Gruyter, Aveskamp \& Verkley (6)

Sulcispora Shoemaker \& C.E. Babc. (2)

Tiarospora Sacc. \& Marchal (3)

Tintelnotia S.A. Ahmed, Hofmüller, M. Seibold \& de Hoog (2)

Vagicola K.W.T. Chethana \& K.D. Hyde (1)

Vittaliana Devadatha, Nikita, A. Baghela \& V.V. Sarma (1)*

Vrystaatia Quaedvl., W.J. Swart, Verkley \& Crous (1)

Wingfieldomyces Y. Marín \& Crous (1)

Wojnowiciella Crous, Hern.-Restr. \& M.J. Wingf. (9)

Xenophaeosphaeria Crous \& M.J. Wingf. (1)

Xenophoma Crous \& Trakunyingcharoen (1)

Xenoseptoria Quaedvl., H.D. Shin, Verkley \& Crous (1)

Yunnanensis Karun., Phook. \& K.D. Hyde (1)*

Pleomassariaceae M.E. Barr

Beverwykella Tubaki (3)

Lichenopyrenis Calat., Sanz \& Aptroot (1)

Myxocyclus Riess (1)

Peridiothelia D. Hawksw. (3)

Prosthemium Kunze (ca. 8)

Pseudotrichia Kirschst. (ca. 8)

Splanchnonema Corda (37)

Pleomonodictydaceae Hern.-Restr., J. Mena \& Gené

Pleomonodictys Hern.-Restr., J. Mena \& Gené (2)

Pleohelicoon Jayasiri, E.B.G. Jones \& K.D. Hyde (2)

Pleosporaceae Nitschke

Allonecte Syd. (3)

Alternaria Nees (ca. 360)*

Bipolaris Shoemaker (69)

Clathrospora Rabenh. (20)

Comoclathris Clem. (30)

Curvularia Boedijn (119)

Decorospora Inderb., Kohlm. \& Volkm.-Kohlm. (1)

Diademosa Shoemaker \& C.E. Babc. (4)*

Dichotomophthora Mehrl. \& Fitzp. ex P.N. Rao (6)

Exserohilum K.J. Leonard \& Suggs (ca. 30)

Extrawettsteinina M.E. Barr (4)

Gibbago E.G. Simmons (1)

Johnalcornia Y.P. Tan \& R.G. Shivas (1)

Paradendryphiella Woudenberg \& Crous (2)

Platysporoides (Wehm.) Shoemaker \& C.E. Babc. (11)

Pleoseptum A.W. Ramaley \& M.E. Barr (1) 
Porocercospora Amaradasa, Amundsen, Madrid \& Crous (1)*

Prathoda Subram. (2)*

Pseudoyuconia Lar.N. Vassiljeva (1)

Pyrenophora Fr. (= Marielliottia Shoemaker) (ca. 95)

Stemphylium Wallr. (ca. 96)*

Tamaricicola Thambug., Camporesi \& K.D. Hyde (1)

Typhicola Crous (1)

Pseudoastrosphaeriellaceae Phook. \& K.D. Hyde

Carinispora K.D. Hyde (2)

Pseudoastrosphaeriella Phook., Z.L. Luo \& K.D. Hyde (6)

Pseudoastrosphaeriellopsis Devadatha, Wanas., Jeewon \& V.V. Sarma (1)*

Pseudoberkleasmiaceae Phukhams. \& K.D. Hyde

Pseudoberkleasmium Tibpromma \& K.D. Hyde (3)

Pseudocoleodictyosporaceae Doilom \& K.D. Hyde

Pseudocoleodictyospora Doilom \& K.D. Hyde (3)

Subglobosporium Doilom \& K.D. Hyde (1)

Pseudolophiotremataceae K.D. Hyde \& Hongsanan

Clematidis Tibpromma, Camporesi \& K.D. Hyde (1)

Pseudolophiotrema A. Hashim. \& Kaz. Tanaka (1)

Pseudomassarinaceae Phukhams \& K.D. Hyde

Pseudomassarina Phukhams. \& K.D. Hyde (1)

Pseudopyrenochaetaceae Valenz.-López, Crous, Stchigel, Guarro \& J.F. Cano

Pseudopyrenochaeta Valenzuela-López, Crous, Stchigel, Guarro \& Cano (2)

Pyrenochaetopsidaceae Valenz.-López, Crous, Cano, Guarro \& Stchigel

Pyrenochaetopsis Gruyter, Aveskamp \& Verkley (7)

Neopyrenochaetopsis Valenz-López, Cano, Guarro \& Stchigel (1)

Xenopyrenochaetopsis Valenz.-Lopez, Crous, Stchigel, Guarro \& Cano (1)

Roussoellaceae Jian K. Liu, Phook., D.Q. Dai \& K.D. Hyde

Appendispora K.D. Hyde (2)

Cytoplea Bizz. \& Sacc. (5)

Elongatopedicellata Jin F. Zhang, Jian K. Liu, K.D. Hyde \& Zi Y. Liu (1)

Immotthia M.E. Barr (2)*

Neoroussoella Jian K. Liu, Phook. \& K.D. Hyde (7)

Pararoussoella Wanas., E.B.G. Jones \& K.D. Hyde (3)*

Pseudoneoconiothyrium Wanas., Phukhams., Camporesi \& K.D. Hyde (1)

Pseudoroussoella Mapook \& K.D. Hyde (2)

Roussoella Sacc. (38)

Roussoellopsis I. Hino \& Katum. (3)

Setoarthopyrenia Mapook \& K.D. Hyde (1)

Xenoroussoella Mapook \& K.D. Hyde (1)

Salsugineaceae K.D. Hyde \& Tibpromma

Acrocordiopsis Borse \& K.D. Hyde (2)

Salsuginea K.D. Hyde (1) 
Shiraiaceae Y.X. Liu, Zi Y. Liu \& K.D. Hyde

Grandigallia M.E. Barr, Hanlin, Cedeño, Parra \& R. Hern. (1)

Rubroshiraia D.Q. Dai \& K.D. Hyde (1)

Shiraia Henn. (1)

\section{Sporormiaceae Munk}

Chaetopreussia Locq.-Lin. (1)

Forliomyces Phukhams., Camporesi \& K.D. Hyde (1)

Pleophragmia Fuckel (1)

Preussia Fuckel (51)

Sparticola Phukhams., Ariyaw., Camporesi \& K.D. Hyde (4)

Sporormia De Not. (29)

Sporormiella Ellis \& Everh. (60)*

Sporormurispora Wanas., Bulgakov, Gafforov \& K.D. Hyde (2)

Westerdykella Stolk (50)

Striatiguttulaceae S.N. Zhang, K.D. Hyde \& J.K. Liu

Longicorpus S.N. Zhang, K.D. Hyde \& J.K. Liu (1)

Striatiguttula S.N. Zhang, K.D. Hyde \& J.K. Liu (2)

Sulcatisporaceae Kaz. Tanaka \& K. Hiray.

Anthosulcatispora Phukhams. \& K.D. Hyde (2)

Magnicamarosporium Kaz. Tanaka \& K. Hiray. (2)

Neobambusicola Crous \& M.J. Wingf. (2)

Parasulcatispora Phukhams. \& K.D. Hyde (1)

Pseudobambusicola Hern.-Restr. \& Crous (1)*

Sulcatispora Kaz. Tanaka \& K. Hiray. (2)

\section{Teichosporaceae M.E. Barr}

Asymmetrispora Thambug. \& K.D. Hyde (2)

Aurantiascoma Thambug. \& K.D. Hyde (1)

Chaetomastia (Sacc.) Berl. (10)

Floricola Kohlm. \& Volkm.-Kohlm. (2)

Loculohypoxylon M.E. Barr (1)

Magnibotryascoma Thambug. \& K.D. Hyde (2)

Misturatosphaeria Mugambi \& Huhndorf (2)

Paulkirkia Wijayaw., Wanas., Tangthir., Camporesi \& K.D. Hyde (1)

Pseudoaurantiascoma Thambug. \& K.D. Hyde (1)

Pseudomisturatosphaeria Thambug. \& K.D. Hyde (1)

Ramusculicola Thambug. \& K.D. Hyde (1)

Sinodidymella J.Z. Yue \& O.E. Erikss. (5)

Teichospora Fuckel (35)

\section{Testudinaceae Arx}

Angustospora Abdel-Aziz (1)

Halotestudina Dayar. \& K.D. Hyde (1)

Lepidosphaeria Parg.-Leduc (1)

Lojkania Rehm (10)

Muritestudina Wanas., E.B.G. Jones \& K.D. Hyde (1)

Neotestudina Segretain \& Destombes (3)

Testudina Bizz. (1)

Ulospora D. Hawksw., Malloch \& Sivan. (1) 
Verruculina Kohlm. \& Volkm.-Kohlm. (1)

Tetraplosphaeriaceae Kaz. Tanaka \& K. Hiray

Byssolophis Clem. (3)

Ernakulamia Subram. (2)

Polyplosphaeria Kaz. Tanaka \& K. Hiray. (5)

Pseudotetraploa Kaz. Tanaka \& K. Hiray. (4)

Quadricrura Kaz. Tanaka, K. Hiray. \& Sat. Hatak. (3)

Shrungabeeja V.G. Rao \& K.A. Reddy (5)

Tetraploa Berk. \& Broome (19)

Triplosphaeria Kaz. Tanaka \& K. Hiray (4)

Thyridariaceae Q. Tian \& K.D. Hyde

Chromolaenomyces Mapook \& K.D. Hyde (1)

Cycasicola Wanas., E.B.G. Jones \& K.D. Hyde (2)

Liua Phook. \& K.D. Hyde (1)*

Parathyridaria Jaklitsch \& Voglmayr (5)

Pseudothyridariella Mapook \& K.D. Hyde (2)

Thyridaria Sacc. (52)

Thyridariella Devadatha, V.V. Sarma, K.D. Hyde, Wanas. \& E.B.G Jones (2)

\section{Torulaceae Corda}

Dendryphion Wallr. (68)

Neotorula Ariyaw., Z.L. Luo \& K.D. Hyde (2)

Rostriconidium Z.L. Luo, K.D. Hyde \& H.Y. Su (2)

Rutola J.L. Crane \& Schokn. (1)

Sporidesmioides Jun F. Li, Phook. \& K.D. Hyde (1)

Torula Pers. (12)

Trematosphaeriaceae K.D. Hyde, Y. Zhang ter, Suetrong \& E.B.G. Jones

Bryosphaeria Döbbeler (9)

Falciformispora K.D. Hyde (4)

Hadrospora Boise (2)

Halomassarina Suetrong, Sakay., E.B.G. Jones, Kohlm., Volkm.-Kohlm. \& C.L. Schoc (1)

Raghukumaria Devadatha, V.V Sarma \& E.B.G Jones (1)

Trematosphaeria Fuckel (20)

Tzeananiaceae H.A. Ariyaw., A.J.L. Phillips \& Chuang

Tzeanania H.A. Ariyaw., A.J.L. Phillips \& Chuang (1)

Wicklowiaceae Ariyaw. \& K.D. Hyde

Wicklowia Raja, A. Ferrer \& Shearer (2)

Zopfiaceae G. Arnaud ex D. Hawksw.

Celtidia J.M. Janse (1)

Coronopapilla Kohlm. \& Volkm.-Kohlm. (2)

Rechingeriella Petr. (2)

Richonia Boud. (1)

Zopfia Rabenh. (5)

Zopfiofoveola D. Hawksw. (1) 
Pleosporales genera incertae sedis

Acuminatispora S.N. Zhang, K.D. Hyde \& J.K. Liu (1)

Aegeanispora E.B.G. Jones \& Abdel-Wahab (1)

Antealophiotrema A. Hashim. \& Kaz. Tanaka (1)

Ascorhombispora L. Cai \& K.D. Hyde (1)

Atradidymella Davey \& Currah (1)

Briansuttonia R.F. Castañeda, Minter \& Saikawa (1)

Camarographium Bubák (7)

Chaetodiplodia P. Karst. (9)

Chaetophoma Cooke (ca. 30)

Cheiromoniliophora Tzean \& J.L. Chen (4)

Crassiperidium M. Matsum. \& Kaz. Tanaka (2)

Cyclothyrium Petr. (2)

Dangeardiella Sacc. \& P. Syd. (2)

Daruvedia Dennis (1)

Dokmaia I. Promputtha (1)

Farasanispora Abdel-Wahab, Bahkali \& E.B.G. Jones (1)

Glaxoa P.F. Cannon (1)

Homostegia Fuckel (2)

Hobus Jaklitsch \& Voglmayr (1)

Inflatispora Y. Zhang ter, J. Fourn. \& K.D. Hyde (2)

Isthmosporella Shearer \& J.L. Crane (1)

Megacapitula J.L. Chen \& Tzean (1)

Megatomentella D.A.C. Almeida, Gusmão \& A.N. Mill. (1)

Neocurreya Thambug. \& K.D. Hyde (5)

Ostropella (Sacc.) Höhn. (5)

Paraepicoccum Matsush. (1)

Paraliomyces Kohlm. (1)

Parameliola Hongsanan, Peršoh \& K.D. Hyde (2)

Perthomyces Crous (1)

Phialophorophoma Linder (1)

Pleosphaerellula Naumov \& Czerepan. (2)

Pseudohendersonia Crous \& M.E. Palm (2)

Pseudopassalora Crous (1)

Pyrenochaeta De Not. (5)

Rebentischia P. Karst. (16)

Repetophragma Subram. (38)

Scleroramularia Batzer \& Crous (6)

Scolecobasidium E.V. Abbott (64)

Setophaeosphaeria Crous \& Y. Zhang ter (6)

Sirodesmium De Not. (ca. 25)

Spiroplana Voglmayr, M.J. Park \& H.D. Shin (1)

Stuartella Fabre (6)

Xenolophium Syd. (ca. 5)

Pleosporomycetidae genus incertae sedis

Hysterographium Corda (3)

Dothideomycetes orders incertae sedis

Abrothallales Pérez-Ort. \& Suija [= Lichenoconiales Diederich, Lawrey \& K.D. Hyde]

Lichenoconiaceae Diederich \& Lawrey [= Abrothallaceae Pérez-Ort. \& Suija]*

Abrothallus De Not (=Epinephroma Zhurb.; Vouauxiomyces Dyko \& D. Hawks.) (42)* 
Acrospermales Minter, Peredo \& A.T. Watson

Acrospermaceae Fuckel

Acrospermum Tode (12)

Gonatophragmium Deighton (17)

Oomyces Berk. \& Broome (7)

Acrospermales genus incertae sedis

Pseudovirgaria H.D. Shin, U. Braun, Arzanlou \& Crous (2)

Asterinales M.E. Barr ex D. Hawksw. \& O.E. Erikss. (= Asterotexales Firmino et al.)

Asterinaceae Hansf.

Asterina Lév. (ca. 1085)

Asterinella Theiss. (ca. 39)

Asterolibertia G. Arnaud (ca. 30)

Asterostomella Speg.

Batistinula Arx (1)

Cirsosia G. Arnaud (18)

Dothidasteromella Höhn. (11)

Echidnodella Theiss. \& Syd. (35)

Halbania Racib. (3)

Meliolaster Höhn. (3)

Parasterinopsis Bat. (3)

Platypeltella Petr. (3)

Prillieuxina G. Arnaud (66)

Pycnocarpon Theiss.

Schenckiella Henn. (1)

Trichasterina G. Arnaud (11)

Trichopeltospora Bat. \& Cif. (2)

Uleothyrium Petr. (3)

Vizellopsis Bat., J.L. Bezerra \& T.T. Barros (1)

Asterotexaceae Firmino, O.L. Pereira \& Crous

Asterotexis Arx (2)

Hemigraphaceae D.Q. Dai \& K.D. Hyde*

Hemigrapha (Müll. Arg.) D. Hawksw. (8)

Lembosiaceae Hosag.

Lembosia Lév. (ca. 200)

Melaspileellaceae D.Q. Dai \& K.D. Hyde* Melaspileella (P. Karst.) Vain. (1)

Morenoinaceae Hongsanan \& K.D. Hyde

Morenoina Theiss. (ca. 25)

Neobueliellaceae Hongsanan \& K.D. Hyde Neobueliella Hongsanan \& K.D. Hyde 
Stictographaceae D.Q. Dai \& K.D. Hyde*

Buelliella Fink (12)

Karschia Körb. (4)

Labrocarpon Etayo \& Pérez-Ort. (1)

Melaspileopsis (Müll. Arg.) Ertz \& Diederich (1)

Stictographa Mudd (2)

Asterinales genera incertae sedis

Andamanomyces Hosag. (1)

Caribaeomyces Cif. (1)

Discopycnothyrium Hongsanan \& K.D. Hyde (1)

Hazslinszkya Körb. (4)

Inocyclus Theiss. \& Syd. (6)

Melanographa Müll. Arg. (1)

Pirozynskiella S. Hughes (3)

Vishnumyces Hosag. (1)

Botryosphaeriales C.L. Schoch, Crous \& Shoemaker

Aplosporellaceae Slippers, Boissin \& Crous

Alanomyces Roh. Sharma (1)*

Aplosporella Speg. (= Bagnisiella Speg.) (10)*

Botryosphaeriaceae Theiss. \& Syd. (= Endomelanconiopsidaceae Tao Yang \& Crous)*

Alanphillipsia Crous \& M.J. Wingf. (5)

Barriopsis A.J.L. Phillips, A. Alves \& Crous (5)

Botryobambusa Phook., J.K. Liu \& K.D. Hyde (2)

Botryosphaeria Ces. \& De Not. (13)

Cophinforma Doilom, J.K. Liu \& K.D. Hyde (2)

Diplodia Fr. (more than 1000 names in MycoBank, 30 known from culture)

Dothiorella Sacc. (389 names in MycoBank, 38 known from culture) (= Spencermartinsia

A.J.L. Phillips, A. Alves \& Crous)*

Endomelanconiopsis Rojas \& Samuels (2)*

Eutiarosporella Crous (7)*

Lasiodiplodia Ellis \& Everh. (35)

Macrophomina Petr. (2)

Marasasiomyces Crous (1)*

Mucoharknessia Crous, R.M. Sánchez \& Bianchin. (2)*

Neodeightonia Booth (6)

Neofusicoccum Crous, Slippers \& A.J.L. Phillips (4)

Neoscytalidium Crous \& Slippers (3)*

Oblongocollomyces Tao Yang \& Crous (1)*

Phaeobotryon Theiss. \& Syd. (4)

Sakireeta Subram. \& K. Ramakr. (1)*

Sardiniella Linaldeddu, A. Alves \& A.J.L. Phillips (1)*

Sphaeropsis Sacc. (more than 600 names in MycoBank, 4 known from culture)

Tiarosporella Höhn. (2)

Melanopsaceae Phillips A.J.L., Slippers, Boissin \& Crous

Melanops Nitschke ex Fuckel (105 names in MycoBank, 4 known from culture)

Phyllostictaceae Fr. (= Pseudofusicoccumaceae Tao Yang \& Crous)

Phyllosticta Pers. (ca. 53) 
Pseudofusicoccum Mohali, Slippers \& M.J. Wingf. (7)*

Planistromellaceae M.E. Barr

Kellermania Ellis \& Everh. (ca. 16)*

Mycosphaerellopsis Höhn. (1)

Planistroma A.W. Ramaley (6)

Umthunziomyces Crous \& M.J. Wingf. (1)*

Saccharataceae Slippers, Boissin \& Crous (= Septorioideaceae Wyka \& Broders)

Neoseptorioides Crous, Jacq. Edwards \& Pascoe (1)*

Pileospora Tanney \& Seifert (1)

Saccharata Denman \& Crous (20)

Septorioides Quaedvl., Verkley \& Crous (2)*

Botryosphaeriales genera incertae sedis

Auerswaldiella Theiss. \& Syd. (7)

Coccostromella Petr. (1)

Leptoguignardia E. Müll. (1)

Metameris Theiss. \& Syd. (5)

Phyllachorella Syd. (8)

Pilgeriella Henn. (2)

Sivanesania W.H. Hsieh \& Chi Y. Chen (1)

Vestergrenia Rehm (3)

Catinellales Ekanayaka, K.D. Hyde \& Ariyaw.

Catinellaceae Ekanayaka, K.D. Hyde \& Ariyaw.

Catinella Boud. (1 or 2)

Cladoriellales Crous

Cladoriellaceae Crous

Cladoriella Crous (5)

Collemopsidiales Pérez-Ort., Garrido-Ben. \& Grube

Xanthopyreniaceae Zahlbr.

Collemopsidium Nyl. (27)

Didymellopsis (Sacc.) Clem. \& Shear (6)

Frigidopyrenia Grube (1)

Xanthopyrenia Bachm. (2)

Zwackhiomacromyces Etayo \& van den Boom (2)

Zwackhiomyces Grube \& Hafellner (35)

Dyfrolomycetales K.L. Pang, K.D. Hyde \& E.B.G. Jones

Pleurotremataceae Walt. Watson

Dyfrolomyces K.D. Hyde, K.L. Pang, Alias, Suetrong \& E.B.G. Jones (8)

Melomastia Nitschke ex Sacc. (4)

Pleurotrema Müll. Arg. (1)

Eremithallales Lücking \& Lumbsch

Melaspileaceae W. Watson (= Eremithallaceae Lücking \& Lumbsch)

Encephalographa A. Massal. (1)

Melaspilea Nyl. $(1+$ c. 75 orphaned) (= Eremithallus Lücking et al.) 
Eremomycetales Pem \& Hyde

Eremomycetaceae Malloch \& Cain

Eremomyces Malloch \& Cain (2)

Rhexothecium Samson \& Mouch. (1)

Eremomycetales genus incertae sedis

Arthrographis G. Cochet ex Sigler (12)

Jahnulales K.L. Pang, Abdel-Wahab, El-Shar., E.B.G. Jones \& Sivichai Aliquandostipitaceae Inderbitzin

Aliquandostipite Inderbitzin (7)

Brachiosphaera Nawawi (2)

Jahnula Kirschst. (19)

Megalohypha A. Ferrer \& Shearer (1)

Neojahnula W. Dong, H. Zhang \& K.D. Hyde (1)

Pseudojahnula W. Dong, H. Zhang \& K.D. Hyde (1)

Xylomyces Goos, R.D. Brooks \& Lamore (8)*

Manglicolaceae Suetrong \& E.B.G. Jones

Manglicola Kohlm. \& E. Kohlm. (1)*

Kirschsteiniotheliales Hern.-Restr., R.F. Castañeda, Gené \& Crous

Kirschsteiniotheliaceae Boonmee \& K.D. Hyde

Kirschsteiniothelia D. Hawksw. (29)

Kirschsteiniotheliales genera incertae sedis

Brachysporiella Bat. (Brachysporiella s. lato.) (15)

Taeniolella S. Hughes sensu lato*

Lembosinales Crous

Lembosinaceae Crous

Lembosina Theiss. (21)

Lichenotheliales K. Knudsen, Muggia \& K.D. Hyde

Lichenotheliaceae Henssen

Lichenothelia D. Hawksw. (27)

Endococcus Nyl. (44)

Microthyriales G. Arnaud

Microthyriaceae Sacc.

Arnaudiella Petr. (12)

Calothyriopsis Höhn. (4)

Chaetothyriothecium Hongsanan \& K.D. Hyde (1)

Hamatispora L.T.H. Yen, K. Yamag. \& K. Ando (1)

Microthyrium Desm. (ca. 180)

Neoanungitea Crous (1)

Paramicrothyrium H.X. Wu \& K.D. Hyde (1)

Pseudomicrothyrium X.Y. Zeng, S. Hongsanan \& K.D. Hyde (1)

Pseudopenidiella Crous \& Koukol (1)

Seynesiella G. Arnaud (5)

Tumidispora Hongsanan \& K.D. Hyde (1) 
Microthyriales genera incertae sedis

Heliocephala V. Rao, K.A. Reddy \& de Hoog (7)

Mitopeltis Speg. (2)

Neoscolecobasidium Crous (1)

Parazalerion Madrid, Gené \& Cano (1)*

Thyriodictyella Cif (1)

Tothia Bat. (2)

Minutisphaerales Raja, Oberlies, Shearer \& A.N. Mill.

Acrogenosporaceae Jayasiri \& K.D. Hyde*

Acrogenospora M.B. Ellis (12)

Minutisphaeraceae Raja, Oberlies, Shearer \& A.N. Mill.

Minutisphaera Shearer, A.N. Mill. \& A. Ferrer (4)

Monoblastiales Lücking, M.P. Nelsen \& K.D. Hyde

Monoblastiaceae Walt. Watson

Acrocordia A. Massal. (6)

Anisomeridium (Müll. Arg.) M. Choisy (ca. 80)

Caprettia Bat. \& H. Maia (8)

Megalotremis Aptroot (12)

Monoblastia Riddle (11)

Trypetheliopsis Asahina (6)

Murramarangomycetales Crous

Murramarangomycetaceae Crous

Murramarangomyces Crous (1)

Muyocopronales Mapook, Boonmee \& K.D. Hyde

Muyocopronaceae K.D. Hyde

Arxiella Papendorf (3)

Leptodiscella Papendorf (5)

Muyocopron Speg. (51)

Mycoleptodiscus Ostaz. (18)

Neocochlearomyces Pinruan, Sommai, Suetrong, J.Z. Groenew. \& Crous (1)

Neomycoleptodiscus Hern.-Restr., J.D.P. Bezerra \& Crous (1)

Paramycoleptodiscus Crous \& M.J. Wingf. (1)

Pseudopalawania Mapook \& K.D. Hyde (1)

Setoapiospora Mapook \& K.D. Hyde (1)

Natipusillales Raja, Shearer, A.N. Mill. \& K.D. Hyde

Natipusillaceae Raja, Shearer \& A.N. Mill.

Natipusilla A. Ferrer, A.N. Mill. \& Shearer (4)

Parmulariales D.Q. Dai \& K.D. Hyde*

Parmulariaceae E. Müll. \& Arx ex M.E. Barr

Aldona Racib. (3)

Aldonata Sivan. \& A.R.P. Sinha (1)

Antoniomyces Inácio (1)

Aulacostroma Syd. \& P. Syd. (5)

Campoa Speg. (4)

Cirsosiopsis Butin \& Speer (1) 
Cocconia Sacc. (13)

Cycloschizon P. Henn. (13)

Cyclostomella Pat. (4)

Dothidasteroma Höhn. (4)

Ferrarisia Sacc. (ca. 8)

Hysterostomella Speg. (23)

Kiehlia Viégas (2)

Mintera Inácio \& P.F. Cannon (1)

Pachypatella Theiss. \& Syd. (1)

Palawaniella Doidge (7)

Parmularia Lév. (6)

Parmulariopsella Sivan. (1)

Parmulariopsis Petr. (1)

Parmulina Theiss. \& Syd. (6)

Placoasterella Sacc. ex Theiss. \& Syd. (4)

Placosoma Syd. (2)

Placostromella Petr. (3)

Pleiostomellina Bat., J.L. Bezerra \& H. Maia (1)

Polycyclina Theiss. \& Syd. (1)

Polycyclus Höhn. (2)

Protothyrium G. Arnaud (4)

Pseudolembosia Theiss. (4)

Rhagadolobiopsis Guatim. \& R.W. Barreto (1)

Rhagadolobium P. Henn. \& Lindau (10)

Rhipidocarpon (Theiss.) Theiss. \& Syd. (1)

Symphaeophyma Speg. (1)

Syrropeltis Bat., J.L. Bezerra \& Matta (1)

Thallomyces H.J. Swart (1)

Viegasella Inácio \& P.F. Cannon (1)

Patellariales D. Hawksw. \& O.E. Erikss.

Patellariaceae Corda

Baggea Auersw. (1)

Banhegyia L. Zeller \& Tóth (2)

Colensoniella Hafellner (1)

Endotryblidium Petr. (1)

Glyphium Nitschke ex F. Lehm. (ca. 4)

Haematomyxa Sacc (2)

Holmiella Petrini, Samuels \& E. Müll. (4)

Hysteropatella Rehm (3)

Hysteropeltella Petr. (1)

Lahmiomyces Cif. \& Tomas. (1)

Lecanidiella Sherwood (1)

Lirellodisca Aptroot (1)

Murangium Seaver (1)

Patellaria Fr. (12)

Poetschia Körb. (4)

Pseudoparodia Theiss. \& Syd. (1)

Rhizodiscina Hafellner (1)

Rimula Velen. (1)

Schrakia Hafellner (1)

Stratisporella Hafellner (1) 
Tryblidaria (Sacc.) Rehm (9)

Phaeotrichales Ariyaw., Jian K. Liu \& K.D. Hyde

Phaeotrichaceae Cain

Echinoascotheca Matsush. (1)

Phaeotrichum Cain \& M.E. Barr (2)

Trichodelitschia Munk (4)

Stigmatodiscales Voglmayr \& Jaklitsch

Stigmatodiscaceae Voglmayr \& Jaklitsch

Stigmatodiscus Voglmayr \& Jaklitsch (= Asterodiscus Voglmayr et al.) (6)*

Strigulales Lücking, M.P. Nelsen \& K.D. Hyde

Strigulaceae Zahlbr. (= Phyllobatheliaceae Bitter \& F. Schill.)

Dichoporis Clem. (18)

Flagellostrigula Lücking \& S.H. Jiang (1)

Flavobathelium Lücking, Aptroot \& G. Thor (1)

Phyllobathelium (Müll. Arg.) Müll. Arg (8)

Phyllocharis Fée (1)

Phyllocraterina Sérus. \& Aptroot (= Phyllocratera Sérus. \& Aptroot) (2)

Phylloporis Clem. (ca. 10)

Puiggariella Speg. (3)

Raciborskiella Höhnel (2)

Racoplaca Fée (5)

Serusiauxiella S.H. Jiang, Lücking \& J.C.Wei (3)

Strigula Fr. (ca. 30)

Swinscowia S.H. Jiang \& Lücking (33)

Tenuitholiascaceae S.H. Jiang, Lücking \& J.C. Wei*

Tenuitholiascus S.H. Jiang, Lücking \& J.C. Wei. (1)

Superstratomycetales van Nieuwenh., Miądl., Houbraken, Adan, Lutzoni \& Samson

Superstratomycetaceae van Nieuwenh., Miądl., Houbraken, Adan, Lutzoni \& Samson

Superstratomyces van Nieuwenh., Miądl. \& Samson (4)

Trypetheliales Lücking, Aptroot \& Sipman

Polycoccaceae Ertz, Hafellner \& Diederich

Clypeococcum D. Hawksw. (ca. 10)

Polycoccum Saut. ex Körb. (ca. 60)

\section{Trypetheliaceae Zenker}

Alloarthopyrenia Phukhams., Lücking \& K.D. Hyde (1)

Aptrootia Lücking \& Sipman (3)

Architrypethelium Aptroot (8)

Astrothelium Eschw. (= Campylothelium Müll.) (ca. 275)

Bathelium Ach. (16)

Bogoriella Zahlbr. (=Distothelia Aptroot) (29)

Constrictolumina Lücking, M.P. Nelsen \& Aptroot (9)

Dictyomeridium Aptroot, M.P. Nelsen \& Lücking (7)

Macroconstrictolumina Lücking, R. Miranda \& Aptroot (5)

Marcelaria Aptroot (= Buscalionia Sambo) (3)

Nigrovothelium Lücking, M.P. Nelsen \& Aptroot (3) 
Novomicrothelia Aptroot, M.P. Nelsen \& Lücking (1)

Polymeridium (Müll. Arg.) R.C. Harris (51)

Polypyrenula D. Hawksw. (1)

Pseudobogoriella Lücking, R. Miranda \& Aptroot (15)

Pseudopyrenula Müll. Arg. (21)

Schummia Lücking, R. Miranda \& Aptroot (1)

Trypethelium Sprengel (16)

Viridothelium Lücking, M.P. Nelsen \& Aptroot (= ?Exiliseptum R.C. Harris fide Hongsanan et al. 2020) (11)

Tubeufiales Boonmee \& K.D. Hyde (= Bezerromycetales J.D.P. Bezerra et al.; = Wiesneriomycetales J.D.P. Bezerra et al.)

Bezerromycetaceae J.D.P. Bezerra, Souza-Motta \& Crous

Bezerromyces J.D.P. Bezerra, Souza-Motta \& Crous (2)

Neorhamphoria Boonmee, E. Hüseyin \& F. Selçuk (1)

Xiliomyces J.D.P. Bezerra, Souza-Motta \& Crous (1)

Tubeufiaceae M.E. Barr

Acanthohelicospora Boonmee \& K.D. Hyde (4)

Acanthophiobolus Berl. (6)

Acanthostigma De Not. (64)

Acanthostigmina Höhn. (7)

Acanthotubeufia Y.Z. Lu \& K.D. Hyde (1)

Aquaphila Goh, K.D. Hyde \& W.H. Ho (2)

Artocarpomyces Subram. (1)

Berkleasmium Zobel (ca. 40)

Bifrontia Norman (2)

Boerlagiomyces Butzin (9)

Camporesiomyces D.P. Wei \& K.D. Hyde (1)

Chaetosphaerulina I. Hino (6)

Chlamydotubeufia Boonmee \& K.D. Hyde (8)

Dematiohelicoma Y.Z. Lu, J.C. Kang \& K.D. Hyde (2)

Dematiohelicomyces Y.Z. Lu, Boonmee \& K.D. Hyde (1)

Dematiohelicosporum Y.Z. Lu, J.K. Liu \& K.D. Hyde (1)

Dematiotubeufia Y.Z. Lu, Boonmee \& K.D. Hyde (1)

Dictyospora Brahaman., Y.Z. Lu, Boonmee \& K.D. Hyde (1)

Discotubeufia Jayasiri, E.B.G. Jones \& K.D. Hyde (1)

Helicangiospora Boonmee, Bhat \& K.D. Hyde (1)

Helicoarctatus Y.Z. Lu, J.C. Kang \& K.D. Hyde (1)

Helicodochium J.S. Monteiro, R.F. Castañeda, A.C. Cruz \& Gusmão (2)

Helicohyalinum Y.Z. Lu, J.K. Liu \& K.D. Hyde (2)

Helicoma Corda (ca. 40)

Helicomyces Link (14)

Helicosporium Nees (ca. 15)

Helicotruncatum Y.Z. Lu, J.C. Kang \& K.D. Hyde (1)

Helicotubeufia Y.Z. Lu \& J.K. Liu (3)

Kamalomyces R.K. Verma, N. Sharma \& Soni (5)

Kevinhydea N.G. Liu, Y.Z. Lu \& J.K. Liu (1)

Manoharachariella Bagyan., N.K. Rao \& Kunwar (4)

Muripulchra Z.L. Luo, Hong Y. Su \& K.D. Hyde (1)

Neoacanthostigma Boonmee, Bhat \& K.D. Hyde (8)

Neochlamydotubeufia Y.Z. Lu, Boonmee \& K.D. Hyde (2) 
Neohelicoma Y.Z. Lu, Boonmee \& K.D. Hyde (1)

Neohelicomyces Z.L. Luo, Bhat \& K.D. Hyde (3)

Neohelicosporium Y.Z. Lu, J.C. Kang \& K.D. Hyde (7)

Neotubeufia Chaiwan, Boonmee, Y.Z. Lu \& K.D. Hyde (1)

Pleurohelicosporium Y.Z. Lu, J.C. Kang \& K.D. Hyde (1)

Podonectria Petch (11)

Pseudohelicomyces Y.Z. Lu, J.K. Liu \& K.D. Hyde (5)

Pseudohelicoon Y.Z. Lu \& K.D. Hyde (2)

Tamhinispora Rajeshkumar \& Rahul Sharma (2)

Thaxteriella Petr. (15)

Thaxteriellopsis Sivan., Panwar \& S.J. Kaur (3)

Tubeufia Penz. \& Sacc. (ca. 60)

Wiesneriomycetaceae Suetrong, Rungjind., Somrith. \& E.B.G. Jones

Parawiesneriomyces Crous \& M.J. Wingf. (1)

Phalangispora Nawawi \& J. Webster (3)

Pseudogliophragma Phadke \& V.G. Rao (1)

Setosynnema D.E. Shaw \& B. Sutton (3)

Speiropsis Tubaki (8)

Wiesneriomyces Koord. (4)

Valsariales Jaklitsch, K.D. Hyde \& Voglmayr

Valsariaceae Jaklitsch, K.D. Hyde \& Voglmayr

Bambusaria Jaklitsch, D.Q. Dai, K.D. Hyde \& Voglmayr (1)

Myrmaecium Nitschke ex Fuckel (ca. 3)

Valsaria Ces. \& De Not. (140 epithets)

Venturiales Y. Zhang ter, C.L. Schoch \& K.D. Hyde

Sympoventuriaceae Y. Zhang ter, C.L. Schoch \& K.D. Hyde

Acroconidiellina M.B. Ellis (4)

Clavatispora Boonmee \& K.D. Hyde (1)

Fusicladium Bonord. (75)

Matsushimaea Subram. (4)

Mycosisymbrium Carris (1)

Ochroconis de Hoog \& Arx (28)

Sympoventuria Crous \& Seifert (3)

Veronaeopsis Arzanlou \& Crous (1)

Verruconis Samerp., H.J. Choi, van den Ende, Horré \& de Hoog (5)

Yunnanomyces Tibpromma \& K.D. Hyde (2)

Venturiaceae E. Müll. \& Arx ex M.E. Barr

Apiosporina Höhn. (6)

Atopospora Petr. (4)

Caproventuria U. Braun (2)

Coleroa (Fr.) Rabenh. (56)

Dimeriella Speg. (51)

Dimerosporiopsis Henn. (1)

Magnohelicospora R.F. Castañeda, Hern.-Restr., Gené \& Guarro (2)

Metacoleroa Petr. (1)

Neocoleroa Petr. (6)

Protoventuria Berl. \& Sacc. (45)

Pseudoanungitea Crous (3)* 
Pseudoparodiella F. Stevens (1)

Tyrannosorus Unter. \& Malloch (1)

Venturia Sacc. (ca. 60)

Venturiales genera incertae sedis

Cylindrosympodioides Crous \& M.J. Wingf. (1)

Cylindrosympodium W.B. Kendr. \& R.F. Castañeda (12)

Lasiobotrys Kunze (9)

Zeloasperisporiales Hongsanan \& K.D. Hyde

Zeloasperisporiaceae Crous

Zeloasperisporium R.F. Castañeda (8)

Dothideomycetes families incertae sedis

Alinaceae Boonmee \& K.D. Hyde

Alina Racib. (1)

Argynnaceae Shearer \& J.L. Crane

Argynna Morgan (1)

Lepidopterella Shearer \& J.L. Crane (2)

Ascoporiaceae Kutorga \& D. Hawksw.

Ascoporia Samuels \& A.I. Romero (1)

Aulographaceae Luttr. ex P.M. Kirk, P.F. Cannon \& J.C. David

Aulographum Lib. (ca. 30)

Echidnodes Theiss. \& Syd. (31)

Lembosiella Sacc. (1)

Thyriopsis Theiss. \& Syd. (3)

Balladynaceae Boonmee \& K.D. Hyde

Balladyna Racib. (41)

Balladynocallia Bat. (3)

Balladynopsis Theiss. \& Syd. (10)

Cleistosphaeraceae Boonmee \& K.D. Hyde

Cleistosphaera Syd. \& P. Syd. (1)

Coccoideaceae P. Henn. ex Sacc. \& D. Sacc.

Coccoidea P. Henn. (4)

Coccoidella Höhn. (9)

Englerodothis Theiss. \& Syd. (3)

Cookellaceae Höhn. ex Saccardo \& Trotter

Cookella Sacc. (4)

Pycnoderma Syd. \& P. Syd. (2)

Dimeriaceae E. Müll. \& Arx ex Arx \& E. Müll.

Dimerium (Sacc. \& P. Syd.) McAlpine (79)

Dubujianaceae D. Pem, Doilom \& K.D. Hyde

Dubujiana D.R. Reynolds \& G.S. Gilbert (1) 
Dysrhynchisceae Boonmee \& K.D. Hyde

Dysrhynchis Clem. (4)

Endosporiaceae D. Pem

Endosporium Tsuneda (2)

Englerulaceae P. Henn.

Allosoma Syd. (5)

Digitosarcinella S. Hughes (1)

Englerula P. Henn. (13)

Goosia B. Song (1)

Parenglerula Höhn. (7)

Rhytidenglerula Höhn. (11)

Sarcinella Sacc. (ca. 70)

Thrauste Theiss. (3)

Eriomycetaceae Huanraluek \& K.D. Hyde

Eriomyces Huanraluek, Thambugala \& K.D. Hyde (1)

Funbolia Crous \& Seifert (1)

Heleiosa Kohlm., Volkm.-Kohlm. \& O.E. Erikss. (1)

Phellinocrescentia Crous \& Decock (1)

Pseudopassalora Pseudopassalora Crous (1)

Homortomycetaceae Thambug., A.J.L. Phillips \& K.D. Hyde Homortomyces Crous \& M.J. Wingf. (2)

Hyalomeliolinaceae Boonmee \& K.D. Hyde

Hyalomeliolina F. Stevens (2)

Leptopeltidaceae Höhn. ex Trotter

Dothiopeltis E. Müll. (2)

Leptopeltis Höhn. (11)

Ronnigeria Petr. (1)

Staibia Bat. \& Peres (1)

Macrovalsariaceae D. Pem, Doilom \& K.D Hyde

Macrovalsaria Petr. (1)

Meliolinaceae S. Hughes

Briania D.R. Reynolds (1)

Meliolina Syd. \& P. Syd. (ca. 40)

Mesnieraceae Arx \& E. Müll.

Bondiella Piroz. (1)

Mesniera Sacc. \& P. Syd. (1)

Stegasphaeria Syd. \& P. Syd. (3)

Naetrocymbaceae Höhn. ex R.C. Harris

Bonaria Bat. (4)

Jarxia D. Hawksw. (2)

Leptorhaphis Körb. (14)

Naetrocymbe Körb. (1) 
Tomasellia A. Massal. (ca. 5)

Nematotheciaceae Boonmee \& K.D. Hyde

Nematothecium Syd. \& P. Syd. (5)

Nematostigma Syd. \& P. Syd. (5)

Ophioparodia Petr. \& Cif. (1)

Neoparodiaceae Boonmee \& K.D. Hyde

Neoparodia Petr. \& Cif. (1)

Palawaniaceae Mapook \& K.D. Hyde

Palawania Syd. \& P. Syd. (2)

Paranectriellaceae S. Boonmee \& K.D. Hyde

Paranectriella (Henn. ex Sacc. \& D. Sacc.) Magnus. (=Araneomyces Höhn.) (9)

Puttemansia Henn. (18)

Parodiellaceae Theiss. \& H. Syd. ex M.E. Barr

Parodiella Speg. (4)

Perisporiopsidaceae E. Müll. \& Arx ex R. Kirschner \& T.A. Hofm. (= Parodiopsidaceae Toro)

Asteronia (Sacc.) Henn. (2)

Byssocallis Syd. (3)

Chevalieropsis G. Arnaud (1)

Parodiellina Henn. ex G. Arnaud (1)

Perisporiopsis Henn. (22)

Phaeodimeriellaceae Boonmee, Mapook \& K.D. Hyde

Phaeodimeriella Speg. (30)

Pododimeriaceae Boonmee \& K.D. Hyde

Chaetoscutula E. Müll. (1)

Pododimeria E. Müll. (4)

Polyclypeolinaceae Boonmee \& K.D. Hyde

Polyclypeolina Bat. \& I.H. Lima (1)

Polystomellaceae Theiss. \& H. Syd.

Dermatodothella Viégas (1)

Dothidella Speg. (2)

Munkiella Speg. (3)

Parastigmatea Doidge (3)

Protoscyphaceae Kutorga \& D. Hawksw.

Protoscypha Syd. (2)

\section{Pseudoperisporiaceae Toro}

Bryomyces Döbbeler (12)

Eudimeriolum Speg. (8)

Lasiostemma Theiss. (5)

Nematostoma Syd. \& P. Syd. (13) 
Pyrenidiaceae Zahlbr.

Pyrenidium Nyl. (11)

Seynesiopeltidaceae K.D. Hyde

Seynesiopeltis F. Stevens \& R.W. Ryan (1)

Stomatogeneceae Boonmee \& K.D. Hyde

Stomatogene Theiss. (3)

Thyrinulaceae X.Y. Zeng, S. Hongsanan \& K.D. Hyde

Blastacervulus H.J. Swart (2)

Paraopeba V.P. Abreu, A.A.M. Gomes, Firmino \& O.L. Pereira (1)

Thyrinula Petr. \& Syd. (= Alysidiella Crous) (1)

Toroaceae Boonmee \& K.D. Hyde

Toroa Syd. (2)

Trichopeltinaceae Bat., C.A.A. Costa \& Cif.

Acrogenotheca Cif. \& Bat. (3)

Brefeldiella Speg. (4)

Saccardinula Speg. (11)

Trichopeltella Höhn. (1)

Trichopeltheca Bat. (2)

Trichopeltina Theiss. (2)

Trichothyrinula Petr. (2)

Trichothyriaceae Theiss.

Lichenopeltella Höhn. (48)

Macrographa Etayo (1)

Pachythyrium G. Arnaud ex Spooner \& P.M. Kirk (1)

Trichothyrium Speg. (12)

Vizellaceae H.J. Swart

Acarella Syd. (1)

Blasdalea Sacc. \& P. Syd. (1)

Vizella Sacc. (11)

Dothideomycetes genera incertae sedis

Acanthorus Bat. \& Cavalc. (1)

Acanthostigmella Höhn. (6)

Achorella Theiss. \& Syd. (10)

Actinomyxa Syd. \& P. Syd. (1)

Alascospora Raja, Violi \& Shearer (1)

Ampullifera Deighton (6)

Anguillosporella U. Braun (2)

Anopeltis Bat. \& Peres (1)

Arkoola J. Walker \& Stovold (1)

Armata W. Yamam. (1)

Ascominuta Ranghoo \& K.D. Hyde (2) 
Asterinema Bat. \& Gayão (3)

Asterodothis Theiss. (1)

Asteromassaria Höhn. (12)

Asteromella Pass. \& Thüm. (ca. 265)

Asteroporum Müll. Arg. (7)

Auerswaldia Sacc. (ca. 20)

Bactrodesmium Cooke (ca. 50)

Bahusakala Subram. (4)

Brachyconidiella R.F. Castañeda \& W.B. Kendr. (1)

Brooksia Hansf. (1)

Bryorella Döbbeler (10)

Bryostroma Döbbeler (8)

Bryothele Döbbeler (2)

Byssogene Syd. (2)

Callebaea Bat. (1)

Calyptra Theiss. \& Syd. (5)

Capillataspora K.D. Hyde (1)

Caryosporella Kohlm. (1)

Catulus Malloch \& Rogerson (1)

Ceramoclasteropsis Bat. \& Cavalc. (2)

Ceratophoma Höhn. (2)

Cercidospora Körb. (101)

Cerodothis Muthappa (1)

Chaetocrea Syd. (1)

Chaetosticta Petr. \& Syd. (3)

Chionomyces Deighton \& Piroz. (7)

Chuppia Deighton (2)

Cilioplea Munk (ca. 10)

Cirsosina Bat. \& J.L. Bezerra (2)

Clavariopsis De Wild. (ca. 5)

Clypeostroma Theiss. \& Syd. (ca. 3)

Cocciscia Norman (2)

Coccochora Höhn. (4)

Coccochorina Hara (2)

Coccodothis Theiss. \& Syd. (2)

Comesella Speg. (1)

Comminutispora A.W. Ramaley (1)

Coniosporium Link (ca. 20)

Crauatamyces Viégas (1)

Crotone Theiss. \& Syd. (1)

Cryomyces Selbmann, de Hoog, Mazzaglia, Friedmann \& Onofri (4)

Cyclotheca Theiss. (9)

Dactuliophora C.L. Leakey (5)

Dawsomyces Döbbeler (2)

Dawsophila Döbbeler (3)

Dermatodothis Racib. ex Theiss. \& Syd. (6)

Dianesea Inácio \& P.F. Cannon (1)

Dictyoasterina Hansf. (1)

Dictyodochium Sivan. (1)

Dictyopeltis Theiss. (6)

Dictyostomiopelta Viégas (1)

Dictyothyriella Speg. (1) 
Dictyothyrina Theiss. (1)

Dictyothyrium Theiss. (2)

Didymocyrtidium Vain. (2)

Didymolepta Munk (2)

Didymopleella Munk (3)

Diplochorina Gutner (1)

Dothichiza Lib. ex Roum. (15)

Dothideopsella Höhn. (1, but more epithets exist)

Dothivalsaria Petr. (1)

Dubitatio Speg. (1)

Echinothecium Zopf (2)

Elmerinula Syd. (1)

Epibelonium E. Müll. (1)

Eriomycopsis Speg. (13)

Eriothyrium Speg. (1, but more epithets exist)

Eupelte Syd. (5)

Excipulariopsis P.M. Kirk \& Spooner (1)

Extrusothecium Matsush. (2)

Fusicladiella Höhn. (5)

Gibbera Fr. (ca. 28)

Gilletiella Sacc. \& P. Syd. (3)

Globoa Bat. \& H. Maia (2)

Globulina Speg. (1 fide Kirk et al. 2008)

Gloeodiscus Dennis (1)

Govindua Bat. \& H. Maia (1)

Griggsia F. Stevens \& Dalbey (1)

Halokirschsteiniothelia Boonmee \& K.D. Hyde (1)

Hansfordiella S. Hughes (8)

Hansfordiellopsis Deighton (5)

Hansfordiopsis Bat. (1)

Harknessiella Sacc. (1)

Helminthopeltis Sousa da Câmara (1)

Heptameria Rehm \& Thuem. (2)

Heptaster Cif., Bat. \& Nascim. (3)

Heteroconium Petr. (21)

Heterosphaeriopsis Hafellner (1)

Hidakaea I. Hino \& Katum. (2)

Hyalocrea Syd. \& P. Syd. (4)

Hyaloscolecostroma Bat. \& J. Oliveira (1)

Hyalosphaera F. Stevens (4)

Hyalotheles Speg. (1)

Hypobryon Döbbeler (7)

Hysteropsis Rehm (4)

Isomunkia Theiss. \& Syd. (1)

Isthmospora F. Stevens (3)

Jaffuela Speg. (1)

Kabatia Bubák (ca. 10)

Keratosphaera H.P. Upadhyay (6)

Kriegeriella Höhn. (4)

Krishnamyces Hosag. (1)

Kullhemia P. Karst. (2)

Kusanobotrys P. Henn. (2) 
Lanatosphaera Matzer (2)

Lautitia S. Schatz (1)

Lazarenkoa Zerova (1)

Lembosiopeltis Bat. \& J.L. Bezerra (2)

Leptomeliola Höhn. (13)

Letendraeopsis K.F. Rodriguez \& Samuels (1)

Leveillella Theiss. \& Syd. (1)

Leveillina Theiss. \& Syd. (2)

Lichenotubeufia Etayo (5)

Licopolia Sacc., Syd. \& P. Syd. (2)

Lignosphaeria Boonmee, Thambug. \& K.D. Hyde (2)

Limaciniopsis Mend. (1)

Lineolata Kohlm. \& Volkm.-Kohlm. (1)

Linopeltis I. Hino \& Katum. (2)

Lophionema Sacc. (9)

Lucidascocarpa A. Ferrer, Raja \& Shearer (1)

Macowaniella Doidgeb (2)

Maheshwaramyces Hosag. (2)

Maireella Syd. \& Maire (ca. 5)

Malacaria Syd. (2)

Manginula G. Arnaud (ca. 5)

Marquesius L.B. Conç., R.F. Castañeda \& Gusmão (1)

Massariola Füisting (2)

Maublancia G. Arnaud (1)

Melioliphila Speg. (7)

Mendoziopeltis Bat. (4)

Microcyclella Theiss. (1)

Microdothella Syd. \& P. Syd. (2)

Monoblastiopsis R.C. Harris \& C.A. Morse (2)

Monodictys S. Hughes (ca. 50)

Monorhizina Theiss. \& Syd. (1)

Montagnella Speg. (9)

Moriolomyces Cif. \& Tomas. (1)

Muricopeltis Viégas (1)

Muroia I. Hino \& Katum. (1)

Mycocryptospora J. Reid \& C. Booth (1)

Mycodidymella C.Z. Wei, Y. Harada \& Katum. (1)

Mycoglaena Höhn. (16)

Mycoporellum Müll. Arg. (7)

Mycoporopsis Müll. Arg. (ca. 10)

Mycothyridium Petr. (30)

Myriangiopsis P. Henn. (2)

Myriostigmella G. Arnaud (1)

Mytilostoma P. Karst. (2)

Myxophora Döbbeler \& Poelt (7)

Nannfeldtia Petr. (2)

Neodactylaria Guevara-Suarez, Deanna A. Sutton, Wiederh. \& Gené (1)

Neopeckia Sacc. (1 fide Kirk et al. 2008)

Neosporidesmium Mercado \& J. Mena (15)

Neottiosporina Subram. (11)

Neoventuria Syd. \& P. Syd. (1)

Ocala Raja \& Shearer (1) 
Oletheriostrigula Huhndorf \& R.C. Harris (1)

Omphalospora Theiss. \& Syd. (2)

Oncopodiella G. Arnaud ex Rifai (13)

Ophioirenina Sawada \& W. Yamam. (1)

Ophiotrichum Kunze (3)

Otthia Nitschke ex Fuckel (11)

Parmulariella P. Henn. (1)

Paropodia Cif. \& Bat. (1)

Passeriniella Berl. (7)

Passerinula Sacc. (1)

Pauahia F. Stevens (1)

Peltaster Syd. \& P. Syd. (8)

Peltasterella Bat. \& H. Maia (1)

Pendulispora M.B. Ellis (1)

Perischizon P. Syd. (3)

Peroschaeta Bat. \& A.F. Vital (1)

Petrakina Cif. (3)

Petrakiopeltis Bat., A.F. Vital \& Cif. (1)

Phacidina Höhn. (1)

Phaeocyrtidula Vain. (2)

Phaeopeltosphaeria Berl. \& Peglion (2)

Phaeosclera Sigler, Tsuneda \& J.W. Carmich. (1)

Phaeosperma Nitschke ex Fuckel (1)

Phaeostigme Syd. \& P. Syd. (6)

Phaeotomasellia Katum. (1)

Phanerococculus Cif. (1)

Philobryon Döbbeler (1)

Philonectria Hara (3)

Phragmaspidium Bat. (3)

Phragmogibbera Samuels \& Rogerson (3)

Phragmoscutella Woron. \& Abramov ex Woron. (1)

Phragmosperma Theiss. \& Syd. (1)

Phycorella Döbbeler (1)

Physalosporopsis Bat. \& H. Maia (1)

Pirozynskia Subram. (1)

Placoasterina Toro (1)

Placodothis Syd. (1)

Placomelan Cif. (1)

Placosphaeria (De Not.) Sacc. (1, but several other epithets exist)

Plagiostromella Höhn. (1)

Plejobolus (E. Bommer et al.) O.E. Erikss. (1 or 2 species)

Plenotrichaius Bat. \& Valle (1)

Pleomerium Speg. (1)

Pleotrichiella Sivan. (1)

Polycyclinopsis Bat., A.F. Vital \& I.H. Lima (1)

Polyrhizon Theiss., Syd. \& P. Syd. (2)

Polysporidiella Petr. (1)

Polystomellopsis F. Stevens (1)

Proliferosphaera T.P. Devi (1)

Pseudoarthrographis Crous \& Thangavel (1)

Pseudomorfea Punith. (1)

Pseudopleospora Petr. (1) 
Punctillum Petr. \& Syd. (1)

Pyrenobotrys Theiss. \& Syd. (1)

Pyrenochium Link (1)

Pyrenocyclus Petr. (1)

Pyrenostigme Syd. (1)

Radulidium Arzanlou, W. Gams \& Crous (3)

Rhizotexis Theiss. \& Syd. (1)

Rhopographus Nitschke ex Fuckel (6)

Rosellinula R. Sant. (4)

Rosenscheldia Speg. (1)

Roumegueria (Sacc.) P. Henn. (1)

Rupestriomyces Lei Su, Li Y. Guo \& Xing Z. Liu (3)

Sapucchaka K. Ramakr. (2)

Saxomyces L. Selbmann \& D. Isola (2)

Scleroconidioma Tsuneda, Currah \& Thormann (1)

Scolecobonaria Bat. (2)

Scolecoxyphium Cif. \& Bat. (5)

Scolionema Theiss. \& Syd. (1)

Semisphaeria K. Holm \& L. Holm (1)

Septoidium G. Arnaud (ca. 7)

Shearia Petr. (2)

Shivamyces Hosag. (2)

Sivanesaniella Gawande \& D.K. Agarwal (1)

Solicorynespora R.F. Castañeda \& W.B. Kendr. (29)*

Soloacrosporiella Crous \& M.J. Wingf. (1)

Spilodochium Syd. (4)

Spissiomyces Lei Su, Li Y. Guo \& Xing Z. Liu (2)

Stegothyrium Höhn. (2)

Stephanotheca Syd. \& P. Syd. (4)

Stigmatodothis Syd. \& P. Syd. (1)

Stigmatophragmia Tehon \& G.L. Stout (1)

Symphaster Theiss. \& Syd. (1)

Taphrophila Scheuer (4)

Teichosporella (Sacc.) Sacc. (26)

Teratoschaeta Bat. \& Fons.) (1)

Tetracrium Henn. (7)

Thalassoascus Ollivier (3)

Thelenidia Nyl. (1)

Thryptospora Petr. (1)

Tilakiella Srinivas. (1)

Tomeoa I. Hino (1)

Torulopsiella Bender (2)

Trematosphaeriopsis Elenkin (1)

Tretospora M.B. Ellis (8)

Trichodothella Petr. (1)

Trichodothis Theiss. \& Syd. (3)

Trichometasphaeria Munk (8)

Trichothyriella Theiss. (1)

Troposporella P. Karst. (4)

Uredinophila Rossman (2)

Wentiomyces Koord. (ca. 50)

Westea H.J. Swart (1) 
Wettsteinina Höhn. (30)

Xenomeris Syd. (11)

Xenosporium Penz. \& Sacc. (18)

Xenostomella Syd. (2)

Xylopezia Höhn. (ca. 3)

Yoshinagaia Henn. (1)

Yoshinagella Höhn. (4)

Eurotiomycetes Tehler ex O.E. Eriksson \& K. Winka

Chaetothyriomycetidae Doweld

Chaetothyriales M.E. Barr

Chaetothyriaceae Hansf. ex M.E. Barr

Actinocymbe Höhn. (3)

Aithaloderma Syd. \& P. Syd. (13)

Aphanophora Réblová \& Unter. (1)

Arthrophiala (D.J. Soares, R.W. Barreto \& U. Braun) W.S. Lisboa, Meir. Silva \& R.W.

Barreto (1)*

Beelia F. Stevens \& R.W. Ryan (3)

Camptophora Réblová \& Unter. (2)*

Ceramothyrium Bat. \& H. Maia (35)

Ceratocarpia Rolland (2)

Chaetothyriomyces Pereira-Carv., Inácio \& Dianese (1)

Chaetothyrium Speg. (51)

Cyphellophoriella Crous \& A.J. Sm. (1)

Euceramia Bat. \& Cif. (3)

Longihyalospora D.S.Tennakoon, C.H Kuo \& K.D. Hyde (2)

Microcallis Syd. (10)

Nullicamyces Crous (1)

Phaeosaccardinula P. Henn. (27)

Stanhughesia Constant. (1)

Treubiomyces Höhn. (7)

Vonarxia Bat. (2)

Yatesula Syd. \& P. Syd. (2)

Coccodiniaceae Höhn. ex O.E. Erikss.

Coccodinium A. Massal. (4)

Dennisiella Bat. \& Cif. (= Microxiphium (Harv. ex Berk. \& Desm.) Thüm.) (9)

Limacinula Höhn. (17)

Cyphellophoraceae Réblová \& Unter.

Anthopsis Fil. March., A. Fontana \& Luppi Mosca (2)*

Cyphellophora G.A. de Vries (25)*

Epibryaceae S. Stenroos \& Gueidan

Epibryon Döbbeler (ca. 40)

\section{Herpotrichiellaceae Munk}

Aculeata W. Dong, H. Zhang \& K.D. Hyde (1)*

Brycekendrickomyces Crous \& M.J. Wingf. (1)

Capronia Sacc. (ca. 81)

Cladophialophora Borelli (35)*

Exophiala J.W. Carmich. (51)* 
Fonsecaea Negroni (8)*

Marinophialophora J.F. Li, Phook. \& K.D. Hyde (1)

Melanoctona Qing Tian, Doilom \& K.D. Hyde (1)

Metulocladosporiella Crous, Schroers, J.Z. Groenew., U. Braun \& K. Schub. (6)

Minimelanolocus R.F. Castañeda \& Heredia (33)*

Phialophora Medlar (7)*

Pleomelogramma Speg. (2)

Rhinocladiella Nannf. (17)

Sorocybe Fr. (3)

Thysanorea Arzanlou, W. Gams \& Crous (2)*

Veronaea Cif. \& Montemart. (20)

\section{Lyrommataceae Lücking}

Lyromma Bat. (7)

Microtheliopsidaceae O.E. Erikss.

Microtheliopsis Müll. Arg. (4)

\section{Paracladophialophoraceae Crous}

Paracladophialophora Crous (2)*

\section{Pyrenotrichaceae Zahlbr}

Pyrenothrix Riddle (2)

Neophaeococcomyces Crous \& M.J. Wingf. (2)

Trichomeriaceae Chomnunti \& K.D. Hyde (= Strelitzianaceae Crous \& M.J. Wingf.)

Arthrocladium Papendorf (4)*

Bradymyces Hubka, Réblová, Selbmann \& M. Kolařík (3)*

Knufia L.J. Hutchison \& Unter. (13)*

Lithohypha Selbmann \& Isola (1)

Lithophila Selbmann \& Isola (1)*

Neostrelitziana Crous \& M.J. Wingf. (1)

Strelitziana Arzanlou \& Crous (8)

Trichomerium Speg. (28)

\section{Chaetothyriales genera incertae sedis}

Atrokylindriopsis Y.R. Ma \& X.G. Zhang (1)

Bacillicladium Hubka, Réblová \& Thureborn (1)*

Lichenodiplis Dyko \& D. Hawksw. (= Laeviomyces D. Hawksw.) (13)

Lichenodiplisiella S.Y. Kondr. \& Kudratov (1)

Melnikomyces Crous \& U. Braun (1)

Minutoexcipula V. Atienza \& D. Hawksw. (7)

Muellerella Hepp (14)*

Pleostigma Kirschst. (9)

Sarcinomyces Lindner (5)

Uncispora R.C. Sinclair \& Morgan-Jones (3)

Phaeomoniellales K.H. Chen, A.E. Arnold, Gueidan \& Lutzoni

Celotheliaceae Lücking, Aptroot \& Sipman (= Phaeomoniellaceae P.M. Kirk)

Aequabiliella Crous (1)

Celerioriella Crous (3)

Celothelium A. Massal. (8) 
Minutiella Crous (1)

Moristroma A.I. Romero \& Samuels (4)

Neophaeomoniella Rooney-Latham \& Crous (3)

Paraphaeomoniella Crous (1)

Phaeomoniella Crous \& W. Gams (2)

Pseudophaeomoniella Nigro, Antelmi \& Crous (2)

Xenocylindrosporium Crous \& Verkley (1)

Pyrenulales Fink ex D. Hawksw. \& O.E. Erikss.

Pyrenulaceae Rabenh.

Anthracothecium Hampe ex A. Massal. (5)

Blastodesmia A. Massal. (1)

Clypeopyrenis Aptroot (2)

Distopyrenis Aptroot (8)

Granulopyrenis Aptroot (6)

Lithothelium Müll. Arg. (28)

Mazaediothecium Aptroot (4)

Pyrenographa Aptroot (1)

Pyrenowilmsia R.C. Harris \& Aptroot (1)

Pyrenula Ach. (= Heufleridium Müll. Arg.; = Stromatothelium Trevis.) (ca. 225)

Pyrgillus Nyl. (8)

Sulcopyrenula $\mathrm{H}$. Harada (5)

Pyrenulales genera incertae sedis

Rhaphidicyrtis Vain. (1)

Xenus Kohlm. \& Volkm.-Kohlm. (1)

Verrucariales Mattick ex D. Hawksw. \& O.E. Erikss.

Adelococcaceae Triebel

Adelococcus Theiss. \& Syd. (4)

Pseudopyrenidium Nav.-Ros., Zhurb. \& Cl. Roux (1)

Sagediopsis Sacc. ex Vain. (10)

Sarcopyreniaceae Nav.-Ros. \& Cl. Roux

Sarcopyrenia Nyl. (11)

Verrucariaceae Zenker

Agonimia Zahlbr. (ca. 20)

Anthracocarpon Breuss (1)

Atla S. Savić \& Tibell (10)

Awasthiella Kr.P. Singh (1)

Bagliettoa A. Massal. (17)

Bellemerella Nav.-Ros. \& Cl. Roux (4)

Catapyrenium Flot. (6)

Clauzadella Nav.-Ros. \& Cl. Roux (1)

Clavascidium Breuss (9)

Dermatocarpon Eschw. (20)

Endocarpon Hedw. (ca. 75)

Flakea O.E. Erikss. (1)

Glomerilla Norman (1)

Haleomyces D. Hawksw. \& Essl. (1)

Halospora (Zschacke) Tomas. \& Cif. (4) 
Henrica de Lesd. (4)

Heterocarpon Müll. Arg. (1)

Heteroplacidium Breuss (12)

Hydropunctaria C. Keller, Gueidan \& Thüs (8)

Involucropyrenium Breuss (9)

Mastodia Hook.f. \& Harv. (= Turgidosculum Kohlm. \& E. Kohlm.) (5)

Moriola Norman (ca. 15)

Neocatapyrenium $\mathrm{H}$. Harada (5)

Normandina Nyl. (= Lauderlindsaya J.C. David \& D. Hawksw.) (3)

Norrlinia Theiss. \& Syd. (2)

Parabagliettoa Gueidan \& Cl. Roux (3)

Phaeospora Hepp ex Stein (14)

Phylloblastia Vain. (12)

Placidiopsis Beltr. (20)

Placidium A. Massal. (28)

Placocarpus Trevis. (5)

Placopyrenium Breuss (22)

Placothelium Müll. Arg. (1)

Plurisperma Sivan. (1)

Polyblastia A. Massal. (ca. $40+$ ca. 50 orphaned)

Psoroglaena Müll. Arg. (17)

Rhabdopsora Müll. Arg. (2)

Scleropyrenium H. Harada (2)

Servitia M.S. Christ. \& Alstrup (1)

Spheconisca (Norman) Norman (ca. 20)

Sporodictyon A. Massal. (5)

Staurothele Norman (ca. 40)

Telogalla Nik. Hoffm. \& Hafellner (2)

Thelidiopsis Vain. (4)

Thelidium A. Massal. (ca. 50 + ca. 50 orphaned)

Trimmatothele Norman ex Zahlbr. (3)

Verrucaria Schrad. (ca. 300)

Verrucula J. Steiner (22)

Verruculopsis Gueidan, Nav.-Ros. \& Cl. Roux (ca. 10)

Wahlenbergiella Gueidan \& Thüs (3)

Willeya Müll. Arg. (12)

Verrucariales genera incertae sedis

Botryolepraria Canals, Hern.-Mar., Gómez-Bolea \& Llimona (2)

Gemmaspora D. Hawksw. \& Halici (1)

Kalbiana Henssen (1)

Merismatium Zopf (10)

Chaetothyriomycetidae family incertae sedis

Rhynchostomataceae Winka \& O.E. Erikss.

Rhynchomeliola Speg. (3)

Rhynchostoma P. Karst. (23)

Coryneliomycetidae A.R. Wood, Damm, J.Z. Groenew., Cheew. \& Crous Coryneliales Seaver \& Chardon

Coryneliaceae Sacc. ex Berl. \& Voglino

Caliciopsis Peck (36) 
Corynelia Ach. (16)

Coryneliopsis Butin (2)

Coryneliospora Fitzp. (2)

Fitzpatrickella Benny, Samuelson \& Kimbr. (1)

Lagenulopsis Fitzp. (1)

Tripospora Sacc. ex Berl. \& Vogl. (5)

Eremascaceae Engl. \& E. Gilg

Eremascus Eidam (2)

Eurotiomycetidae Geiser \& Lutzoni

Arachnomycetales Gibas, Sigler \& Currah

Arachnomycetaceae Gibas, Sigler \& Currah

Arachnomyces Massee \& E.S. Salmon (10)

Onychocola Sigler (4)

Eurotiales G.W. Martin ex Benny \& Kimbr.

Aspergillaceae Link (= Monascaceae J. Schröt.)

Aspergillago Samson, Houbraken \& Frisvad (1)

Aspergillus P. Micheli ex Haller (428)

Dichlaena Durieu \& Mont. (4)

Hamigera Stolk \& Samson (9)

Leiothecium Samson \& Mouch. (2)

Monascus Tiegh. (38)

Penicillago Guevara-Suarez, Gené \& Dania García (1)

Penicilliopsis Solms (15)

Penicillium Link (467)

Phialomyces P.C. Misra \& P.H.B. Talbot (5)

Pseudopenicillium Guevara-Suarez, Cano \& Guarro (2)

Sclerocleista Subram. (2)

Sclerocleista Subram. (2)

Xerochrysium Pitt (2)

Xeromyces Fraser (1)

Elaphomycetaceae Tul. ex Paol.

Elaphomyces Nees (101)

Pseudotulostoma O.K. Miller \& T. Henkel (2)

Thermoascaceae Apinis

Paecilomyces Bainier (10)

Thermoascus Miehe (5)

Trichocomaceae E. Fisch.

Chaetotheca Zukal (2)

Dendrosphaera Pat. (1)

Rasamsonia Houbraken \& Frisvad (11)

Sagenomella W. Gams (8)

Talaromyces C.R. Benj. (149)

Thermomyces Tsikl. (6)

Trichocoma Jungh. (2)

Onygenales Cif. ex Benny \& Kimbr. 
Ajellomycetaceae Unter., J.A. Scott \& Sigler

Blastomyces Gilchrist \& W.R. Stokes (=Ajellomyces McDonough \& A.L Lewis; Emmonsia Cif. \& Montemart.) (9)

Emmonsiellopsis Y. Marín, Stchigel, Guarro \& Cano (2)

Emergomyces Dukik, Sigler \& de Hoog (5)*

Histoplasma Darling (4 epithets in Index Fungorum 2020)

Lacazia Taborda, V.A. Taborda \& McGinnis (1)

Paracoccidioides F.P. Almeida (6)

Arthrodermataceae Currah

Arthroderma Curr. \& Berk. (32)

Ctenomyces Eidam (7)

Epidermophyton Sabour. (1)

Guarromyces Y Gräser \& de Hoog (1)

Lophophyton Matr. \& Dassonv. (1)

Microsporum Gruby (3)

Nannizzia Stockdale (9)

Paraphyton Y Gräser, Dukik \& de Hoog (3)

Shanorella R.K. Benj. (1)

Trichophyton Malmsten (16)

Ascosphaeraceae L.S. Olive \& Spiltoir

Arrhenosphaera Stejskal (1)

Ascosphaera L.S. Olive \& Spiltoir (27)

Bettsia Skou (2)

Gymnoascaceae Baran.

Aciascus Doweld (1)

Amaurascopsis Guarro, Gené \& De Vroey (1)

Arachniotus J. Schröt. (21)

Gymnascella Peck (9)

Gymnoascoideus G.F. Orr, K. Roy \& G.R. Ghosh (1)

Gymnoascus Baran. (=Narasimhella Thirum. \& P.N. Mathur) (26)

Kraurogymnocarpa Udagawa \& Uchiyama (1)

Mallochia Arx \& Samson (4)

Oncocladium Wallr. (1)

Orromyces Sur \& G.R. Ghosh (1)

Nannizziopsidaceae Guarro, Stchigel, Deanna A. Sutton \& Cano

Nannizziopsis Currah (16)

\section{Onygenaceae Berk.}

Amauroascus J. Schröt. (ca. 15)

Aphanoascus Zukal (18)

Apinisia La Touche (3)

Arachnotheca Arx (1)

Ascocalvatia Malloch \& Cain (1)

Auxarthron G.F. Orr \& Kuehn (13)

Auxarthronopsis Rah. Sharma, Y. Gräser \& S.K. Singh (2)

Bifidocarpus Cano, Guarro \& R.F. Castañeda (2)

Byssoonygena Guarro, Punsola \& Cano (1)

Castanedomyces Cano, L.B. Pitarch \& Guarro (1) 
Chlamydosauromyces Sigler, Hambl. \& Paré (1)

Chrysosporium Corda (66)

Coccidioides G.W. Stiles (6)

Kuehniella G.F. Orr (2)

Leucothecium Arx \& Samson (3)

Malbranchea Sacc. (23)

Myotisia Kubátová, M. Kolařík \& Hubka (1)

Myriodontium Samson \& Polon. (1)

Neoarachnotheca Ulfig, Cano \& Guarro (1)

Neogymnomyces G.F. Orr (2)

Onygena Pers. (10)

Ophidiomyces Sigler, Hambl. \& Paré (1)

Paranannizziopsis Sigler (4)

Pectinotrichum Varsavsky \& G.F. Orr (2)

Polytolypa J.A. Scott \& Malloch (1)

Pseudoamauroascus Cano, M. Solé \& Guarro (1)

Renispora Sigler \& J.W. Carmich. (2)

Sporendonema Desm. (2)

Testudomyces Cano, M. Solé \& Guarro (1)

Uncinocarpus Sigler \& G.F. Orr (2)

Xanthothecium Arx \& Samson (1)

Spiromastigaceae Guarro, Cano \& Stchigel

Pseudospiromastix Guarro, Stchigel \& Cano (1)

Sigleria Hirooka, Tanney \& Seifert (2)

Spiromastigoides Doweld (8)

Spiromastix Kuehn \& G.F. Orr (5)

Onygenales genera incertae sedis

Arthropsis Sigler, M.T. Dunn \& J.W. Carmich. (4)

Ovadendron Sigler \& J.W. Carmich. (1)*

Sphaerosporium Schwein. sensu lato (2)*

Eurotiomycetidae genera incertae sedis

Azureothecium Matsush. (1)

Calyptrozyma Boekhout \& Spaay (1)

Pisomyxa Corda (1)

Samarospora Rostr. (1)

Veronaia Benedek (2)

Mycocaliciomycetidae Tibell

Mycocaliciales Tibell \& Wedin

Mycocaliciaceae A.F.W. Schmidt (= Sphinctrinaceae M. Choisy)

Brunneocarpos Giraldo \& Crous (1)

Chaenothecopsis Vain. (ca. 40)

Mycocalicium Vain. ex Reinke (12)

Phaeocalicium A.F.W. Schmidt (11)

Pyrgidium Nyl. (3)

Sphinctrina Fr. (ca. 9)

Stenocybe (Nyl.) Körb. (14)

Sclerococcomycetidae Réblová, Unter. \& W. Gams 
Sclerococcales Réblová, Unter. \& W. Gams

Dactylosporaceae Bellem. \& Hafellner (= Sclerococcaceae Réblová, Unter. \& W. Gams)

Cylindroconidiis H. Zhang \& X.D. Yu (1)

Fusichalara S. Hughes \& Nag Raj (5)*

Longimultiseptata H. Zhang \& W. Dong (2)

Rhopalophora Réblová, Unter. \& W. Gams (1)

Sclerococcum Fr. (= Dactylospora Körb.) (ca. 80)*

Eurotiomycetes genus incertae sedis

Neocladophialophora Crous \& R.K. Schumach. (1)

Geoglossomycetes Zheng Wang, C.L. Schoch \& Spatafora

Geoglossales Zheng Wang, C.L. Schoch \& Spatafora

Geoglossaceae Corda

Geoglossum Pers. (40)

Glutinoglossum Hustad, A.N. Mill., Dentinger \& P.F. Cannon (13)

Hemileucoglossum Arauzo (5)

Leucoglossum S. Imai (2)

Maasoglossum K.S. Thind \& R. Sharma (2)

Sabuloglossum Hustad, A.N. Mill., Dentinger \& P.F. Cannon (1)

Trichoglossum Boud. (19)

Geoglossomycetes genera incertae sedis

Nothomitra Maas Geest. (3)*

Sarcoleotia S. Ito \& S. Imai (3)*

Laboulbeniomycetes Engler

Herpomycetales Haelew. \& Pfister*

Herpomycetaceae I.I. Tav.

Herpomyces Thaxt. (26)

Laboulbeniales Lindau

Ceratomycetaceae S. Colla

Autoicomyces Thaxt. (28)

Ceratomyces Thaxt. (32)

Drepanomyces Thaxt. (1)

Eusynaptomyces Thaxt. (5)

Helodiomyces F. Picard (1)

Phurmomyces Thaxt. (1)

Plectomyces Thaxt. (1)

Rhynchophoromyces Thaxt. (8)

Synaptomyces Thaxt. (1)

Tettigomyces Thaxt. (16)

Thaumasiomyces Thaxt. (3)

Thripomyces Speg. (2)

Euceratomycetaceae I.I. Tav.

Cochliomyces Speg. (2)

Colonomyces R.K. Benj. (2)

Euceratomyces Thaxt. (1)

Euzodiomyces Thaxt. (2)

Pseudoecteinomyces W. Rossi (1) 
Laboulbeniaceae G. Winter

Acallomyces Thaxt. (3)

Acompsomyces Thaxt. (6)

Acrogynomyces Thaxt. (6)

Amorphomyces Thaxt. (15)

Amphimyces Thaxt. (1)

Apatelomyces Thaxt. (1)

Apatomyces Thaxt. (1)

Aphanandromyces W. Rossi (1)

Aporomyces Thaxt. (11)

Arthrorhynchus Kolen. (3)

Asaphomyces Thaxt. (2)

Autophagomyces Thaxt. (17)

Benjaminiomyces I.I. Tav. (4)

Blasticomyces I.I. Tav. (3)

Bordea Maire (15)

Botryandromyces I.I. Tav. \& T. Majewski (2)

Camptomyces Thaxt. (8)

Cantharomyces Thaxt. (29)

Capillistichus Santam. (1)

Carpophoromyces Thaxt. (1)

Cesariella W. Rossi \& Santam. (1)

Chaetarthriomyces Thaxt. (3)

Chaetomyces Thaxt. (2)

Chitonomyces Peyr. (ca. 98)

Clematomyces Thaxt. (5)

Clonophoromyces Thaxt. (2)

Columnomyces R.K. Benj. (1)

Compsomyces Thaxt. (7)

Coreomyces Thaxt. (22)

Corethromyces Thaxt. (ca. 85)

Corylophomyces R.K. Benj. (5)

Cryptandromyces Thaxt. (= Peyerimhoffiella Maire) (19)

Cucujomyces Speg. (20)

Cupulomyces R.K. Benj. (= Balazucia R.K. Benj.) (1)

Dermapteromyces Thaxt. (1)

Diandromyces Thaxt. (2)

Diaphoromyces Thaxt. (5)

Diclonomyces Thaxt. (3)

Dimeromyces Thaxt. (118)

Dimorphomyces Thaxt. (32)

Dioicomyces Thaxt. (32)

Diphymyces I.I. Tav. (25)*

Diplomyces Thaxt. (3)

Diplopodomyces W. Rossi \& Balazuc (6)

Dipodomyces Thaxt. (2)

Distolomyces Thaxt. (3)

Dixomyces I.I. Tav. (14)

Ecteinomyces Thaxt. (1)

Enarthromyces Thaxt. (1)

Eucantharomyces Thaxt. (26)

Euhaplomyces Thaxt. (1) 
Eumonoicomyces Thaxt. (2)

Euphoriomyces Thaxt. (15)

Filariomyces Shanor (1)

Gloeandromyces Thaxt. (4)

Haplomyces Thaxt. (3)

Hesperomyces Thaxt. (8)*

Histeridomyces Thaxt. (6)

Homaromyces R.K. Benj. (1)

Hydraeomyces Thaxt. (1)

Hydrophilomyces Thaxt. (12)

Idiomyces Thaxt. (1)

Ilyomyces F. Picard (2)

Ilytheomyces Thaxt. (15)

Kainomyces Thaxt. (3)

Kleidiomyces Thaxt. (4)

Kruphaiomyces Thaxt. (1)

Kyphomyces I.I. Tav. (14)

Laboulbenia Mont. \& C.P. Robin (= Scalenomyces I.I. Tav.) (ca. 633)*

Limnaiomyces Thaxt. (3)

Majewskia Y.B. Lee \& Sugiyama (1)

Meionomyces Thaxt. (6)

Microsomyces Thaxt. (2)

Mimeomyces Thaxt. (16)

Misgomyces Thaxt. (4)

Monandromyces R.K. Benj. (11)

Monoicomyces Thaxt. (47)

Nanomyces Thaxt. (48)

Neohaplomyces R.K. Benj. (3)

Nycteromyces Thaxt. (2)

Opilionomyces Santam., Enghoff, Gruber \& Reboleira (1)*

Ormomyces I.I. Tav. (1)

Osoriomyces Terada (1)

Parvomyces Santam. (1)

Peyritschiella Thaxt. (47)

Phalacrichomyces R.K. Benj. (2)

Phaulomyces Thaxt. (14)

Picardella I.I. Tav. (2)

Polyandromyces Thaxt. (1)

Polyascomyces Thaxt. (1)

Porophoromyces Thaxt. (1)

Prolixandromyces R.K. Benj. (20)

Pselaphidomyces Speg. (1)

Rhachomyces Thaxt. (ca. 75)

Rhipidiomyces Thaxt. (1)

Rhizomyces Thaxt. (10)

Rhizopodomyces Thaxt. (7)

Rickia Cavara (144)

Rodaucea W. Rossi \& Santam. (2)

Rossiomyces R.K. Benj. (1)

Sandersoniomyces R.K. Benj. (1)

Scaphidiomyces Thaxt. (5)

Scelophoromyces Thaxt. (1) 
Scepastocarpus Santam. (1)

Siemaszkoa I.I. Tav. \& Maj. (7)

Smeringomyces Thaxt. (4)

Sphaleromyces Thaxt. (3)

Stemmatomyces Thaxt. (2)

Stichomyces Thaxt. (7)

Stigmatomyces H. Karst. (= Fanniomyces T. Majewski) (150)

Sugiyamaemyces I.I. Tav. \& Balazuc (1)

Symplectromyces Thaxt. (3)

Sympodomyces R.K. Benj. (1)

Synandromyces Thaxt. (9)

Tavaresiella T. Majewski (4)

Teratomyces Thaxt. (11)

Tetrandromyces Thaxt. (6)

Thaxterimyces Santam., Reboleira \& Enghoff (1)

Trenomyces Chatton \& F. Picard (11)

Triainomyces W. Rossi \& A. Weir (1)

Triceromyces T. Majewski (5)

Trochoideomyces Thaxt. (1)

Troglomyces S. Colla (8)

Zeugandromyces Thaxt. (4)

Zodiomyces Thaxt. (4)

Laboulbeniales genera incertae sedis

Cainomyces Thaxt. (1)

Coreomycetopsis Thaxt. (1)

Gliocephalis Matr. (2)

Pyxidiophorales P.F. Cannon

Pyxidiophoraceae Arnold

Mycorhynchidium Malloch \& Cain (1)

Pleurocatena G. Arnaud ex Aramb., Gamundí, W. Gams \& G.R.W. Arnold (3)

Pyxidiophora Bref. \& Tavel (17)

Laboulbeniomycetes genus incertae sedis

Laboulbeniopsis Thaxt. (1)

Lecanoromycetes O.E. Erikss. \& Winka

Acarosporomycetidae V. Reeb, Lutzoni \& Cl. Roux

Acarosporales V. Reeb, Lutzoni \& Cl. Roux

Acarosporaceae Zahlbr.

Acarospora A. Massal. (200)

Caeruleum Arcadia (2)

Glypholecia Nyl. (1)

Lithoglypha Brusse (1)

Myriospora Nägeli ex Uloth (9)

Pleopsidium Körb. (4)

Polysporina Vězda (10)

Sarcogyne Flot. (28)

Thelocarpella Nav.-Ros. \& Cl. Roux (1)

Timdalia Hafellner (1)

Trimmatothelopsis Zschacke (1) 
Eigleraceae Hafellner

Eiglera Hafellner (2)

Lecanoromycetidae P.M. Kirk, P.F. Cannon, J.C. David \& Stalpers ex Miądl., Lutzoni \& Lumbsch

Caliciales Bessey

Caliciaceae Chevall.

Acolium (Ach.) Gray (5)

Acroscyphus Lév. (1)

Allocalicium M. Prieto \& Wedin (1)

Amandinea M. Choisy ex Scheid. \& M. Mayrhofer (35)

Australiaena Matzer, H. Mayrhofer \& Elix (1)

Baculifera Marbach (14)

Buellia De Not. (= Dirinastrum Müll. Arg.) (300)

Caliciella Vain. (1)

Calicium Pers. (= Cyphelium Ach.) (ca. 30)

Chrismofulvea Marbach (4)

Ciposia Marbach (1)

Cratiria Marbach (ca. 20)

Culbersonia Essl. (1)*

Dermatiscum Nyl. (3)

Dermiscellum Hafellner, H. Mayrhofer \& Poelt (1)

Dimelaena Norman (10)

Diploicia A. Massal. (ca. 12)

Diplotomma Flot. (ca. 30)

Dirinaria (Tuck.) Clem. (ca. 35)

Endohyalina Marbach (10)

Fluctua Marbach (1)

Gassicurtia Fée (30)

Hypoflavia Marbach (3)

Monerolechia Trevis. (4)

Orcularia (Malme) Kalb \& Giralt (4)

Pseudothelomma M. Prieto \& Wedin (2)

Pyxine Fr. (ca. 75)

Redonia C.W. Dodge (2)

Santessonia Hale \& Vobis (10)

Sculptolumina Marbach (4)

Sphinctrinopsis Woron. (1)

Stigmatochroma Marbach (9)

Tetramelas Norman (16)

Texosporium Nádv. ex Tibell \& Hofsten (1)

Thelomma A. Massal. (5)

Tholurna Norman (1)

Physciaceae Zahlbr.

Anaptychia Körb. (ca. 15)

Coscinocladium Kunze (2)

Heterodermia Trevis. (ca. 90)

Hyperphyscia Müll. Arg. (9)

Kashiwadia S.Y. Kondr. (1)

Leucodermia Kalb (10)

Mischoblastia A. Massal. (3) 
Mobergia H. Mayrhofer \& Sheard (1)

Oxnerella S.Y. Kondr., Lökös \& Hur (1)

Phaeophyscia Mob. (66)

Phaeorrhiza H. Mayrhofer \& Poelt (2)

Physcia (Schreb.) Michaux (ca. 80)

Physciella Essl. (4)

Physconia Poelt (ca. 25)

Polyblastidium Kalb (18)

Rinodina (Ach.) Gray (ca. 300)

Rinodinella H. Mayrhofer \& Poelt (6)

Tornabea Oesth. (1)

\section{Lecanorales Nannf.}

Bruceomycetaceae Rikkinen \& A.R. Schmidt

Bruceomyces Rikkinen (4)

Resinogalea Rikkinen \& A.R. Schmidt (1)*

\section{Catillariaceae Hafellner}

Austrolecia Hertel (1)

Catillaria A. Massal. (ca. 30 + several orphaned names)

Placolecis Trevis. (1)

Solenopsora A. Massal. (11)

Xanthopsorella Kalb \& Hafellner (1)

Cladoniaceae Zenker (= Squamarinaceae Hafellner, = Stereocaulaceae Chevall.)*

Calathaspis I.M. Lamb \& W.A. Weber (1)

Carassea S. Stenroos (1)

Cetradonia J.C. Wei \& Ahti (1)

Cladia Nyl. (ca. 27)

Cladonia Hill ex P. Browne (ca. 500)

Gymnoderma Nyl. (3)

Herteliana P. James (3)

Hertelidea Printzen \& Kantvilas (6)

Heteromyces Müll. Arg. (1)

Lepraria Ach. (76)

Metus D.J. Galloway \& P. James (3)

Notocladonia S. Hammer (2)

Paralecia Brackel, Greiner, Peršoh \& Rambold (1)

Pilophorus Th. Fr. (17)

Pulchrocladia S. Stenroos, Pino-Bodas, Lumbsch \& Ahti (3)

Pycnothelia Duf. (2)

Sphaerophoropsis Vain. (2)

Squamarina Poelt (25)

Squamella S. Hammer (1)

Stereocaulon Hoffm. (ca. 140)

Thysanothecium Mont. \& Berk. (3)

Xyleborus R.C. Harris \& Ladd (1)

\section{Gypsoplacaceae Timdal}

Gypsoplaca Timdal (5)

Haematommataceae Hafellner 
Haematomma A. Massal. (ca. 50)

Lecanoraceae Körb. (= Carbonicolaceae Bendiksby \& Timdal)

Adelolecia Hertel \& Hafellner (4)*

Ameliella Fryday \& Coppins (2)

Bryodina Hafellner (2)

Bryonora Poelt (11)

Carbonicola Bendiksby \& Timdal (3)

Cladidium Hafellner (2)

Claurouxia D. Hawksw. (1)

Clauzadeana $\mathrm{Cl}$. Roux (1)

Edrudia W.P. Jordan (1)

Frutidella Kalb (3)*

Huea C.W. Dodge \& G.E. Baker (=Carbonea (Hertel) Hertel) (20)

Japewia Tønsberg (3)*

Japewiella Printzen (7)

Lecanora Ach. (ca. 550)

Lecidella Körb. (80)

Maronina Hafellner \& R.W. Rogers (6)

Maronora Kalb \& Aptroot (1)

Miriquidica Hertel \& Rambold (30)

Myriolecis Clements (43)

Palicella Rodr. Flakus \& Printzen (4)

Protoparmeliopsis Choisy (= Sedelnikovaea S.Y. Kondr., M.H. Jeong \& Hur) (ca. 20)

Psorinia Gotth. Schneid. (2)

Punctonora Aptroot (2)

Pyrrhospora Körb. (7)

Rhizoplaca Zopf (11)

Sagema Poelt \& Grube (1)

Traponora Aptroot (8)

Vainionora Kalb (9)

Malmideaceae Kalb, Rivas Plata \& Lumbsch

Cheiromycina B. Sutton (4)

Crustospathula Aptroot (4)*

Kalbionora Sodamuk, S.D. Leav. \& Lumbsch (1)

Malmidea Kalb, Rivas Plata \& Lumbsch (52)

Savoronala Ertz, Eb. Fisch., Killmann, Razafindr. \& Sérus (1)

Sprucidea M.Cáceres, Aptroot \& Lücking (4)

Zhurbenkoa Flakus, Etayo, Pérez-Ortega \& Rodr. Flakus (3)

Megalariaceae Hafellner

Catillochroma Kalb (2)

Megalaria Hafellner (ca. 30)*

Parmeliaceae Zenker

Alectoria Ach. (= Gowardia Halonen, Myllys, Velmala \& Hyvärinen) (9)

Allantoparmelia (Vain.) Essl. (3)

Anzia Stizenb. (34)

Arctoparmelia Hale (5)

Asahinea W.L. Culb. \& C.F. Culb. (2)

Austromelanelixia Divakar, A. Crespo \& Lumbsch (5) 
Austroparmelina A. Crespo, Divakar \& Elix (13)

Brodoa Goward (3)

Bryocaulon Kärnefelt (4)

Bryoria Brodo \& D. Hawksw. (ca. 52)

Bulbothrix Hale (62)

Canoparmelia Elix \& Hale (35)

Cetraria Ach. (= Allocetraria Kurok. \& M.J. Lai, = Cetrariella Kärnefelt \& Thell, = Usnocetraria M.J. Lai \& J.C. Wei, = Vulpicida Mattson \& M.J. Lai) (35)

Cetrelia W.L. Culb. \& C.F. Culb. (18)

Coelopogon Brusse \& Karnefelt (2)

Cornicularia (Schreb.) Ach. (1)

Dactylina Nyl. (2)

Davidgallowaya Aptroot (1)

Dolichousnea (Y. Ohmura) Articus (3)

Emodomelanelia Divakar \& A. Crespo (1)

Esslingeriana Hale \& M.J. Lai (1)

Eumitria Stirt. (13)

Evernia Ach. (10)

Everniopsis Nyl. (1)

Flavoparmelia Hale (32)

Flavopunctelia Hale (5)

Himantormia I.M. Lamb (2)

Hypogymnia (Nyl.) Nyl. (90)

Hypotrachyna (Vain.) Hale (262)

Imshaugia F.C. Mey. (1)

Letharia (Th. Fr.) Zahlbr. (9)

Lethariella (Motyka) Krog (11)

Masonhalea Kärnefelt (2)*

Melanelia Essl. (2)

Melanelixia O. Blanco, A. Crespo, Divakar, Essl., D. Hawksw. \& Lumbsch (11)

Melanohalea O. Blanco, A. Crespo, Divakar, Essl., D. Hawksw. \& Lumbsch (22)

Menegazzia A. Massal. (70)

Montanelia Divakar, A. Crespo, Wedin \& Essl. (5)

Myelochroa (Asahina) Elix \& Hale (30)

Neoprotoparmelia Garima Singh, Lumbsch \& I. Schmitt (14)

Nephromopsis Müll. Arg. (= Ahtiana Goward; = Arctocetraria Kärnefelt \& Thell; = Cetrariopsis Kurok.; = Flavocetraria Kärnefelt \& Thell; = Flavocetrariella D.D. Awasthi; = Kaernefeltia Thell \& Goward; = Tuckermanella Essl.; = Tuckermannopsis Gyeln.) (62)

Nesolechia A. Massal. (ca. 2)

Nipponoparmelia (Kurok.) K.H. Moon, Y. Ohmura \& Kashiw. (4)

Nodobryoria Common \& Brodo (3)

Notoparmelia A. Crespo, Ferencová \& Divakar (16)

Omphalodium Meyen \& Flot. (4)

Omphalora T.H. Nash \& Hafellner (1)

Oropogon Th. Fr. (42)

Pannoparmelia (Müll. Arg.) Darb. (5)

Parmelia Ach (43)

Parmelina Hale (10)

Parmelinella Elix \& Hale (8)

Parmeliopsis (Nyl.) Nyl. (3)

Parmotrema A. Massal. (= Crespoa (D. Hawksw.) Lendemer \& B.P. Hodk.) (255)

Parmotremopsis Elix \& Hale (2) 
Phacopsis Tul. (10)

Platismatia W.L. Culb. \& C.F. Culb. (11)

Pleurosticta Petr. (2)

Protoparmelia M. Choisy (11)

Protousnea (Motyka) Krog (8)

Pseudephebe M. Choisy (2)

Pseudevernia Zopf (4)

Pseudoparmelia Lynge (15)

Psiloparmelia Hale (13)

Punctelia Krog (48)

Relicina (Hale \& Kurok.) Hale (59)

Remototrachyna Divakar \& A. Crespo (19)

Raesaenenia D. Hawksw. (1)

Sulcaria Bystr. (5)

Usnea Dill. ex Adans. (355)

Xanthoparmelia (Vain.) Hale (822)

Pilocarpaceae Zahlbr.

Aquacidia Aptroot (3)*

Badimiella Malcolm \& Vězda (1)

Baflavia Lücking (1)

Bapalmuia Sérus. (22)

Barubria Vězda (2)

Brasilicia Lücking, Kalb \& Serus. (6)

Bryogomphus Lücking, W.R. Buck, Sérus. \& L.I. Ferraro (1)

Byssolecania Vain. (7)

Byssoloma Trevis. (60)

Calopadia Vězda (27)

Calopadiopsis Lücking \& R. Sant. (2)

Eugeniella Lücking, Sérus. \& Kalb (11)

Fellhanera Vězda (ca. 100)

Fellhaneropsis Sérus. \& Coppins (9)

Kantvilasia P.M. McCarthy, Elix \& Sérus. (1)

Lasioloma R. Sant. (9)

Leimonis R.C. Harris (2)

Loflammia Vězda (5)

Loflammiopsis Lücking \& Kalb (1)

Logilvia Vězda (1)

Micarea Fr. (102)

Podotara Malcolm \& Vězda (1)

Pseudocalopadia Lücking (1)

Roccellinastrum Follmann (7)

Schadonia Körb. (4)*

Septotrapelia Aptroot \& Chaves (4)

Sporopodiopsis Sérus. (2)

Sporopodium Mont. (24)

Szczawinskia A. Funk (5)

Tapellaria Müll. Arg. (23)

Tapellariopsis Lücking (1)

Psilolechiaceae S. Stenroos, Miądl. \& Lutzoni

Psilolechia A. Massal. (4) 
Psoraceae Zahlbr.

Brianaria S. Ekman \& M. Svensson (4)

Glyphopeltis Brusse (1)

Protoblastenia (Zahlbr.) J. Steiner (30)

Protomicarea Hafellner (2)

Psora Hoffm. (35)

Psorula Gotth. Schneid. (1)

Ramalinaceae C. Agardh*

Auriculora Kalb (1)

Bacidia De Not. (= Bacidiopsora Kalb) (230)*

Bacidina Vězda (12)

Badimia Vězda (20)

Bellicidia Kistenich, Timdal, Bendiksby \& Ekman (1)*

Biatora Fr. (= Myrionora R.C. Harris; = Ivanpisutia S.Y. Kondr., Lőkös \& Hur) (42)*

Bibbya J.H. Willis (10)*

Bilimbia De Not. (= Myxobilimbia Hafellner) (6)

Cenozosia A. Massal. (1)

Cliostomum Fr. (25)

Echidnocymbium Brusse (1)

Eschatogonia Trevis. (7)

Heppsora D.D. Awasthi \& K. Singh (1)

Jarmania Kantvilas (2)

Kiliasia Hafellner (9)*

Krogia Timdal (7)

Lecania A. Massal. (50)

Lueckingia Aptroot \& Umana (1)

Mycobilimbia Rehm (5)*

Myelorrhiza Verdon \& Elix (2)*

Niebla Rundel \& Bowler (23)

Parallopsora Kistenich, Timdal \& Bendiksby (3)*

Phyllopsora Müll. Arg. (= Crocynia (Ach.) A. Massal.) (75)*

Physcidia Tuck. (10)

Ramalina Ach. (230)

Rolfidium Moberg (3)

Scutula Tul. (=Karsteniomyces D. Hawksw.; = Libertiella Speg. \& Roum.) (43)*

Sporacestra A. Massal. (1)*

Stirtoniella D.J. Galloway, Hafellner \& Elix (1)

Thalloidima A. Massal. (17)*

Thamnolecania (Vain.) Gyeln. (1)

Tibellia Vězda \& Hafellner (1)

Toninia A. Massal. (=Arthrosporum A. Massal.) (85)*

Toniniopsis Frey (7)

Tylothallia P. James \& H. Kilias (3)*

Waynea Moberg (7)

Ramboldiaceae S. Stenroos, Miądl. \& Lutzoni

Ramboldia Kantvilas \& Elix (34)

Scoliciosporaceae Hafellner

Scoliciosporum A. Massal. (15) 


\section{Sphaerophoraceae Fr.}

Austropeltum Henssen, H. Döring \& Kantvilas (1)

Bunodophoron A. Massal. (25)

Calycidium Stirt. (2)

Leifidium Wedin (1)

Neophyllis F. Wilson (2)

Sphaerophorus Pers. (8)

\section{Tephromelataceae Hafellner}

Calvitimela Hafellner (11)

Mycoblastus Norman (10)

Tephromela M. Choisy (ca. 30)

Violella T. Sprib. (2)

Lecanorales genera incertae sedis

Catinaria Vain. (2)*

Compsocladium I.M. Lamb (2)*

Coronoplectrum Brusse (1)

Corticiruptor Wedin \& Hafellner (2)

Lichenosticta Zopf (5)

Myochroidea Printzen, T. Sprib. \& Tønsberg (4)*

Neopsoromopsis Gyeln. (1)

Nimisiostella Calat., Barreno \& O.E. Erikss. (1)

Psoromella Gyeln. (1)

Puttea S. Stenroos \& Huhtinen (4)

Ramalea Nyl. (4)

Tasmidella Kantvilas, Hafellner \& Elix (1)*

Umbilithecium Etayo (1)

Umushamyces Etayo (1)

Lecideales Vain.

Lecideaceae Chevall.

Amygdalaria Norman (11)

Bahianora Kalb (1)

Bellemerea Hafellner \& Cl. Roux (10)

Bryobilimbia Fryday (6)*

Catarrhospora Brusse (2)

Cecidonia Triebel \& Rambold (2)

Clauzadea Hafellner \& Bellem. (7)

Cryptodictyon A. Massal. (2)

Eremastrella Vogel (2)

Farnoldia Hertel (6)

Immersaria Rambold \& Pietschm. (8)

Koerberiella Stein (2)

Labyrintha Malcolm, Elix \& Owe-Larss. (1)

Lecidea Ach. (ca. 100)

Lecidoma Gotth. Schneid. \& Hertel (1)

Melanolecia Hertel (7)

Pachyphysis R.C. Harris \& Ladd (1)

Paraporpidia Rambold \& Pietschm. (3)

Poeltiaria Hertel (8)

Poeltidea Hertel \& Hafellner (3) 
Porpidia Körb. (51)

Porpidinia Timdal (1)

Pseudopannaria (B. de Lesd.) Zahlbr. (1)

Rhizolecia Hertel (1)

Romjularia Timdal (1)

Schizodiscus Brusse (1)

Stenhammarella Hertel (1)

Stephanocyclos Hertel (1)

Xenolecia Hertel (2)

Lopadiaceae Hafellner

Lopadium Körb. (10)

Leprocaulales Lendemer \& B.P. Hodk.

Leprocaulaceae Lendemer \& B.P. Hodk.

Halecania M. Mayrhofer (22)

Leprocaulon Nyl. (ca. 10)

Speerschneidera Trevis. (1)

Peltigerales W. Watson

Coccocarpiaceae Henssen ex Henssen

Coccocarpia Pers. (ca. 50)

Peltularia R. Sant. (4)

Spilonema Bornet (4)

\section{Collemataceae Zenker}

Blennothallia Trevis. (4)

Callome Otálora \& Wedin (1)

Collema F.H. Wigg. (ca. 35)

Enchylium (Ach.) Gray (11)

Lathagrium (Ach.) Gray (10)

Leptogium (Ach.) Gray (ca. 110)

Pseudoleptogium Müll. Arg. (1)

Rostania Trevis. $(3+4$ orphaned species)*

Scytinium (Ach.) Gray (49)

Koerberiaceae T. Sprib. \& Muggia

Henssenia Ertz, R.S. Poulsen \& Søchting (4)*

Koerberia A. Massal. (2)

Vestergrenopsis Gyeln. (2)

Massalongiaceae Wedin, P.M. Jørg. \& E. Wiklund.

Leptochidium M. Choisy (2)

Massalongia Körb. ( $2+6$ orphaned species)

Polychidium (Ach.) Gray (1)

Pannariaceae Tuck.

Austrella P.M. Jørg. (3)

Degelia Arv. \& D.J. Galloway (16)

Erioderma Feé (32)

Fuscoderma (D.J. Galloway \& P.M. Jørg.) P.M. Jørg. \& D.J. Galloway (5)

Fuscopannaria P.M. Jørg. (= Kroswia P.M. Jørg.) (58) 
Gibbosporina Elvebakk, S.G. Hong \& P.M. Jørg. (13)

Homothecium A. Massal. (4)

Joergensenia Passo, S. Stenroos \& Calvelo (1)

Leciophysma Th. Fr. (2)

Leightoniella Henssen (1)

Leioderma Nyl. (7)

Lepidocollema Vain. (22)

Leptogidium Nyl. (3)

Nebularia P.M. Jørg. (2)

Nevesia P.M.Jørg, L. Lindblom, Wedin \& S. Ekman (1)

Pannaria Del. ex Bory (ca. 40)

Parmeliella Müll. Arg. (ca. 40)

Pectenia P.M. Jørg. (4)

Physma A. Massal. (12)

Protopannaria (Gyeln.) P.M. Jørg. \& S. Ekman (7)

Psoroma Michaux (ca. 70)

Psoromaria Nyl. ex Nyl. (= Degeliella P.M. Jørg.) (2)

Psoromidium Stirt. (2)

Ramalodium Nyl. (6)

Siphulastrum Müll. Arg. (4)

Staurolemma Körb. (3)

Steineropsis T. Sprib. \& Muggia (1)

Peltigeraceae Dumort. (= Lobariaceae Chevall.; = Nephromataceae Wetm. ex J.C. David \& D. Hawksw.)*

Crocodia Link (5)

Dendriscosticta Moncada \& Lücking (5)

Lobaria (Schreb.) Hoffm. (ca. 60)

Lobariella Yoshim. (35)

Lobarina Nyl. ex Cromb. (15)

Nephroma Ach. (ca. 36)

Parmostictina Nyl. (15)

Peltigera Willd. (ca. 100)

Podostictina Clem. (5)

Pseudocyphellaria Vain. (ca. 100)

Ricasolia De Not. (15)

Solorina Ach. (ca. 10)

Sticta (Schreb.) Ach. (ca. 200)

Yarrumia D.J. Galloway (2)

Yoshimuriella Moncada \& Lücking (8)

Placynthiaceae Å.E. Dahl

Hertella Henssen (3)

Placynthiopsis Zahlbr. (1)

Placynthium (Ach.) Gray (ca. 20)

Vahliellaceae Wedin

Vahliella P.M. Jørg. (10)

Peltigerineae genus incertae sedis

Erinacellus T. Sprib., Muggia \& Tønsberg (2) 
Rhizocarpales Miądl. \& Lutzoni ex Miądl. \& Lutzoni ex Miadl. \& Lutzoni

Rhizocarpaceae M. Choisy \& Hafellner

Catolechia Flot. (1)

Epilichen Clem. (2)

Poeltinula Hafellner (2)

Rhizocarpon Ramond ex DC. (225)

Sporastatiales Lumbsch \& Leavitt*

Sporastatiaceae Bendiksby \& Timdal

Sporastatia A. Massal. (4)

Toensbergia Bendiksby \& Timdal (1)

Teloschistales D. Hawksw. \& O.E. Erikss.

Brigantiaeaceae Hafellner \& Bellem. (= Letrouitiaceae Bellem. \& Hafellner)*

Brigantiaea Trevis. (26)

Letrouitia Hafellner \& Bellem. (18)

Megalosporaceae Vězda ex Hafellner \& Bellem.

Megaloblastenia Sipman (2)

Megalospora Meyen (36)

Sipmaniella Kalb (1)

Teloschistaceae Zahlbr.

Amundsenia Søchting, Garrido-Ben., Arup \& Frödén (2)

Apatoplaca Poelt \& Hafellner (1)

Athallia Arup, Frödén \& Søchting (= ?Coppinsiella S. Y. Kondr. et al.; = ?Fominiella S. Y.

Kondr., Upreti \& Hur) (17)

Austroplaca Søchting, Frödén \& Arup (10)

Blastenia A. Massal. (11)

Brownliella S.Y. Kondr., Kärnefelt, Elix, A. Thell \& Hur (4)

Bryoplaca Søchting, Frödén \& Arup (3)

Calogaya Arup, Frödén \& Søchting (= Lazarenkoella S.Y. Kondr. et al.; = Seawardiella

S.Y. Kondr. et al.) (19)

Caloplaca Th. Fr. (351)

Catenarina Søchting, Søgaard, Arup, Elvebakk \& Elix (3)

Cephalophysis (Hertel) H. Kilias (1)

Cerothallia Arup, Frödén \& Søchting (4)

Charcotiana Søchting, Garrido-Ben. \& Arup (1)

Dijigiella S.Y. Kondr. \& L. Lőkös (2)

Dufourea Ach. (= Xanthodactylon P.A. Duvign.) (25)

Eilifdahlia S.Y. Kondr., Kärnefelt, Elix, A. Thell \& Hur (2)

Fauriea S.Y. Kondr., Lökös \& Hur (2)

Filsoniana S.Y. Kondr., Kärnefelt, Elix, A. Thell \& Hur (= Harusavskia S.Y. Kondr.; = Nevilleiella S.Y.Kondr. \& Hur; = Thelliana S.Y. Kondr. et al.) (9)

Flavoplaca Arup, Søchting \& Frödén (28)

Follmannia C.W. Dodge (2)

Franwilsia S.Y. Kondr., Kärnefelt, Elix, A. Thell \& Hur (3)

Gondwania Søchting, Frödén \& Arup (4)

Gyalolechia A. Massal. (= Hanstrassia S.Y. Kondr.; = Laundonia S. Y. Kondr., L. Lőkös \& Hur; = Lazarenkoiopsis S.Y. Kondr., L. Lőkös \& Hur; = Opeltia S.Y. Kondr. \& L. Lőkös; = Oxneriopsis S.Y. Kondr., D. Upreti \& Hur) (40)

Haloplaca Arup, Søchting \& Frödén (31) 
Hosseusiella S.Y. Kondr., L. Lőkös, Kärnefelt \& A. Thell (3)

Huneckia S.Y. Kondr., Elix, Kärnefelt, A. Thell \& Hur (2)

Ioplaca Poelt (2)

Jasonhuria S.Y. Kondr., Lőkös \& S.O. Oh (1)

Josefpoeltia S.Y. Kondr. \& Kärnefelt (3)

Kaernefia S.Y. Kondr., Elix, A. Thell \& Hur (3)

Leproplaca (Nyl.) Nyl. (7)

Loekoesia S.Y. Kondr., S.O. Oh \& Hur (1)

Marchantiana S.Y. Kondr., Kärnefelt, Elix, A. Thell \& Hur (= Streimanniella S.Y. Kondr. et al.) (5)

Olegblumia S.Y. Kondr., Lőkös \& Hur (1)

Orientophila Arup, Søchting \& Frödén (4)

Pachypeltis Søchting, Arup \& Frödén (4)

Parvoplaca Arup, Søchting \& Frödén (6)

Polycauliona Hue (= ? Tomnashia S.Y. Kondr. \& Hur) (18)

Pyrenodesmia A. Massal. (6)

Rehmaniella S.Y. Kondr. et al. (1)

Rufoplaca Arup, Søchting \& Frödén (6)

Rusavskia S.Y. Kondr. \& Kärnefelt (= ? Zeroviella S.Y. Kondr. \& J.-S. Hur) (19)

Scutaria Søchting, Arup \& Frödén (1)

Seirophora Poelt (ca. 8)

Shackletonia Søchting, Frödén \& Arup (5)

Sirenophila Søchting, Arup \& Frödén (= Elixjohnia S.Y. Kondr. \& Hur; = Tarasginia S.Y.

Kondr. et al.) (14)

Solitaria Arup, Søchting \& Frödén (1)

Squamulea Arup, Søchting \& Frödén (= Huriella S.Y. Kondr. \& D. Upreti) (8)

Stellarangia Frödén, Arup \& Søchting (3)

Tassiloa S.Y. Kondr., Kärnefelt, A. Thell, Elix \& Hur (2)

Teloschistes Norman (ca. 24)

Teloschistopsis Frödén, Søchting \& Arup (3)

Teuvoahtiana S.Y. Kondr. \& Hur (3)

Upretia S.Y. Kondr., A. Thell \& Hur

Usnochroma Søchting, Arup \& Frödén (2)

Variospora Arup, Søchting \& Frödén (16)

Villophora Søchting, Arup \& Frödén (= Tayloriella S.Y. Kondr. et al.; = Tayloriellina S.Y.

Kondr. et al.) (4)

Wetmoreana Arup, Søchting \& Frödén (3)

Xanthocarpia A. Massal. \& De Not. (12)

Xanthomendoza S.Y. Kondr. \& Kärnefelt (20)

Xanthopeltis R. Sant. (1)

Xanthoria (Fr.) Th. Fr. (10)

Yoshimuria S.Y. Kondr., Kärnefelt, Elix, A. Thell \& Hur (= Ikaeria S.Y. Kondr., D. Upreti \& Hur) (4)

Teloschistales genus incertae sedis

Malcolmiella Vězda (1)

Lecanoromycetidae familis incertae sedis

Biatorellaceae M. Choisy ex Hafellner \& Casares-Porcel

Biatorella De Not. (ca. 30)

Helocarpaceae Hafellner 
Helocarpon Fr. (3)

Pachyascaceae Poelt ex P.M. Kirk, P.F. Cannon \& J.C. David

Pachyascus Poelt \& Hertel (1)

Ostropomycetidae V. Reeb, Lutzoni \& Cl. Roux

Baeomycetales Lumbsch, Huhndorf \& Lutzoni. (= Arctomiales S. Stenroos, Miądl. \& Lutzoni; = Hymeneliales S. Stenroos, Miądl. \& Lutzoni; = Trapeliales B.P. Hodk. \& Lendemer)*

Arctomiaceae Th. Fr.

Arctomia Th. Fr. (14)

Gregorella Lumbsch (1)

Steinera Zahlbr. (14)*

Wawea Henssen \& Kantvilas (1)

Arthrorhaphidaceae Poelt \& Hafellner

Arthrorhaphis Th. Fr. (13)

Baeomycetaceae Dumort.

Ainoa Lumbsch \& I. Schmitt (2)

Anamylopsora Timdal (1)

Baeomyces Pers. (10)

Parainoa Resl \& T. Sprib. (1)

Phyllobaeis Gierl \& Kalb (6)

Cameroniaceae Kantvilas \& Lumbsch

Cameronia Kantvilas (2)

\section{Hymeneliaceae Körb.}

Hymenelia Kremp. (26)

Ionaspis Th. Fr. (7)

Tremolecia M. Choisy (6)

Protothelenellaceae Vězda, H. Mayrhofer \& Poelt (= Thrombiaceae Poelt \& Vězda ex J.C. David \& D. Hawksw.)*

Mycowinteria Sherwood (3)

Protothelenella Räsenen (11)

Thrombium Wallr. (5)

Trapeliaceae M. Choisy ex Hertel

Amylora Rambold (1)

Aspiciliopsis (Müll. Arg.) M. Choisy (1)

Coppinsia Lumbsch \& Heibel (1)

Ducatina Ertz \& Søchting (1)*

Lignoscripta B.D. Ryan (1)

Orceolina Hertel (2)

Placopsis (Nyl.) Linds. (ca. 60)

Placynthiella Elenkin (7)

Rimularia Nyl. (4)*

Sarea Fr. (2)

Trapelia M. Choisy (24)

Trapeliopsis Hertel \& Gotth. Schneid. (20) 
Xylographaceae Tuck.

Lambiella Hertel (12)

Lithographa Nyl. (10)

Ptychographa Nyl. (1)

Xylographa (Fr.) Fr. (2)

Graphidales Bessey

Diploschistaceae Zahlbr.

Acanthothecis Clem. (ca. 60)

Acanthotrema Frisch (5)

Aggregatorygma M. Cáceres, Aptroot \& Lücking (1)

Ampliotrema Kalb ex Kalb (12)

Asteristion Leight. (9)

Austrotrema I. Medeiros, Lücking \& Lumbsch (3)

Borinquenotrema Merc.-Díaz, Lücking \& Parnmen (1)

Byssotrema M. Cáceres (1)

Carbacanthographis Staiger \& Kalb (28)

Compositrema Rivas Plata, Lücking \& Lumbsch (4)

Corticorygma M. Cáceres, S.C. Feuerst., Aptroot \& Lücking (1)

Diploschistes Norman (33)

Fibrillithecis A. Frisch (15)

Gintarasia Kraichak, Lücking \& Lumbsch (5)

Glaucotrema Rivas Plata \& Lumbsch (5)

Gyrotrema A. Frisch (6)

Heiomasia Nelsen, Lücking \& Rivas Plata (3)

Melanotopelia Lumbsch \& Mangold (4)

Melanotrema A. Frisch (12)

Myriochapsa M. Cáceres, Lücking \& Lumbsch (3)

Myriotrema Fée (55)

Nadvornikia Tibell (5)

Nitidochapsa Parnmen, Lücking \& Lumbsch (5)

Ocellularia G. Mey. (ca. 400)

Phaeographopsis Sipman (3)

Pseudoramonia Kantvilas \& Vězda (4)

Redingeria A. Frisch (8)

Reimnitzia Kalb (1)

Rhabdodiscus Vain. (35)

Sanguinotrema Lücking (1)

Schizotrema Mangold \& Lumbsch (6)

Stegobolus Mont. (16)

Topeliopsis Kantvilas \& Vězda (20)

Wirthiotrema Rivas Plata, Kalb, Frisch \& Lumbsch (5)

Xalocoa Kraichak, Lücking \& Lumbsch (1)

Fissurinaceae (Rivas Plata, Lücking \& Lumbsch) B.P. Hodk.

Clandestinotrema Rivas Plata, Lücking \& Lumbsch (17)

Cruentotrema Rivas Plata, Papong, Lumbsch \& Lücking (6)

Dyplolabia A. Massal. (5)

Enigmotrema Lücking (1)

Fissurina Fée (ca. 155)

Pycnotrema Rivas Plata \& Lücking (2) 
Gomphillaceae Walt. Watson

Actinoplaca Müll. Arg. (2)

Aderkomyces Bat. (30)

Aplanocalenia Lücking, Sérus. \& Vězda (1)

Arthotheliopsis Vain. (5)

Asterothyrium Müll. Arg. (32)

Aulaxina Fée (14)

Calenia Müll. Arg. (30)

Caleniopsis Vězda \& Poelt (2)

Corticifraga D. Hawksw. \& R. Sant. (7)*

Diploschistella Vain. (4)

Echinoplaca Fée (40)

Ferraroa Lücking, Sérus. \& Vězda (1)

Gomphillus Nyl. (6)

Gyalectidium Müll. Arg. (52)

Gyalidea Lettau (50)

Gyalideopsis Vězda (91)

Hippocrepidea Sérus. (1)

Jamesiella Lücking, Sérus. \& Vězda (4)

Lithogyalideopsis Lücking, Sérus. \& Vězda (4)

Paratricharia Lücking (1)

Paragyalideopsis Etayo (4)

Phyllogyalidea Lücking \& Aptroot (2)

Psorotheciopsis Rehm (7)

Rolueckia Papong, Thammath. \& Boonpr. (2)

Taitaia Suija, Kaasalainen, Kirika \& Rikkinen (1)*

Tricharia Fée (ca. 30)

Graphidaceae Dumort.

Allographa Chevall. (183)*

Amazonotrema Kalb \& Lücking (1)

Anomalographis Kalb (2)

Anomomorpha Nyl. ex Hue (8)

Creographa A. Massal. (1)

Cryptoschizotrema Aptroot, Lücking \& M. Cáceres (1)

Diaphorographis A.W. Archer \& Kalb (2)

Diorygma Eschw. (74)

Flegographa A. Massal. (1)

Glyphis Ach. (7)

Graphis Adans. (ca. 275)

Halegrapha Rivas Plata \& Lücking (9)

Hemithecium Trevis. (ca. 50)

Kalbographa Lücking (5)

Leiorreuma Eschw. (18)

Malmographina M. Cáceres, Rivas Plata \& Lücking (1)

Mangoldia Lücking, Parnmen \& Lumbsch (2)

Pallidogramme Staiger, Kalb \& Lücking (13)

Phaeographis Müll. Arg. (ca. 180)

Platygramme Fée (30)

Platythecium Staiger (27)

Pliariona A. Massal. (= Phaeographina Müll. Arg.) (1)

Polistroma Clemente (1) 
Pseudochapsa Parnmen, Lücking \& Lumbsch (18)

Pseudotopeliopsis Parnmen, Lücking \& Lumbsch (4)

Sarcographa Fée (37)

Sarcographina Müll. Arg. (6)

Schistophoron Stirt. (5)

Thalloloma Trevis. (20)

Thecaria Fée (4)

Thecographa A. Massal. (3)

Redonographaceae (Lücking, Tehler \& Lumbsch) Lumbsch (Bas.: Redonographoideae Lücking, Tehler \& Lumbsch, Am. J. Bot. 100: 846 2013)

Gymnographopsis C.W. Dodge (2)

Redonographa Lücking, Tehler \& Lumbsch (4)

Thelotremataceae Stizenb.

Astrochapsa Parnmen, Lücking \& Lumbsch (28)

Chapsa A. Massal. (ca. 60)

Chroodiscus (Müll. Arg.) Müll. Arg. (17)

Crutarndina Parnmen, Lücking \& Lumbsch (1)

Leucodecton A. Massal. (31)

Paratopeliopsis Merc.-Díaz, Lücking \& Parnmen (1)

Thelotrema Ach. (= Tremotylium Nyl.) (106)

Gyalectales Henssen ex D. Hawksw. \& O.E. Erikss.

Coenogoniaceae (Fr.) Stizenb.

Coenogonium Ehrenb. ex Nees (ca. 91)

Gyalectaceae (A. Massal.) Stizenb.

Gyalecta Ach. (= Cryptolechia A. Massal.) (50)

Ramonia Stizenb. (24)

Semigyalecta Vain. (1)

Phlyctidaceae Poelt \& Vězda ex J.C. David \& D. Hawksw.

Phlyctis (Wallr.) Flot. (20)

Psathyrophlyctis Brusse (1)

Sagiolechiaceae Baloch, Lücking, Lumbsch \& Wedin

Rhexophiale Th. Fr. (1)

Sagiolechia A. Massal. (3)

Trichotheliaceae Bitter \& F. Schill. (= Porinaceae Walt. Watson; = Porinaceae Rchb.)

Clathroporina Müll. Arg. (ca. 25)

Flabelloporina Sobreira, M. Cáceres \& Lücking (1)

Myeloconis P.M. McCarthy \& Elix (4)

Porina Müll. Arg. (ca. 145)

Pseudosagedia (Müll. Arg.) Choisy (80)

Segestria Fr. (70)

Trichothelium Müll. Arg. (40)

Ostropales Nannf.

Odontotremataceae D. Hawksw. \& Sherwood

Claviradulomyces P.R. Johnst., D.C. Park, H.C. Evans, R.W. Barreto \& D.J. Soares (1) 
Coccomycetella Höhn. (2)

Odontotrema Nyl. (7)

Odontura Clem. (1)

Paschelkiella Sherwood (1)

Potriphila Döbbeler (3)

Rogellia Döbbeler (2)

Stromatothecia D.E. Shaw \& D. Hawksw. (1)

Tryblis Clem. (2)

Xerotrema Sherwood \& Coppins (2)

Phaneromycetaceae Gamundí \& Spinedi

Phaneromyces Speg. \& Har. ex Speg. (2)

Spirographaceae Flakus, Etayo \& Miadlikowska

Spirographa Zahlbr. (5)

\section{Stictidaceae Fr.}

Absconditella Vězda (12)

Acarosporina Sherwood (5)

Biostictis Petr. (5)

Carestiella Bres. (1)

Conotremopsis Vězda (1)

Cryptodiscus Corda (= Lettauia D. Hawksw. \& R. Sant.) (9)*

Cyanodermella O.E. Erikss. (2)

Delpontia Penz. \& Sacc. (1)

Dendroseptoria Alcalde (3)

Fitzroyomyces Crous (1)

Geisleria Nitschke (1)

Glomerobolus Kohlm. \& Volkm.-Kohlm. (1)

Ingvariella Guderley \& Lumbsch (1)

Karstenia Fr. (10)

Lillicoa Sherwood (4)

Nanostictis M.S Christ. (ca. 8)

Neofitzroyomyces Crous (1)

Ostropa Fr. (1)

Propoliopsis Rehm (1)

Robergea Desm. (8)

Schizoxylon Pers. (ca. 35)

Sphaeropezia Sacc. (= Lethariicola Grummann) (19)

Stictis Pers. (4)

Stictophacidium Rehm (3)

Thelopsis Nyl. (9)

Topelia P.M. Jørg. \& Vězda (6)

Trinathotrema Lücking, Rivas Plata \& Mangold (3)

Xyloschistes Vain. ex Zahlbr. (1)

Ostropales genera incertae sedis

Aabaarnia Diederich (1)

Biazrovia Zhurb. \& Etayo (1)

Elongaticonidia W.J. Li, E. Camporesi \& K.D. Hyde (1)

Epicladonia D. Hawksw. sensu lato (2)*

Normanogalla Diederich (1) 
Paralethariicola Calat., Etayo \& Diederich (1)

Pertusariales M. Choisy ex D. Hawksw. \& O.E. Erikss.

Agyriaceae Corda (= Miltideaceae Hafellner)*

Agyrium Fr. (3)

Miltidea Stirt. (1)

Coccotremataceae Henssen ex J.C. David \& D. Hawksw.

Coccotrema Müll. Arg. (16)

Gyalectaria I. Schmitt, Kalb \& Lumbsch (3)

Parasiphula Kantvilas \& Grube (7)

\section{Icmadophilaceae Triebel}

Dibaeis Clem. (ca. 14)

Endocena Cromb. (=Chirleja Lendemer \& B.P. Hodk.) (2)

Icmadophila Trevis. (4)

Pseudobaeomyces M. Satì (1)

Siphula Fr. (26)

Siphulella Kantvilas, Elix \& P. James (1)

Thamnolia Ach. ex Schaerer (4)

Megasporaceae Lumbsch

Aspicilia A. Massal. (ca. 200)

Circinaria Link (ca. 40)

Lobothallia (Clauzade \& Cl. Roux) Hafellner (12)

Megaspora (Clauzade \& Cl. Roux) Hafellner \& V. Wirth (4)

Sagedia Ach. (ca. 30)

Teuvoa Sohrabi \& S. Leavitt (5)

Microcaliciaceae Tibell*

Microcalicium Vain. (4)

Ochrolechiaceae R.C. Harris ex Lumbsch \& I. Schmitt

Ochrolechia A. Massal. (60)

Pertusariaceae Körb. ex Körb.

Loxosporopsis Henssen (1)

Pertusaria DC. (ca. 400)*

Thamnochrolechia Aptroot \& Sipman (1)

Varicellariaceae B.P. Hodk., R.C. Harris \& Lendemer ex Lumbsch \& Leavitt Varicellaria Nyl. (8)*

Variolariaceae Fée ex Zenker

Lepra Scop. (= Marfloraea S.Y. Kondr., Lökös \& Hur) (94)

Sarrameanales B.P. Hodk. \& Lendemer

Sarrameanaceae Hafellner

Loxospora A. Massal. (13)

Sarrameana Vězda \& P. James (1)

Schaereriales Lumbsch \& Leavitt 
Schaereriaceae M. Choisy ex Hafellner

Schaereria Körb. (= Hafellnera Houmeau \& Cl. Roux) (16)

Thelenellales Lumbsch \& Leavitt

Thelenellaceae O.E. Erikss. ex H. Mayrhofer

Aspidothelium Vain. (17)

Chromatochlamys Trevis. (3)

Thelenella Nyl. (30)

Ostropomycetidae family incertae sedis

Epigloeaceae Zahlbr.

Epigloea Zukal (12)

Ostropomycetidae genera incertae sedis

Amphorothecium P.M. McCarthy, Kantvilas \& Elix (1)

Anzina Scheid. (1)

Aspilidea Hafellner (1)

Bachmanniomyces D. Hawksw. (8)

Dictyocatenulata Finley \& E.F. Morris (1)

Malvinia Döbbeler (1)

Pleiopatella Rehm (1)

Umbilicariomycetidae Bendiksby, Hestmark \& Timdal

Umbilicariales J.C. Wei \& Q.M. Zhou

Elixiaceae Lumbsch

Elixia Lumbsch (2)

Meridianelia Kantvilas \& Lumbsch (1)

Fuscideaceae Hafellner

Fuscidea V. Wirth \& Vězda (ca. 40)

Hueidea Kantvilas \& P.M. McCarthy (1)

Maronea A. Massal. (13)

Orphniospora Körb. (4)

Ophioparmaceae R.W. Rogers \& Hafellner

Boreoplaca Timdal (1)

Hypocenomyce M. Choisy (3)

Ophioparma Norman (9)

Ropalosporaceae Hafellner

Ropalospora A. Massal. (9)

Umbilicariaceae Chevall.

Fulgidea Bendiksby \& Timdal (2)

Umbilicaria Hoffm. (= Lasallia Mérat) (ca. 90) *

Xylopsora Bendiksby \& Timdal (2)

Lecanoromycetes order incertae sedis

Micropeltidales X.Y. Zeng, H.X. Wu \& K.D. Hyde

Micropeltidaceae Clem. \& Shear*

Anariste Syd. (1)

Caudella Syd. \& P. Syd. (2) 
Cyclopeltella Petr. (1)

Dictyopeltella Bat. \& I.H. Lima (2)

Haplopeltheca Bat., J.L. Bezerra \& Cavalc. (1)

Micropeltis Mont. (ca. 110)

Neopeltella Petr. (1)

Scolecopeltidium F. Stevens \& Manter (ca. 80)

Stomiopeltis Theiss. (25)

Stomiopeltopsis Bat. \& Cavalc. (2)

Stomiotheca Bat. (2)

\section{Turquoiseomycetales Crous}

Turquoiseomycetaceae Crous

Turquoiseomyces Crous (1)

Lecanoromycetes genera incertae sedis

Argopsis Th. Fr. (1)

Ascographa Velen. (1)

Bartlettiella D.J. Galloway \& P.M. Jørg. (1)

Bouvetiella Øvstedal (1)

Buelliastrum Zahlbr. (1)

Haploloma Trevis. (1)

Hosseusia Gyeln. (3)

Korfiomyces Iturr. \& D. Hawksw. (1)

Maronella M. Steiger (1)

Notolecidea Hertel (1)

Petractis Fr. (3)

Piccolia A. Massal. (ca. 7)

Ravenelula Speg. (1)

Robincola Velen. (1)

Roburnia Velen. (1)

Leotiomycetes O.E. Erikss. \& Winka

Chaetomellales Crous \& Denman

Chaetomellaceae Baral, P.R. Johnst. \& Rossman

Chaetomella Fuckel (26)

Pilidium Kunze (23)

Sphaerographium Sacc. (23)

Synchaetomella Decock \& Seifert (3)

Cyttariales Luttr. ex Gamundí

Cyttariaceae Speg.

Cyttaria Berk. (13)

Helotiales Nannf. ex Korf \& Lizoň

Amorphothecaceae Parbery*

Amorphotheca Parbery (21 fide Baral 2016)

Arachnopezizaceae Hosoya, J.G. Han \& Baral

Arachnopeziza Fuckel (35)

Arachnoscypha Boud. (1)

Austropezia Spooner (1)

Eriopezia (Sacc.) Rehm (21) 
Parachnopeziza Korf (8)

Ascocorticiaceae J. Schrot

Ascocorticiellum Julich \& B. de Vries (1)

Ascocorticium Bref. (2)

Ascosorus P. Henn. \& Ruhland (1)

Ascodichaenaceae D. Hawksw. \& Sherwood

Ascodichaena Butin (2)

Delpinoina Kuntze (1)

Bloxamiaceae Locq.

Bloxamia Berk. \& Broome (10)

Bryoglossaceae Ekanayaka \& Hyde

Bryoclaviculus L. Ludw., P.R. Johnst. \& Steel (1)

Bryoglossum Redhead (2)

"Crocicreas" multicuspidatum (1)

Neocudoniella S. Imai (3)

"Roseodiscus" formosus (1)

Calloriaceae Marchand

Aivenia Svrcek (4)

Calloria Fr. (28)

Chaetonaevia Arx (3)

Diplonaevia Sacc. (33)

Duebenia Fr. (6)

Eupropolella Hohn. (8)

Hyalacrotes (Korf \& L.M. Kohn) Raitv. (5)

Iridinea Velen. (2)

Laetinaevia Nannf. (19)

Loricella Velen. (6)

Micropodia Boud. (15)

Naeviella (Rehm) Clem. (3)

Naeviopsis B. Hein (14)

Ploettnera Henn. (6)

Cenangiaceae Rehm (= Hemiphacidiaceae Korf)*

Cenangiopsis Rehm (9)

Cenangium Fr. (47)

Chlorencoelia J.R. Dixon (4)

Crumenulopsis J.W. Groves (3)

Encoelia (Fr.) P. Karst. (38)

Fabrella Kirschst. (1)

Heyderia Link (4)

Rhabdocline Syd. (7)

Sarcotrochila Hohn. (7)

Trochila Fr. (33)

Velutarina Korf (3)

Chlorociboriaceae Baral \& P.R. Johnst.*

Chlorociboria Seaver ex C.S. Ramamurthi, Korf \& L.R. Batra (23) 
Chlorospleniaceae Ekanayaka \& Hyde

Chlorosplenium Fr. (17)

Chrysodiscaceae Baral \& Haelew.*

Chrysodisca Baral, Polhorský \& G. Marson (1)

\section{Cordieritidaceae Sacc.}

Ameghiniella Speg. (2)

Annabella Fryar, Haelew. \& D.E.A. Catches. (1)

Austrocenangium Gamundí (2)

Cordierites Mont. (5)

Diplocarpa Massee (1)

Diplolaeviopsis Giralt \& D. Hawksw. (3)

"Encoelia" fimbriata Spooner \& Trigaux (1)

"Encoelia" heteromera (Mont.) Nannf. (1)

Ionomidotis E.J. Durand ex Thaxt. (4)

Llimoniella Hafellner \& Nav.-Ros. (21)

Macroskyttea Etayo, Flakus, Suija \& Kukwa (1)

Midotiopsis Henn. (2)

Rhymbocarpus Zopf (10)

Sabahriopsis Crous \& M.J. Wingf. (1)

Skyttea Sherwood, D. Hawksw. \& Coppins (30)

Skyttella D. Hawksw. \& R. Sant. (2)

Thamnogalla D. Hawksw. (1)

Unguiculariopsis Rehm (29)

\section{Dermateaceae Fr.}

Coleophoma Hohn. (= Parafabraea Chen Chen et al.) (30)*

Corniculariella P. Karst. (3)

Dermea Fr. (24)

Gelatinoamylaria Prasher \& R. Sharma (1)*

Neodermea W.J. Li, D.J. Bhat \& K.D. Hyde (1)

Neofabraea H.S. Jacks. (9)

Neogloeosporidina W.J. Li, Camporesi \& K.D. Hyde (1)

Pezicula Tul. \& C. Tul. (92)

Phlyctema Desm. (60)

Pseudofabraea Chen Chen, Verkley \& Crous (1)

Rhizodermea Verkley \& Zijlstra (1)

Schizothyrioma Hohn (4)

Verkleyomyces Y. Marın \& Crous (1)

Xenochalara M.J. Wingf. \& Crous (1)

Discinellaceae Ekanayaka \& K.D. Hyde*

Articulospora Ingold (6)

Cladochasiella Marvanova (1)

Discinella Boud. (13)

Fontanospora Dyko (4)

Gyoerffyella Kol (10)

Lemonniera De Wild. (8)

Margaritispora Ingold (2)

Naevala B. Hein (5)

Pezoloma Clem. (14) 
Pseudopezicula Korf (2)

Tetrachaetum Ingold (1)

Varicosporium W. Kegel (9)

\section{Drepanopezizaceae Baral*}

Blumeriella Arx (7)

Diplocarpon F.A. Wolf (7)

Drepanopeziza (Kleb.) Hohn. (5)

Felisbertia Viegas (7)

Leptotrochila P. Karst. (15)

Pseudopeziza Fuckel (2)

Spilopodia Boud. (4)

Spilopodiella E. Mull. (1)

Erysiphaceae Tul. \& C. Tul.

Arthrocladiella Vassilkov (1)

Blumeria Golovin ex Speer (1)

Brasiliomyces Viegas (6)

Bulbomicroidium Marm., S. Takam. \&U. Braun (1)*

Caespitotheca S. Takam. \& U. Braun (1)

Cystotheca Berk. \& Curtis (9)

Erysiphe DC. (478)

Golovinomyces (U. Braun) Heluta (66)

Leveillula G. Arnaud (49)

Microidium (To-anun \& S. Takam.) To-anun \& S. Takam. (3)

Neoerysiphe U. Braun (15)

Parauncinula S. Takam. \& U. Braun (4)

Phyllactinia Lev. (117)

Pleochaeta Sacc. \& Speg. (5)

Podosphaera Kunze (124)

Pseudoidium Y.S. Paul \& J.N. Kapoor (80)

Queirozia Viegas \& Cardoso (1)

Sawadaea Miyabe (10)

Takamatsuella U. Braun \& A. Shi (1)

Typhulochaeta Ito \& Hara (4)

\section{Gelatinodiscaceae S.E. Carp}

Ascocoryne J.W. Groves \& D.E. Wilson (8)

Ascotremella Seaver (2)

Chloroscypha Seaver (14)

Didymocoryne Sacc. \& Trotter (1)

Neobulgaria Petr. (11)

Ombrophila Fr. (11)

Phaeangellina Dennis (1)

Skyathea Spooner \& Dennis (1)

Xerombrophila Baral (1)

\section{Godroniaceae Baral}

Ascocalyx Naumov (4)

Atropellis Zeller \& Goodd. (4)

Godronia Moug. \& Lev. (30)

Gremmeniella M. Morelet (3) 
Grovesiella M. Morelet (2)*

\section{Helotiaceae Rehm}

Ascoconidium Seaver (3)

Bisporella Sacc. (19)

Bryoscyphus Spooner (19)

Calycella (Sacc.) Sacc. (1)

Cudoniella Sacc. (31)

Cyathicula De Not. (30)

Dicephalospora Spooner (4)

Dimorphospora Tubaki (1)

Discorehmia Kirschst. (5)

Eubelonis Hohn. (2)

Filosporella Nawawi (6)

Geniculospora Sv. Nilsson ex Marvanová \& Sv. Nilsson (2)

Glarea Bills \& Palaez (2)

Gloeotinia M. Wilson, Noble \& E.G. Gray (2)

Graddonia Dennis (7)

Gremmenia Korf (4)

Helicodendron Peyronel (3)

Hymenoscyphus Gray (170)

Hymenotorrendiella P.R. Johnst., Baral \& R. Galán (9)

Muscicola Velen. (1)

Mycofalcella Marvanová, Om-Kalth. \& J. Webster (2)

Mytilodiscus Kropp \& S.E. Carp. (1)

Neocrinula Crous (2)

Phaeohelotium Kanouse (41)

Pithyella Boud. (8)

Pseudohelotium Fuckel (50)

Pseudoniptera Velen. (25)

Roesleria Thüm. \& Pass. (4)*

Scytalidium Pesante (30)

Symphyosirinia E.A. Ellis (6)

Tatraea Svrcek (2)

Tricladium Ingold (25)

Xylogramma Wallr. (18)

\section{Heterosphaeriaceae Rehm}

Heterosphaeria Grev. (7)

\section{Hyaloscyphaceae Nannf.*}

Aeruginoscyphus Dougoud (7)*

Ambrodiscus S.E. Carp. (1)

Amicodisca Svrcek (6)

Arbusculina Marvanova \& Descals (3)

Asperopilum Spooner (1)

"Chalara" longipes (Preuss) Cooke (1)

Clathrosphaerina Beverw. (2)

Crucellisporiopsis Nag Raj (3)

Dematioscypha Svrcek (4)

Dimorphotricha Spooner (1)

Echinula Graddon (1) 
Endoscypha Syd. (1)

Fuscolachnum J.H. Haines (7)

Gamarada D.J. Midgley \& Tran-Dinh (1)

Graddonidiscus Raitv. \& R. Galan (3)

Grahamiella Spooner (3)

Haplographium Berk. \& Broome (15)

Hegermila Raitv. (4)

Hyalopeziza Fuckel (15)

Hyaloscypha Boud. (45)

Hyphodiscus Kirschst. (16)

Hyphopeziza J.G. Han, Hosoya \& H.D. Shin (1)

Incrupila Raitv. (10)

Meliniomyces Hambl. \& Sigler (3)

Mycoarthris Marvanova \& P.J. Fisher (1)

Olla Velen. (2)

Polaroscyphus Huhtinen (1)

Proprioscypha Spooner (1)

Protounguicularia Raitv. \& Galan (10)

Pseudaegerita J.L. Crane \& Schokn. (7)*

Psilocistella Svrcek (10)

Rhizoscyphus W.Y. Zhuang \& Korf (1)

Scolecolachnum Guatim., R.W. Barreto \& Crous (2)*

Thindiomyces Arendh. \& R. Sharma (1)

Unguiculariella K.S. Thind \& R. Sharma (1)

Unguiculella Hohn (17)

Venturiocistella Raitv (7)

Lachnaceae (Nannf.) Raitv.

Albotricha Raitv. (19)

Belonidium Mont. \& Dur. (1)

Brunnipila Baral (10)

Capitotricha (Raitv.) Baral (10)

Dasyscyphella Tranzschel (1)

Erioscyphella Kirschst. (10)

Incrucipulum Baral (6)

Lachnellula P. Karst. (40)

Lachnopsis Guatim., R.W. Barreto \& Crous (2)*

Lachnum Retz. (50)

Lasiobelonium Ellis \& Everh. (20)

Neodasyscypha Sukova \& Spooner (2)

Perrotia Boud. (19)

Proliferodiscus J.H. Haines \& Dumont (8)

Solenopezia Sacc. (7)

Trichopeziza Fuckel (30)

Tubolachnum Velen (2)

Velebitea I. Kušan, Matočec \& Jadan (1)

Leptodontidiaceae Hern.-Restr., Crous \& Gené

Leptodontidium de Hoog. (11)

Loramycetaceae Dennis ex Digby \& Goos

Loramyces W. Weston (2) 
Obtectodiscus E. Müll., Petrini \& Samuels (2)

Mitrulaceae Rchb.

Mitrula Fr. (16)

\section{Mollisiaceae Rehm}

Barrenia E. Walsh \& N. Zhang (2)*

Bulbomollisia Graddon (1)

Cheirospora Moug. \& Fr. (2)*

Cystodendron Bubak (2)

Discocurtisia Nannf. (12)

Fuscosclera Hern.-Restr., J. Mena \& Gené (1)*

Mollisia (Fr.) P. Karst. (130)

Neotapesia E. Mull. \& Hutter (3)

Niptera Fr. (10)

Nipterella Starback ex Dennis (2)

Phialocephala W.B. Kendr. (37)*

Pseudonaevia Dennis \& Spooner (2)

Sarconiptera Raitv. (1)

Scutobelonium Graddon (1)

Scutomollisia Nannf. (14)

Tapesia (Pers.) Fuckel (110)*

Trimmatostroma Corda (30)

Variocladium Descals \& Marvanova (1)

Myxotrichaceae Currah*

Byssoascus Arx (1)

"Malbranchea" flavorosea Sigler \& J.W. Carmich. (1)

Myxotrichum Kunze (17)

Oidiodendron Robak (26)

Neolauriomycetaceae Crous*

Exochalara W. Gams \& Hol.-Jech. (3)

Lareunionomyces Crous \& M.J. Wingf. (4)

Neolauriomyces Crous (1)

Pezizellaceae Velen.

Allophylaria (P. Karst.) P. Karst. (6)

Antinoa Velen. (8)

Calycellina Hohn (45)

Calycina Nees ex Gray (30)

Chalara (Corda) Rabenh. (99)

Ciliolarina Svrcek (1)

Curviclavula G. Delgado, F.A. Fernández \& A.N. Mill. (1)

Hamatocanthoscypha Svrcek (3)

Hyalodendriella Crous (1)

Micropeziza Fuckel (12)

Microscypha Syd. \& P. Syd. (6)

Mollisina Hohn. ex Weese (11)

Mollisinopsis Arendh. \& R. Sharma (3)

Moserella Poder \& Scheuer (1)

Phaeoscypha Spooner (1) 
Phialina Höhn. (6)*

Poculinia Spooner (1)

Psilachnum Hohn. (28)

Rodwayella Spooner (3)

Scleropezicula Verkley (6)

Velutaria Fuckel (1)

Xenopolyscytalum Crous (1)

Zymochalara Guatim., R.W. Barreto \& Crous (2)*

Ploettnerulaceae Kirschst.

Cadophora Lagerb. \& Melin (15)

Collembolispora Marvanova \& Pascoal (2)

Cylindrosporium Grev. (168)*

Dennisiodiscus Svrcek (10)

Lasiomollisia Raitv. \& Vesterh. (1)

Mastigosporium Riess (4)

Mycochaetophora Hara \& Ogawa (2)

Nothophacidium J. Reid \& Cain (1)

Oculimacula Crous \& W. Gams (6)

Pirottaea Sacc. (28)

Pyrenopeziza Fuckel (3)

Rhynchosporium Heinsen ex A.B. Frank (5)

Rutstroemiaceae Holst-Jensen, L.M. Kohn \& T. Schumach.*

Bicornispora Checa, Barrasa, M.N. Blanco \& A.T. Martínez (2)

Dencoeliopsis Korf (2)

Lambertella Hohn. (6)

Lanzia Sacc. (1)

Pseudolanzia Baral \& G. Marson (1)*

Rutstroemia P. Karst. (100)

Torrendiella Boud. \& Torrend (3)

Sclerotiniaceae Whetzel ex Whetzel

Amphobotrys Hennebert (1)

Botrytis P. Micheli ex Pers. (3)

Ciboria Fuckel (21)

Ciborinia Whetzel (16)

Cristulariella Hohn. (5)

Cudoniopsis Speg. (1)

Dumontinia L.M. Kohn (5)

Elliottinia L.M. Kohn (1)

Grovesinia M.N. Cline, J.L. Crane \& S.D. Cline (2)

Haradamyces Masuya, Kusunoki, Kosaka \& Aikawa (1)

Kohninia Holst-Jensen, Vrålstad \& T. Schumach. (1)

Martininia Dumont \& Korf (1)

Monilinia Honey (30)

Mycopappus Redhead \& G.P. White (3)*

Myrioconium Syd. \& P. Syd. (10)

Myriosclerotinia N.F. Buchw. (10)

Ovulinia Weiss (9)

Phaeosclerotinia Hori (1)

Piceomphale Svrcek (1) 
Pseudociboria Kanouse (1)

Pycnopeziza W.L. White \& Whetzel (5)

Redheadia Y. Suto \& Suyama (1)

Sclerencoelia Pärtel \& Baral (3)*

Scleromitrula S. Imai (6)

Sclerotinia Fuckel (15)

Sclerotium Tode (100)

Seaverinia Whetzel (2)

Septotinia Whetzel ex J.W. Groves \& M.E. Elliott (2)

Streptotinia Whetzel (3)

Stromatinia (Boud.) Boud. (15)

Valdensia Peyronel (3)

Vibrisseaceae Korf

Acephala Grunig \& T.N. Sieber (2)

Chlorovibrissea L.M. Kohn (4)

Leucovibrissea (A. Sanchez) Korf (1)

Pocillum De Not. (1)

Vibrissea Fr (34)

Helotiales genera incertae sedis

Acidea Hujslova \& M. Kolarık (1)

Acidomelania E. Walsh \& N. Zhang (1)

Algincola Velen. (1)

Amylocarpus Curr. (1)

Angelina Fr. (1)

Apiculospora Wijayaw., Camporesi, A.J.L. Phillips \& K.D. Hyde (1)

Aquadiscula Shearer \& J.L. Crane (2)

Aquapoterium Raja \& Shearer (1)

Ascluella DiCosmo, Nag Raj \& W.B. Kendr. (1)

Ascoclavulina Otani (8)

Banksiamyces G. Beaton (4)

Belonioscyphella Hohn. (4)

Benguetia Syd. \& P. Syd. (1)

Bioscypha Syd. (2)

Brachyalara Reblova \& W. Gams (1)

Brefeldochium Verkley (1)

Bulgariella P. Karst. (4)

Bulgariopsis Henn. (2)

Calycellinopsis W.Y. Zhuang (1)

Capillipes R. Sant. (1)

Capricola Velen. (1)

Cashiella Petr. (3)

Cejpia Velen. (3)

Cenangiumella J. Frohl. \& K.D. Hyde (1)

Chloroepilichen Etayo (1)

Chlorospleniella P. Karst. (1)

Chondroderris Maire (1)

Ciliella Sacc. \& P. Syd. (1)

Cistella Quel. (50)

Clathrosporium Nawawi \& Kuthub. (1)

Coleosperma Ingold (1) 
Colipila Baral \& Guy Garcıa (2)

Comesia Sacc. (3)

Cornuntum Velen. (1)

Coronellaria P. Karst. (4)

Criserosphaeria Speg. (1)

Crocicreas Fr. (4)

Crucellisporium M.L. Farr (3)

Crumenella P. Karst. (1)

Cryptohymenium Samuels \& L.M. Kohn (1)

Cryptopezia Hohn. (1)

Dactylaria Sacc. (100)

Dawsicola Dobbeler (1)

Dermateopsis Nannf. (2)

Didonia Velen. (5)

Didymascella Maire \& Sacc. (5)

Discomycella Hohn. (1)

Durella Tul. \& C. Tul. (22)*

Echinodiscus Etayo \& Diederich (2)

Encoeliopsis Nannf. (4)

Episclerotium L.M. Kohn (2)

Erikssonopsis M. Morelet (1)

Fulvoflamma Crous (1)

Gloeopeziza Zukal (8)

Godroniopsis Diehl \& E.K. Cash (3)

Gorgoniceps (P. Karst.) P. Karst. (3)

Grimmicola Dobbeler \& Hertel (1)

Grovesia Dennis (1)

Hemiglossum Pat. (2)

Humicolopsis Cabral \& S. Marchand (2)

Hydrocina Scheuer (1)

Hymenobolus Durieu \& Mont. (3)

Hyphoscypha Velen. (1)

Hysteronaevia Nannf. (12)

Hysteropezizella Hohn. (26)

Hysterostegiella Hohn. (10)

Infundichalara Reblova \& W. Gams (2)

Involucroscypha Raitv. (10)

Jacobsonia Boedijn (1)

Korfia J. Reid \& Cain (1)

Lareunionomyces Crous \& M.J. Wingf. (2)

Larissia Raitv. (1)

Lasseria Dennis (1)

Lemalis Fr. (3)

Libartania Nag Raj (2)

Livia Velen. (1)

Masseea Sacc. (4)

Melanopeziza Velen. (1)

Merodontis Clem. (1)

Microdiscus Sacc. (1)

Mitrulinia Spooner (1)

Monochaetiellopsis B. Sutton \& DiCosmo (2)

Mycosphaerangium Verkley (3) 
Obconicum Velen. (2)

Obscurodiscus Raitv. (1)

Orbiliopsis (Sacc. \& D. Sacc.) Syd. \& P. Syd. (2)

Otwaya G. Beaton (12)

Pachydisca Boud. (32)*

Parencoelia Petr. (4)

Patellariopsis Dennis (5)

Patinellaria H. Karst. (1)

Peltigeromyces Möller (3)

Pestalopezia Seaver (3)

Pezolepis Syd. (2)

Pezomela Syd. (1)

Phacidiella P. Karst. (1)

Phaeofabraea Rehm (1)

Phaeopyxis Rambold \& Triebel (1)

Phragmonaevia Rehm (16)*

Piceomphale Svrček (1)

Pleoscutula Vou. (3)

Podophacidium Niessl (2)

Polydesmia Boud. (7)

Polyphilus D.G. Knapp, Ashrafi, W. Maier \& Kovács (2)

Potridiscus Dobbeler \& Triebel (1)

Pseudohelotium Fuckel (50)

Pseudolachnum Velen. (1)

Pseudomitrula Gamund1 (1)

Pseudopeltis L. Holm \& K. Holm (1)

Pseudotryblidium Rehm (1)

Psilophana Syd. (1)

Pteromyces E. Bommer, M. Rousseau \& Sacc. (1)

Pubigera Baral, Gminder \& Svrček (1)

Radotinea E. Bommer, M. Rousseau \& Sacc. (1)

Rhexocercosporidium U. Braun (2)

Rhizocladosporium Crous \& U. Braun (1)

Rhizothyrium Naumov (1)

Rommelaarsia Baral \& Haelew. (1)

Roseodiscus Baral (4)

Sageria A. Funk (1)

Sambucina Velen. (1)

Sarcomyces Massee (1)

Sclerocrana Samuels \& L.M. Kohn (4)

Scutulopsis Velen. (1)

Soosiella Hujslova \& M. Kolarık (1)

Sorokina Sacc. (1)

Sorokinella J. Frohl. \& K.D. Hyde (2)

Spirosphaera Beverw. (8)

Stamnaria Fuckel (7)

Stilbopeziza Speg. (1)

Strossmayeria Schulzer (20)

Tetracladium De Wild. (10)

Thedgonia B. Sutton (6)

Themisia Velen. (8)

Tovariella Syd. (1) 
Trichohelotium Killerm. (2)

Triposporium Corda (14)

Unguicularia Hohn. (7)*

Urceolella Boud. (44)

Vandijckella Sand.-Den. (1)

Waltonia Saho (1)

Woodiella Sacc. \& P. Syd. (3)

Xeromedulla Korf \& W.Y. Zhuang (3)

Zugazaea Korf, Iturr. \& Lizoň (1)

Lahmiales O.E. Erikss.

Lahmiaceae O.E. Erikss.

Lahmia Korb. (2)

Lauriomycetales Hern.-Restr., R.F. Castañeda \& Guarro

Lauriomycetaceae Hern.-Restr., R.F. Castañeda \& Guarro

Lauriomyces R.F. Castaneda (11)

Leotiales Korf \& Lizoň

Cochlearomycetaceae Crous

Cochlearomyces Crous (1)*

Satchmopsis B. Sutton \& Hodges (4)

Leotiaceae Corda

Halenospora E.B.G. Jones (1)

Leotia Pers. (23)

Microglossum Gillet (26)

Miniancora Marvanova \& Barl. (1)

Mniaeciaceae Baral*

Epithamnolia Zhurb. (7)*

Mniaecia Boud. (3)

Tympanidaceae Baral \& Quijada

Claussenomyces Kirschst. (15)*

Collophorina Damm \& Crous (7)

Durandiella Seaver (15)

Gelatinosporium Peck (12)*

Myriodiscus Boedijn (2)

Pragmopora A. Massal. (8)

Tympanis Fr. (64)

Leotiales genera incertae sedis

Aotearoamyces P.R. Johnst., J.A. Cooper \& Quijada (1)*

Alatospora Ingold (4)

Flagellospora Ingold (6)*

Lichinodiales M. Prieto, M. Schultz, Olariaga \& Wedin

Lichinodiaceae M. Prieto, M. Schultz, Olariaga \& Wedin Lichinodium Nyl. (4)* 
Marthamycetales. R. Johnst. \& Baral*

Marthamycetaceae Baral, Lantz, Hustad \& Minter

Cyclaneusma DiCosmo, Peredo \& Minter (2)

Marthamyces Minter (18)

Mellitiosporiella Hohn. (3)

Mellitiosporium Corda (10)

Naemacyclus Fuckel (13)

Phragmiticola Sherwood (1)

Propolina Sacc. (1)

Propolis (Fr.) Corda (8)

Ramomarthamyces P.R. Johnst. (4)*

Medeolariales Korf

Medeolariaceae Korf

Medeolaria Thaxt (1)

Micraspidales Quijada \& Tanney*

Micraspidaceae Quijada \& Tanney

Micraspis Darker (3)

Phacidiales C.E. Bessey*

Helicogoniaceae Baral

Calloriopsis Syd. \& P. Syd. (1)

Eleutheromycella Hohn. (1)

Eleutheromyces Fuckel (2)

Gelatinipulvinella Hosoya \& Y. Otani (1)

Gelatinopsis Rambold \& Triebe (8)

Geltingia Alstrup \& D. Hawksw. (1)

Helicogonium W.L. White (19)

Phacidiaceae Fr.

Allantophomopsiella Crous (1)

Allantophomopsis Petr. (4)

Bulgaria Fr. (12)

Darkera H.S. Whitney, J. Reid \& Piroz. (5)

Lophophacidium Lagerb. (2)

Phacidiopycnis Potebnia (6)

Phacidium Fr. (40)

Pseudophacidium P. Karst. (11)

Starbaeckia Rehm ex Starback (1)

Phacidiales genus incertae sedis

Coma Nag Raj \& W.B. Kendr. (1)

Rhytismatales M.E. Barr ex Minter

Cudoniaceae P.F. Cannon

Cudonia Fr. (20)

Spathularia Pers. (10)

Rhytismataceae Chevall.

Bifusella Hohn. (9)

Bifusepta Darker (1) 
Bivallum P.R. Johnst. (7)

Canavirgella W. Merr, Wenner \& Dreisbach (1)

Cavaraella Speg. (1)

Ceratophacidium J. Reid \& Piroz. (1)

Cerion Massee (2)

Coccomyces De Not. (119)

Colpoma Wallr. (14)

Criella (Sacc.) Sacc. \& P. Syd. (2)

Cryptomyces Grev. (3)

Davisomycella Darker (11)

Discocainia J. Reid \& A. Funk (4)

Duplicaria Fuckel (1)

Duplicariella B. Erikss. (1)

Elytroderma Darker (3)

Gelineostroma H.J. Swart (2)

Heufleria Auersw. (2)*

Hypoderma De Not. (56)

Hypodermella Tubeuf (3)

Hypodermellina Höhn. (1)

Hypohelion P.R. Johnst. (4)

Lasiostictella Sherwood (1)

Lirula Darker (12)

Lophodermella Hohn. (9)

Lophodermium Chevall. (185)

Macroderma Hohn. (2)

Meloderma Darker (5)

Moutoniella Penz. \& Sacc. (1)

Mycomelanea Velen. (1)

Myriophacidium Sherwood (6)

Nematococcomyces C.L. Hou, M. Piepenbr. \& Oberw. (2)

Neococcomyces Y.R. Lin, C.T. Xiang \& Z.Z. Li (3)

Neophacidium Petr. (2)

Nothorhytisma Minter, P.F. Cannon, A.I. Romero \& Peredo (1)

Parvacoccum R.S. Hunt \& A. Funk (1)

Phaeophacidium P. Henn. \& Lindau (3)

Ploioderma Darker. (8)

Propolidium Sacc. (15)

Pseudographis Nyl. (10)*

Pseudorhytisma Juel (1)

Pureke P.R. Johnst. (1)

Rhytisma Fr. (30)

Soleella Darker (7)

Sporomega Corda (1)

Terriera B. Erikss. (34)

Therrya Sacc. (7)

Tryblidiopsis P. Karst. (5)

Virgella Darker (1)

Vladracula P.F. Cannon, Minter \& Kamal (2)

Xyloschizon Syd. (2)

Zeus Minter \& Diamandis (1)

Triblidiaceae Rehm* 
Huangshania O.E. Erikss. (2)

Triblidium Rebent. (13)

Rhytismatales genera incertae sedis

Apiodiscus Petr. (1)

Bonanseja Sacc. (1)

Didymascus Sacc. (2)

Haplophyse Theiss. (1)

Irydyonia Racib. (1)

Laquearia Fr. (2)

Mycosymbioces J.L. Frank (1)

Nymanomyces P. Henn. (2)

Pseudotrochila Hohn. (1)

Thelebolales P.F. Cannon

Pseudeurotiaceae Malloch \& Cain

Connersia Malloch (1)

Geomyces Traaen (9)

Gymnostellatospora Udagawa, Uchiy. \& Kamiya (6)*

Leuconeurospora Malloch \& Cain (2)

Neelakesa Udaiyan \& Hosag. (3)

Pleuroascus Massee \& E.S. Salmon (3)

Pseudeurotium J.F.H. Beyma (8)

Pseudogymnoascus Raillo (12)*

Thelebolaceae (Brumm.) Eckblad

Antarctomyces Stchigel \& Guarro (2)

Ascophanus Boud. (56)

Ascozonus (Renny) E.C. Hansen (9)

Caccobius Kimbr. (1)

Cleistothelebolus Malloch \& Cain (1)

Coprobolus Cain \& Kimbr. (1)

Leptokalpion Brumm. (1)

Pseudascozonus Brumm. (1)

Ramgea Brumm. (2)

Thelebolus Tode (16)

Leotiomycetes genera incertae sedis

Adelodiscus Syd. (1)

Bagnisimitrula S. Imai (1)

Callerascus Whitton, K.D. Hyde \& McKenzie (1)

Deltopyxis Baral \& G. Marson (1)

Epicladonia D. Hawksw. (5)

Gorgomyces M. Gonczol \& Revay (2)

Helicocentralis Sri-indr., Chuaseehar., Boonyuen, K. Yamag., Suetrong \& C.K.M. Tsui (1)

Helotiella Sacc. (17)

Holwaya Sacc. (2)

Leohumicola N.L. Nick. (7)

Melanormia Korb. (1)

Metapezizella Petr. (1)

Ocotomyces H.C. Evans \& Minter (1)

Patinella Sacc. (25)* 
Phyllopezis Petr. (1)

Physmatomyces Rehm (1)

Polydiscina Syd. (1)

Psilothecium Clem. (1)

Schnablia Sacc. \& P. Syd. (1)

Trullula Ces. (5)

Lichinomycetes $\mathrm{V}$. Reeb, Lutzoni \& Cl. Roux

Lichinales Henssen \& Büdel

Gloeoheppiaceae Henssen

Gloeoheppia Gyeln. (5)

Gudelia Henssen (1)

Pseudopeltula Henssen (1)

\section{Lichinaceae Nyl.}

Anema Nyl. ex Forssell (21)

Calotrichopsis Vain. (4)

Corynecystis Brusse (1)

Cryptothele Th. Fr. (7)

Digitothyrea P. Moreno \& Egea (3)

Edwardiella Henssen (1)

Ephebe Fr. (13)

Finkia Vain. (1)

Gyrocollema Vain. (2)

Heppia Nägeli (4)

Jenmania W. Wächt. (2)

Lecidopyrenopsis Vain. (1)

Lemmopsis (Vain.) Zahlbr. (3)

Lempholemma Körb. (35)

Leprocollema Vain. (3)

Lichina C. Agardh (9)

Lichinella Nyl. (30)

Mawsonia C.W. Dodge (1)

Metamelanea Henssen (3)

Paulia Feé (10)

Peccania A. Massal. ex Arnold (3)

Phloeopeccania J. Steiner (4)

Phylliscidiopsis Sambo (1)

Phylliscidium Forssell (1)

Phyllisciella Henssen \& Büdel (3)

Phylliscum Nyl. (8)

Porocyphus Körb. (8)

Pseudarctomia Gyeln. (1)

Pseudoheppia Zahlbr. (1)

Pseudopaulia M. Schultz (1)

Psorotichia A. Massal. (50)

Pterygiopsis Vain. (17)

Pyrenocarpon Trevis. (1)

Pyrenopsis Nyl. (40)

Solorinaria (Vain.) Gyeln. (1)

Stromatella Henssen (1)

Synalissa Fr. (30) 
Thallinocarpon A.E. Dahl (2)

Thelignya A. Massal. (2)

Thermutis Fr. (2)

Thermutopsis Henssen (1)

Thyrea A. Massal. (13)

Zahlbrucknerella Herre (10)

\section{Peltulaceae Büdel}

Peltula Nyl. (32)

Orbiliomycetes O.E. Erikss. \& Baral

Orbiliales Baral, O.E. Erikss., G. Marson \& E. Weber

Orbiliaceae Nannf.

Arthrobotrys Corda (ca. 100+)

Dactylella Grove (31)

Dactylellina M. Morelet (= Gamsylella M. Scholler et al.) (26)

Drechslerella Subram. (ca. 7)

Dwayaangam Subram. (8)

Helicoon Morgan (ca. 15)

Hyalorbilia Baral \& G. Marson (40)

Orbilia Fr. (ca. 400)

Pseudotripoconidium Z.F. Yu \& K.Q. Zhang (1)

Pseudorbilia Y. Zhang, Z.F. Yu, Baral \& K-Q Zhang (1)

Retiarius D.L. Olivier (4)

Vermispora Deighton \& Piroz. (7)

Orbiliales genus incertae sedis

Microdochiella Hern.-Restr. \& Crous (1)

Orbiliomycetes genus incertae sedis

Mycoceros D. Magyar \& Z. Merényi (1)*

Pezizomycetes O.E. Erikss. \& Winka

Pezizales J. Schröt.

Ascobolaceae Boud. ex Sacc.

Ascobolus Pers. (ca. 70)

Cleistoiodophanus J.L. Bezerra \& Kimbr. (1)

Cubonia Sacc. (ca. 7)

Saccobolus Boud. (33)

Thecotheus Boud. (23)*

Ascodesmidaceae J. Schröt.

Ascodesmis Tiegh. ( 10)

Cephaliophora Thaxt. (2)*

Chalazion Dissing \& Sivertsen (3)

Coprotiella Jeng \& J.C. Krug (1)

Dictyocoprotus J.C. Krug \& R.S. Khan (1)

Eleutherascus Arx (4)

Lasiobolus Sacc. (11)

Luciotrichus R. Galán \& Raitv. (1)

Ochotrichobolus Kimbr. \& Korf (1)

Trichobolus (Sacc.) Kimbr. \& Cain (6) 
Caloscyphaceae Harmaja

Caloscypha Boud. (2)

Chorioactidaceae Pfister

Chorioactis Kupfer ex Eckblad (1)

Desmazierella Lib. (2)

Neournula Paden \& Tylutki (2)

Pseudosarcosoma M. Carbone, Agnello \& P. Alvarado (1)

Trichaleurina Rehm (3)

Wolfina Seaver ex Eckblad (2)

Discinaceae Benedix

Discina (Fr.) Fr. (20)

Gymnohydnotrya B.C. Zhang \& Minter (3)

Gyromitra Fr. (25)

Hydnotrya Berk. \& Broome (11)

Pseudorhizina Jacz. (3)

Glaziellaceae J.L. Gibson

Glaziella Berk. (1)

Helvellaceae Fr.

Balsamia Vittad. (21)

Barssia Gilkey (8)

Helvella L. (ca. 80)

Underwoodia Peck (2)

Wynnella Boud. (3)

Kallistoskyphaceae Ekanayaka, K.D. Hyde, Q. Zhao \& E.B.G. Jones Kallistoskypha Pfister, Agnello, Lantieri \& LoBuglio (1)

Karstenellaceae Harmaja

Karstenella Harmaja (1)

Morchellaceae H.G.L. Reichenbach

Disciotis Boud. (3)

Fischerula Mattir. (2)

Imaia Trappe \& Kovács (1)

Kalapuya M.J. Trappe, Trappe \& Bonito (1)

Leucangium Quél. (1)

Morchella Dill. ex Pers. ( 60)

Verpa Sw. (4)

Pezizaceae Dumort. (= Carbomycetaceae Trappe)

Adelphella Pfister, Matočec \& I. Kušan (1)

Amylascus Trappe (1)

Antrelloides P.S. Catches. \& D.E.A. Catches. (1)

Aquapeziza D.M. Hu, L. Cai \& K.D. Hyde (1)

Boudiera Cooke (10)

Calongea Healy, Bonito \& Trappe (1)*

Carbomyces Gilkey (3)

Cazia Trappe (2) 
Delastria Tul. \& C. Tul. (6)

Elderia McLennan (1)

Eremiomyces Trappe \& Kagan-Zur (3)

Galactinia (Cooke) Boud. (ca. 5)

Hapsidomyces J.C. Krug \& Jeng (1)

Hydnobolites Tul. \& C. Tul. (ca. 6)

Hydnotryopsis Gilkey (4)

Iodophanus Korf (15)

Iodowynnea Medel, Guzmán \& S. Chacón (1)

Kalaharituber Trappe \& Kagan-Zur (1)

Lepidotia Boud. (1)

Luteoamylascus Cabero, P. Alvarado \& G. Moreno (1)

Marcelleina Brumm., Korf \& Rifai (11)

Mattirolomyces E. Fisch. (5)

Mycoclelandia Trappe \& G.W. Beaton (2)

Pachyella Boud. (12)

Pachyphlodes Zobel (ca. 10)*

Peziza Dill. ex Fr. (ca. 120)

Plicaria Fuckel (10)

Plicariella (Sacc.) Rehm (2)*

Rhodopeziza Hohmeyer \& Moravec (1)

Ruhlandiella P. Henn. (7)

Sarcopeziza Loizides, Agnello \& P. Alvarado (1)*

Sarcosphaera Auersw. (4)

Sphaerozone Zobel (1)

Stouffera Kovács \& Trappe (1)

Temperantia K. Hansen, Healy \& Kovács (1)

Terfezia (Tul. \& C. Tul.) Tul. \& C. Tul. (19)

Tirmania Chatin (3)

Ulurua Trappe, Claridge \& Kovács (1)

Pseudombrophilaceae Ekanayaka, K.D. Hyde, Q. Zhao \& E.B.G. Jones

Heydenia Fresen. (3)

Lasiobolidium Malloch \& Cain (7)

Orbicula Cooke (1)

Pseudombrophila Boud. (37)

Pulvinulaceae Ekanayaka, K.D. Hyde, Q. Zhao \& E.B.G. Jones

Lazuardia Rifai (1)

Pseudoboubovia U. Lindem., M. Vega, B. Perić \& R. Tena (1)

Pulvinula Boud. ( 30)

Pyronemataceae Corda (= Otideaceae Eckblad)

Acervus Kanouse (9)

Aleuria Fuckel (ca. 10)

Aleurina Massee (ca. 10)

Anthracobia Boud. (ca. 10)

Arpinia Berthet (4)

Ascosparassis Kobayasi (1)

Byssonectria P. Karst. (7)*

Chaetothiersia B.A. Perry \& Pfister (1)

Cheilymenia Boud. (67) 
Cupulina Dougoud, Van Vooren \& M. Vega (2)

Diehliomyces Gilkey (1)

Eoaleurina Korf \& W.Y. Zhuang (1)

Galeoscypha Svrček \& J. Moravec (1)

Genabea Tul. \& C. Tul. (4)

Genea Vittad. (ca. 40)

Geneosperma Rifai (2)

Geopora Harkn. (ca. 20)

Gilkeya M.E. Sm., Trappe \& Rizzo (1)

Hoffmannoscypha Stielow, Göker \& Klenk (1)*

Humaria Fuckel (ca. 10)

Jafnea Korf (2)

Lamprospora De Not. (ca. 50)

Lasiocupulina Van Vooren \& M. Vega (1)

Lathraeodiscus Dissing \& Sivertsen (1)

Lotinia Pérez-Butrón Fern.-Vic. \& P. Alvarado (1)*

Melastiza Boud. (ca. 10)

Micronematobotrys Xiang Sun \& L.D. Guo (1)

Miladina Svrček (1)

Monascella Guarro \& Arx (1)

Myrmecocystis Harkn. (7)*

Neottiella (Cooke) Sacc. (ca. 5)

Octospora Hedw. (ca. 50)

Octosporopsis U. Lindem. \& M. Vega (2)

Otidea (Pers.) Bonord. (ca. 52)

Oviascoma Y.J. Yao \& Spooner (1)

Parascutellinia Svrček (6)

Paratricharina Van Vooren, U. Lindemann, M. Vega, Ribes, Illescas \& Matočec (1)

Paratrichophaea Trigaux (5)

Petchiomyces E. Fisch. \& Mattir. (1)

Picoa Vittad. (2)*

Planamyces Crous \& Decock (1)

Pseudaleuria Lusk (2)

Pseudotricharina Van Vooren, Tello \& M. Vega (3)

Pyronema Carus (3)

Pyropyxis Egger (1)

Ramsbottomia W.D. Buckley (3)

Rhizoblepharia Rifai (2)

Scutellinia (Cooke) Lambotte (70)

Selenaspora R. Heim \& Le Gal (1)

Sepultariella Van Vooren, U. Lindemmann \& Healy (2)*

Smardaea Svrček (9)

Smarodsia Raitv. \& Vimba (1)

Sowerbyella Nannf. (17)

Sphaerosporella (Svrček) Svrček \& Kubička (3)

Sphaerosporium Schwein. sensu stricto $(1)^{*}$

Spooneromyces T. Schumach. \& J. Moravec (5)

Tricharina Eckblad (=Ascorhizoctonia Chin S. Yang \& Korf) (12)

Trichophaea Boud. (26)

Trichophaeopsis Korf \& Erb (4)

Warcupia Paden \& J.V. Cameron (1)

Wenyingia Zheng Wang \& Pfister (1) 
Wilcoxina Chin S. Yang \& Korf (5)

Rhizinaceae Bonord.

Phymatotrichopsis Hennebert (1)

Psilopezia Berk. (7)

Rhizina Fr. (1)

Sarcoscyphaceae LeGal ex Eckblad

Aurophora Rifai (1)

Cookeina Kuntze (11)

Geodina Denison (1)

Kompsoscypha Pfister (4)

Microstoma Bernstein (7)

Nanoscypha Denison (8)

Phillipsia Berk. ( 20)

Pithya Fuckel (2)

Pseudopithyella Seaver (2)

Sarcoscypha (Fr.) Boud. (18)

Thindia Korf \& Waraitch (1)

Wynnea Berk. \& M.A. Curtis (7)

Sarcosomataceae Kobayasi

Conoplea Pers. (11)

Donadinia Bellem. \& Mel.-Howell (5)

Galiella Nannf. \& Korf (9)

Korfiella D.C. Pant \& V.P. Tewari (1)

Plectania Fuckel (ca. 20)

Pseudoplectania Fuckel (4)

Sarcosoma Casp. (5)

Strumella Fr. (8)

Urnula Fr. (9)

Strobiloscyphaceae Ekanayaka, K.D. Hyde, Q. Zhao \& E.B.G. Jones Strobiloscypha N.S. Weber \& Denison (2)

Tarzettaceae Ekanayaka, K.D. Hyde, Q. Zhao \& E.B.G. Jones

Densocarpa Gilkey (2)*

Geopyxis (Pers.) Sacc. (7)

Hydnocystis Tul. (= Stephensia Tul. \& C. Tul.) (5)*

Hypotarzetta Donadini (1)

Paurocotylis Berk. (4)

Tarzetta (Cooke) Lambotte (ca. 10)

Tuberaceae Dumort.

Choiromyces Vittad. (5)

Dingleya Trappe (6)

Labyrinthomyces Boedijn (1)

Nothojafnea Rifai (2)

Paradoxa Mattir. (2)

Reddellomyces Trappe, Castellano \& Malajczuk (4)

Tuber P. Micheli ex F.H. Wigg. (ca. 120) 
Pezizales genera incertae sedis

Aparaphysaria Speg. (1)

Ascocalathium Eidam ex J. Schröt. (1)

Boubovia Svrček (5)*

Boudierella Sacc. (1)

Cidaris Fr. (1)

Coprotus Korf ex Korf \& Kimbr. (33)*

Dennisiopsis Subram. \& Chandras. (2)

Filicupula Y.J. Yao \& Spooner (1)

Heydenia Fresen.

Hiemsia Svrček (2)

Leucoscypha Boud (4)

Microeurotium Ghatak (1)

Moravecia Benkert, Caillet \& Moyne (2)

Mycoarctium K.P. Jain \& Cain (2)

Mycogalopsis Gjurašin (1)

Octosporella Döbbeler (9)

Orcadia G.K. Sutherl. (1)

Sphaerosoma Klotzsch (3)

Sordariomycetes O.E. Erikss. \& Winka

Diaporthomycetidae Senan., Maharachch. \& K.D. Hyde

Annulatascales M.J. D'souza, Maharachch. \& K.D. Hyde

Annulatascaceae S.W. Wong, K.D. Hyde \& E.B.G. Jones

Annulatascus K.D. Hyde (18)

Annulusmagnus J. Campb. \& Shearer (1)

Aqualignicola Ranghoo, C.K.M. Tsui \& K.D. Hyde (2)

Ascitendus J. Campb. \& Shearer (2)

Ayria Fryar \& K.D. Hyde (2)

Cataractispora K.D. Hyde, S.W. Wong \& E.B.G. Jones (5)

Chaetorostrum Zelski, Raja, A.N. Mill. \& Shearer (1)

Longicollum Zelski, F.R. Barbosa, Raja, A.N. Mill. \& Shearer (1)

Submersisphaeria K.D. Hyde (5)

Vertexicola K.D. Hyde, Ranghoo \& S.W. Wong (3)

Annulatascales genus incertae sedis

Clohiesia K.D. Hyde (3)

Atractosporales H. Zhang, K.D. Hyde \& Maharachch.

Atractosporaceae H. Zhang, K.D. Hyde \& Maharachch.

Atractospora Réblová \& J. Fourn. (5)

Rubellisphaeria Réblová \& J. Fourn. (1)

Conlariaceae H. Zhang, K.D. Hyde \& Maharachch.

Conlarium F. Liu \& L. Cai (3)

Riomyces A. Ferrer, A.N. Mill., Sarmiento \& Shearer (1)

Pseudoproboscisporaceae H. Zhang, K.D. Hyde \& Maharachch.

Diluviicola K.D. Hyde, S.W. Wong \& E.B.G. Jones (2)

Pseudoproboscispora Punith. (3)

Calosphaeriales M.E. Barr 
Calosphaeriaceae Munk

Calosphaeria Tul. \& C Tul. (114)

Flabellascus Réblová (1)

Jattaea Berl (27)

Togniniella Réblová, L. Mostert, W. Gams \& Crous (1)

Pleurostomataceae Réblová, L. Mostert, W. Gams \& Crous

Pleurostoma Tul. \& C. Tul. (7)

Calosphaeriales genera incertae sedis

Calosphaeriopsis Petr. (1)

Enchnoa Fr. (21)

Kacosphaeria Speg. (1)

Sulcatistroma A.W. Ramaley (1)

Diaporthales Nannf.

Apiosporopsidaceae Senan., Maharachch. \& K.D. Hyde

Apiosporopsis (Traverso) Mariani. (3)

Apoharknessiaceae Senan., Maharachch. \& K.D. Hyde

Apoharknessia Crous \& S.J. Lee (3)

Lasmenia Speg. (5)

Asterosporiaceae Senan., Maharachch. \& K.D. Hyde

Asterosporium Kunze (5)

Auratiopycnidiellaceae Senan., Maharachch. \& K.D. Hyde

Auratiopycnidiella Crous \& Summerell (1)

Coryneaceae Corda (=Pseudovalsaceae M.E. Barr)

Coryneum Nees (30)

Cryphonectriaceae Gryzenh. \& M.J. Wingf.

Amphilogia Gryzenh., H.F. Glen \& M.J. Wingf. (2)

Aurantioporthe G. Beier \& R.A. Blanchette (1)

Aurantiosacculus Dyko \& B. Sutton (3)

Aurapex Gryzenh. \& M.J. Wingf. (1)

Aurifilum Begoude, Gryzenh. \& Jol. Roux (1)

Capillaureum M.E.S. Oliveira (1)

Celoporthe Nakab., Gryzenh., Jol. Roux \& M.J. Wingf. (2)

Chromendothia Lar.N. Vassiljeva (2)

Chrysofolia Crous \& M.J. Wingf. (1)

Chrysomorbus S.F. Chen (1)

Chrysoporthe Gryzenh. \& M.J. Wingf. (9)

Corticimorbus S.F. Chen \& M.J. Wingf. (1)

Cryphonectria (Sacc.) Sacc. \& D. Sacc. (1)

Cryptometrion Gryzenh. \& M.J. Wingf. (1)

Diversimorbus S.F. Chen \& J. Roux (1)

Endothia Fr. (2)

Eriocamporesia R.H. Perera, Samarak. \& K.D. Hyde (1)

Foliocryphia Cheew. \& Crous (2)

Holocryphia Gryzenh. \& M.J. Wingf. (1) 
Immersiporthe S.F. Chen, M.J. Wingf. \& Jol. Roux (1)

Latruncellus M. Verm., Gryzenh. \& Jol. Roux (1)

Luteocirrhus C.F. Crane \& T.I. Burgess (1)

Mastigosporella Höhn. (=Wuestneiopsis J. Reid \& Dowsett) (5)

Microthia Gryzenh. \& M.J. Wingf. (2)

Myrtonectria Marinc., D.B. Ali \& J. Roux (1)

Rostraureum Gryzenh. \& M.J. Wingf. (2)

Ursicollum Gryzenh. \& M.J. Wingf. (1)

Wuestneia Auersw. ex Fuckel (13)

Cytosporaceae Fr. (= Valsaceae Tul. \& C. Tul.)

Cryptascoma Ananthap. (2)

Cytospora Ehrenb. (123)

Pachytrype Berl. ex M.E. Barr, J.D. Rogers \& Y.M. Ju (1)

Paravalsa Ananthap. (1)

Waydora B. Sutton (1)

Xenotypa Petr. (1)

Diaporthaceae Höhn. ex Wehm.

Apioporthella Petr. (1)

Apiosphaeria Höhn. (5)

Chaetoconis Clem. (1)

Chiangraiomyces Senan. \& K.D. Hyde (1)

Diaporthe Nitschke (= Allantoporthe Petr.; = Clypeoporthella Petr.) (173)

Hyaliappendispora Senan., Camporesi \& K.D. Hyde (1)

Leucodiaporthe M.E. Barr \& Lar.N. Vassiljeva (1)

Massariothea Syd. (10)

Mazzantia Mont. (4)

Ophiodiaporthe Y.M. Ju, H.M. Hsieh, C.H. Fu, Chi Y. Chen \& T.T. Chang (1)

Paradiaporthe Senan., Camporesi \& K.D. Hyde (1)

Phaeocytostroma Petr. (4)

Phaeodiaporthe Petr. (2)

Pustulomyces D.Q. Dai, Bhat \& K.D. Hyde (1)

Stenocarpella Syd. \& P. Syd. (2)

Diaporthosporellaceae C.M. Tian \& Q. Yang*

Diaporthosporella C.M. Tian \& Q. Yang (1)

Diaporthostomataceae X.L. Fan \& C.M. Tian*

Diaporthostoma X.L. Fan \& C.M. Tian (1)*

Dwiroopaceae K.V. Xavier, A.N. KC, J.Z. Groenew., Vallad \& Crous

Dwiroopa Subram. \& Muthumary (2)

Erythrogloeaceae Senan., Maharachch. \& K.D. Hyde

Chrysocrypta Crous \& Summerell (1)

Dendrostoma X.L. Fan \& C.M. Tian (4)*

Disculoides Crous, Pascoe, I.J. Porter \& Jacq. Edwards (2)

Erythrogloeum Petr. (2)

Gnomoniaceae G. Winter

Alnecium Voglmayr \& Jaklitsch (2) 
Ambarignomonia Sogonov (1)

Amphiporthe Petr. (=Amphicytostroma Petr.) (2)

Anisomyces Theiss. \& Syd. (5)

Apiognomonia Höhn. (=Discula Sacc.) (28)

Apioplagiostoma M.E. Barr (3)

Asteroma DC. (54)

Bagcheea E. Müll. \& R. Menon (2)

Chadefaudiomyces Kamat (1)

Clypeoporthe Höhn. (5)

Cryptosporella Sacc. (ca. 26)

Dictyoporthe Petr. (4)

Diplacella Syd. (2)

Ditopella De Not. (16)

Ditopellopsis J. Reid \& C. Booth (4)

Flavignomonia C.M. Tian, Qin Yang \& N. Jiang (1)*

Gloeosporidina Petr. (6)

Gnomonia Ces. \& De Not. (ca. 70)

Gnomoniella Sacc. (=Cylindrosporella Höhn.) (ca. 70)

Gnomoniopsis Berl. (25)

Maculatipalma J. Fröhlich \& K.D. Hyde (1)

Mamianiella Höhn. (=Anisogramma Theiss. \& Syd.; = Mamiania Ces \& De Not.) (2)

Marsupiomyces Senan. \& K.D. Hyde (2)

Millerburtonia Cif. (1)

Occultocarpon L.C. Mejía \& Zhu L. Yang (1)

Ophiognomonia (Sacc.) Sacc. (49)

Phragmoporthe Petr. (1)

Phylloporthe Syd. (2)

Plagiostoma Fuckel (52)

Pleuroceras Riess. (12)

Sirococcus Preuss (5)

Spataporthe Bronson, Klymiuk, Stockey \& Tomescu (1)

Tenuignomonia Minosh., D.M. Walker \& Hirooka (1)

Uleoporthe Petr. (1)

Valsalnicola D.M. Walker \& Rossman (1)

Vismaya V.V. Sarma \& K.D. Hyde (1)

\section{Harknessiaceae Crous}

Harknessia Cooke (ca. 50)

Mebarria J. Reid \& C. Booth (1)

Juglanconidaceae Voglmayr \& Jaklitsch (=Melanosporellaceae C.M. Tian \& Z. Du) Juglanconis Voglmayr \& Jaklitsch (4)

Lamproconiaceae Norph., T.C. Wen \& K.D. Hyde

Hercospora Fr. (= Rabenhorstia Fr.) (1)

Lamproconium (Grove) Grove (1)

\section{Macrohilaceae Crous}

Macrohilum H.J. Swart (1)

Melanconidaceae G. Winter

Melanconis Tul. \& C. Tul. (1) 
Melanconiellaceae Senan., Maharachch. \& K.D. Hyde

Dicarpella Syd. \& P. Syd. (7)

Greeneria Scribn. \& Viala (3)

Massariovalsa Sacc. (=Melanconiopsis Ellis \& Everh.) (4)

Melanconiella Sacc. (2)

Microascospora Senan. \& K.D. Hyde (2)

Septomelanconiella Samarak. \& K.D. Hyde (1)*

Sheathospora X.L. Fan (1)*

Sphaeronaemella P. Karst. sensu lato (10)

Neomelanconiellaceae Crous

Neomelanconiella Crous (1)*

Phaeoappendicosporaceae Crous \& M.J. Wingf.

Phaeoappendicospora Senan., Q.R. Li \& K.D. Hyde (1)

Neophaeoappendicospora Crous \& M.J. Wingf. (1)

Prosopidicolaceae Senan. \& K.D. Hyde

Prosopidicola Crous \& C.L. Lennox (2)

Pseudomelanconidaceae C.M. Tian \& X.L. Fan*

Pseudomelanconis C.M. Tian \& X.L. Fan (1)*

Neopseudomelanconis C.M. Tian \& N. Jiang (1)

Pseudoplagiostomataceae Cheew., M.J. Wingf. \& Crous

Pseudoplagiostoma Cheew., M.J. Wingf. \& Crous (7)

Schizoparmaceae Rossman

Coniella Höhn. (34)

Stilbosporaceae Link

Crinitospora B. Sutton \& Alcorn (1)

Natarajania Pratibha \& Bhat (1)

Stegonsporium Corda (8)

Stilbospora Pers. (20)

Sydowiellaceae Lar.N. Vassiljeva

Alborbis Senan. \& K.D. Hyde (1)

Breviappendix Senan. \& K.D. Hyde (3)

Cainiella E. Müll. (2)

Calosporella J. Schröt (1)

Caudospora Starbäck (2)

Chapeckia M.E. Barr (2)

Hapalocystis Auersw. ex Fuckel (9)

Italiomyces Senan., Camporesi \& K.D. Hyde (1)

Lambro Racib. (3)

Paragnomonia Senan. \& K.D. Hyde (1)

Ranulospora Senan., Camporesi \& K.D. Hyde (1)

Rossmania Lar.N. Vassiljeva (2)

Sillia P. Karst. (9)

Sydowiella Petr. (11)

Tenuiappendicula Senan., Camporesi \& K.D. Hyde (1) 
Tortilispora Senan. \& K.D. Hyde (3)

Synnemasporellaceae X.L. Fan \& J.D.P. Bezerra*

Synnemasporella X.L. Fan \& J.D.P. Bezerra (2)*

Tubakiaceae U. Braun, J.Z. Groenew. \& Crous*

Apiognomonioides U. Braun, J.Z. Groenew. \& Crous (1)

Involutscutellula U. Braun \& C. Nakash. (1)

Oblongisporothyrium U. Braun \& C. Nakash. (1)

Paratubakia U. Braun \& C. Nakash. (2)

Racheliella Crous \& U. Braun (2)

Saprothyrium U. Braun, Crous \& J.Z. Groenew. (1)

Sphaerosporithyrium U. Braun, Crous, O. Moreno-Rico \& Marm. (1)

Tubakia B. Sutton (25)

Diaporthales genera incertae sedis

Ceratoporthe Petr. (1)

Cryptoleptosphaeria Petr. (1)

Cryptonectriella (Höhn.) Weese (2)

Cryptonectriopsis (Höhn.) Weese (1)

Cytomelanconis Naumov (1)

Diaporthella Petr. (5)

Diatrypoidiella Manohar., Kunwar \& D.K. Agarwa (1)

Ditopellina J. Reid \& C. Booth (1)

Durispora K.D. Hyde (2)

Exormatostoma Gray (10 epithets in Index Fungorum 2020)

Fremineavia Nieuwl. (1)

Gibellia Sacc. (1)

Gyrostroma Naumov (3)

Hyalorostratum Raja \& Shearer (1)

Hypophloeda K.D. Hyde \& E.B.G. Jones (1)

Hypospilina (Sacc) Traverso (4)

Kapooria J. Reid \& C. Booth (1)

Keinstirschia J. Reid \& C. Booth (1)

Kensinjia J. Reid \& C. Booth (1)

Lollipopaia Inderb. (1)

Macrodiaporthe Petr. (1)

Melanamphora Lafl. (1)

Phragmodiaporthe Wehm. (3)

Phruensis Pinruan (1)

Plagiophiale Petr. (2)

Plagiostigme Syd. (1)

Prostratus Sivan., W.H. Hsieh \& Chi Y. Chen (1)

Pseudocryptosporella J. Reid \& C. Booth (1)

Pseudothis Theiss. \& Syd. (12)

Pseudovalsella Höhn. (2)

Savulescua Petr. (1)

Skottsbergiella Petr. (1)

Sphaerognomoniella Naumov \& Kusnezowa (1)

Stioclettia Dennis (1)

Trematovalsa Jacobesco (1)

Wehmeyera J. Reid \& C. Booth (1) 
Distoseptisporales Z.L. Luo, K.D. Hyde \& H.Y. Su

Distoseptisporaceae K.D. Hyde \& McKenzie

Distoseptispora K.D. Hyde, McKenzie \& Maharachch. (18)

Magnaporthales Thongk., Vijaykr. \& K.D. Hyde

Ceratosphaeriaceae Z.L. Luo, H.Y. Su \& K.D. Hyde

Ceratosphaeria Niessl. (24)

Magnaporthaceae P.F. Cannon

Bifusisporella R.M.F. Silva, R.J.V. Oliveira, J.D.P. Bezerra, Souza-Motta \& G.A. Silva (1)*

Budhanggurabania P. Wong, Khemmuk \& R.G. Shivas (1)

Buergenerula Syd. (1)

Bussabanomyces Klaubauf, M.-H. Lebrun \& Crous (1)

Ceratosphaerella Huhndorf, Greif, Mugambi \& A.N. Mill. (2)

Clasterosphaeria Sivan. (2)

Clasterosporium Schwein (41)

Clavatisporella K.D. Hyde (1)

Falciphora J. Luo \& N. Zhang (1)

Falciphoriella M. Hern.-Restr. \& Crous (1)

Gaeumannomycella M. Hern.-Restr. \& Crous (2)

Gaeumannomyces Arx \& D.L. Olivier (20)

Herbampulla Scheuer \& Nograsek (1)

Kohlmeyeriopsis Klaubauf, M.-H. Lebrun \& Crous (1)

Magnaporthiopsis J. Luo \& N. Zhang (7)

Muraeriata Huhndorf, Greif, Mugambi \& A.N. Mill. (2)

Nakataea Hara (8)

Neogaeumannomyces D.Q. Dai \& K.D. Hyde (1)

Omnidemptus P.F. Cannon \& Alcorn (3)

Plagiosphaera Petr. (1)*

Pseudophialophora J. Luo \& N. Zhang (9)

Pyriculariopsis M.B. Ellis (9)

Slopeiomyces Klaubauf, M.-H. Lebrun \& Crous (1)

Ophioceraceae Klaubauf, E.G. LeBrun \& Crous

Ophioceras Sacc. (50)

Pseudohalonectriaceae Hongsanan \& K.D. Hyde

Pseudohalonectria Minoura \& T. Muroi (16)

Pyriculariaceae Klaubauf, E.G. LeBrun \& Crous

Bambusicularia Klaubauf, M.-H. Lebrun \& Crous (1)

Barretomyces Klaubauf, M.-H. Lebrun \& Crous (1)

Deightoniella S. Hughes (20)

Macgarvieomyces Klaubauf, M.-H. Lebrun \& Crous (3)

Neocordana Hern.-Rest. \& Crous (6)

Neopyricularia Klaubauf, M.-H. Lebrun \& Crous (1)

Proxipyricularia Klaubauf, M.-H. Lebrun \& Crous (2)

Pseudopyricularia Klaubauf, M.-H. Lebrun \& Crous (7)

Pyricularia Sacc. (84)

Pyriculariomyces Y. Marín, M.J. Wingf. \& Crous (1)

Xenopyricularia Klaubauf, M.-H. Lebrun \& Crous (1) 
Myrmecridiales Crous

Myrmecridiaceae Crous

Myrmecridium Arzanlou, W. Gams \& Crous (14)

Neomyrmecridium Crous (2)

Xenodactylariaceae Crous

Xenodactylaria Crous (1)*

Ophiostomatales Benny \& Kimbr.

Kathistaceae Malloch \& M. Blackw.

Kathistes Malloch \& M. Blackw. (3)

Mattirolella S. Colla (2)

Termitariopsis M. Blackw., Samson \& Kimbr. (1)

Ophiostomataceae Nannf.

Afroraffaele C.C. Bateman, Y.T. Huang \& D.R. Simmons (1)

Aureovirg J.A. van der Linde, Z.W. de Beer \& Jol. Roux (1)

Ceratocystiopsis H.P. Upadhyay \& W.B. Kendr. (5)

Fragosphaeria Shear (2)

Graphilbum H.P. Upadhyay \& W.B. Kendr. (13)

Hawksworthiomyces Z.W. de Beer, Marinc. \& M.J. Wingf. (4)

Klasterskya Petr. (3)

Leptographium Lagerb. \& Melin (= Grosmannia Gold.) (74)

Ophiostoma Syd. \& P. Syd. (= Hyalorhinocladiella H.P. Upadhyay \& W.B. Kendr.; = Pesotum J.L. Crane \& Schokn.) (134)

Raffaelea Arx \& Hennebert (33)

Sporothrix Hektoen \& C.F. Perkins (79)

Spumatoria Massee \& E.S. Salmon (1)

Subbaromyces Hesselt. (2)

Phomatosporales Senan., Maharachch. \& K.D. Hyde

Phomatosporaceae Senan. \& K.D. Hyde

Lanspora K.D. Hyde \& E.B.G. Jones (2)

Phomatospora Sacc. (ca. 100)

Tenuimurus Senan., Camporesi \& K.D. Hyde (1)

Sporidesmiales Crous

Sporidesmiaceae Fr.

Sporidesmium Link (ca. 330)

Tirisporellales Suetrong, E.B.G. Jones \& K.L. Pang

Tirisporellaceae Suetrong, E.B.G. Jones \& K.L. Pang

Bacusphaeria Norlail., Alias \& Suetrong (1)

Thailandiomyces Pinruan, Sakay., K.D. Hyde \& E.B.G. Jones (1)

Tirisporella E.B.G. Jones, K.D. Hyde \& Alias (1)

Togniniales Senan., Maharachch. \& K.D. Hyde

Togniniaceae Réblová, L. Mostert, W. Gams \& Crous

Conidiotheca Réblová \& L Mostert (1)

Phaeoacremonium W. Gams, Crous \& M.J. Wingf. (65)

Xenospadicoidales Hern.-Restr., J. Mena \& Gené 
Xenospadicoidaceae Hern.-Restr., J. Mena \& Gené (= Lentomitellaceae H. Zhang, K.D. Hyde \& Maharachch)*

Calyptosphaeria Réblová \& A.N. Mill. (4)

Lentomitella Höhn. (13)

Neospadicoides Z.L. Luo (3)

Spadicoides S. Hughes (=Xenospadicoides Hern.-Restr., J. Mena \& Gené; Pseudodiplococcium Hern.-Restr., J. Mena \& Gené) (45)*

Torrentispora K.D. Hyde, W.H. Ho, E.B.G. Jones (= Fusoidispora Vijaykr., Jeewon \& K.D. Hyde; = Pseudoannulatascus Z.L. Luo, Maharachch. \& K.D. Hyde) (9)*

Diaporthomycetidae families incertae sedis

Barbatosphaeriaceae H. Zhang, K.D. Hyde \& Maharachch.*

Barbatosphaeria Réblová (9)

Ceratostomella Sacc. (18)

Xylomelasma Réblová (4)

Papulosaceae Winka \& O.E. Erikss.

Brunneosporella V.M. Ranghoo \& K.D. Hyde (1)

Fluminicola S.W. Wong, K.D. Hyde \& E.B.G. Jones (4)

Papulosa Kohlm \& Volkm-Kohlm (1)

Wongia Khemmuk, Geering \& R.G. Shivas (3)

Rhamphoriaceae Réblová*

Rhamphoria Niessl (15)*

Rhamphoriopsis Réblová \& Gardiennet (1)*

Rhodoveronaea Arzanlou, W. Gams \& Crous (1)*

Xylolentia Réblová (1)*

Thyridiaceae O.E. Erikss \& J.Z. Yue

Pleurocytospora Petr. (3)

Thyridium Nitschke (34)

Trichosphaeriaceae G. Winter

Brachysporium Sacc. (25)

Collematospora Jeng \& Cain (1)

Coniobrevicolla Réblová (1)

Eriosphaeria Sacc. (24)

Koorchaloma Subram. (= Kananascus Nag Raj) (11)

Rizalia Syd. \& P. Syd. (6)

Schweinitziella Speg. (4)

Setocampanula Sivan. \& W.H. Hsieh (1)

Trichosphaeria Fuckel (20)

Unisetosphaeria Pinnoi, E.B.G. Jones, McKenzie \& K.D. Hyde (1)

Woswasiaceae H. Zhang, K.D. Hyde \& Maharachch.

Cyanoannulus Raja, J. Campb. \& Shearer (1)

Woswasia Jaklitsch, Réblová \& Voglmayr (1)

Xylochrysis Réblová (1)

Diaporthomycetidae genera incertae sedis

Aquapteridospora Jiao Yang, K.D. Hyde \& Maharachch. (1)

Aquaticola W.H. Ho, C.K.M. Tsui, Hodgkiss \& K.D. Hyde (5) 
Aquimonospora J. Yang \& K.D. Hyde (1)*

Fusoidispora D. Vijaykr., Jeewon \& K.D. Hyde (1)

Platytrachelon Réblová (1)

Proliferophorum G.N. Wang, H. Zhang \& Senan. (1)*

Pseudoconlarium N.G. Liu, K.D. Hyde \& J.K. Liu (1)

Pseudostanjehughesia J. Yang \& K.D. Hyde (1)

Hypocreomycetidae O.E. Erikss. \& Winka

Coronophorales Nannf. (= Melanosporales N. Zhang \& M. Blackw.)

Bertiaceae Smyk

Bertia De Not. (48)

Gaillardiella Pat. (6)

Ceratostomataceae G. Winter

Arxiomyces P.F. Cannon \& D. Hawksw. (3)

Dactylidispora Y. Marín, Stchigel, Guarro \& Cano (3)

Echinusitheca Y. Marín, Stchigel, Dania García, Guarro, A.N. Mill. \& Cano (1)

Erythrocarpon Zukal (1)

Harzia Costantin (10)

Melanospora Corda (=Gonatobotrys Corda) (69)

Microthecium Corda (=Pteridiosperma J.C. Krug \& Jeng) (ca. 20)

Pseudomicrothecium Y. Marín, Stchigel, Guarro \& Cano (1)

Pustulipora P.F. Cannon (1)

Rhytidospora Jeng \& Cain (5)

Scopinella Lév. (9)

Setiferotheca Matsush. (1)

Syspastospora P.F. Cannon \& D. Hawksw. (4)

Vittatispora P. Chaudhary, J. Campb., D. Hawksw. \& K.N. Sastry (1)

Chaetosphaerellaceae Huhndorf, A.N. Mill. \& F.A. Fernández

Chaetosphaerella E. Müll. \& C. Booth (4)

Crassochaeta Réblová (2)

Spinulosphaeria Sivan. (2)

Coronophoraceae Höhn.

Coronophora Fuckel (2)

Nitschkiaceae (Fitzp.) Nannf.

Acanthonitschkea Speg. (10)

Biciliosporina Subram. \& Sekar (1)

Botryola Bat. \& J.L. Bezerra (1)

Fracchiaea Sacc. (35)

Groenhiella Jørg. Koch, E.B.G. Jones \& S.T. Moss (1)

Janannfeldtia Subram. \& Sekar (1)

Lasiosphaeriopsis D. Hawksw. \& Sivan. (7)

Loranitschkia Lar.N. Vassiljeva (1)

Neochaetosphaerella Lar.N. Vassiljeva, S.L. Stephenson \& Chernyshev (4)

Neotrotteria Sacc. (1)

Nitschkia G.H. Otth ex P. Karst. (66)

Rhagadostoma Körb. (7)

Rhagadostomella Etayo (1)

Tortulomyces Lar.N. Vassiljeva, S.L. Stephenson, Chernyshev \& K.D. Hyde (1) 
Scortechiniaceae Huhndorf, A.N. Mill. \& F.A. Fernández

Biciliospora Petr. (1)

Coronophorella Höhn. (1)

Cryptosphaerella Sacc. (20)

Euacanthe Theiss. (2)

Neofracchiaea Teng (1)

Pseudocatenomycopsis Crous \& L.A. Shuttlew. (1)

Scortechinia Sacc. (9)

Scortechiniella Arx \& E. Müll. (1)

Scortechiniellopsis Sivan. (1)

Tympanopsis Starbäck (1)

Coronophorales genera incertae sedis

Papulaspora Preuss (33)

Sphaerodes Clem. (9)

Falcocladiales R.H. Perera, Maharachch., Somrith., Suetrong \& K.D. Hyde

Falcocladiaceae Somrith., E.B.G. Jones \& K.L. Pang

Falcocladium S.F. Silveira, Alfenas, Crous \& M.J. Wingf. (5)

Glomerellales Chadef. ex Réblová, W. Gams \& Seifert

Australiascaceae Réblová \& W. Gams

Monilochaetes Halst. ex Harter (8)

Glomerellaceae Locq. ex Seifert \& W. Gams

Colletotrichum Corda (ca. 895)

Malaysiascaceae Tibpromma \& K.D. Hyde

Malaysiasca Crous \& M.J. Wingf. (1)

Plectosphaerellaceae W. Gams, Summerb. \& Zare

Acremoniisimulans Tibpromma \& K.D. Hyde (1)

Acrostalagmus Corda (13)

Brunneochlamydosporium Giraldo López \& Crous (4)

Brunneomyces A. Giraldo, Gené \& Guarro (3)

Chlamydosporiella Giraldo López \& Crous (1)

Chordomyces Bilanenko, Georgieva \& Grum-Grzhim. (2)

Furcasterigmium Giraldo López \& Crous (1)

Fuscohypha Giraldo López \& Crous (1)

Gibellulopsis Bat. \& H. Maia (3)

Lectera P.F. Cannon (6)

Longitudinalis Tibpromma \& K.D. Hyde (1)

Musicillium Zare \& W Gams (2)

Musidium Giraldo López \& Crous (1)

Nigrocephalum Giraldo López \& Crous (1)

Paragibellulopsis Giraldo López \& Crous (1)

Paramusicillium Giraldo López \& Crous (1)

Phialoparvum Giraldo López \& Crous (1)

Plectosphaerella Kleb. (17)

Sayamraella Giraldo López \& Crous (1)

Sodiomyces A.A. Grum-Grzhim., Debets \& Bilanenko (5)

Stachylidium Link (7) 
Summerbellia Giraldo López \& Crous (1)

Theobromium Giraldo López \& Crous (1)

Verticillium Nees (81)

Reticulascaceae Réblová \& W. Gams

Blastophorum Matsush. (5)

Cylindrotrichum Bonord. (23)

Kylindria DiCosmo, S.M. Berch \& W.B. Kendr. (11)

Sporoschismopsis Hol-Jech. \& Hennebert (8)

Glomerellales genus incertae sedis

Ascocodinaea Samuels, Cand. \& Magni (2)

Hypocreales Lindau

Bionectriaceae Samuels \& Rossman

Acremonium Link (ca. 150)

Anthonectria Döbbeler (1)

Aphanotria Döbbeler (1)

Battarrina (Sacc.) Clem. \& Shear (1)

Bryocentria Döbbeler (15)

Bryotria Döbbeler \& P.G. Davison (2)

Bullanockia Crous (1)

Chrysonectria Lechat \& J. Fourn. (1)*

Clibanites (P. Karst.) P. Karst. (1)

Clonostachys Corda (78)

Dimerosporiella Speg. (8)

Fusariella Sacc. (17)

Geonectria Lechat \& J. Fourn. (1)*

Geosmithia J. Pitt (24)

Gliomastix Guég. (24)

Globonectria Etayo (1)

Gracilistilbella Seifert (4)

Halonectria E.B.G. Jones (1)

Heleococcum P.M. Jørg. (5)

Hydropisphaera Dumort (29)

Ijuhya Starbäck (22)

Kallichroma Kohlm. \& Volkm.-Kohlm. (4)

Laniatria Döbbeler \& P.G. Davison (1)

Lasionectria (Sacc.) Cooke (23)

Lasionectriella Lechat \& J. Fourn. (2)*

Mycoarachis Malloch \& Cain (2)

Mycocitrus Möller (3)

Nectriella Nitschke ex Fuckel (84)

Nectriopsis Maire (70)

Nigrosabulum Malloch \& Cain (1)

Ochronectria Rossman \& Samuels (3)

Ovicuculispora Etayo (2)

Paracylindrocarpon Crous, Roets \& L. Lombard (4)

Paranectria Sacc. (4)

Periantria Döbbeler \& P.G. Davison (2)

Peristomialis (W. Phillips) Boud. (6)

Pronectria Clem. (44) 
Protocreopsis Yoshim Doi (12)

Roumegueriella Speg. (4)

Selinia P. Karst. (6)

Stephanonectria Schroers \& Samuels (1)

Stilbocrea Pat. (7)

Stromatonectria Jaklitsch \& H. Voglmayr (1)

Synnemellisia N.K. Rao, Manohar. \& Goos (2)

Trichonectria Kirschst. (19)

Verrucostoma Hirooka, Tak. Kobay. \& P. Chaverri (2)

Xanthonectria Lechat, J. Fourn. \& P.-A. Moreau (1)*

Calcarisporiaceae Jing Z. Sun, X.Z. Liu \& K.D. Hyde

Calcarisporium Preuss (8)

Clavicipitaceae (Lindau) Earle ex Rogerson

Aciculosporium I. Miyake (=Neoclaviceps J.F. White, Bills, S.C. Alderman \& Spatafora) (4)

Aschersonia Mont. (=Hypocrella Sacc. fide Hyde et al. 2020) (170+)

Atkinsonella Diehl (2)

Balansia Speg. (49)

Cavimalum Yoshim. Doi, Dargan \& K.S. Thind (2)

Claviceps Tul. (111)

Collarina A. Giraldo, Gené \& Guarro (1)

Conoideocrella D. Johnson, G.H. Sung, Hywel-Jones \& Spatafora (3)

Corallocytostroma Y.N. Yu \& Z.Y. Zhang (2)

Dussiella Pat. (3)

Ephelis Fr. (4)

Epichlö̈ (Fr.) Tul. \& C. Tul. (75)

Epicrea Petr. (1)

Helicocollum Luangsa-ard (3)

Helminthascus Tranzschel (1)

Heteroepichloë E. Tanaka, C. Tanaka, Gafur \& Tsuda (2)

Konradia Racib. (2)

Loculistroma F. Patt \& Charles (1)

Metapochonia Kepler, S.A. Rehner \& Humber (6)

Metarhiziopsis D.W. Li, R.S. Cowles \& C.R. Vossbrinck (1)

Metarhizium Sorokīn (= Chamaeleomyces Sigler; = Metacordyceps G.H. Sung, J.M. Sung, Hywel-Jones \& Spatafora; = Nomuraea Maubl.; = Stereocrea Syd. \& P. Syd.) (78)

Moelleriella Bres. (57)

Mycomalus A. Möller (1)

Mycophilomyces Crous \& M.J. Wingf. (1)

Myriogenospora G.F. Atk. (4)

Neobarya Lowen (12)

Neocordyceps Kobayasi (1)

Nigelia Luangsa-ard (2)

Nigrocornus Ryley \& Langdon (1)

Orbiocrella D. Johnson, G.H. Sung, Hywel-Jones \& Spatafora (1)

Parepichloë J.F. White \& P.V. Reddy (4)

Periglandula U. Steiner, E. Leistner \& Leuchtm. (2)

Pochonia Bat. \& O.M. Fonseca (4)

Pseudomeria G.L. Barron (1)

Regiocrella Chaverri \& K.T. Hodge (2)

Romanoa Thirum. (1) 
Rotiferophthora G.L. Barron (27)

Samuelsia Chaverri \& K.T. Hodge (6)

Shimizuomyces Kobayasi (2)

Sphaerocordyceps Kobayasi (2)

Tyrannicordyceps Kepler \& Spatafora (5)

Ustilaginoidea Bref. (19)

Cocoonihabitaceae W.Y. Zhuang \& Z.Q. Zeng Cocoonihabitus W.Y. Zhuang \& Z.Q. Zeng (1)

Cordycipitaceae Kreisel ex G.H. Sung, J.M. Sung, Hywel-Jones \& Spatafora Akanthomyces Lebert (= Torrubiella Boud., = Lecanicillium W. Gams \& Zare) (21)* Amphichorda Fr. (1)

Ascopolyporus Möller (7)

Beauveria Vuill. (54)

Beejasamuha Subram. \& Chandrash. (1)

Blackwellomyces Spatafora \& Luangsa-ard (2)

Cordyceps (Fr.) Link (= Isaria Pers.; = Microhilum H.Y. Yip \& A.C. Rath) (498)

Coremiopsis Sizova \& Suprun (2)

Engyodontium de Hoog (5)

Gibellula Cavara (=Granulomanus de Hoog \& Samson) (29)

Hevansia Luangsa-ard, Hywel-Jones \& Spatafora (8)

Hyperdermium J.F. White, R.F. Sullivan, Bills \& Hywel-Jones (3)

Leptobacillium Zare \& W. Gams (1)

Parengyodontium C.C. Tsang, J.F.W. Chan, W.M. Pong, J.H.K. Chen, A.H.Y. Ngan, M.

Cheung, C.K.C. Lai, D.N.C. Tsang, S.K.P. Lau \& P.C.Y. Woo (1)

Pseudogibellula Samson \& H.C. Evans (1)

Samsoniella Mongkols., Noisrip., Thanakitp., Spatafora \& Luangsa-ard (3)

Simplicillium W. Gams \& Zare (12)

Flammocladiellaceae Crous, L. Lombard \& R.K. Schumach.

Flammocladiella Crous, L. Lombard \& R.K. Schumach. (2)

\section{Hypocreaceae De Not.}

Arachnocrea Z. Moravec. (3)

Dialhypocrea Speg. (1)

Escovopsioides H.C. Evans \& J.O. Augustin (1)

Escovopsis J.J. Muchovej \& Della Lucia (14)

Hypocreopsis P. Karst. (14)

Hypomyces (Fr.) Tul. \& C. Tul. (ca. 150)

Kiflimonium Summerb., J.A. Scott, Guarro \& Crous (1)

Lichenobarya Etayo, Diederich \& Lawrey (1)

Mycogone Link (28)

Protocrea Petch (6)

Rogersonia Samuels \& Lodge (1)

Sepedonium Link (13)

Sphaerostilbella (Henn.) Sacc. \& D. Sacc (13)

Sporophagomyces K. Põldmaa \& Samuels (3)

Stephanoma Wallr. (?6)

Trichoderma Pers. (400+)

Verticimonosporium Matsush. (3) 
Myrotheciomycetaceae Crous

Emericellopsis J.F.H. Beyma (23)

Leucosphaerina Arx (2)

Myrotheciomyces Crous (1)

Trichothecium Link (9)

Nectriaceae Tul. \& C. Tul.

Albonectria Rossman \& Samuels (1)

Allantonectria Earle (1)

Allonectella Petr. (2)

Aphanocladium W. Gams (4)

Aquanectria L. Lombard \& Crous (3)

Atractium Link (3)*

Baipadisphaeria Pinruan (1)

Bisifusarium L. Lombard, Crous \& W. Gams (7)

Calonectria De Not. (400)

Calostilbe Sacc. \& Syd. (4)

Campylocarpon Halleen, Schroers \& Crous (3)

Chaetonectrioides Matsush. (1)

Chaetopsina Rambelli (19)

Coccinonectria Lombard \& Crous (2)

Corallomycetella Henn. (4)

Corallonectria C. Herrera \& P. Chaverri (1)

Corinectria C. González \& P. Chaverri (3)

Cosmospora Rabenh. (50)

Cosmosporella S.K. Huang, R. Jeewon \& K.D. Hyde (1)

Curvicladiella Decock \& Crous (1)

Cyanochyta Höhn. (1)

Cyanonectria Samuels \& Chaverri (2)

Cyanophomella Höhn. (1)

Cylindrocladiella Boesew. (45)

Cylindrodendrum Bonord. (4)

Dacryoma Samuels (2)

Dactylonectria L. Lombard \& Crous (14)

Dematiocladium Allegr., Aramb., Cazau \& Crous (2)

Fusarium Link (ca. 120)

Fusicolla Bonord (18)

Geejayessia Schroers, Gräfenhan \& Seifert (7)

Gliocephalotrichum J.J. Ellis \& Hesselt. (13)

Gliocladiopsis S.B. Saksena (15)

Ilyonectria P. Chaverri \& C. Salgado (23)

Macroconia (Wollenw.) Gräfenhan, Seifert \& Schroers (5)

Mariannaea G. Arnaud ex Samson (22)

Microcera Desm. (4)

Murinectria M. Niranjan \& V.V. Sarma (4)

Nalanthamala Subram. (6)

Nectria (Fr.) Fr. (29)

Neocosmospora E.F. Sm. (84)

Neonectria Wollenw. (30)

Neothyronectria Crous \& Thangavel (2)

Ophionectria Sacc. (39)

Pandanaceomyces Tibpromma \& K.D. Hyde (1) 
Paracremonium L. Lombard \& Crous (5)

Payosphaeria W.F. Leong (1)

Penicillifer Emden (7)

Persiciospora P.F. Cannon \& D. Hawksw. (4)

Pleiocarpon L. Lombard \& D. Aiello (3)

Pleogibberella Sacc. (3)

Pleurocolla Petr. (1)

Pseudoachroiostachys Tibpromma \& K.D. Hyde (1)

Pseudocosmospora C. Herrera \& P. Chaverri (13)

Pseudonectria Seaver (17)

Rectifusarium L. Lombard, Crous \& W. Gams (2)

Rugonectria P. Chaverri \& Samuels (5)

Sarcopodium Ehrenb. (22)

Stylonectria Höhn. (5)

Thelonectria P. Chaverri \& C.G. Salgado (46)

Thyronectria Sacc. (41)

Varicosporella Lechat \& J. Fourn. (1)

Varicosporellopsis Lechat \& J. Fourn. (1)*

Volutella Fr. (127)

Xenoacremonium Lombard \& Crous (2)

Xenocylindrocladium Decock, Hennebert \& Crous (3)

Xenogliocladiopsis Crous \& W.B. Kendr. (2)

Xenoleptographium Marinc., T.A. Duong, Z.W. de Beer \& M.J. Wingf. (1)

Xenonectriella Weese (18)

Niessliaceae Kirschst.

Atronectria Etayo (1)

Circinoniesslia Samuels \& M.E. Barr (1)

Cryptoniesslia Scheuer (1)

Eucasphaeria Crous (2)

Hyaloseta A.W. Ramaley (1)

Malmeomyces Starb. (1)

Melchioria Penz. \& Sacc. (6)

Miyakeomyces Hara (1)

Myrmaeciella Lindau (2)

Myrtacremonium Crous (1)

Neoeucasphaeria Crous (1)

Niesslia Auersw. (43)

Paraniesslia K.M. Tsui, K.D. Hyde \& Hodgkiss (2)

Pseudohyaloseta Tibpromma \& K.D. Hyde (1)

Pseudonectriella Petr. (1)

Pseudorhynchia Höhn. (2)

Rosasphaeria Jaklitsch \& Voglmayr (1)

Taiwanascus Sivan \& H.S. Chang (2)

Trichosphaerella E. Bommer, M. Rousseau \& Sacc. (=Neorehmia Höhn.; = Oplothecium

Syd.) (4)

Valetoniella Höhn. (3)

Valetoniellopsis Samuels \& M.E. Barr (1)

Ophiocordycipitaceae G.H. Sung, J.M. Sung, Hywel-Jones \& Spatafora

Drechmeria W. Gams \& H.B. Jansson (12)

Harposporium Lohde (37) 
Hirsutella Pat. (50+)

Hymenostilbe Petch (12)

Ophiocordyceps Petch (263)

Paraisaria Samson \& B.L. Brady (11)

Perennicordyceps Matočec \& I. Kušan (4)*

Polycephalomyces Kobayasi (18)*

Purpureocillium Luangsa-ard, Hywel-Jones, Houbraken \& Samson (5)

Tolypocladium W. Gams (47)

Sarocladiaceae L. Lombard

Parasarocladium Summerb., J.A. Scott, Guarro \& Crous (4)

Sarocladium W. Gams \& D. Hawksw. (22)

Stachybotryaceae L. Lombard \& Crous

Achroiostachys L. Lombard \& Crous (6)

Albifimbria L. Lombard \& Crous (5)

Albosynnema E.F. Morris (2)

Alfaria Crous, Montaño-Mata \& García-Jim. (13)

Alfariacladiella Crous \& R.K. Schumach. (1)

Brevistachys L. Lombard \& Crous (5)

Capitofimbria L. Lombard \& Crous (1)

Cymostachys L. Lombard \& Crous (3)

Didymostilbe Henn. (14)

Digitiseta Gordillo \& Decock (4)*

Dimorphiseta L. Lombard \& Crous (1)

Globobotrys L. Lombard \& Crous (1)

Grandibotrys L. Lombard \& Crous (3)

Gregatothecium L. Lombard \& Crous (1)

Hyalinostachys C.G. Lin \& K.D. Hyde (1)

Inaequalispora L. Lombard \& Crous (3)

Kastanostachys L. Lombard \& Crous (1)

Koorchalomella Chona, Munjal \& J.N. Kapoor (2)

Melanopsamma Niessl (ca. 5)

Memnoniella Höhn. (9)

Myrothecium Tode (2)

Myxospora L. Lombard \& Crous (6)

Neomyrothecium L. Lombard \& Crous (1)

Paramyrothecium L. Lombard \& Crous (14)

Parasarcopodium Melnik, S.J. Lee \& Crous (3)

Parvothecium L. Lombard \& Crous (2)

Peethambara Subram. \& Bhat (1)

Pseudoornatispora Tibpromma \& K.D. Hyde (1)

Septomyrothecium Matsush. (4)

Sirastachys L. Lombard \& Crous (9)

Smaragdiniseta L. Lombard \& Crous (1)

Stachybotrys Corda (12 phylogenetically studied, 81 epithets remain be studied)

Striatibotrys L. Lombard \& Crous (7)

Striaticonidium L. Lombard \& Crous (5)

Tangerinosporium L. Lombard \& Crous (1)

Virgatospora Finley (2)

Xenomyrothecium L. Lombard \& Crous (1)

Xepicula Nag Raj (4) 
Xepiculopsis Nag Raj (2)

Tilachlidiaceae Lombard \& Crous

Psychronectria J. Pawłowska, Istel, Wrzosek, D. Hawksw. (47)

Septofusidium W. Gams (5)

Tilachlidium Preuss (1)

Hypocreales genera incertae sedis

Acremoniopsis A. Giraldo, Gené \& Guarro (1)

Berkelella (Sacc.) Sacc. (2)

Bulbithecium Udagawa \& T Muroi (1)

Cephalosporiopsis Peyronel (10)

Chondronectria Etayo, Flakus \& Kukwa (1)

Cylindronectria Etayo (1)

Diploöspora Grove (ca. 7)

Gynonectria Döbbeler (1)

Hapsidospora Malloch \& Cain (2)

Haptospora G.L. Barron (3)

Illosporiopsis D. Hawksw. (1)

Illosporium Mart. (17)

Leptobarya Etayo (2)

Lichenopenicillus Etayo (1)

Metadothella Henn. (1)

Munkia Speg. (4)

Neomunkia Petr. (1)

Peloronectria Möller (3)

Pseudoacremonium Crous (1)

Pseudoidriella Crous \& R.G. Shivas (1)

Pseudomeliola Speg. (10)

Rodentomyces Doveri, Pecchia, Sarrocco \& Vannacci (1)

Roselliniella Vain (19)

Saksenamyces A.N. Rai \& P.N. Singh (1)

Sedecimiella K.L. Pang, Alias \& E.B.G. Jones (1)

Stanjemonium W. Gams, O'Donnell, Schroers \& M. Chr. (4)

Stilbella Lindau (61)

Ticonectria Döbbeler (3)

Tilakidium Vaidya, C.D. Naik \& Rathod (1)

Jobellisiales M.J. D’souza \& K.D. Hyde

Jobellisiaceae Réblová

Jobellisia M.E. Barr (8)

Microascales Luttr. ex Benny \& Kimbr.

Ceratocystidaceae Locq. ex Réblová, W. Gams \& Seifert

Ambrosiella Brader ex Arx \& Hennebert (10)

Berkeleyomyces W.J. Nel, Z.W. de Beer, T.A. Duong \& M.J. Wingf. (2)

Bretziella Z.W. de Beer, Marinc., T.A. Duong \& M.J. Wingf. (1)

Ceratocystis Ellis \& Halst. (105)

Chalaropsis Peyronel (3)

Davidsoniella Z.W. de Beer, T.A. Duong \& M.J. Wingf. (4)

Endoconidiophora Münch (9)

Huntiella Z.W. de Beer, T.A. Duong \& M.J. Wingf. (29) 
Meredithiella McNew, C. Mayers \& T.C. Harr. (3)

Phialophoropsis L.R. Batra emend. T.C. Harr. (2)

Thielaviopsis Went. (7)

Chadefaudiellaceae Faurel \& Schotter ex Benny \& Kimbr.

Chadefaudiella Faurel \& Schotter (2)

Faurelina Locq-Lin. (4)

Gondwanamycetaceae Réblová, W. Gams \& Seifert

Custingophora Stolk (1)

Knoxdaviesia M.J. Wingf., P.S. van Wyk \& Marasas. (5)

Graphiaceae De Beer

Graphium Corda (20)

Halosphaeriaceae E. Müll \& Arx ex Kohlm.

Alisea J. Dupont \& E.B.G. Jones (1)

Amphitrite S. Tibell (1)

Aniptodera Shearer \& M. Miller (21)

Aniptosporopsis (K.D. Hyde) K.L. Pang (1)

Anisostagma K.R.L. Petersen \& Jørg. Koch (1)

Antennospora Meyers (2)

Appendichordella R.G. Johnson, E.B.G. Jones \& S.T. Moss (1)

Arenariomyces Höhnk (5)

Ascosacculus J. Campbell, J.L. Anderson \& Shearer (1)

Bathyascus Kohlm. (5)

Carbosphaerella I. Schmidt (2)

Ceriosporopsis Linder (9)

Chadefaudia Feldm.-Maz. (6)

Corallicola Volkm.-Kohlm. \& Kohlm. (1)

Corollospora Werderm (= Cirrenalia Meyers \& R.T. Moore; = Sigmoidea J.L. Crane) (25)

Cucullosporella K.D. Hyde \& E.B.G. Jones (1)

Ebullia K.L. Pang (1)

Fluviatispora K.D. Hyde (3)

Gesasha Abdel-Wahab \& Nagah. (3)

Haiyanga K.L. Pang \& E.B.G. Jones (1)

Haligena Kohlm. (1)

Halosarpheia Kohlm. \& E. Kohlm. (8)

Halosphaeria Linder (1)

Halosphaeriopsis T.W. Johnson (1)

Havispora K.L. Pang \& Vrijmoed (1)

Iwilsoniella E.B.G.Jones (1)

Kitesporella Jheng \& K.L. Pang (1)

Kochiella Sakay., K.L. Pang \& E.B.G. Jones (1)

Lautisporopsis E.B.G. Jones, Yusoff \& S.T. Moss (1)

Lignincola Höhnk (2)

Limacospora Jørg. Koch \& E.B.G. Jones (1)

Luttrellia Shearer (4)

Magnisphaera J. Campb., J.L. Anderson \& Shearer (2)

Marinospora A.R. Caval. (2)

Moana Kohlm. \& Volkm.-Kohlm. (1)

Morakotiella Sakay. (1) 
Naïs Kohlm. (3)

Natantispora J. Campb., J.L. Anderson \& Shearer (3)

Nautosphaeria E.B.G. Jones (1)

Neptunella K.L. Pang \& E.B.G. Jones (1)

Nereiospora E.B.G. Jones, R.G. Johnson \& S.T. Moss. (2)

Nimbospora Jørg. Koch (1)

Nohea Kohlm. \& Volkm.-Kohlm. (3)

Oceanitis Kohlm. (4)

Ocostaspora E.B.G. Jones, R.G. Johnson \& S.T. Moss (1)

Okeanomyces K.L. Pang \& E.B.G. Jones (1)

Ondiniella E.B.G. Jones, R.G. Johnson \& S.T. Moss (1)

Ophiodeira Kohlm. \& Volkm.-Kohlm. (1)

Paraaniptodera K.L. Pang, C.L. Lu, W.T. Ju \& E.B.G. Jones (1)

Phaeonectriella R.A. Eaton \& E.B.G. Jones (1)

Praelongicaulis E.B.G. Jones, Abdel-Wahab \& K.L. Pang (1)

Panorbis J. Campb., J.L. Anderson \& Shearer (1)

Pileomyces K.L. Pang \& Jheng (1)

Pseudolignincola Chatmala \& E.B.G. Jones (1)

Remispora Linder (5)

Saagaromyces K.L. Pang \& E.B.G. Jones (3)

Sablicola E B.G. Jones, K.L. Pang \& Vrijmoed (1)

Thalassogena Kohlm. \& Volkm.-Kohlm. (1)

Thalespora Chatmala \& E.B.G. Jones (1)

Tinhaudeus K.L. Pang, S.Y. Guo \& E.B.G. Jones (1)

Tirispora E.B.G. Jones \& Vrijmoed (1)

Toriella Sakay., K.L. Pang \& E.B.G. Jones (1)

Trailia G.K. Sutherl. (1)

Trichomaris Hibbits, G.C. Hughes \& Sparks (1)

Tubakiella Sakay., K.L. Pang \& E.B.G. Jones (1)

Tunicatispora K.D. Hyde (1)

\section{Microascaceae Luttr. ex Malloch}

Acaulium Sopp (4)

Brachyconidiellopsis Decock, R.F. Castañeda \& Adhikari (1)

Canariomyces Arx (3)

Cephalotrichum Link (37)

Doratomyces Corda (3)

Echinobotryum Corda (2)

Enterocarpus Locq.-Lin. (2)

Fairmania Sacc. (1)

Gamsia M. Morelet (5)

Kernia Nieuwl. (14)

Lomentospora Hennebert \& B.G. Desai (1)

Lophotrichus R.K. Benj. (8)

Microascus Zukal (60)

Parascedosporium Gilgado, Gené, Cano \& Guarro (2)

Petriella Curzi (8)

Pseudallescheria Negroni \& I. Fisch. (8)

Pseudoscopulariopsis Sand.-Den., Gené \& Guarro (2)

Rhinocladium Sacc. \& Marchal (11)

Scedosporium Sacc. ex Castell. \& Chalm. (12)

Scopulariopsis Bainier (87) 
Wardomyces F.T. Brooks \& Hansf. (11)

Wardomycopsis Udagawa \& Furuya (5)

Yunnania H.Z. Kong (3)

Triadelphiaceae Y.Z. Lu, J.K. Liu, Z.L. Luo \& K.D. Hyde

Synnematotriadelphia Chuaseehar., Somrith., Nuankaew \& Boonyuen (2)

Triadelphia Shearer \& J.L. Crane (18)*

Microascales genera incertae sedis

Bisporostilbella Brandsb. \& E.F. Morris (1)

Cephalotrichiella Crous (1)

Cornuvesica C.D. Viljoen, M.J. Wingf. \& K. Jacobs (4)

Gabarnaudia Samson \& W. Gams (2)

Sporendocladia G. Arnaud ex Nag Raj \& W.B. Kendr. (7)

Pararamichloridiales Crous

Pararamichloridiaceae Crous

Pararamichloridium Crous (2)

Torpedosporales E.B.G. Jones, Abdel-Wahab \& K.L. Pang

Etheirophoraceae Rungjind., Somrith. \& Suetrong

Etheirophora Kohlm. \& Volkm.-Kohlm. (3)

Swampomyces Kohlm. \& Volkm. (2)

Juncigenaceae E.B.G. Jones, Abdel-Wahab \& K.L. Pang

Elbamycella A. Poli, E. Bovio, V. Prigione \& G.C. Varese (1)

Fulvocentrum E.B.G. Jones \& Abdel-Wahab (3)

Juncigena Kohlm Kohlm., Volkm.-Kohlm. \& O.E. Erikss. (2)

Khaleijomyces Abdel-Wahab (1)*

Marinokulati E.B.G. Jones \& K.L. Pang (1)

Moheitospora Abdel-Wahab, Abdel-Aziz \& Nagah. (2)

Torpedosporaceae E.B.G. Jones \& K.L. Pang

Torpedospora Meyers (3)

Hypocreomycetidae genera incertae sedis

Campylospora Ranzoni (5)

Dendroclathra Voglmayr \& G. Delgado (2)

Lulworthiomycetidae Dayar., E.B.G. Jones \& K.D. Hyde

Koralionastetales Kohlm., Volkm.-Kohlm., J. Campb. \& Inderb.

Koralionastetaceae Kohlm. \& Volkm.-Kohlm.

Koralionastes Kohlm. \& Volkm.-Kohlm. (5)

Pontogeneia Kohlm. (8)

Lulworthiales Kohlm., Spatafora \& Volkm.-Kohlm.

Lulworthiaceae Kohlm., Spatafora \& Volkm.-Kohlm.

Cumulospora I. Schmidt (2)

Halazoon Abdel-Aziz, Abdel-Wahab \& Nagah. (2)

Haloguignardia A. Cribb \& J. Cribb (1)

Hydea K.L. Pang \& E.B.G Jones (1)

Kohlmeyeriella E.B.G. Jones, R.G. Johnson \& S.T. Moss (2) 
Lindra I. Wilson (2)

Lulwoana Kohlm., Volkm.-Kohlm., J. Campb., Spatafora \& Gräfenhan (1)

Lulwoidea Kohlm., Volkm.-Kohlm., J. Campb., Spatafora \& Gräfenhan (1)

Lulworthia G.K. Sutherl(32)

Matsusporium E.B.G. Jones \& K.L. Pang (1)

Moleospora Abdel-Wahab, Abdel-Aziz \& Nagah (1)

Moromyces Abdel-Wahab, K.L. Pang, Nagah., Abdel-Aziz \& E.B.G. Jones (1)

Orbimyces Linder (1)

Rostrupiella Jørg Koch, K.L. Pang \& E.B.G. Jones. (1)

Sammeyersia S.Y. Guo, E.B.G. Jones \& K.L. Pang (1)

Pisorisporiomycetidae Bundhun, Maharachch. \& K.D. Hyde

Pisorisporiales Réblová \& J. Fourn.

Pisorisporiaceae Réblová \& J. Fourn.

Achroceratosphaeria Réblová, Fourn., K.D. Hyde \& Ranghoo (2)

Pisorisporium Réblová \& J. Fourn. (2)

Savoryellomycetidae Hongsanan, K.D. Hyde \& Maharachch.

Conioscyphales Réblová \& Seifert

Conioscyphaceae Réblová \& Seifert

Conioscypha Höhn. (16)

Fuscosporellales Jing Yang, Bhat \& K.D. Hyde

Fuscosporellaceae Jing Yang, Bhat \& K.D. Hyde

Bactrodesmiastrum Hol.-Jech. (5)

Fuscosporella Jing Yang (2)

Mucispora Jing Yang (2)

Parafuscosporella Jing Yang \& K.D. Hyde (3)

Plagiascoma Réblová \& J. Fourn. (1)

Pseudoascotaiwania Jing Yang, Bhat \& K.D. Hyde (1)

Pleurotheciales Réblová \& Seifert

Pleurotheciaceae Réblová \& Seifert

Adelosphaeria Réblová (1)

Anapleurothecium Hern.-Restr., R.F. Castañeda \& Gené (1)

Helicoön Morgan (28)

Melanotrigonum Réblová (1)

Monotosporella S. Hughes (4)

Neomonodictys Y.Z. Lu, C.G. Lin \& K.D. Hyde (1)

Phaeoisaria Höhn. (23)

Phragmocephala E.W. Mason \& S. Hughes (15)

Pleurotheciella Réblová (11)

Pleurothecium Höhn. (11)

Sterigmatobotrys Oudem. (6)

Savoryellales Boonyuen, Suetrong, Sivichai, K.L. Pang \& E.B.G. Jones

Savoryellaceae Jaklitsch \& Réblová

Ascotaiwania Sivan. \& H.S. Chang (= Neoascotaiwania Hern.-Restr., R.F. Castañeda \& Guarro fide Dayarathne et al. 2019) (9)

Canalisporium Nawawi \& Kuthub. (= Ascothailandia Sri-indr., Boonyuen, Sivichai \& E.B.G. Jones) (15)

Rhexoacrodictys W.A. Baker \& Morgan-Jones (5)* 
Savoryella E.B.G. Jones \& R.A. Eaton (11)

Sordariomycetidae O.E. Erikss \& Winka (= Meliolomycetidae P.M. Kirk \& K.D. Hyde)* Boliniales P.F. Cannon

Boliniaceae Rick

Apiocamarops Samuels \& J.D. Rogers (4)

Apiorhynchostoma Petr. (4)

Camaropella Lar.N. Vassiljeva (2)

Camarops P. Karst. (= Bolinia (Nitschke) Sacc.) (28)

Cornipulvina Huhndorf, A.N. Mill., F.A. Fernández \& Lodge (1)

Endoxyla Fuckel(3)

Mollicamarops Lar.N. Vassiljeva (1)

Neohypodiscus J.D. Rogers, Y.M. Ju \& Læssøe (3)

Pseudovalsaria Spooner (3)

Cephalothecales Maharachch. \& K.D. Hyde

Cephalothecaceae Höhn.

Albertiniella Kirschst. (2)

Cephalotheca Fuckel (ca. 10)

Cryptendoxyla Malloch \& Cain (2)

Phialemonium W. Gams \& McGinnis (6)

Victoriomyces D. Davolos, B. Pietrangeli, A.M. Persiani \& O. Maggi (1)

Chaetosphaeriales Huhndorf, A.N. Mill. \& F.A. Fernández

Chaetosphaeriaceae Réblová, M.E. Barr \& Samuels

Adautomilanezia Gusmão, S.S. Silva, Fiuza, L.A. Costa \& T.A.B. Santos (1)

Anacacumisporium Y.R. Ma \& X.G. Zhang (1)

Ascochalara Réblová (1)

Bahusutrabeeja Subram. \& Bhat (6)

Brunneodinemasporium Crous \& R.F. Castañeda (2)

Catenularia Grove (13)

Chaetosphaeria Tul. \& C. Tul. (ca. 150)

Chloridium Link (= Melanopsammella Höhn.) (ca. 30)

Codinaea Maire (15)

Conicomyces R.C. Sinclair, Eicker \& Morgan-Jones (4)

Craspedodidymum Hol.-Jech. (14)

Cryptophiale Piroz. (ca. 20)

Cryptophialoidea Kuthub. \& Nawawi (5)

Dendrophoma Sacc. (ca. 100)

Dictyochaeta Speg. (84)

Dictyochaetopsis Aramb. \& Cabello (14)

Dinemasporium Lév. (35)

Eucalyptostroma Crous \& M.J. Wingf. (2)

Exserticlava S. Hughes (7)

Hemicorynespora M.B. Ellis (12)

Infundibulomyces Plaingam, Somrith. \& E.B.G. Jones (2)

Kionochaeta P.M. Kirk \& B. Sutton (13)

Lecythothecium Réblová \& Winka (1)

Menispora Pers. (14)

Menisporopsis S. Hughes (ca. 10)

Miyoshiella Kawam. (3)

Morrisiella Saikia \& A.K. Sarbhoy (1) 
Nawawia Marvanová (7)

Neopseudolachnella A. Hashim. \& Kaz. Tanaka (3)

Paliphora Sivan. \& B. Sutton (7)

Phialosporostilbe Mercado \& J. Mena (5)

Polynema Lév. (13)

Pseudodinemasporium A. Hashim. \& Kaz. Tanaka (1)

Pseudolachnea Ranoj. (5)

Pseudolachnella Teng (18)

Pyrigemmula D. Magyar \& R. Shoemaker (1)

Rattania Prabhug. \& Bhat (1)

Sporoschisma Berk. \& Broome (15)

Striatosphaeria Samuels \& E. Müll. (1)

Tainosphaeria F.A. Fernández \& Huhndorf (3)

Thozetella Kuntze (22)

Umbrinosphaeria Réblová (1)

Verhulstia Hern.-Rest. (1)

Zanclospora S. Hughes \& W.B. Kendr. (10)

Helminthosphaeriaceae Samuels, Cand. \& Magni.

Echinosphaeria A.N. Mill. \& Huhndorf (14)

Endophragmiella B. Sutton (ca. 80)

Helminthosphaeria Fuckel (ca. 20)

Hilberina Huhndorf \& A.N. Mill. (ca. 20)

Ruzenia O. Hilber (1)

Synaptospora Cain (5)

Tengiomyces Réblová (1)

Leptosporellaceae Konta \& K.D. Hyde

Leptosporella Penz. \& Sacc. (17)

Linocarpaceae Konta \& K.D. Hyde

Linocarpon Syd. \& P. Syd. (42)

Neolinocarpon K.D. Hyde (13)

Chaetosphaeriales genera incertae sedis

Calvolachnella Marinc., T.A. Duong \& M.J. Wingf. (1)

Caudatispora J. Fröhl. \& K.D. Hyde (2)

Erythromada Huhndorf, A.N. Mill., F.A. Fernández \& Lodge (1)

Lasiosphaeriella Sivan. (6)

Neoleptosporella Phukhams., Perera \& K.D. Hyde (2)

Neonawawia Jing Yang, K.D. Hyde \& J.K. Liu (1)

Rimaconus Huhndorf, F.A. Fernández, Joanne E. Taylor \& K.D. Hyde (2)

Coniochaetales Huhndorf, A.N. Mill. \& F.A. Fernández (= Cordanales M. Hern.-Rest. \& Crous)

Coniochaetaceae Malloch \& Cain

Barrina A.W. Ramaley (1)

Coniochaeta (Sacc.) Cooke (82)

\section{Cordanaceae Nann.}

Cordana Preuss (19)

Coniochaetales genera incertae sedis 
Cannonia J.E. Taylor \& K.D. Hyde (1)

Pseudogliomastix W. Gams (1)

Meliolales Gäum. ex D. Hawksw. \& O.E. Erikss.

Armatellaceae Hosag.

Armatella Theiss. \& Syd. (19)

Meliolaceae G.W. Martin ex Hansf.

Amazonia Theiss. (60)

Appendiculella Höhn. (70)

Asteridiella McAlpine (2)

Cryptomeliola S. Hughes \& Piroz. (3)

Endomeliola S. Hughes \& Piroz. (1)

Irenopsis F. Stevens (150)

Meliola Fr. (1700)

Setameliola D.R. Reynolds (17)

Phyllachorales M.E. Barr

Phaeochoraceae K.D. Hyde, P.F. Cannon \& M.E. Barr

Cocoicola K.D. Hyde (5)

Phaeochora Höhn. (4)

Phaeochoropsis K.D. Hyde \& P.F. Cannon (4)

Serenomyces Petr. (4)

Phaeochorellaceae Guterres, Galvão-Elias \& Dianese

Phaeochorella Theiss. \& Syd. (6)

Phyllachoraceae Theiss. \& H. Syd.

Ascovaginospora Fallah, Shearer \& W.D. Chen (1)

Brobdingnagia K.D. Hyde \& P.F. Cannon (4)

Camarotella Theiss. \& Syd. (8)

Coccodiella Hara (27)

Cyclodomus Höhn. (5)

Deshpandiella Kamat \& Ullasa (1)

Diachora Müll. Arg. (4)

Diatractium Syd. \& P. Syd. (4)

Erikssonia Penz. \& Sacc. (5)

Fremitomyces P.F. Cannon \& H.C. Evans (2)

Geminispora Pat. (2)

Gibellina Pass. Ex Roum. (2)

Imazekia Tak. Kobay. \& Y. Kawabe (1)

Isothea Fr. (4)

Lichenochora Hafellner (44)

Lindauella Rehm (1)

Linochora Höhn. (37)

Lohwagia Petr. (3)

Maculatifrondes K.D. Hyde (1)

Malthomyces K.D. Hyde \& P.F. Cannon (2)

Muelleromyces Kamat \& Anahosur (1)

Neoflageoletia J. Reid \& C. Booth (1)

Neophyllachora Dayar. \& K.D. Hyde (4)

Ophiodothis Sacc. (6) 
Ophiodothella (Henn.). Höhn. (31)

Orphnodactylis Malloch \& Mallik (2)

Oxodeora K.D. Hyde \& P.F. Cannon (1)

Parberya C.A. Pearce \& K.D. Hyde (2)

Petrakiella Syd. (1)

Phycomelaina Kohlm. (1)

Phyllachora Nitschke ex Fuckel(1513)

Phylleutypa Petr. (3)

Phyllocrea Höhn. (3)

Pseudothiella Petr. (1)

Pseudothiopsella Petr. (1)

Pterosporidium W.H. Ho \& K.D. Hyde (2)

Rehmiodothis Theiss. \& Syd. (10)

Retroa P.F. Cannon (2)

Rhodosticta Woron. (2)

Rikatlia P.F. Cannon (1)

Schizochora Syd. \& P. Syd. (3)

Sphaerodothella C.A. Pearce \& K.D. Hyde (1)

Sphaerodothis (Sacc. \& P. Syd.) Shear (43)

Stigmatula (Sacc.) Syd. \& P. Syd. (10)

Stigmochora Theiss. \& Syd. (12)

Stromaster Höhn. (1)

Tamsiniella S.W. Wong, K.D. Hyde, W.H. Ho \& S.J. Stanley (1)

Telimenella Petr. (3)

Telimenochora Sivan. (1)

Trabutia Sacc. \& Roum. (1)

Tribulatia J.E. Taylor, Hyde \& E.B.G. Jones (1)

Uropolystigma Maubl. (1)

Vitreostroma P.F. Cannon (3)

Zimmermanniella Henn. (1)

Telimenaceae Mardones, T. Trampe \& M. Piepenbr Telimena Racib. (14)

Phyllachorales genus incertae sedis

Marinosphaera K.D. Hyde (1)

Pseudodactylariales Crous

Pseudodactylariaceae Crous

Pseudodactylaria Crous (3)

Sordariales Chad. ex D. Hawksw. \& O.E. Erikss.

Chaetomiaceae $\mathrm{G}$. Winter

Achaetomium J.N. Rai, Tewari \& Mukerji (16)

Acrophialophora Edward (17)

Allobotryotrichum M. Raza \& L. Cai (1)

Amesia X. Wei Wang, Samson \& Crous (4)

Arcopilus X. Wei Wang, Samson \& Crous (5)

Arxotrichum A. Nováková \& M. Kolařik (2)

Botryotrichum Sacc. \& Marchal (11)

Brachychaeta X. Wei Wang \& Houbraken (1)

Carteria X. Wei Wang \& Houbraken (1) 
Chaetomium Kunze (359)

Chrysanthotrichum X. Wei Wang \& Houbraken (4)

Chrysocorona X. Wei Wang \& Houbraken (1)

Collariella X. Wei Wang, Samson \& Crous (9)

Condenascus X. Wei Wang \& Houbraken (1)

Corynascella Arx \& Hodges (1)

Crassicarpon Y. Marín, Stchigel, Guarro \& Cano (3)

Dichotomopilus X. Wei Wang, Samson \& Crous (12)

Floropilus X. Wei Wang \& Houbraken (1)

Guanomyces M.C. Gonzáles, Hanlin \& Ulloa (1)

Humicola Traaen (86)

Hyalosphaerella X. Wei Wang \& Houbraken (1)

Madurella Brumpt (15)

Melanocarpus Arx (5)

Microthielavia X. Wei Wang \& Houbraken (1)

Myceliophthora Costantin (4)

Ovatospora X. Wei Wang, Samson \& Crous (6)

Parathielavia X. Wei Wang \& Houbraken (3)

Pseudothielavia X. Wei Wang \& Houbraken (4)

Remersonia Samson \& Seifert (2)*

Retroconis de Hoog \& Bat. Vegte (1)*

Staphylotrichum J.A. Mey. \& Nicot (8)

Stolonocarpus X. Wei Wang \& Houbraken (1)

Subramaniula Arx (9)

Thermothelomyces Y. Marín, Stchigel, Guarro \& Cano (4)

Thermothielavioides X. Wei Wang \& Houbraken (1)

Thielavia Zopf (47)

Trichocladium Harz (44)

Lasiosphaeriaceae Nannf.

Anopodium Lundq. (2)

Apiosordaria Arx \& W. Gams (31)

Apodospora Cain \& J.H. Mirza (6)

Apodus Malloch \& Cain (2)

Arnium Nitschke ex G. Winter (34)

Bellojisia Réblová (1)

Biconiosporella Schaumann (1)

Bombardia (Fr.) P. Karst. (43)

Bombardioidea C. Moreau ex N. Lundqv. (5)

Camptosphaeria Fuckel (4)

Cercophora Fuckel (77)

Corylomyces Stchigel, M. Calduch \& Guarro (1)

Diffractella Guarro, P.F. Cannon \& Aa (1)

Diplogelasinospora Cain (4)

Emblemospora Jeng \& J.C. Krug (2)

Eosphaeria Höhn. (2)

Episternus Górz \& Boroń (1)

Fimetariella N. Lundq. (9)

Immersiella A.N. Mill. \& Huhndorf(2)

Jugulospora N. Lundq. (1)

Lasiosphaeria Ces. \& De Not. (229)

Mammaria Ces. ex Rabenh. (2) 
Periamphispora J.C. Krug (1)

Ramophialophora M. Calduch, Stchigel, Gené \& Guarro (4)*

Rinaldiella Deanna A. Sutton, Y. Marín, Guarro \& E.H. Thomps (1)

Schizothecium Corda (31)

Strattonia Cif. (11)

Thaxteria Sacc. (8)

Tripterosporella Subram. \& Lodha (5)

Zopfiella G. Winter (22)

Zygopleurage Boedijn (3)

Zygospermella Cain (3)

Podosporaceae X. Wei Wang \& Houbraken

Cladorrhinum Sacc. \& Marchal (13)*

Triangularia Boedijn (7)

Podospora Ces. (92)

Sordariaceae G. Winter

Copromyces N. Lundq. (1)

Effetia Bartoli, Maggi \& Persiani (1)

Guilliermondia Boud. (1)

Neurospora Shear \& B.O. Dodge (= Gelasinospora Dowding) (60)

Pseudoneurospora Dania García, Stchigel \& Guarro (2)

Sordaria Ces. \& De Not. (37)

Stellatospora T. Ito \& A. Nakagiri (1)

Sordariales genera incertae sedis

Abyssomyces Kohlm (1)

Acanthotheciella Höhn. (3)

Ascolacicola Ranghoo \& K.D. Hyde (1)

Bombardiella Höhn. (1)

Coronatomyces Dania García, Stchigel \& Guarro (1)

Cuspidatispora Shearer \& Bartolata (1)

Globosphaeria D. Hawksw. (1)

Isia D. Hawksw \& Manohar (2)

Lasiosphaeris Clem. (3)

Lunulospora Ingold (2)

Lockerbia K.D. Hyde (2)

Nitschkiopsis Nannf. \& R. Sant. (1)

Onygenopsis Henn. (1)

Phaeosporis Clem. (2)

Reconditella Matzer \& Hafellner (1)

Rhexodenticula W.A. Baker \& Morgan-Jones (4)

Rhexosporium Udagawa \& Furuya (1)

Roselliniomyces Matzer \& Hafellner (7)

Roselliniopsis Matzer \& Hafellner (7)

Stromatographium Höhn. (= Fluviostroma Samuels \& E. Müll.) (2)

Utriascus Réblová (1)

Ypsilonia Lév. (3)

Sordariomycetidae family incertae sedis

Batistiaceae Samuels \& K.F. Rodrigues

Batistia Cif. (1) 
Sordariomycetidae genera incertae sedis

Arecacicola Joanne E. Taylor, J. Fröhl. \& K.D. Hyde (1)

Bullimyces A. Ferrer, A.N. Mill., Sarmiento \& Shearer (3)

Cancellidium Tubaki (2)

Ceratolenta Réblová (1)

Chaetosphaerides Matsush. (1)

Cryptophyllachora L. Kiss, Kovács \& R.G. Shivas (2)*

Hanliniomyces Raja \& Shearer (1)

Hydromelitis A. Ferrer, A.N. Mill., Sarmiento \& Shearer (1)

Merugia Rogerson \& Samuels (1)

Mycomedusiospora G.C. Carroll \& Munk (1)

Myxocephala G. Weber, Spaaij \& Oberw. (1)

Nigromammilla K.D. Hyde \& J. Fröhl. (1)

Phaeotrichosphaeria Sivan. (4)

Phragmodiscus Hansf. (2)

Pseudobotrytis Krzemien. \& Badura (2)

Xylariomycetidae O.E. Erikss \& Winka

Amphisphaeriales D. Hawksw. \& O.E. Erikss.

Amphisphaeriaceae G. Winter

Amphisphaeria Ces. \& De Not. (66)

Griphosphaerioma Höhn. (2)

Lepteutypa Petr. (14)

Apiosporaceae K.D. Hyde, J. Fröhl., Joanne E. Taylor \& M.E. Barr

Appendicospora K.D. Hyde (2)

Arthrinium Kunze (74)

Dictyoarthrinium S. Hughes (6)

Endocalyx Berk. \& Broome (8)

Nigrospora Zimm. (ca. 20)

Beltraniaceae Nann.

Beltrania Penz. (17)

Beltraniella Subram. (25)

Beltraniopsis Bat. \& J.L. Bezerra (11)

Hemibeltrania Piroz. (13)

Parapleurotheciopsis P.M. Kirk (5)

Porobeltraniella Gusmão (2)

Pseudobeltrania Henn. (9)

Subramaniomyces Varghese \& V.G. Rao (3)

Subsessila C.G. Lin \& K.D. Hyde (1)

Clypeophysalosporaceae Giraldo \& Crous

Bagadiella Cheew. \& Crous (4)

Clypeophysalospora H.J. Swart (1)

Neophysalospora Crous \& M.J. Wingf. (1)

Plectosphaera Theiss. (28)

Cylindriaceae Crous \& L. Lombard*

Cylindrium Bonord (6)

Hansfordiaceae Crous 
Hansfordia S. Hughes (7)

Hyponectriaceae Petr.

Apiothyrium Petr. (2)

Arecomyces K.D. Hyde (10)

Arwidssonia B. Erikss. (2)

Cesatiella Sacc. (3)

Chamaeascus L. Holm, K. Holm \& M.E. Barr (1)

Discosphaerina Höhn. (21)

Exarmidium P. Karst. (14)

Frondicola K.D. Hyde (1)

Hyponectria Sacc. (30)

Lichenoverruculina Etayo (1)

Micronectria Speg. (4)

Papilionovela Aptroot (1)

Pellucida Dulym., Sivan., P.F. Cannon \& Peerally (1)

Phragmitensis M.K.M. Wong, Poon \& K.D. Hyde (2)

Physalospora Niessl (37)

Rachidicola K.D. Hyde \& J. Fröhl. (1)

Xenothecium Höhn. (1)

Iodosphaeriaceae O. Hilber

Iodosphaeria Samuels (8)

Melogrammataceae G. Winter

Melogramma Fr. (20)

Phlogicylindriaceae Senan. \& K.D. Hyde

Ciferriascosea Senan., Bhat, Camporesi \& K.D. Hyde (2)

Idriellomyces Crous (1)

Phlogicylindrium Crous, Summerb. \& Summerell (5)

Pseudomassariaceae Senan. \& K.D. Hyde

Leiosphaerella Höhn. (15)

Pseudapiospora Petr. (3)

Pseudomassaria Jacz. (24)

Pseudomassariella Petr (1)

Pseudotruncatellaceae Crous

Pseudotruncatella R.H. Perera, Camporesi, Maharachch. \& K.D. Hyde (2)

Sporocadaceae Corda*

Allelochaeta Petr. (50)

Annellolacinia B. Sutton (2)

Bartalinia Tassi (19)

Broomella Sacc. (2)

Ciliochorella Syd. (4)

Dilophospora Desm. (ca. $2+$ few orphaned names)

Diploceras (Sacc.) Died (2)

Disaeta Bonar (1)

Discosia Lib. (ca. 17)

Distononappendiculata F. Liu, L. Cai \& Crous (3) 
Diversimediispora F. Liu, L. Cai \& Crous (1)

Doliomyces Steyaert (3)

Heterotruncatella F. Liu, L. Cai \& Crous (17)

Hyalotiella Papendorf (6)

Hymenopleella Munk (= Dyrithiopsis L. Cai, Jeewon \& K.D. Hyde; = Neotruncatella Hyang B. Lee \& T.T.T. Nguyen) (7)

Immersidiscosia Kaz. Tanaka, Okane \& Hosoya (1)

Monochaetia (Sacc.) Allesch. (ca. 30)

Morinia Berl. \& Bres. (=Zetiasplozna Nag Raj) (2)

Neopestalotiopsis Maharachch., K.D. Hyde \& Crous (33)

Nonappendiculata F. Liu, L. Cai \& Crous (1)

Parabartalinia F. Liu, L. Cai \& Crous (1)

Pestalotiopsis Steyaert (ca. 100)

Pseudopestalotiopsis Maharachch., K.D. Hyde \& Crous (12)

Pseudosarcostroma F. Liu, L. Cai \& Crous (1)

Robillarda Sacc. (ca. 15)

Sarcostroma Cooke (28)

Seimatosporium Corda (ca. 100)

Seiridium Nees (20)

Sporocadus Corda (49)

Strickeria Körb. (10)

Synnemapestaloides T. Handa \& Y. Harada (2)

Truncatella Steyaert (13)

Xenoseimatosporium F. Liu, L. Cai \& Crous (1)

Vialaeaceae P.F. Cannon

Vialaea Sacc. (50)

Amphisphaeriales genus incertae sedis

Chitonospora E. Bommer, M. Rousseau \& Sacc. (1)

Delonicicolales R.H. Perera, Maharachch. \& K.D. Hyde

Delonicicolaceae R.H. Perera, Maharachch. \& K.D. Hyde

Delonicicola R.H. Perera, Maharachch. \& K.D. Hyde (1)

Furfurella Voglmayr \& Jaklitsch (3)

Xylariales Nannf.

Anungitiomycetaceae Crous

Anungitiomyces Crous (1)

Barrmaeliaceae Voglmayr \& Jaklitsch*

Barrmaelia Rappaz. (8)

Entosordaria (Sacc.) Höhn. (ca. 18)

Castanediellaceae Hern.-Restr., Guarro \& Crous

Castanediella Hern.-Restr., Crous \& M.J. Wingf. (12)

Clypeosphaeriaceae G. Winter

Aquasphaeria K.D. Hyde (1)

Apioclypea K.D. Hyde (7)

Brunneiapiospora K.D. Hyde, J. Fröhl. \& Joanne E. Taylor (9)

Clypeosphaeria Fuckel (37) 
Crassoascus Checa, Barrasa \& A.T. Martínez (3)

Palmaria K.D. Hyde, J. Fröhl. \& Joanne E. Taylor (1)

Coniocessiaceae Asgari \& Zare

Coniocessia Dania García, Stchigel, D. Hawksw. \& Guarro (5)

Paraxylaria Wanas., E.B.G. Jones, Gafforov \& K.D. Hyde (1)

Diatrypaceae Nitschke

Allocryptovalsa Senwanna, Phook. \& K.D. Hyde (2)

Anthostoma Nitschke (ca. 101)

Cryptosphaeria Ces \& De Not. (48)

Cryptovalsa Ces. \& De Not. ex Fuckel (43)

Diatrypasimilis J.J. Zhou \& Kohlm. (1)

Diatrype Fr. (ca. 244)

Diatrypella (Ces. \& De Not.) De Not. (ca. 115)

Echinomyces Rappaz (2)

Endoxylina Romell (16)

Eutypa Tul. \& C. Tul. (ca. 131)

Eutypella (Nitschke) Sacc. (ca. 196)

Halodiatrype Dayar. \& K.D. Hyde (3)

Halocryptovalsa Dayar. \& K.D. Hyde (2)

Leptoperidia Rappaz (4)

Libertella Desm. (ca. 72)

Monosporascus Pollack \& Uecker (4)

Neoeutypella M. Raza, Q.J. Shang, Phook. \& L. Cai (1)*

Pedumispora K.D. Hyde \& E.B.G. Jones (1)

Peroneutypa Berl. (30)

Quaternaria Tul. \& C. Tul. (14)

Fasciatisporaceae S.N. Zhang, K.D. Hyde \& J.K. Liu

Fasciatispora K.D. Hyde (11)

Graphostromataceae M.E. Barr, J.D. Rogers \& Y.M. Ju

Biscogniauxia Kuntze (ca. 76)

Camillea Fr. (50)

Graphostroma Piroz. (1)

Obolarina Pouzar (2)

Vivantia J.D. Rogers, Y.M. Ju \& Cand. (1)

\section{Hypoxylaceae DC.}

Annulohypoxylon Y.M. Ju, J.D. Rogers \& H.M. Hsieh (ca. 60)

Anthocanalis Daranag., Camporesi \& K.D. Hyde (1)

Chlorostroma A.N. Mill., Lar.N. Vassiljeva \& J.D. Rogers (3)

Daldinia Ces. \& De Not. (ca. 67)

Durotheca Læssøe, Srikit., Luangsa-ard \& M. Stadler (4)*

Entonaema Möller (10)

Hypomontagnella Sir, L. Wendt \& C. Lambert (4)*

Hypoxylon Bull. (141)

Jackrogersella L. Wendt, Kuhnert \& M. Stadler (6)

Natonodosa Heredia (1)

Phylacia Lév. (12)

Pyrenomyxa Morgan(3) 
Pyrenopolyporus Lloyd (5)

Rhopalostroma D. Hawksw. (11)

Rostrohypoxylon J. Fourn. \& M. Stadler (1)

Ruwenzoria J. Fourn., M. Stadler, Læssøe \& Decock (1)

Thamnomyces Ehrenb. (11)

Theissenia Maubl. (8)

Thuemenella Penz. \& Sacc. (10)

Induratiaceae Samarak., Thongbai, K.D. Hyde \& M. Stadler

Emarcea Duong, Jeewon \& K.D. Hyde (3)

Induratia Samuels, E. Müll. \& Petrini (26)

Leptosilliaceae Voglmayr \& Jaklitsch

Leptosillia Höhn. (=Cresporhaphis M.B. Aguirre) (9)*

Lopadostomataceae Daranag. \& K.D. Hyde

Creosphaeria Theiss. (3)

Jumillera J.D. Rogers, Y.M. Ju \& F. San Martín (8)

Lopadostoma (Nitschke) Traverso (27)

Whalleya J.D. Rogers, Y.M. Ju \& F. San Martín (2)

Microdochiaceae Hern.-Restr., Crous \& J.Z. Groenew.

Idriella P.E. Nelson \& S. Wilh. (=Monographella Petr.) (24)

Microdochium Syd. (38)

Selenodriella R.F. Castañeda \& W.B. Kendr. (7)

Myelospermataceae K.D. Hyde \& S.W. Wong

Myelosperma Syd. \& P. Syd. (5)

Nothodactylariaceae Crous

Nothodactylaria Crous (1)

Oxydothidaceae Konta \& K.D. Hyde

Oxydothis Penz. \& Sacc. (79)

Polystigmataceae Höhn. ex Nannf.*

Polystigma DC. (23)

Pseudosporidesmiaceae Crous

Pseudosporidesmium K.D. Hyde \& McKenzie (2)

Requienellaceae Boise*

Acrocordiella O.E. Erikss. (2)

Lacrymospora Aptroot (1)

Parapyrenis Aptroot (8)

Requienella Fabre (8)

Xyladictyochaetaceae Crous \& Hern.-Restr*

Xyladictyochaeta Hern.-Restr., R.F. Castañeda \& Gené (1)

Xylariaceae Tul. \& C. Tul.

Abieticola Hyang B. Lee (1) 
Amphirosellinia Y.M. Ju, J.D. Rogers, H.M. Hsieh \& Lar.N. Vassiljeva (6)

Anthostomella Sacc. (ca. 100)

Anthostomelloides Tibpromma \& K.D. Hyde (5)

Ascotricha Berk. (27)

Astrocystis Berk. \& Broome (24)

Brunneiperidium Daranag., Camporesi \& K.D. Hyde (2)

Collodiscula I. Hino \& Katum. (5)

Coniolariella Dania García, Stchigel \& Guarro (5)

Engleromyces Henn. (2)

Entalbostroma J.D. Rogers \& P.R. Johnst. (1)

Entoleuca Syd. (3)

Euepixylon Füisting (2)

Halorosellinia Whalley, E.B.G. Jones, K.D. Hyde \& Læssøe (3)

Helicogermslita Lodha \& D. Hawksw. (9)

Hypocopra (Fr) J. Kickx f. (58)

Hypocreodendron Henn. (1)

Kretzschmaria Fr. (ca. 57)

Kretzschmariella Viégas (2)

Leprieuria Læssøe, J.D. Rogers \& Whalley (1)

Lunatiannulus Daranag., Camporesi \& K.D. Hyde (1)

Nemania Gray (57)

Podosordaria Ellis \& Holw. (35)

Poronia Willd. (ca. 24)

Rosellinia De Not. (ca. 359)

Sarcoxylon Cooke (6)

Squamotubera Henn. (1)

Stilbohypoxylon Henn. (12)

Vamsapriya Gawas \& Bhat (8)

Virgaria Nees (11)

Wawelia Namysl. (5)

Xylaria Hill ex Schrank (ca. 571)

Zygosporiaceae J.F. Li, Phook. \& K.D. Hyde.

Zygosporium Mont. (25)

Xylariales genera incertae sedis

Adomia S. Schatz (1)

Alloanthostomella Daranag., Camporesi \& K.D. Hyde (1)

Anungitea B. Sutton (22)

Ascotrichella Valldos. \& Guarro (1)

Basifimbria Subram. \& Lodha (1)

Biporispora J.D. Rogers, Y.M. Ju \& Cand. (1)

Botryohypoxylon Samuels \& J.D. Rogers (1)

Castellaniomyces Senan., Camporesi \& K.D. Hyde (1)

Chaenocarpus Rebent. (4)

Circinotrichum Nees (15)

Cryptostroma P.H. Greg. \& S. Waller (1)

Cyanopulvis J. Fröhl. \& K.D. Hyde (1)

Diamantinia A.N. Mill., Læssøe \& Huhndorf (1)

Gigantospora B.S. Lu \& K.D. Hyde (1)

Guestia G.J.D. Sm. \& K.D. Hyde (1)

Gyrothrix (Corda) Corda (22) 
Hadrotrichum Fuckel (22)

Idriellopsis Hern.-Restr. \& Crous (1)

Kirstenboschia Quaedvl., Verkley \& Crous (1)

Lanceispora Nakagiri, Okane, Tad. Ito \& Katum. (2)

Lasiobertia Sivan. (2)

Leptomassaria Petr. (4)

Melanographium Sacc. (11)

Neoanthostomella D.Q. Dai \& K.D. Hyde (2)

Neoidriella Hern.-Restr. \& Crous (1)

Nipicola K.D. Hyde (4)

Occultitheca J.D. Rogers \& Y.M. Ju (1)

Ophiorosellinia J.D. Rogers, A. Hidalgo, F.A. Fernández \& Huhndorf (1)

Palmicola K.D. Hyde (4)

Pandanicola K.D. Hyde (2)

Paraidriella Hern.-Restr. \& Crous (1)

Paramphisphaeria F.A. Fernández, J.D. Rogers, Y.M. Ju, Huhndorf \& L. Umaña (1)

Paraphysalospora Crous (1)

Paucithecium Lloyd (1)

Pidoplitchkoviella Kiril. (1)

Polyancora Voglmayr \& Yule (1)

Polyscytalum Riess (28)

Poroleprieuria M.C. González, Hanlin, Ulloa \& Elv. Aguirre (1)

Pseudoanthostomella Daranag., Camporesi \& K.D. Hyde (5)

Pseudophloeospora Crous \& R.G. Shivas (2)

Pseudosubramaniomyces Crous (1)

Pulmosphaeria Joanne E. Taylor, K.D. Hyde \& E.B.G. Jones (1)

Pyriformiascoma Daranag., Camporesi \& K.D. Hyde (1)

Roselymyces Fiuza, C.R. Silva, R.F. Castañeda \& Gusmão (1)*

Sabalicola K.D. Hyde (1)

Spirodecospora B.S. Lu, K.D. Hyde \& W.H. Ho (2)

Sporidesmina Subram. \& Bhat (1)

Striatodecospora D.Q. Zhou, K.D. Hyde \& B.S. Lu (1)

Stromatoneurospora S.C. Jong \& E.E. Davis (2)

Surculiseries Okane (1)

Synnemadiella Crous \& M.J. Wingf. (1)

Tristratiperidium Daranag., Camporesi \& K.D. Hyde (1)

Xenoanthostomella Mapook \& K.D. Hyde (1)

Xylocrea Möller (2)

Xylotumulus J.D. Rogers, Y.M. Ju \& Cand. (1)

Yuea O.E. Erikss. (1)

Xylariomycetidae family incertae sedis

Cainiaceae J.C. Krug

Alishanica A. Karunarathna, C.H. Kuo \& K. D. Hyde (1)

Amphibambusa D.Q. Dai \& K.D. Hyde (1)

Arecophila K.D. Hyde (14)

Atrotorquata Kohlm. \& Volkm.-Kohlm. (2)

Cainia Arx \& E. Müll. (6)

Seynesia Sacc. (ca. 46)

Xylariomycetidae genus incertae sedis

Calceomyces Udagawa \& S. Ueda (1) 
Sordariomycetes orders incertae sedis

Amplistromatales M.J. D'souza, Maharachch. \& K.D. Hyde Amplistromataceae Huhndorf, A.N. Mill., Greif \& Samuels* Acidothrix Hujslová \& M. Kolařík (1)

Amplistroma Huhndorf, A.N. Mill., Greif \& Samuels (9)

Wallrothiella Sacc. (ca. 10)

Catabotryaceae Petr. ex M.E. Barr Catabotrys Theiss. \& Syd. (3)

Parasympodiellales Hern.-Restr., Gené, R.F. Castañeda \& Crous

Parasympodiellaceae Hern.-Restr., Gené, Guarro \& Crous

Parasympodiella Ponnappa (10)

Spathulosporales Kohlm.

Hispidicarpomycetaceae Nakagiri

Hispidicarpomyces Nakagiri (1)

Spathulosporaceae Kohlm.

Retrostium Nakagiri \& Tad Ito (1)

Spathulospora A.R. Caval. \& T.W. Johnson (4)

Tracyllalales Crous

Tracyllaceae Crous

Tracylla (Sacc.) Tassi (3)

Vermiculariopsiellales Hern.-Restr., J. Mena, Gené \& Crous

Vermiculariopsiellaceae Hern.-Restr, J. Mena, Gené \& Crous

Vermiculariopsiella Bender (22)

Sordariomycetes families incertae sedis

Acrodictyaceae J.W. Xia \& X.G. Zhang*

Acrodictys M.B. Ellis (25)

Junewangiaceae J.W. Xia \& X.G. Zhang*

Dictyosporella Abdel-Aziz (2)

Junewangia W.A. Baker \& Morgan-Jones (6)

Lautosporaceae Kohlm., Volkm.-Kohlm. \& O.E. Erikss

Lautospora K. D. Hyde \& E.B.G. Jones (2)

Obryzaceae Körb.

Obryzum Wallr. (3)

Sordariomycetes genera incertae sedis

Acerbiella Sacc. (4)

Acrospermoides Miller \& G.E. Thomps. (2)

Ameromassaria Hara (1)

Amphisphaerellula Gucevič (1)

Amphisphaerina Höhn. (3 epithets in Index Fungorum 2020)

Amphorulopsis Petr. (1)

Amylis Speg. (1) 
Anisomycopsis I. Hino \& Katum. (1)

Antennopsis R. Heim (1)*

Anthostomaria (Sacc.) Theiss. \& Syd. (1)

Anthostomellina L.A. Kantsch. (2)

Apodothina Petr. (1)

Apogaeumannomyces Matsush. (1)

Aquadulciospora Fallah \& Shearer (1)

Areolospora S.C. Jong \& E.E. Davis (2 epithets in Index Fungorum 2020)

Aropsiclus Kohlm. \& Volkm.-Kohlm. (1)

Ascorhiza Lecht.-Trinka (1)

Ascoyunnania L. Cai \& K.D. Hyde (1)

Atrogeniculata J.S. Monteiro, Gusmão \& R.F. Castañeda (1)

Aulospora Speg. (1)

Azbukinia Lar.N. Vassiljeva (1)

Bactrosphaeria Penz. \& Sacc. (1)

Basidiobotrys Höhn. (1)

Biciliopsis Diederich (2)

Bombardiastrum Pat. (1)

Boothiella Lodhi \& Mirza (1)

Botryosporium Corda (11)

Brenesiella Syd. (1)

Byrsomyces Cavalc. (1)

Byssotheciella Petr. (2)

Caleutypa Petr. (1)

Caproniella Berl. (1)

Chaetoamphisphaeria Hara (1)

Charonectria Sacc. (3)

Ciliofusospora Bat. \& J.L. Bezerra (1)

Clypeoceriospora Sousa da Câmara (1)

Clypeosphaerulina Sousa da Câmara (1)

Cryptoascus Petri (2)

Cryptomycella Höhn. (2)

Cryptomycina Höhn. (2)

Cucurbitopsis Bat. \& Cif. (1)

Curvatispora V.V. Sarma \& K.D. Hyde (1)

Dasysphaeria Speg. (1)

Delpinoëlla Sacc. (1)

Diacrochordon Petr. (1)

Didymobotryum Sacc. (6)

Duradens Samuels \& Rogerson (1)

Ellisembia Subram. (ca. 60)

Esfandiariomyces Ershad (1)

Fantasmomyces Dong Hyeon Lee, Marinc., Z.W. de Beer \& M.J. Wingf. (1)

Farrowia D. Hawksw. (3)

Fassia Dennis (1)

Flammispora Pinruan, Sakay., K.D. Hyde \& E.B.G. Jones (2)

Frondisphaeria K.D. Hyde (2)

Hapsidascus Kohlm. \& Volkm.-Kohlm. (1)

Hassea Zahlbr. (1)

Heliastrum Petr. (1)

Hyaloderma Speg. (1)

Hyalotiopsis Punith. (1) 
Hydronectria Kirschst. (1)

Immersisphaeria Jaklitsch (1)

Iraniella Petr. (1)

Konenia Hara (1)

Kravtzevia Schwartzman (1)

Kurssanovia Kravtzev (1)

Lecythiomyces Doweld (1)

Leptosacca Syd. (1)

Leptosphaerella Speg. (14 epithets in Index Fungorum 2020)

Mangrovispora K.D. Hyde \& Nageire (1)

Marisolaris Jørg. Koch \& E.B.G. Jones (1)

Microcyclephaeria Bat. (1)

Mirannulata Huhndorf, F.A. Fernández, A.N. Mill. \& Lodge (2)

Mycothermus D.O. Natvig, J.W. Taylor, A. Tsang, M.I. Hutch. \& A.J. Powell ex X. Wei

Wang, Houbraken \& D.O. Natvig (2)

Natantiella Réblová (1)

Naumovela Kravtzev (2)

Neocryptospora Petr. (1)

Neoeriomycopsis Crous \& M.J. Wingf. (1)

Neolamya Theiss. \& Syd. (3)

Neothyridaria Petr. (1)

Ophiomassaria Jacz. (1)

Ophiomeliola Starbäck (3)

Paoayensis Cabanela, Jeewon \& K.D. Hyde (2)

Paradiplococcium Hern.-Restr., J. Mena \& Gené (1)

Paramicrodochium Hern.-Restr. \& Crous (1)

Pareutypella Y.M. Ju \& J.D. Rogers (2)

Phialemoniopsis Perdomo, Dania García, Gené, Cano \& Guarro (6)

Phragmeriella Hansf. (1)

Phyllocelis Syd. (2)

Pleocryptospora J. Reid \& C. Booth (1)

Pleosphaeria Speg. (24)

Pleurophragmium Costantin (22)

Protocucurbitaria Naumov (1)

Pulvinaria Bon. (2)

Pumilus Viala \& Marsais (1)

Rehmiomycella E. Müll. (1)

Rhamphosphaeria Kirschst. (1)

Rhizophila K.D. Hyde \& E.B.G. Jones (1)

Rhopographella (Henn.) Sacc. \& Trotter (2)

Rhynchosphaeria (Sacc.) Berl. (5)

Rivulicola K.D. Hyde (3)

Romellina Petr. (1)

Saccardoëlla Speg. (15)

Sartorya Vuill. (9 epithets in Index Fungorum 2020)

Scharifia Petr. (1)

Scoliocarpon Nyl. (1 epithets in Index Fungorum 2020)

Scotiosphaeria Sivan. (1)

Selenosporella G. Arnaud ex MacGarvie (12)

Servazziella J. Reid \& C. Booth (1)

Sporoctomorpha J.V. Almeida \& Sousa da Câmara (1)

Stanjehughesia Subram. (16) 
Stearophora L. Mangin \& Viala (1)

Steganopycnis Syd. \& P. Syd. (1)

Stegophorella Petr. (1)

Stellosetifera Matsush. (1)

Stereosphaeria Kirschst. (1)

Stomatogenella Petr. (1)

Sungaiicola Fryar \& K.D. Hyde (1)

Synsphaeria Bon. (4 epithets in Index Fungorum 2020)

Teracosphaeria Réblová \& Seifert (1)

Thelidiella Fink (1)

Thyridella (Sacc.) Sacc. (3)

Thyrotheca Kirschst. (1 epithets in Index Fungorum 2020)

Trichospermella Speg. (2)

Trichosphaeropsis Bat. \& Nasc. (1)

Tulipispora Révay \& Gönczöl (1)

Tunstallia Agnihothr. (3 epithets in Index Fungorum 2020)

Urosporella G.F. Atk. (5)

Urupe Viégas (1)

Vleugelia J. Reid \& C. Booth (1)

Xenodium Syd. (1)

Zalerion R.T. Moore \& Meyers (6)

Xylonomycetes Gazis \& P. Chaverri

Symbiotaphrinales Baral \& E. Weber

Symbiotaphrinaceae Baral \& E. Weber

Symbiotaphrina Kühlw. \& Jurzitza ex W. Gams \& Arx (17)*

Xylonales Gazis \& P. Chaverri

Xylonaceae Gazis \& P. Chaverri

Trinosporium Crous \& Decock (1)

Xylona Gazis \& P. Chaverri (1)

Xylobotryomycetes Voglmayr \& Jaklitsch

Xylobotryales Voglmayr \& Jaklitsch

Cirrosporiaceae Voglmayr \& Jaklitsch

Cirrosporium S. Hughes (1)

Xylobotryaceae Voglmayr \& Jaklitsch

Xylobotryum Pat. (2)

Pezizomycotina orders incertae sedis

Thelocarpales Lücking \& Lumbsch

Thelocarpaceae Zukal

Sarcosagium A. Massal. (1)

Thelocarpon Nyl (25)

Vezdaeales Lumbsch \& Lücking

Vezdaeaceae Poelt \& Vězda ex J.C. David \& D. Hawksw.

Vezdaea Tsch.-Woess \& Poelt (12)

Pezizomycotina family incertae sedis

Harpidiaceae Vězda ex Hafellner 
Euopsis Nyl. (2)

Harpidium Körb. (3)

Pezizomycotina genera incertae sedis

Angatia Syd. (5)

Biatoridium J. Lahm ex Körb. (3)

Cyanoporina Groenh. (1)

Melanophloea P. James \& Vězda (1)

Milospium D. Hawksw. (4)

Oevstedalia Ertz \& Diederich (1)

Psammina Sacc. \& M. Rousseau ex E. Bommer \& M. Rousseau (8)

Pygmaeosphaera Etayo \& Diederich (3)

Pyrenocollema Reinke (1)

Solanella Vaňha (1)*

Wadeana Coppins \& P. James (2)

Saccharomycotina O.E. Erikss. \& Winka

Saccharomycetes O.E. Erikss. \& Winka*

Saccharomycetales Kudrjanzev

Alloascoideaceae Kurtzman \& Robnett

Alloascoidea Kurtzman \& Robnett (2)

Ascoideaceae J. Schröter

Ascoidea Bref. (4)

Cephaloascaceae L.R. Batra

Cephaloascus Hanawa (2)

Debaryomycetaceae Kurtzman \& M. Suzuki

Babjeviella Kurtzman \& M. Suzuki (1)

Debaryomyces Lodder \& Kreger-van Rij (15)

Hemisphaericaspora Hui, Ren, Chen, Li, Zhan \& Niu (2)

Kurtzmaniella M.A. Lachance \& W.T. Starmer (5)

Lodderomyces Van der Walt (2)*

Meyerozyma Kurtzman \& M. Suzuki (8)

Millerozyma Kurtzman \& M. Suzuki (5)

Priceomyces Kurtzman \& M. Suzuki (8)

Scheffersomyces Kurtzman \& M. Suzuki (18)

Schwanniomyces Klöcker emend. M. Suzuki \& Kurtzman (7)

Spathaspora N.H. Nguyen, S.O. Suh \& M. Blackwell (11)

Suhomyces M. Blackwell \& Kurtzman (26)

Yamadazyma Billon-Grand (23)

Dipodascaceae Engl. \& E. Gilg

Dipodascus Lagerh. (14)

Galactomyces Redhead \& Malloch (5)

Geotrichum Link (8)

Magnusiomyces Zender (7)

Saprochaete Coker \& Shanor ex D.T.S. Wagner \& Dawes (10)*

Lipomycetaceae E.K. Novák \& Zsolt

Dipodascopsis Batra \& P. Millner emend. Kurtzman, Albertyn \& Basehoar-Powers (3) 
Kockiozyma Jindam., Yukphan \& Y. Yamada (1)

Limtongia Jindam., Am-in, Yukphan \& Y. Yamada (1)

Lipomyces Lodder \& Kreger (16)

Myxozyma Van der Walt, Weijman \& von Arx (12)

Metschnikowiaceae T. Kamienski*

Clavispora Rodr. Mir. (4)

Kodamaea Y. Yamada, T. Suzuki, Matsuda \& Mikata emend. Rosa, Lachance, Starmer, Barker, Bowles \& Schlag-Edler (8)

Metschnikowia T. Kamienski (64)

Phaffomycetaceae Y. Yamada, H. Kawas., Nagats., Mikata \& Tats. Seki

Barnettozyma Kurtzman, Robnett \& Basehoar-Powers (9)

Cyberlindnera Minter (27)

Phaffomyces Y. Yamada (4)

Starmera Y. Yamada, Higashi, Ando \& Mikata (7)

Wickerhamomyces Kurtzman, Robnett \& Basehoar-Powers (31)

Pichiaceae Zender

Brettanomyces Kufferath \& van Laer (3)

Dekkera Van der Walt (2)*

Komagataella Y. Yamada, Matsuda, Maeda \& Mikata (6)

Kregervanrija Kurtzman (3)

Kuraishia Y. Yamada, Maeda \& Mikata (9)

Martiniozyma Kurtzman (2)

Ogataea Y. Yamada, K. Maeda \& Mikata (46)

Pachysolen Boidin \& Adzet (1)

Pichia E.C. Hansen (27)

Saturnispora Z.W. Liu \& Kurtzman (21)

Saccharomycetaceae G. Winter

Citeromyces Santa Maria (4)

Cyniclomyces Van der Walt \& D.B. Scott (1)

Eremothecium Borzi emend. Kurtzman (5)

Hagleromyces Sousa, Morais, Lachance \& Rosa (1)

Kazachstania Zubcova (44)

Kluyveromyces Van der Walt (6)

Lachancea Kurtzman (10)

Nakaseomyces Kurtzman (2)

Naumovozyma Kurtzman (3)

Saccharomyces Meyen (10)

Tetrapisispora Ueda-Nishimura \& K. Mikata emend. Kurtzman (9)

Torulaspora Lindner (8)

Vanderwaltozyma Kurtzman (4)

Yueomyces Q.M. Wang, L. Wang, M. Groenewald \& T. Boekhout (1)

Zygosaccharomyces B.T.P. Barker (11)

Zygotorulaspora Kurtzman (4)

Saccharomycodaceae Kudrjanzev

Hanseniaspora Zikes (17)*

Saccharomycodes E.C. Hansen (2) 
Saccharomycopsidaceae Arx \& Van der Walt Ambrosiozyma Van der Walt (14)

Saccharomycopsis Schiønning (19)

Trichomonascaceae Kurtzman \& Robnett*

Blastobotrys Klopotek (23)

Diddensiella Péter, Dlauchy \& Kurtzman (1)

Groenewaldozyma Kurtzman (3)

Spencermartinsiella Péter, Dlauchy, Tornai-Lehoczki, M. Suzuki \& Kurtzman (4)

Starmerella Rosa \& Lachance (44)

Sugiyamaella Kurtzman \& Robnett (27)

Trichomonascus H.S. Jackson emend. Kurtzman \& Robnett (6)*

Wickerhamiella Van der Walt (39)

Zygoascus M.T. Sm. (8)

Trigonopsidaceae M.A. Lachance \& C.P. Kurtzman

Botryozyma Shann \& M.T. Sm. emend. Lachance \& Kurtzman (4)*

Tortispora Lachance \& Kurtzman (8)

Trigonopsis Schachner emend. Kurtzman \& Robnett (4)

Saccharomycetales genera incertae sedis

Aciculoconidium D.S. King \& S.C. Jong (1)

Candida Berkhout (316)*

Coccidiascus Chatton (1)

Conidiascus Holterm. (1)

Danielozyma Kurtzman \& Robnett (2)

Deakozyma Kurtzman \& Robnett (2)

Diutina Khunnamwong, Lertwattanasakul, Jindam., Limtong \& Lachance (10)

Endomyces Reess (4)

Hyphopichia von Arx \& van der Walt (12)

Macrorhabdus Tomaszewski, Logan, Snowden, Kurtzman \& Phalen. (1)

Metahyphopichia Sipiczki \& Pfliegler (1)

Middelhovenomyces Kurtzman \& Robnett (2)

Nadsonia Syd. (3)

Nakazawaea Y. Yamada, Maeda \& Mikata (13)

Oscarbrefeldia Holterm. (1)*

Peterozyma Kurtzman \& Robnett (2)

Phialoascus Redhead \& Malloch (1)

Sporopachydermia Rodr. Mir. (3)

Teunomyces Kurtzman \& M. Blackwell (12)

Wickerhamia Soneda (1)

Yarrowia Van der Walt \& Arx (12)

TAPHRINOMYCOTINA O.E. Erikss. \& Winka

Archaeorhizomycetes Rosling \& T.Y. James

Archaeorhizomycetales Rosling \& T.Y. James

Archaeorhizomycetaceae Rosling \& T.Y. James

Archaeorhizomyces Rosling \& T.Y. James (2)

Neolectomycetes O.E. Erikss. \& Winka

Neolectales Landvik, O.E. Erikss., Gargas \& P. Gust.

Neolectaceae Redhead 
Neolecta Speg. (3)

Pneumocystomycetes O.E. Erikss. \& Winka Pneumocystidales O.E. Erikss.

Pneumocystidaceae O.E. Erikss.

Pneumocystis P. Delanoë \& Delanoë (5)

Schizosaccharomycetes O.E. Erikss. \& Winka

Schizosaccharomycetales O.E. Erikss.

Schizosaccharomycetaceae Beij. ex Klöcker

Schizosaccharomyces Lindner (4)

Taphrinomycetes O.E. Erikss. \& Winka

Taphrinales Gäum. \& C.W. Dodge

Protomycetaceae Gray

Buerenia M.S. Reddy \& C.L. Kramer (4)

Protomyces Unger (ca. 10)

Protomycopsis Magnus (5)

Saitoella Goto, Sugiy., Hamam. \& Komag. (2)

Taphridium Lagerh. \& Juel ex Juel (2)

Volkartia Maire (1)

Taphrinaceae Gäum.

Taphrina Fr. (ca. 95)

Ascomycota families incertae sedis

Aphanopsidaceae Printzen \& Rambold

Aphanopsis Nyl. ex Syd. (1)

Steinia Körb. (3)

Diporothecaceae R.K. Mibey \& D. Hawksw.

Diporotheca C.C. Gordon \& C.G. Shaw (4)

Eoterfeziaceae G.F. Atk.

Acanthogymnomyces Udagawa \& Uchiyama (1)

Eoterfezia G.F. Atk. (2)

Mucomassariaceae Petr. \& Cif.

Mucomassaria Petr. (1)

Saccardiaceae Höhn.

Ascolectus Samuels \& Rogerson (1)

Cyanodiscus E. Müll. \& M.L. Farr (2)

Henningsiella Rehm (2)

Phillipsiella Cooke (7)

Pseudodiscus Arx \& E. Müll. (1)

Saccardia Cooke (3)

Schenckiella P. Henn. (1)

Seuratiaceae Vuill. ex M.E. Barr

Seuratia Pat. (5)

Seuratiopsis Woron. (1) 
Strangosporaceae S. Stenroos, Miądl. \& Lutzoni Strangospora Körb. (ca. 11)

Ascomycota genera incertae sedis

Abropelta B. Sutton (1)

Acarellina Bat. \& H. Maia (1)

Acaroconium Kocourk. \& D. Hawksw. (1)

Acarocybe Syd. (3)

Acarocybella M.B. Ellis (1)

Acarocybellina Subram. (1)

Acarocybiopsis J. Mena, A. Hern.-Gut. \& Mercado (1)

Acaropeltis Petr. (1)

Achoropeltis Syd. (1)

Acleistia Bayl. Ell. (1)

Acontium Morgan (4)

Acrodictyella W.A. Baker \& Partr. (1)

Acrodictyopsis P.M. Kirk (1)

Acrodontiella U. Braun \& Scheuer (1)

Acrophragmis Kiffer \& Reisinger (4)

Acrospeira Berk. \& Broome (1)

Acrostaurus Deighton \& Piroz. (1)

Actinocladium Ehrenb. (6)

Actinotexis Arx (1)

Actinothecium Ces. (5)

Actinothyrium Kunze (10)

Acumispora Matsush. (5)

Agaricodochium X.J. Liu (1)

Agarwalomyces R.K. Verma \& Kamal (1)

Agrabeeja Subram. (1)

Agyriella Sacc. (2)

Agyriellopsis Höhn. (3)

Ahmadia Syd. (1)

Ajrekarella Kamat \& Kalani (1)

Alatosessilispora K. Ando \& Tubaki (1)

Alciphila Harmaja (1)

Algonquinia R.F. Castañeda \& W.B. Kendr. (1)

Allantophomoides S.L. Wei \& T.Y. Zhang (1)

Alloneottiosporina Nag Raj (2)

Allophoron Nádv. (1)

Allothyriella Bat., Cif. \& Nascim. (3)

Allothyrina Bat. \& J.L. Bezerra (1)

Allothyriopsis Bat., Cif. \& H. Maia (1)

Alpakesa Subram. \& K. Ramakr. (4)

Alpakesiopsis Abbas, B. Sutton, Ghaffar \& A. Abbas (1)

Alveariospora Meir. Silva, R.F. Castañeda, O.L. Pereira \& R.W. Barreto (1)

Alveophoma Alcalde (1)

Alysidiopsis B. Sutton (5)

Amallospora Penz. (1)

Amblyosporium Fresen. (4)

Ameroconium U. Braun \& Zhurb. (1)

Amerodiscosiella M.L. Farr (1)

Amerodiscosiellina Bat. \& Cavalc. (1) 
Amerosporiopsis Petr. (1)

Amerosympodula Matsush. (1)

Amoenodochium Peláez \& R.F. Castañeda (1)

Amoenomyces R.F. Castañeda, Saikawa \& Hennebert (1)

Amphichaetella Höhn. (1)

Amphophialis R.F. Castañeda, W.B. Kendr. \& Guarro (1)

Amphoropycnium Bat. (1)

Ampullicephala R.F. Castañeda, Minter \& M. Stadler (1)

Ampulliferina B. Sutton (2)

Amylogalla Suija, Motiej. \& Kantvilas (1)

Anabahusakala Carmo, J.S. Monteiro, Gusmão \& R.F. Castañeda (1)

Anacraspedodidymum C.R. Silva, R.F. Castañeda \& Gusmão (2)

Anaexserticlava Santa Izabel, R.F. Castañeda \& Gusmão (1)

Anaphysmene Bubák (2)

Anarhyma M.H. Pei \& Z.W. Yuan (1)

Anaselenosporella Heredia, R.F. Castañeda \& R.M. Arias (2)

Anaseptoidium R.F. Castañeda, Heredia \& R.M. Arias (1)

Anaverticicladus P.O. Costa, Malosso \& R.F. Castañeda (1)

Ancoraspora Mig. Rodr. (1)

Ancorasporella J. Mena, Mercado \& Heredia (1)

Angiopomopsis Höhn. (1)

Angulimaya Subram. \& Lodha (1)

Angulospora Sv. Nilsson (1)

Annellodentimyces Matsush. (1)

Annellodochium Deighton (1)

Annellophorella Subram. (5)

Annellospermosporella P.R. Johnst. (1)

Antennatula Fr. ex F. Strauss (10)

Anthracoderma Speg. (3)

Antimanoa Syd. (1)

Antromyces Fresen. (4)

Anulohypha Cif. (1)

Anungitopsis R.F. Castañeda \& W.B. Kendr. (7)

Aoria Cif. (1)

Aphanofalx B. Sutton (2)

Apiocarpella Syd. \& P. Syd. (8)

Apiotypa Petr. (1)

Apogloeum Petr. (1)

Apomelasmia Grove (8)

Aporellula B. Sutton (2)

Aposporella Thaxt. (1)

Apostrasseria Nag Raj (2)

Arachnophora Hennebert (11)

Arachnospora R.F. Castañeda, Minter \& Camino (1)

Arborillus Munt.-Cvetk. \& Gómez-Bolea (1)

Arborispora K. Ando (4)

Arcuadendron Sigler \& J.W. Carmich. (2)

Ardhachandra Subram. \& Sudha (3)

Argentinomyces Peña \& Arambarri (1)

Argopericonia B. Sutton \& Pascoe (2)

Aristastoma Tehon (1)

Arthrobotryum Ces. (5) 
Arthrocristula Sigler, M.T. Dunn \& J.W. Carmich. (1)

Arthromoniliphora S.S. Silva, Gusmão \& R.F. Castañeda (1)

Arthrosporium Sacc. (2)

Arthrowallemia R.F. Castañeda, Dania García \& Guarro (2)

Articulophora C.J.K. Wang \& B. Sutton (1)

Artocarpomyces Subram. (1)

Ascochytopsis Henn. (5)

Ascochytulina Petr. (3)

Ascofascicula Matsush. (6)

Ascomauritiana V.M. Ranghoo \& K.D. Hyde (1)

Ascosubramania Rajendran (1)

Ashtaangam Subram. (1)

Aspilaima Bat. \& H. Maia (1)

Astelechia Cif. (2)

Asterinothyriella Bat. \& Cif. (3)

Asterinothyrium Bat., Cif. \& H. Maia (1)

Asteroconium Syd. \& P. Syd. (2)

Asteroglobulus Brackel (2)

Asteromyces F. Moreau \& V. Moreau (1)

Asterophoma D. Hawksw. (1)

Asteroscutula Petr. (1)

Asterostomopora Bat. \& H. Maia (1)

Asterostomopsis Bat., Cif. \& H. Maia (1)

Asterostomula Theiss. (4)

Asterostomulina Bat., J.L. Bezerra \& H. Maia (1)

Astomella Thirum. (1)

Astronatelia Bat. \& H. Maia (1)

Atractilina Dearn. \& Barthol. (2)

Atractobolus Tode (1)

Atrosetaphiale Matsush. (1)

Atrosynnema J.W. Xia, X.G. Zhang \& Z. Li (1)

Aurosphaeria Sun J. Lee, Strobel, Eisenman, Geary, P.N. Vargas \& S.A. Strobel (1)

Avesicladiella W.P. Wu, B. Sutton \& Gange (2)

Avettaea Petr. \& Syd. (3)

Bacillopeltis Bat. (1)

Bactridium Kunze (15)

Bactrodesmiella M.B. Ellis (2)

Baculospora Zukal (1)

Badarisama Kunwar, J.B. Manandhar \& J.B. Sinclair (1)

Bahuchashaka Subram. (1)

Bahugada K.A. Reddy \& Vasant Rao (2)

Bahukalasa Subram. \& Chandrash. (1)

Balaniopsis P.M. Kirk (4)

Balanium Wallr. (1)

Barbarosporina Kirulis (1)

Barnettella D. Rao \& P. Rag. Rao (1)

Basauxia Subram. (1)

Batistina Peres (1)

Batistospora J.L. Bezerra \& M.M.P. Herrera (1)

Beauveriphora Matsush. (1)

Beccopycnidium F. Stevens (1)

Beejadwaya Subram. (1) 
Belemnospora P.M. Kirk (7)

Bellulicauda B. Sutton (2)

Beltramono Rashmi Dubey, A.K. Pandey bis \& Manohar. (1)

Beltraniomyces Manohar., D.K. Agarwal \& Rao (1)

Beniowskia Racib. (4)

Benjpalia Subram. \& Bhat (1)

Berggrenia Cooke (2)

Bhadradriella Nagaraju, Kunwar \& Manohar. (1)

Bhadradriomyces Sureshk., Manohar. \& Kunwar (1)

Bharatheeya D'Souza \& Bhat (3)

Bhatia W.A. Baker \& Morgan-Jones (2)

Bibanasiella R.F. Castañeda \& W.B. Kendr. (1)

Bicoloromyces Heuchert, U. Braun \& D. Hawksw. (1)

Biflagellospora Matsush. (1)

Biflagellosporella Matsush. (1)

Biflua Jørgen Koch \& E.B.G. Jones (1)

Bimeris Petr. (1)

Bioconiosporium Bat. \& J.L. Bezerra (2)

Biophomopsis Petr. (3)

Bisbyopeltis Bat. \& A.F. Vital (1)

Bispora Corda (31)

Bisseomyces R.F. Castañeda (1)

Blastocatena Subram. \& Bhat (2)

Blastodictys M.B. Ellis (1)

Blastofusarioides Matsush. (1)

Blastophorella Boedijn (1)

Blastophragma Subram. (4)

Blennoria Moug. \& Fr. (4)

Blennoriopsis Petr. (1)

Bleptosporium Steyaert (4)

Blodgettia Harv. (2)

Bostrichonema Ces. (4)

Botryoderma Papendorf \& H.P. Upadhyay (4)

Botryodiplodina Dias \& Sousa da Câmara (1)

Botryomonilia Goos \& Piroz. (1)

Botryostroma Höhn. (2)

Brachycephala J.S. Monteiro, Gusmão \& R.F. Castañeda (1)

Brachydesmiella G. Arnaud ex S. Hughes (8)

Brachysporiellina Subram. \& Bhat (2)

Brachysporiopsis Yanna, W.H. Ho \& K.D. Hyde (1)

Braunomyces V.A. Melnik \& Crous (1)

Brefeldiopycnis Petr. \& Cif. (1)

Brencklea Petrak (1)

Brevicatenospora R.F. Castañeda, Minter \& Saikawa (1)

Briosia Cavara (6)

Brycekendrickia Nag Raj (1)

Bryophytomyces Cif. (1)

Bulbilopycnis Matsush. (1)

Bulbocatenospora R.F. Castañeda \& Iturr. (1)

Bullaserpens Bat., J.L. Bezerra \& Cavalc. (1)

Cacumisporium Preuss (9) 
Caeruleoconidia Zhurb. \& Pino-Bodas (= Caeruleoconidia Zhurb. \& Diederich 2015 nom. inv.) (2)

Calcarispora Marvanová \& Marvan (1)

Calceispora Matsush. (2)

Callistospora Petr. (1)

Calocline Syd. (1)

Calongeomyces D. Hawksw. \& Etayo (1)

Camaroglobulus Speer (1)

Camaropycnis E.K. Cash (1)

Camarosporellum Tassi (1)

Camarosporiopsis Abbas, B. Sutton \& Ghaffar (1)

Camposporidium Nawawi \& Kuthub. (3)

Candelabrum Beverw. (7)

Candelosynnema K.D. Hyde \& Seifert (1)

Capitorostrum Bat. (1)

Capnocheirides J.L. Crane \& S. Hughes (1)

Capnofrasera S. Hughes (1)

Capsicumyces Gamundí et al. (1)

Carnegieispora Etayo \& F. Berger (1)

Carnia Bat. (1)

Carrismyces R.F. Castañeda \& Heredia (1)

Casaresia Gonz. Frag. (1)

Castanedaea W.A. Baker \& Partr. (1)

Catenocuneiphora Matsush. (1)

Catenophora Luttr. (3)

Catenophoropsis Nag Raj \& W.B. Kendr. (1)

Catenosubulispora Matsush. (1)

Catenosynnema Kodsueb, K.D. Hyde \& W.H. Ho (1)

Catenulaster Bat. \& C.A.A. Costa (1)

Catinopeltis Bat. \& C.A.A. Costa (1)

Cecidiomyces U. Braun \& Zhurb. (1)

Ceeveesubramaniomyces J. Pratibha, K.D. Hyde \& Bhat (1)

Ceratocladium Corda (2)

Ceratophorum Sacc. (2)

Ceratopycnis Höhn. (2)

Ceratosporella Höhn. (19)

Ceratosporium Schwein. (11)

Ceuthodiplospora Died. (1)

Ceuthosira Petr. (1)

Ceuthosporella Petr. \& Syd. (1)

Chaetendophragmia Matsush. (7)

Chaetoblastophorum Morgan-Jones (1)

Chaetochalara B. Sutton \& Piroz. (7)

Chaetocytostroma Petr. (1)

Chaetodiplis Clem. (1)

Chaetodiplodina Speg. (2)

Chaetopeltaster Katum. (1)

Chaetophiophoma Speg. (1)

Chaetoplaca Syd. \& P. Syd. (1)

Chaetopsis Grev. (7)

Chaetopyrena Pass. (2)

Chaetoseptoria Tehon. (1) 
Chalarodendron C.J.K. Wang \& B. Sutton (1)

Chalarodes McKenzie (2)

Chantransiopsis Thaxt. (3)

Characonidia Bat. \& Cavalc. (1)

Charomyces Seifert (2)

Chasakopama Manohar., Bagyan., N.K. Rao \& Kunwar (1)

Cheilaria Lib. (1)

Cheiroidea W.A. Baker \& Morgan-Jones (1)

Cheiromycella Höhn. (3)

Cheiromyceopsis Mercado \& J. Mena (1)

Cheiromyces Berk. \& M.A. Curtis (6)

Cheiropolyschema Matsush. (2)

Chiastospora Riess (1)

Chithramia Nag Raj (1)

Chlamydopsis Hol.-Jech. \& R.F. Castañeda (1)

Choanatiara DiCosmo (2)

Choreospora Constant. \& R. Sant. (1)

Chrysachne Cif. (2)

Chrysalidopsis Steyaert (1)

Chryseidea Onofri (1)

Ciferria Gonz. Frag. (1)

Ciferrina Petr. (1)

Ciferriopeltis Bat. \& H. Maia (1)

Ciferrioxyphium Bat. \& H. Maia (2)

Ciliochora Höhn. (2)

Ciliophora Petr. (2)

Ciliophorella Petr. (2)

Ciliosporella Petr. (2)

Circinoconiopsis A. Hern.-Gut. (1)

Circinoconis Boedijn (1)

Cissococcomyces Brain (1)

Civisubramaniania Vittal \& Dorai (2)

Cladoconidium Bandoni \& Tubaki (1)

Cladoniicola Diederich, van den Boom \& Aptroot (2)

Cladosphaera Dumort. (1)

Cladosporiopsis S.C. Ren \& X.G. Zhang (1)

Clasteropycnis Bat. \& Cavalc. (1)

Clathroconium Samson \& H.C. Evans (2)

Clauzadeomyces Diederich (1)

Clavariana Nawawi (1)

Cleistocystis Sousa da Câmara (1)

Cleistonium Speer (1)

Cleistophoma Petr. \& Syd. (2)

Clypeochorella Petr. (1)

Clypeolum Speg. (8)

Clypeopatella Petr. (1)

Clypeophialophora Bat. \& Peres (1)

Clypeopycnis Petr. (3)

Clypeoseptoria F. Stevens \& P.A. Young (3)

Clypeostagonospora Punith. (1)

Coccogloeum Petr. (1)

Codonmyces Calat. \& Etayo (1) 
Colemaniella Agnihothr. (1)

Coleodictyospora Charles (2)

Coleoseptoria Petr. (1)

Colispora Marvanová (3)

Colletoconis de Hoog \& Aa (1)

Colletosporium Link (1)

Collostroma Petr. (1)

Columnodomus Petr. (1)

Columnothyrium Bubák (1)

Comatospora Piroz. \& Shoemaker (1)

Comocephalum Syd. (1)

Complexipes C. Walker (2)

Condylospora Nawawi (4)

Coniambigua Etayo \& Diederich (1)

Conioscyphopsis Goh \& K.D. Hyde (1)

Coniothyrina Syd. (1)

Conjunctospora Udagawa \& Uchiy. (1)

Conostoma Bat. \& J.L. Bezerra (2)

Conostroma Moesz (3)

Consetiella Hol.-Jech. \& Mercado (1)

Coremiella Bubák \& K. Krieg. (1)

Cornucopiella Höhn. (2)

Cornutispora Piroz. (9)

Cornutostilbe Seifert (1)

Coronospora M.B. Ellis (4)

Corynecercospora V.K. Pal, M. Akhtar, N. Ahmad, Kamal \& D.K. Agarwal (1)

Coryneliella Har. \& P. Karst. (1)

Corynesporella Munjal \& H.S. Gill (11)

Corynesporina Subram. (1)

Corynesporopsis P.M. Kirk (16)

Costanetoa Bat. \& J.L. Bezerra (1)

Crandallia Ellis \& Sacc. (4)

Craneomyces Morgan-Jones, R.C. Sinclair \& Eicker (1)

Craspedodidimella F.R. Barbosa, R.F. Castañeda \& Gusmão (1)*

Creodiplodina Petr. (1)

Creonecte Petr. (1)

Creoseptoria Petr. (1)

Creothyriella Bat. \& C.A.A. Costa (1)

Cribropeltis Tehon (1)

Crinigera I. Schmidt (1)

Crousobrauniella Sh. Kumar, Raghv. Singh, D.P. Singh \& Kamal (1)

Crustodiplodina Punith. (1)

Cryptoceuthospora Petr. (2)

Cryptocoryneopsis B. Sutton (1)

Cryptosporium Kunze (25)

Cryptumbellata Udagawa \& Uchiy. (1)

Ctenosporium R. Kirschner (1)

Cubasina R.F. Castañeda (2)

Culicidospora R.H. Petersen (2)

Culicinomyces Couch, Romney \& B. Rao (3)

Curucispora Matsush. (3)

Curvulariopsis M.B. Ellis (1) 
Cyanopatella Petr. (1)

Cyanopyrenia Harada (1)

Cyclomarsonina Petr. (1)

Cylindrogloeum Petr. (1)

Cylindromyces Manohar., D.K. Agarwal \& N.K. Rao (1)

Cylindrothyrium Maire (1)

Cylindroxyphium Bat. \& Cif. (1)

Cyrtidium Vain (1)

Cyrtidula Minks (ca. 5)

Cyrtopsis Vain. (1)

Cystodium Fée (1)

Cystotricha Berk. \& Broome (1)

Cytodiscula Petr. (1)

Cytogloeum Petr. (1)

Cytonaema Höhn. (2)

Cytoplacosphaeria Petr. (2)

Cytosphaera Died. (2)

Cytosporella Sacc. (32)

Cyttariella Palm (1)

Dactylifera Alcorn (1)

Dactylosporium Harz (2)

Dasysticta Speg. (2)

Davisiella Petr. (2)

Dearnessia Bubák (1)

Deichmannia Alstrup \& D. Hawksw. (1)

Delortia Pat. \& Gaillard (3)

Dendrodomus Bubák (1)

Dendrographiella Agnihothr. (1)

Dendrographium Massee (8)

Dendrospora Ingold (10)

Dendrosporium Plakidas \& Edgerton ex J.L. Crane (2)

Dendryphiosphaera Lunghini \& Rambelli (4)

Dennisographium Rifai (2)

Denticularia Deighton (7)

Dentocircinomyces R.F. Castañeda \& W.B. Kendr. (1)

Descalsia A. Roldán \& Honrubia (1)

Desertella Mouch. (2)

Desmidiospora Thaxt. (3)

Dexhowardia J.J. Taylor (1)

Diaboliumbilicus I. Hino \& Katum. (1)

Diademospora B.E. Söderstr. \& Bååth (1)

Diarimella B. Sutton (3)

Dichelostroma Bat. \& Peres (1)

Dicholobodigitus G.P. White \& Illman (1)

Dichotomophthoropsis M.B. Ellis (2)

Dichotophora Whitton, K.D. Hyde \& McKenzie (2)

Dictyoceratosporella Y.R. Ma \& X.G. Zhang (3)*

Dictyodesmium S. Hughes (4)

Dictyophrynella Bat. \& Cavalc. (1)

Dictyopolyschema M.B. Ellis (1)

Dictyorostrella U. Braun (1)

Dictyospiropes M.B. Ellis (1) 
Dictyotrichocladium Fiuza, Gusmão \& R.F. Castañeda (1)

Didymochaetina Bat. \& J.L. Bezerra (1)

Didymochora Höhn. (1)

Didymopsis Sacc. \& Marchal (5)

Didymosporina Höhn. (1)

Diedickea Syd. \& P. Syd. (3)

Digicatenosporium S.M. Leão, Gusmão \& R.F. Castañeda (1)

Digitodochium Tubaki \& Kubono (1)

Digitopodium U. Braun et al. (1)

Digitoramispora R.F. Castañeda \& W.B. Kendr. (4)

Dimastigosporium Faurel \& Schotter (2)

Diplocladiella G. Arnaud ex M.B. Ellis (8)

Diplodinis Clem. (1)

Diplodinula Tassi (1)

Diploplenodomus Died. (2)

Diplosporonema Höhn. (1)

Diplozythiella Died. (1)

Dipyrgis Clem. (1)

Discogloeum Petr. (1)

Discomycetoidea Matsush. (1)

Discosiellina Subram. \& K.R.C. Reddy (1)

Discosporina Höhn. (1)

Discotheciella Syd. \& P. Syd (1)

Discozythia Petr. (1)

Dissitimurus E.G. Simmons, McGinnis \& Rinaldi (1)

Distophragmia R.F. Castañeda, S.M. Leão \& Gusmão (1)

Ditangifibula G.C. Adams (1)

Domingoella Petr. \& Cif. (4)

Dothideodiplodia Murashk. (1)

Dothioropsis Riedl (1)

Drepanospora Berk. \& M.A. Curtis (1)

Drudeola Kuntze (1)

Drumopama Subram. (1)

Dryosphaera Jørg. Koch \& E.B.G. Jones (3)

Dualomyces Matsush. (2)

Dwayabeeja Subram. (3)

Dwayaloma Subram. (1)

Dwayalomella Brisson, Piroz. \& Pauzé (1)

Dwibahubeeja N. Srivast., A.K. Srivast. \& Kamal (1)

Dwibeeja Subram. (1)

Dwiroopella Subram. \& Muthumary (1)

Ebollia Minter \& Caine (1)

Echinocatena R. Campb. \& B. Sutton (1)

Echinochondrium Samson \& Aa (1)

Echinoconidiophorum Pereira-Carv. \& Dianese (1)

Eiona Kohlm. (1)

Elachopeltella Bat. \& Cavalc. (2)

Elattopycnis Bat. \& Cavalc. (1)

Elegantimyces Goh, C.K.M. Tsui \& K.D. Hyde (1)

Elletevera Deighton (2)

Ellisembiopsis T.S. Santa Izabel \& Gusmão (2)

Ellismarsporium R.F. Castañeda \& X.G. Zhang (7) 
Elotespora R.F. Castañeda \& Heredia (1)

Embryonispora G.Z. Zhao (1)

Enantioptera Descals (2)

Endobotrya Berk. \& M.A. Curtis (1)

Endobotryella Höhn. (1)

Endocolium Syd. (1)

Endoconospora Gjaerum (2)

Endocoryneum Petr. (3)

Endogenospora R.F. Castañeda, O. Morillo \& Minter (1)

Endomelanconium Petr. (4)

Endophragmiopsis M.B. Ellis (2)

Endoplacodium Petr. (1)

Endoramularia Petr. (1)

Endosporoideus W.H. Ho, Yanna, K.D. Hyde \& Goh (1)

Endozythia Petr. (1)

Enerthidium Syd. (1)

Engelhardtiella A. Funk (1)

Enridescalsia R.F. Castañeda \& Guarro (1)

Enthallopycnidium F. Stevens (1)

Entoderma Hanula, Andreadis \& M. Blackw. (1)

Epaphroconidia Calat. \& V. Atienza (1)

Ephelidium C.W. Dodge \& E.D. Rudolph (1)

Epiclinium Fr. (2)

Epicoccospora Budathoki \& S.K. Singh (2)

Episporogoniella U. Braun (1)

Epistigme Syd. (2)

Epithyrium (Sacc.) Trotter (2)

Eriocercospora Deighton (3)

Eriocercosporella Rak. Kumar, A.N. Rai \& Kamal ex U. Braun (2)

Eriospora Berk. \& Broome (1)

Erispora Pat. (1)

Esteya J.Y. Liou, J.Y. Shih \& Tzean (1)

Evanidomus Caball. (1)

Everhartia Sacc. \& Ellis (6)

Everniicola D. Hawksw. (1)

Eversia J.L. Crane \& Schokn. (2)

Excipularia Sacc. (2)

Exophoma Weedon (1)

Exosporella Höhn. (1)

Exosporodiella Ganie, Azam \& A.H. Wani (1)

Fairmaniella Petr. \& Syd. (1)

Farriolla Norman (1)

Favostroma B. Sutton \& E.M. Davison (1)

Feltgeniomyces Diederich (4)

Fenestroconidia Calat. \& Etayo (1)

Fissuricella Pore, D'Amatao \& Ajello (1)

Flabellocladia Nawawi (2)

Flabellospora Alas. (6)

Flosculomyces B. Sutton (2)

Frigidispora K.D. Hyde \& Goh (1)

Fujimyces Minter \& Caine (2)

Fuligomyces Morgan-Jones \& Kamal (4) 
Fumagopsis Speg. (2)

Furcaspora Bonar (2)

Fusamen (Sacc.) P. Karst. (2)

Fuscophialis B. Sutton (4)

Fusticeps J. Webster \& R.A. Davey (5)

Gaeumanniella Petr. (1)

Gallaicolichen Serux. \& Lücking (1)

Gampsonema Nag Raj (1)

Gangliophora Subram. (1)

Gangliostilbe Subram. \& Vittal (5)

Garnaudia Borowska (3)

Gaubaea Petr. (2)

Gelatinocrinis Matsush. (1)

Gelatinopycnis Dyko \& B. Sutton (1)

Geminoarcus K. Ando (3)

Gemmulina Descals \& Marvanová (1)

Gilmaniella G.L. Barron (9)

Glaphyriopsis B. Sutton \& Pascoe (2)

Glioannellodochium Matsush. (1)

Glioblastocladium Matsush. (1)

Globoconidiopsis G.F. Sepúlveda, Pereira-Carv. \& Dianese (1)

Globoconidium G.F. Sepúlveda, Pereira-Carv. \& Dianese (1)

Gloeocoryneum Weindlm. (3)

Gloeodes Colby (1)

Gloeosporiella Cavara (1)

Gloiosphaera Höhn. (2)

Glutinium Fr. (2)

Goidanichiella G.L. Barron ex W. Gams (5)

Gonatobotryum Sacc. (4)

Goniopila Marvanová \& Descals (1)

Goosiella Morgan-Jones, Kamal \& R.K. Verma (1)

Goosiomyces N.K. Rao \& Manohar. (2)

Grallomyces F. Stevens (1)

Graphiothecium Fuckel (6)

Groveolopsis Boedijn (6)

Guarroa M. Calduch, Gené, Heredia \& R.F. Castañeda (1)

Guedea Rambelli \& Bartoli (3)

Guelichia Speg. (6)

Gymnoxyphium Cif., Bat. \& I.J. Araújo (6)

Gyrophthorus Hafellner \& Sancho (3)

Hadronema Syd. \& P. Syd. (4)

Hadrosporium Syd. (2)

Halysiomyces E.G. Simmons (1)

Hansfordiopeltis Bat. \& C.A.A. Costa (5)

Hansfordiopeltopsis M.L. Farr (1)

Hapalosphaeria Syd. (1)

Haplariopsis Oudem. (2)

Haplobasidion Erikss. (3)

Haplolepis Syd. (3)

Haptocara Drechsler (1)

Harmoniella V.N. Boriss. (2)*

Harpographium Sacc. (5) 
Harpostroma Höhn. (1)

Hawksworthiana U. Braun (4)

Heimiodora Nicot (1)

Helensiella Minter, R.F. Castañeda \& Heredia (1)

Helhonia B. Sutton (1)

Helicofilia Matsush. (2)

Helicogoosia Hol.-Jech. (1)

Helicominopsis Deighton (2)

Helicorhoidion S. Hughes (6)

Helicosingula P.S. van Wyk, Marasas, Baard \& Knox-Dav. (1)

Helicothyrium I. Hino \& Katum. (1)

Helicoubisia Lunghini \& Rambelli (1)

Heliscella Marvanová (2)

Heliscina Marvanová (2)

Helminthosporiomyces G.F. Sepúlveda, Pereira-Carv. \& Dianese (1)

Helochora Sherwood (1)

Hemicorynesporella Subram. (1)

Hemidothis Syd. \& P. Syd. (1)

Hemisphaeropsis Petr. (1)

Hendersoniella Tassi (1)

Hendersonina E.J. Butler (1)

Hendersoniopsis Höhn. (1)

Hendersonula Speg. (20)

Hendersonulina Petr. (1)

Henfellra Halici, D. Hawksw., Z. Kocak. \& M. Kocak (1)

Henicospora P.M. Kirk \& B. Sutton (6)

Herposira Syd. (1)

Herreromyces R.F. Castañeda \& W.B. Kendr. (1)

Heterocephalum Thaxt. (2)

Heterosporiopsis Petr. (1)

Heuflera Bail (1)

Hexacladium D.L. Olivier (1)

Himantia Pers. (4)

Hinoa Hara \& I. Hino (2)

Hirudinaria Ces. (2)

Hobsoniopsis D. Hawksw. (1)

Hoehneliella Bres. \& Sacc. (2)

Holubovaea Mercado (2)

Homalopeltis Bat. \& Valle (1)

Hoornsmania Crous (1)

Hormiactis Preuss (5)

Hormiscioideus M. Blackw. \& Kimbr. (1)

Hormocephalum Syd. (1)

Hormographis Guarro, Punsola \& Arx (1)

Hughesinia J.C. Lindq. \& Gamundí (3)

Hyalobelemnospora Matsush. (1)

Hyalocamposporium Révay \& J. Gönczöl (4)

Hyalocephalotrichum Nagaraju, Kunwar, Sureshk. \& Manohar. (1)

Hyalocladium Mustafa (1)

Hyalocylindrophora J.L. Crane \& Dumont (3)

Hyalodermella Speg. (1)

Hyalodictyum Woron. (1) 
Hyalohelicomina T. Yokoy. (1)

Hyalopleiochaeta R.F. Castañeda, Guarro \& Cano (1)

Hyalopyrenia $\mathrm{H}$. Harada (1)

Hyalosynnema Matsush. (1)

Hyalothyridium Tassi (1)

Hydrometrospora J. Gönczöl \& Révay (1)

Hymenella Fr. (11)

Hymeniopeltis Bat. (3)

Hymenobactron (Sacc.) Höhn.

Hymenobia Nyl. (1)

Hymenopsis Sacc. (13)

Hyphodiscosia Lodha \& K.R.C. Reddy (5)

Hyphodiscosioides Matsush. (1)

Hyphopolynema Nag Raj (6)

Hyphostereum Pat. (1)

Hyphothyrium B. Sutton \& Pascoe (1)

Hyphozyma de Hoog \& M.T. Sm. (4)

Hypnotheca Tommerup (1)

Hypocline Syd. (1)

Hypodermina Höhn. (1)

Hypogloeum Petr. (1)

Hypotrachynicola Etayo (1)

Hysteridium P. Karst. (1)

Hysterodiscula Petr. (1)

Hysteropycnis Hilitzer (1)

Ialomitzia Gruia (1)

Idiocercus B. Sutton (2)

Igneocumulus A.W. Ramaley (10)

Imicles Shoemaker \& Hambl. (6)

Impudentia Vujanović (1)

Inesiosporium R.F. Castañeda \& W. Gams (2)

Inifatiella R.F. Castañeda (1)

Intercalarispora J.L. Crane \& Schokn. (1)

Intralichen D. Hawksw. \& M.S. Cole (4)*

Ionophragmium Peres (1)

Irpicomyces Deighton (3)

Isariella Henn. (2)

Ischnostroma Syd. \& P. Syd. (1)

Isthmoconidium Etayo \& Fr. Berger (1)

Isthmolongispora Matsush. (11)

Isthmophragmospora Kuthub. \& Nawawi (2)

Isthmotricladia Matsush. (3)

Ityorhoptrum P.M. Kirk (4)

Iyengarina Subram. (3)

Jahniella Petr. (3)

Javonarxia Subram. (2)

Jayarambhatia J. Pratibha (1)

Jerainum Nawawi \& Kuthub. (1)

Jubispora B. Sutton \& H.J. Swart (1)

Junctospora Minter \& Hol.-Jech. (1)

Kalamarospora G. Delgado (1)

Kalchbrenneriella Diederich \& M.S. Christ. (1) 
Kaleidosporium Van Warmelo \& B. Sutton (1)

Kamatella Anahosur (1)

Kamatia V.G. Rao \& Subhedar (1)

Kameshwaromyces Kamal, R.K. Verma \& Morgan-Jones (2)

Katherinomyces Khodos. (1)

Keissleriomyces D. Hawksw. (1)

Kendrickiella K. Jacobs \& M.J. Wingf. (1)

Ketubakia Kamat, Varghese \& V.G. Rao (1)

Kiliophora Kuthub. \& Nawawi (3)

Kionocephala P.M. Kirk (1)

Kmetia Bres. \& Sacc. (1)

Kmetiopsis Bat. \& Peres (1)

Knemiothyrium Bat. \& J.L. Bezerra (1)

Kodonospora K. Ando (1)

Kolletes Kohlm. \& Volkm.-Kohlm. (1)

Kontospora A. Roldán et al. (1)

Korunomyces Hodges \& F.A. Ferreira (3)

Kostermansinda Rifai (4)

Kostermansindiopsis R.F. Castañeda (1)

Kramabeeja G.V. Rao \& K.A. Reddy (1)

Kramasamuha Subram. \& Vittal (1)

Kreiseliella Braun (1)

Kumanasamuha P. Rag. Rao \& D. Rao (5)

Kutilakesa Subram. (2)

Kyphophora B. Sutton (1)

Lacellina Sacc. (3)

Lacellinopsis Subram. (3)

Laciniocladium Petri (1)

Lagenomyces Cavalc. \& A.A. Silva (1)

Lambdasporium Matsush. (3)

Lambinonia Sérus. \& Diederich (1)

Laocoön J.C. David (1)

Lappodochium Matsush. (1)

Lasiodiplodiella Zambett. (3)

Lasiothyrium Syd. \& P. Syd. (1)

Lasmeniella Petr. \& Syd. (13)

Latericonis G.V. Rao, K.A. Reddy \& de Hoog (1)

Lateriramulosa Matsush. (5)

Laterispora Uecker, W.A. Ayers \& P.B. Adams (1)

Lawalreea Diederich (1)

Lecaniocola Brain (1)

Lecanostictopsis B. Sutton \& Crous (4)

Leeina Petr. (1)

Leightoniomyces D. Hawksw. \& B. Sutton (2)

Lembuncula Cif. (1)

Lemkea Morgan-Jones \& R.C. Sinclair (1)

Lepisticola W. Gams (1)

Leprieurinella Bat. \& H. Maia (1)

Leptascospora Speg. (1)

Leptochlamys Died. (1)

Leptodermella Höhn. (1)

Leptophyllosticta I.E. Brezhnev (2) 
Leptostromella (Sacc.) Sacc. (2)

Leptothyrella Sacc. (10)

Leptothyrina Höhn. (1)

Leptothyrium Kunze (2)

Leucoconiella Bat., H. Maia \& Peres (1)

Leucoconis Theiss. \& Syd. (1)

Leucodochium Syd. \& P. Syd. (1)

Leuliisinea Matsush. (2)

Lichenobactridium Diederich \& Etayo (1)

Lichenohendersonia Calat. \& Etayo (3)

Lichenopeziza Zukal (1)

Lichenophoma Keissl. (2)

Lichenopuccinia D. Hawksw. \& Hafellner (1)

Lichenostella Calat. \& Etayo (1)

Linkosia A. Hern. Gut. \& B. Sutton (12)

Linochorella Syd. \& P. Syd. (1)

Linodochium Höhn. (5)

Listeromyces Penz. \& Sacc. (1)

Lithopythium Bornet \& Flahault (3)

Lobatopedis P.M. Kirk (5)

Loliomyces Maire (1)

Lomaantha Subram. (3)

Lomachashaka Subram. (5)

Ludwigomyces Kirschst. (1)

Luxuriomyces R.F. Castañeda (1)

Luzfridiella R.F. Castañeda \& W.B. Kendr. (1)

Lylea Morgan-Jones (6)

Lysotheca Cif. (6)

Mackenziella Yanna \& K.D. Hyde (1)

Macroallantina Speer (1)

Macrodiplodia Sacc. (2)

Macrotrichum Grev. (2)

Magmopsis Nyl. (1)

Mahabalella B. Sutton \& S.D. Patil (4)

Manginella Bat. \& H. Maia (2)

Mapletonia B. Sutton (1)

Margarinomyces Laxa (1 fide Kirk et al. 2008)

Martinellisia V.G. Rao \& Varghese (1)

Massalongina Bubák (2)

Matsushimiella R.F. Castañeda \& Heredia (2)

Matsushimomyces V.G. Rao \& Varghese (2)

Medusamyces G.L. Barron \& Szijarto (1)

Megalodochium Deighton (4)

Megaloseptoria Naumov (1)

Melanocephala S. Hughes (5)

Melanophoma Papendorf \& J.W. du Toit (1)

Melophia Sacc. (4)

Menidochium R.F. Castañeda \& W.B. Kendr. (1)

Mercadomyces J. Mena (1)

Merismella Syd. (6)

Metadiplodia Syd. (40)

Metazythia Petr. (1) 
Metazythiopsis M. Morelet (1)

Microblastosporon Cif. (1)

Microclava F. Stevens (5)

Microdiscula Höhn. (2)

Microdothiorella C.A.A. Costa \& Sousa da Câmara (1)

Microhendersonula Dias \& Sousa da Câmara (1)

Micromastia Speg. (2)

Microperella Höhn. (1)

Micropustulomyces R.W. Barreto (1)

Microtyle Speg. (1)

Microxyphiella Speg. (15)

Microxyphiopsis Bat. (2)

Mindoa Petr. (2)

Minimidochium B. Sutton (8)

Minteriella Heredia, R.F. Castañeda \& R.M. Arias (1)

Minutophoma D. Hawksw. (1)

Mirandina G. Arnaud ex Matsush. (ca. 10)

Miricatena Punith. \& Spooner (2)

Mirimyces Nag Raj (1)

Monochaetiella E. Castell. (3)

Monochaetinula Muthumary, Abbas \& B. Sutton (6)

Monochaetopsis Pat. (1)

Monodia Breton \& Faurel (2)

Monodidymaria U. Braun (5)

Monodisma Alcorn (1)

Monostichella Höhn. (15)

Moorella P. Rag. Rao \& D. Rao (3)

Moralesia Urries (1)

Morrisographium M. Morelet (8)

Mucosetospora M. Morelet (1)

Muiogone Thaxt. (2)

Muirella R. Sprague (1)

Murogenella Goos \& E.F. Morris (3)

Mycelephas R.F. Castañeda (2)

Mycocentrodochium K. Matsush. \& Matsush. (1)

Mycoënterolobium Goos (3)

Mycohypallage B. Sutton (2)

Mycopara Bat. \& J.L. Bezerra (1)

Mycospraguea U. Braun \& Rogerson (1)

Mycosticta Höhn. (1)

Mycosylva M.C. Tulloch (3)

Mycotodea Kirschst. (14)

Mycousteria M.L. Farr (2)

Myiocoprula Petr. (2)

Myriellina Höhn. (2)

Myrmecomyces Jouvenaz \& Kimbr. (1)

Myrotheciastrum Abbas \& B. Sutton (1)

Mystrosporiella Munjal \& Kulshr. (4)

Myxoparaphysella Caball. (2)

Myxosporella Sacc. (1)

Myxosporidiella Negru (1)

Myxostomellina Syd. (1) 
Myxothyriopsis Bat. \& A.F. Vital (1)

Myxothyrium Bubák \& Kabát (1)

Naemosphaera P. Karst. (1)

Naemosphaerella Höhn. (2)

Nagrajia R.F. Castañeda \& W.B. Kendr. (1)

Nagrajomyces Mel'nik (1)

Nakatopsis Whitton, McKenzie \& K.D. Hyde (2)

Nanoschema B. Sutton (1)

Naothyrsium Bat. (1)

Necraphidium Cif. (1)

Nematogonum Desm. (1)

Nematographium Goid. (5)

Nemozythiella Höhn. (1)

Neoalpakesa Punith. (1)

Neoarbuscula B. Sutton (1)

Neobarclaya Sacc. (2)

Neodiplodina Petr. (1)

Neofuckelia Zeller \& Goodd. (1)

Neoheteroceras Nag Raj (2)

Neojohnstonia B. Sutton (2)

Neoligniella Naumov (4)

Neomarssoniella U. Braun (1)

Neomelanconium Petr. (3)

Neoovularia U. Braun (6)

Neopeltis Syd. (3)

Neopericonia Kamal, A.N. Rai \& Morgan-Jones (1)

Neophoma Petr. \& Syd. (2)

Neoplaconema B. Sutton (2)

Neopodoconis Rifai (3)

Neoramularia U. Braun (9)

Neospegazzinia Petr. \& Syd. (2)

Neottiospora Desm. (2)

Neozythia Petr. (1)

Neta Shearer \& J.L. Crane (10)

Nidulispora Nawawi \& Kuthub. (1)

Nigrolentilocus R.F. Castañeda \& Heredia (6)

Nigromacula Etayo (1)

Nigropuncta D. Hawksw. (2)

Nosophloea Fr. (3)

Nothospora Peyronel (1)

Novozymia W.P. Wu (1)

Nummospora E. Müll. \& Shoemaker (1)

Nusia Subram. (2)

Nyctalospora E.F. Morris (1)

Nypaella K.D. Hyde \& B. Sutton (2)

Obeliospora Nawawi \& Kuthub. (5)

Obstipipilus B. Sutton (1)

Octopodotus Kohlm. \& Volkm.-Kohlm. (1)

Odontodictyospora Mercado (1)

Oedothea Syd. (1)

Ojibwaya B. Sutton (1)

Omega B. Sutton \& Minter (1) 
Oncopodium Sacc. (12)

Oncospora Kalchbr. (8)

Oncosporella P. Karst. (1)

Oncostroma Bat. \& Marasas (1)

Onychophora W. Gams, P.J. Fisher \& J. Webster (1)

Oothyrium Syd. (1)

Ophiosira Petr. (1)

Orphanocoela Nag Raj (3)

Ostracoderma Fr. (3)

Ostracodermidium Mukerji (1)

Oswaldina Rangel (1)

Paathramaya Subram. (5)

Pachycladina Marvanová (3)

Palawaniopsis Bat., Cif. \& Nascim. (1)

Papilionospora V.G. Rao \& B. Sutton (1)

Pappimyces B. Sutton \& Hodges (1)

Paraaoria R.K. Verma \& Kamal (1)

Paraarthrocladium Matsush. (1)

Parablastocatena Y.D. Zhang \& X.G. Zhang (1)

Paraceratocladium R.F. Castañeda (6)

Parachionomyces Thaung (1)

Paracostantinella Subram. \& Sudha (1)

Paracryptophiale Kuthub. \& Nawawi (2)

Paracytospora Petr. (1)

Paradendryphiopsis M.B. Ellis (5)

Paradidymobotryum C.J.K. Wang \& B. Sutton (1)

Paradiplodia Speg. ex Trotter (6)

Paradischloridium Bhat \& B. Sutton (1)

Paradiscula Petr. (1)

Paraëpicoccum Matsush. (1)

Parafulvia Kamal, A.N. Rai \& Morgan-Jones (1)

Parahaplotrichum W.A. Baker \& Partr. (1)

Paraharknessia Matsush. (1)

Parahyalotiopsis Nag Raj (1)

Paramassariothea Subram. \& Muthumary (1)

Paramenisporopsis Matsush. (1)

Parapericonia M.B. Ellis (2)

Parapericoniella U. Braun, Heuchert \& K. Schub. (1)

Paraphaeoisaria de Hoog \& Morgan-Jones (1)

Parapithomyces Thaung (1)

Parapyricularia M.B. Ellis (4)

Pararobillarda Matsush. (1)

Parasphaeropsis Petr. (1)

Parastigmatellina Bat. \& C.A.A. Costa (1)

Paratetraploa M.K.M. Wong \& K.D. Hyde (1)

Paratomenticola M.B. Ellis (2)

Paratrichoconis Deighton \& Piroz. (4)

Paraulocladium R.F. Castañeda (2)

Paspalomyces Linder (1)

Patriciomyces D. Hawksw. (1)

Pazschkeella Syd. \& P. Syd.

Peethasthabeeja P. Rag. Rao (1) 
Pellionella (Sacc.) Sacc. (1)

Peltasterinostroma Punith. (1)

Peltasteropsis Bat. \& H. Maia (7)

Peltistroma Henn. (1)

Peltistromella Höhn. (1)

Peltosoma Syd. (1)

Peltostromellina Bat. \& A.F. Vital (1)

Peltostromopsis Bat. \& A.F. Vital (1)

Penzigomyces Subram. (13)

Perelegamyces R.F. Castañeda \& W.B. Kendr. (1)

Perizomella Syd. (1)

Pestalozziella Sacc. \& Ellis ex Sacc. (4)

Petrakiopsis Subram. \& K.R.C. Reddy (1)

Phacostroma Petr. (1)

Phacostromella Petr. (1)

Phaeoblastophora Partr. \& Morgan-Jones (2)

Phaeocandelabrum R.F. Castañeda, Gusmão, Guarro \& Iturr. (3)

Phaeodactylium Agnihothr. (7)

Phaeodiscula Cub. (1)

Phaeodomus Höhn. (3)

Phaeohiratsukaea Udagawa \& Iwatsu (1)

Phaeoidiomyces Dorn.-Silva \& Dianese (2)

Phaeolabrella Speg. (1)

Phaeomonilia R.F. Castañeda, Heredia \& R.M. Arias (5)

Phaeomonostichella Keissl. ex Petr. (1)

Phaeophloeosporella Crous \& B. Sutton (1)

Phaeophomopsis Höhn. (1)

Phaeoschizotrichum C.R. Silva, Gusmão \& R.F. Castañeda (1)

Phaeostalagmus W. Gams (7)

Phaeostilbelloides Armando, Z.M. Chaves \& Dianese (1)

Phaeothyrium Petr. (1)

Phaeotrichoconis Subram. (8)

Phaeoxyphiella Bat. \& Cif. (7)

Phellostroma Syd. \& P. Syd. (1)

Phialoarthrobotryum Matsush. (2)

Phialogeniculata Matsush. (4)

Phialophaeoisaria Matsush. (1)

Phialostele Deighton (1)

Phialotubus R.Y. Roy \& Leelav. (1)

Phloeosporina Höhn. (1)

Phlyctaeniella Petr. (2)

Phomachora Petr. \& Syd. (2)

Phomachorella Petr. (1)

Phomatosporella Tak. Kobay. \& K. Sasaki (1)

Phomyces Clem. (1)

Phragmoconidium G.F. Sepúlveda, Pereira-Carv. \& Dianese (1)

Phragmopeltis Henn. (5)

Phragmospathula Subram. \& N.G. Nair (3)

Phragmospathulella J. Mena \& Mercado (1)

Phthora d'Hérelle (1)

Phylloedium Fr. (1)

Phyllohendersonia Tassi (25) 
Physalidiella Rulamort (2)

Physalidiopsis R.F. Castañeda \& W.B. Kendr. (1)

Piggotia Berk. \& Broome (3)

Pinatubo J.B. Manandhar \& Mew (1)

Piperivora Siboe, P.M. Kirk \& P.F. Cannon (1)

Piricauda Bubák (8)

Piricaudilium Hol.-Jech. (2)

Piricaudiopsis J. Mena \& Mercado (1)

Pirispora Faurel \& Schotter (1)

Pirostomella Sacc. (2)

Pithosira Petr. (1)

Pittostroma Kowalski \& T.N. Sieber (1)

Placella Syd. (1)

Placodiplodia Bubák (2)

Placonema (Sacc.) Petr. (3)

Placonemina Petr. (1)

Placosphaerina Maire (1)

Placothea Syd. (1)

Placothyrium Bubák (1)

Plagiostigmella Petr. (1)

Plasia Sherwood (1)

Plectonaemella Höhn. (1)

Plectopeltis Syd. (1)

Plectophomopsis Petr. (1)

Plectopycnis Bat. \& A.F. Vital (4)

Plectosira Petr. (1)

Plectronidiopsis Nag Raj (1)

Plectronidium Nag Raj (4)

Plenocatenulis Bat. \& Cif. (1)

Plenophysa Syd. \& P. Syd. (1)

Plenotrichopsis Bat. (1)

Plenotrichum Syd. (2)

Plenozythia Syd. \& P. Syd. (2)

Pleocouturea G. Arnaud (2)

Plesiospora Drechsler (1)

Pleurodesmospora Samson, W. Gams \& H.C. Evans (1)

Pleurodiscula Höhn. (1)

Pleurodomus Petr. (1)

Pleuropedium Marvanová \& S.H. Iqbal (3)

Pleurophomopsis Petr. (7)

Pleuroplaconema Petr. (2)

Pleuroplacosphaeria Syd. (1)

Pleurostromella Petr. (15)

Pleurotheciopsis B. Sutton (6)

Pleurothyriella Petr. \& Syd. (1)

Pleurovularia R. Kirschner \& U. Braun (1)

Pocillopycnis Dyko \& B. Sutton (1)

Podoplaconema Petr. (1)

Podosporiella Ellis \& Everh. (4)

Podosporiopsis Jian Ma, X.G. Zhang \& R.F. Castañeda (2)

Podosporium Schwein. (67)

Poikilosperma Bat. \& J.L. Bezerra (1) 
Polybulbophiale Goh \& K.D. Hyde (1)

Polychaetella Speg. (3)

Polycladium Ingold (1)

Polydesmus Mont. (14)

Polyetron Bat. \& Peres (1)

Polylobatispora Matsush. (3)

Polyrostrata T.P. Devi \& N. Mathur (2)

Polystomellomyces Bat. (1)

Polystratorictus Matsush. (2)

Polytretophora Mercado (3)

Porocladium Descals (1)

Poropeltis Henn. (1)

Porophilomyces U. Braun (1)

Porosubramaniania Hol.-Jech. (2)

Porrectotheca Matsush. (1)

Potamomyces K.D. Hyde (1)

Proboscispora Punith. (1)

Protostegiomyces Bat. \& A.F. Vital (1)

Protostroma Bat. (1)

Pseudoacrodictys W.A. Baker \& Morgan-Jones (14)

Pseudoanguillospora S.H. Iqbal (3)

Pseudoaristastoma Suj. Singh (1)

Pseudoasperisporium U. Braun (3)

Pseudobasidiospora Dyko \& B. Sutton (1)

Pseudocanalisporium R.F. Castañeda \& W.B. Kendr. (1)

Pseudocenangium P. Karst. (1)

Pseudochuppia Kamal et al. (1)

Pseudoclathrosphaerina Voglmayr (2)

Pseudoconium Petr. (1)

Pseudocytoplacosphaeria Punith. \& Spooner (1)

Pseudocytospora Petr. (1)

Pseudodichomera Höhn. (3)

Pseudodidymaria U. Braun (3)

Pseudodiplodia (P. Karst.) Sacc. (45)

Pseudodiscula Laubert (2)

Pseudofuscophialis Sivan. \& H.S. Chang (1)

Pseudogaster Höhn. (1)

Pseudographiella E.F. Morris (3)

Pseudohepatica P.M. Jørg. (1)

Pseudomicrodochium B. Sutton (8)

Pseudoneottiospora Faurel \& Schotter (2)

Pseudopatellina Höhn. (1)

Pseudopeltistroma Katum. (1)

Pseudoperitheca Elenkin (1)

Pseudopetrakia M.B. Ellis (2)

Pseudophloeosporella U. Braun (1)

Pseudophragmotrichum W.P. Wu, B. Sutton \& Gange (1)

Pseudopolystigmina Murashk. (2)

Pseudoramularia Matsush. (2)

Pseudorhizopogon Kobayasi (1)

Pseudoschizothyra Punith. (1)

Pseudosigmoidea K. Ando \& N. Nakam. (2) 
Pseudostegia Bubák (1)

Pseudothyrium Höhn. (1)

Pseudotorula Subram. (3)

Pseudotracylla B. Sutton \& Hodges (2)

Pseudotrichoconis W.A. Baker \& Morgan-Jones (1)

Pseudozythia Höhn. (1)

Psilosphaeria Cooke (1)

Pteromycula P. Cannon (1)

Pterulopsis Wakef. \& Hansf. (1)

Pterygosporopsis P.M. Kirk (2)

Pucciniospora Speg. (1)

Pulchromyces Hennebert (1)

Pullospora Faurel \& Schotter (2)

Pulvinella A.W. Ramaley (1)

Punctillina Toro (1)

Pycmaeosphaera Etayo \& Diederich (3)

Pycnidioarxiella Punith. \& N.D. Sharma (1)

Pycnidiopeltis Bat. \& C.A.A. Costa (1)

Pycnis Bref. (1)

Pycnodactylus Bat., A.A. Silva \& Cavalc. (1)

Pycnodallia Kohlm. \& Volkm.-Kohlm. (1)

Pycnoharknessia Matsush. (1)

Pycnomma Syd. (1)

Pycnomoreletia Rulamort (2)

Pycnoseynesia Kuntze (1)

Pycnothera N.D. Sharma \& G.P. Agarwal (1)

Pycnothyriella Bat. (2)

Pycnothyrium Diederich (6)

Pyramidospora Sv. Nilsson (9)

Pyrenyllium Clem. (2)

Pyrgostroma Petr. (2)

Pyripnomyces Cavalc. (1)

Quadracaea Lunghini, Pinzari \& Zucconi (3)

Quadricladium Nawawi \& Kuthub. (1)

Quasidiscus B. Sutton (1)

Quasiphloeospora B. Sutton, Crous \& Shamoun (1)

Queenslandia Bat. \& H. Maia (5)

Quezelia Faurel \& Schotter (1)

Raciborskiomyces Siemaszko (4)

Radiatispora Matsush. (1)

Raizadenia S.L. Srivast. (1)

Ramakrishnanella Kamat \& Ullasa ex Ullasa (1)

Ramicapitulum Whitton, K.D. Hyde \& McKenzie (1)

Ramicephala Voglmayr \& G. Delgado (1)

Ramoconidiifera B. Sutton, Carmarán \& A.I. Romero (2)

Redbia Deighton \& Piroz. (5)

Refractohilum D. Hawksw. (5)

Repetoblastiella R.F. Castañeda, Minter \& M. Stadler (1)

Rhabdoclema Syd. (2)

Rhabdogloeopsis Petr. (2)

Rhabdostromella Höhn. (1)

Rhabdostromina Died. (3) 
Rhexoampullifera P.M. Kirk (3)

Rhexoprolifer Matsush. (1)

Rhinotrichella G. Arnaud ex de Hoog (4)

Rhipidocephalum Trail (2)

Rhizosphaerina B. Sutton (2)

Rhodesia Grove (2)

Rhodesiopsis B. Sutton \& R. Campb. (2)

Rhodothallus Bat. \& Cif. (2)

Rhombostilbella Zimm. (2)

Rhopalocladium Schroers, Samuels \& W. Gams (1)

Rhynchodiplodia Briosi \& Farneti (1)

Rhynchomyces Willk. (1)

Rhynchoseptoria Unamuno (1)

Rhynchosporina Arx (2)

Riclaretia Peyronel (1)

Rileya A. Funk (1)

Robakia Petr. (1)

Rogergoosiella A. Hern.-Gut. \& J. Mena (1)

Roscoepoundia Kuntze (1)

Rosulomyces S. Marchand \& Cabral (1)

Rota Bat., Cif. \& Nascim. (1)

Ruggieria Cif. \& Montemart. (1)

Saania Zhurb. (1)

Sadasivania Subram. (3)

Sanjuanomyces R.F. Castañeda \& W.B. Kendr. (1)

Sarcinosporon D.S. King \& S.C. Jong (1)

Sarcoexcipula Etayo (1)

Sarcophoma Höhn. (3)

Sarophorum Syd. \& P. Syd. (1)

Satchmopsis B. Sutton \& Hodges (1)

Sativumoides S.C. Ren, Jian Ma \& X.G. Zhang (1)

Scaphidium Clem. (1)

Sceptrifera Deighton (1)

Schizothyra Bat. \& C.A.A. Costa (1)

Schizothyrella Thüm. (1)

Schizothyropsis Bat. \& A.F. Vital (1)

Schizotrichum McAlpine (1)

Schroeteria G. Winter (1)

Schwarzmannia Pisareva (1)

Scirrhophoma Petr. (1)

Sclerographiopsis Deighton (1)

Sclerographium Berk. (4)

Scleromeris Syd. (3)

Sclerophoma Höhn. (30)

Scleropycnis Syd. \& P. Syd. (2)

Sclerozythia Petch (1)

Scolecobasidiella M.B. Ellis (2)

Scolecobeltrania Iturr., R.F. Castañeda \& Rob. Fernández (1)

Scolecodochium K. Matsush. \& Matsush. (1)

Scolecosporiella Petr. (6)

Scolecotheca Søchting \& B. Sutton (1)

Scolecozythia Curzi (1) 
Scoliotidium Bat. \& Cavalc. (1)

Scopaphoma Dearn. \& House (1)

Scopulariella Gjaerum (1)

Scothelius Bat., J.L. Bezerra \& Cavalc. (1)

Scutisporus K. Ando \& Tubaki (1)

Scutopeltis Bat. \& H. Maia (2)

Scutopycnis Bat. (2)

Seimatosporiopsis B. Sutton, Ghaffer \& Abbas (2)

Selenosira Petr. (1)

Selenosporopsis R.F. Castañeda \& W.B. Kendr. (1)

Semipseudocercospora J.M. Yen (2)

Septocytella Syd. (1)

Septogloeum Sacc. (2)

Septomyxella (Höhn.) Höhn. (1)

Septopatella Petr. (1)

Septosporiopsis W.A. Baker \& Morgan-Jones (1)

Septosporium Corda (5)

Septotrullula Höhn. (2)

Sessiliospora D. Hawksw. (1)

Setolibertella Punith. \& Spooner (1)

Setophiale Matsush. (1)

Setosporella Mustafa \& Abdul-Wahid (1)

Seychellomyces Matsush. (1)

Seynesiopsis Henn. (1)

Shawiella Hansf. (1)

Sheariella Petr. (1)

Sheathnema Dubey \& Moonambeth (2)

Shivomyces Hosag. (2)

Siamia V. Robert, Decock \& R.F. Castañeda (1)

Sigmatomyces Sacc. \& P. Syd. (1)

Simmonsiella J.L. Crane \& A.N. Mill. (1)

Sirexcipula Bubák (1)

Sirocyphis Clem. (1)

Sirogloea Petr. (1)

Siroligniella Naumov (1)

Sirophoma Höhn. (3)

Siroplacodium Petr. (6)

Siropleura Petr. (1)

Siroscyphellina Petr. (2)

Sirosperma Syd. \& P. Syd. (2)

Sirosphaera Syd. \& P. Syd. (2)

Sirosporonaemella Naumov (1)

Sirothecium P. Karst. (3)

Sirothyriella Höhn. (2)

Sirothyrium Syd. \& P. Syd. (1)

Sirozythia Höhn. (2)

Sirozythiella Höhn. (1)

Sitochora H.B.P. Upadhyay (1)

Slimacomyces Minter (2)

Soloacrospora W.B. Kendr. \& R.F. Castañeda (2)

Solosympodiella Matsush. (8)

Soloterminospora Matsush. (1) 
Spermatoloncha Speg. (1)

Spermatoloncha Speg. (1)

Spermochaetella Cif. (1)

Spermospora R. Sprague (9)

Spermosporella Deighton (4)

Sphaeridium Fresen. (5)

Sphaeriostromella Bubák (1)

Sphaeriothyrium Bubák (2)

Sphaeromma H.B.P. Upadhyay (2)

Sphaeronaema Fr. (50)

Sphaerophoma Petr. (2)

Sphaerulomyces Marvanová (1)

Spinulospora Deighton (1)

Spiralum J.L. Mulder (2)

Spiropes Cif. (ca. 40)

Splanchospora Lar.N. Vassiljeva (1)

Spondylocladiella Linder (2)

Spondylocladiopsis M.B. Ellis (2)

Sporhaplus H.B.P. Upadhyay (1)

Sporidesmiopsis Subram. \& Bhat (6)

Sporoglena Sacc. (1)

Sporophiala P. Rag. Rao (3)

Sporotretophora Whitton, McKenzie \& K.D. Hyde (1)

Stachybotryella Ellis \& Barthol. (3)

Stachybotryna Tubaki \& T. Yokoy. (6)

Stagonopatella Petr. (1)

Stagonopsis Sacc. (4)

Stagonosporina Tassi (1)

Stagonostromella Petr. \& Syd. (1)

Staheliella Emden (2)

Stalagmochaetia Cif. \& Bat. (2)

Stanhughesiella R.F. Castañeda \& D.W. Li (1)

Stauronema (Sacc.) Syd., P. Syd. \& E.J. Butler (5)

Stauronematopsis Abbas, B. Sutton \& Ghaffar (1)

Staurophoma Höhn. (1)

Stegonsporiopsis Van Warmelo \& B. Sutton (1)

Stellifraga Alstrup \& Olech (1)

Stellomyces Morgan-Jones, R.C. Sinclair \& Eicker (2)

Stellopeltis Bat. \& A.F. Vital (2)

Stellospora Alcorn \& B. Sutton (2)

Stellothyriella Bat. \& Cif. (2)

Stenocephalopsis Chamuris \& C.J.K. Wang (1)

Stenocladiella Marvanová \& Descals (1)

Stenospora Deighton (1)

Stephembruneria R.F. Castañeda (1)

Stevensonula Petr. (1)

Stictopatella Höhn. (1)

Stictosepta Petr. (1)

Stigmatellina Bat. \& H. Maia (1)

Stigmea Fr. (1)

Stigmella Lév. (28)

Stigmopeltis Syd. (2) 
Stilbellula Boedijn (1)

Stilbodendron Syd. \& P. Syd. (1)

Stilbophoma Petr. (1)

Strasseriopsis B. Sutton \& Tak. Kobay. (1)

Stratiphoromyces Goh \& K.D. Hyde (2)

Striosphaeropsis Verkley \& Aa (1)

Stromatocrea W.B. Cooke (1)

Stromatopogon Zahlbr. (3)

Stromatopycnis A.F. Vital (1)

Stromatostysanus Höhn. (3)

Strongylothallus Bat. \& Cif. (1)

Stygiomyces Coppins \& S.Y. Kondr. (1)

Stylaspergillus B. Sutton, Alcorn \& P.J. Fisher (1)

Subhysteropycnis Wedin \& Hafellner (1)

Subicularium M.L. Farr \& Goos (1)

Subulispora Tubaki (8)

Suttoniella S. Ahmad (3)

Suttonina H.C. Evans (1)

Syamithabeeja Subram. \& Natarajan (1)

Sylviacollaea Cif. (1)

Symphysos Bat. \& Cavalc. (1)

Sympodiella W.B. Kendr. (5)

Sympodiocladium Descals (1)

Sympodioclathra Voglmayr (1)

Sympodioplanus R.C. Sinclair \& Boshoff (3)

Sympodiosynnema J.W. Xia \& X.G. Zhang (1)

Synchronoblastia Uecker \& F.L. Caruso (1)

Syncladium Rabenh. (1)

Synnemacrodictys W.A. Baker \& Morgan-Jones (1)

Synnemaseimatoides K. Matsush. \& Matsush. (1)

Synnematomyces Kobayasi (1)

Synostomina Petr. (1)

Syphosphaera Dumort. (1)

Systremmopsis Petr. (1)

Taeniolina M.B. Ellis (6)

Talekpea Lunghini \& Rambelli (1)

Talpapellis Alstrup \& M.S. Cole (4)

Tandonea M.D. Mehrotra (1)

Tarsodisporus Bat. \& A.A. Silva (1)

Tectacervulus A.W. Ramaley (1)

Telioclipeum Viégas (1)

Temerariomyces B. Sutton (1)

Teratosperma Syd. \& P. Syd. (11)

Termitaria Thaxt. (6)

Tetrabrachium Nawawi \& Kuthub. (1)

Tetrabrunneospora Dyko (1)

Tetracoccosporium Szabó (4)

Tetrameronycha Speg. ex W. Rossi \& M. Blackw. (1)

Tetranacriella Kohlm. \& Volkm.-Kohlm. (1)

Tetranacrium H.J. Huds. \& B. Sutton (1)

Tetraposporium S. Hughes (2)

Textotheca Matsush. (1) 
Thaptospora B. Sutton \& Pascoe (3)

Thirumalacharia Rathaiah (1)

Tholomyces Matsush. (1)

Thoracella Oudem. (1)

Thrinacospora Petr. (1)

Thyriostromella Bat. \& C.A.A. Costa (1)

Thyrostromella Höhn. (3)

Thyrsidiella Höhn. ex Höhn. (2)

Thyrsidina Höhn. (1)

Tiarosporellivora Punith. (1)

Ticogloea G. Weber et al. (2)

Ticosynnema R.F. Castañeda, Granados \& Mardones (1)

Titaea Sacc. (23)

Titaeopsis B. Sutton \& Deighton (1)

Titaeospora Bubák (2)

Tomenticola Deighton (1)

Tompetchia Subram. (1)

Toxosporiella B. Sutton (1)

Toxosporiopsis B. Sutton \& Sellar (1)

Toxosporium Vuill. (2)

Trematophoma Petr. (2)

Tremellidium Petr. (1)

Tretendophragmia Subram. (1)

Tretocephala Subram. (1)

Tretolylea Cantillo, R.F. Castañeda \& Gusmão (1)

Tretospeira Piroz. (1)

Tretovularia Deighton (1)

Tribolospora D.A. Reid (1)

Tricellula Beverw. (8)

Trichobolbus Bat. (1)

Trichobotrys Penz. \& Sacc. (4)

Trichoconis Clem. (21)

Trichodiscula Vouaux (1)

Trichodochium Syd. (3)

Trichomatoclava G.F. Sepúlveda, Pereira-Carv. \& Dianese (1)

Trichomatomyces Dorn.-Silva \& Dianese (1)

Trichomatosphaera Pereira-Carv., G.F. Sepúlveda \& Dianese (1)

Trichopeltulum Speg. (1)

Trichoseptoria Cavara (2)

Trichosporiella Kamyschko (4)

Trichosporodochium Dorn.-Silva \& Dianese (1)

Trichotheca P. Karst. (1)

Tricladiella K. Ando \& Tubaki (1)

Tricladiopsis Descals (2)

Tricladiospora Nawawi \& Kuthub. (3)

Tricornispora Bonar (1)

Trifurcospora K. Ando \& Tubaki (2)

Trigonosporium Tassi (2)

Tripoconidium Subram. (1)

Triposporina Höhn. (2)

Triramulispora Matsush. (3)

Triscelophorus Ingold (8) 
Triscelosporium Nawawi \& Kuthub. (1)

Trisulcosporium H.J. Huds. \& B. Sutton (1)

Tromeropsis Sherwood (1)

Troposporium Harkn. (1)

Troposporopsis Whitton, McKenzie \& K.D. Hyde (2)

Tryblidiopycnis Höhn. (1)

Tryssglobulus B. Sutton \& Pascoe (1)

Tuberculispora Deighton \& Piroz. (1)

Tunicago B. Sutton \& Pollack (2)

Turturconchata J.L. Chen, T.L. Huang \& Tzean (2)

Tympanosporium W. Gams (1)

Uberispora Piroz. \& Hodges (4)

Ubrizsya Negru (1)

Ulocoryphus Michaelides, L. Hunter \& W.B. Kendr. (1)

Umbellidion B. Sutton \& Hodges (1)

Uniseta Ciccar. (1)

Urohendersonia Speg. (5)

Urohendersoniella Petr. (1)

Uvarispora Goos \& Piroz. (1)

Vagnia D. Hawksw. \& Miądl. (1)

Vanakripa Bhat et al. (9)

Vanbeverwijkia Agnihothr. (1)

Vanderystiella Henn. (1)

Vanterpoolia A. Funk (1)

Vasudevella Chona et al. (1)

Velloziomyces Armando, Z.M. Chaves \& Dianese (1)

Velutipila D. Hawksw. (1)

Ventrographium H.P. Upadhyay, Cavalc. \& A.A. Silva (1)

Venustocephala Matsush. (2)

Venustosynnema R.F. Castañeda \& W.B. Kendr. (3)

Veracruzomyces Mercado, Guarro, Heredia \& J. Mena (1)

Veramycella G. Delgado (1)

Veramyces Matsush. (1)

Verdipulvinus A.W. Ramaley (1)

Veronaella Subram. \& K.R.C. Reddy (1)

Veronidia Negru (1)

Verrucariella S. Ahmad (1)

Verrucaster Tobler (1)

Verrucophragmia Crous, M.J. Wingf. \& W.B. Kendr. (1)

Verticicladus Matsush. (3)

Vesicladiella Crous \& M.J. Wingf. (1)

Vesiculohyphomyces Armando, Pereira-Carv. \& Dianese (1)

Vestigium Piroz. \& Shoemaker (2)

Virgariella S. Hughes (11)

Viridiannula Etayo (1)

Vittalia Gaws \& Bhat (1)

Vizellopsidites M.A. Khan, M. Bera \& Bera (1)

Vouauxiella Petr. \& Syd. (3)

Waihonghopes Yanna \& K.D. Hyde (1)

Wardinella Bat. \& Peres (1)

Websteromyces W.A. Baker \& Partr. (2)

Weufia Bhat \& B. Sutton (1) 
Wolkia Ramsb. (1)

Xenidiocercus Nag Raj (1)

Xenochora Petr. (1)

Xenodomus Petr. (1)

Xenoheteroconium Bhat, W.B. Kendr. \& Nag Raj (1)

Xenokylindria DiCosmo, S.M. Berch \& W.B. Kendr. (2)

Xenomyxa Syd. (1)

Xenopeltis Syd. \& P. Syd. (1)

Xenoplaca Petr. (1)

Xenostroma Höhn. (1)

Xeroconium D. Hawksw. (1)

Xiphomyces Syd. \& P. Syd. (2)

Xiuguozhangia K. Zhang, R.F. Castañeda, Jian Ma \& L.G. Ma (5)

Xylochia B. Sutton (2)

Xyloglyphis Clem. (1)

Xylohypha (Fr.) E.W. Mason (6)

Xylohyphopsis W.A. Baker \& Partr. (3)

Yalomyces Nag Raj (6)

Yinmingella Goh, C.K.M. Tsui \& K.D. Hyde (1)

Ypsilomyces D.A.C. Almeida \& Gusmão (1)

Yuccamyces Gour, Dyko \& B. Sutton (6)

Zakatoshia B. Sutton (2)

Zebrospora McKenzie (1)

Zelandiocoela Nag Raj (1)

Zelodactylaria A.C. Cruz, Gusmão \& R.F. Castañeda (1)

Zelopelta B. Sutton \& R.D. Gaur (1)

Zelosatchmopsis Nag Raj (1)

Zetesimomyces Nag Raj (1)

Zevadia J.C. David \& D. Hawksw. (1)

Zilingia Petr. (1)

Zinzipegasa Nag Raj (1)

Zopheromyces B. Sutton \& Hodges (1)

Zunura Nag Raj (1)

Zythia Fr. (1)

Zyxiphora B. Sutton (1)

BASIDIOBOLOMYCOTA Doweld

Basidiobolomycetes Doweld*

Basidiobolales Jacz. \& P.A. Jacz.*

Basidiobolaceae Engl. \& E. Gilg

Basidiobolus Eidam (10)

Schizangiella J. Dwyer, B. Burwell, Humber, C. Mcleod, M. Fleetwood \& T. Johnson bis (1)

BASIDIOMYCOTA R.T. Moore

Basidiomycota R.T. Moore

Agaricomycotina Doweld

Agaricomycetes Doweld

Agaricales Underw.

Agaricaceae Chevall.

Abstoma G. Cunn. (8)

Acutocapillitium P. Ponce de León (3) 
Agaricus L. (ca. 500)

Arachnion Schwein. (13)

Barcheria T. Lebel (1)

Battarrea Pers. (3)

Battarreoides T. Herrera (1)

Calvatiopsis Hollós (1)

Chamaemyces Battarra ex Earle (2)

Chlamydopus Speg. (1)

Chlorolepiota Sathe \& S.D. Deshp. (3)

Chlorophyllum Massee (19)

Clarkeinda Kuntze (5)

Clavogaster Henn. (2)

Coniolepiota Vellinga (1)

Coprinus Pers. (ca. 17)

Crucispora E. Horak (2)

Cystolepiota Singer (ca. 12)

Dictyocephalos L.M. Underwood ex V.S. White (1)

Disciseda Czern. (15)

Echinoderma (Locq. ex Bon) Bon (ca. 15)

Endolepiotula Singer (1)

Eriocybe Vellinga (1)

Gasterellopsis Routien (1)

Glyptoderma R. Heim \& Perr.-Bertr. (1)

Heinemannomyces Watling (2)

Hiatulopsis Singer \& Grinling (2)

Holocotylon Lloyd (3)

Hymenagaricus Heinem. (20)

Janauaria Singer (1)

Japonogaster Kobayasi (1)

Lepiota (Pers.) Gray (ca. 450)

Leucoagaricus Locq. ex Singer (ca. 135)

Leucocoprinus Pat. (ca. 50)

Lycoperdopsis Henn. (1)

Macrolepiota Singer (ca. 40)

Melanophyllum Velen. (3)

Metrodia Raithelh. (2)

Micropsalliota Höhn. (ca. 70)

Montagnea Fr. (5)

Mycenastrum Desv. (18)

Neosecotium Singer \& A.H. Sm. (2)

Panaeolopsis Singer (4)

Phellorinia Berk. (1)

Phyllogaster Pegler (1)

Podaxis Desv. (10)

Pseudoauricularia Kobayasi (1)

Pseudolepiota Z.W. Ge (1)

Queletia Fr. (2)

Rugosospora Heinem. (2)

Schinzinia Fayod (1)

Schizostoma Ehrenb. ex Lév. (1)

Singerina Sathe \& S.D. Deshp. (1)

Smithiogaster J.E. Wright (1) 
Smithiomyces Singer (3)

Termiticola E. Horak (1)

Tulostoma Pers. (ca. 83)

Xanthagaricus (Heinem.) Little Flower, Hosag. \& T.K. Abraham (12)

Xerocoprinus Maire (1)

Amanitaceae E.-J. Gilbert

Amanita Pers. (ca. 570)

Catatrama Franco-Mol. (2)

Limacella Earle (ca. 15)

Limacellopsis Zhu L. Yang, Q. Cai \& Y.Y. Cui (2)

Zhuliangomyces Redhead (5)

Biannulariaceae Jülich

Anupama K.N.A. Raj, K.P.D. Latha \& Manim. (1)

Callistosporium Singer (14)

Catathelasma Lovejoy (4)

Guyanagarika Sánchez-García, T.W. Henkel \& Aime (3)

Macrocybe Pegler \& Lodge (7)

Pleurocollybia Singer (6)

Pseudolaccaria Vizzini, Contu\& Z.W. Ge (1)

\section{Bolbitiaceae Singer}

Agrogaster D.A. Reid (1)

Bolbitius Fr. (ca. 70)

Conocybe Fayod (ca. 221)

Cyttarophyllopsis R. Heim (1)

Descolea Singer (ca. 15)

Galerella Earle (8)

Galeropsis Velen. (9)

Gymnoglossum Massee (1)

Pholiotina Fayod (56)

Ptychella Roze \& Boud. (1)

Rhodoarrhenia Singer (8)

Tubariella E. Horak \& Hauskn. (1)

Tubariopsis R. Heim (1)

Tympanella E. Horak (1)

Wielandomyces Raithelh. (1)

Broomeiaceae Zeller

Broomeia Berk. (2)

\section{Chromocyphellaceae Knudsen}

Chromocyphella De Toni \& Levi (5)

\section{Clavariaceae Chevall.}

Camarophyllopsis Herink (26)

Clavaria Vaill. ex L. (32)

Clavicorona Doty (10)

Clavulinopsis Overeem (34)

Hirticlavula J.H. Petersen \& Læssøe (1)

Hodophilus R. Heim (13) 
Hyphodontiella $\AA$. Strid (2)

Lamelloclavaria Birkebak \& Adamčík (1)

Ramariopsis (Donk) Corner (48)

Setigeroclavula R.H. Petersen (1)

Cortinariaceae R. Heim ex Pouzar

Cortinarius (Pers.) Gray (ca. 2250)

Protoglossum Massee (8)

Pyrrhoglossum Singer (12)

Quadrispora Bougher \& Castellano (3)

Stephanopus M.M. Moser \& E. Horak (5)

Crassisporiaceae Vizzini, Consiglio \& M. Marchetti

Crassisporium Matheny, P.-A. Moreau \& Vizzini (3)

Romagnesiella Contu, Matheny, P.-A. Moreau, Vizzini \& A. de Haan (2)

Crepidotaceae (S. Imai) Singer

Crepidotus (Fr.) Staude (ca. 200)

Episphaeria Donk (1)

Nanstelocephala Oberw. \& R.H. Petersen (1)

Pellidiscus Donk (3)

Pleuroflammula Singer (10)

Simocybe P. Karst. (26)

\section{Cyphellaceae Lotsy}

Asterocyphella W.B. Cooke (3)

Campanophyllum Cifuentes \& R.H. Petersen (1)

Catilla Pat. (1)

Cheimonophyllum Singer (4)

Chondrostereum Pouzar (4)

Cunninghammyces Stalpers (2)

Cyphella Fr. (2)

Gloeocorticium Hjortstam \& Ryvarden (1)

Gloeostereum S. Ito \& S. Imai (1)

Granulobasidium Jülich (1)

Hyphoradulum Pouzar (1)

Incrustocalyptella Agerer (3)

Phaeoporotheleum (W.B. Cooke) W.B. Cooke (2)

Seticyphella Agerer (3)

Sphaerobasidioscypha Agerer (2)

Thujacorticium Ginns (1)

\section{Cystostereaceae Jülich}

Cericium Hjortstam (1)

Crustomyces Jülich (3)

Cystidiodontia Hjortstam (2)

Cystostereum Pouzar (7)

Parvobasidium Jülich (3)

Parvodontia Hjortstam \& Ryvarden (2)

Rigidotubus J. Song, Y.C. Dai \& B.K. Cui (1)

Entolomataceae Kotl. \& Pouzar 
Clitocella Kluting, T.J. Baroni \& Bergemann (6)

Clitopilopsis Maire (2)

Clitopilus (Fr. ex Rabenh.) P. Kumm. (ca. 140)

Entocybe T.J. Baroni, V. Hofst. \& Largent (10)

Entoloma P. Kumm. (ca. 1800)

Rhodocybe Maire (ca. 50)

Rhodophana Kühner (7)

Hemigasteraceae Gäum. \& C.W. Dodge

Hemigaster Juel (1)

Hydnangiaceae Gäum. \& C.W. Dodge

Hydnangium Wallr. (ca. 20)

Laccaria Berk. \& Broome (ca. 85)

Maccagnia Mattir. (1)

Podohydnangium G.W. Beaton, Pegler \& T.W.K. Young (1)

\section{Hygrophoraceae Lotsy}

Acantholichen P.M. Jørg. (6)

Aeruginospora Höhn. (2)

Ampulloclitocybe Redhead, Lutzoni, Moncalvo \& Vilgalys (3)

Aphroditeola Redhead \& Manfr. Binder (1)

Arrhenia Fr. (ca. 36)

Cantharocybe H.E. Bigelow \& A.H. Sm. (3)

Chromosera Redhead, Ammirati \& Norvell (5)

Chrysomphalina Clémençon (4)

Cora Fr. (189)

Corella Vain. (2)

Cuphophyllus (Donk) Bon (ca. 25)

Cyphellostereum D.A. Reid (9)

Dictyonema C. Agardh ex Kunth (28)

Eonema Redhead, Lücking \& Lawrey (1)

Gliophorus Herink (ca. 17)

Haasiella Kotl. \& Pouzar (2)

Humidicutis (Singer) Singer (12)

Hygroaster Singer (3)

Hygrocybe (Fr.) P. Kumm. (ca. 120)

Hygrophorus Fr. (ca. 200)

Lichenomphalia Redhead, Lutzoni, Moncalvo \& Vilgalys (14)

Neohygrocybe Herink (5)

Porpolomopsis Bresinsky (5)

Pseudoarmillariella Singer (3)

Semiomphalina Redhead (1)

SinohygrocybeC.Q. Wang, Ming Zhang \& T.H. Li (1)

\section{Hymenogastraceae Vittad.}

Anamika K.A. Thomas, Peintner, M.M. Moser \& Manim. (3)

Flammula (Fr.) P. Kumm. (ca. 10)

Galerina Earle (ca. 250)

Gymnopilus P. Karst. (ca. 200)

Hebeloma (Fr.) P. Kumm. (ca. 190)

Hymenogaster Vittad. (c.170) 
Naucoria (Fr.) P. Kumm. (30)

Phaeocollybia R. Heim (ca. 80)

Psathyloma Soop, J.A. Cooper \& Dima (2)

Psilocybe (Fr.) P. Kumm. (ca. 326)

\section{Inocybaceae Jülich}

Auritella Matheny \& Bougher (8)

Inocybe (Fr.) Fr. (ca. 1000)

Tubariomyces Esteve-Rav. \& Matheny (3)

\section{Limnoperdaceae G.A. Escobar}

Limnoperdon G.A. Escobar (1)

Lycoperdaceae Chevall.

Apioperdon (Kreisel \& D. Krüger) Vizzini (1)

Bovista Pers. Bryoperdon Vizzini (ca. 58)

Calbovista Morse ex M.T. Seidl (1)

Calvatia Fr. (ca. 43)

Gastropila Homrich \& J.E. Wright (4)

Lycoperdon Pers. (ca. 55)

Morganella Zeller (7)

\section{Lyophyllaceae Jülich}

Asterophora Ditmar (3)

Blastosporella T.J. Baroni \& Franco-Mol. (1)

Calocybe Kühner ex Donk (46)

Calocybella Vizzini, Consiglio \& Setti (4)

Clitolyophyllum Sesli, Vizzini \& Contu (1)

Gerhardtia Bon (ca. 7)

Hypsizygus Singer (3)

Lyophyllopsis Sathe \& J.T. Daniel (1)

Lyophyllum P. Karst. (ca. 60)

Myochromella V. Hofst., Clémençon, Moncalvo \& Redhead (2)

Ossicaulis Redhead \& Ginns (2)

Rugosomyces Raithelh. (ca. 12)

Sagaranella V. Hofst., Clémençon, Moncalvo \& Redhead (4)

Sphagnurus Redhead \& V. Hofst. (1)

Tephrocybe Donk (ca. 47)

Tephrocybella Picillo, Vizzini \& Contu (1)

Termitomyces R. Heim (ca. 34)

Tricholomella Zerova ex Kalamees (1)

\section{Macrocystidiaceae Kühner}

Macrocystidia Joss. (5)

Marasmiaceae Roze ex Kühner

Amyloflagellula Singer (4)

Brunneocorticium Sheng H. Wu (1)

Campanella Henn. (ca. 39)

Chaetocalathus Singer (ca. 20)

Crinipellis Pat. (ca. 65)

Hymenogloea Pat. (1) 
Marasmius Fr. (ca. 600)

Moniliophthora H.C. Evans, Stalpers, Samson \& Benny (7)

Neocampanella Nakasone, Hibbett \& Goranova (1)

Tetrapyrgos E. Horak (18)

\section{Mycenaceae Overeem}

Atheniella Redhead, Moncalvo, Vilgalys, Desjardin \& B.A. Perry (7)

Cruentomycena R.H. Petersen, Kovalenko \& O.V. Morozova (3)

Decapitatus Redhead \& Seifert (1)

Favolaschia (Pat.) Pat. (ca. 54)

Flabellimycena Redhead (1)

Heimiomyces Singer (ca. 7)

Hemimycena Singer (ca. 60)

Hydropus Kühner ex Singer (ca. 100)

Mycena (Pers.) Roussel (ca. 600)

Mycopan Redhead, Moncalvo \& Vilgalys (1)

Panellus P. Karst. (ca. 55)

Resinomycena Redhead \& Singer (ca. 10)

Roridomyces Rexer (9)

Sarcomyxa P. Karst. (2)

Tectella Earle (3)

Xeromphalina Kühner \& Maire (ca. 32)

Mythicomycetaceae Vizzini, Consiglio \& M. Marchetti

Mythicomyces Redhead \& A.H. Sm. (1)

Stagnicola Redhead \& A.H. Sm. (1)

Niaceae Jülich

Digitatispora Doguet (2)

Flagelloscypha Donk (ca. 25)

Halocyphina Kohlm. \& E. Kohlm. (1)

Lachnella Fr. (6)

Maireina W.B. Cooke (ca. 18)

Merismodes Earle (20)

Nia R.T. Moore \& Meyers (3)

Peyronelina P.J. Fisher, J. Webster \& D.F. Kane (1)

Woldmaria W.B. Cooke (1)

\section{Omphalotaceae Bresinsky}

Anthracophyllum Ces. (12)

Caripia Kuntze (1)

Connopus R.H. Petersen (1)

Gymnopanella Sand.-Leiva, J.V. McDonald \& Thorn (1)

Gymnopus (Pers.) Gray (ca. 325)

Hymenoporus Tkalčec, Mešić \& Chun Y. Deng (1)

Lentinula Earle (8)

Marasmiellus Murrill (ca. 260)

Mycetinis Earle (15)

Neonothopanus R.H. Petersen \& Krisai (3)

Omphalotus Fayod (6)

Rhodocollybia Singer (ca. 35)

Paragymnopus J.S. Oliveira (6) 
Pusillomyces J.S. Oliveira (3)

Physalacriaceae Corner

Anastrophella E. Horak \& Desjardin (3)

Armillaria (Fr.) Staude (39)

Cibaomyces Zhu L. Yang, Y.J. Hao \& J. Qin (1)

Cribbea A.H. Sm. \& D.A. Reid (5)

Cryptomarasmius T.S. Jenkinson \& Desjardin (15)

Cylindrobasidium Jülich (7)

Cyptotrama Singer (16)

Dactylosporina (Clémençon) Dörfelt (5)

Desarmillaria (Herink) R. A. Koch \& Aime (2)

Epicnaphus Singer (2)

Flammulina P. Karst. (14)

Gloiocephala Massee (ca. 40)

Guyanagaster T.W. Henkel, M.E. Sm. \& Aime (2)

Hymenopellis R.H. Petersen (ca. 50)

Laccariopsis Vizzini (1)

Manuripia Singer (1)

Mucidula Pat. (2)

Mycaureola Maire \& Chemin (1)

Naiadolina Redhead, Labbé \& Ginns (1)

Oudemansiella Speg. (ca. 20)

Paraxerula R.H. Petersen (4)

Physalacria Peck (33)

Ponticulomyces R.H. Petersen (2)

Protoxerula R.H. Petersen (1)

Rhizomarasmius R.H. Petersen (5)

Rhodotus Maire (2)

Strobilurus Singer (10)

Xerula Maire (ca. 17)

Pleurotaceae Kühner

Agaricochaete Eichelb. (4)

Hohenbuehelia Schulzer (ca. 50)

Lignomyces R.H. Petersen \& Zmitr. (1)

Pleurotus (Fr.) P. Kumm. (25)

Resupinatus Nees ex Gray (33)

Pluteaceae Kotl. \& Pouzar

Pluteus Fr. (ca. 500)

Volvariella Speg. (ca. 50)

Volvopluteus Vizzini, Contu \& Justo (4)

Porotheleaceae Murrill

Phloeomana Redhead (6)

Porotheleum Fr. (ca. 16)

Psathyrellaceae Vilgalys, Moncalvo \& Redhead

Coprinellus P. Karst. (70)

Coprinopsis P. Karst. (ca. 150)

Cystoagaricus Singer (7) 
Gasteroagaricoides D.A. Reid (1)

Homophron (Britzelm.) Örstadius \& E. Larss. (3)

Hormographiella Guarro \& Gené (3)

Kauffmania Örstadius \& E. Larss. (1)

Lacrymaria Pat. (14)

Macrometrula Donk \& Singer (1)

Parasola Redhead, Vilgalys \& Hopple (ca. 27)

Psathyrella (Fr.) Quél. (ca. 420)

Rhacophyllus Berk. \& Broome (1)

Typhrasa Örstadius \& E. Larss. (2)

Pseudoclitocybaceae Vizzini, Consiglio, P.-A. Moreau \& P. Alvarado Bonomyces Vizzini (3)

Cleistocybe Ammirati, A.D. Parker \& Matheny (5)

Clitopaxillus G. Moreno, Vizzini, Consiglio \& P. Alvarado (2)

Harmajaea Dima, P. Alvarado \& Kekki (3)

Musumecia Vizzini \& Contu (4)

Pogonoloma (Singer) Sánchez-García (3)

Pseudoclitocybe (Singer) Singer (16)

\section{Pterulaceae Corner}

Actiniceps Berk. \& Broome (6)

Allantula Corner (1)

Aphanobasidium Jülich (17)

Chaetotyphula Corner (7)

Coronicium J. Erikss. \& Ryvarden (5)

Deflexula Corner (ca. 11)

Lepidomyces Jülich (2)

Merulicium J. Erikss. \& Ryvarden (1)

Parapterulicium Corner (3)

Pterula Fr. (ca. 50)

Pterulicium Corner (1)

Radulomyces M.P. Christ. (10)

Radulotubus Y.C. Dai, S.H. He \& C.L. Zhao (1)

Schizophyllaceae Quél.

Auriculariopsis Maire (3)

Porodisculus Murrill (2)

Schizophyllum Fr. (6)

Stephanosporaceae Oberw. \& E. Horak

Athelidium Oberw. (3)

Cristinia Parmasto (10)

Lindtneria Pilát (10)

Mayamontana Castellano, Trappe \& Lodge (1)

Stephanospora Pat. (6)

Strophariaceae Singer \& A.H. Sm.

Agrocybe Fayod (ca. 100)

Bogbodia Redhead (1)

Brauniella Rick ex Singer (1)

Deconica (W.G. Sm.) P. Karst. (44) 
Hypholoma (Fr.) P. Kumm. (ca. 45)

Leratiomyces Bresinsky \& Manfr. Binder ex Bridge, Spooner, Beever \& D.C. Park (13)

Melanotus Pat. (ca. 33)

Pholiota (Fr.) P. Kumm. (ca. 157)

Protostropharia Redhead, Moncalvo \& Vilgalys (14)

Pseudogymnopilus Raithelh. (1)

Stropharia (Fr.) Quél. (ca. 20)

Tricholomataceae R. Heim ex Pouzar

Albomagister Sánchez-García, Birkebak \& Matheny (2)

Corneriella Sánchez-García (3)

Dennisiomyces Singer (5)

Dermoloma J.E. Lange ex Herink (ca. 25)

Leucopaxillus Boursier (ca. 16)

Porpoloma Singer (ca. 13)

Pseudobaeospora Singer (ca. 26)

PseudoporpolomaVizzini \& Consiglio (1)

Pseudotricholoma (Singer) Sánchez-García \& Matheny (2)

Tricholoma (Fr.) Staude (ca. 210)

Tubariaceae Vizzini

Cyclocybe Velen. (6)

Flammulaster Earle (10)

Hemistropharia Jacobsson \& E. Larss. (1)

Pachylepyrium Singer (1)

Phaeomarasmius Scherff. (ca. 20)

Pleuromyces Dima, P.-A. Moreau \& V. Papp (1)*

Tubaria (W.G. Sm.) Gillet (ca. 21)

Typhulaceae Jülich

Lutypha Khurana, K.S. Thind \& Berthier (1)

Macrotyphula R.H. Petersen (6)

Tygervalleyomyces Crous (1)

Typhula (Pers.) Fr. (ca. 100)

Agaricales genera incertae sedis

Acanthocorticium Baltazar, Gorjón \& Rajchenb. (1)

Acinophora Raf. (1)

Aleurocystis Lloyd ex G. Cunn. (3)

Amparoina Singer (2)

Amylolepiota Harmaja (1)

Aphyllotus Singer (1)

Arthromyces T.J. Baroni \& Lodge (2)

Arthrosporella Singer (1)

Asproinocybe R. Heim (5)

Aspropaxillus Kühner \& Maire (3)

Atractosporocybe P. Alvarado, G. Moreno \& Vizzini (2)

Austroclitocybe Raithelh. (2)

Austroomphaliaster Garrido (1)

Baeospora Singer (13)

Callistodermatium Singer (1)

Calyptella Quél. (20) 
Caulorhiza Lennox (3)

Cellypha Donk (10)

Cephaloscypha Agerer (1)

Cercopemyces T.J. Baroni, Kropp \& V.S. Evenson (3)

Clavomphalia E. Horak (1)

Clitocybe (Fr.) Staude (ca. 300)

Clitocybula (Singer) Singer ex Métrod (25)

Coccobotrys Boud. \& Pat. (2)

Collybia (Fr.) Staude (3)

Conchomyces Overeem (2)

Crucibulum Tul. \& C. Tul. (7)

Cyathus Haller (ca. 59)

Cymatella Pat. (4)

Cymatellopsis Parmasto (1)

Cynema Maas Geest. \& E. Horak (1)

Cyphellocalathus Agerer (1)

Cystoderma Fayod (ca. 36)

Cystodermella Harmaja (16)

Deigloria Agerer (5)

Delicatula Fayod (ca. 3)

Dendrocollybia R.H. Petersen \& Redhead (1)

Dendrothele Höhn. \& Litsch. (58)

Disporotrichum Stalpers (1)

Fayodia Kühner (ca. 10)

Fibulochlamys A.I. Romero \& Cabral (2)

Fissolimbus E. Horak (1)

Fistulina Bull. (9)

Floccularia Pouzar (6)

Gamundia Raithelh. (ca. 7)

Gerronema Singer (58)

Giacomia Vizzini \& Contu (1)

Glabrocyphella W.B. Cooke (12)

Gloioxanthomyces Lodge, Vizzini, Ercole \& Boertm. (2)

Gramincola Velen. (1)

Hemipholiota (Singer) Bon (2)*

Henningsomyces Kuntze (ca. 21)

Hispidocalyptella E. Horak \& Desjardin (1)

Hygrophorocybe Vizzini \& Contu (1)

Infundibulicybe Harmaja (22)

Lactocollybia Singer (20)

Lecanocybe Desjardin \& E. Horak (1)

Lepista (Fr.) W.G. Sm. (ca. 50)

Lepistella T.J. Baroni \& Ovrebo (ca. 50)

Leucocalocybe X.D. Yu \& Y.J. Yao (1)

Leucocortinarius (J.E. Lange) Singer (1)

Leucocybe Vizzini, P. Alvarado, G. Moreno \& Consiglio (3)

Leucoinocybe Singer ex Antonín, Borovička, Holec \& Kolařík (3)

Leucopholiota (Romagn.) O.K. Mill., T.J. Volk \& Bessette (2)

Lignomphalia Antonín, Borovička, Holec \& Kolařík (1)

Lulesia Singer (3)

Lycogalopsis E. Fisch. (1)

Megacollybia Kotl. \& Pouzar (9) 
Melanoleuca Pat. (ca. 60)

Melanomphalia M.P. Christ. (1)

Meottomyces Vizzini (1)

Mesophelliopsis Bat. \& A.F. Vital (1)

Metraria (Cooke) Cooke \& Massee (2)

Metulocyphella Agerer (2)

Mucronella Fr. (8)

Mycenella (J.E. Lange) Singer (10)

Mycoalvimia Singer (1)

Mycocalia J.T. Palmer (7)

Mycospongia Velen. (1)

Myxomphalia Hora (ca. 2)

Neoclitocybe Singer (11)

Neopaxillus Singer (6)

Nidula V.S. White (6)

Nidularia Fr. (3)

Nochascypha Agerer (3)

Notholepista Vizzini \& Contu (1)

Omphaliaster Lamoure (7)

Omphalina Quél. (ca. 50)

Palaeocephala Singer (1)

Panaeolina Maire (2)

Panaeolus (Fr.) Quél. (15)

Paralepistopsis Vizzini (2)

Peglerochaete Sarwal \& Locq. (1)

Pegleromyces Singer (1)

Phaeodepas D.A. Reid (2)

Phaeolepiota Maire ex Konrad \& Maubl. (1)

Phaeomycena R. Heim ex Singer \& Digilio (5)

Phaeopholiota Locq. \& Sarwal (1)

Phlebonema R. Heim (1)

Phlebophyllum R. Heim (1)

Phyllotopsis E.-J. Gilbert \& Donk ex Singer (5)

Physocystidium Singer (1)

Pleurella E. Horak (1)

Pleurocybella Singer (5)

Plicatura Peck (1)

Polygaster Fr. (1)

Pseudoclitopilus Vizzini \& Contu (2)

Pseudofistulina O. Fidalgo \& M. Fidalgo (3)

Pseudohiatula (Singer) Singer (ca. 5)

Pseudohygrophorus Velen. (1)

Pseudolasiobolus Agerer (1)

Pseudoomphalina (Singer) Singer (ca. 6)

Pseudotyphula Corner (1)

Radulomycetopsis Dhingra, Priyanka \& J. Kaur (1)

Rectipilus Agerer (11)

Rhizocybe Vizzini, G. Moreno, P. Alvarado \& Consiglio (4)

Rimbachia Pat. (11)

Ripartitella Singer (1)

Ripartites P. Karst. (5)

Secotium Kunze (ca. 10) 
Singerocybe Harmaja (7)

Skepperiella Pilát (4)

Squamanita Imbach (10)

Stanglomyces Raithelh. (1)

Stemastrum Raf. (1)

Stromatocyphella W.B. Cooke (3)

Tephroderma Contu \& Musumeci (1)

Trichocybe Vizzini (1)

Tricholomopsis Singer (ca. 33)

Tricholosporum Guzmán (7)

Trogia Fr. (ca. 94)

Ugola Adans. (3)

Vanromburghia Holterm. (1)

Verrucospora E. Horak (2)

Amylocorticiales K.H. Larss., Manfr. Binder \& Hibbett Amylocorticiaceae Jülich

Amyloathelia Hjortstam \& Ryvarden (3)

Amylocorticiellum Spirin \& Zmitr. (4)

Amylocorticium Pouzar (11)

Amyloxenasma (Oberw.) Hjortstam \& Ryvarden (6)

Anomoloma Niemelä \& K.H. Larss. (6)

Anomoporia Pouzar (8)

Ceraceomyces Jülich (16)

Irpicodon Pouzar (1)

Plicaturopsis D.A. Reid (2)

Podoserpula D.A. Reid (2)

Serpulomyces (Zmitr.) Zmitr. (1)

Atheliales Jülich

Atheliaceae Jülich

Amphinema P. Karst. (4)

Athelia Pers. (32)

Athelicium K.H. Larss. \& Hjortstam (2)

Athelocystis Hjortstam \& Ryvarden (1)

Athelopsis Oberw. ex Parmasto (14)

Butlerelfia Weresub \& Illman (1)

Byssocorticium Bondartsev \& Singer (11)

Elaphocephala Pouzar (1)

Hypochnella J. Schröt. (2)

Hypochniciellum Hjortstam \& Ryvarden (1)

Leptosporomyces Jülich (15)

Lobulicium K.H. Larss. \& Hjortstam (1)

Lyoathelia Hjortstam \& Ryvarden (1)

Melzericium Hauerslev (3)

Mycostigma Jülich (1)

Piloderma Jülich (6)

Pteridomyces Jülich (4)

Taeniospora Marvanová (2)

Tretomyces K.H. Larss., Kotir. \& Saaren. (2)

Tylospora Donk (2) 
Auriculariales J. Schröt.

Auriculariaceae Fr.

Amphistereum Spirin \& Malysheva (2)

Auricularia Bull. (ca. 21)

Eichleriella Bres. (ca. 14)

Elmerina Bres. (7)

Exidia Fr. (ca. 26)

Exidiopsis (Bref.) Möller (ca. 30)

Fibulosebacea K. Wells \& Raitv. (1)

Heterochaete Pat. (ca. 40)

Heteroradulum Lloyd ex Spirin \& Malysheva (7)

Protodaedalea Imazeki (2)

Pseudostypella McNabb (1)

Sclerotrema Spirin \& Malysheva (1)

Hyaloriaceae Lindau

Helicomyxa R. Kirschner \& Chee J. Chen (1)

Hyaloria Möller (3)

Myxarium Wallr. (14)

Auriculariales genera incertae sedis

Basidiodendron Rick (ca. 15)

Bourdotia (Bres.) Bres. \& Torrend (1)

Ceratosebacina P. Roberts (3)

Dendrogloeon Spirin \& Miettinen (1)

Ductifera Lloyd (ca. 11)

Endoperplexa P. Roberts (6)

Gelacantha V. Malysheva \& Spirin (1)

Grammatus H.S. Yuan \& C. Decock (2)

Guepinia Fr. (1)

Hauerslevia P. Roberts (1)

Heterorepetobasidium Chee J. Chen \& Oberw. (2)

Heteroscypha Oberw. \& Agerer (1)

Hyalodon V. Malysheva \& Spirin (2)

Hydrophana V. Malysheva \& Spirin (1)

Metabourdotia L.S. Olive (1)

Microsebacina P. Roberts (2)

Mycostilla Spirin \& V. Malysheva (1)

Myxariellum Spirin \& V. Malysheva (2)

Ofella Spirin \& V. Malysheva (1)

Porpopycnis R. Kirschner (1)

Protoacia Spirin \& V. Malysheva (1)

Protodontia Höhn. (3)

Protograndinia Rick (1)

Protohydnum Möller (3)

Protomerulius Möller (7)

Protoradulum Rick (1)

Pseudohydnum P. Karst. (1)

Renatobasidium Hauerslev (1)

Stypella Möller (4)

Stypellopsis Spirin \& V. Malysheva (2)

Tremellacantha Jülich (1) 
Boletales E.-J. Gilbert

Boletaceae Chevall.

Afroboletus Pegler \& T.W.K. Young (8)

Afrocastellanoa M.E. Sm. \& Orihara (1)

Alessioporus Gelardi, Vizzini \& Simonini (2)

Aureoboletus Pouzar (33)

Australopilus Halling \& N.A. Fechner (1)

Austroboletus (Corner) Wolfe (ca. 36)

Baorangia G. Wu \& Zhu L. Yang (4)

Binderoboletus T.W. Henkel \& M.E. Sm. (1)

Boletellus Murrill (ca. 50)

Boletochaete Singer (5)

Boletus L. (ca. 350)

Borofutus Hosen \& Zhu L. Yang (1)

Bothia Halling, T.J. Baroni \& Manfr. Binder (2)

Buchwaldoboletus Pilát (11)

Butyriboletus Arora \& J.L. Frank (ca. 25)

Caloboletus Vizzini (14)

Carolinigaster M.E. Sm. \& S. Cruz (1)

Castellanea T.W. Henkel \& M.E. Sm. (1)

Chalciporus Bataille (ca. 30)

Chamonixia Rolland (8)

Chiua Y.C. Li \& Zhu L. Yang (4)

Corneroboletus N.K. Zeng \& Zhu L. Yang (1)

Costatisporus T.W. Henkel \& M.E. Sm. (1)

Crocinoboletus N.K. Zeng, Zhu L. Yang \& G. Wu (2)

Cupreoboletus Simonini, Gelardi \& Vizzini (1)

Cyanoboletus Gelardi, Vizzini \& Simonini (7)

Durianella Desjardin, A.W. Wilson \& Manfr. Binder (1)

Erythrophylloporus Ming Zhang \& T.H. Li (1)

Fistulinella Henn. (ca. 25)

Gastroboletus Lohwag (14)

Gastroleccinum Thiers (1)

Guyanaporus T.W. Henkel \& M.E. Sm. (1)

Gymnogaster J.W. Cribb (1)

Harrya Halling, Nuhn \& Osmundson (6)

Heimioporus E. Horak (14)

Heliogaster Orihara \& K. Iwase (1)

Hemileccinum Šutara (5)

Hortiboletus Simonini, Vizzini \& Gelardi (7)

Hourangia Xue T. Zhu \& Zhu L. Yang (4)

Hymenoboletus Y.C. Li \& Zhu L. Yang (1)

Imleria Vizzini (5)

Imperator G. Koller, Assyov, Bellanger, Bertéa, Loizides, G. Marques, P.-A. Moreau, J.A.

Muñoz, Oppicelli, Puddu \& F. Richard (3)

Indoporus A. Parihar, K. Das, Hembrom \& Vizzini (1)

Ionosporus O. Khmelnitsky (2)

Jimtrappea T.W. Henkel, M.E. Sm. \& Aime (2)

Kombocles Castellano, T.W. Henkel \& Dentinger (1)

Lanmaoa G. Wu \& Zhu L. Yang (7)

Leccinellum Bresinsky \& Manfr. Binder (17)

Leccinum Gray (ca. 130) 
Mackintoshia Pacioni \& Sharp (1)

Mucilopilus Wolfe (1)

Mycoamaranthus Castellano, Trappe \& Malajczuk (3)

Neoboletus Gelardi, Simonini \& Vizzini (11)

Nigroboletus Gelardi, Vizzini, E. Horak, T.H. Li \& Ming Zhang (1)

Octaviania Vittad. (ca. 40)

Parvixerocomus G. Wu \& Zhu L. Yang (2)

Paxillogaster E. Horak (1)

Phylloboletellus Singer (1)

Phyllobolites Singer (1)

Phylloporus Quél. (ca. 90)

Porphyrellus E.-J. Gilbert (ca. 20)

Pseudoaustroboletus Y.C. Li \& Zhu L. Yang (3)

Pseudoboletus Šutara (2)

Pulchroboletus Gelardi, Vizzini \& Simonini (1)

Pulveroboletus Murrill (38)

Retiboletus Manfr. Binder \& Bresinsky (12)

Rheubarbariboletus Vizzini, Simonini \& Gelardi (2)

Rhodactina Pegler \& T.W.K. Young (3)

Rossbeevera T. Lebel, Orihara \& N. Maek. (10)

Royoungia Castellano, Trappe \& Malajczuk (6)

Rubroboletus Kuan Zhao \& Zhu L. Yang (14)

Rugiboletus G. Wu \& Zhu L. Yang (2)

Setogyroporus Heinem. \& Rammeloo (1)

Singerocomus T.W. Henkel \& M.E. Sm. (2)

Singeromyces M.M. Moser (1)

Solioccasus Trappe, Osmundson, Manfr. Binder, Castellano \& Halling (1)

Spongiforma Desjardin, Manfr. Binder, Roekring \& Flegel (2)

Spongispora G. Wu, S.M.L. Lee, E. Horak \& Zhu L. Yang (1)

Strobilomyces Berk. (ca. 27)

Suillellus Murrill (23)

Sutorius Halling, Nuhn \& N.A. Fechner (2)

Tengioboletus G. Wu \& Zhu L. Yang (2)

Tubosaeta E. Horak (6)

Turmalinea Orihara \& N. Maek. (4)

Tylocinum Y.C. Li \& Zhu L. Yang (1)

Tylopilus P. Karst. (ca. 100)

Veloporphyrellus L.D. Gómez \& Singer (7)

Wakefieldia Corner \& Hawker (2)

Xanthoconium Singer (10)

Xerocomellus Šutara (17)

Xerocomus Quél. (ca. 120)

Zangia Y.C. Li \& Zhu L. Yang (6)

Boletinellaceae P.M. Kirk, P.F. Cannon \& J.C. David

Boletinellus Murrill (1)

Phlebopus (R. Heim) Singer (14)

Calostomataceae E. Fisch.

Calostoma Desv. (16)

Coniophoraceae Ulbr. 
Chrysoconia McCabe \& G.A. Escobar (1)

Coniophora DC. (20)

Coniophoropsis Hjortstam \& Ryvarden (2)

Gyrodontium Pat. (3)

Sedecula Zeller (1)

Diplocystidiaceae Kreisel

Astraeus Morgan (11)

Diplocystis Berk. \& M.A. Curtis (2)

Endogonopsis R. Heim (1)

Tremellogaster E. Fisch. (1)

Gasterellaceae Zeller

Gasterella Zeller \& L.B. Walker (1)

Gomphidiaceae Maire ex Jülich

Chroogomphus (Singer) O.K. Mill. (25)*

Cystogomphus Singer (1)

Gomphidius Fr. (10)

Gomphogaster O.K. Mill. (1)

Gyroporaceae (Singer) Manfr. Binder \& Bresinsky

Gyroporus Quél. (24)

Hygrophoropsidaceae Kühner

Hygrophoropsis (J. Schröt.) Maire ex Martin-Sans (16)

Leucogyrophana Pouzar (13)

Paxillaceae Lotsy

Alpova C.W. Dodge (16)

Austrogaster Singer (4)

Gyrodon Opat. (10)

Hoehnelogaster Lohwag (1)

Hydnomerulius Jarosch \& Besl (1)

Meiorganum R. Heim (3)

Melanogaster Corda (ca. 26)

Neoalpova Vizzini (1)

Paragyrodon (Singer) Singer (1)

Paxillus Fr. (19)

Protogastraceae Zeller

Protogaster Thaxt. (1)

Rhizopogonaceae Gäum. \& C.W. Dodge

Fevansia Trappe \& Castellano (1)

Rhizopogon Fr. (ca. 157)

Rhopalogaster J.R. Johnst. (1)

\section{Sclerodermataceae Corda}

Chlorogaster Læssøe \& Jalink (1)

Favillea Fr. (2)

Horakiella Castellano \& Trappe (2) 
Pisolithus Alb. \& Schwein. (17)

Scleroderma Pers. (ca. 46)

Serpulaceae Jarosch \& Bresinsky

Austropaxillus Bresinsky \& Jarosch (9)

Gymnopaxillus E. Horak (4)

Serpula (Pers.) Gray (ca. 11)

Suillaceae Besl \& Bresinsky

Psiloboletinus Singer (1)

Suillus Gray (ca. 60)

Tapinellaceae C. Hahn

Bondarcevomyces Parmasto (1)

Pseudomerulius Jülich (4)

Tapinella E.-J. Gilbert (2)

Boletales genera incertae sedis

Corditubera Henn. (5)

Corneromyces Ginns (2)

Marthanella States \& Fogel (1)

Phaeoradulum Pat. (1)

Cantharellales Gäum.

Aphelariaceae Corner

Aphelaria Corner (20)

Phaeoaphelaria Corner (1)

Tumidapexus D.A. Crawford (1)

Botryobasidiaceae Jülich

Acladium Link (20)

Allescheriella Henn. (5)

Alysidium Kunze (4)

Botryobasidium Donk (ca. 58)

Suillosporium Pouzar (4)

Ceratobasidiaceae G.W. Martin

Ceratobasidium D.P. Rogers (ca. 19)

Ceratoporia Ryvarden \& de Meijer (1)

Ceratorhiza R.T. Moore (7)

Rhizoctonia DC. (ca. 50)

Scotomyces Jülich (1)

Thanatephorus Donk (12)

Hydnaceae Chevall.

Burgoa Goid. (9)

Burgella Diederich \& Lawrey (2)

Burgellopsis Diederich \& Lawrey (1)

Cantharellus Adans.ex Fr. (ca. 300)

Clavulina J. Schröt. (ca. 75)

Corallofungus Kobayasi (2)

Craterellus Pers. (ca. 80) 
Gloeoтисro R.H. Petersen (10)

Hydnum L. (49)

Ingoldiella D.E. Shaw (3)

Membranomyces Jülich (1)

Multiclavula R.H. Petersen (13)

Neoburgoa Diederich, E. Zimm. \& Lawrey (1)

Parastereopsis Corner (1)

Osteomorpha G. Arnaud ex Watling \& W.B. Kendr. (1)

Paullicorticium J. Erikss. (5)

Repetobasidiellum J. Erikss. \& Hjortstam (1)

Repetobasidium J. Erikss. (12)

Rogersiomyces J.L. Crane \& Schokn. (2)

Sistotrema Fr. (ca. 55)

Sistotremella Hjortstam (3)

Oliveoniaceae P. Roberts

Oliveonia Donk (5)

Tulasnellaceae Juel

Pseudotulasnella Lowy (1)

Tulasnella J. Schröt. (ca. 70)

Cantharellales genera incertae sedis

Boidinella Nakasone (2)

Bulbilla Diederich, Flakus \& Etayo (1)

Clavulicium Boidin (3)

Minimedusa Weresub \& P.M. LeClair (3)

Odontiochaete Rick (1)

Radulochaete Rick (2)

Schildia Franchi \& M. Marchetti (1)

Stilbotulasnella Oberw. \& Bandoni (1)

Corticiales K.H. Larss.

Corticiaceae Herter

Capillosclerotium Prameela \& Deeba (1)

Corticirama Pilát (2)

Corticium Pers. (25)

Erythricium J. Erikss. \& Hjortstam (6)

Galzinia Bourdot (9)

Giulia Tassi (1)

Laetisaria (Burds. (7)

Lawreymyces Lücking \& Moncada (7)

Marchandiomyces Diederich \& D. Hawksw. (3)

Necator Massee (1)

Tretopileus B.O. Dodge (3)

Waitea Warcup \& P.H.B. Talbot (1)

Dendrominiaceae Ghobad-Nejhad

Dendrominia Ghobad-Nejhad \& Duhem (4)

Punctulariaceae Donk

Dendrocorticium M.J. Larsen \& Gilb. (9) 
Punctularia Pat. (2)

Punctulariopsis Ghobad-Nejhad (4)

Vuilleminiaceae Maire ex Lotsy

Australovuilleminia Ghobad-Nejhad \& Hallenb. (1)

Cytidia Quél. (5)

Vuilleminia Maire (8)

Corticiales genera incertae sedis

Ambivina Katz (1)

Amylobasidium Ginns (1)

Leptocorticium Hjortstam \& Ryvarden (8)

Melzerodontia Hjortstam \& Ryvarden (3)

Nothocorticium Gresl. \& Rajchenb. (1)

Papyrodiscus D. A. Reid (1)

Ripexicium Hjortstam (1)

Geastrales K. Hosaka \& Castellano

Geastraceae Corda

Geasteroides Long (1)

Geastrum Pers. (130)

Myriostoma Desv. (4)

Nidulariopsis Greis (2)

Phialastrum Sunhede (1)

Schenella T. Macbr. (4)

Sphaerobolus Tode (3)

Sclerogastraceae Locq. ex P. M. Kirk

Sclerogaster R. Hesse (11)

Geastrales genus incertae sedis

Boninogaster Kobayasi (1)

Gloeophyllales Thorn

Gloeophyllaceae Jülich

Boreostereum Parmasto (4)

Campylomyces Nakasone (2)

Chaetodermella Rauschert (1)

Gloeophyllum P. Karst. (13)

Griseoporia Ginns (2)

Heliocybe Redhead \& Ginns (1)

Hispidaedalea Y. C. Dai \& S.H. He (1)

Mycothele Jülich (1)

Neolentinus Redhead \& Ginns (14)

Osmoporus Singer (2)

Stiptophyllum Ryvarden (1)

Veluticeps Cooke (12)

Gloeophyllales genus incertae sedis

Pileodon P. Roberts \& Hjortstam (2)

Gomphales Jülich 
Clavariadelphaceae Corner

Beenakia D. A. Reid (7)

Clavariadelphus Donk (20)

Gomphaceae Donk

Araeocoryne Corner (1)

Ceratellopsis Konrad \& Maubl. (9)

Delentaria Corner (1)

Destuntzia Fogel \& Trappe (5)

Gautieria Vittad. (37)

Gloeocantharellus Singer (12)

Gomphus Pers. (7)

Phaeoclavulina Brinkmann (41)

Protogautieria A. H. Sm. (2)

Pseudogomphus R. Heim (1)

Ramaria Fr. ex Bonord. (230)

Ramaricium J. Erikss. (5)

Terenodon Maas Geest. (1)

Turbinellus Earle (5)

Lentariaceae Jülich

Hydnocristella R.H. Petersen (2)

Kavinia Pilát (4)

Lentaria Corner (19)

Hymenochaetales Oberw.

Hymenochaetaceae Donk

Arambarria Rajchenb. \& Pildain (1)

Asterodon Pat. (1)

Aurificaria D.A. Reid (2)

Botryodontia (Hjortstam \& Ryvarden) Hjortstam (6)

Clavariachaete Corner (2)

Coltricia Gray (40)

Coltriciella Murrill (13)

Coniferiporia L.W. Zhou \& Y. C. Dai (3)

Cylindrosporus L.W. Zhou (1)

Deviodontia (Parmasto) Hjortstam \& Ryvarden (1)

Dichochaete Parmasto (2)

Erythromyces Hjortstam \& Ryvarden (1)

Fomitiporella Murrill (13)

Fomitiporia Murrill (46)

Fulvifomes Murrill (33)

Fuscoporia Murrill (62)

Hastodontia (Parmasto) Hjortstam \& Ryvarden (2)

Hydnochaete Bres. (1)

Hymenochaete Lév. (149)

Hymenochaetopsis S. H. He \& Jiao Yang (16)

Inocutis Fiasson \& Niemelä (9)

Inonotopsis Parmasto (1)

Inonotus P. Karst. (120)

Mensularia Lázaro Ibiza (6)

Neomensularia F. Wu, L. W. Zhou \& Y.C. Dai (4) 
Nothophellinus Rajchenb. (1)

Onnia P. Karst. (8)

Phellinidium (Kotl.) Fiasson \& Niemelä (5)

Phellinopsis Y. C. Dai (10)

Phellinotus Drechsler-Santos, Robledo \& Rajchenb. (2)

Phellinus Quél. (202)

Phellopilus Niemelä, T. Wagner \& M. Fisch. (1)

Phylloporia Murrill (38)

Porodaedalea Murrill (14)

Pseudoinonotus T. Wagner \& M. Fisch. (8)

Pyrrhoderma Imazeki (2)

Sanghuangporus Sheng H. Wu, L.W. Zhou \& Y. C. Dai (13)

Tropicoporus L.W. Zhou, Y. C. Dai \& Sheng H. Wu (12)

Tubulicrinis Donk (34)

Xanthoporia Murrill (3)

Neoantrodiellaceae Y.C. Dai, B. K. Cui, Jia J. Chen \& H. S. Yuan

Neoantrodiella Y. C. Dai, B. K. Cui, Jia J. Chen \& H.S. Yuan (2)

Nigrofomitaceae Jülich

Nigrofomes Murrill (3)

Oxyporaceae Zmitr. \& V. Malysheva

Oxyporus (Bourdot \& Galzin) Donk (18)

Rickenellaceae Vizzini

Alloclavaria Dentinger \& D. J. McLaughlin (1)

Atheloderma Parmasto (2)

Contumyces Redhead, Moncalvo, Vilgalys \& Lutzoni (3)

Cotylidia P. Karst. (10)

Globulicium Hjortstam (1)

Peniophorella P. Karst. (25)

Resinicium Parmasto (8)

Rickenella Raithelh. (10)

Schizoporaceae Jülich

Alutaceodontia (Parmasto) Hjortstam \& Ryvarden (1)

Basidioradulum Nobles (1)

Echinoporia Ryvarden (3)

Fibrodontia Parmasto (6)

Hyphodontia J. Erikss. (86)

Lagarobasidium Jülich (5)

Leucophellinus Bondartsev \& Singer (1)

Paratrichaptum Corner (1)

Poriodontia Parmasto (1)

Rogersella Liberta \& A.J. Navas (1)

Odontiopsis Hjortstam \& Ryvarden (2)

Schizopora Velen. (7)

Xylodon (Pers.) Gray (60)

Hymenochaetales genera incertae sedis

Caeruleomyces Stalpers (1) 
Cantharellopsis Kuyper (1)

Cyanotrama Ghobad-Nejhad \& Y.C. Dai (1)

Fibricium J. Erikss. (5)

Ginnsia Sheng H. Wu \& Hallenb. (1)

Gyroflexus Raithelh. (1)

Kurtia Karasiński (3)

Lawrynomyces Karasiński (1)

Muscinupta Redhead, Lücking \& Lawrey (1)

Physodontia Ryvarden \& H. Solheim (1)

Sidera Miettinen \& K. H. Larss. (6)

Skvortzovia Bononi \& Hjortstam (1)

Subulicium Hjortstam \& Ryvarden (3)

Trichaptum Murrill (27)

Tsugacorticium Nakasone \& Burds. (1)

Hysterangiales K. Hosaka \& Castellano

Gallaceaceae Locq. ex P. M. Kirk

Austrogautieria E. L. Stewart \& Trappe (7)

Gallacea Lloyd (6)

Hallingea Castellano (3)

Hysterangiaceae E. Fisch.

Aroramyces Castellano \& Verbeken (5)

Circulocolumella S. Ito \& S. Imai (1)

Clathrogaster Petri (2)

Hysterangium Vittad. (54)

Mesophelliaceae Jülich

Andebbia Trappe, Castellano \& Amar. (1)

Castoreum Cooke \& Massee (3)

Chondrogaster Maire (2)

Gummiglobus Trappe, Castellano \& Amar. (2)

Gummivena Trappe \& Bougher (1)

Malajczukia Trappe \& Castellano (8)

Mesophellia Berk. (15)

Nothocastoreum G. W. Beaton (1)

Phallogastraceae Locq.

Phallogaster Morgan (1)

Protubera Möller (13)

Trappeaceae P.M. Kirk

Phallobata G. Cunn. (1)

Restingomyces Sulzbacher, Grebenc \& Baseia (1)

Trappea Castellano (1)

Jaapiales Manfr. Binder, K. H. Larss. \& Hibbett

Jaapiaceae Manfr. Binder, K. H. Larss. \& Hibbett

Jaapia Bres. (2)

Lepidostromatales B.P. Hodk. \& Lücking

Lepidostromataceae Ertz, Eb. Fisch., Killmann, Sérus. \& Lawrey 
Ertzia B.P. Hodk. \& Lücking (1)

Lepidostroma Mägd. \& S. Winkl. (1)

Sulzbacheromyces B. P. Hodk. \& Lücking (6)

Phallales E. Fisch.

Claustulaceae G. Cunn.

Claustula K.M. Curtis (1)

Gelopellis Zeller (6)

Kjeldsenia W. Colgan, Castellano \& Bougher (1)

Phlebogaster Fogel (2)

Pseudogelopellis K. Tao \& B. Liu (1)

\section{Gastrosporiaceae Pilát}

Gastrosporium Mattir. (2)

\section{Phallaceae Corda}

Abrachium Baseia \& T. S. Cabral (1)

Aporophallus Möller (1)

Aseroë Labill. (2)

Blumenavia Möller (3)

Calvarula Zeller (1)

Clathrus P. Micheli ex L. (20)

Colus Cavalier \& Séchier (4)

Echinophallus Henn. (1)

Endoclathrus B. Liu, Yin H. Liu \& Z.J. Gu (1)

Endophallus M. Zang \& R. H. Petersen (1)

Ileodictyon Tul. \& C. Tul. (2)

Itajahya Möller (4)

Kobayasia S. Imai \& A. Kawam. (1)

Laternea Turpin (2)

Ligiella J.A. Sáenz (1)

Lysurus Fr. (30)

Mutinus Fr. (21)

Neolysurus O. K. Mill., Ovrebo \& Burk (1)

PhallusJunius ex L. (34)

Protuberella S. Imai \& A. Kawam. (1)

Pseudoclathrus B. Liu \& Y.S. Bau (5)

Pseudocolus Lloyd (2)

Staheliomyces E. Fisch. (1)

Staurophallus Mont. (1)

Stephanophallus MacOwan (1)

Xylophallus (Schltdl.) E. Fisch. (2)

Phallales genera incertae sedis

Saprogaster Fogel \& States (1)

Vandasia Velen. (1)

Polyporales Gäum.

Cerrenaceae Miettinen, Justo \& Hibbett

Cerrena Gray (7)

Irpiciporus Murrill (1)

Pseudolagarobasidium J. C. Jang \& T. Chen (7) 
Radulodon Ryvarden (11)

Dacryobolaceae Jülich

Amylocystis Bondartsev \& Singer ex Singer (1)

Dacryobolus Fr. (7)

Jahnoporus Nuss (4)

Oligoporus Bref. (15)

Osteina Donk (1)

Postia Fr. (40)

Spongiporus Murrill (7)

Fomitopsidaceae Jülich*

Adustoporia Audet (1)

Anthoporia Karasiński \& Niemelä (1)

Antrodia P. Karst. (80)

Antrodiopsis Audet (1)

Brunneoporus Audet (5)

Buglossoporus Kotl. \& Pouzar (9)

Daedalea Pers. (12)

Dentiporus Audet (1)

Flavidoporia Audet (3)

Fomitopsis P. Karst. (40)

Fragifomes B. K. Cui, M.L. Han \& Y. C. Dai (1)

Laricifomes Kotl. \& Pouzar (1)

Lentoporia Audet (1)

Neoantrodia Audet (13)

Neolentiporus Rajchenb. (2)

Niveoporofomes B. K. Cui, M. L. Han \& Y. C. Dai (1)

Ranadivia Zmitr. (5)*

Resinoporia Audet (11)

Rhizoporia Audet (1)

Rhodofomes Kotl. \& Pouzar (5)

Rhodofomitopsis B.K. Cui, M. L. Han \& Y. C. Dai (4)

Rubellofomes B. K. Cui, M.L. Han \& Y. C. Dai (2)

Subantrodia Audet (2)

Ungulidaedalea B. K. Cui, M.L. Han \& Y. C. Dai (1)

Wolfiporia Ryvarden \& Gilb. (6)

Fragiliporiaceae Y. C. Dai, B.K. Cui \& C. L. Zhao

Fragiliporia Y. C. Dai, B.K. Cui \& C. L. Zhao (1)

Gelatoporiaceae Miettinen, Justo \& Hibbett

Cinereomyces Jülich (2)

Gelatoporia Niemelä (2)

Obba Miettinen \& Rajchenb. (2)

Sebipora Miettinen (1)

Grifolaceae Jülich

Aegis Gómez-Montoya, Rajchenb. \& Robledo (1)

Grifola Gray (5)

Hyphodermataceae Jülich 
Hyphoderma Fr. (20)

Incrustoporiaceae Jülich

Gloeoporellus Zmitr. (1)*

Incrustoporia Domanski (5)

Piloporia Niemelä (2)

Skeletocutis Kotl. \& Pouzar (40)

Tyromyces P. Karst. (41)

Irpicaceae Spirin \& Zmitr.

Byssomerulius Parmasto (8)

Ceriporia Donk (ca. 50)

Cytidiella Pouzar (2)

Efibula Sheng H. Wu (18)

Emmia Zmitr., Spirin \& Malysheva (2)

Flavodon Ryvarden (3)

Gloeoporus Mont. (13)

Hydnopolyporus D. A. Reid (2)

Irpex Fr. (10)

Leptoporus Quél. (1)

Meruliopsis Bondartsev (4)

Raduliporus Spirin \& Zmitr. (1)

Resiniporus Zmitr. (2)*

Trametopsis Tomšovský (4)

Ischnodermataceae Jülich

Ischnoderma P. Karst. (10)

Laetiporaceae Jülich

Kusaghiporia J. Hussein, S. Tibell \& Tubuhwa (1)*

Laetiporus Murrill (15)

Phaeolus (Pat.) Pat. (3)

Meripilaceae Jülich

Meripilus P. Karst. (5)

Pseudonadsoniella T. O. Kondr. \& S. Y. Kondr. (1)

Rigidoporus Murrill (30)

\section{Meruliaceae Rea}

Aurantiopileus Ginns, D. L. Lindner \& T. J. Baroni (3)

Aurantiporus Murrill (6)

Ceriporiopsis Domański (40)

Climacodon P. Karst. (7)

Crustodontia Hjortstam \& Ryvarden (1)

Geesterania Westphalen, Tomšovský \& Rajchenb. (2)*

Hermanssonia Zmitr. (1)*

Hydnophanerochaete Sheng H. Wu \& C.C. Chen (1)*

Hydnophlebia Parmasto (5)

Lilaceophlebia (Parmasto) Spirin \& Zmitr. (2)

Luteoporia F. Wu, Jia J. Chen \& S. H. He (1)

Merulius Fr. (150)

Mycoacia Donk (16) 
Mycoaciella J. Erikss. \& Ryvarden (5)

Odoria V. Papp \& Dima (1)

Pappia Zmitr. (1)*

Phlebia Fr. (60)

Phlebiporia Jia J. Chen, B. K. Cui \& Y. C. Dai (1)

Physisporinus P. Karst. (15)

Sarcodontia Schulzer (1)

Scopuloides (Massee) Höhn. \& Litsch. (5)

Stereophlebia Zmitr. (1)*

Panaceae Miettinen, Justo \& Hibbett

Cymatoderma Jungh. (11)

Panus Fr. (20)

Phanerochaetaceae Jülich

Bjerkandera P. Karst. (5)

Crepatura C.L. Zhao (1)*

Donkia Pilát (1)

Efibulella Zmitr. (1)

Geliporus Yuan Yuan, Jia J. Chen \& S. H. He (1)

Hapalopilus P. Karst. (11)

Hyphodermella J. Erikss. \& Ryvarden (7)

Odontoefibula C. C. Chen \& Sheng H. Wu (1)*

Oxychaete Miettinen (1)

Phaeophlebiopsis D. Floudas \& Hibbett (3)

Phanerina Miettinen (1)

Phanerochaete P. Karst. (80)

Phlebiopsis Jülich (22)

Pirex Hjortstam \& Ryvarden (1)

Porostereum Pilát (15)

Rhizochaete Gresl., Nakasone \& Rajchenb. (13)

Riopa D.A. Reid (3)

Terana Adans. (1)

Podoscyphaceae D.A. Reid

Abortiporus Murrill (4)

Podoscypha Pat. (36)

Pouzaroporia Vampola (1)

Polyporaceae Fr. ex Corda

Abundisporus Ryvarden (8)

Amauroderma Murrill (40)

Atroporus Ryvarden (3)

Australoporus P.K. Buchanan \& Ryvarden (1)

Bresadolia Speg. (4)*

Cerarioporia F. Wu, L.W. Zhou \& J. Si (1)

Cerioporus Quél. (15)

Cinereomycetella Zmitr. (1)*

Colospora Miettinen \& Spirin (2)

Coriolopsis Murrill (19)

Cryptoporus (Peck) Shear (2)

Daedaleopsis J. Schröt. (7) 
Datronia Donk (9)

Datroniella B.K. Cui, Hai J. Li \& Y.C. Dai (6)

Dentocorticium (Parmasto) M.J. Larsen \& Gilb. (3)*

Dextrinoporus H.S. Yuan (1)*

Dichomitus D.A. Reid (13)

Donkioporia Kotl. \& Pouzar (2)

Donkioporiella L.W. Zhou (1)

Earliella Murrill (1)

Echinochaete D.A. Reid (5)

Endopandanicola Tibpromma \& K.D. Hyde (1)

Epithele (Pat.) Pat. (17)

Epithelopsis Jülich (2)

Favolus Fr. (20)

Flammeopellis Y.C. Dai, B.K. Cui \& C.L. Zhao (1)

Fomes (Fr.) Fr. (3)

Fomitella Murrill (2)

Globifomes Murrill (1)

Foraminispora Robledo, Costa-Rezende \& Drechsler-Santos (1)

Funalia Pat. (10)

Furtadoa Costa-Rezende, Robledo \& Drechsler-Santos (3)

Ganoderma P. Karst. (180)

Grammothele Berk. \& M.A. Curtis (20)

Grammothelopsis Jülich (7)

Haddowia Steyaert (3)

Haploporus Bondartsev \& Singer (13)

Hexagonia Fr. (17)

Hornodermoporus Teixeira (2)

Humphreya Steyaert (4)

Laccocephalum Mc Alpine \& Tepper (5)

Leifiporia Y.C. Dai, F. Wu \& C.L. Zhao (2)

Lentinus Fr. (55)

Lignosus Lloyd ex Torrend (8)

Lopharia Kalchbr. \& MacOwan (7)

Megasporia B.K. Cui, Y.C. Dai \& Hai J. Li (10)

Megasporoporia Ryvarden \& J.E. Wright (3)

Megasporoporiella B.K. Cui, Y.C. Dai \& Hai J. Li (5)

Melanoderma B.K. Cui \& Y.C. Dai (2)

Microporellus Murrill (20)

Microporus P. Beauv. (13)

Mollicarpus Ginns (1)

Murinicarpus B.K. Cui \& Y.C. Dai (2)

Myriothele Nakasone (1)

Navisporus Ryvarden (8)

Neodatronia B.K. Cui, Hai J. Li \& Y.C. Dai (2)

Neodictyopus Palacio, Robledo, Reck \& Drechsler-Santos (3)

Neofavolus Sotome \& T. Hatt. (4)

Neofomitella Y.C. Dai, Hai J. Li \& Vlasák (3)

Pachykytospora Kotl. \& Pouzar (4)

Perenniporia Murrill (100)

Perenniporiella Decock \& Ryvarden (5)

Perenniporiopsis C.L. Zhao (1)

Phaeotrametes Lloyd ex J. E. Wright (1) 
Picipes Zmitr. \& Kovalenko (16)

Pilatotrama Zmitr. (1)*

Podofomes Pouzar (3)

Polyporopsis Audet (1)

Polyporus [P. Micheli ex Adans.] Fr. (35)

Porogramme (Pat.) Pat. (4)

Pseudofavolus Pat. (4)

Pseudomegasporoporia X.H. Ji \& F. Wu (1)

Pseudopiptoporus Ryvarden (2)

Pyrofomes Kotl. \& Pouzar (8)

Rubroporus Log.-Leite, Ryvarden \& Groposo (1)

Sparsitubus L.W. Hsu \& J.D. Zhao (1)

Szczepkamyces Zmitr. (1)*

Theleporus Fr. (9)

Thermophymatospora Udagawa, Awao \& Abdullah (1)

Tinctoporellus Ryvarden (4)

Tomophagus Murrill (2)

Trametes Fr. (70)

Truncospora Pilát (23)

Vanderbylia D.A. Reid (7)

Yuchengia B.K. Cui \& K.T. Steffen (1)

Sparassidaceae Jülich

Crustoderma Parmasto (16)

Pycnoporellus Murrill (2)

Sparassis Fr. (7)

\section{Steccherinaceae Parmasto}

Antella Miettinen (3)

Antrodiella Ryvarden \& I. Johans. (50)

Atraporiella Ryvarden (2)

Austeria Miettinen (1)

Butyrea Miettinen (2)

Cabalodontia Piątek (5)

Caudicicola Miettinen, M. Kulju \& Kotir. (1)

Citripora Miettinen (2)

Elaphroporia Z.Q. Wu \& C.L. Zhao (1)

Flabellophora G. Cunn. (18)

Flaviporus Murrill (14)

Frantisekia Spirin \& Zmitr. (4)

Junghuhnia Corda (35)

Lamelloporus Ryvarden (1)

Loweomyces (Kotl. \& Pouzar) Jülich (6)

Metuloidea G. Cunn. (5)

Mycorrhaphium Maas Geest. (6)

Niemelaea Zmitr., Ezhov \& Khimich (5)

Nigroporus Murrill (3)

Steccherinum Gray (40)

Trullella Zmitr. (6)*

Xanthoporus Audet (2) 
Polyporales genera incertae sedis

Aegeritopsis Höhn. (1)

Amaropostia B.K. Cui, L.L. Shen \& Y.C. Dai (2)

Amaurohydnum Jülich (1)

Amauromyces Jülich (1)

Amethicium Hjortstam (1)

Amyloporia Singer (5)

Aquascypha D.A. Reid (1)

Auriporia Ryvarden (4)

Australicium Hjortstam \& Ryvarden (2)

Australohydnum Jülich (2)

Austrolentinus Ryvarden (1)

Bourdotiella Duhem \& Schultheis (1)

Bulbillomyces Jülich (1)

Calcipostia B.K. Cui, L.L. Shen \& Y.C. Dai (1)

Candelabrochaete Boidin (12)

Climacocystis Kotl. \& Pouzar (2)

Columnodontia Jülich (1)

Conohypha Jülich (2)

Coralloderma D.A. Reid (2)

Cordochaete Sanyal, Samita, Dhingra \& Avn. P. Singh (1)

Cryptomphalina R. Heim (1)

Cyanodontia Hjortstam (1)

Cyanosporus McGinty (1)

Cystidiopostia B.K. Cui, L.L. Shen \& Y.C. Dai (3)

Dendrophlebia Dhingra \& Priyanka (1)

Diacanthodes Singer (3)

Diplomitoporus Domański (25)

Erastia Niemelä \& Kinnunen (1)

Faerberia Pouzar (1)

Fibroporia Parmasto (10)

Fuscopostia B.K. Cui, L.L. Shen \& Y.C. Dai (4)

Gilbertsonia Parmasto (1)

Globosomyces Jülich (1)

Globuliciopsis Hjortstam \& Ryvarden (2)

Gyrophanopsis Jülich (2)

Henningsia Möller (5)

Hymenogramme Mont. \& Berk. (1)

Hyphodontiastra Hjortstam (1)

Hypochnicium J. Erikss. (30)

Inflatostereum D.A. Reid (2)

Irpicochaete Rick (1)

Laetifomes T. Hatt. (1)

Macrohyporia I. Johans. \& Ryvarden (2)

Meruliophana Duhem \& Buyck (1)

Mycoleptodonoides Nikol. (4)

Mycorrhaphoides Hembrom, K. Das \& Hallenb. (1)

Nigrohydnum Ryvarden (1)

Phaneroites Hjortstam \& Ryvarden (1)

Phanerodontia Hjortstam \& Ryvarden (4)

Phlebiella P.Karst. (20)

Piptoporellus B.K. Cui, M.L. Han \& Y.C. Dai (3) 
Pseudofibroporia Yuan Y. Chen, B.K. Cui \& Y.C. Dai (1)

Repetobasidiopsis Dhingra \& Avn. P. Singh (1)

Rhodonia Niemelä (1)

Rickiopora Westphalen, Tomšovský \& Rajchenb. (1)

Roseofavolus T. Hatt. (1)

Roseograndinia Hjortstam \& Ryvarden (1)

Ryvardenia Rajchenb. (2)

Sarcoporia P. Karst. (9)

Skeletohydnum Jülich (1)

Sparassiella Schwarzman (1)

Spathulina Pat. (1)

Spongioides Lázaro Ibiza (1)

Spongipellis Pat. (8)

Stegiacantha Maas Geest. (1)

Taiwanofungus Sheng H. Wu, Z.H. Yu, Y.C. Dai \& C.H. Su (2)

Uncobasidium Hjortstam \& Ryvarden (2)

Russulales Kreisel ex P. M. Kirk, P. F. Cannon \& J. C. David

Albatrellaceae Nuss

Albatrellopsis Teixeira (8)

Albatrellus Gray (22)

Byssoporia M.J. Larsen \& Zak (1)

Leucogaster R. Hesse (20)

Leucophleps Harkn. (3)

Mycolevis A.H. Sm. (1)

Polyporoletus Snell (4)

Scutiger Paulet (10)

Auriscalpiaceae Maas Geest.

Amylonotus Ryvarden (6)

Artomyces Jülich (17)

Auriscalpium Gray (8)

Dentipratulum Domański (3)

Lentinellus P. Karst. (30)

Stalpersia Parmasto (1)

Bondarzewiaceae Kotl. \& Pouzar

Amylaria Corner (1)

Amylosporus Ryvarden (12)

Bondarzewia Singer (14)

Gloiodon P. Karst. (3)

Heterobasidion Bref. (15)

Laurilia Pouzar (2)

Lauriliella Nakasone \& S.H. He (2)

Stecchericium D.A. Reid (7)

Wrightoporia Pouzar (32)

\section{Echinodontiaceae Donk}

Echinodontiellum S.H. He \& Nakasone (1)

Echinodontium Ellis \& Everh. (4)

Larssoniporia Y.C. Dai, Jia J. Chen \& B.K. Cui (2) 
Hericiaceae Donk

Dentipellicula Y.C. Dai \& L.W. Zhou (3)

Dentipellis Donk (7)

Hericium Pers. (23)

Laxitextum Lentz (3)

Pseudowrightoporia Y.C. Dai, Jia J. Chen \& B.K. Cui (10)

Wrightoporiopsis Y.C. Dai, Jia J. Chen \& B.K. Cui (5)

Hybogasteraceae Jülich

Hybogaster Singer (1)

Peniophoraceae Lotsy

Amylofungus Sheng H. Wu (2)

Asterostroma Massee (19)

Baltazaria Leal-Dutra, Dentinger \& G.W. Griff. (4)

Dendrophora (Parmasto) Chamuris (3)

Dichostereum Pilát (11)

Duportella Pat. (13)

Entomocorticium H.S. Whitney, Bandoni \& Oberw. (1)

Gloiothele Bres. (12)

Lachnocladium Lév. (40)

Licrostroma P.A. Lemke (1)

Metulodontia Parmasto (1)

Peniophora Cooke (60)

Sceptrulum K.H. Larss. (1)

Scytinostroma Donk (35)

Vararia P. Karst. (50)

Vesiculomyces E. Hagstr. (1)

Russulaceae Lotsy

Boidinia Stalpers \& Hjortstam (11)

Gloeopeniophorella Rick (6)

Lactarius Pers. (450)

Lactifluus (Pers.) Roussel (207)

Multifurca Buyck \& V. Hofst. (10)

Pseudoxenasma K.H. Larss. \& Hjortstam (1)

Russula Pers. (>3000)

\section{Stereaceae Pilát}

Acanthobasidium Oberw. (6)

Acanthofungus Sheng H. Wu, Boidin \& C.Y. Chien (6)

Acanthophysellum Parmasto (14)

Acanthophysium (Pilát) G. Cunn. (20)

Aleurobotrys Boidin (10)

Aleurodiscus Rabenh. ex J. Schröt. (27)

Aleuromyces Boidin \& Gilles (1)

Amylohyphus Ryvarden (1)

Amylosporomyces S. S. Rattan (2)

Confertextum Priyanka \& Dhingra (2)

Conferticium Hallenb. (4)

Dextrinocystidium Sheng H. Wu (2)

Gloeocystidiellum Donk (8) 
Gloeocystidiopsis Jülich (1)

Gloeomyces Sheng H. Wu (3)

Gloeosoma Bres. (1)

Matula Massee (2)

Megalocystidium Jülich (7)

Neoaleurodiscus Sheng H. Wu (2)

Scotoderma Jülich (1)

Stereum Hill ex Pers. (40)

Xylobolus P. Karst. (10)

Xenasmataceae Oberw.

Xenasma Donk (16)

Xenasmatella Oberw. (14)

Xenosperma Oberw. (4)

Russulales genera incertae sedis

Aleurocystidiellum P.A. Lemke (3)

Dentipellopsis Y.C. Dai \& L.W. Zhou (1)

Dichantharellus Corner (2)

Dichopleuropus D. A. Reid (1)

Gloeoasterostroma Rick (1)

Gloeodontia Boidin (8)

Gloeohypochnicium (Parmasto) Hjortstam (2)

Haloaleurodiscus N. Maek., Suhara \& K. Kinjo (1)

Laeticutis Audet (1)

Neoalbatrellus Audet (4)

Perplexostereum Ryvarden \& S. Tutka (1)

Polypus Audet (1)

Scopulodontia Hjortstam (3)

Scytinostromella Parmasto (6)

Xeroceps Audet (2)

Sebacinales M. Weiss, Selosse, Rexer, A. Urb. \& Oberw.

Sebacinaceae K. Wells \& Oberw.

Chaetospermum Sacc. (4)

Ditangium P. Karst. (3)

Efibulobasidium K. Wells (1)

Globulisebacina Oberw., Garnica \& K. Riess (2)

Helvellosebacina Oberw., Garnica \& K. Riess (2)

Paulisebacina Oberw., Garnica \& K. Riess (1)

Sebacina Tul. \& C. Tul. (17)

Tremelloscypha D.A. Reid (4)

Serendipitaceae M. Weiss, Waller, A. Zuccaro \& Selosse Serendipita P. Roberts (11)

Stereopsidales Sjökvist, E. Larss., B.E. Pfeil \& K.H. Larss.

Stereopsidaceae Sjökvist, E. Larss., B.E. Pfeil \& K.H. Larss.

Stereopsis D.A. Reid (15)

Thelephorales Corner ex Oberw.

Bankeraceae Donk 
Bankera Coker \& Beers ex Pouzar (8)

Boletopsis Fayod (10)

Corneroporus T. Hatt. (1)

Hydnellum P. Karst. (39)

Sarcodon Quél. ex P. Karst. (49)

Thelephoraceae Chevall.

Amaurodon J. Schröt (10)

Lenzitopsis Malençon \& Bertault (2)

Phellodon P. Karst. (18)

Polyozellus Murrill (1)

Pseudotomentella Svrček (17)

Skepperia Berk. (5)

Thelephora Ehrh. ex Willd. (50)

Tomentella Pers. ex Pat. (100)

Tomentellopsis Hjortstam (8)

Thelephorales genus incertae sedis

Thelephorella P. Karst. (1)

Trechisporales K.H. Larss.

Hydnodontaceae Jülich

Brevicellicium K. H. Larss. \& Hjortstam (13)

Dextrinocystis Gilb. \& M. Blackw. (2)

Dextrinodontia Hjortstam \& Ryvarden (1)

Hydnodon Banker (1)

Litschauerella Oberw. (3)

Luellia K.H. Larss. \& Hjortstam (3)

Porpomyces Jülich (1)

Scytinopogon Singer (5)

Sistotremastrum J. Erikss. (6)

Sphaerobasidium Oberw. (3)

Subulicystidium Parmasto (20)

Trechispora P. Karst. (48)

Tubulicium Oberw. (7)

Tremellodendropsidales Vizzini

Tremellodendropsidaceae Jülich

Tremellodendropsis (Corner) D.A. Crawford (8)

Agaricomycetes genera incertae sedis

Akenomyces G. Arnaud ex D. Hornby (1)

Aldridgea Massee (1)

Arthrodochium R.F. Castañeda \& W.B. Kendr. (1)

Arualis Katz (1)

Blasiphalia Redhead (1)

Bridgeoporus T.J. Volk, Burds. \& Ammirati (2)

Cenangiomyces Dyko \& B. Sutton (1)

Ceraceopsis Hjortstam \& Ryvarden (1)

Cilicia Fr. (2)

Corticomyces A.I. Romero \& S. E. López (1)

Cruciger R. Kirschner \& Oberw. (1) 
Dendrosporomyces Nawawi, J. Webster \& R.A. Davey (1)

Ellula Nag Raj (1)

Fibulocoela Nag Raj (1)

Fibulotaeniella Marvanová \& Bärl. (1)

Geotrichopsis Tzean \& Estey (1)

Gloeosynnema Seifert \& G. Okada (2)

Glomerulomyces A.I. Romero \& S.E. López (1)

Glutinoagger Sivan. \& Watling (1)

Hallenbergia Dhingra \& Priyanka (1)

Heteroacanthella Oberw. (3)

Intextomyces J. Erikss. \& Ryvarden (4)

Korupella Hjortstam \& P. Roberts (1)

Loreleia Redhead, Moncalvo, Vilgalys \& Lutzoni (3)

Minostroscyta Hjortstam \& Ryvarden (1)

Mylittopsis Pat. (1)

Myriococcum Fr. (1)

Odonticium Parmasto (7)

Pagidospora Drechsler (1)

Phlyctibasidium Jülich (1)

Purpureocorticium S.H. Wu (1)

Pycnovellomyces R.F. Castañeda (1)

Riessia Fresen. (5)

Riessiella Jülich (2)

Taiwanoporia T.T. Chang \& W.N. Chou (1)

Titaeella G. Arnaud ex K. Ando \& Tubaki (1)

Trechinothus E.C. Martini \& Trichiès (1)

Trimitiella Dhingra (1)

Tubulicrinopsis Hjortstam \& Kotir. (4)

Xerotus Fr. (4)

\section{Bartheletiomycetes Thines}

Bartheletiales Thines

Bartheletiaceae R. Bauer, Scheuer, M. Lutz \& Grube

Bartheletia G. Arnaud ex Scheuer, R. Bauer, M. Lutz, Stabenth., Melnik \& Grube (1)

Dacrymycetes Doweld

Dacrymycetales Henn.

Cerinomycetaceae Jülich

Cerinomyces G. W. Martin (13)

Dacrymycetaceae J. Schröt.

Calocera (Fr.) Fr. (18)

Cerinosterus R.T. Moore (1)

Dacrymyces Nees (50)

Dacryonaema Nannf. (1)

Dacryopinax G.W. Martin (24)

Dacryoscyphus R. Kirschner \& Zhu L. Yang (1)

Ditiola Fr. (10)

Femsjonia Fr. (7)

Guepiniopsis Pat. (8)

Heterotextus Lloyd (6) 
Unilacrymales Shirouzu, Tokum. \& Oberw.

Unilacrymaceae Shirouzu, Tokum. \& Oberw.

Unilacryma Shirouzu, Tokum. \& Oberw. (1)

Tremellomycetes Doweld

Cystofilobasidiales Fell, Roeijmans \& Boekhout

Cystofilobasidiaceae K. Wells \& Bandoni

Cystofilobasidium Oberw. \& Bandoni (8)

Mrakiaceae X.Z. Liu, F.Y. Bai, M. Groenew. \& Boekhout

Itersonilia Derx (3)

Krasilnikovozyma X.Z. Liu, F.Y. Bai, M. Groenew. \& Boekhout (3)

Mrakia Y. Yamada \& Komag. (12)

Phaffia M.W. Mill., Yoney. \& Soneda (1)

Tausonia Babeva (3)

Udeniomyces Nakase \& Takem. (4)

Vustinia Kachalkin, Turchetti \& Yurkov (1)

Filobasidiales Jülich

Filobasidiaceae L.S. Olive

Filobasidium L.S. Olive (9)

Goffeauzyma X.Z. Liu, F.Y. Bai, M. Groenew. \& Boekhout (6)

Heterocephalacria Berthier (8)

Naganishia S. Goto (8)

Syzygospora G.W. Martin (2)

Piskurozymaceae X.Z. Liu, F.Y. Bai, M. Groenew. \& Boekhout

Piskurozyma X.Z. Liu, F.Y. Bai, M. Groenew. \& Boekhout (12)

Solicoccozyma X.Z. Liu, F.Y. Bai, M. Groenew. \& Boekhout (7)

Holtermanniales Libkind, Wuczk., Turchetti \& Boekhout

Holtermanniaceae Redhead

Holtermannia Sacc. \& Traverso (8)

Holtermanniella Libkind, Wuczk., Turchetti \& Boekhout (5)

Tremellales Fr.

Bulleraceae X. Zh. Liu, F.Y. Bai, M. Groenew. \& Boekhout

Bullera Derx (4)

Fonsecazyma X.Z. Liu, F.Y. Bai, M. Groenew. \& Boekhout (3)

Genolevuria X.Z. Liu, F.Y. Bai, M. Groenew. \& Boekhout (4)

Pseudotremella X.Z. Liu, F.Y. Bai, A.M. Yurkov, M. Groenew. \& Boekhout (4)

Bulleribasidiaceae X. Z. Liu, F.Y. Bai, M. Groenew. \& Boekhout

Bulleribasidium J.P. Samp., M. Weiss \& R. Bauer (11)

Derxomyces F.Y. Bai \& Q.M. Wang (24)

Dioszegia Zsolt (18)

Hannaella F.Y. Bai \& Q.M. Wang (11)

Nielozyma X.Z. Liu, F.Y. Bai, M. Groenew. \& Boekhout (2)

Vishniacozyma X.Z. Liu, F.Y. Bai, M. Groenew. \& Boekhout (11)

Carcinomycetaceae Oberw. \& Bandoni

Carcinomyces Oberw. \& Bandoni (3) 
Cryptococcaceae Kütz. ex Castell. \& Chalm.

Cryptococcus Vuill. (12)

Kwoniella Statzell \& Fell (14)

Cuniculitremaceae J.P. Samp., R. Kirschner \& M. Weiss

Fellomyces Y. Yamada \& I. Banno (4)

Kockovaella Nakase, I. Banno \& Y. Yamada (19)

Sterigmatosporidium G. Kraep. \& U. Schulze (1)

Naemateliaceae X. Z. Liu, F. Y. Bai, M. Groenew. \& Boekhout

Dimennazyma X. Z. Liu, F. Y. Bai, M. Groenew. \& Boekhout (1)

Naematelia Fr. (4)

Phaeotremellaceae A.M. Yurkov \& Boekhout

Gelidatrema A.M. Yurkov, X.Z. Liu, F.Y. Bai, M. Groenew. \& Boekhout (1)

Phaeotremella Rea (11)

Phragmoxenidiaceae Oberw. \& R. Bauer

Phragmoxenidium Oberw. (1)

Rhynchogastremaceae Oberw. \& B. Metzler

Papiliotrema J.P. Samp., M. Weiss \& R. Bauer (30)

Rhynchogastrema B. Metzler \& Oberw. (9)

Tetragoniomyces Oberw. \& Bandoni (1)

Sirobasidiaceae Lindau

Fibulobasidium Bandoni (3)

Tremellaceae Fr.

Hormomyces Bonord. (6)

Mycocryptococcus Pollacci \& Nann. (1)

Tremella Pers. (>500)

Trimorphomycetaceae X. Z. Liu, F.Y. Bai, M. Groenew. \& Boekhout Carlosrosaea A.M. Yurkov, X.Z. Liu, F.Y. Bai, M. Groenew. \& Boekhout (3) Saitozyma X.Z. Liu, F.Y. Bai, M. Groenew. \& Boekhout (4)

Sugitazyma A.M. Yurkov, X.Z. Liu, F.Y. Bai, M. Groenew. \& Boekhout (1) Trimorphomyces Bandoni \& Oberw. (2)

Tremellales genera incertae sedis

Biatoropsis Räsänen (4)

Dictyotremella Kobayasi (1)

Neotremella Lowy (1)

Sigmogloea Bandoni \& J.C. Krug (1)

Sirobasidium Lagerh. \& Pat. (8)

Sirotrema Bandoni (3)

Tremellina Bandoni (1)

Xenolachne D.P. Rogers (2)

Trichosporonales Boekhout \& Fell

Tetragoniomycetaceae Oberw. \& Bandoni

Bandonia A.M. Yurkov, X.Z. Liu, F.Y. Bai, M. Groenew. \& Boekhout (1) 
Cryptotrichosporon Okoli \& Boekhout (5)

Takashimella Q.M. Wang (4)

\section{Trichosporonaceae Nann.}

Apiotrichum Stautz (21)

Cutaneotrichosporon X.Z. Liu, F.Y. Bai, M. Groenew. \& Boekhout (15)

Effuseotrichosporon A.M. Yurkov, X.Z. Liu, F.Y. Bai, M. Groenew. \& Boekhout (1)

Haglerozyma X.Z. Liu, F.Y. Bai, M. Groenew. \& Boekhout (1)

Pascua Takashima, Manabe, Nishimura, Sriswasdi, Ohkuma, Iwasaki \& Sugita (1)

Prillingera Takashima, Manabe, Nishimura, Sriswasdi, Ohkuma, Iwasaki \& Sugita (1)

Trichosporon Behrend (12)

Vanrija R. T. Moore (9)

Tremellomycetes genera incertae sedis

Heteromycophaga P. Roberts (2)

Phyllopta (Fr.) Fr. (1)

Trichosporonoides Haskins \& J.F.T. Spencer (6)

Pucciniomycotina R. Bauer, Begerow, J.P. Samp., M. Weiss \& Oberw.

Agaricostilbomycetes R. Bauer, Begerow, J.P. Samp., M. Weiss \& Oberw.

Agaricostilbales Oberw. \& R. Bauer

Agaricostilbaceae Oberw. \& R. Bauer

Agaricostilbum J.E. Wright (4)

Pseudobensingtonia F.Y. Bai, Q.M. Wang, M. Groenewald \& Boekhout (2)

Sterigmatomyces Fell (5)

Chionosphaeraceae Oberw. \& Bandoni

Ballistosporomyces Nakase, G. Okada \& Sugiy. (4)

Chionosphaera D.E. Cox (6)

Cystobasidiopsis R. Bauer, B. Metzler, Begerow \& Oberw. (3)

Kurtzmanomyces Y. Yamada, Itoh, H. Kawas., I. Banno \& Nakase (4)

Stilbum Tode (10)

Kondoaceae R. Bauer, Begerow, J. P. Samp., M. Weiss \& Oberw.

Bensingtonia Ingold (5)

Kondoa Y. Yamada, Nakagawa \& I. Banno (10)

Ruineniaceae Q.M. Wang, F.Y. Bai, M. Groenew. \& Boekhout

Ruinenia Q.M. Wang, F.Y. Bai, M. Groenew. \& Boekhout (5)

Agaricostilbales genera incertae sedis

Jianyunia Q. M. Wang, F. Y. Bai, M. Groenew. \& Boekhout (1)

Mycogloea L. S. Olive (7)

Atractiellomycetes R. Bauer, Begerow, J. P. Samp., M. Weiss \& Oberw.

Atractiellales Oberw. \& Bandoni

Atractogloeaceae Oberw. \& R. Bauer

Atractogloea Oberw. \& Bandoni (1)

\section{Hoehnelomycetaceae Jülich}

Basidiopycnis Oberw., R. Kirschner, R. Bauer, Begerow \& Arenal (1) 
Proceropycnis M. Villarreal, Arenal, V. Rubio, Begerow, R. Bauer, R. Kirschner \& Oberw. (2)

\section{Phleogenaceae Gäum.}

Atractidochium Oono, Urbina \& Aime (1)

Atractiella Sacc. (7)

Bourdotigloea Aime (9)

Helicogloea Pat. (25)

Hobsonia Berk. ex Massee (2)

Phleogena Link (1)

Saccosoma Spirin (9)

Classiculomycetes R. Bauer, Begerow, J. P. Samp., M. Weiss \& Oberw.

Classiculales R. Bauer, Begerow, Oberw. \& Marvanová

Classiculaceae R. Bauer, Begerow, Oberw. \& Marvanová

Classicula R. Bauer, Begerow, Oberw. \& Marvanová (2)

Jaculispora H. J. Huds. \& Ingold (1)

Cryptomycocolacomycetes R. Bauer, Begerow, J.P. Samp., M. Weiss \& Oberw.

Cryptomycocolacales Oberw. \& R. Bauer

Cryptomycocolacaceae Oberw. \& R. Bauer

Colacosiphon R. Kirschner, R. Bauer \& Oberw. (1)

Cryptomycocolax Oberw. \& R. Bauer (1)

Cystobasidiomycetes R. Bauer, Begerow, J. P. Samp., M. Weiss \& Oberw.

Buckleyzymales R.L. Zhao \& K.D. Hyde

Buckleyzymaceae Q. M. Wang, F.Y. Bai, M. Groenew. \& Boekhout

Buckleyzyma Q. M. Wang, F.Y. Bai, M. Groenew. \& Boekhout (5)

Cystobasidiales R. Bauer, Begerow, J.P. Samp., M. Weiss \& Oberw.

Cystobasidiaceae Gäum.

Cystobasidium (Lagerh.) Neuhoff (20)

Halobasidium Z. Guo, Y.R. Wang, Q.C. Hou, W.C. Li, H. J. Zhao, Z. H. Sun \& Z.D. Zhang

(1)

Occultifur Oberw. (?9)

Erythrobasidiales R. Bauer, Begerow, J.P. Samp., M. Weiss \& Oberw.

Erythrobasidiaceae Denchev

Bannoa Hamam. (4)

Erythrobasidium Hamam, Sugiy. \& Komag. (3)

Erythrobasidiales genera incertae sedis

Cyphobasidium Millanes, Diederich \& Wedin (2)

Cyrenella Goch. (1)

Hasegawazyma Q.M. Wang, F.Y. Bai, M. Groenew. \& Boekhout (1)

Naohideales R. Bauer, Begerow, J.P. Samp., M. Weiss \& Oberw.

Naohideaceae Denchev

Naohidea Oberw. (1)

Sakaguchiales R.L. Zhao \& K. D. Hyde

Sakaguchiaceae Q.M. Wang, F.Y. Bai, M. Groenew. \& Boekhout 
Sakaguchia Y. Yamada, K. Maeda \& Mikata (5)

Cystobasidiomycetes families incertae sedis

Microsporomycetaceae Q.M. Wang, F. Y. Bai, M. Groenew. \& Boekhout

Microsporomyces Q.M. Wang, F. Y. Bai, M. Groenew. \& Boekhout (4)

Symmetrosporaceae Q.M. Wang, F.Y. Bai, M. Groenew. \& Boekhout

Symmetrospora Q.M. Wang, F.Y. Bai, M. Groenew. \& Boekhout (10)

Cystobasidiomycetes genus incertae sedis

Queiroziella C.R. Félix, J.D.P. Bezerra, R.P. Neves \& Landell (1)

Microbotryomycetes R. Bauer, Begerow, J.P. Samp., M. Weiss \& Oberw.

Heterogastridiales Oberw. \& R. Bauer

Heterogastridiaceae Oberw. \& R. Bauer

Hyalopycnis Höhn. (1)

Krieglsteinera Pouzar (1)

Pycnopulvinus Toome \& Aime (1)

Kriegeriales Toome \& Aime

Camptobasidiaceae R.T. Moore

Camptobasidium Marvanová \& Suberkr. (1)

Glaciozyma Turchetti, Connell, Thomas-Hall \& Boekhout (4)

Kriegeriaceae Toome \& Aime

Kriegeria Bres. (1)

Meredithblackwellia Toome \& Aime (1)

Phenoliferia Q.M. Wang, F.Y. Bai, M. Groenew. \& Boekhout (4)

Yamadamyces Q.M. Wang, F.Y. Bai, M. Groenew. \& Boekhout (1)

Leucosporidiales Sampaio, M. Weiss \& Bauer

Leucosporidiaceae Sampaio, M. Weiss \& Bauer

Leucosporidium Fell, Statzell, I.L. Hunter \& Phaff (11)

Microbotryales R. Bauer \& Oberw.

Microbotryaceae R.T. Moore

Bauerago Vánky (9)

Microbotryum Lév. (100)

Sphacelotheca de Bary (50)

Zundeliomyces Vánky (1)

Ustilentylomataceae R. Bauer \& Oberw.

Aurantiosporium M. Piepenbr., Vánky \& Oberw. (4)

Fulvisporium Vánky (1)

Microbotryozyma S.O. Suh, D.A. Maslov, Molestina \& J.J. Zhou (1)

Ustilentyloma Savile (4)

Sporidiobolales Doweld

Sporidiobolaceae R.T. Moore

Rhodosporidiobolus Q.M. Wang, F.Y. Bai, M. Groenew. \& Boekhout (11)

Rhodotorula F.C. Harrison (15)

Sporobolomyces Kluyver \& C.B. Niel (10) 
Microbotryomycetes families incertae sedis

Chrysozymaceae Q.M. Wang, F.Y. Bai, M. Groenew. \& Boekhout

Bannozyma Q.M. Wang, F.Y. Bai, M. Groenew. \& Boekhout (2)

Chrysozyma Q.M. Wang, F.Y. Bai, M. Groenew. \& Boekhout (2)

Fellozyma Q.M. Wang, F.Y. Bai, M. Groenew. \& Boekhout (1)

Hamamotoa Q.M. Wang, F.Y. Bai, M. Groenew. \& Boekhout (4)

Colacogloeaceae Q.M. Wang, F.Y. Bai, M. Groenew. \& Boekhout

Colacogloea Oberw. \& Bandoni (13)

Microbotryomycetes genera incertae sedis

Atractocolax R. Kirschner, R. Bauer \& Oberw. (1)

Curvibasidium Samp. \& Golubev (3)

Heitmania X.Z. Liu, F.Y. Bai, M. Groenew. \& T. Boekhout (3)

Libkindia Mašínová, A. Pontes, J.P. Samp. \& Baldrian (1)

Oberwinklerozyma Q.M. Wang, F.Y. Bai, M. Groenew. \& Boekhout (3)

Pseudohyphozyma Q.M. Wang, F.Y. Bai, M. Groenew. \& Boekhout (3)

Pseudoleucosporidium V. de Garcia, M.A. Coelho, T. Maia, L.H. Rosa, A.B.M. Vaz, C.A.

Rosa, J.P. Samp., P. Gonç., M.R. Van Broock \& Libkind (1)

Sampaiozyma Q.M. Wang, F.Y. Bai, M. Groenew. \& Boekhout (2)

Slooffia Q.M. Wang, F.Y. Bai, M. Groenew. \& Boekhout (4)

Spencerozyma Q.M. Wang, F.Y. Bai, M. Groenew. \& Boekhout (1)

Trigonosporomyces Q.M. Wang, F.Y. Bai, M. Groenew. \& Boekhout (1)

Udeniozyma Q.M. Wang, F.Y. Bai, M. Groenew. \& Boekhout (1)

Vonarxula Q.M. Wang, F.Y. Bai, M. Groenew. \& Boekhout (1)

Yunzhangia Q.M. Wang, F.Y. Bai, M. Groenew. \& Boekhout (2)

Yurkovia Mašínová, A. Pontes, J.P. Samp. \& Baldrian (1)

Mixiomycetes R. Bauer, Begerow, J.P. Samp., M. Weiss \& Oberw.

Mixiales R. Bauer, Begerow, J.P. Samp., M. Weiss \& Oberw.

Mixiaceae C.L. Kramer

Mixia C.L. Kramer (1)

Pucciniomycetes R. Bauer, Begerow, J.P. Samp., M. Weiss \& Oberw.

Helicobasidiales R. Bauer, Begerow, J.P. Samp., M. Weiss \& Oberw.

Helicobasidiaceae P.M. Kirk

Helicobasidium Pat. (6)

Tuberculina Tode ex Sacc. (26)

Pachnocybales R. Bauer, Begerow, J.P. Samp., M. Weiss \& Oberw.

Pachnocybaceae Oberw. \& R. Bauer

Pachnocybe Berk. (1)

Platygloeales R.T. Moore

Eocronartiaceae Jülich

Eocronartium G.F. Atk. (1)

Herpobasidium Lind (6)

Jola Möller (1)

Platycarpa Couch (2)

Ptechetelium Oberw. \& Bandoni (1) 
Platygloeaceae Racib.

Glomerogloea Doweld (1)

Glomopsis D.M. Hend. (2)

Insolibasidium Oberw. \& Bandoni (1)

Platygloea J. Schröt. (16)

Pucciniales Clem. \& Shear

Chaconiaceae Cummins \& Y. Hirats.

Achrotelium Syd. (5)

Aplopsora Mains (6)

Botryorhiza Whetzel \& Olive (1)

Ceraceopsora Kakish., T. Sato \& S. Sato (1)

Chaconia Juel (12)

Goplana Racib. (13)

Maravalia Arthur (41)

Olivea Arthur (8)

Telomapea G.F. Laundon (1)

\section{Coleosporiaceae Dietel}

Ceropsora B.K. Bakshi \& Suj. Singh (1)

Chrysomyxa Unger (38)

Coleosporium Lév. (125)

Diaphanopellis P.E. Crane (2)

Gallowaya Arthur (3)

\section{Cronartiaceae Dietel}

Cronartium Fr. (34)

Endocronartium Y. Hirats. (2)

Peridermium (Link) J.C. Schmidt \& Kunze (50)

\section{Melampsoraceae Dietel}

Melampsora Castagne (100)

Mikronegeriaceae Cummins \& Y. Hirats.

Blastospora Dietel (5)

Chrysocelis Lagerh. \& Dietel (5)

Mikronegeria Dietel (3)

Phakopsoraceae Cummins \& Hirats. f.

Aeciure Buriticá \& J.F. Hennen (1)

Arthuria H.S. Jacks. (6)

Cerotelium Arthur (27)

Crossopsora Syd. \& P. Syd. (16)

Dasturella Mundk. \& Khesw. (3)

Kweilingia Teng (4)

Macabuna Buriticá \& J.F. Hennen (7)

Monosporidium Barclay (3)

Newinia Thaung (3)

Nothoravenelia Dietel (3)

Phakopsora Dietel (116)

Phragmidiella Henn. (8)

Pucciniostele Tranzschel \& K.L. Kom. (4) 
Scalarispora Buriticá \& J.F. Hennen (1)

Uredopeltis Henn. (7)

\section{Phragmidiaceae Corda}

Arthuriomyces Cummins \& Y. Hirats. (3)

Campanulospora Salazar-Yepes, Pardo-Card. \& Buriticá (1)

Gerwasia Racib. (19)

Gymnoconia Lagerh. (4)

Hamaspora Körn. (15)

Joerstadia Gjaerum \& Cummins (4)

Kuehneola Magnus (22)

Morispora Salazar-Yepes, Pardo-Card. \& Buriticá (1)

Phragmidium Link (100)

Physonema Lév. (1)

Scutelliformis Salazar-Yepes, Pardo-Card. \& Buriticá (1)

Trachyspora Fuckel (5)

Xenodochus Schltdl. (2)

Pileolariaceae Cummins \& Y. Hirats.

Atelocauda Arthur \& Cummins (3)

Pileolaria Castagne (16)

Skierka Racib. (13)

Uromycladium McAlpine (11)

Pucciniaceae Chevall.

Allodus Arthur (1)

Chrysella Syd. (1)

Chrysocyclus Syd. (3)

Chrysopsora Lagerh. (1)

Cleptomyces Arthur (1)

Coleopucciniella Hara ex Hirats. (2)

Corbulopsora Cummins (3)

Cumminsiella Arthur (8)

Cystopsora E.J. Butler (2)

Endophyllum Lév. (43)

Gymnosporangium R. Hedw. ex DC.(64)

Kernella Thirum. (1)

Miyagia Miyabe ex Syd. \& P. Syd. (3)

Polioma Arthur (5)

Puccinia Pers. (3300)

Ramakrishnania Ramachar \& Bhagyan. (1)

Roestelia Rebent. (15)

Stereostratum Magnus (1)

Uromyces (Link) Unger (1500)

Xenostele Syd. \& P. Syd. (4)

Zaghouania Pat. (2)

Pucciniastraceae Gäum. ex Leppik

Hyalopsora Magnus (21)

Melampsorella J. Schröt. (2)

Melampsoridium Kleb. (11)

Milesia F.B. White 1878 (20) 
Milesina Magnus (65)

Naohidemyces S. Sato, Katsuya \& Y. Hirats. (2)

Peridiopsora Kamat \& Sathe (2)

Pucciniastrum G.H. Otth (50)

Thekopsora Magnus (7)

Uredinopsis Magnus (30)

Pucciniosiraceae Cummins \& Y. Hirats.

Alveolaria Lagerh. (2)

Baeodromus Arthur (6)

Ceratocoma Buriticá \& J.F. Hennen (1)

Chardoniella F. Kern (4)

Cionothrix Arthur (5)

Didymopsora Dietel (6)

Dietelia Henn. (13)

Gambleola Massee (1)

Pucciniosira Lagerh. (17)

Trichopsora Lagerh. (1)

Raveneliaceae Leppik

Allotelium Syd. (1)

Anthomyces Dietel (1)

Anthomycetella Syd. \& P. Syd. (1)

Apra J.F. Hennen \& F.O. Freire (1)

Bibulocystis J. Walker, Beilharz, Pascoe \& Priest (3)

Cumminsina Petr. (1)

Cystomyces Syd. (1)

Diabole Arthur (1)

Diabolidium Berndt (1)

Dicheirinia Arthur (14)

Diorchidiella J.C. Lindq. (2)

Diorchidium Kalchbr. (20)

Endoraecium Hodges \& D.E. Gardner (22)

Esalque J.F. Hennen, Figueiredo \& A.A. Carvalho (1)

Hapalophragmium Syd. \& P. Syd. (18)

Kernkampella Rajendren (8)

Lipocystis Cummins (1)

Nyssopsora Arthur (11)

Ravenelia Berk. (250)

Sphenospora Dietel (6)

Spumula Mains (7)

Triphragmiopsis Naumov (3)

Triphragmium Link (7)

Ypsilospora Cummins (3)

Sphaerophragmiaceae Cummins \& Y. Hirats.

Austropuccinia Beenken (1)

Sphaerophragmium Magnus (24)

\section{Uncolaceae Buriticá}

Calidion Syd. \& P. Syd. (4)

Uncol Buriticá \& P.A. Rodr. (1) 
Uropyxidaceae (P. Syd. \& Syd.) Cummins \& Y. Hirats.

Canasta A.A. Carvalho \& J.F. Hennen (3)

Dasyspora Berk. \& M.A. Curtis (13)

Didymopsorella Thirum. (2)

Dipyxis Cummins \& J.W. Baxter (2)

Kimuromyces Dianese, L.T.P. Santos, R.B. Medeiros \& Furlan. (1)

Leucotelium Tranzschel (3)

Macruropyxis Azbukina (2)

Mimema H.S. Jacks. (1)

Ochropsora Dietel (3)

Phragmopyxis Dietel (4)

Poliomopsis A.W. Ramaley (1)

Porotenus Viégas (7)

Prospodium Arthur (84)

Sorataea Syd. (8)

Tranzschelia Arthur (19)

Uropyxis J. Schröt. (15)

Pucciniales genera incertae sedis

Aecidiconium Vuill. (1)

Aecidiolum Unger (12)

Aecidium Pers. (ca. 800)

Caeoma Link (ca. 50)

Caetea Salazar-Yepes \& A.A. Carvalho (1)

Cerradoa J.F. Hennen \& Y. Ono (1)

Coleopuccinia Pat. (1)

Desmella Syd. \& P. Syd. (4)

Desmellopsis J.M. Yen (1)

Desmosorus Ritschel, Oberw. \& Berndt (1)

Edythea H.S. Jacks. (5)

Elateraecium Thirum., F. Kern \& B.V. Patil (3)

Flaminia Sacc. \& P. Syd. (1)

Hemileia Berk. \& Broome (55)

Hennenia Buriticá (1)

Intrapes J.F. Hennen \& Figueiredo (1)

Masseeëlla Dietel (6)

Mehtamyces Mundk. \& Thirum. (1)

Phragmotelium Syd. (10)

Puccorchidium Beenken (2)

Schroeteriaster Magnus (4)

Sphenorchidium Beenken (2)

Uraecium Arthur (12)

Uredo Pers. (600)

Septobasidiales Couch ex Donk

Septobasidiaceae Racib.

Aphelariopsis Jülich (2)

Auriculoscypha D.A. Reid \& Manim. (1)

Coccidiodictyon Oberw. (1)

Johncouchia S. Hughes \& Cavalc. (1)

Septobasidium Pat. (200)

Uredinella Couch (2) 
Spiculogloeomycetes Q.M. Wang, F.Y. Bai, M. Groenew. \& Boekhout Spiculogloeales R. Bauer, Begerow, J.P. Samp., M. Weiss \& Oberw.

Spiculogloeaceae Denchev

Phyllozyma Q.M. Wang, F.Y. Bai, M. Groenew. \& Boekhout (7)

Spiculogloea P. Roberts (5)

Tritirachiomycetes Aime \& Schell

Tritirachiales Aime \& Schell

Tritirachiaceae Aime \& Schell

Tritirachium Limber (4)

Paratritirachium Beguin, Pyck \& Detandt (2)

Pucciniomycotina genera incertae sedis

Kryptastrina Oberw (1)

Paraphelaria Corner (2)

Zygogloea P. Roberts (1)

Ustilaginomycotina Doweld

Exobasidiomycetes Begerow, M. Stoll \& R. Bauer

Ceraceosorales Begerow, M. Stoll \& R. Bauer

Ceraceosoraceae Denchev \& R.T. Moore

Ceraceosorus B.K. Bakshi (3)

Doassansiales R. Bauer \& Oberw.

Doassansiaceae R.T. Moore ex P.M. Kirk, P.F. Cannon \& J.C. David

Burrillia Setch. (4)

Doassansia Cornu (12)

Doassinga Vánky, R. Bauer \& Begerow (1)

Entylomaster Vánky \& R.G. Shivas (2)

Heterodoassansia Vánky (8)

Nannfeldtiomyces Vánky (2)

Narasimhania Thirum. \& Pavgi (1)

Pseudodermatosorus Vánky (2)

Pseudodoassansia (Setch.) Vánky (2)

Pseudotracya Vánky (1)

Tracya Syd. \& P. Syd. (2)

Melaniellaceae R. Bauer, Vánky, Begerow \& Oberw.

Melaniella R. Bauer, Vánky, Begerow \& Oberw. (2)

Rhamphosporaceae R. Bauer \& Oberw.

Rhamphospora D.D. Cunn. (2)

Entylomatales R. Bauer \& Oberw.

Entylomataceae R. Bauer \& Oberw.

Entyloma de Bary (163)

Tilletiopsis Derx (3)

Exobasidiales Henn.

Brachybasidiaceae Gäum.

Brachybasidium Gäum. (1)

Dicellomyces L.S. Olive (4) 
Kordyana Racib. (8)

Meira Boekhout, Scorzetti, Gerson \& Sztejnb. (4)

Proliferobasidium J.L. Cunn. (1)

Cryptobasidiaceae Malençon ex Donk

Acaromyces Boekhout, Scorzetti, Gerson \& Sztejnb. (1)

Botryoconis Syd. \& P.Syd. (2)

Clinoconidium Pat. (6)

Coniodictyum Har. \& Pat. (1)

Drepanoconis J. Schröt. \& Henn. (3)

Phacellula Syd. (1)

Exobasidiaceae J. Schröt.

Arcticomyces Savile (1)

Austrobasidium Palfner (1)

Exobasidium Woronin (51)

Muribasidiospora Kamat \& Rajendren (3)

Graphiolaceae Clem. \& Shear

Graphiola Poit. (12)

Stylina Syd. \& P. Syd. (1)

Laurobasidiaceae Pinruan, Sommai, Suetrong, Somrith. \& E.B.G. Jones Laurobasidium Jülich (2)

Georgefischeriales R. Bauer, Begerow \& Oberw.

Eballistraceae R. Bauer, Begerow, A. Nagler \& Oberw.

Eballistra R. Bauer, Begerow, A. Nagler \& Oberw. (4)

Georgefischeriaceae R. Bauer, Begerow \& Oberw.

Georgefischeria Thirum. \& Naras. (4)

Jamesdicksonia Thirum., Pavgi \& Payak (20)

Gjaerumiaceae R. Bauer, M. Lutz \& Oberw.

Gjaerumia R. Bauer, M. Lutz \& Oberw. (3)

Tilletiariaceae R.T. Moore

Phragmotaenium R. Bauer, Begerow, A. Nagler \& Oberw. (5)

Tilletiaria Bandoni \& B.N. Johri (1)

Tolyposporella G.F. Atk. (6)

Golubeviales Q.M. Wang, Begerow, F.Y. Bai \& Boekhout

Golubeviaceae Q.M. Wang, F.Y. Bai, Begerow \& Boekhout

Golubevia Q.M. Wang, F.Y. Bai, Begerow \& Boekhout (1)

Microstromatales R. Bauer \& Oberw.

Microstromataceae Jülich

Microstroma Niessl (16)

Quambalariaceae Z.W. de Beer, Begerow \& R. Bauer

Quambalaria J.A. Simpson (7) 
Volvocisporiaceae Begerow, R. Bauer \& Oberw.

Volvocisporium Begerow, R. Bauer \& Oberw. (2)

Microstromatales genera incertae sedis

Jaminaea Sipiczki \& Kajdacsi ex T. Kij. \& Aime (4)

Parajaminaea T. Kij. \& Aime (2)

Pseudomicrostroma T. Kij. \& Aime (3)

Sympodiomycopsis Sugiy., Tokuoka \& Komag. (3)

Robbauerales Boekhout, Begerow, Q.M. Wang \& F.Y. Bai

Robbaueraceae Boekhout, Begerow, Q.M. Wang \& F.Y. Bai

Robbauera Boekhout, Begerow, Q.M. Wang \& F.Y. Bai (1)

Tilletiales Kreisel ex R. Bauer \& Oberw.

Erratomycetaceae Denchev \& T. Denchev

Erratomyces M. Piepenbr. \& R. Bauer (5)

Tilletiaceae J. Schröt.

Conidiosporomyces Vánky (3)

Ingoldiomyces Vánky (1)

Neovossia Körn. (1)

Oberwinkleria Vánky \& R. Bauer (1)

Salmacisia D.R. Huff \& A. Chandra (1)

Tilletia Tul. \& C. Tul. (179)

Malasseziomycetes Q.M. Wang \& F.Y. Bai

Malasseziales R.T. Moore

Malasseziaceae Denchev \& R.T. Moore

Malassezia Baillon (18)

Moniliellomycetes Q.M. Wang, F.Y. Bai \& Boekhout

Moniliellales Q.M. Wang, F.Y. Bai \& Boekhout

Moniliellaceae Q.M. Wang, F.Y. Bai \& Boekhout Moniliella Stolk \& Dakin (15)

Ustilaginomycetes R. Bauer, Oberw. \& Vánky

Uleiellales Garnica, K. Riess, M. Schön, H. Butin, M. Lutz, Oberw. \& R. Bauer

Uleiellaceae Vánky Uleiella J. Schröt. (2)

Urocystidales R. Bauer \& Oberw.

Doassansiopsidaceae Begerow, R. Bauer \& Oberw.

Doassansiopsis (Setch.) Dietel (14)

Fereydouniaceae S. Nasr, Soudi, H.D.T. Nguyen, M. Lutz \& Piątek Fereydounia S. Nasr, M.R. Soudi, H.D.T. Nguyen, M. Lutz \& Piątek (1)

Floromycetaceae S. Nasr, Soudi, H.D.T. Nguyen, M. Lutz \& Piątek Antherospora R. Bauer, M. Lutz, Begerow, Piątek \& Vánky (12)

Floromyces Vánky, M. Lutz \& R. Bauer (1)

Glomosporiaceae Cif. 
Thecaphora Fingerh. (61)

Mycosyringaceae $\mathrm{R}$. Bauer \& Oberw.

Mycosyrinx Beck (4)

Urocystidaceae Begerow, R. Bauer \& Oberw.

Flamingomyces R. Bauer, M. Lutz, Piątek, Vánky \& Oberw. (1)

Melanoxa M. Lutz, Vánky \& R. Bauer (2)

Melanustilospora Denchev (2)

Mundkurella Thirum. (5)

Urocystis Rabenh. ex Fuckel (166)

Ustacystis Zundel (2)

Vankya Ershad (3)

Ustilaginales $\mathrm{G}$. Winter

Anthracoideaceae Denchev

Anthracoidea Bref. (112)

Cintractia Cornu (13)

Dermatosorus Sawada ex L. Ling (6)

Farysia Racib. (23)

Farysporium Vánky (1)

Heterotolyposporium Vánky (2)

Kuntzeomyces Henn. Ex Sacc. \& P. Syd. (2)

Leucocintractia M. Piepenbr., Begerow \& Oberw. (4)

Moreaua Liou \& H.C. Cheng (39)

Orphanomyces Savile (3)

Pilocintractia Vánky (2)

Planetella Savile (1)

Portalia V. González, Vánky \& Platas (1)

Schizonella J. Schröt. (5)

Stegocintractia M. Piepenbr., Begerow \& Oberw. (6)

Testicularia Klotzsch (3)

Tolyposporium Woronin ex J. Schröt. (5)

Trichocintractia M. Piepenbr. (1)

Ustanciosporium Vánky (22)

Cintractiellaceae Vánky

Cintractiella Boedijn (2)

Clintamraceae Vánky

Clintamra Cordas \& Durán (1)

Geminaginaceae Vánky

Geminago Vánky \& R. Bauer (1)

Melanotaeniaceae Begerow, R. Bauer \& Oberw.

Exoteliospora R. Bauer, Oberw. \& Vánky (1)

Melanotaenium de Bary (9)

Yelsemia J. Walker (4)

Pericladiaceae Vánky

Pericladium Pass. (3) 
Ustilaginaceae Tul. \& C. Tul.

Anthracocystis Bref. (134)

Macalpinomyces Langdon \& Full. (41)

Moesziomyces Vánky (8)

Sporisorium Ehrenb. ex Link (195)

Tranzscheliella Lavrov (17)

Ustilago (Pers.) Roussel (170)

Websdaneaceae Vánky

Restiosporium Vánky (21)

Websdanea Vánky (1)

Ustilaginales genera incertae sedis

Ahmadiago Vánky (1)

Aizoago Vánky (2)

Anomalomyces Vánky, M. Lutz \& R.G. Shivas (2)

Bambusiomyces Vánky (1)

Centrolepidosporium R.G. Shivas \& Vánky (1)

Dirkmeia F.Y. Bai, Q.M. Wang, Begerow \& Boekhout (1)

Eriocaulago Vánky (2)

Eriocortex Vánky \& R.G. Shivas (1)

Eriosporium Vánky (2)

Franzpetrakia Thirum. \& Pavgi (3)

Kalmanozyma Q.M. Wang, F.Y. Bai, Begerow \& Boekhout (3)

Langdonia McTaggart \& R.G. Shivas (8)

Melanopsichium Beck (2)

Mycosarcoma Bref (5)

Parvulago R. Bauer, M. Lutz, Piątek, Vánky \& Oberw. (1)

Pattersoniomyces Piątek, M. Lutz \& C.A. Rosa (1)

Shivasia Vánky, M. Lutz \& Piątek (1)

Stollia McTaggart \& R.G. Shivas (5)

Triodiomyces McTaggart \& R.G. Shivas (6)

Yunchangia L. Guo \& B. Xu (1)

Violaceomycetales Albu, Toome \& Aime

Violaceomycetaceae Albu, Toome \& Aime

Violaceomyces Albu, Toome \& Aime (1)

Ustilaginomycetes genus incertae sedis

Capitulocladosporium L.Y. Sun, X. Sun \& L.D. Guo (1)

Wallemiomycotina Doweld

Wallemiomycetes Zalar, de Hoog \& Schroers

Geminibasidiales H.D.T. Nguyen, N.L. Nick. \& Seifert

Geminibasidiaceae H.D.T. Nguyen, N.L. Nick. \& Seifert

Basidioascus Matsush. (3)

Geminibasidium H.D.T. Nguyen, N.L. Nick. \& Seifert (2)

Wallemiales Zalar, de Hoog \& Schroers

Wallemiaceae R.T. Moore

Wallemia Johan-Olsen (8) 
Wallemiomycetes genus incertae sedis

Chernovia A.M. Yurkov \& Begerow (1)

Basidiomycota genera incertae sedis

Anastomyces W.P. Wu, B. Sutton \& Gange (1)

Anguillomyces Marvanová \& Bärl. (1)

Arcispora Marvanová \& Bärl. (1)

Arrasia Bernicchia, Gorjón \& Nakasone (1)

Brevicellopsis Hjortstam \& Ryvarden (1)

Celatogloea P. Roberts (1)

Cystogloea P. Roberts (1)

Microstella K. Ando \& Tubaki (1)

Neotyphula Wakef. (1)

Radulodontia Hjortstam \& Ryvarden (1)

Restilago Vánky (1)

Blastocladiomycota T.Y. James

Blastocladiomycetes Doweld

Blastocladiales H.E. Petersen

Blastocladiaceae H.E. Petersen

Allomyces E.J. Butler (13)

Blastocladia Reinsch (31)

Blastocladiopsis Sparrow (2)

Catenariaceae Couch

Catenophlyctis Karling (2)

Nematoceromyces Doweld (3)

Paraphysodermataceae Doweld

Paraphysoderma Boussiba, Zarka \& T.Y. James (1)

Sorochytriaceae Dewel

Sorochytrium Dewel (1)

Blastocladiales genus incertae sedis

Endoblastidium Codreanu (1)

Callimastigales Doweld

Callimastigaceae Fonseca

Callimastix Weissenb. (2)

Catenomycetales Doweld

Catenomycetaceae Doweld

Catenomyces A.M. Hanson (2)

Coelomomycetaceae Couch

Coelomomyces Keilin (66)

Coelomycidium Debais. (2)

Blastocladiomycetes genus incertae sedis

Microallomyces R. Emers. \& J.A. Robertson (1) 
Physodermatomycetes Tedersoo, Sanchez-Ramirez, Kõljalg, Bahram, M. Döring, Schigel, T.W. May, M. Ryberg \& Abarenkov

Physodermatales Caval.-Sm.

Physodermataceae Sparrow

Physoderma Wallr. (99)

Calcarisporiellomycota Tedersoo, Sanchez-Ramirez, Kõljalg, Bahram, M. Döring, Schigel, T.W. May, M. Ryberg \& Abarenkov

Calcarisporiellomycotina Tedersoo, Sanchez-Ramirez, Kõljalg, Bahram, M. Döring, Schigel, T.W. May, M. Ryberg \& Abarenkov

Calcarisporiellomycetes Tedersoo, Sanchez-Ramirez, Kõljalg, Bahram, M. Döring, Schigel, T.W. May, M. Ryberg \& Abarenkov

Calcarisporiellales Tedersoo, Sanchez-Ramirez, Kõljalg, Bahram, M. Döring, Schigel, T.W. May, M. Ryberg \& Abarenkov

Calcarisporiellaceae Tedersoo, Sanchez-Ramirez, Kõljalg, Bahram, M. Döring, Schigel, T.W. May, M. Ryberg \& Abarenkov

Calcarisporiella de Hoog (1)

Echinochlamydosporium X.Z. Jiang, H.Y. Yu, M.C. Xiang, X.Y. Liu \& Xing Z. Liu (1)

Caulochytriomycota Doweld

Caulochytriomycetes Doweld

Caulochytriales Doweld

Caulochytriaceae Subram.

Caulochytrium Voos \& L.S. Olive (2)

Chytridiomycota Doweld

Chytridiomycetes Caval.-Sm.

Chytridiales Cohn

Asterophlyctaceae Doweld

Asterophlyctis H.E. Petersen (2)

Wheelerophlyctis P.M Letcher, M.J. Powell, W.J. Davis (2)*

\section{Chytridiaceae Nowak.}

Chytridium A. Braun (143)

Dendrochytridium Letcher, Longcore \& M.J. Powell (1)

Dinochytrium Lesham, Letcher \& M.J. Powell (1)

Irineochytrium Letcher, Longcore \& M.J. Powell (1)

Polyphlyctis Karling (3)

Zopfochytrium M.J. Powell, Longcore, Letcher (1)*

\section{Chytriomycetaceae Letcher}

Avachytrium Vélez \& Letcher (1)

Chytriomyces Karling (33)

Entophlyctis A. Fisch. (29)

Fayochytriomyces W.J. Davis, Letcher, Longcore \& M.J. Powell (1)

Obelidium Nowak. (3)

Odontochytrium Vélez \& Letcher (1)

Pendulichytrium K. Seto \& Degawa (1)

Physocladia Sparrow (1)

Podochytrium Pfitzer (7)

Rhizoclosmatium H.E. Petersen (4)

Siphonaria H.E. Petersen (3) 
Phlyctochytriaceae Doweld

Phlyctochytrium J. Schröt. (73)

Phlyctorhizaceae Doweld

Phlyctorhiza A.M. Hanson (3)

Pseudorhizidiaceae Doweld

Pseudorhizidium M.J. Powell, Letcher \& Longcore (1)

Scherffeliomycetaceae Doweld

Scherffeliomyces Sparrow (4)

Zygorhizidiaceae Doweld

Zygorhizidium Löwenthal (12)

Chytridiales genus incertae sedis

Delfinachytrium Vélez \& Letcher (1)

Nephridiophagales Doweld

Nephridiophagaceae R. Radek, Letcher, Wijayaw., P.M. Kirk \& K.D. Hyde

Coleospora Gibbs (1)

Nephridiophaga Ivanić (12)

Oryctospora Purrini \& Weiser (1)

Peltomyces Léger (1)

Polyphagales Doweld

Polyphagaceae F. Maekawa

Polyphagus Nowak. (15)

Saccopodiales Doweld

Saccopodiaceae Jacz. \& P.A. Jacz.

Saccopodium Sorokīn (1)

Chytridiomycetes families incertae sedis

Amoebochytriaceae Doweld

Amoebochytrium Zopf (1)

Sparrowiaceae Doweld

Sparrowia Willoughby (2)

Sphaeromonadaceae Doweld

Sphaeromonas E. Liebet. (6)

Tetrachytriaceae Doweld

Tetrachytrium Sorokīn (1)

\section{Thalassochytriaceae Doweld}

Thalassochytrium Nyvall, M. Pedersén \& Longcore (1)

Chytridiomycetes genera incertae sedis

Aphanistis Sorokīn (2)

Bertramia Mesnil \& Caullery (3) 
Blyttiomyces A.F. Bartsch (11)

Canteria Karling (1)

Dangeardia Schröd. (11)

Dangeardiana Valkanov ex A. Batko (4)

Dictyomorpha Mullins (2)

Gamolpidium Vlădescu (2)

Ichthyochytrium Plehn (1)

Loborhiza A.M. Hanson (1)

Macrochytrium Minden (1)

Megachytrium Sparrow (1)

Mitochytridium P.A. Dang. (2)

Mucophilus Plehn (1)

Nowakowskia Borzí (1)

Olpidiaster Pascher (4)

Perolpidium Doweld (2)

Physorhizophidium Scherff. (1)

Plasmophagus De Wild. (3)

Pseudopileum Canter (1)

Rhizidiocystis Sideris (1)

Rhizosiphon Scherff. (4)

Rhopalophlyctis Karling (1)

Riethophlyctis Doweld (1)

Saccomyces Serbinow (2)

Sagittospora Lubinsky (1)

Scherffeliomycopsis Geitler (1)

Schizolpidium Doweld (1)

Septolpidium Sparrow (1)

Septosperma Whiffen ex R.L. Seym. (5)

Solutoparies Whiffen ex W.H. Blackw. \& M.J. Powell (1)

Sorokinocystis Doweld (1)

Sporophlyctidium Sparrow (2)

Sporophlyctis Serbinow (2)

Trematophlyctis Pat. (1)

Truittella Karling (1)

Volvorax Doweld (1)

Zygochytrium Sorokīn (1)

Zygophlyctis Doweld (1)

Cladochytriomycetes Tedersoo, Sanchez-Ramirez, Kõljalg, Bahram, M. Döring, Schigel, T.W. May, M. Ryberg \& Abarenkov

Cladochytriales Mozl.-Standr.

Catenochytridiaceae Doweld

Catenochytridium Berdan (6)

Cladochytriaceae J. Schröt.

Cladochytrium Nowak. (51)

Endochytriaceae Sparrow ex D.J.S. Barr

Diplophlyctis J. Schröt. (12)

Endochytrium Sparrow (7)

Nowakowskiellaceae Sparrow ex Mozl.-Standr. 
Nowakowskiella J. Schröt. (18)

Septochytriaceae Mozl.-Standr.

Septochytrium Berdan (5)

Cladochytriales genera incertae sedis

Allochytridium D.J.S. Barr \& Désauln. (2)

Cylindrochytridium Karling (2)

Nephrochytrium Karling (8)

Lobulomycetes Tedersoo, Sanchez-Ramirez, Kõljalg, Bahram, M. Döring, Schigel, T.W. May, M. Ryberg \& Abarenkov

Lobulomycetales D.R. Simmons

Alogomycetaceae Doweld

Alogomyces D.R. Simmons \& Letcher (1)

Lobulomycetaceae D.R. Simmons

Clydaea D.R. Simmons (1)

Cyclopsomyces K. Seto \& Degawa (1)

Lobulomyces D.R. Simmons (2)

Maunachytrium D.R. Simmons (1)

Lobulomycetales genus incertae sedis

Algochytrops Doweld (1)

Mesochytriomycetes Tedersoo, Sanchez-Ramirez, Kõljalg, Bahram, M. Döring, Schigel, T.W. May, M. Ryberg \& Abarenkov

Gromochytriales Karpov \& Aleoshin

Gromochytriaceae Karpov \& Aleoshin

Gromochytrium Karpov \& Aleoshin (1)

Mesochytriales Doweld

Mesochytriaceae Doweld

Mesochytrium B.V. Gromov, Mamkaeva \& Pljusch (1)

Polychytriomycetes Tedersoo, Sanchez-Ramirez, Kõljalg, Bahram, M. Döring, Schigel, T.W. May, M. Ryberg \& Abarenkov

Polychytriales Longcore \& D.R. Simmons

Arkayaceae Doweld

Arkaya Longcore \& D.R. Simmons (2)

Polychytriaceae Doweld

Karlingiomyces Sparrow (8)

Lacustromyces Longcore (1)

Neokarlingia Longcore \& D.R. Simmons (1)

Polychytrium Ajello (1)

Rhizophydiomycetes Tedersoo, Sanchez-Ramirez, Kõljalg, Bahram, M. Döring, Schigel, T.W. May, M. Ryberg \& Abarenkov

Rhizophydiales Letcher

Alphamycetaceae Letcher

Alphamyces Letcher (1) 
Betamyces Letcher (1)

Gammamyces Letcher (1)

Angulomycetaceae Letcher

Angulomyces Letcher (1)

Aquamycetaceae Letcher

Aquamyces Letcher (1)

Batrachochytriaceae Doweld

Batrachochytrium Longcore, Pessier \& D.K. Nichols (2)

Collimycetaceae K. Seto \& Degawa

Collimyces K. Seto \& Degawa (1)

Coralloidiomycetaceae Doweld

Coralloidiomyces Letcher (1)

Dinomycetaceae Karpov \& Guillou

Dinomyces Karpov \& Guillou (1)

Globomycetaceae Letcher

Globomyces Letcher (1)

Urceomyces Letcher (1)

Gorgonomycetaceae Letcher

Gorgonomyces Letcher (1)

Halomycetaceae Letcher \& M.J. Powell

Halomyces Letcher \& M.J. Powell (1)

Paludomyces Letcher \& M.J. Powell (1)

Paranamyces Letcher \& M.J. Powell (1)

Ulkenomyces Letcher \& M.J. Powell (1)

Kappamycetaceae Letcher

Kappamyces Letcher \& M.J. Powell (1)

Operculomycetaceae Doweld

Operculomyces M.J. Powell, Letcher \& Longcore (1)

Pateramycetaceae Letcher

Pateramyces Letcher (1)

Protrudomycetaceae Letcher

Protrudomyces Letcher (1)

Rhizophydiaceae Letcher

Rhizophydium Schenk ex Rabenh. (218)

Staurastromycetaceae S. Van den Wyngaert, K. Seto \& K. Rojas

Staurastromyces Van den Wyngaert, K. Seto \& K. Rojas (1) 
Terramycetaceae Letcher

Boothiomyces Letcher (1)

Terramyces Letcher (1)

Uebelmesseromycetaceae M.J. Powell \& Letcher

Uebelmesseromyces M.J. Powell \& Letcher (1)

Rhizophydiales genus incertae sedis

Homolaphlyctis Longcore, Letcher \& T.Y. James (1)

Rhizophlyctidomycetes Tedersoo, Sanchez-Ramirez, Kõljalg, Bahram, M. Döring, Schigel, T.W. May, M. Ryberg \& Abarenkov

Rhizophlyctidales Letcher

Arizonaphlyctidaceae Letcher

Arizonaphlyctis Letcher (1)

Borealophlyctidaceae Letcher

Borealophlyctis Letcher (2)

Rhizophlyctidaceae H.E. Petersen

Rhizophlyctis A. Fisch. (31)

Sonoraphlyctidaceae Letcher

Sonoraphlyctis Letcher (1)

Spizellomycetes Tedersoo, Sanchez-Ramirez, Kõljalg, Bahram, M. Döring, Schigel, T.W. May, M. Ryberg \& Abarenkov

Spizellomycetales D.J.S. Barr

Powellomycetaceae D.R. Simmons

Fimicolochytrium D.R. Simmons \& Longcore (2)

Geranomyces D.R. Simmons (4)

Powellomyces Longcore (2)

Thoreauomyces D.R. Simmons \& Longcore (1)

Spizellomycetaceae D.J.S. Barr

Barromyces M.J. Powell \& Letcher (1)

Brevicalcar Letcher \& M.J. Powell (1)

Bulbosomyces Letcher \& Longcore (1)

Gaertneriomyces D.J.S. Barr (4)

Gallinipes Letcher \& M.J. Powell (3)

Kochiomyces D.J.S. Barr (1)

Spizellomyces D.J.S. Barr (8)

Triparticalcar D.J.S. Barr (2)

Synchytriomycetes Tedersoo, Sanchez-Ramirez, Kõljalg, Bahram, M. Döring, Schigel, T.W. May,

M. Ryberg \& Abarenkov

Synchytriales Doweld

Synchytriaceae J. Schröt.

Carpenterophlyctis Doweld (2)

Endodesmidium Canter (1)

Johnkarlingia Pavgi \& S.L. Singh (1)

Synchytrium de Bary \& Woronin (255) 
Synchytriales genus incertae sedis

Micromyces P.A. Dang. (19)

Chytridiomycota genera incertae sedis

Achlyella Lagerh. (1)

Coenomyces K.N. Deckenb. (1)

Achlyogeton Schenk (4)

Entomophthoromycota Humber

Entomophthoromycotina Humber

Entomophthoromycetes Humber

Entomophthorales G. Winter

Ancylistaceae J. Schröt.

Ancylistes Pfitzer (6)

Conidiobolus Bref. (54)

Macrobiotophthora Reukauf (2)

Completoriaceae Humber

Completoria Lohde (1)

Entomophthoraceae Nowak.

Batkoa Humber (10)

Entomophaga A. Batko (22)

Entomophthora Fresen. (63)

Erynia (Nowak. ex A. Batko) Remaud. \& Hennebert (27)

Eryniopsis Humber (5)

Furia (A. Batko) Humber (16)

Massospora Peck (15)

Orthomyces Steinkr., Humber \& J.B. Oliv. (1)

Strongwellsea A. Batko \& J. Weiser (3)

Tarichium Cohn sensu stricto (26)

Zoophthora A. Batko (38)

Meristacraceae Humber

Meristacrum Drechsler (= Tabanomyces Couch, R.J. Andrejeva, Laird \& Nolan) (2)

Neozygitomycetes Humber

Neozygitales Humber

Neozygitaceae Ben Ze'ev, R.G. Kenneth \& Uziel

Apterivorax S. Keller (2)

Neozygites Witlaczil (22)

Tarichium Cohn pro parte (27)

Thaxterosporium Ben Ze'ev \& R.G. Kenneth (1)

Entorrhizomycota R. Bauer, Garnica, Oberw., Riess, Weiß \& Begerow

Entorrhizomycetes Begerow, M. Stoll \& R. Bauer

Entorrhizales R. Bauer \& Oberw.

Entorrhizaceae R. Bauer \& Oberw.

Entorrhiza C.A. Weber (c.15)

Talbotiomycetales K. Riess, R. Bauer, R. Kellner, Kemler, Piątek, Vánky \& Begerow 
Talbotiomycetaceae K. Riess, R. Bauer, R. Kellner, Kemler, Piątek, Vánky \& Begerow Talbotiomyces Vánky, R. Bauer \& Begerow (1)

Glomeromycota C. Walker \& A. Schüssler

Archaeosporomycetes Sieverd., G.A. Silva, B.T. Goto \& Oehl

Archaeosporales C. Walker \& A. Schüssler

Ambisporaceae C. Walker, Vestberg \& A. Schüssler (= Appendicisporaceae C. Walker, Vestberg \& A. Schüssler)

Ambispora C. Walker, Vestberg \& A. Schüssler (basionym Appendicispora Spain, Oehl \& Sieverding) (11)

Archaeosporaceae J.B. Morton \& D. Redecker

Archaeospora J.B. Morton \& D. Redecker (6)

Intraspora Oehl \& Sieverd. (1)

Palaeospora Oehl, Palenz., Sánchez-Castro \& G.A. Silva (1)

Geosiphonaceae Engl. \& E. Gilg

Geosiphon F. Wettst. (1)

Glomeromycetes Caval.-Sm. emend. Oehl, G.A. Silva, B.T. Goto \& Sieverd.

Diversisporales C. Walker \& A. Schüssler emend. Oehl, G.A. Silva \& Sieverd.

Acaulosporaceae J.B. Morton \& Benny

Acaulospora Gerd. \& Trappe (= Kuklospora Oehl \& Sieverd.) (57)

Diversisporaceae C. Walker \& A. Schüssler

Corymbiglomus Błaszk. \& Chwat (3)

Desertispora Błaszk., Kozłowska, Ryszka, Al-Yahya’ei \& Symanczik (1)

Diversispora C. Walker \& A. Schüssler (21)

Otospora Oehl, Palenz. \& N. Ferrol (1)

Redeckera C. Walker \& A. Schüssler (6)

Sieverdingia Błaszk., Niezgoda \& B.T. Goto (1)

Tricispora Oehl, Sieverd., G.A. Silva \& Palenz. (1)

Pacisporaceae C. Walker, Błaszk., A. Schüssler \& Schwarzott Pacispora Sieverd. \& Oehl (7)

Sacculosporaceae Oehl, Sieverd., G.A. Silva, B.T. Goto, Sánchez-Castro \& Palenz.

Sacculospora Oehl, Sieverd., G.A. Silva, B.T. Goto, I.C. Sánchez \& Palenz. (2)

Gigasporales S.P. Gautam \& U.S. Patel (= Gigasporales Sieverd., G.A. Silva, B.T. Goto \& Oehl)

Dentiscutataceae F.A. Souza, Oehl \& Sieverd.

Dentiscutata Sieverd., F.A. Souza \& Oehl (9)

Fuscutata Oehl, F.A. Souza \& Sieverd. (5)

Quatunica F.A. Souza, Sieverd. \& Oehl (1)

Gigasporaceae J.B. Morton \& Benny

Gigaspora Gerd. \& Trappe (7)

Intraornatosporaceae B.T. Goto \& Oehl

Intraornatospora B.T. Goto, Oehl \& G.A. Silva (1)

Paradentiscutata B.T. Goto, Oehl \& G.A. Silva (2) 
Racocetraceae Oehl, Sieverd. \& F.A. Souza

Cetraspora Oehl, F. A. Souza \& Sieverd. (8)

Racocetra Oehl, F.A. Souza \& Sieverd. (13)

Scutellosporaceae Sieverd., F.A. Souza \& Oehl

Bulbospora Oehl\& G.A. Silva (1)

Orbispora Oehl, G.A. Silva \& D.K. Silva (2)

Scutellospora C. Walker \& F.E. Sanders (10)

Glomerales J.B. Morton \& Benny emend. Oehl, G.A. Silva, B.T. Goto \& Sieverd.

Entrophosporaceae Oehl \& Sieverd.

Albahypha Oehl, G.A. Silva, B.T. Goto \& Sieverd. (2)

Claroideoglomus C. Walker \& A. Schüssler (6)

Entrophospora R.N. Ames \& R.W. Schneid. (2)*

Glomeraceae Piroz. \&Dalpé emend. Oehl, G.A. Silva \& Sieverd.

Dominikia Błaszk., Chwat \&Kovács (11)

Funneliglomus Corazon-Guivin, G.A. Silva \& Oehl (1)

Funneliformis C. Walker \& A. Schüssler emend. Oehl, G.A. Silva \& Sieverd. (11)

Glomus Tul. \& C. Tul. emend. Oehl, G.A. Silva \& Sieverd. (49)

Halonatospora Błaszk., Niezgoda, B.T. Goto \& Kozłowska (1)

Kamienskia Błaszk., Chwat \& Kovács (1)

Microdominikia Oehl, Corazon-Guivin \& G.A. Silva (1)

Microkamienskia Corazon-Guivin, G.A. Silva \& Oehl (3)*

Nanoglomus Corazon-Guivin, G.A. Silva \& Oehl (1)

Oehlia Błaszk., Kozłowska, Niezgoda, B.T. Goto \& Dalpé (1)

Orientoglomus G.A. Silva, Oehl \& Corazon-Guivin (1)

Rhizoglomus Sieverd., G.A. Silva \& Oehl (22)*

Sclerocystis Berk. \& Broome (8)

Sclerocarpum B.T. Goto, Błaszk., Niezgoda, Kozłowska \& Jobim (1)

Septoglomus Sieverd., G.A. Silva \& Oehl (13)

Simiglomus Sieverd., G.A. Silva \& Oehl (1)

Viscospora Sieverd. Oehl \& G.A. Silva (1)

Paraglomeromycetes Oehl, G.A. Silva, B.T. Goto \& Sieverd.

Paraglomerales C. Walker \& A. Schüssler

Paraglomeraceae J.B. Morton \& D. Redecker

Paraglomus J.B. Morton \& D. Redecker (8)

Innospora Błaszk., Kovács, Chwat \& Kozłowska (1)

Pervetustaceae Błaszk., Chwat, Kozłowska, Symanczik \& Al-Yahya’ei

Pervetustus Błaszk., Chwat, Kozłowska, Symanczik \& Al-Yahya'ei (1)

Kickxellomycota Tedersoo, Sanchez-Ramirez, Kõljalg, Bahram, M. Döring, Schigel, T.W. May, M. Ryberg \& Abarenkov

Asellariomycetes Tedersoo, Sanchez-Ramirez, Kõljalg, Bahram, M. Döring, Schigel, T.W. May, M. Ryberg \& Abarenkov

Asellariales Manier ex Manier \& Lichtw.

Asellariaceae Manier ex Manier \& Lichtw.

Asellaria R.A. Poiss. (9)

Asellariales genus incertae sedis 
Barbatosporomycetes Tedersoo, Sanchez-Ramirez, Kõljalg, Bahram, M. Döring, Schigel, T.W. May, M. Ryberg \& Abarenkov

Barbatosporales Doweld

Barbatosporaceae Doweld

Barbatospora M.M. White, Siri \& Lichtw. (1)

Dimargaritomycetes Tedersoo, Sanchez-Ramirez, Kõljalg, Bahram, M. Döring, Schigel, T.W. May, M. Ryberg \& Abarenkov

Dimargaritales R.K. Benj.

Dimargaritaceae R.K. Benj.

Dimargaris Tiegh. (7)

Dispira Tiegh. (4)

Tieghemiomyces R.K. Benj. (2)

Dimargaritales genus incertae sedis

Spinalia Vuill. (1)

Harpellomycetes Tedersoo, Sanchez-Ramirez, Kõljalg, Bahram, M. Döring, Schigel, T.W. May, M. Ryberg \& Abarenkov

Harpellales Lichtw. \& Manier

Harpellaceae L. Léger \& Duboscq ex P.M. Kirk \& P.F. Cannon

Carouxella Manier, Rioux \& Whisler (2)

Harpella L. Léger \& Duboscq (7)

Harpellomyces Lichtw. \& S.T. Moss (4)

Klastostachys Lichtw., M.C. Williams \& M.M. White (1)

Stachylina L. Léger \& M. Gauthier (40)

Stachylinoides Lichtw. \& López-Lastra (1)

Legeriomycetaceae Pouzar

Austrosmittium Lichtw. \& M.C. Williams (5)

Bactromyces William \& Strongman (1)

Baetimyces L.G. Valle \& Santam. (1)

Bojamyces Longcore (3)

Capniomyces S.W. Peterson \& Lichtw. (3)

Caudomyces Lichtw., Kobayasi \& Indoh (3)

Coleopteromyces Ferrington, Lichtw. \& López-Lastra (1)

Dacryodiomyces Lichtw. (1)

Ejectosporus S.W. Peterson, Lichtw. \& M.C. Williams (1)

Ephemerellomyces M.M. White \& Lichtw. (1)

Furculomyces Lichtw. \& M.C. Williams (3)

Gauthieromyces Lichtw. (3)

Genistelloides S.W. Peterson, Lichtw. \& B.W. Horn (5)

Genistellospora Lichtw. (6)

Glotzia M. Gauthier ex Manier \& Lichtw. (7)

Graminella L. Léger \& M. Gauthier ex Manier (3)

Laculus William \& Strongman (1)

Lancisporomyces Santam. (5)

Legerioides M.M. White (1)

Legeriomyces Pouzar (11)

Legeriosimilis M.C. Williams, Lichtw., M.M. White \& J.K. Misra (8) 
Orphella L. Léger \& M. Gauthier (12)

Pennella Manier (8)

Plecopteromyces Lichtw., Ferrington \& López-Lastra (3)

Pseudoharpella Ferrington, M.M. White \& Lichtw. (1)

Pteromaktron Whisler (2)

Simuliomyces Lichtw. (1)

Sinotrichium Juan Wang (1)

Smittium R.A. Poiss. (1)

Spartiella Tuzet \& Manier ex Manier (3)

Stipella L. Léger \& M. Gauthier (2)

Stypomyces Doweld (2)

Tectimyces L.G. Valle \& Santam. (3)

Trichozygospora Lichtw. (1)

Trifoliellum Strongman \& M.M. White (1)

Zancudomyces Yan Wang, Tretter, Lichtw. \& M.M. White (1)

Zygopolaris S.T. Moss, Lichtw. \& Manier (2)

Zygopolaropsis Hirok. Sato \& Degawa (1)

Harpellales genus incertae sedis

Trissocladomyces Doweld (1)

Kickxellomycetes Tedersoo, Sanchez-Ramirez, Kõljalg, Bahram, M. Döring, Schigel, T.W. May, M. Ryberg \& Abarenkov

Kickxellales Kreisel ex R.K. Benj.

Kickxellaceae Linder

Coemansia Tiegh. \& G. Le Monn. (25)

Dipsacomyces R.K. Benj. (1)

Kickxella Coem. (1)

Linderina Raper \& Fennell (2)

Martensella Coem. (1)

Martensiomyces J.A. Mey. (1)

Mycoemilia Kurihara, Degawa \& Tokum. (1)

Myconymphaea Kurihara, Degawa \& Tokum. (1)

Pinnaticoemansia Kurihara \& Degawa (1)

Spirodactylon R.K. Benj. (1)

Spiromyces R.K. Benj. (2)

Ramicandelaberomycetes Tedersoo, Sanchez-Ramirez, Kõljalg, Bahram, M. Döring, Schigel, T.W. May, M. Ryberg \& Abarenkov

Ramicandelaberales Doweld

Ramicandelaberaceae Doweld

Ramicandelaber Y. Ogawa, S. Hayashi, Degawa \& Yaguchi (4)

Kickxellomycotina genera incertae sedis

Aenigmatospora R.F. Castañeda, Saikawa, Guarro \& M. Calduch (1)

Ballocephala Drechsler (1)

Zygnemomyces K. Miura (2)

Monoblepharomycota Doweld

Hyaloraphidiomycetes Doweld

Hyaloraphidiales Doweld 
Hyaloraphidiaceae Doweld Hyaloraphidium Korshikov (1)

Monoblepharidomycetes J.H. Schaffn.

Monoblepharidales Sparrow

Gonapodyaceae H.E. Petersen ex P.M. Kirk, P.F. Cannon \& J.C. David

Gonapodya A. Fisch. (5)

Monoblepharella Sparrow (5)

Harpochytriaceae Wille

Harpochytrium Lagerh. (12)

Monoblepharidaceae A. Fisch.

Monoblepharis Cornu (15)

Oedogoniomycetaceae D.J.S. Barr

Oedogoniomyces Kobayasi \& M. Ôkubo (1)

Telasphaerulaceae Longcore \& T.Y. James

Telasphaerula Longcore \& T.Y. James (1)

Sanchytriomycetes Tedersoo, Sanchez-Ramirez, Kõljalg, Bahram, M. Döring, Schigel, T.W. May, M. Ryberg \& Abarenkov

Sanchytriales Tedersoo, Sanchez-Ramirez, Kõljalg, Bahram, M. Döring, Schigel, T.W. May, M. Ryberg \& Abarenkov

Sanchytriaceae Karpov \& Aleoshin

Amoeboradix Karpov, López-García, Mamkaeva \& Moreira (1)

Sanchytrium Karpov \& Aleoshin (1)

Mortierellomycota Tedersoo, Sanchez-Ramirez, Kõljalg, Bahram, M. Döring, Schigel, T.W. May, M. Ryberg \& Abarenkov

Mortierellomycotina Kerst. Hoffm., K. Voigt \& P.M. Kirk

Mortierellomycetes Doweld

Mortierellales Caval.-Sm.

Mortierellaceae A. Fisch.

Aquamortierella Embree \& Indoh (1)

Dissophora Thaxt. (3)

Gamsiella (R.K. Benj.) Benny \& M. Blackw. (1)

Lobosporangium M. Blackw. \& Benny (1)

Modicella Kanouse (2)

Mortierella Coem. (112)*

Mucoromycota Doweld

Mucoromycotina Benny

Endogonomycetes Doweld

Endogonales Jacz. \& P.A. Jacz.

Densosporaceae Desirò, M.E. Sm., Bidartondo, Trappe \& Bonito

Densospora McGee (9)*

Endogonaceae Paol.

Endogone Link (26)

Jimgerdemannia Trappe (2) 
Peridiospora C.G. Wu \& Suh J. Lin (2)

Sclerogone Warcup (1)

Sphaerocreas Sacc. \& Ellis (4)

Mucoromycetes Doweld

Mucorales Fr.

Backusellaceae K. Voigt \& P.M. Kirk

Backusella Hesselt. \& J.J. Ellis (13)

Choanephoraceae J. Schröt.

Blakeslea Thaxt. (2)

Choanephora Curr. (2)

Gilbertella Hesselt. (2)

Poitrasia P.M. Kirk (1)

Cunninghamellaceae Naumov ex R.K. Benj.

Absidia Tiegh. (20)

Chlamydoabsidia Hesselt. \& J.J. Ellis (1)

Cunninghamella Matr. (13)

Gongronella Ribaldi (6)

Halteromyces Shipton \& Schipper (1)

Hesseltinella H.P. Upadhyay (1)

Lentamycetaceae K. Voigt \& P.M. Kirk

Lentamyces Kerst. Hoffm. \& K. Voigt (4)

Lichtheimiaceae Kerst. Hoffm., Walther \& K. Voigt

Circinella Tiegh. \& G. Le Monn. (11)

Dichotomocladium Benny \& R.K. Benj. (5)

Fennellomyces Benny \& R.K. Benj. (4)

Lichtheimia Vuill. (7)

Phascolomyces Boedijn ex Benny \& R.K. Benj. (1)

Rhizomucor Lucet \& Costantin (6)

Thamnostylum Arx \& H.P. Upadhyay (4)

Thermomucor Subrahm., B.S. Mehrotra \& Thirum. (1)

Zychaea Benny \& R.K. Benj. (1)

Mucoraceae Dumort.

Actinomucor Schostak. (1)

Ambomucor R.Y. Zheng \& X.Y. Liu (3)

Benjaminiella Arx (3)

Chaetocladium Fresen. (2)

Cokeromyces Shanor (1)

Dicranophora J. Schröt. (1)

Ellisomyces Benny \& R.K. Benj. (1)

Helicostylum Corda (2)

Hyphomucor Schipper \& Lunn (1)

Isomucor J.I. Souza, Pires-Zottar. \& Harakava (2)

Kirkiana L.S. Loh, Kuthub. \& Nawawi (1)

Kirkomyces Benny (1)

Mucor Fresen. (91)*

Nawawiella L.S. Loh \& Kuthub. (1) 
Parasitella Bainier (1)

Pilaira Tiegh. (7 and 1 subspecies)

Pirella Bainier (2)

Rhizopodopsis Boedijn (1)

Thamnidium Link (1)

Tortumyces L.S. Loh (2)

Mycocladaceae Kerst. Hoffm.

Mycocladus Beauverie (1)

Mycotyphaceae Benny \& R.K. Benj.

Mycotypha Fenner (4)

Phycomycetaceae Arx

Phycomyces Kunze (3)

Spinellus Tiegh. (5)

Pilobolaceae Corda

Pilobolus Tode (10 and 1 subspecies)

Utharomyces Boedijn ex P.M. Kirk \& Benny (1 sp. and 1 subspecies)

Radiomycetaceae Hesselt. \& J.J. Ellis

Radiomyces Embree (3)

Rhizopodaceae K.voigt \& P.M. Kirk

Rhizopus Ehrenb. (13)

Sporodiniella Boedijn (1)

Syzygites Ehrenb. (1)

Saksenaeaceae Hesselt. \& J.J. Ellis

Apophysomyces P.C. Misra (5)

Saksenaea S.B. Saksena (5)

Syncephalastraceae Naumov ex R.K. Benj.

Protomycocladus Schipper \& Samson (1)

Syncephalastrum J. Schröt. (2)

Umbelopsidomycetes Tedersoo, Sanchez-Ramirez, Kõljalg, Bahram, M. Döring, Schigel, T.W.

May, M. Ryberg \& Abarenkov

Umbelopsidales Spatafora \& Stajich

Umbelopsidaceae W. Gams \& W. Mey.

Umbelopsis Amos \& H.L. Barnett (16)

Mucoromycotina genera incertae sedis

Bifiguratus Torr.-Cruz \& Porras-Alfaro (1)

Mucorodium K.W. Zaleski (1)

Palaeoendogone Strullu-Derr., Kenrick, Pressel, Duckett, J.P. Rioult \& Strullu (1)

Planticonsortium C. Walker \& D. Redecker (1)

Mucoromycota genus incertae sedis

Nothadelphia Degawa \& W. Gams 
Neocallimastigomycota M.J. Powell

Neocallimastigomycetes M.J. Powell

Neocallimastigales J.L. Li, I.B. Heath \& L. Packer

Neocallimastigaceae I.B. Heath (= Piromonadaceae Doweld; = Anaeromycetaceae Doweld)

Anaeromyces Breton, Bernalier, Dusser, Fonty, B. Gaillard \& J. Guillot (4)

Buwchfawromyces T.M. Callaghan \& G.W. Griff. (1)

Caecomyces J.J. Gold (5)

Cyllamyces Ozkose, B.J. Thomas, D.R. Davies, G.W. Griff. \& Theodorou (1)

Feramyces Radwa Hanafy, Mostafa Elshahed \& Noha Youssef (1)

Liebetanzomyces Joshi, G.W. Griff. \& Dagar (1)

Neocallimastix Vávra \& Joyon ex I.B. Heath (7)

Oontomyces Dagar (1)

Orpinomyces D.J.S. Barr, H. Kudo, Jakober \& K.J. Cheng (2)

Pecoramyces Hanafy, N.H. Youssef, G.W. Griff. \& Elshahed (1)

Piromyces J.J. Gold, I.B. Heath \& Bauchop (= Piromonas E. Liebet.) (6)

Olpidiomycota Doweld

Olpidiomycetes Doweld

Olpidiales Caval.-Sm.

Olpidiaceae J. Schröt.

Chytridhaema Moniez (1)

Cibdelia Juel (1)

Leiolpidium Doweld (5)

Olpidium (A. Braun) J. Schröt. (ca. 50)

Rozellomycota Doweld

Rudimicrosporea Sprague

Metchnikovellida Vivier

Amphiacanthidae Larsson

Amphiacantha Caullery \& Mesnil (3)

Metchnikovellidae Caullery \& Mesnil emend. Larsson

Amphiamblys Caullery \& Mesnil (7)

Caulleryetta Dogiel (8)

Desportesia Issi \& Voronin (1)

Metchnikovella Caullery \& Mesnil (8)

Microsporidea Corliss \& Levine

Amblyosporida Tokarev \& Issi

Amblyosporidae Weiser emend. Tokarev \& Issi

Aedispora Kilochitskii (1)

Amblyospora Hazard \& Oldacre (90)

Andreanna Simakova, Vossbrinck \& Andreadis (1)

Becnelia Tonka \& Weiser (1)

Crepidulospora Simakova, Pankova \& Issi (1)

Cristulospora Khodzhaeva \& Issi (3)

Culicospora Weiser (2)

Culicosporella Weiser (1)

Dimeiospora Simakova, Pankova \& Issi (1)

Edhazardia Becnel, V. Sprague \& Fukuda (1)

Hyalinocysta Hazard \& Oldacre (1)

Intrapredatorus Chen, Kuo \& Wu (1) 
Novothelohania Andreadis, Simakova, Vossbrinck, Shepard \& Yurchenko (1)

Parastempellia Khodzhaeva (2)

Parathelohania Codreanu (25)

Trichoctosporea Larsson (1)

Tricornia Pell \& Canning (1)

Caudosporidae Weiser emend. Tokarev \& Issi

Binucleospora Bronnvall \& Larsson (1)

Caudospora J. Weiser (1)

Flabelliforma Canning, R. Killick-Kendrick \& Killick-Kendrick (4)

Myrmecomorba R.M. Plowes, J.J. Becnel, E.G. LeBrun, D.H. Oi, S.M. Valles, N.T. Jones \&

L.E. Gilbert (1)

Neoflabelliforma Morris \& Freeman (2)

Octosporea Flu (18)

Polydispyrenia Canning \& Hazard (2)

Ringueletium Garcia (1)

Scipionospora Bylén \& Larsson (1)

Weiseria Doby \& Saguez (3)

Gurleyidae Sprague emend. Tokarev \& Issi

Agglomerata Larsson\& Yan (5)

Binucleata Refardt, Decaestecker, Johnson \& Vávra (1)

Conglomerata Vavra, Fiala, Krylova, Petrusek, Hylis (1)

Episeptum Larsson (6)

Gurleya Doflein (10)

Lanatospora Voronin (4)

Larssonia Vidtmann \& Sokolova (2)

Marssoniella Lemmermann (1)

Norlevinea Vávra (1)

Paraepiseptum Hyliš, Oborník, Nebesářová \& Vávra (4)

Pseudoberwaldia Vavra, Fiala, Krylova, Petrusek, Hylis (1)

Senoma Simakova, Pankova, Tokarev \& Issi (1)

Zelenkaia Hyliš, Oborník, Nebesářová \& Vávra (1)

Amblyosporida genera incertae sedis

Alfvenia Larsson (4)

Hazardia Weiser (2)

Multilamina Becnel, Scheffrahn, Vossbrinck \& Bahder (1)

Takaokaspora Andreadis, Takaoka, Otsuka \& Vossbrinck (1)

Trichotuzetia Vávra, Larsson \& Baker (1)

Neopereziida Tokarev \& Issi

Berwaldiidae Simakova, Tokarev \& Issi

Berwaldia Larsson (4)

Fibrillanosema Slothouber Galbreath, Smith, Terry, Becnel \& Dunn (1)

Neopereziidae Voronin emend. Issi, Tokarev, Seliverstova \& Voronin

Bacillidium Janda (5)

Bryonosema Canning, Refardt, Vossbrinck, Okamura \& Curry (2)

Neoperezia Issi \& Voronin (2)

Pseudonosema Canning, Refardt, Vossbrinck, Okamura \& Curry (1)

Schroedera Morris \& Adams (2) 
Trichonosema Canning, Refardt, Vossbrinck, Okamura \& Curry (2)

Tubulinosematidae Franzen, Fischer, Schröder, Schölmerich \& Schneuwly emend. Tokarev \& Issi

Anncaliia Issi, Krylova \& Nikolaeva (6)

Kneallhazia Sokolova \& Fuxa (2)

Tubulinosema Franzen, Fischer, Schröder, Schölmerich \& Schneuwly (5)

Neopereziida genera incertae sedis

Janacekia Larsson (6)

Systenostrema Hazard \& Oldacre (5)

Ovavesiculida Tokarev \& Issi

Ovavesiculidae Sprague, Becnel \& Hazard emend. Tokarev \& Issi

Antonospora Fries, Paxton, Tengo, Slemenda, da Silva, \& Pieniazek (2)

Ovavesicula Andreadis \& Hanula (1)

Paranosema Sokolova, Dolgikh, Morzhina, Nassonova, Issi, Terry, Ironside, Smith (4)

Ovavesiculida genus incertae sedis

Nematocida Troemel, Félix, Whiteman, Barrière \& Ausubel (1)

Glugeida Gurley emend. Tokarev \& Issi

Facilisporidae Jones, Prosperi-Porta \& Kim

Facilispora Jones, Prosperi-Porta \& Kim (1)

Glugeidae Gurley emend. Tokarev \& Issi

Alloglugea Paperna \& Lainson (1)

Amazonspora Azevedo \& Matos (1)

Glugea Thélohan (40)

Ichthyosporidium Caullery \& Mesnil (5)

Johenrea Lange, Becnel, Razafindratiana, Przybyszewski \& Razafindrafara (1)

Loma Morrison \& Sprague (12)

Parapleistophora Issi, Kadyrova, Pushkar, Khodzhaeva \& Krylova (1)

Pseudoloma J.L. Matthews, A.M.V. Br., K. Larison, J.K. Bishop-Stewart, P. Rogers \& M.L.

Kent (6)

Myosporidae Stentiford, Bateman, Small, Moss, Shields, Reece \& Tuck

Myospora Stentiford, Bateman, Small, Moss, Shields, Reece \& Tuck (1)

Pereziidae Loubes, Maurand, Comps \& Campillo emend. Tokarev \& Issi

Ameson Sprague (2)

Nadelspora Olson, Tiekotter \& Reno (1)

Perezia Léger \& Duboscq (12)

Pernicivesicula Bylén \& Larsson (1)

Pleistophoridae Doflein emend. Tokarev \& Issi

Dasyatispora Diamant, Goren, Yokeş, Galil, Klopman, Huchon, Szitenberg \& Karhan (1)

Heterosporis Schubert (4)

Myosporidium Baquero, Rubio, Moura, Pieniazek \& Jordana (1)

Ovipleistophora Pekkarinen, Lom \& Nilsen (2)

Pleistophora Gurley (10)

Trachipleistophora Hollister, Canning, Weidner, Field, Kench \& Marriott (3) 
Vavraia Weiser (10)

Spragueidae Weissenberg emend. Tokarev \& Issi

Apotaspora Sokolova \& Overstreet (1)

Inodosporus Overstreet \& Weidner (2)

Microgemma Ralphs \& Matthews (6)

Spraguea Weissenberg (2)

Potaspora Casal, Matos, Teles-Grilo \& Azevedo (3)

Pseudokabatana Liu, Stentiford, Voronin, Sato, Li\& Zhang (1)

Tetramicra Matthews \& Matthews (1)

Thelohaniidae Hazard \& Oldacre emend. Tokarev \& Issi

Bohuslavia Larsson (1)

Chapmanium Hazard \& Oldacre (4)

Coccospora Wallr. (1)

Cucumispora Ovcharenko, Bacela, Wilkinson, Ironside, Rigaud \& Wattier (2)

Hyperspora Stentiford, Ramilo, Abollo, Kerr, Bateman, Feist, Bass \& Villalba (1)

Napamichum Larsson (3)

Nudispora Larsson (1)

Octotetraspora Issi, Kadyrova, Pushkar, Khodzhaeva \& Krylova (1)

Ormieresia Vivarès, Bouix \& Manier (1)

Orthothelohania Codreanu \& Codreanu-Balcescu (1)

Paradoxium Stentiford, Ross, Kerr, Bass \& Bateman (1)

Pegmatheca Hazard \& Oldacre (2)

Resiomeria Larsson (1)

Spherospora Garcia (1)

Thelohania Henneguy (50)

Unikaryonidae Sprague emend. Tokarev \& Issi

Canningia Weiser, Wegensteiner \& Žižka (2)

Dictyocoela Terry, Smith, Sharpe, Rigaud, Littlewood, Ironside, Rollinson, Bouchon, MacNeil, Dick \& Dunn (8)

Larssoniella Weiser \& David (2)

Unikaryon Canning, Lai \& Lie (18)

Glugeida genus incertae sedis

Triwangia Wang, Nai, Chih Wang, Solter, Hsu, Wang \& Lo (1)

Nosematida Labbe emend. Tokarev \& Issi

Encephalitozoonidae Voronin

Encephalitozoon Levaditi, Nicolau \& Schoen (12)

Mockfordia Sokolova, Sokolov \& C.E. Carlton (1)

Enterocytozoonidae Cali \& Owen emend. Tokarev \& Issi

Desmozoon Freeman \& Sommerville (3)

Enterocytozoon Desportes, Le Charpentier, Galian, Bernard, Cochand-Priollet, Lavergne,

Ravisse \& Modigliani (2)

Enterospora Stentiford, Bateman, Longshaw \& Feist (2)

Hepatospora Stentiford, Bateman, Dubuffet, Chambers \& Stone (1)

Nucleospora Hedrick, Groff \& Baxa (3)

Obruspora Diamant, Rothman, Goren, Galil, Yokes, Szitenberg \& Huchon (1) 
Heterovesiculidae Lange, Macvean, Henry \& Streett

Heterovesicula Lange, Macvean, Henry \& Streett (1)

Mrazekiidae Léger \& Hesse emend. Tokarev \& Issi

Agmasoma Hazard \& Oldacre (3)

Anostracospora Rode, Landes, Lievens, Flaven, Segard, Jabbour-Zahab, Michalakis, Agnew, Vivarés \& Lenormand (1)

Euplotespora Fokin, Di Giuseppe, Erra \& Dini (1)

Helmichia Larsson (5)

Hrabyeia Lom \& Dyková (1)

Jirovecia Weiser (7)

Mrazekia Léger \& Hesse (17)

Rectispora Larsson (1)

Nosematidae Tokarev, Huang, Solter, Malysh, Becnel \& Vossbrinck

Nosema Nägeli (20)

Vairimorpha Pilley (15)

Ordosporidae Larsson, Ebert \& Vávra

Ordospora Larsson, Ebert \& Vávra (2)

Nosematida genera incertae sedis

Alternosema Lipa, Tokarev, Issi (1)

Anisofilariata Tokarev, Voronin, Seliverstova, Dolgikh, Pavlova, Ignatieva \& Issi (1)

Crispospora Tokarev, Voronin, Seliverstova, Pavlova \& Issi (1)

Cystosporogenes Canning, Barker, Nicholas \& Page (4)

Endoreticulatus Brooks, Becnel \& Kennedy (5)

Enterocytospora Rode, Landes, Lievens, Flaven, Segard, Jabbour-Zahab, Michalakis, Agnew, Vivarés \& Lenormand (1)

Enteropsectra Zhang, Sachse, Prevost, Luallen, Troemel \& Felix(2)

Glugoides Larsson, Ebert, Vávra \& Voronin (1)

Liebermannia Sokolova, Lange \& Fuxa (3)

Orthosomella Canning, Wigley \& Barker (2)

Pancytospora Zhang, Sachse, Prevost, Luallen, Troemel \& Felix (2)

Parahepatospora Bojko, Clark, Bass, Dunn, Stewart-Clark, Stebbing \& Stentiford (1)

Percutemincola Nishikori, Setiamarga, Tanji, Kuroda, Shiraishi \& Okashi-Kobayashi (1)

Sporanauta Ardila-Garcia \& Fast (1)

Vittaforma Silveira \& Canning (1)

Microsporidia families incertae sedis

Abelsporidae Azevedo

Abelspora Azevedo (1)

Areosporiidae Stentiford, Bateman, Feist, Oyarzún, Uribe, Palacios \& Stone

Areospora Stentiford, Bateman, Feist, Oyarzún, Uribe, Palacios \& Stone (1)

Burenellidae Jouvenaz \& Hazard

Burenella Jouvenaz \& Hazard (1)

Pilosporella Hazard \& Oldacre (2)

Tabanispora Bykova, Sokolova \& Issi (2)

Cougourdellidae Poisson 
Cougourdella Hesse (7)

Cylindrosporidae Issi \& Voronin

Cylindrospora Issi \& Voronin (2)

Duboscqiidae R. Sprague

Duboscqia Pérez (11)

Mitoplistophora Codreanu (1)

Pulicispora Vedmed, Krylova \& Issi (1)

Tardivesicula Larsson\& Bylén (1)

Trichoduboscqia Léger (1)

\section{Golbergiidae Issi}

Golbergia Weiser (1)

Krishtalia Kilochitskii (1)

Simuliospora Khodzhaeva, Krylova \& Issi (2)

Microfilidae Sprague, Becnel \& Hazard

Microfilum Faye, Toguebaye \& Bouix (1)

Neonosemoidiidae Faye, Toguebaye \& Bouix

Neonosemoides Faye \& Toguebaye (4)

Pleistosporidiidae Codreanu-Balcescu \& Codreanu

Pleistosporidium Codreanu-Balcescu \& Codreanu (1)

Pseudopleistophoridae Sprague

Pseudopleistophora Sprague (1)

Steinhausia Sprague, Ormières \& Manier (4)

Striatosporidae Issi \& Voronin

Striatospora Issi \& Voronin (1)

Telomyxidae Léger \& Hesse

Telomyxa Léger \& Hesse (4)

Toxoglugeidae Larsson

Toxoglugea Léger \& Hesse (15)

Toxospora Voronin (2)

Tuzetiidae Sprague, Tuzet \& Maurand

Nelliemelba Larsson (1)

Pankovaia Simakova, Tokarev \& Issi (1)

Paratuzetia Poddubnaya, Tokarev \& Issi (1)

Tuzetia Maurand, Fize, Vernick \& Michel (7)

Microsporidia genera incertae sedis

Auraspora Weiser \& Purrini (1)

Baculea Loubès \& Akbarieh (1)

Burkea Sprague (2)

Chytridioides Tregouboff (1)

Ciliatosporidium Foissner \& Foissner (1) 
Cryptosporina Hazard \& Oldacre (1)

Evlachovaia Voronin (1)

Geusia Rühl \& Korn (1)

Gurleyides Voronin (1)

Hamiltosporidium Haag, Larsson, Refardt \& Ebert (2)

Hirsutosporos Batson (1)

Holobispora Voronin (1)

Issia Weiser (3)

Kinorhynchospora Adrianov \& Rybakov (1)

Mariona Stempell (1)

Merocinta Pell \& Canning (1)

Microsporidium Balbiani (120)

Myxocystis Mrazek (1)

Nematocenator Sapir, Dillman, Connon, Grupe, Ingels, Mundo-Ocampo, Levin, Bladwin,

Orphan \& Sternberg (1)

Nosemoides Vinckier (5)

Pyrotheca Hesse (4)

Spiroglugea Léger \& Hesse (1)

Stempellia Léger \& Hesse (19)

Wittmannia Czaker (1)

Rozellomycota orders incertae sedis

Chytridiopsidea Weiser

Buxtehudidae Larsson

Jiroveciana Larsson (1)

Buxtehudea Larsson (1)

Chytridiopsidae Sprague, Ormières \& Manier

Acarispora Radek and Alberti (1)

Chytridiopsis Schneider (11)

Intexta Larsson, Steiner \& Bjørnson (1)

Nolleria Beard, Butler \& Becnel (1)

Sheriffia Larsson (1)

Hesseidae Ormières \& Sprague

Hessea Ormières \& Sprague (1)

Rozellomycota genera incertae sedis

Mitosporidium Haag, James, Pombert, Larsson, Schaer, Refardt \& Ebert (2)

Morellospora Corsaro, Walochnik, Venditti, Hauröder \& Michel (1)

Nucleophaga Dangeard (2)

Paramicrosporidium Corsaro, Walochnik, Venditti, Steinmann, Müller \& Michel (1)

Rozella Cornu (20)

${ }^{\#}$ Microsporidium is a collective genus which incorporate species with uncertain genus allocation

Zoopagomycota Gryganskyi, M.E. Sm., Spatafora \& Stajich

Zoopagomycetes Doweld

Zoopagales Bessey ex R.K. Benj.

Cochlonemataceae Dudd.

Aenigmatomyces R. F. Castañeda \& W.B. Kendr. (1)

Amoebophilus P.A. Dang. (4) 
Aplectosoma Drechsler (1)

Bdellospora Drechsler (1)

Cochlonema Drechsler (11)

Endocochlus Drechsler (4)

Euryancale Drechsler (4)

Helicocephalidaceae Boedijn

Brachymyces G.L. Barron (1)

Helicocephalum Thaxt. (6)

Rhopalomyces Corda (11)

Verrucocephalum Degawa (1)

Piptocephalidaceae J. Schröt.

Kuzuhaea R.K. Benj. (1)

Piptocephalis de Bary (ca. 25)

Syncephalis Tiegh. \& G. Le Monn. (ca. 55)

Sigmoideomycetaceae Benny, R.K. Benj. \& P.M. Kirk

Reticulocephalis Benny, R.K. Benj. \& P.M. Kirk (2)

Sigmoideomyces Thaxt. (1)

Sphondylocephalum Stalpers (1)

Thamnocephalis Blakeslee (3)

Zoopagaceae Drechsler

Acaulopage Drechsler (27)

Cystopage Drechsler (9)

Lecophagus M.W. Dick (2)

Stylopage Drechsler (17)

Tentaculophagus Doweld (1)

Zoopage Drechsler (11)

Zoophagus Sommerst. (4)

Zoopagales genus incertae sedis

Massartia De Wild. (1)

Zoopagomycotina genus incertae sedis

Basidiolum Cienk. (1)

\section{Outline of Fossil fungi}

The legitimate fungal genera known so far are listed below (with number of species in each genus in brackets). Here we list genera based on Saccardoan System (Table 4), fossil fungal sporophores, mycelia and other fungal remains (Table 5) and modern fungal genera to which fossil species have been assigned (Table 6).

Table 4 Fossil fungal spores (according to Saccardoan System).

\begin{tabular}{lll}
\hline $\begin{array}{l}\text { Fungi } \\
\text { Imperfecti }\end{array}$ & Family & Genera \\
\hline & Amerosporae & Asyregraamspora Locq. \& Sal.-Cheb. (1) \\
& & Basidiosporites Elsik (4)* \\
& & Biporipsilonites Kalgutkar \& Janson. (11)* \\
\hline
\end{tabular}


Table 4 Continued.

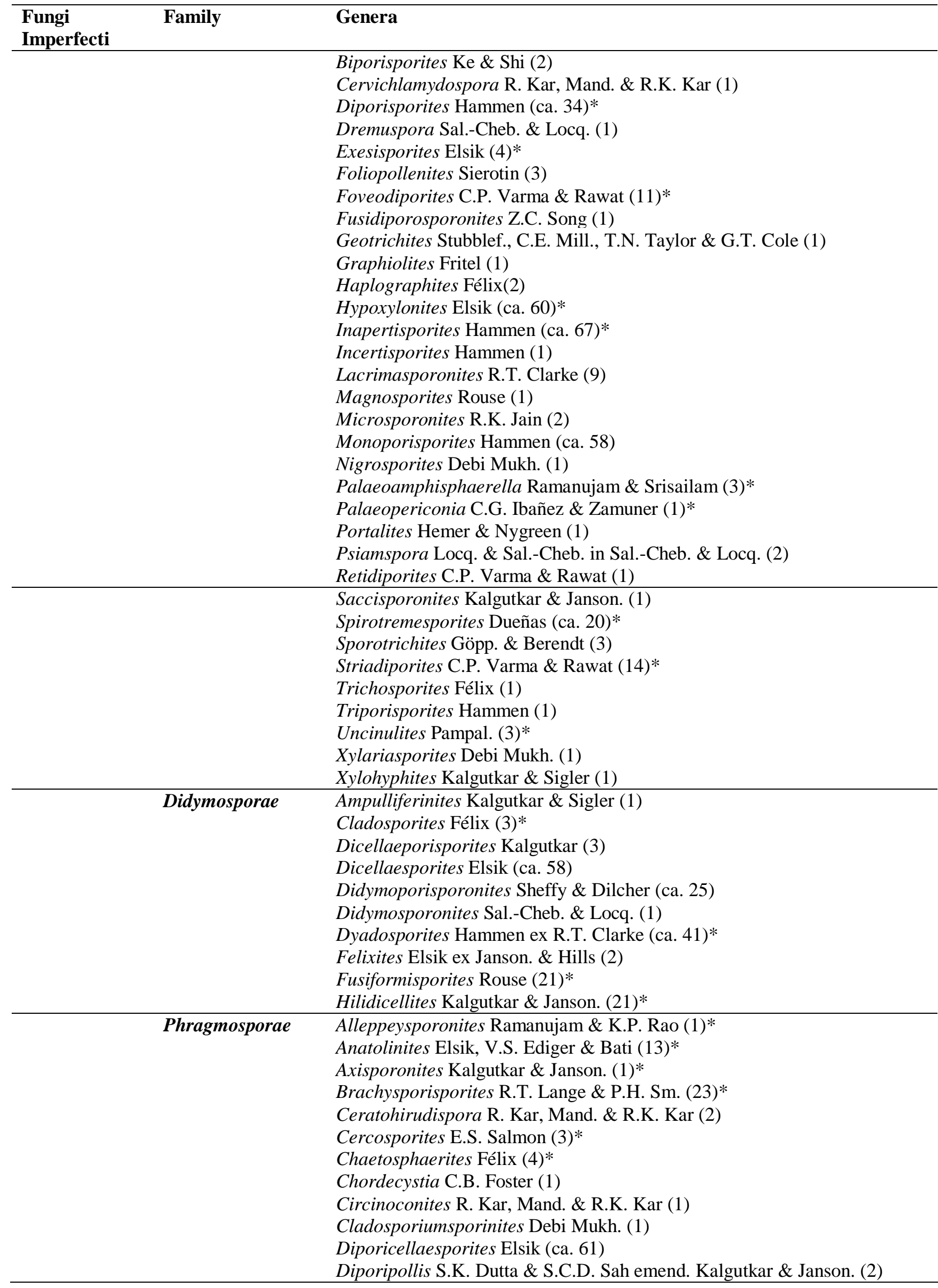


Table 4 Continued.

\begin{tabular}{|c|c|c|}
\hline $\begin{array}{l}\text { Fungi } \\
\text { Imperfecti }\end{array}$ & Family & Genera \\
\hline & & $\begin{array}{l}\text { Dwayabeejaesporonites Debi Mukh. (1) } \\
\text { Edmundmasonaesporites Debi Mukh. (1) } \\
\text { Foveoletisporonites Ramanujam \& K.P. Rao (3) } \\
\text { Fractisporonites R.T. Clarke (9) } \\
\text { Heterocystinella Cookson \& Eisenack (1) } \\
\text { Jansoniisporites Kalgutkar (1) } \\
\text { Kumarisporites Kalgutkar \& Janson. (1)* } \\
\text { Mathurisporites Kalgutkar \& Janson. (2)* } \\
\text { Monilites Pampal. (1) } \\
\text { Multicellaesporites Elsik emend. P. Kumar (ca. 14) } \\
\text { Multicellites Kalgutkar \& Janson. (48) } \\
\text { Ornasporonites Ramanujam \& K.P. Rao (1)* } \\
\text { Paragranatisporites Zhong Y. Zhang (5) } \\
\text { Phialophoronites Debi Mukh. (1) } \\
\text { Pluricellaesporites Hammen (ca. 72)* } \\
\text { Quilonia K.P. Jain \& R.C. Gupta emend. Kalgutkar \& Janson. (11) } \\
\text { Ramasricellites Kalgutkar \& Janson. (2)* } \\
\text { Reduviasporonites L.R. Wilson (9) } \\
\text { Reticellites D.L.E. Glass, D.D. Br. \& Elsik (1) } \\
\text { Scolecosporites R.T. Lange \& P.H. Sm. (4) } \\
\text { Tripithonites Sat. K. Srivastava \& Al-Tayyar (2) } \\
\text { Tympanicysta Malme (1) } \\
\text { Varmasporites Kalgutkar \& Janson. (1)* }\end{array}$ \\
\hline & Dictyosporae & $\begin{array}{l}\text { Centonites Peppers (1) } \\
\text { Ctenosporites Elsik \& Janson. (3) } \\
\text { Dictyosporites Félix emend. Kalgutkar \& Janson. (ca. 20) } \\
\text { Dictyostromata R. Kar, Mand. \& R.K. Kar (2) } \\
\text { Kutchiathyrites R.K. Kar emend. Kalgutkar \& Janson. (7)* } \\
\text { Lirasporis R. Potonié \& S.C.D. Sah (3) } \\
\text { Octosporites Sal.-Cheb. \& Locq. (1) } \\
\text { Palambages Wetzel (3) } \\
\text { Papulosporonites Schmied. \& G. Schwab (7)* } \\
\text { Polyadosporites Hammen (ca. 9) } \\
\text { Polycellaesporonites Anil Chandra, R.K. Saxena \& Setty (7)* } \\
\text { Staphlosporonites Sheffy \& Dilcher (c.21)* }\end{array}$ \\
\hline & Helicosporae & $\begin{array}{l}\text { Colligerites K.P. Jain \& R.K. Kar (3)* } \\
\text { Elsikisporonites } \text { P. Kumar (1) } \\
\text { Helicominites } \text { Barlinge \& Paradkar (1) } \\
\text { Helicoönites Kalgutkar \& Sigler (1) } \\
\text { Helicosporiates Kalgutkar \& Sigler (1) } \\
\text { Involutisporonites R.T. Clarke (ca. 8) } \\
\text { Palaeocirrenalia Ramanujam \& Srisailam (3) } \\
\text { Paleoslimacomyces Kalgutkar \& Sigler (3)* } \\
\text { Retihelicosporonites Ramanujam \& K.P. Rao (1)* } \\
\end{array}$ \\
\hline & Staurosporae & $\begin{array}{l}\text { Eoglobella W.H. Bradley (1) } \\
\text { Frasnacritetrus Taug. (7)* } \\
\text { Mossopisporites Kalgutkar \& Janson. (1)* } \\
\text { Pesavis Elsik \& Janson. (3) } \\
\text { Spegazzinites Félix (3) } \\
\text { Tribolites W.H. Bradley (2)* } \\
\text { Trihyphites Kalgutkar \& Janson. (1)* } \\
\text { Triporicellaesporites Ke \& Shi (4)* }\end{array}$ \\
\hline
\end{tabular}


Table 5 Fossil fungal fructifications, mycelia and other fungal remains.

\begin{tabular}{|c|c|c|}
\hline Phylum & Order & Genera \\
\hline \multirow[t]{56}{*}{ Ascomycota } & Botryosphaeriales & Guignardiacarpites Debi Mukh. (1) \\
\hline & Capnodiales & Mycosphaerellascoidetes Debi Mukh. (1) \\
\hline & Dothideales & Cucurbitariaceites R.K. Kar, R.Y. Singh \& S.C.D. Sah (2)* \\
\hline & & Leptosphaerites Richon (2) \\
\hline & & Palaeoleptosphaeria Barlinge \& Paradkar (1) \\
\hline & & Perisporiacites Félix (4) \\
\hline & Erysiphales & Erisiphites Pampal. (1) \\
\hline & & Meliolinites Selkirk (9) \\
\hline & & Meliostroma R. Kar, Mand. \& R.K. Kar (1) \\
\hline & & Palaeosclerotium G.W. Rothwell (1)* \\
\hline & & Perisporites Pampal. (2) \\
\hline & Eurotiales & Coleocarpon Stubblef., T.N. Taylor, C.E. Miller \& G.T. Cole (1) \\
\hline & & Cryptocolax R.A. Scott (2) \\
\hline & & Memnonillasporonites Debi Mukh. (1) \\
\hline & & Mycocarpon S.A. Hutch. (7)* \\
\hline & & Roannaisia T.N. Taylor, Galtier \& Axsmith (1) \\
\hline & & Sporocarpon Will. (13)* \\
\hline & & Traquairia Carruth. ex Scott (4) \\
\hline & Hysteriales & Hysterites Unger (16) \\
\hline & Microthyriales & Appendicisporonites R.K. Saxena \& S. Khare (1) \\
\hline & & Asterinites Doub. \& D. Pons ex Kalgutkar \& Janson. (2) \\
\hline & & Asterothyrites Cookson (16) \\
\hline & & Brefeldiellites Dilcher (2) \\
\hline & & Caldesites Puri (1) \\
\hline & & Callimothallus Dilcher (11) \\
\hline & & Cribrites R.T. Lange (1) \\
\hline & & Dictyotopileos Dilcher (1) \\
\hline & & Euthythyrites Cookson (4) \\
\hline & & Haplopeltis Theiss. (5) \\
\hline & & Kalviwadithyrites M.R. Rao (1) \\
\hline & & Koshalia S. Sarkar \& V. Prasad (1) \\
\hline & & Mariusia D. Pons \& Boureau (1) \\
\hline & & Melanosporites Pampal. (1) \\
\hline & & Microthyriacites Cookson (19) \\
\hline & & Microthyrites Pampal. (1) \\
\hline & & Molinaea Doub. \& D. Pons (1) \\
\hline & & Palmellathyrites Locq., D. Pons \& Sal.-Cheb. (1) \\
\hline & & Parmathyrites K.P. Jain \& R.C. Gupta (5) \\
\hline & & Pelicothallos Dilcher (1) \\
\hline & & Phragmothyrites W.N. Edwards (24)* \\
\hline & & Plochmopeltinites Cookson (3) \\
\hline & & Polyhyphaethyrites R. Srivast. \& R.K. Kar (1) \\
\hline & & Ratnagiriathyrites R.K. Saxena \& N.K. Misra (1)* \\
\hline & & Spinosporonites R.K. Saxena \& S. Khare (1)* \\
\hline & & Stomiopeltites Alvin \& M.D. Muir (3) \\
\hline & & Trichopeltinites Cookson (5) \\
\hline & & Trichothyrites Rosend. (13)* \\
\hline & & Ussurithyrites Krassilov (1) \\
\hline & Patellariales & Rhytidhysteriumites Debi Mukh. (1) \\
\hline & Pezizales & Ascodesmisites Trivedi, Chaturv. \& C.L. Verma (1)\ \\
\hline & & Paleomorchella Poinar (1) \\
\hline & & Pezizites Göpp. \& Berendt (4) \\
\hline & Phyllachorales & Paleoserenomyces Currah, Stockey \& B.A. LePage (1) \\
\hline & Pleosporales & Cryptodidymosphaerites Currah, Stockey \& B.A. LePage (1)* \\
\hline & & Dictyosporiuminites Debi Mukh. (1) \\
\hline & & Pleosporites Y. Suzuki (1) \\
\hline
\end{tabular}


Table 5 Continued.

\begin{tabular}{|c|c|c|}
\hline Phylum & Order & Genera \\
\hline \multirow{35}{*}{ Basidiomycota } & Sphaeriales & Diploneurospora K.P. Jain \& R.C. Gupta (1)* \\
\hline & & Palaeosordaria Sahni \& H.S. Rao (1) \\
\hline & & Petrosphaeria Stopes \& H. Fujii (1) \\
\hline & & Valsarites Puri (1) \\
\hline & Uredinales & Aeciosporonites Debi Mukh. (1) \\
\hline & Xylariales. & Chaethomites Pampal. (1) \\
\hline & & Sphaerites Unger (48) \\
\hline & Incertae sedis & Cephalothecoidomyces G. Worobiec, Neumann \& E. Worobiec (1) \\
\hline & Agaricales. & Archaeomarasmius Hibbett, D. Grimaldi \& Donoghue (1) \\
\hline & & Coprinites Poinar \& Singer $(1)^{*}$ \\
\hline & & Gondwanagaricites Heads, A.N. Mill \& J.L. Crane (1) \\
\hline & & Protomycena Hibbett, D .Grimaldi \& Donoghue (1) \\
\hline & Polyporales. & Eopolyporoides Rigby (1) \\
\hline & & Phellinites Singer \& S. Archang. (1) \\
\hline & & Pseudopolyporus Hollick (1) \\
\hline & & Trametites A. Straus (3) \\
\hline & Pucciniales. & Shuklania J.N. Dwivedi (1) \\
\hline & Sphaeropsidales & Archephoma Kyoto Watanabe, H. Nishida \& Tak. Kobay. (1) \\
\hline & & Ascochytites Barlinge \& Paradkar (2)* \\
\hline & & Deccanodia Singhai (1) \\
\hline & & $\begin{array}{l}\text { Diplodites D.N. Babajan \& Tasl. ex Kalgutkar, Nambudiri \& Tidwell } \\
(5)^{*}\end{array}$ \\
\hline & & Entopeltacites Selkirk (6) \\
\hline & & Meniscoideisporites Kyoto Watanabe, H. Nishida \& Tak. Kobay. (1) \\
\hline & & Mohgaonidium Singhai (1) \\
\hline & & Palaeocytosphaera R.B. Singh \& G.V. Patil (1) \\
\hline & & Palaeophoma Singhai (1)* \\
\hline & & Phomites Fritel (2) \\
\hline & & Rabenhorstinidium R.B. Singh \& G.V. Patil (1) \\
\hline & Uredinales & Aecidites Debey \& Ettingsh. (4) \\
\hline & & Aeciosporonites Debi Mukh. (1) \\
\hline & & Hapalophragmites Ramanujam \& Ramachar (1) \\
\hline & & Milesites Ramanujam \& Ramachar (1) \\
\hline & & Pucciniasporonites Ramanujam \& Ramachar (1) \\
\hline & Ustilaginales & Chlamydosporites Paradkar (1) \\
\hline & & Teliosporites R. Kar, Mand. \& R.K. Kar (2) \\
\hline \multirow[t]{6}{*}{ Chytridiomycota } & Chytridiales & Grilletia Renault \& C.E. Bertrand (1) \\
\hline & & Guizhounema X. Mu (1) \\
\hline & & Krispiromyces T.N. Taylor, Hass \& W. Remy (1) \\
\hline & & Lyonomyces T.N. Taylor, Hass \& W. (1) \\
\hline & & Milleromyces T.N. Taylor, Hass \& W. Remy (1) \\
\hline & & Oochytrium Renault (1) \\
\hline \multirow[t]{6}{*}{ Mucoromycota } & Endogonales & Chlamydospora R. Kar, Mand. \& R.K. Kar (1) \\
\hline & & Endochaetophora J.F. White \& T.N. Taylor (1) \\
\hline & & Gigasporites Carlie J. Phipps \& T.N. Taylor (1) \\
\hline & & Palaeogigaspora R. Kar, Mand. \& R.K. Kar (1) \\
\hline & & Palaeomycites Mesch. (21)* \\
\hline & & Udaria A. Gupta (2) \\
\hline Mucoromycota & & Lithoтисоrites R. Kar, Mand. \& R.K. Kar (2) \\
\hline $\begin{array}{l}\text { genera incertae } \\
\text { sedis }\end{array}$ & & \\
\hline \multirow[t]{5}{*}{ Mycelia Sterilia } & & Animikiea Bargh. (1) \\
\hline & & Archaeorestis Bargh. (1) \\
\hline & & Celyphus Batten (1) \\
\hline & & Dendromyceliates K.P. Jain \& R.K. Kar (2) \\
\hline & & Entosphaeroides Bargh. (1) \\
\hline
\end{tabular}


Table 5 Continued.

\begin{tabular}{|c|c|c|}
\hline Phylum & Order & Genera \\
\hline $\begin{array}{l}\text { Fossil fungi } \\
\text { incertae sedis }\end{array}$ & & 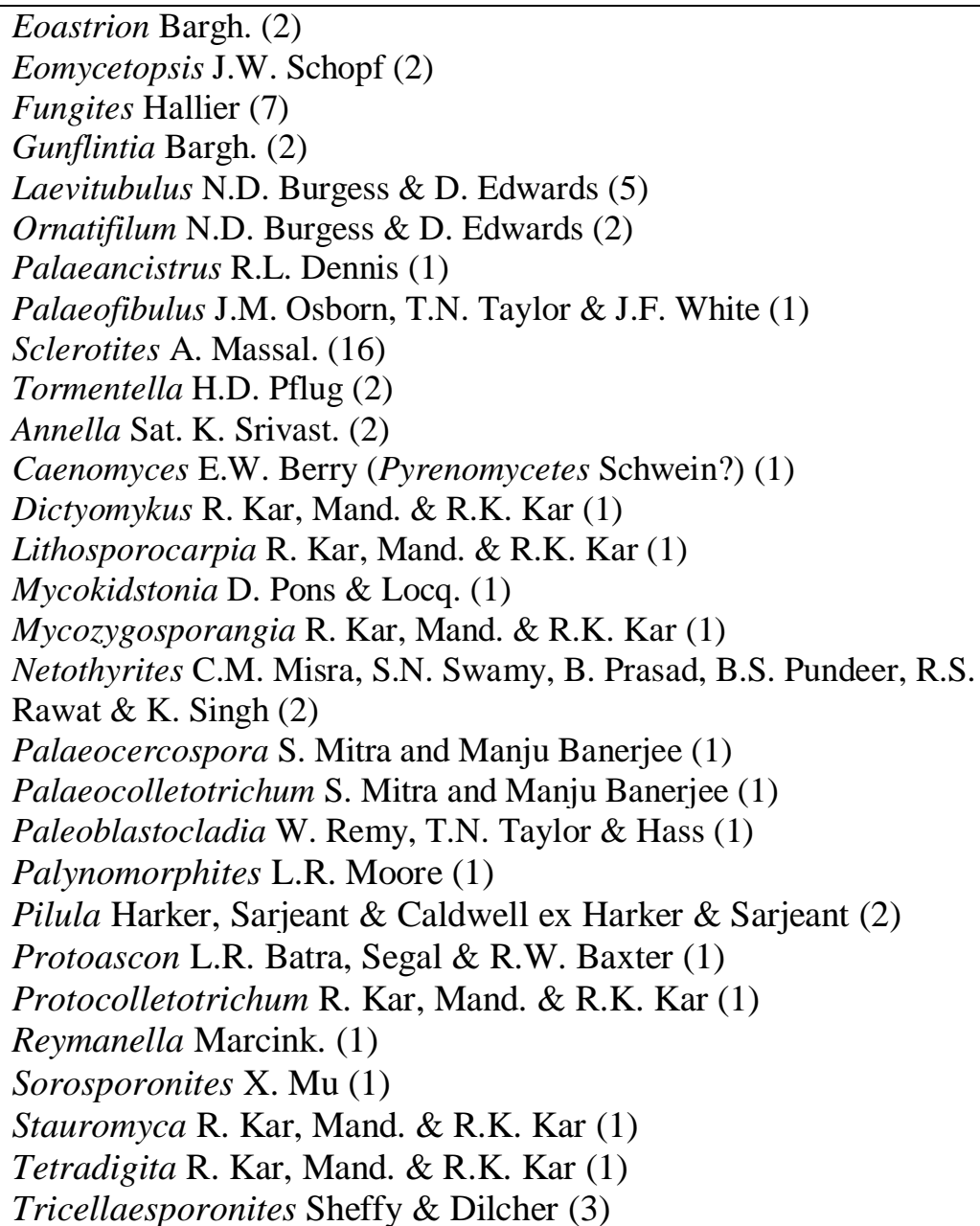 \\
\hline
\end{tabular}

Table 6 Modern fungal genera to which fossil species have been assigned.

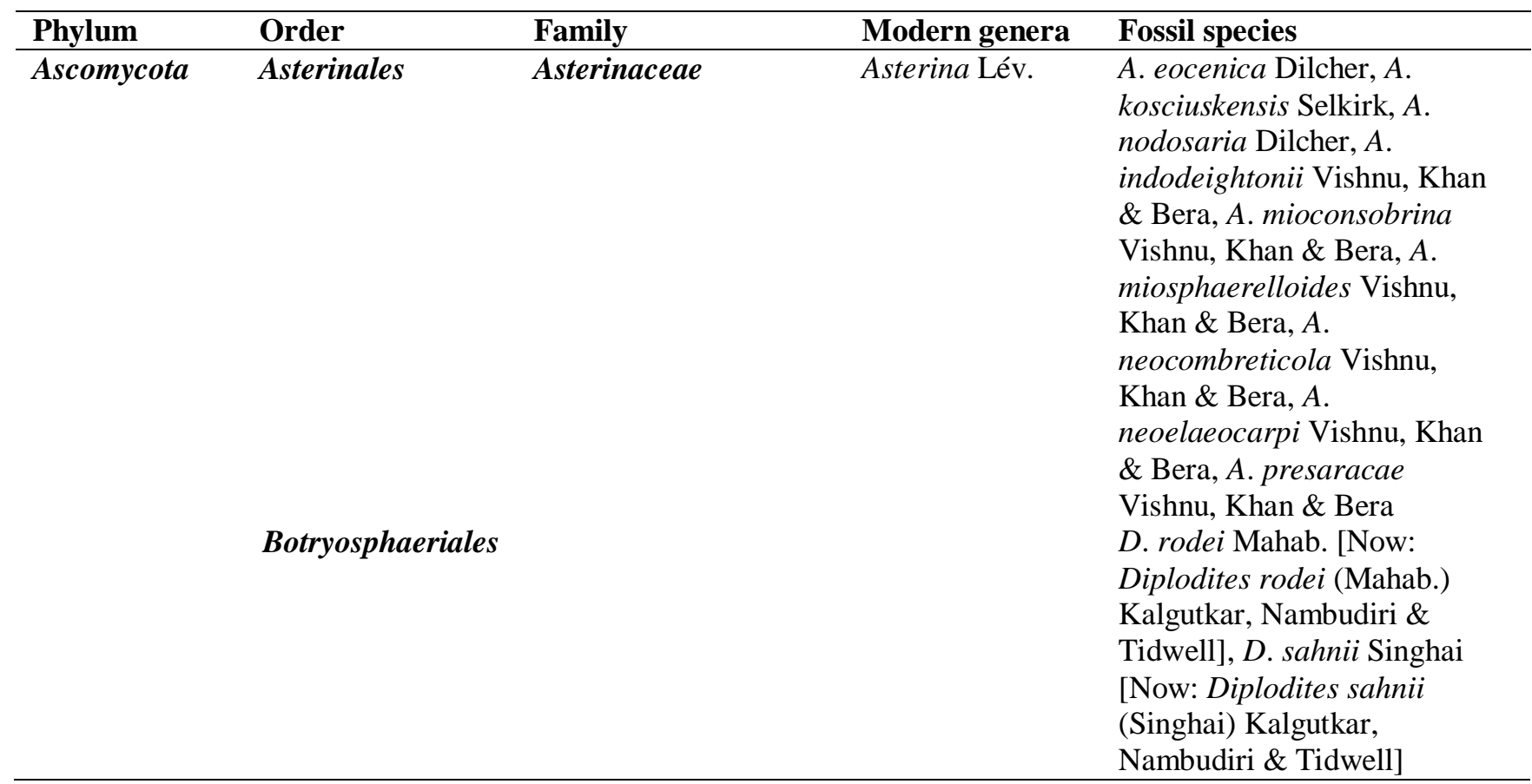


Table 6 Continued.

\begin{tabular}{|c|c|c|c|c|}
\hline Phylum & Order & Family & Modern genera & Fossil species \\
\hline & $\begin{array}{l}\text { Capnodiales } \\
\text { Chaetosphaeriales }\end{array}$ & $\begin{array}{l}\text { Mycosphaerellaceae } \\
\text { Chaetosphaeriaceae }\end{array}$ & $\begin{array}{l}\text { Ramularia Sacc. } \\
\text { Chaetosphaeria } \\
\text { Tul. \& C. Tul. }\end{array}$ & $\begin{array}{l}\text { R. oblongispora Casp. } \\
\text { C. elsikii M.J. Pound, J.M.K. } \\
\text { O'Keefe, N.B. Nuñez Otaño, } \\
\text { J.B. Riding }\end{array}$ \\
\hline & Eurotiales & Aspergillaceae & Penicillium Link & P. curtipes Berk. \\
\hline & Helotiales & Mollisiaceae & $\begin{array}{l}\text { Trimmatostroma } \\
\text { Corda. }\end{array}$ & $\begin{array}{l}\text { Trimmatostroma } \\
\text { intertrappea K.S. Patil \& } \\
\text { Datar }\end{array}$ \\
\hline & Hypocreales & Bionectriaceae & $\begin{array}{l}\text { Acremonium } \\
\text { Link }\end{array}$ & A. succineum Casp. \\
\hline & & Ceratostomatacea & $\begin{array}{l}\text { Gonatobotrys } \\
\text { Corda }\end{array}$ & G. primigenius Casp. \\
\hline & Laboulbeniales & Laboulbeniaceae & $\begin{array}{l}\text { Stigmatomyces } \\
\text { H. Karst. }\end{array}$ & $\begin{array}{l}\text { Stigmatomyces succini } \mathrm{W} \text {. } \\
\text { Rossi, Kotrba \& Triebel }\end{array}$ \\
\hline & Lecanorales & Sphaerophoraceae & $\begin{array}{l}\text { Sphaerophorus } \\
\text { Pers. }\end{array}$ & S. moniliformis Menge \\
\hline & Meliolales & Meliolaceae & Meliola Fr. & $\begin{array}{l}\text { M. anfracta Dilcher [Now: } \\
\text { Meliolinites anfractus } \\
\text { (Dilcher) Kalgutkar \& } \\
\text { Janson.], M. spinksii Dilcher } \\
\text { [Now: Meliolinites spinksii } \\
\text { (Dilcher) Selkirk] }\end{array}$ \\
\hline & Microthyriales & Microthyriaceae & $\begin{array}{l}\text { Trichopeltina } \\
\text { Theiss. }\end{array}$ & T. exporrecta Dilcher \\
\hline & Mycocaliciales & Mycocaliciaceae & $\begin{array}{l}\text { Chaenothecopsis } \\
\text { Vain. }\end{array}$ & $\begin{array}{l}\text { C. bitterfeldensis Rikkinen } \\
\& \text { Poinar }\end{array}$ \\
\hline & Pleosporales & Didymellaceae & Epicoccum Link & $\begin{array}{l}\text { E. deccanense R. Srivast., } \\
\text { Kapgate \& S. Chatterjee }\end{array}$ \\
\hline & & Pleosporaceae & $\begin{array}{l}\text { Alternaria Nees } \\
\text { ex Fr. }\end{array}$ & $\begin{array}{l}\text { A. malayensis Trivedi \& } \\
\text { C.L. Verma [Now: } \\
\text { Pluricellaesporites } \\
\text { malayensis (Trivedi \& C.L. } \\
\text { Verma) Kalgutkar \& } \\
\text { Janson.] }\end{array}$ \\
\hline & & Torulaceae & $\begin{array}{l}\text { Torula Pers. ex } \\
\text { Fr. }\end{array}$ & $\begin{array}{l}\text { T. globulifera Casp., } T \text {. } \\
\text { heteromorpha Casp., } T \text {. } \\
\text { mengeana Casp. \& R. Klebs } \\
\text { in Casp. }\end{array}$ \\
\hline & Sporidesmiales & Sporidesmiaceae & $\begin{array}{l}\text { Sporidesmium } \\
\text { Link ex Fr. }\end{array}$ & S. henryense Dilcher \\
\hline & Taphrinales & Protomycetaceae & $\begin{array}{l}\text { Protomyces } \\
\text { Unger }\end{array}$ & P. protogenes $\mathrm{W} . \mathrm{Sm}$. \\
\hline & Trichosphaeriales & Trichosphaeriaceae & $\begin{array}{l}\text { Brachysporium } \\
\text { Sacc. }\end{array}$ & $\begin{array}{l}\text { B. minutum Trivedi \& C.L. } \\
\text { Verma [Now: } \\
\text { Pluricellaesporites minutus } \\
\text { (Trivedi \& C.L. Verma) ex } \\
\text { Kalgutkar \& Janson.] }\end{array}$ \\
\hline & $\begin{array}{l}\text { Dothideomycetes } \\
\text { family incertae } \\
\text { sedis }\end{array}$ & Vizellaceae & Vizella Sacc. & $\begin{array}{l}V \text {. discontinua Selkirk, } V \text {. } \\
\text { memorabilis (Dilcher) } \\
\text { Selkirk }\end{array}$ \\
\hline & Incertae sedis & Incertae sedis & $\begin{array}{l}\text { Desmidiospora } \\
\text { Thaxt. } \\
\text { Manginula } \mathrm{G} . \\
\text { Arnaud }\end{array}$ & $\begin{array}{l}\text { D. marginiconvoluta } \\
\text { Kalgutkar } \\
\text { M. maegdefraui Lange } \\
\text { [Now: Entopeltacites } \\
\text { maegdefraui (Lange) } \\
\text { Selkirk], M. memorabilis }\end{array}$ \\
\hline
\end{tabular}


Table 6 Continued.

\begin{tabular}{|c|c|c|c|c|}
\hline Phylum & Order & Family & Modern genera & Fossil species \\
\hline & & & & $\begin{array}{l}\text { (Dilcher) Lange [Now: Vizella } \\
\text { memorabilis (Dilcher) } \\
\text { Selkirk], M. osbornii Lange } \\
\text { [Now: Entopeltacites osbornii } \\
\text { (Lange) Selkirk] }\end{array}$ \\
\hline & & & Sarcophoma & S. deccani R.B. Singh \& G.V. \\
\hline & & & Höhn. & Patil \\
\hline & & & $\begin{array}{l}\text { Tetracoccosporiu } \\
m \text { Szabó }\end{array}$ & T. eоcenum Biradar \& Mahab. \\
\hline & & & $\begin{array}{l}\text { Monotosporella } \mathrm{S} . \\
\text { Hughes }\end{array}$ & $\begin{array}{l}\text { M. doerfeltii Sadowski, } \\
\text { Beimforde, Gube \& A.R. } \\
\text { Schmidt }\end{array}$ \\
\hline & & & $\begin{array}{l}\text { Rhexoampullifera } \\
\text { (M.B. Ellis) P.M. }\end{array}$ & $\begin{array}{l}\text { R. stogieana M.J. Pound, } \\
\text { J.M.K. O'Keefe, N.B. Nuñez }\end{array}$ \\
\hline & & & Kirk \& C.M. Kirk & Otaño, J.B. Riding, R. sufflata \\
\hline & & & & $\begin{array}{l}\text { M.J. Pound, J.M.K. O'Keefe, } \\
\text { N.B. Nuñez Otaño, J.B. Riding }\end{array}$ \\
\hline \multirow[t]{7}{*}{ Basidiomycota } & Agaricostilbales & Chionosphaeraceae & $\begin{array}{l}\text { Stilbum Tode ex } \\
\text { Fr. }\end{array}$ & S. succini Casp. \\
\hline & Boletales & Sclerodermataceae & $\begin{array}{l}\text { Scleroderma } \\
\text { Pers. }\end{array}$ & S. echinosporites Rouse \\
\hline & Cantharellales & Hydnaceae & $\begin{array}{l}\text { Hydnum L. ex } \\
\text { Fr. }\end{array}$ & H. argillae R. Ludw. \\
\hline & Geastrales & Geastraceae & Geastrum Pers. & $\begin{array}{l}\text { G. tepexense Magallon-Puebla } \\
\text { \& Cevallos-Ferriz }\end{array}$ \\
\hline & Nidulariales & Nidulariaceae & $\begin{array}{l}\text { Nidula V.S. White } \\
\text { Cyathus Haller }\end{array}$ & $\begin{array}{l}\text { N. baltica Poinar } \\
\text { C. dominicanus Poinar }\end{array}$ \\
\hline & $\begin{array}{l}\text { Polyporales } \\
\text { Urocystidales }\end{array}$ & $\begin{array}{l}\text { Polyporaceae } \\
\text { Urocystidaceae }\end{array}$ & $\begin{array}{l}\text { Fomes (Fr.) Fr. } \\
\text { Mundkurella } \\
\text { Thirum. }\end{array}$ & $\begin{array}{l}\text { F. idahoensis R.W. Br. } \\
\text { M. mohgaoensis Chitaley \& } \\
\text { Yawale }\end{array}$ \\
\hline & Ustilaginales & Ustilaginaceae & $\begin{array}{l}\text { Ustilago (Pers.) } \\
\text { Roussel }\end{array}$ & $\begin{array}{l}\text { U. deccani Chitaley \& Yawale } \\
\text { [Now: Inapertisporites } \\
\text { deccani (Chitaley \& Yawale) } \\
\text { Kalgutkar \& Janson.] }\end{array}$ \\
\hline $\begin{array}{l}\text { Chytridiomyco } \\
\text { ta }\end{array}$ & Chytridiales & Chytriomycetaceae & $\begin{array}{l}\text { Entophlyctis A. } \\
\text { Fisch. }\end{array}$ & $\begin{array}{l}\text { E. willoughbyi W.H. Bradley } \\
\text { [Now: Desmidiospora } \\
\text { willoughbyi (W.H. Bradley) } \\
\text { D.L.E. Glass, D.D. Br. \& } \\
\text { Elsik] }\end{array}$ \\
\hline $\begin{array}{l}\text { Fungi incertae } \\
\text { sedis }\end{array}$ & & & $\begin{array}{l}\text { Patoullardiella } \\
\text { Speg. }\end{array}$ & P. imbricata Dilcher \\
\hline
\end{tabular}

Outline of fungus-like organisms

Obazoa Brown et al.

Opisthokonta Cavalier-Smith

Holomycota Liu et al. $=$ Nucletmycea Brown et al.

Nucleariae Tedersoo et al.

Fonticulida Tedersoo et al.

Fonticulea Tedersoo et al.

Fonticulida Cavaler-Smith

Fonticulidae Worley, Raper \& Hohl

Fonticula Worley, Raper \& M. Hohl 
Diaphoretickes Adl et al.

S A R Burki et al. emend. Adl et al.

Rhizaria Cavalier-Smith

Endomyxa Cavalier-Smith

Phytomyxea Engler \& Prantl

Plasmodiophorida Cook

Plasmodiophoridae Loeblich \& Tappan

Ligniera Maire \& A. Tison

Plasmodiophora Worona

Polymyxa Ledingham

Sorosphaerula Neuh. \& Kirchm.

Spongospora Brunch.

Woronina Cornu

Phagomyxida Cavalier-Smith

Phagomyxidae Cavalier-Smith

Maullinia I. Maier, E.R. Parodi, Westermeier \& D.G. Müll

Phagomyxa Karling

Cercozoa Cavalier-Smith

Sainouroidea Schuler et al.

Guttulinopsidae L.S. Olive

Guttulinopsis E.W. Olive

Straminipila M.W. Dick

Labyrinthulomycota Whittaker

Labyrinthulomycetes Dick

Labyrinthulales E.A. Bessey

Aplanochytriaceae Leander ex Cavalier-Smith

Aplanochytrium Bahnweg \& Sparrow

Stellarchytriaceae Bennett et al. ad int.

Stellarchytrium FioRito \& Leander

Labyrinthulaceae Haeckel

Labyrinthula Cienk.

Oblongichytridiales Bennett et al. ad int.

Oblongichytridiaceae Cavalier-Smith

Oblongichytrium R. Yokoy. \& D. Honda

Thraustochytriales Sparrow

Althornidiaceae Jones and Alderman

Althornia E.B.G. Jones \& Alderman

Thraustochytriacae Sparrow ex Cejp

Aurantiochytrium R. Yokoy. \& D. Honda

Botryochytrium R. Yokoy., Salleh \& D. Honda

Japanochytrium

Monorhizochytrium K. Doi \& D. Honda

Parietichytrium R. Yokoy., Salleh \& D. Honda

Schizochytrium S. Goldst. \& Belsky ex Raghuk. 
Sicyoidochytrium R. Yokoy., Salleh \& D. Honda

Thraustochytrium Sparrow

Ulkenia A. Gaertn. ex M.W. Dick

Amphitremida Gomaa et al.

Amphitremidae Poch

Amphitrema Archer

Archerella Loeblich \& Tappan

Paramphitrema Valkanov

Diplophrydae Cavalier-Smith

Diplophrys J.S.F. Barker

Amphifilida Cavalier-Smith

Amphifilidae Cavalier-Smith

Amphifila Caval.-Sm.

Sorodiplophryidae Cavalier-Smith

Fibrophrys Takahashi et al.

Sorodiplophrys L.S. Olive \& Dykstra

Hyphochytriomycota Whittaker

Hyphochytriomycetes Sparrow

Hyphochytriales Bessey ex Sparrow

Hyphochytriaceae Fischer

Canteriomyces Sparrow

Cystochytrium Ivimey Cook

Hyphochytrium Zopf

Rhizidiomycetaceae Karling ex Kirk, Cannon \& David

Latrostium Zopf

Reessia Fisch

Rhizidiomyces Zopf

Oomycota Arx

Peronosporomycetes M.W. Dick

Albuginales Thines

Albuginaceae Schroet.

Albugo (Pers.) Roussel (40)

Pustula Thines (11)

Wilsoniana Thines (5)

Peronosporales A.N. Beketov

Peronosporaceae de Bary

Basidiophora Roze \& Cornu (3)

Baobabopsis R.G. Shivas, Y.P. Tan, Telle \& Thines (2)

Benua Constant. (1)

Bremia Regel (15)

Calycofera R. Bennett \& Thines (2)

Eraphthora Telle \& Thines (1)

Graminivora Thines (1)

Halophytophthora H.H. Ho \& S.C. Jong (6) 
Hyaloperonospora Constant. (35)

Nothophytophthora T. Jung, Scanu, Bakonyi \& M. Horta Jung (6)

Novotelnova Voglmayr \& Constant. (1)

Paraperonospora Constant. (9)

Perofascia Constant. (2)

Peronospora Corda (350)

Peronosclerospora (S. Ito) Hara (15)

Phytophthora de Bary (150)

Phytopythium Abad, de Cock, Bala, Robideau, A.M. Lodhi \& Lévesque (25)

Plasmopara J. Schröt. (150)

Plasmoverna Constant., Voglmayr, Fatehi \& Thines (7)

Poakatesthia Thines (1)

Protobremia Voglmayr, Riethm., Göker, Weiss \& Oberw. (1)

Pseudoperonospora Rostov. (9)

Sclerophthora Thirum., C.G. Shaw \& Naras. (5)

Sclerospora J. Schröt. (2)

Viennotia Göker, Voglmayr, Riethm., M. Weiss \& Oberw. (1)

\section{Pythiaceae Schroet.}

Elongisporangium Uzuhashi, Tojo \& Kakish. (5)

Globisporangium Uzuhashi, Tojo \& Kakish. (70)

Lagena Vanterp. \& Ledingham (1)

Lagenidium Schenk (40)

Myzocytiopsis M.W. Dick (18)

Myzocytium Schenk (2)

Pilasporangium (Uzuhashi \& Tojo) Uzuhashi, Tojo \& Kakish. (1)

Pythiogeton Minden (16)

Pythium Pringsh. (200)

\section{Salisapiliaceae}

Salisapilia Hulvey, Nigrelli, Telle, Lamour \& Thines (9)

Rhipidiales M.W. Dick

Rhipidiaceae Cejp

Aqualinderella Emerson \& Weston (1)

Araiospora Thaxt. (4)

Mindeniella Kanouse (2)

Nellymyces A. Batko (1)

Rhipidium Cornu (6)

Sapromyces Fritsch (4)

Salispinaceae R. Bennett \& Thines

Salispina Marano, A.L. Jesus \& Pires-Zottar. (4)

Peronosporomycetes genera incertae sedis

Kawakamia Miyabe (4)

Paralagenidium Grooters, C.F.J. Spies, de Cock \& Lévesque (2)

Trachysphaera Tabor \& Bunting (1)

Saprolegniomycetes Thines \& Beakes

Leptomitales Kanouse

Atkinsiellaceae Sparrow 
Atkinsiella Vishniac (1)

Bolbea Buaya \& Thines (1)

\section{Leptomitaceae Kütz}

Apodachlya Pringsh. (5)

Apodachlyella Indoh (1)

Blastulidium Pérez (1)

Leptomitus C. Agardh (11)

\section{Ectrogellaceae Cejp}

Crypticola Humber, Frances \& A.W. Sweeney (1)

Ectrogella Zopf (8)

Lagenisma Schnepf (1)

Saprolegniales K. Prantl

Achlyaceae ined.

Achlya Nees (80)

Brevilegnia Coker \& Couch (16)

Dictyuchus Leitg. (9)

Thraustotheca Humphrey (4)

Saprolegniaceae Warm.

Aplanopsis Höhnk (1)

Calyptralegnia Coker (3)

Couchia W.W. Martin (3)

Isoachlya Kauffmann (9)

Newbya M.W. Dick \& M.A. Spencer (13)

Protoachlya Coker (7)

Pythiopsis de Bary (7)

Saprolegnia Nees (80)

Scoliolegnia M.W. Dick (5)

Verrucalvaceae M.W. Dick

Aphanomyces de Bary (40)

Aquastella Glockling \& D.P. Molloy (2)

Geolegnia Coker (4)

Leptolegnia de Bary (9)

Pachymetra B.J. Croft \& M.W. Dick (1)

Plectospira Drechsler (4)

Verrucalvus P. Wong \& M.W. Dick (1)

Saprolegniomycetes genera incertae sedis

Aphanomycopsis Scherff. (6)

Brevilegniella M.W. Dick (1)

Cornumyces M.W. Dick (8)

Clamydomycium M.W. Dick (7)

Ducellieria Teiling (1)

Eurychasmopsis Canter \& M.W. Dick (1)

Leptolegniella Huneycutt (7)

Nematophthora Kerry \& D.H. Crump (1)

Pythiella Couch (3)

Sommerstorffia Arnaudov (1) 
Oomycota orders incertae sedis Anisolpidiales M.W. Dick Anisolpidiaceae Karling Anisolpidium Karling (7)

Diatomophthoraceae Buaya \& Thines Diatomophthora Buaya \& Thines (3)

Eurychasmales Sparrow

Eurychasmataceae Petersen Eurychasma Magnus (3)

Haliphthorales ined.

Haliphthoraceae Vishniac

Halioticida Muraosa \& Hatai (1)

Halocrusticida K. Nakam. \& Hatai (7)

Haliphthoros Vishniac (3)

Haptoglossales M.W. Dick

Haptoglossaceae M.W. Dick

Haptoglossa Drechsler (12)

Miraculales ined.

Miraculaceae Buaya, Hanic \& Thines

Miracula Buaya, Hanic \& Thines (2)

Olpidiopsidales M.W. Dick

Olpidiopsidaceae Sparrow

Olpidiopsis Cornu (12)

Pontismatales Thines

Postismataceae H.E. Petersen

Petersenia Sparrow (3)

Pontisma H.E. Petersen (10)

Sirolpidium H.E. Petersen (7)

Rozellopsidales M.W. Dick

Rozellopsidaceae M.W. Dick

Rozellopsis Karling (5)

Amorphea Adl et al.

Amoebozoa Lühe

Evosea Kang et al.

Eumycetozoa L.S. Olive

Dictyosteliomycetes Doweld

Acytosteliales S. Baldauf, S. Sheikh \& Thulin

Acytosteliaceae Raper ex Raper \& Quinlan

Acytostelium Raper

Heterostelium S. Baldauf, S. Sheikh \& Thulin

Rostrostelium S. Baldauf, S. Sheikh \& Thulin 
Cavenderiaceae S. Baldauf, S. Sheikh \& Thulin

Cavenderia S. Baldauf, S. Sheikh \& Thulin

Dictyosteliales L.S. Olive ex P.M. Kirk et al.

Dictyosteliaceae Rostaf. ex Cooke

Dictyostelium Bref.

Polysphondylium Bref.

Raperosteliaceae S. Baldauf, S. Sheikh \& Thulin

Hagiwaraea S. Baldauf, S. Sheikh \& Thulin

Raperostelium S. Baldauf, S. Sheikh \& Thulin

Speleostelium S. Baldauf, S. Sheikh \& Thulin

Tieghemostelium S. Baldauf, S. Sheikh \& Thulin

Dictyosteliales genus incertae sedis

Coremiostelium S. Baldauf, S. Sheikh, Thulin \& Spiegel

Dictyosteliomycetes genera incertace sedis

Coenonia Tiegh.

Synstelium S. Baldauf, S. Sheikh \& Thulin

Ceratiomyxomycetes D. Hawksw., B. Sutton \& Ainsw. in Leontyev et al. (2019)

Ceratiomyxales G.W. Martin ex M.L. Farr \& Alexop.

Ceratiomyxaceae J. Schröt.

Ceratiomyxa J. Schröt.

Protosporangiaceae Leontyev, Stephenson, Schnittler, Shchepin, Novozhilov

Clastostelium L.S. Olive \& Stoian.

Protosporangium L.S. Olive \& Stoian.

Myxomycetes G. Winter

Lucisporomycetidae Leontyev, Schnittler, S.L. Stephenson, Novozhilov \& Shchepin

Cribrariales T. Macbr.

Cribrariaceae Corda

Cribraria Pers.

Licaethalium Rostaf.

Lindbladia Fr.

Reticulariales Leontyev, Schnittler, S.L. Stephenson, Novozhilov \& Shchepin

Reticulariaceae Chevall. ex Corda

Alwisia Berk. \& Broome (6)

Lycogala Adans.

Reticularia Bull.

Tubifera J.F. Gmel.

Siphoptychium Rostaf.

Thecotubifera Leontyev, Schnittler, S.L. Stephenson \& Novozh.

Liceales E. Jahn

Liceaceae Chevall.

Licea Schrad.

Listerella E. Jahn 
Trichiales T. Macbr.

Dianemataceae T. Macbr.

Calomyxa Nieuwl.

Dianema Rex

Dictydiaethalium Rostaf.

Prototrichia Rostaf.

Trichiaceae Chevall.

Arcyodes O.F. Cook

Arcyria F.H. Wigg.

Cornuvia Rostaf.

Hemitrichia Rostaf.

Metatrichia Ing

Oligonema Rostaf.

Perichaena Fr.

Trichia Haller

Lucisporomycetidae genera incertae sedis

Arcyriatella Hochg. \& Gottsb.

Calonema Morgan

Minakatella G. Lister

Trichioides Novozh., Hoof \& Jagers

Columellomycetidae Leontyev, Schnittler, S.L. Stephenson, Novozhilov \& Shchepin Echinosteliopsidales Shchepin, Leontyev, Schnittler, S.L. Stephenson, Novozhilov Echinosteliopsidaceae L.S. Olive

Echinosteliopsis Reinhardt \& L.S. Olive

Echinosteliales G.W. Martin

Echinosteliaceae Rostaf. ex Cooke

Barbeyella Meyl.

Echinostelium de Bary

Semimorula E.F. Haskins, McGuinn. \& C.S. Berry

Clastodermatales Leontyev, Schnittler, S.L. Stephenson, Novozhilov \& Shchepin Clastodermataceae Alexop. \& T.E. Brooks

Clastoderma A. Blytt.

Meridermatales Leontyev, Schnittler, S.L. Stephenson, Novozhilov \& Shchepin

Meridermataceae Leontyev, Schnittler, S.L. Stephenson, Novozhilov \& Shchepin Meriderma Mar. Mey. \& Poulain

Stemonitidales T. Macbr.

Amaurochaetaceae Rostaf. ex Cooke

Amaurochaete Rostaf.

Brefeldia Rostaf.

Comatricha Preuss

Enerthenema Bowman

Paradiacheopsis Hertel.

Stemonaria Nann.-Bremek., R. Sharma \& Y. Yamam.

Stemonitopsis (Nann.-Bremek.) Nann.-Bremek. 
Stemonitidaceae Fr.

Macbrideola H.C. Gilbert

Stemonitis Gled.

Symphytocarpus Ing \& Nann.-Bremek.

Physarales T. Macbr.

Lamprodermataceae T. Macbr.

Collaria Nann.-Bremek.

Colloderma G. Lister

Diacheopsis Meyl.

Elaeomyxa Hagelst.

Lamproderma Rostaf.

Didymiaceae Rostaf. ex Cooke

Diderma Pers.

Didymium Schrad.

Lepidoderma de Bary

Mucilago Battarra

Physaraceae Chevall.

Badhamia Berk.

Craterium Trentep.

Fuligo Haller

Kelleromyxa Eliasson

Leocarpus Link

Physarella Peck.

Physarina Höhn.

Physarum Pers.

Willkommlangea Kuntze

Columellomycetidae genera incertae sedis

Diachea Fr.

Leptoderma G. Lister

Paradiachea Hertel

Protophysarum M. Blackw. \& Alexop.

Trabrooksia H.W. Keller

Variosea Cavalier-Smith et al.

Protosteliida Olive \& Stoian. sensu Shadwick et Spiegel in Adl et al. 2012

Protosteliidae Olive \& Stoian., emend Spiegel

Protostelium L.S. Olive \& Stoian.

Fractovitellida Lahr et al. sensu Kang et al. 2017

Schizoplasmodiidae Shadwick \& Spiegel in Adl et al.

Ceratiomyxella L.S. Olive \& Stoian.

Nematostelium L.S. Olive \& Stoian.

Schizoplasmodium L.S. Olive \& Stoian.

Soliformoviidae Lahr \& Katz

Soliformovum Spiegel

Cavosteliida Shadwick \& Spiegel in Adl et al. 
Cavosteliidae S.L. Olive

Cavostelium S.L. Olive

Schizoplasmodiopsis S.L. Olive

Tychosporium Spiegel

Tubulinea Smirnov et al.

Elardia Kang et al.

Euamoebida Lepşi

Copromyxidae L.S. Olive \& Stoian.

Copromyxa Zopf

Discosea Cavalier-Smith et al. sensu Smirnov et al. 2011

Flabellinea Smirnov et al.

Thecamoebida Schaeffer

Sappinia P.A. Dang.

Vannellida Smirnov et al.

Protosteliopsis L.S. Olive \& Stoian.

Centramoebia Cavalier-Smith et al.

Acanthopodida Page

Acanthamoeba Volkonsky

Luapelamoeba Shadwick et al.

Pellitida Smirnov \& Cavalier-Smith sensu Kang et al. 2017

Endostelium L.S. Olive, W.E. Benn. \& Deasey

Discoba Simpson in Hampl et al.

Heterolobosea Page \& Blanton

Tetramitia Cavalier-Smith

Eutetramitia Hanousková et al.

Acrasidae Poche

Acrasis Tiegh. (incl. Pocheina A.R. Loebl. \& Tappan)

\section{Discussion}

Alternative classification of Leotiomycetes (Authors: A.H. Ekanayaka \& K.D. Hyde)

The arrangement of Leotiomycetes in Ekanayaka et al. (2019) and Johnston et al et al. (2019) are based on morphological interpretations and phylogenies using different data, however, they are generally congruent. Johnston et al. (2019) used three phylogenetic reconstructions, one based on 3156 single-copy genes for 49 taxa, the second based on 15 genes for 279 taxa, and the third based on ITS alone for 568 taxa. Ekanayaka et al. (2019) used five genes with 482 taxa. There are, however, some differences and therefore the outline from Ekanayaka et al. (2019) with modifications is given below with notes. Understandably, if different taxa were used in the Ekanayaka et al. (2019) and Johnston et al. (2019) phylogenies, different conclusions have been drawn and therefore the classification of Leotiomycetes is not settled. We hope that by providing an alternative outline, this will initiate positive discussion and further research with fresh collections to resolve inconsistencies. However, with insufficient taxa in this class having been sequenced it will take several years before the classification is stabilized.

Taxa with notes in this section are indicated by $\#$.

Chaetomellales Crous \& Denman

Chaetomellaceae Baral, P.R. Johnst. \& Rossman 
Chaetomella Fuckel (26)

Corniculariella P. Karst. (1)

Pilidium Kunze (23)

Sphaerographium Sacc. (23)

Synchaetomella Decock \& Seifert (3)

Xeropilidium Baral \& Pärtel (1)

Cyttariales Luttr. ex Gamundí

Cordieritidaceae Sacc."

Ameghiniella Speg. (2)

Annabella Fryar, Haelew., \& D.E.A. Catches. (1)

Austrocenangium Gamundí (2)

Cordierites Mont. (5)

Diplocarpa Massee (2)

Diplolaeviopsis Giralt \& D. Hawksw. (3)

Gelatinopsis Rambold \& Triebel (8)

Ionomidotis E.J. Durand ex Thaxt. (13)

Llimoniella Hafellner \& Nav.-Ros. (19)

Macroskyttea Etayo, Flakus, Suija \& Kukwa (1)

Midotiopsis Henn. (2)

Phaeangella (Sacc.) Massee (11)

Rhymbocarpus Zopf (12)

Rhizocladosporium Crous \& U. Braun (1)

Skyttea Sherwood, D. Hawksw. \& Coppins (30)

Skyttella D. Hawksw. \& R. Sant. (2)

Sabahriopsis Crous \& M.J. Wingf. (1)

Thamnogalla D. Hawksw. (1)

Unguiculariopsis Rehm (25)

Cyttariaceae Lév.

Cyttaria Berk. (13)

Deltopyxidaceae Ekanayaka \& K.D. Hyde ${ }^{\#}$

Deltopyxis Baral \& G. Marson (1)

Phaeopyxis Rambold \& Triebel (1)

Erysiphales Gwynne-Vaughan ${ }^{\#}$

Amorphothecaceae Parbery (= Myxotrichaceae Locq. ex Currah fide Ekanayaka et al. 2019)

Amorphotheca Parbery (1)

Brefeldochium Verkley (1)

Byssoascus Arx (2)

Myxotrichum Kunze (12)

Oidiodendron Robak (ca. 30)

Polydesmia Boud. (7)

Hormoconis Arx \& G.A. de Vries (2)

Erysiphaceae Tul. \& C. Tul.

Arthrocladiella Vassilkov (1)

Blumeria Golovin ex Speer (1)

Brasiliomyces Viégas (6)

Bulbomicroidium Marm., S. Takam. \& U. Braun (1)

Caespitotheca S. Takam. \& U. Braun (1) 
Cystotheca Berk. \&M.A. Curtis (9)

Erysiphe R. Hedw. ex DC. (478)

Golovinomyces (U. Braun) V.P. Heluta (66)

Leveillula G. Arnaud (49)

Microidium (To-anun \& S. Takam.) To-anun \& S. Takam. (3)

Neoerysiphe U. Braun (15)

Oidiopsis Scalia (ca. 12)

Oidium Link (ca. 200+)

Ovulariopsis Pat. \& Har. (ca. 13)

Parauncinula S. Takam. \& U. Braun (4)

Phyllactinia Lév. (117)

Pleochaeta Sacc. \& Speg. (5)

Podosphaera Kunze (124)

Pseudoidium Y.S. Paul \& J.N. Kapoor (ca. 80)

Queirozia Viégas \& Cardoso (1)

Sawadaea Miyabe (10)

Takamatsuella U. Braun \& A. Shi (1)

Typhulochaeta S. Ito \& Hara (ca. 4)

Helotiales Nannf. ex Korf \& Lizoň

Arachnopezizaceae Hosoya, J.G. Han \& Baral

Arachnopeziza Fuckel (15)

Arachnoscypha Boud. (3)

Austropezia Spooner (1)

Eriopezia (Sacc.) Rehm (30)

Durella Tul. \& C. Tul. (5)

Bryoglossaceae Ekanayaka \& K.D. Hyde

Bryoclaviculus L. Ludw., P.R. Johnst. \& Steel (1)

Bryoglossum Redhead (2)

Neocudoniella S. Imai (2)

Chlorociboriaceae Baral \& P.R. Johnst."

Chlorociboria Seaver ex C.S. Ramamurthi, Korf \& L.R. Batra (22)

Chlorospleniaceae Ekanayaka \& K.D. Hyde

Chlorosplenium Fr. (5)

Chrysodiscaceae Baral \& Haelew.

Chrysodisca Baral, Polhorský \& G. Marson (1)

Discinellaceae Ekanayaka \& K.D. Hyde

Articulospora Ingold (ca. 5)

Acidea Hujslová \& M. Kolařík (1)

Cladochasiella Marvanová (1)

Discinella Boud. (ca. 15)

Fontanospora Dyko (1)

Gyoerffyella Kol (10)

Lemonniera De Wild. (7)

Naevala B. Hein (6)

Margaritispora Ingold (1)

Pezoloma Clem. (ca. 15)

Tetrachaetum Ingold (3) 
Drepanopezizaceae Baral (=Drepanopezizaceae Bat. \& H. Maia; Nom. inval., Arts 32.1(c), 36, 39.1 (Melbourne))

Blumeriella Arx (= Higginsia Nannf.; = Phloeosporella Höhn., = Microgloeum Petr.) (7)

Diplocarpon F.A. Wolf (7)

Drepanopeziza (Kleb.) Höhn. (= Gloeosporidiella Petr.) (5)

Felisbertia Viégas (7)

Leptotrochila P. Karst. (= Ephelina Sacc.; = Fabraea Sacc.; = Sporonema Desm.) (15)

Pseudopezicula Korf (2)

Spilopodia Boud. (= Holmiodiscus Svrček; = Melanodiscus Höhn.) (ca. 4)

Spilopodiella E. Müll. (1)

Thedgonia B. Sutton (6)

Gelatinodiscaceae S.E. Carp

Ascocoryne J.W. Groves \& D.E. Wilson (= Didymocoryne Sacc. \& Trotter) (8)

Ascotremella Seaver (2)

Chloroscypha Seaver (14)

Dimorphospora Tubaki (1)

Helicodendron Peyronel (3)

Neobulgaria Petr. (11)

Phaeangellina Dennis (1)

Skyathea Spooner \& Dennis (1)

\section{Godroniaceae Baral}

Ascocalyx Naumov (4)

Atropellis Zeller \& Goodd. (4)

Godronia Moug. \& Lév. (ca. 30)

Gremmeniella M. Morelet (3)

Grovesiella M. Morelet (2)

Helotiaceae Rehm (= Roesleriaceae Y.J. Yao \& Spooner fide Ekanayaka et al. 2019)

Amylocarpus Curr. (1)

Asterocalyx Höhn. (1)

Ascoconidium Seaver (3)

Bryoscyphus Spooner (19)

Calycella sensu (Sacc.) Sacc. (1)

Crocicreas Fr. (5)

Eubelonis Clem. (2)

Cudoniella Sacc. (31)

Cyathicula De Not. (30)

Dicephalospora Spooner (4)

Endoscypha Syd. (1)

Discorehmia Kirschst. (5)

Glarea Bills \& Paláez (2)

Gloeotinia M. Wilson, Noble \& E.G. Gray (2)

Helicocentralis Sri-indr., Chuaseehar., Boonyuen, K. Yamag., Suetrong \& C.K.M. Tsui (1)

Hymenoscyphus Gray (170)

Hymenotorrendiella P.R. Johnst., Baral \& R. Galán (9)

Lanzia Sacc. (55)

Muscicola Velen. (1)

Mytilodiscus Kropp \& S.E. Carp. (1)

Ombrophila Fr. (11) 
Phaeohelotium Kanouse (41)

Pirottaea Sacc. (28)

Pithyella Boud. (8)

Pseudoniptera Velen. (25)

Roesleria Thüm. \& Pass. (ca. 10)

Roeslerina Redhead (3)

Symphyosirinia E.A. Ellis (6)

Tatraea Svrcek (2)

Torrendiella Boud. \& Torrend (3)

Xylogramma Wallr. (18)

\section{Heterosphaeriaceae Rehm}

Heterosphaeria Grev. (7)

Hyaloscyphaceae Nannf.

Ambrodiscus S.E. Carp. (1)

Aeruginoscyphus Dougoud (7)

Arbusculina Marvanová \& Descals (3)

Clathrosphaerina Beverw. (2)

Curviclavula G. Delgado, F.A. Fernández \& A.N. Mill. (1)

Dimorphotricha Spooner (1)

Echinula Graddon (1)

Glutinomyces Nor. Nakam. (1)

Graddonidiscus Raitv. \& R. Galán (3)

Grahamiella Spooner (3)

Hegermila Raitv. (4)

Hyaloscypha Boud. (45)

Hyalodendriella Crous (1)

Hyphopeziza J.G. Han, Hosoya \& H.D. Shin (1)

Incrupila Raitv. (10)

Meliniomyces Hambl. \& Sigler (3)

Olla Velen. (2)

Parachnopeziza Korf (8)

Polaroscyphus Huhtinen (1)

Proprioscypha Spooner (1)

Protounguicularia Raitv. \& R. Galán (10)

Pseudaegerita J.L. Crane \& Schokn. (7)

Psilocistella Svrcek (10)

Rhizoscyphus W.Y. Zhuang \& Korf (1)

Scytalidium Pesante (ca. 30)

Thindiomyces Arendh. \& R. Sharma (1)

Unguiculariella K.S. Thind \& R. Sharma (1)

Unguiculella Höhn. (17)

Hydrocinaceae Ekanayaka \& K.D. Hyde

Clathrosporium Nawawi \& Kuthub. (1)

Filosporella Nawawi (6)

Hydrocina Scheuer (1)

Varicosporium W. Kegel (11)

Xerombrophila Baral (1)

Lachnaceae Raitv. 
Albotricha Raitv. (19)

Asperopilum Spooner (1)

Belonidium Mont. \& Dur. (1)

Brunnipila Baral (10)

Capitotricha (Raitv.) Baral (10)

Crucellisporiopsis Nag Raj (3)

Crucellisporium M.L. Farr (3)

Dasyscyphella Tranzschel (1)

Erioscyphella Kirschst. (10)

Incrucipulum Baral (6)

Lachnellula P. Karst. (40)

Lachnum Retz. (50)

Lachnopsis Guatim., R.W. Barreto \& Crous (1)

Neodasyscypha Suková \& Spooner (2)

Perrotia Boud. (19)

Proliferodiscus J.H. Haines \& Dumont (8)

Tubolachnum Velen. (2)

Velebitea I. Kušan, Matočec \& Jadan (1)

Loramycetaceae Dennis ex Digby \& Goos

Acidomelania E. Walsh \& N. Zhang (1)

Loramyces W. Weston (2)

Obtectodiscus E. Müll., Petrini \& Samuels (2)

Mitrulaceae Rchb.

Mitrula Fr. (8)

Mollisiaceae Rehm

Bulbomollisia Graddon (1)

Cystodendron Bubák (2)

Discocurtisia Nannf. (12)

Mollisia (Fr.) P. Karst. (130)

Neotapesia E. Müll. \& Hütter (3)

Niptera Fr. (10)

Nipterella Starbäck ex Dennis (2)

Phialocephala W.B. Kendr. (37)

Pseudonaevia Dennis \& Spooner (2)

Pyrenopeziza Fuckel (3)

Sarconiptera Raitv. (1)

Scutobelonium Graddon (1)

Scutomollisia Nannf. (14)

Tapesia (Pers.) Fuckel (ca. 25)

Trimmatostroma Corda (30)

Variocladium Descals \& Marvanová (1)

Ploettnerulaceae Kirschst.

Cadophora Lagerb. \& Melin (15)

Collembolispora Marvanová \& Pascoal (2)

Cylindrosporium Grev. (3)

Dennisiodiscus Svrcek (10)

Lasiomollisia Raitv. \& Vesterh. (1)

Mastigosporium Riess (4)

Mycochaetophora Hara \& Ogawa (2) 
Nothophacidium J. Reid \& Cain (1)

Oculimacula Crous \& W. Gams (6)

Peltigeromyces A. Möller (3)

Ploettnerula Kirschst. (1)

Pseudopeziza Fuckel (4)

Rhexocercosporidium U. Braun (2)

Rhynchosporium Heinsen ex A.B. Frank (5)

Ypsilina J. Webster, Descals \& Marvanová (1)

Solenopeziaceae Ekanayaka \& K.D. Hyde

Geniculospora Sv. Nilsson ex Marvanová \& Sv. Nilsson (2)

Graddonia Dennis (7)

Halenospora E.B.G. Jones (1)

Lasiobelonium Ellis \& Everh. (20)

Mycofalcella Marvanová, Om-Kalth. \& J. Webster (2)

Solenopezia Sacc. (7)

Trichopeziza Fuckel (30)

Trichopezizella Dennis ex Raitv. (12)

Tricladium Ingold (25)

Vibrisseaceae Korf

Acephala Grünig \& T.N. Sieber (2)

Cheirospora Moug. \& Fr. (2)

Diplococcium Grove (30)

Fuscosclera Hern.-Restr., J. Mena \& Gené (1)

Gorgoniceps (P. Karst.) P. Karst. (3)

Leucovibrissea (A. Sánchez) Korf (1)

Pocillum De Not. (1)

Strossmayeria Schulzer (= Pseudospiropes M.B. Ellis) (16)

Srinivasanomyces S. Rana \& S.K. Singh (1)

Vibrissea Fr. (34)

Helotiales genera incertae sedis

Aquapoterium Raja \& Shearer (1)

Arboricolonus S. Bien \& Damm (1)

Barrenia E. Walsh \& N. Zhang (2)

Brackelia Zhurb. (1)

Bulgariella P. Karst. (4)

Cecidioskyttea Etayo (1)

Chlorovibrissea L.M. Kohn (4)

Colipila Baral \& Guy García (2)

Connersia Malloch (1)

Cryptocline Petr. (20)

Encoeliopsis Nannf. (4)

Gamarada D.J. Midgley \& Tran-Dinh (1)

Larissia Raitv. (1)

Lemalis Fr. (3)

Libartania Nag Raj (2)

Merodontis Clem. (1)

Mitrulinia Spooner (1)

Mollisiopsis Rehm (7)

Muscia Gizhitsk (1) 
Patellariopsis Dennis (5)

Pestalopezia Seaver (3)

Phacidiella P. Karst. (1)

Pleuroascus Massee \& E.S. Salmon (11)

Pseudomitrula Gamundí (1)

Sambucina Velen. (1)

Sarcomyces Massee (1)

Unguicularia Höhn. (7)

Lahmiales O.E. Erikss.

Lahmiaceae O.E. Erikss.

Lahmia Körb. (1)

Lauriomycetales Hern.-Restr., R.F. Castañeda \& Guarro

Lauriomycetaceae Hern.-Restr., R.F. Castañeda \& Guarro

Lauriomyces R.F. Castañeda (11)

Leotiales Korf \& Lizoň

Cochlearomycetaceae Crous ${ }^{\#}$

Cochlearomyces Crous (1)

Leotiaceae Corda

Geocoryne Korf (2)

Leotia Pers. (4)

Microglossum Gillet (ca. 10)

Thuemenidium Kuntze (1)

Mniaeciaceae Baral

Mniaecia Boud. (= Epiglia Boud.) (6)

Tympanidaceae Baral \& Quijada

Aotearoamyces P.R. Johnst., J.A. Cooper \& Quijada (1)

Claussenomyces Kirschst. (ca. 19)

Collophorina Damm \& Crous (6)

Durandiella Seaver (15)

Gelatinosporium Peck (15)

Myriodiscus Boedijn (1)

Pragmopora A. Massal. (7)

Tympanis Fr. (ca. 27)

Leotiales genus incertae sedis

Gelatinomyces Sanoam., Sanoam., Jitjak, Rodtong \& Whalley (2)

Lichinodiales M. Prieto, M. Schultz, Olariaga \& Wedin

Lichinodiaceae M. Prieto, M. Schultz, Olariaga \&Wedin

Lichinodium Nyl. (4)

Mycosymbioces J.L. Frank (1)

Lichinodiales genera incertae sedis

Epithamnolia Zhurb. (6)

Fulvoflamma Crous (1)

Marthamycetales P.R. Johnst. \& Baral 
Marthamycetaceae Baral, Lantz, Hustad \& Minter

Cyclaneusma DiCosmo, Peredo \& Minter (2)

Marthamyces Minter (13)

Mellitiosporiella Höhn. (2)

Mellitiosporium Corda (11)

Naemacyclus Fuckel (6)

Phragmiticola Sherwood (1)

Propolina Sacc. (1)

Propolis (Fr.) Corda (ca. 9)

Medeolariales Korf ${ }^{\#}$

Ascocorticiaceae J. Schröt.

Ascocorticiellum Jülich \& B. de Vries (3)

Ascocorticium Bref. (8)

Ascosorus P. Henn. \& Ruhland (1)

Ascodichaenaceae D. Hawksw. \& Sherwood

Ascodichaena Butin (2)

Delpinoina Kuntze (2)

Dermateaceae Fr.

Arctomollisia Raitv. (2)

Cashiella Petr. (3)

Davidhawksworthia Crous (1)

Dermea Fr. (25)

Gelatinoamylaria Prasher \& R. Sharma (1)

Neofabraea H.S. Jacks. (7)

Pezicula Tul. \& C. Tul. (50)

Phlyctema Desm. (30)

Pseudofabraea Chen Chen, Verkley \& Crous (1)

Rhizodermea Verkley \& J.D. Zijlstra (1)

Schizothyrioma Höhn. (1)

Verkleyomyces Y. Marín \& Crous (1)

Medeolariaceae Korf

Medeolaria Thaxt. (1)

Medeolariales genera incertae sedis

Coleophoma Höhn. (35)

Parafabraea Chen Chen, Verkley \& Crous (2)

Phacidiales C.E. Bessey

Helicogoniaceae Baral

Eleutheromycella Höhn. (1)

Eleutheromyces Fuckel (1)

Gelatinipulvinella Hosoya \& Y. Otani (2)

Geltingia Alstrup \& D. Hawksw. (1)

Helicogonium W.L. White (19)

Humicolopsis Cabral \& S. Marchand (2)

Phacidiaceae Fr. (= Bulgariaceae Fr.; = Phacidiostromataceae Höhn. fide Jaklitsch et al. 2016a) 
Allantophomopsiella Crous (1)

Allantophomopsis Petr. (8)

Bulgaria Fr. (2)

Calvophomopsis J.B. Tanney \& Seifer (1)

Ceuthospora Grev. (ca. 100)"

Cornibusella J.B. Tanney \& Seifer (1)

Darkera H.S. Whitney, J. Reid \& Piroz. (5)

Gloeopycnis J.B. Tanney \& Seifer (1)

Phacidiopycnis Potebnia (5)

Phacidium Fr. (ca. 200)

Potebniamyces Smerlis (1)

Pseudophacidium P. Karst. (5)

Starbaeckia Rehm ex Starbäck (1)

Strasseria Bres. \& Sacc. (1)

\#Ekanayaka et al. (2019) accepted these genera in Phacidiaceae

Phacidiales genus incertae sedis

Coma Nag Raj \& W.B. Kendr. (2)

Rhytismatales M.E. Barr ex Minter

Rhytismataceae Chevall. (= Hypodermataceae Rehm; = Cryptomycetaceae Höhn. nom. inval. fide Jaklitsch et al. 2016a; = Cudoniaceae P.F. Cannon fide Ekanayaka et al. 2019)

Angelina Fr. (1)

Apiodiscus Petr. (1)

Bifusella Höhn. (11)

Bifusepta Darker (1)

Bivallum P.R. Johnst. (7)

Bonanseja Sacc. (1)

Canavirgella W. Merr, Wenner \& Dreisbach (1)

Cavaraella Speg. (1)

Ceratophacidium J. Reid \& Piroz. (1)

Cerion Massee (2)

Coccomyces De Not. (118)

Colpoma Wallr. (14)

Criella (Sacc.) Henn. (2)

Cryptomyces Grev. (1)

Cudonia Fr. (9)

Davisomycella Darker (10)

Didymascus Sacc. (1)

Discocainia J. Reid \& A. Funk (4)

Duplicaria Fuckel (1)

Duplicariella B. Erikss. (1)

Elytroderma Darker (1)

Gelineostroma H.J. Swart (1)

Haplophyse Theiss. (10)

Heufleria Auersw. (1)

Hypoderma De Not. (1)

Hypodermella Tubeuf (3)

Hypodermellina Höhn. (1)

Hypohelion P.R. Johnst. (3)

Irydyonia Racib. (1) 
Laquearia Fr. (1)

Lasiostictella Sherwood (1)

Lirula Darker (3)

Lophodermella Höhn. (9)

Lophodermium Chevall. (145)

Lophophacidium Lagerb. (5)

Macroderma Höhn. (1)

Meloderma Darker (9)

Moutoniella Penz. \& Sacc. (1)

Mycomelanea Velen. (1)

Myriophacidium Sherwood (6)

Nematococcomyces C.L. Hou, M. Piepenbr. \& Oberw. (9)

Neococcomyces Y.R. Lin, C.T. Xiang \& Z.Z. Li (3)

Neophacidium Petr. (2)

Nothorhytisma Minter, P.F. Cannon, A.I. Romero \& Peredo (3)

Nymanomyces Henn. (1)

Parvacoccum R.S. Hunt \& A. Funk (1)

Phaeophacidium P. Henn. \& Lindau (4)

Ploioderma Darker (7)

Propolidium Sacc. (2)

Pseudorhytisma Juel (1)

Pseudotrochila Höhn. (2)

Pureke P.R. Johnst. (7)

Rhytisma Fr. (21)

Soleella Darker (7)

Spathularia Pers. (ca. 12)

Sporomega Corda (7)

Terriera B. Erikss. (26)

Therrya Sacc. (7)

Triblidiopsis P. Karst. (2)

Tryblidiopsis P. Karst. (3)

Virgella Darker (1)

Vladracula P.F. Cannon, Minter \& Kamal (2)

Xyloschizon Syd. (2)

Zeus Minter \& Diamandis (2)

Triblidiaceae Rehm

Huangshania O.E. Erikss. (2)

Pseudographis Nyl. (3)

Triblidium Rebent. (6)

Thelebolales P.F. Cannon

Thelebolaceae A. Engler (=Pseudeurotiaceae Malloch \& Cain fide Ekanayaka et al. 2019)

Antarctomyces Stchigel \& Guarro (1)

Ascophanus Boud. (20)

Ascozonus (Renny) E.C. Hansen (6)

Caccobius Kimbr. (6)

Cleistothelebolus Malloch \& Cain (13)

Coprobolus Cain \& Kimbr. (1)

Geomyces Traaen (ca. 10)

Gymnostellatospora Udagawa, Uchiy. \& Kamiya (ca. 6)

Holwaya Sacc. (= Crinula Fr.) (2) 
Leptokalpion Brumm. (2)

Leuconeurospora Malloch \& Cain (3)

Neelakesa Udaiyan \& Hosag. (3)

Patinella Sacc. (1)

Pseudascozonus Brumm. (1)

Pseudeurotium J.F.H. Beyma (6)

Pseudogymnoascus Raillo (ca. 10)

Ramgea Brumm. (2)

Thelebolus Tode (13)

Thelebolales genera incertae sedis

Alatospora Ingold (4)

Gorgomyces M. Gönczöl \& Révay (2)

Miniancora Marvanová \& Bärl. (1)

Leotiomycetes families incertae sedis

Cenangiaceae $\mathrm{Rehm}^{\#}$

Cenangium Fr. (30)

Moellerodiscus Henn. (7)

Piceomphale Svrcek (1)

Calloriaceae L. Marchand $^{\#}$

Aivenia Svrček (4)

Belonioscyphella Höhn. (4)

Calloria Fr. (5)

Chaetonaevia Arx (3)

Cistella Quél. (45)

Dactylaria Sacc. (ca. 100)

Diplonaevia Sacc. (23)

Duebenia Fr. (5)

Eupropolella Höhn. (7)

Hyalacrotes (Korf \& L.M. Kohn) Raitv. (2)

Iridinea Velen. (2)

Laetinaevia Nannf. (15)

Leohumicola N.L. Nick. (7)

Loricella Velen. (2)

Micropodia Boud. (2)

Mycoarthris Marvanová \& P.J. Fisher (1)

Naeviella (Rehm) Clem. (3)

Naeviopsis B. Hein (3)

Ploettnera Henn. (5)

Polyphilus D.G. Knapp, Ashrafi, W. Maier \& Kovács (2)

Psilachnum Höhn. (28)

Rodwayella Spooner (3)

Rommelaarsia Baral \& Haelew. (1)

Roseodiscus Baral (6)

Stamnaria Fuckel (5)

Tetracladium De Wild. (11)

Urceolella Boud. (24)

Hamatocanthoscyphaceae Ekanayaka \& K.D. Hyde Brachyalara Réblová \& W. Gams (1) 
Chalara (Corda) Rabenh. (ca. 100)

Ciliolarina Svrček (1)

Gremmenia Korf (4)

Hamatocanthoscypha Svrček (3)

Infundichalara Réblová \& W. Gams (2)

Microscypha Syd. \& P. Syd. (6)

Pseudohelotium Fuckel (50)

Xenochalara M.J. Wingf. \& Crous (1)

Xenopolyscytalum Crous (1)

\section{Hemiphacidiaceae Korf $^{\#}$}

Calycellinopsis W.Y. Zhuang (1)

Cenangiopsis Rehm (2)

Chlorencoelia J.R. Dixon (4)

Crumenulopsis J.W. Groves (1)

Didymascella Maire \& Sacc. (5)

Encoelia (Fr.) P. Karst. (40)

Fabrella Kirschst. (1)

Heyderia Link (6)

Hysterostegiella Höhn. (10)

Korfia J. Reid \& Cain (1)

Rhabdocline Syd. (4)

Sarcotrochila Höhn. (4)

Trochila Fr. (15)

Velutarina Korf (3)

Hyphodiscaceae Ekanayaka \& K.D. Hyde

Fuscolachnum J.H. Haines (7)

Hyalopeziza Fuckel (15)

Hyphodiscus Kirschst. (16)

Soosiella Hujslová \& M. Kolařík (1)

Scolecolachnum Guatim., R.W. Barreto \& Crous (2)

Venturiocistella Raitv. (7)

Leptodontidiaceae Hern.-Restr., Crous \& Gené

Leptodontidium de Hoog. (11)

\section{Neocrinulaceae Crous}

Neocrinula Crous (2)

\section{Neolauriomycetaceae Crous ${ }^{\#}$}

Exochalara W. Gams \& Hol.-Jech. (3)

Lareunionomyces Crous \& M.J. Wingf. (2)

Neolauriomyces Crous (1)

Pezizellaceae Velen. (= Bloxamiaceae Locq. ex Hern.-Restr., Gené, R.F. Castañeda, J. Mena, Crous \& Guarro fide Ekanayaka et al. 2019)

Allophylaria (P. Karst.) P. Karst. (6)

Antinoa Velen. (8)

Bisporella Sacc. (19)

Bloxamia Berk. \& Broome (19)

Calycellina Höhn (45) 
Calycina Nees ex Gray (= Pezizella Fuckel) (30)

Micropeziza Fuckel (12)

Mollisina Höhn. ex Weese (11)

Mollisinopsis Arendh. \& R. Sharma (3)

Moserella Pöder \& Scheuer (1)

Orbiliopsis (Sacc. \& D. Sacc.) Syd. \& P. Syd. (2)

Phaeoscypha Spooner (1)

Phialina Höhn. (ca. 13)

Triposporium Corda (14)

Poculinia Spooner (1)

Scleropezicula Verkley (6)

Velutaria Fuckel (1)

Xiambola Minter \& Hol.-Jech. (1)

Zymochalara Guatim., R.W. Barreto \& Crous (2)

Rutstroemiaceae Holst-Jensen, L.M. Kohn \& T. Schumach. ${ }^{\#}$

Bicornispora Checa, Barrasa, M.N. Blanco \& A.T. Martínez (2)

Dencoeliopsis Korf (2)

Lambertella Höhn. (6)

Neometulocladosporiella Crous \& M.J. Wingf. (1)

Rutstroemia P. Karst. (ca. 100)

Sclerotiniaceae Whetzel ${ }^{\#}$

Amerosporium Speg. (31)

Amphobotrys Hennebert (1)

Banksiamyces G. Beaton (4)

Botrytis P. Micheli ex Pers. (3)

Ciboria Fuckel (21)

Ciborinia Whetzel (ca. 16)

Clarireedia L.A. Beirn, B.B. Clarke, C. Salgado \& J.A. Crouch (4)

Coprotinia Whetzel (1)

Cristulariella Höhn. (5)

Cudoniopsis Speg. (1)

Dumontinia L.M. Kohn (5)

Elliottinia L.M. Kohn (1)

Grovesinia M.N. Cline, J.L. Crane \& S.D. Cline (2)

Haradamyces Masuya, Kusunoki, Kosaka \& Aikawa (1)

Kohninia Holst-Jensen, Vrålstad \& T. Schumach. (1)

Martininia Dumont \& Korf (1)

Monilinia Honey (30)

Mycopappus Redhead \& G.P. White (4)

Myrioconium Syd. \& P. Syd. (10)

Myriosclerotinia N.F. Buchw. (10)

Ovulinia Weiss (9)

Phaeosclerotinia Hori (1)

Poculum Velen. (ca. 22)

Pseudociboria Kanouse (1)

Pycnopeziza W.L. White \& Whetzel (5)

Redheadia Y. Suto \& Suyama (1)

Sclerencoelia Pärtel \& Baral (3)

Scleromitrula S. Imai (6)

Sclerotinia Fuckel (15) 
Sclerotium Tode (100)

Seaverinia Whetzel (2)

Septotinia Whetzel ex J.W. Groves \& M.E. Elliott (2)

Streptotinia Whetzel (3)

Stromatinia (Boud.) Boud. (15)

Valdensia Peyronel (3)

Valdensinia Peyronel (1)

Vandijckellaceae Sandoval-Denis

Vandijckella Sandoval-Denis (1)

Leotiomycetes family incertae sedis

Porodiplodiaceae Crous

Porodiplodia Crous (1)

Leotiomycetes genera incertae sedis

Adelodiscus Syd. (3)

Algincola Velen. (1)

Apiculospora Wijayaw., Camporesi, A.J.L. Phillips \& K.D. Hyde (1)

Aquadiscula Shearer \& J.L. Crane (2)

Ascluella DiCosmo, Nag Raj \& W.B. Kendr. (1)

Ascoclavulina Otani (8)

Bagnisimitrula S. Imai (1)

Benguetia Syd. \& P. Syd. (1)

Bioscypha Syd. (2)

Bulgariopsis Henn. (2)

Callerascus Whitton, K.D. Hyde \& McKenzie (1)

Calloriopsis Syd. \& P. Syd. (2)

Capillipes R. Sant. (1)

Capricola Velen. (1)

Cejpia Velen. (3)

Cenangiumella J. Fröhl. \& K.D. Hyde (1)

Chloroepilichen Etayo (1)

Chlorospleniella P. Karst. (1)

Chondroderris Maire (1)

Ciliella Sacc. \& P. Syd. (1)

Coleosperma Ingold (1)

Comesia Sacc. (3)

Cornuntum Velen. (1)

Coronellaria P. Karst. (4)

Criserosphaeria Speg. (1)

Crumenella P. Karst. (1)

Cryptohymenium Samuels \& L.M. Kohn (1)

Cryptopezia Höhn. (1)

Dawsicola Döbbeler (1)

Dermateopsis Nannf. (2)

Didonia Velen. (5)

Discomycella Höhn. (1)

Echinodiscus Etayo \& Diederich (2)

Epicladonia D. Hawksw. sensu stricto (3)

Episclerotium L.M. Kohn (2)

Erikssonopsis M. Morelet (1) 
Flagellospora Ingold (6)

Gloeopeziza Zukal (8)

Godroniopsis Diehl \& E.K. Cash (3)

Grimmicola Döbbeler \& Hertel (1)

Grovesia Dennis (1)

Helotiella Sacc. (1)

Hemiglossum Pat. (2)

Hymenobolus Durieu \& Mont. (3)

Hyphoscypha Velen. (1)

Hysteronaevia Nannf. (12)

Hysteropezizella Höhn. (19)

Involucroscypha Raitv. (10)

Jacobsonia Boedijn (1)

Lasseria Dennis (1)

Livia Velen. (1)

Masseea Sacc. (4)

Melanopeziza Velen. (1)

Melanormia Körb. (1)

Metapezizella Petr. (1)

Micraspis Darker (3)

Microdiscus Sacc. (1)

Monochaetiellopsis B. Sutton \& DiCosmo (2)

Mycosphaerangium Verkley (3)

Obconicum Velen. (2)

Obscurodiscus Raitv. (1)

Ocotomyces H.C. Evans \& Minter (1)

Otwaya G.W. Beaton (12)

Pachydisca Boud. (1)

Parencoelia Petr. (4)

Patinellaria P. Karst. (1)

Pezolepis Syd. (2)

Pezomela Syd. (1)

Phaeofabraea Rehm (1)

Phragmonaevia Rehm (?1)

Phyllopezis Petr. (1)

Physmatomyces Rehm (1)

Pleoscutula Vouaux (3)

Podophacidium Niessl (2)

Polydiscidium Wakef. (7)

Polydiscina Syd. (1)

Potridiscus Döbbeler \& Triebel (1)

Pseudolachnum Velen. (1)

Pseudopeltis L. Holm \& K. Holm (1)

Pseudotryblidium Rehm (1)

Psilophana Syd. (1)

Psilothecium Clem. (1)

Pteromyces E. Bommer, M. Rousseau \& Sacc. (1)

Pubigera Baral, Gminder \& Svrček (1)

Radotinea Velen. (1)

Rhizocalyx Petr. (1)

Rhizothyrium Naumov (1)

Riedera Fr. (1) 
Sageria A. Funk (1)

Schnablia Sacc. \& P. Syd. (1)

Sclerocrana Samuels \& L.M. Kohn (4)

Scutulopsis Velen. (1)

Sinofavus W.Y. Zhuang (1)

Sorokina Sacc. (1)

Sorokinella J. Fröhl. \& K.D. Hyde (2)

Spirosphaera Beverw. (8)

Stilbopeziza Speg. (1)

Themisia Velen. (8)

Tovariella Syd. (1)

Trichohelotium Killerm. (2)

Trizodia Laukka (1)

Trullula Ces. (15)

Waltonia Saho (1)

Woodiella Sacc. \& P. Syd. (3)

Xeromedulla Korf \& W.Y. Zhuang (3)

Zugazaea Korf, Iturr. \& Lizoň (1)

\section{Notes for alternative classification of Leotiomycetes}

Amorphothecaceae (= Myxotrichaceae) - Ekanayaka et al. (2019) placed this family under Erysiphales, considering its morphological similarity with other taxa in Erysiphales.

The authors accept the synonymy of Myxotrichaceae under Amorphothecaceae as the close phylogenetic relatedness of these two families were shown in the phylogeny provided in Ekanayaka et al. (2019) (A.H. Ekanayaka \& K.D. Hyde).

Calloriaceae and Pezizellaceae - In other classification systems, Calloriaceae and Pezizellaceae are members of Helotiales. Ekanayaka et al. (2019) placed Calloriaceae and Pezizellaceae under Rhytismatales based on their phylogeny. Johnston et al. (2019) placed these families within Helotiales. However, the placement of Calloriaceae is not clear. In addition, whereas Pezizellaceae was retrieved as a monophyletic family in the "pezizelloid clade" of the 15-gene tree, taxa from this family are polyphyletic in the ITS tree. In the present alternative classification for Leotiomycetes we accept these families under Leotiomycetes families incertae sedis, until we have more data to provide a stable classification (A.H. Ekanayaka \& K.D. Hyde).

Cenangiaceae, Chlorociboriaceae, Hemiphacidiaceae, Rutstroemiaceae, Sclerotiniaceae Those families formed a well-supported distinct clade in Ekanayaka et al. (2019). Therefore, here we keep them in Leotiomycetes families incertae sedis until we have more data (A.H. Ekanayaka \& K.D. Hyde).

Cochlearomycetaceae - Within the phylogeny of Ekanayaka et al. (2019), this family clustered within Leotiales, and also Johnston et al. (2019) suggested its position should be within Leotiales based on their ITS phylogeny. As a result, we placed this family in Leotiales (A.H. Ekanayaka \& K.D. Hyde).

Cordieritidaceae - Taxa of this family differ from other Helotiales by having a unique ionomidotic reaction (solubility of excipular pigments in $\mathrm{KOH}$ ). Hence, Jaklitsch et al. (2016) suggested a separate phylogenetic position for this family away from other Helotiales. In Johnston et al. (2019), this family clustered in the "sclerotinoid clade" within Helotiales in their 15-gene tree. In the phylogeny of Ekanayaka et al. (2019), however, this family grouped sister to 
Deltopyxidaceae within Cyttariales. Therefore, here we place this family under Cyttariales until we have more data to confirm its placement (A.H. Ekanayaka \& K.D. Hyde).

Deltopyxidaceae - In the phylogeny of Ekanayaka et al. (2019), this family grouped sister to Cordieritidaceae with strong statistical support within Cyttariales. We are unable to compare with Johnston et al. (2019), as these authors did not include taxa from this family (A.H. Ekanayaka \& K.D. Hyde).

Erysiphales - In Ekanayaka et al. (2019), this order formed a distinct clade, while in Johnston et al. (2019) its placement was within Helotiales both in the genomic-scale and 15-gene trees. However, we believe that this order is distinct as it has a unique morphology, which differs from taxa in Leotiomycetes. Further studies are needed to resolve this inconsistency (A.H. Ekanayaka \& K.D. Hyde).

Leptodontidiaceae - This family sat in a well-supported clade away from Helotiales in the phylogeny provided by Ekanayaka et al. (2019). We cannot compare its placement with Johnston et al. (2019) as their phylogenies did not include taxa from this family. Therefore, we placed this family in Leotiomycetes families incertae sedis until more data are available to provide a stable classification (A.H. Ekanayaka \& K.D. Hyde).

Medeolariales - Ascocorticiaceae, Ascodichaenaceae, Dermateaceae, Medeolariaceae Ekanayaka et al. (2019) accommodated these families in Medeolariales based on morphological similarities and phylogenetic analysis. In Johnston et al. (2019), Dermateaceae formed the basalmost clade within Helotiales. However, Johnston et al. (2019) did not include Ascocorticiaceae, Ascodichaenaceae and Medeolariaceae in their phylogeny, thus, we were unable to compare their placements in Leotiomycetes. Future studies are needed to resolve the placement of these families (A.H. Ekanayaka \& K.D. Hyde).

Neolauriomycetaceae - This family produces a well-supported clade within "Sclerotiniales" in the phylogeny of Ekanayaka et al. (2019). We cannot compare its placement with Johnston et al. (2019) as their phylogenies did not include taxa from this family. Therefore, we placed this family under Leotiomycetes families incertae sedis until more data become available to provide a stable classification (A.H. Ekanayaka \& K.D. Hyde).

\section{Multi-locus phylogenies and comprehensive treatments of the Xylariales (Author: M. Stadler)}

Wendt et al. (2018) provided a multigene genealogy of the stromatic families of the Xylariales, including a significant number of representative species of the main lineages in the Xylariaceae and four DNA loci. This study resulted in the segregation of the Xylariaceae sensu lato in the traditional definition, and the Hypoxylaceae were resurrected and amended. Moreover, the genera Biscogniauxia and Camillea were transferred to the Graphostromataceae. The molecular phylogeny corresponds with the distribution of secondary metabolites and types of conidiogenous structures, while the ascospore morphology, which had traditionally constituted the salient feature to define the family, had to be abandoned. Notably, the Lopadostomaceae also contains genera with similar ascospores that had previously been accommodated in the Xylariaceae sensu lato and some genera like Whalleya and Jumillera were transferred to Lopadostomataceae.

Wendt et al. (2018) also proposed to exclude several genera of which no information on the asexual morph and no molecular data are available, from the Xylariaceae and place them at interim in Xylariales incertae sedis. Moreover, they resurrected the genus Pyrenonopolyporus for some species formerly placed in Hypoxylon with massive stromata and long tubular perithecia and segregated the new genus Jackrogersella from Annulohypoxylon as an outcome of their polyphasic study. Concurrently with the study by Wendt et al. (2018), Daranagama et al. (2018) 
provided a very comprehensive overview on the families of the "stromatic" Xylariales, which roughly comprise the genera that had traditionally been classified in the Xylariaceae according to the traditional concept of the late 1990s, which was based on ascospore morphology. In the first comprehensive study of this type, they provided illustrations of most of the type species or other representative species and revised the history of their taxonomy. A molecular phylogeny was also presented, using data from additional taxa that represented genera that were not included in the paper by Wendt et al. (2018). Therefore, certain deviations from the concept were observed. In addition Daranagama et al. (2018) retained some genera in the Xylariaceae that were expelled from the family in the concurrent study. Helaly et al. (2018) reviewed the taxonomy, ecology and in particular the secondary metabolite production of the Xylariales. Even though no taxonomic novelties are provided in this paper, the records of endophytic and marine strains of Xylariales that had previously been reported in the literature to produce novel bioactive compounds were revised and numerous incongruities between the data published in chemistry journals and the current taxonomy were found. The authors gave an overview on the current taxonomic status of the taxonomy of the order in relation to the numbers of known metabolites and pointed out some genera and families that deserve further study as they seem to be hitherto unexplored. Recently, the genus Dematophora has been resurrected and divided from Rosellinia based on a comparison of molecular data, morphology of the asexual morphs and chemotaxonomic evidence (Wittstein et al. 2020), and all the serious pathogens like D. necatrix and D. bunodes now no longer belong to Rosellinia, which mainly comprises saprotrophic and endophytic species. Finally, Samarakoon et al. (2020) have recently found out that the sexual morph of the economically important endophytic genus Muscodor is close to Induratia, which was erected earlier and takes preference over the latter genus. They provided a polyphasic study involving a multi-locus phylogeny, divergence time estimations, morphological studies and comparison of volatile secondary metabolite profiles, which resulted in the recgnisiton of a new family Induratiaceae in the order Xylariales. This family is sister to the Xylariaceae sensu stricto and also includes the genus Emarcea. All names in Muscodor were newly combined in Induratia, and two new species were described with their full life cycle, comprising both the asexual and sexual morph.

\section{Placement and phylogenies of Laboulbeniomycetes (Author: D. Haelewaters)}

The placement of Laboulbeniomycetes has been traditionally problematic (Blackwell et al. 2020). First considered as cuticle hairs or even parasitic worms, life history studies and molecular phylogenetic analyses have helped in placing these fungi among filamentous Ascomycota. The first phylogenetic analysis of the class by Weir \& Blackwell (2001) was based on SSU rDNA and supported the placement of Laboulbeniales-Pyxidiophora as a separate clade within Ascomycota, sister to Sordariomycetes although without statistical support for this sister relationship. Schoch et al. (2009) were the first ones to obtain support for the sister relationship between Laboulbeniomycetes and Sordariomycetes based on a six-locus dataset. Their results were later confirmed by Goldmann \& Weir (2018) as well as Haelewaters et al. (2019b) who proposed the informal taxon 'laboulbeniomyceta' as a descriptor of the most recent common ancestor of both classes.

To date, limited sequence data are available for members of Laboulbeniomycetes. An NCBI GenBank search for 'Laboulbeniomycetes' resulted in 727 sequences only, the majority of which are SSU sequences (17 February 2020). An SSU-based phylogeny by Goldmann \& Weir (2018) including 65 isolates resulted in evidence for multiple clades in the class. Haelewaters et al. (2019b), based on a three-locus dataset with 61 isolates, described the third order in the class, Herpomycetales, to accommodate the genus Herpomyces. Blackwell et al. (2020) presented a phylogenetic reconstruction of the Laboulbeniomycetes from a concatenated SSU-LSU dataset of 75 isolates and found high support for 5 clades: orders Herpomycetales, Laboulbeniales and Pyxidiophorales, in addition to two unnamed clades, Chantransiopsis clade (Chantransiopsis sp., Tetrameronycha spp., Subbaromyces splendens) and Laboulbeniopsis clade (with Laboulbeniopsis termitarius). Finally, several recent studies have pointed at the polyphyly of accepted higher taxa 
in the Laboulbeniales order (Goldmann \& Weir 2018, Haelewaters et al. 2018b), and so future integration of molecular data will undoubtedly change the classification of the order and by extension the whole class.

\section{Structural and functional organization of Microsporidia (Author: Yuri S. Tokarev)}

Microsporidia is a monophyletic group of highly specialized intracellular parasites which are ultimately dependent upon and being able to develop only within the host cell (Vavra \& Lukes 2013). Microsporidia infect Metazoa and some protists: Gregarina, Ciliata, Paramyxea (Fokin et al. 2008, Sokolova et al. 2013, Larsson 2014, Cali et al. 2017, Stentiford et al. 2017). Host switching between representatives of different families (Ghani et al. 2013, Malysh et al. 2018a, 2019), orders (Schuld et al. 1999, Ovcharenko et al. 2017, Malysh et al. 2018b), classes (Hinney et al. 2016) and phyla may occur (Franzen et al. 2006, Ironside et al. 2008, Nylund et al. 2010, Choudhary et al. 2011, Meissner et al. 2012). Parasite-host interactions are diverse, ranging from asymptomatic presence in the form of a latent infection to devastating epizootics especially in arthropods and fishes (Bader et al. 1998, Stentiford et al. 2013, Sokolova et al. 2015, Yu et al. 2019). Routes of transmission include alimentary and transovarial/ transplacental infection, transfer from host to host by parasites etc. (Becnel \& Andreadis 1999, Dunn \& Smith, 2001, Didier et al. 2004, Wang-Peng et al. 2018).

Typical life cycle includes merogonial (for parasite multiplication) and sporogonial sequence (for multiplication and spore formation). The cells multiply either by binary or multiple fission. The merogonial developmental stages are single cells or plasmodia delineated with a plasma membrane (Dunn \& Smith 2001). In early sporogonial stages, cytoplasm becomes more condensed, electron dense material is deposited on the cell surface which further transforms into the spore wall. Other primordial spore structures emerge in late sporogonial stages (Issi et al. 2012a, Sokolova et al. 2015, Cali \& Takvorian 2014). The nuclear apparatus is mono- or diplokaryotic, the number of nuclei varies from one to many (in plasmodia). In some species the transformation of the nuclear apparatus from one type to another one takes place (sometimes accompanied by meiosis) in the course of life cycle (Lee et al. 2014). The parasites develop either in direct contact with the host cell cytoplasm or within a parasitophorous vacuole which is derived from the membranes of endoplasmic reticulum, Golgi complex or nuclear envelope of the host cell (Bohne et al. 2011, Issi et al. 2012b, Vavra \& Lukes 2013).

The spore is a specialized parasite cell which serves for invasion into the host cell, dissemination over the host organism and spreading within the host population (Vavra \& Lukes 2013). The spore wall is a complex chitin- and protein-rich structure consisting of inner electrontranslucent endospore layer with an underlying plasma membrane and outer electron-dense exospore layer (Bigliardi \& Sacchi 2001). The main distinctive feature of microsporidia spore is the extrusion apparatus, which includes polar tube (polar filament), polar cap (polar sac anchoring disc complex), polaroplast and posterior vacuole (Fig. 2) (Vavra \& Larsson 2014). The polar tube is attached to the apical pole of the spore by the polar cap (Franzen 2004, Xu \& Weiss 2005). The length, diameter and structure of polar tube vary greatly among species. Most often, the polar tube is thin and long, exceeding the length of the spore, with proximal and distal parts straight and coiled, respectively (Delbac \& Polonais 2008, Issi et al. 2012a). 


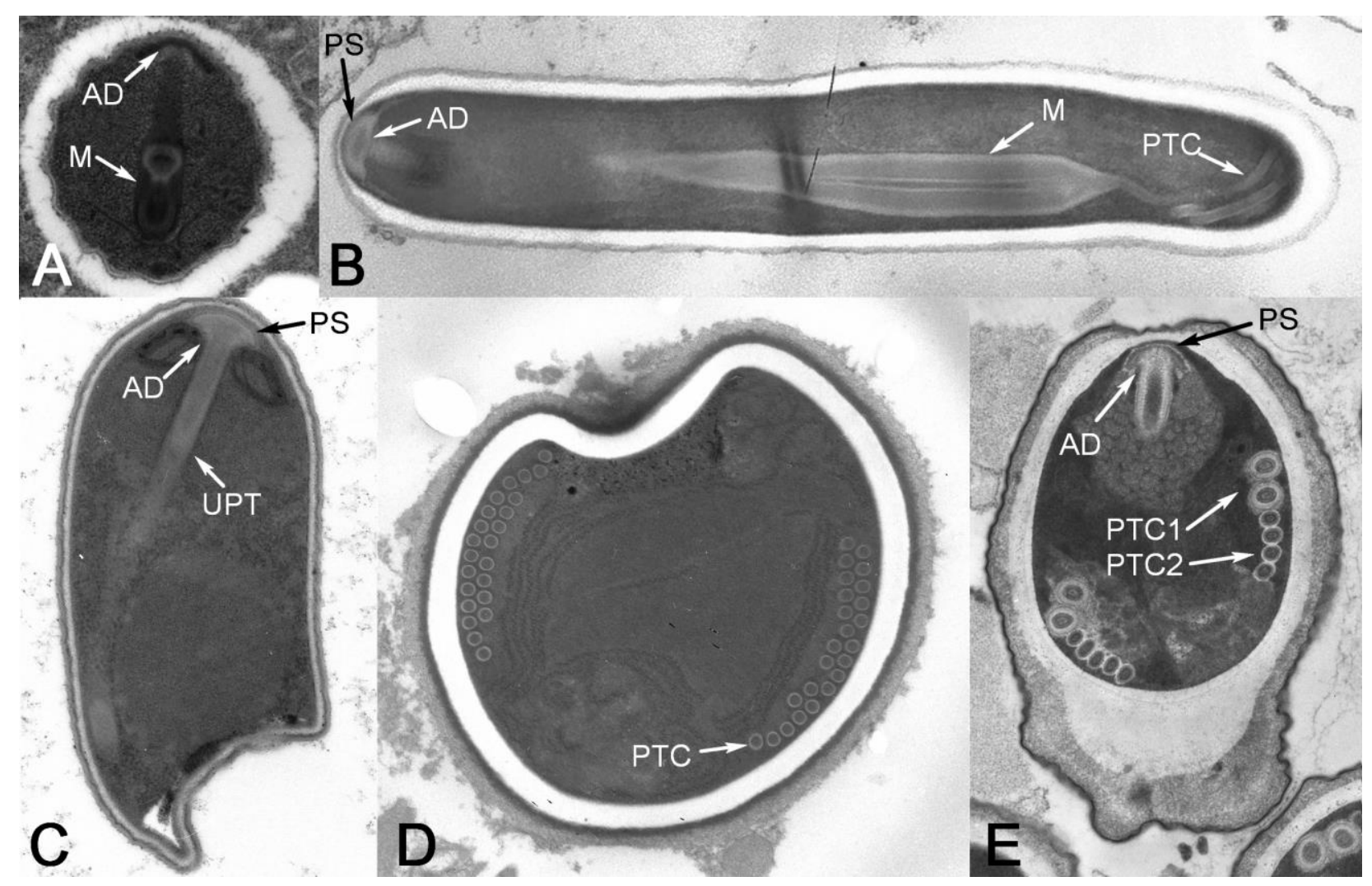

Figure 2 - Spore structure of Microsporidia with different types of polar tube. A Metchnikovella incurvata with a manubrial polar tube. B Mrazekia macrocyclopis with a manubrial polar tube containing a coiled distal part. C Helmichia lacustris with an uncoiled polar tube (UPT). D Neoperezia sp. with numerous coils of isofilar polar tube. E Crepidulospora beklemishevi with anterior (PTC1) and posterior (PTC2) polar tube coils of different structure and size. AD anchoring disc; M - manubrium; PS - polar sac; PTC - polar tube coils. Figs 2A, 2E are reproduced with a kind permission of Yuliya Y. Sokolova (Institute of Cytology RAS, St. Petersburg, Russia) and Anastasia V. Simakova (Tomsk State University, Tomsk, Russia), respectively.

The number of coils ranges from 3 to 30. In isofilar polar tube, the diameter is the same throughout the tube length. It is either coiled (Fig. 2D) or uncoiled (Fig. 2C), as its length exceeds or do not exceed the spore length, respectively (Issi et al. 2012a, Tokarev et al. 2012). In anisofilar polar tube, several posterior coils are of lesser diameter and sometimes of different electron density (Tokarev et al. 2010). In heterofilar polar tube, the anterior coils are remarkably bigger as compared to the posterior ones and their structure is different (Fig. 2E) (Vavra \& Larsson 2014). The manubrium is thick and short, not exceeding the spore length, cylindrical, usually thicker at central or distal 'bulbal' part, which is interconnected with manubrial cisternae associated with vesicles and/or short tubules (Fig. 2A) (Issi et al. 2010, Sokolova et al. 2013). Another type of polar tube is clavate, which is thick and short, not exceeding the spore length, thicker at distal 'bulbar' part, which is continued into thin polar tube with several coils (Fig. 2B). This clavate polar tube is sometimes referred to as "manubrium" (Issi et al. 2010, which is not correct. The manubrium is present in Rudimicrosporea (Sokolova et al. 2013), being different in its structure and supposed to be a primitive form of the canonical polar tube of higher Microsporidia (Vivier 1975). The polaroplast is a lamellar or vesicular (or combined), occupying the anterior part of the spore and surrounding the straight part of the polar tube (Vavra \& Larsson 2014). Its function is unclear and participation in extrusion process is supposed. The spores are formed as separate units ("free spores") or by packets enclosed within a common envelope of parasite origin, so called sporophorous vesicle (Dunn \& Smith 2001, Sokolova et al. 2015). 

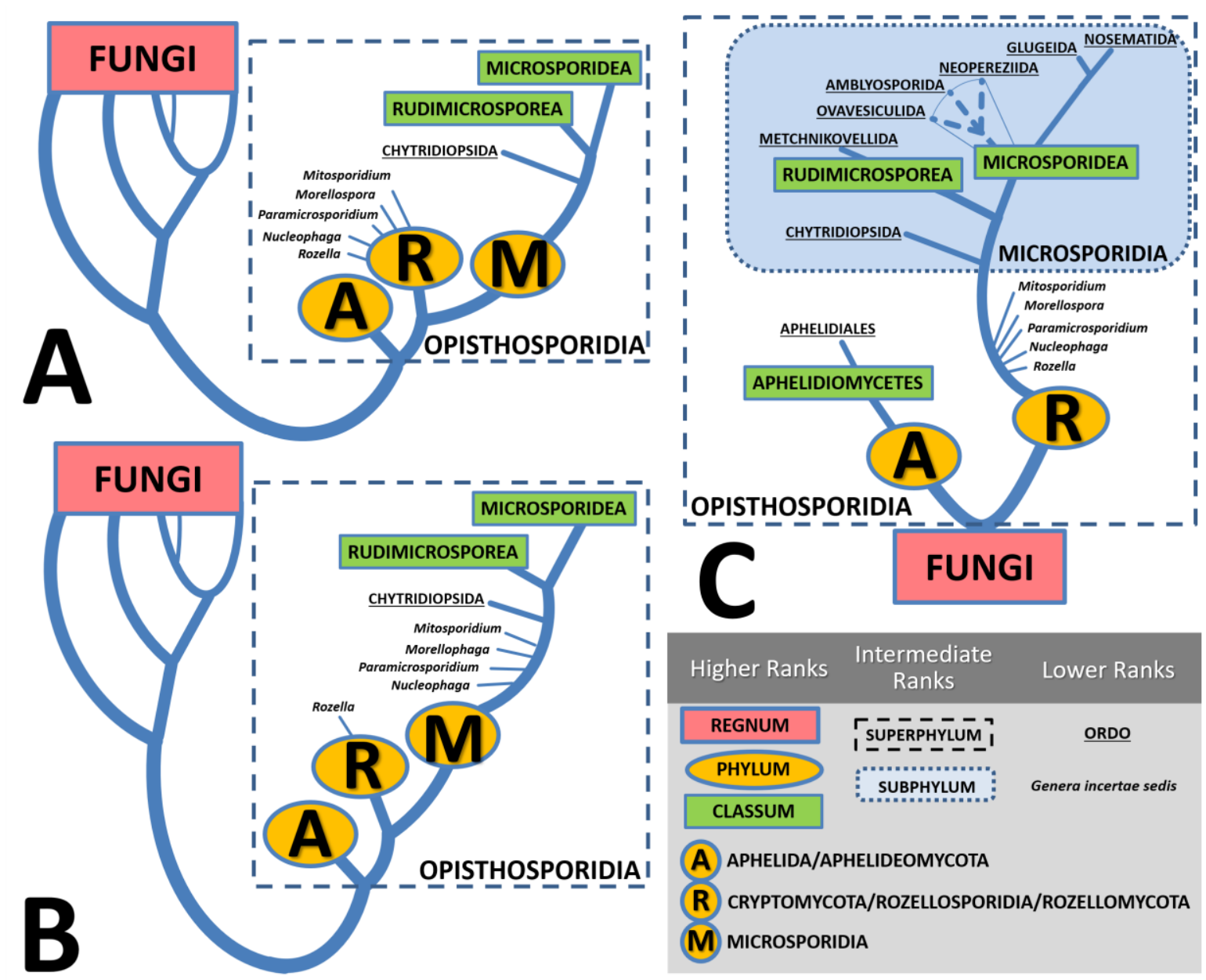

Figure 3 - Alternative hypotheses of phylogenetic and taxonomic relationships within Fungs-like representatives of Aphelida (Aphelidiomycota) - Rozellomycota (Rozellidal Cryptomycotal Rozellosporidia) - Microsporidia (ARM) clade and its relations to Fungi. The schematic phylogenetic trees follow the recent phylogenetic reconstructions by Karpov et al. (2014), Tedersoo et al. (2018), Bass et al. (2018), Corsaro et al. (2019, 2020). Three alternative schemes are presented as nowadays there is no consensus among the specialists. A Karpov et al. (2014) proposed the superphylum Opisthosporidia for the ARM clade, which was considered as a sister to Fungi. Several new genera related to Rozella and Microsporidia have been added to the tree more recently (Corsaro et al. 2014a, b, 2016, 2019, 2020, Haag et al. 2014). B In the interpretation of Bass et al. (2018) the boundaries of Microsporidia should be expanded to include all these genera except Rozella. C According to Tedersoo et al. (2018); Wijayawardene et al. (2018) ARM clade should be included into kingdom Fungi, and phylum Microsporidia should be merged with Rozellomycota. To further conserve Microsporidia as a monophyletic taxon within Rozellomycota it has been redefined as a subphylum. The labels for taxon ranks and phyla abbreviations are explained in the right lower corner. The synonymic names for the phyla are indicated: Aphelidiomycota Tedersoo et al $2018=$ Aphelida Karpov, Aleoshin \& Mikhailov $2014=$ Rozellida Lara, Moreiro, López-García, 2010 = Rozellomycota James \& Berbee, 2011 emend. Corsaro \& Michel 2014 = Cryptomycota Jones \& Richards 2011 emend. Karpov \& Aleoshin 2014 = Rozellosporidia Karpov, Torruella, Moreira, Mamkaeva, López-García 2017.

Many of the basic physiological functions and biochemical processes in Microsporidia are reduced as they mostly rely on the host cell. As a result of deep adaptation to intracellular parasitism, structural organization of prespore stages is simplified, and many biochemical 
pathways are absent. Basic nutrients are imported from the host cell including ATP (Tsaousis et al. 2008). The physiological processes in the host cell are altered and can be managed by the actively proliferating parasites to provide the latter with enough nutrients including sugars, aminoand fatty acids (Cuomo et al. 2012, Senderskiy et al. 2014). Exploitation of host cell metabolic pathways results in reorganization of host cell organelles: mitochondria, endoplasmatic reticulum and Golgi complex, which come into contact with the parasite cells (Simakova et al. 2005, Tokarev et al. 2010, Issi et al. 2012b, Vavra \& Lukes 2013). Other adaptations to survival within the host cell include alteration of host cell cycle, suppression of host cell apoptosis (MartinHernandez et al. 2017, Sokolova et al. 2019) and renewal of infected epithelial cells (Issi 1986).

Mitochondria are reduced to mitosomes which lack genome (Williams et al. 2002). Vesicular transport of cargo proteins is absent (Beznoussenko et al. 2007). The genomes of Microsporidia are reduced and compact, many genes and gene families are lost and the sequences of ribosomal RNA and protein-coding genes are shortened and highly derived (Peyretaillade et al. 2015). The genome size is ranged from 2.3 to $\sim 50 \mathrm{Mb}$, in the vast majority of sequenced species it does not exceed $15 \mathrm{Mb}$ (Keeling et al. 2014).

\section{Phylogenetic approaches and current status of Rozellomycota (Author: Yuri S. Tokarev)}

With the advent of molecular phylogeny studies, major groups within Microsporidia Tree of Life were primarily recognized and polyphyletic nature of traditional high rank taxa was demonstrated (Fig. 3) (Vossbrinck \& Debrunner-Vossbrinck 2005, Vossbrinck et al. 2014). Later on, some basal or sister groups of parasites were examined using molecular phylogenetic and phylogenomic approaches. In particular, relationships between Aphelida, Rozellomycota and Microsporidia were established (Karpov et al. 2014). Several new representatives of Rozellomycota were discovered and examined (Haag et al. 2014, Corsaro et al. 2014a, b, 2016, Quandt et al. 2017). Genome surveys of Metchnikovellida were provided (Mikhailov et al. 2016, Galindo et al. 2018) confirming basal position of this lineage within Microsporidia and demonstrating shared genomic signatures with other Microsporidia. Notably, the order of Metchnikovellida was always referred to as "primitive Microsporidia" (class Rudimicrosporea) on the basis of ultrastructural features, such as poorly developed or absent polaroplast and absent posterior vacuole as well as specific structure of polar tube, so called manubrium. Finally, phylogenetic position of Chytridiopsis typographi (order Chytridiopsidea) was also resolved (Corsaro et al. 2019), showing more basal position than Metchnikovellida. In this parasite species, only the sequences of rRNA genes are available, showing less compact organization as compared to other Microsporidia. In addition, spore structure of C. typographi is described as "unique" as compared to other Microsporidia (Purrini \& Weiser 1985) and essentially, the developmental sequence of $C$. typographi includes a unique budding stage, unknown for other species of Microsporidia, which multiply by fission of cells only (Tonka et al. 2010). Thus, ultrastructural and developmental traits support the basal position of Chytridiopsida inferred in the phylogenetic reconstructions. Here the order Chytridiopsida includes the composition of the families and genera suggested by Larsson (2014) with exclusion of Burkeidae, which is less similar to the chytridiopsids and was attributed to this group only temporaly. The mentioned taxon is provisionally placed to Microsporidia genera incertae sedis as possessing more canonical ultrastructure.

Recently, Bass et al. (2018) proposed taxonomic expansion of Microsporidia to include unclassified genera of Cryptomycota (Rozellomycota). This assumption was based upon monophyletic arrangement of Microsporidia and sister taxa, though the vast majority of sequences used in that analysis were short (below $400 \mathrm{bp}$ ). We prefer to preserve Microsporidia as a more compact group unless more robust phylogenies are available. Thus, such representatives of Rozellomycota as Nucleophaga, Paramicrosporidium, Morellospora and Mitosporidium are not considered here as Microsporidia.

Upon following the ranking of Tedersoo et al. (2016) who proposed Rozellomycota (including Microsporidia) and Aphelidiomycota as phyla in Kingdom Fungi, the phylum 
Microsporidia becomes obsolete. To recognize this group as a monophyletic lineage of highly specialized intracellular parasites with specific developmental, structural and genetic features (see above, Structural and functional organization of Microsporidia), we propose an intermediate rank of subphylum for this taxon. The superphylum Opisthosporidia proposed by Karpov et al. (2014) is also retained to include Aphelidiomycota and Rozellomycota. However recent transcriptomic data provide poor support for the monophyly of Opisthosporidia (Toruella et al. 2018), and in future the aphelids may be excluded and the higher ranks may be again reshuffled.

The Microsporidia Tree of Life consists of five major clades (Vossbrinck \& DebrunnerVossbrinck 2005, Vossbrinck et al. 2014). To accommodate these clades within a formal classorder-family system, the following changes to the taxonomy of Microsporidia are proposed. Orders Dissociodihaplophasida and Meiodihaplophasida are suppressed as polyphyletic. Orders Glugeida and Nosematida are redefined for the purposes of the current revision. Three new orders, Amblyosporida ord. nov., Neopereziida ord. nov. and Ovavesiculida ord. nov. are introduced in this study. All these orders are referred to as distinct monophyletic clades of the Rozellomycota Tree of Life, each presented by the type family: Glugeidae, Nosematidae, Amblyosporidae, Neopereziidae and Ovavesiculidae, respectively, and related taxa. Order Chytridiopsidea is excluded from Microsporidia (see the outline) and family Enterocytozoonidae is transferred to Nosematida. Allocation of order Metchnikovellida to class Rudimicrosporea is also supported.

Each phylogenetic lineage corresponding to the taxa of class, order or family rank contains representatives with diverse features. This makes it difficult to define these taxa using developmental, structural or ecological features, so that primary taxonomic allocation of species is based upon phylogeny.

Classification of Glomeromycota (Authors: B.T. Goto \& N. Wijayawardene)

Outline of arbuscular mycorrhizal fungi (AMF) was initially organized in Gerdemann \& Trappe (1974) and updated by Schenck \& Peréz (1990). Initially, AMF fungi have been included in one order (i.e. Glomerales) in Zygomycota (Morton \& Benny 1990). However, Schüßler et al. (2001) established new phylum, Glomeromycota to accommodate AMF. Since Schüßler et al. (2001), taxonomy of AMF has been rapidly updating over the last years (Oehl et al. 2011a, b, c, d, e, f, Goto et al. 2012, Błaszkowski et al. 2015, 2018a, b, 2019a, b, Corazon-Guivin et al. 2019a, b, Jobim et al. 2019). Oehl et al. (2008) introduced Dentiscutataceae, Racocetraceae and Scutellosporaceae and later Oehl et al. (2011b) introduced Gigasporales, to include these families along with Gigasporaceae. At the same time, Oehl et al. (2011b) introduced two new classes (i.e. Archaeosporomycetes and Paraglomeromycetes) thus currently Glomeromycota comprises three classes.

Morton \& Msiska (2010) did not recognize families introduced in Oehl et al. (2008) and Gigasporales was not accepted in Wijayawardene et al. (2018b). Nevertheless, phylogenetic reconstructions using different genes (TUB2, RPB1, ITS, SSU and LSU rRNA) (e.g. Goto et al. 2012, Mello et al. 2012, Silva et al. 2012, Marinho et al. 2014, de Souza et al. 2018, Tedersoo et al. 2018) supported the monophyletic nature of the families and or genera proposed by Oehl et al (2008). Hence, we conclude that higher level classification of families in Glomeromycota is debatable. Therefore, in this study, we include an alternative classification for Glomeromycota which was included in Wijayawardene et al. (2018b).

\section{Alternative classification for Glomeromycota}

Glomeromycota C. Walker \& A. Schüßler

Archaeosporomycetes Sieverd., G.A. Silva, B.T. Goto \& Oehl

Archaeosporales C. Walker \& A. Schüßler

Ambisporaceae C. Walker, Vestberg \& A. Schüßler

Ambispora C. Walker, Vestberg \& A. Schüßler (10) 
Archaeosporaceae J.B. Morton \& D. Redecker

Archaeospora J.B. Morton \& D. Redecker (= Intraspora Oehl \& Sieverd.) (3)

Palaeospora Oehl, Palenz., Sánchez-Castro \& G.A. Silva (1)

Geosiphonaceae Engl. \& E. Gilg

Geosiphon F. Wettst. (1)

Glomeromycetes Caval.-Sm.

Diversisporales C. Walker \& A. Schüßler

Acaulosporaceae J.B. Morton \& Benny

Acaulospora Gerd. \& Trappe (= Kuklospora Oehl \& Sieverd.) (57)

Diversisporaceae C. Walker \& A. Schüßler

Corymbiglomus Błaszk. \& Chwat (2)

Desertispora Błaszk., Kozłowska, Ryszka, Al-Yahya’ei \& Symanczik (1)

Diversispora C. Walker \& A. Schüßler (18)

Otospora Oehl, Palenz. \& N. Ferrol (1)

Redeckera C. Walker \& A. Schüßler (3)

Tricispora Oehl, Sieverd., G.A. Silva \& Palenz. (1)

Gigasporaceae J.B. Morton \& Benny

Bulbospora Oehl \& G.A. Silva (1)

Cetraspora Oehl, F. A. Souza \& Sieverd. (6)

Dentiscutata Sieverd., F.A. Souza \& Oehl (7)

Gigaspora Gerd. \& Trappe (7)

Intraornatospora B.T. Goto, Oehl \& G.A. Silva (1)

Paradentiscutata B.T. Goto, Oehl \& G.A. Silva (2)

Racocetra Oehl, F.A. Souza \& Sieverd. (14)

Scutellospora C. Walker \& F.E. Sanders (21)

Pacisporaceae C. Walker, Błaszk., A. Schüßler \& Schwarzott

Pacispora Sieverd. \& Oehl (6)

Sacculosporaceae Oehl, Sieverd., G.A. Silva, B.T. Goto, Sánchez-Castro \& Palenz.

Sacculospora Oehl, Sieverd., G.A. Silva, B.T. Goto, I.C. Sánchez \& Palenz. (2)

Glomerales J.B. Morton \& Benny

Claroideoglomeraceae C. Walker \& A. Schüßler

Claroideoglomus C. Walker \& A. Schüßler (8)

Glomeraceae Piroz. \& Dalpé

Dominikia Błaszk., Chwat \& Kovács (13)

Funneliformis C. Walker \& A. Schüßler (16)

Glomus Tul. \& C. Tul. (55)

Halonatospora Błaszk., Niezgoda, B.T. Goto \& Kozłowska (1)

Kamienskia Błaszk., Chwat \& Kovács (1)

Oehlia Błaszk., Kozłowska, Niezgoda, B.T.Goto \& Dalpé (1)

Rhizoglomus Sieverd., G.A. Silva \& Oehl (19)

Rhizophagus P.A. Dang. (ca. 19)

Sclerocystis Berk. \& Broome (10)

Sclerocarpum B.T. Goto, Błaszk., Niezgoda, Kozłowska \& Jobim (1)

Septoglomus Sieverd., G.A. Silva \& Oehl (10) 
Simiglomus Sieverd., G.A. Silva \& Oehl (1)

Glomeromycetes genus incertae sedis

Entrophospora R.N. Ames \& R.W. Schneid. (3)

Paraglomeromycetes Oehl, G.A. Silva, B.T. Goto \& Sieverd.

Paraglomerales C. Walker \& A. Schüßler

Paraglomeraceae J.B. Morton \& D. Redecker

Paraglomus J.B. Morton \& D. Redecker (1)

Innospora Błaszk., Kovács, Chwat \& Kozłowska (7)

Pervetustaceae Błaszk., Chwat, Kozłowska, Symanczik \& Al-Yahya'ei

Pervetustus Błaszk., Chwat, Kozłowska, Symanczik \& Al-Yahya’ei (1)

Oomycota (Author: M. Thines, with contributions from H.P. Grossart)

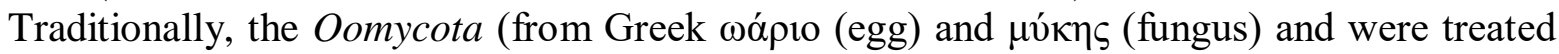
with other osmotrophic aseptate organisms in the polyphyletic "Phycomycetes". However, already at the turn from the $19^{\text {th }}$ to the $20^{\text {th }}$ century, it was known that the oomycetes differed in various aspects, and chlor-zinc-iodine solution, which gives a blue staining with cellulose, was widely used to identify oomycete thalli in their hosts (e.g. Petersen 1905). Already in 1939, just one year, after the first electron microscope became commercially available, Vlk (1939) reported that the zoospores of Saprolegnia did not resemble motile spores of fungi, but rather of heterokont algae. Since then, it was widely recognised that oomycetes belonged within heterokonta, which were suggested as an independent kingdom by Leedale (1974), largely congruent with the kingdom Straminipila, introduced by Dick (2001). By emphasizing periplastid protein targeting, cytoskeletal and periplastid evolution, Cavalier-Smith 2018 proposed a new classification, assuming that Heterokonta, Alveolata, Rhizaria, and Hacrobia (cryptophytes and haptophytes) are a monophylum which could be recognized as a re-circumscribed kingdom Chromista. However, there is still some controversy on the precise phylogenetic relationships between the different groups, in particular haptophytes and cryptomonads may not belong together with the heterokonts or the SAR clade, but be associated with the Archaeplastida (Burki et al. 2007, 2016).

Thus, oomycetes are currently best placed in the kingdom Straminipila and its phylum Oomycota.The kingdom Straminipila forms a major line of eukaryotes (e.g. van den Hoek et al. 1995) currently containing more than 25,000 known species, most of which belong to the two major phyla with photosynthetic members, Phaeophyta and Bacillariophyta. The osmotrophic Oomycota are, with about 1700 recognised species, the next largest phylum of the Straminipila. The main chracterisitic of the Straminipila is the formation of stiff hairs, one of the two flagella, which strengthens and reverses its thrust, rendering oomycete spores excellent swimmers. These stiff "straw hairs" gave their name to the whole group, derived from "stramen" (straw) and "pilus" (hair). The majority of the species of the Oomycota are parasitic (Beakes \& Thines 2017), with Phytophthora infestans, the organism that triggered the Irish Potato Famine, being its most prominent member (Yoshida et al. 2013). Most Oomycota show an asexual reproduction via zoospores formed in zoosporangia or, in case of some genera of the obligate biotrophic downy mildes, such as Peronospora, Hyaloperonospora, and Bremia, via multinucleate, wind-dispersed conidiosporangia. Apart from the holocarpic oomycetes, in which the entire thallus is converted into a zoosporangium, asexual sporangia and conidiosporangia are formed by specialized parts of the mycelium, i.e. sporogenous hyphae, sporangiophores or sporangiophores (Beakes \& Thines 2017). The holocarpic nature is the ancestral form for the whole group, but likey derived in members of Lagenidiales (Buaya \& Thines 2020). Sexual reproduction in most of the species of the crown classes, Peronosporomycetes and Saprolegniomycetes, is by oogamy (gametangiogamy), whereby the zygote often converts into a thick-walled resting spore. However, in many species of the Leptomitales sexual reproduction is cryptic, e.g. by zoomeiospore fusion in 
Lagenisma coscinodisci (Schnepf et al. 1977). Leptomitales are also unusual in that some have a significant amount of chitin in addition to glucans and cellulose in their cell walls (Lin \& Aronson 1970). The orders of the oomycetes that branch before the crown classes are, with the exception of Haliphthorales not forming an extended hyphal network but, apart from the fragmenting thalli in some members of Pontismatales (Buaya et al. 2019), only simple globose to sacculate thalli that convert into zoosporangia. The oospore-like resting structures formed by Olpidiopsidales are likely reflecting convergent evolution to the crown groups. The oomycetes have likely origninated in the marine environment more than 500 million years ago, have colonised limnic and terrestrial habitats several times independently and are now found in almost any environment in which eukaryotic organisms strive (Marano et al. 2016). Most of the known species belong to the probably monophyletic downy mildews (Sharma et al. 2015), which are obligate biotrophic plant parasites that are similar to other obligate parasite groups, have diversified by host jumps, radiation and subsequent speciation (Choi \& Thines 2015, Thines 2019). However, it can be assumed that across all oomycete groups only a small fraction of the existing species are known.

\section{Myxomycetes (Author: D. Leontyev)}

Even the limited phylogenetic data currently available support the conclusion that some very conspicuous characters traditionally considered as important for the classification of myxomycetes have evolved several times independently. This is the case for both the formation and reduction of the stalk and the capillitium, the crystallization of lime deposits, and the evolution of compound fruiting bodies (Leontyev et al. 2014, Leontyev \& Schnittler 2017). All of these characters were used in the traditional system of classification to delimit genera, families and even orders (as was the case for the order Liceales s. $l$., which was characterized by the absence of capillitium). However, in the light of the phylogenetic data, other characters appear to be better predictors of evolutionary relationships than the traditional criteria. These are, for example, the attachment of the capillitium to the peridium, details of stalk formation, color of the spore mass, or the presence of spore-like cells within the stalk. These new criteria were used to provide emended descriptions for some of the traditional myxomycete taxa in the recently published classification by Leontyev et al. (2019).

\section{Dictyostelids (Author: D. Leontyev)}

The new system of classification for dictyostelids works well for the genus level; however, it relies on $18 \mathrm{~S}$ gene markers to distinguish species, and this does not always separate out some morphologically similar but genetically different taxa. Moreover, Coremiostelium and Synstelium still occupy a problematic position in the entire phylogeny (Sheikh et al. 2018). A consideration of morphological characteristics is essential for evaluating new species of dictyostelids. Identifying new genetic markers to use for distinguishing dictyostelids at the species level would be exceedingly worthwhile.

\section{Hidden taxa of the fungal tree of life (Authors: F.Q. Brearley \& D. Haelewaters)}

Whereas four to five million species of fungi are estimated to be found across the globe, we have only described around 144,000 of these (i.e. less than 2\%) (Willis et al. 2018). The question remains where are all these fungi yet to be described can be found? asa Lücking (2017) suggest that these missing fungi will be found in: i) habitats that are naturally diverse yet poorly explored, e.g., tropical forests; ii) cryptic taxa, those that are morphologically indistinguishable; and iii) in fungal collections that might contain cryptic or new species hidden under current names. These three categories are not mutually exclusive and, with the rapid rise of next-generation sequencing, we can also add iv) molecular novelties. A final category that is often neglected is the study of natural history collections of organisms other than fungi (plant herbaria, dried insect collections).

Large collections of dried, pinned insects have been dubbed "treasure troves" for the study of ectoparasitic fungi, such as the Laboulbeniales (Laboulbeniomycetes). During a study of different systematic insect collections around the world, Laboulbeniales fruit bodies were 
discovered on 1,937 of 45,785 specimens (Haelewaters \& Rossi 2017). This and other insect collections-based works have led to the description of multiple new species (e.g., Santamaria et al. 2016, Haelewaters \& Rossi 2017), a better understanding of host usage patterns and global number of Laboulbeniales species (Weir \& Hammond 1997), and estimates of parasite prevalences on a given host through time (Haelewaters et al. 2017). Likewise, plant herbaria can be screened for fungal "hitchhikers" inadvertently sampled along with the plant host (Lang et al. 2019). Examples are the causal agent of potato late blight (Phytophthora infestans), rust fungi, downy mildews, etc. Herbarium specimens allow for identification of fungal strains based on morphology and DNA sequence data, which can be coupled with host plant studies to provide a complete overview of host-pathogen dynamics.

Given that fungi are often microscopic, morphologically similar among species, occupy hidden habitats, and are often recalcitrant in axenic culture, it is difficult to find them, but perhaps they are simply 'hiding' in material already collected. Lücking et al. (2014) found that a single species of Cora was actually more than one-hundred species following more careful morphological observation and sequencing. This restudy/resequencing of currently known taxa is likely to increase the number of known species. An increasing number of examples of cryptic diversity is coming to light with the advances of molecular phylogenetic studies, in different groups, e.g., Eurotiomycetes (Pringle et al. 2005), Laboulbeniomycetes (Haelewaters et al. 2018a), Lecanoromycetes (Singh et al. 2015) and Leotiomycetes (Grünig et al. 2008) in Ascomycota; Agaricomycetes (Stefani et al. 2014, Accioly et al. 2019), and Ustilaginomycetes (Li et al. 2017) in Basidiomycota.

Because of the decreasing price and increasing availability, large-scale sequencing studies offer the opportunity to mined data for new species. These large-scale sequencing studies offer the opportunity to find new species as such studies regularly have a sizeable proportion of sequences that can only assigned at a phylum or even kingdom level. Indeed, the rate of accumulation of novel/unassigned sequences is massively outpacing the rate with which taxonomists can describe new species, especially given the lack of taxonomic expertise among, for example, the basal fungal clades.

Whilst some have called for a sequence-based taxonomy (Hibbett et al. 2016) we recognise that this is a controversial subject and, instead, note that these sequences can be used in a 'taxonomic feedback' capacity to identify new taxa. For example, Rosling et al. (2011) and Jones et al. (2011) described the class of ubiquitous soil-inhabiting fungi, the Archaeorhizomycetes, and the basal fungal phylum the Cryptomycota, respectively. Capitalising on the global sequencing of soil samples by Tedersoo et al. (2014), Tedersoo et al. (2017) re-analysed samples and found nearly 40 strongly supported new clades (roughly equivalent to orders) in the fungal tree of life. About half of these were in the basal clades (particularly Rozellomycota) and about half were found in tropical habitats. This study looked at soil samples only but fungi associated with other habitats such as aquatic fungi or endophytic fungi would benefit from further study - aquatic habitats may be particularly rich in new clades and taxa given the association of basal clades with aquatic habitats. The rich resource of DNA extracted from the millions of plant leaves stored in herbaria can also be fruitfully mined to find new plant-associated fungi (e.g. Datlof et al. 2017, Daru et al. 2018).

Another possibility is that the molecular markers we are currently using are not sufficient to delineate the full range of 'species' (OTUs, RSVs etc.) - this is certainly the case for Glomeromycota where the ITS region is not sufficiently phylogenetically informative and development and comparison of suitable primers has occupied a lot of energy (e.g. Kohout et al. 2014).

Whilst some have called for a molecular taxonomy, we recognise that this is controversial and, as yet, not incorporated into relevant taxonomic codes. Therefore, discovery of new physical specimens is required and the work of Henkel et al. (2012) and Hyde et al. (2018b) illustrate how dedicated work of mycologists over a number of years can lead to the discoveries and descriptions of numerous new fungal species. Indeed, Truong et al. (2017) show how this can be done with 
straightforward short collecting expeditions of underexplored habitats (Nothofagus forest of South America in this case) combined with taxonomic work and sequencing of relevant material to advance our knowledge of fungal diversity in a comprehensive way.

Notes

Abrothallus De Not.

The phylogenetic reconstruction by Suija et al. (2015) showed that Abrothallus accommodates the monotypic Epinephroma Zhurb. and made a new combination based on that. Pérez-Ortega et al. (2011) showed that Vouauxiomyces Dyko \& D. Hawksw. represents an asexual stage of Abrothallus. Synonymization with Abrothallus was done in Rossman et al. (2016) (A. Suija).

Abrothallaceae Pérez-Ort. \& Suija

Diederich et al. (2018) proposed to synonymize Licheniconiaceae with Abrothallaceae as several studies beforehand (e.g. Liu et al. 2017) have revealed close relationships of these two monotypic families. However, Hngsanan et al. (2020) accepted Licheniconiaceae over Abrothallaceae (A. Suija \& N. Wijayawardene).

Acrodictyaceae J.W. Xia \& X.G. Zhang

Xia et al. (2017) established Acrodictyaceae and Junewangiaceae in Sordariomycetes (J. Ma).

Acrogenosporaceae Jayasiri \& K.D. Hyde

Based on a multi-gene phylogeny, Jayasiri et al. (2018) showed that this is a distinct family within the Minutisphaerales (S. Fryar).

Aculeata W. Dong, H. Zhang \& K.D. Hyde

Dong et al. (2018) introduced this genus in Herpotrichiellaceae based on phylogenetic analyses and distinct morphological characters (Q. Tian).

Adelolecia Hertel \& Hafellner Timdal).

Kistenich et al. (2018) transferred this genus from Ramalinaceae to Lecanoraceae (E.

Aeruginoscyphus Dougoud

Dougoud (2012) introduced this genus within Hyaloscyphaceae to accommodate Peziza sericea (D. Haelewaters).

Agyriaceae Corda

Kraichak et al. (2018a) regarded that Miltideaceae Hafellner as a synonym of Agyriaceae (N. Wijayawardene).

Alanomyces Roh. Sharma

Sharma et al. (2017) showed that this genus is a separate lineage in Aplosporellaceae and introduced a new genus with a single species (A.J.L. Phillips).

Alleppeysporonites Ramanujam \& K.P. Rao (fossil).

This genus shows striking similarity to the dematiaceous fungus Grallomyces F. Stevens (Barnett 1956, Ellis 1971, Subramanian 1971) (R.K. Saxena).

Allographa Chevall. 
Lücking \& Kalb (2018) resurrected Allographa from synonymy of Graphis (M. Kukwa).

\section{Alternaria Nees}

Currently, Alternaria has about with 589 legitimate species epithets (MycoBank 2019). More than 150 of them have been synonymized or assigned as "indistinguishable as unique" by Simmons (2007) since their descriptions and illustrations are scanty while the type material is unavailable. Remaining list of species should be reduced by sixty five synonymic names due to revisions of Woudenberg et al. $(2014,2015)$. Thirteen species should be transferred to Alternaria from abolished genera Ulocladium and Nimbya (Gannibal 2018, Gannibal \& Lawrence 2018). Thus the genus Alternaria at the moment contains 366 accepted and recognizable species. Not all of them have been subjected to molecular phylogenetic studies (P.B. Gannibal).

\section{Amarenomyces O.E. Erikss.}

Rossman et al. (2015) treated Amarenomyces as a synonym of Amarenographium. Based on molecular analyses coupled with morphological characteristic, Wijayawardene et al. (2016) introduced Amarenographium ammophilae Wanas.et al.; whereas, Hyde et al. (2017) introduced Amarenomyces dactylidis Mapook et al. Multigene phylogenetic analyses revealed that these two genera were not congeneric, thus Hyde et al. (2017) reinstated Amarenomyces (R. Phookamsak).

\section{Amazonotheca Bat. \& H. Maia}

Phookamsak et al. (2016) treated the genus in Dothideomycetes, genera incertae sedis based on herbarium study from Dr. Lima's collection (P-4. Serra do Veado-Serra do Navio, 24 August 1961, Lima J.A. (Leg.), A.C. Batista A.C. \& Xavier Filho L. (det.), URM 28927). The type specimen was deposited in the Universidade Federal de Pernambuco (URM), Brazil but it could not be loaned (R. Phookamsak).

\section{Amorphothecaceae Parbery}

Amorphothecaceae is a monotypic family that was previously placed in Leotiomycetes genera incertae sedis (Baral 2016). Ekanayaka et al. (2019) retrieved it as sister to Erisyphaceae but this placement was without support. Strong support came with the 15-gene tree of Johnston et al. (2019), in which Amorphothecaceae was placed in the pezizelloid clade of Helotiales (D. Haelewaters).

Anatolinites Elsik, V.S. Ediger \& Bati (fossil)

Spores of Anatolinites have a tendency to break apart along the septa. Kalgutkar \& Jansonius (2000) considered Cupulisporonites Z.C. Song \& Liu Cao a junior taxonomic synonym of Anatolinites (R.K. Saxena).

Annabella Fryar, Haelew., \& D.E.A. Catches.

Fryar et al. (2019) introduced this genus for A. australiensis Fryar, Haelew., \& D.E.A. Catches. from mangrove wood with perithecioid hyaline to yellowish apothecia. The genus is confirmed as a member of Cordieritidaceae based on the molecular phylogenetic analysis of a concatenated dataset of three ribosomal nuclear loci (D. Haelewaters).

Antennopsis R. Heim

Guswenrivo et al. (2018) used the partial 18S rRNA gene to place this genus within Ascomycota and found that it is positioned within Sordariomycetes. It has a sister relationship to Graphium euwallaceae (Graphiaceae), but we are reluctant in formally placing Antennopsis in this family, because the phylogeny was based only on 18S (D. Haelewaters).

Anthopsis Fil. March. et al. 
Phylogenetic analyses placed this genus in Cyphellophoraceae, Chaetothyriales. The type species, A. deltoidea, clusters within a large Cyphellophora clade and therefore Anthopsis might be a synonym of Cyphellophora. The synonymy, however, has not yet been properly proposed. Anthopsis currently includes three species, A. deltoidea, A. catenata and A. microspora, but DNA sequence data proved that A. catenata is not a member of Chaetothyriales (Moussa et al. 2017). No DNA sequence data is available for A. microspora (H. Madrid).

Aotearoamyces P.R. Johnst., J.A. Cooper \& Quijada

Quijada et al. (2018a) introduced this genus for A. nothofagi, a species from fallen wood in Nothofagaceae forests in New Zealand. It was placed in the family Tympanidaceae within the order Phacidiales based on the phylogenetic analysis of three ribosomal nuclear loci. Based on a larger 15-locus analysis, Johnston et al. (2019) placed Aotearoamyces within Leotiales genera incertae sedis with high support (D. Haelewaters).

\section{Aplosporella Speg.}

Slippers et al. (2013) suggested that this genus is the sexual morph of Aplosporella, but they declined to make a formal synonymy. Phillips et al. (2019) formally placed Bagnisiella as a synonym of Aplosporella (A.J.L. Phillips).

Appendicisporonites R.K. Saxena \& S. Khare (fossil).

Appendicisporonites appears to be related to the setose pycnidia found in some Coelomycetes Grove (R.K. Saxena).

\section{Aquacidia Aptroot}

Aptroot et al. (2018) introduced this genus and confirmed its placement in Pilocarpaceae (N. Wijayawardene).

Aquimonospora J. Yang \& K.D. Hyde

Yang et al. (2019) introduced this monotypic genus to accommodate the new species Aquimonospora tratensis. A new genus and species collected from freshwater habitat in Thailand (S. Tibpromma).

\section{Arthrocladium Papendorf}

This genus comprises four species which form a monophyletic group in Trichomeriaceae. They are associated mainly with plant material and occasionally also with opportunistic infections in humans (Nascimento et al. 2016) (H. Madrid).

Arthrophiala (D.J. Soares, R.W. Barreto \& U. Braun) W.S. Lisboa et al.

This monotypic genus was recently erected to accommodate the plant pathogenic fungus Pseudocercospora arthrospora. DNA sequence data revealed that this fungus is a member of Chaetothyriales (Crous et al. 2016), while true Pseudocercospora species belong in Capnodiales. In the protologue, Arthrophiala was considered as a member of Chaetothyriaceae, but its definitive phylogenetic placement may require further study (H. Madrid).

Ascochytites Barlinge \& Paradkar (fossil)

The genus name Ascochytites was first used by Teterevnikova-Babaian \& Taslakhchian (1973), but not then validly published due to lack of illustration of the spores (R.K. Saxena).

Ascodesmisites Trivedi, Chaturv. \& C.L. Verma (fossil)

The fossil specimen shows close affinity with family Pezizaceae but differs from the latter by the absence of exciple. Minute fruiting bodies and various sexual stages somewhat resemble 
those of Ascodesmis Tiegh. In fossil specimen, the male and female sex organs are found growing on two different hyphae indicating that the fungus could be dioecious (R.K. Saxena).

Asterinites Doub. \& D. Pons ex Kalgutkar \& Janson. (fossil)

Jansonius \& Hills (1976) remarked: "Two species described; until one of these is selected as type species, the genus is not validly published. The genus seems to be intended for mycelia, rather than definite fruiting bodies". The lectotype was designated by Kalgutkar \& Jansonius (2000) (R.K. Saxena).

Asterothyrites Cookson emend. Kalgutkar \& Janson. (fossil)

The lectotype was selected by Jansonius \& Hills (1976). Kalgutkar \& Jansonius (2000) emended the generic diagnosis. Paramicrothallites K.P. Jain \& R.C. Gupta is a junior taxonomic synonym of Asterothyrites (Kalgutkar \& Jansonius 2000) (R.K. Saxena).

Astragalicola Jaklitsch \& Voglmayr

Jaklitsch et al. (2018) proposed this new genus based on morphological and molecular data (S. Fryar).

Atractium Syd. \& P. Syd.

For many years, this genus was considered as a synonym of Fusarium, but a multilocus phylogenetic study by Gräfenhan et al. (2011) proved that Atractium represents a distinct genus in Nectriaceae (H. Madrid).

Axisporonites Kalgutkar \& Janson. (fossil)

Kalgutkar and Jansonius (2000) designated Multicellaesporites indicus P. Kumar (Now: Axisporonites indicus (P. Kumar) Kalgutkar \& Janson.) as type of this monotypic genus (R.K. Saxena).

Bacidia De Not.

Kistenich et al. (2018) treated Bacidiopsora Kalb as synonym of Bacidia (E. Timdal).

Bacillicladium Hubka, Réblová \& Thureborn

This monotypic genus, typified by $B$. lobatum, forms a distinct lineage in Chaetothyriales which might represent an undescribed family (Réblová et al. 2016) (H. Madrid).

\section{Baeomycetales Lumbsch et al.}

Kraichak et al. (2018a) regarded that Arctomiales S. Stenroos et al., Hymeneliales S.

Stenroos et al and Trapeliales B.P. Hodk. \& Lendemer as synonyms of Baeomycetales (N. Wijayawardene).

\section{Bahusandhika Subram.}

Liu et al. (2018a) transferred this genus to the new family, Lentimurisporaceae, based on a multi-gene phylogeny (S. Fryar).

\section{Barrenia E. Walsh \& N. Zhang}

Johnston et al. (2019) accepted this genus in Mollisiaceae (D. Haelewaters).

\section{Barrmaeliaceae Voglmayr \& Jaklitsch}

Voglmayr et al. (2018) recognised the family Barrmaeliaceae with the genera Barrmaelia and Entosordaria from phylogenetic analyses of a combined DNA data matrix containing ITS, LSU, rpb2 and tub2 sequences of representative Xylariales including the type species of both genera. They also established that the morphologically similar genus Clypeosphaeria belongs to 
Xylariaceae sensu stricto. The other genera of the family Clypeosphaeraceae aside from Clypeosphaeria, however, still need to be studied by methods of polyphasic taxonomy to assure their affinities to the Xylariaceae. The study by Vogmayr et al. (2018) also revealed that some DNA sequence data of the genera Alloanthostomella, Neoanthostomella, and Pseudoanthostomella that are available in the public domain were erroneous, which gave rise to some serious doubt as to their familiar affinities. These genera are therefore placed in Xylariales Incertae sedis until the phylogenetic position has been verified based on new DNA sequence data (M. Stadler).

Basidiobolales Jacz. \& P.A. Jacz.

Basidiobolales Caval.-Sm., Biological Reviews Cambridge 73: 246 (1998) is an isonym (K. Bensch).

Basidiobolomycetes Doweld

Basidiobolomycetes Humber, Mycotaxon 120: 484 (2012) is an isonym, Doweld was published already in 2001; see IF and MB (K. Bensch).

Basidiosporites Elsik (fossil)

Kalgutkar \& Jansonius (2000) considered Amepiospora Sal.-Cheb. \& Locq. a later synonym of this genus (R.K. Saxena).

Bellicidia Kistenich, Timdal, Bendiksby \& S.Ekman

Kistenich et al. (2018) introduced this genus in Ramalinaceae (E. Timdal).

Biatora Fr.

Kistenich et al. (2018) reduced Ivanpisutia S.Y. Kondr., Lőkös \& Hur and Myrionora R.C. Harris under this genus (E. Timdal).

Bibbya J.H.Willis

Kistenich et al. (2018) resurrected this genus in Ramalinaceae (E. Timdal).

Bifusisporella R.M.F. Silva et al.

Silva et al (2019) placed this new genus in the family Magnaporthaceae based on molecular and morphological characters. This genus shown the phylogenetic inference related to Omnidemptus but differ in by having an asexual morph with sporodochial conidiomata (Cannon \& Alcorn 1994) (S. Tibpromma).

Biporipsilonites Kalgutkar \& Janson. (fossil)

Spores in this genus can be differentiated from Diporisporites Hammen by having pore chambers. Kalgutkar \& Jansonius (2000) designated Diporicellaesporites belluloides Z.C. Song (Now: Biporipsilonites belluloides (Z.C. Song) Kalgutkar \& Janson.) as type of this genus (R.K. Saxena).

Botryosphaeriaceae Theiss. \& Syd.

See under Endomelanconiopsis E.I. Rojas \& Samuels Tao (A.J.L. Phillips).

Botryozyma Shann \& M.T. Sm. emend. Lachance \& Kurtzman

Kurtzman \& Boekhout (2017) include Ascobotryozyma J. Kerrigan, M.T. Sm. \& J.D. Rogers (under Botryozyma) to comply with the Melbourne Code (W.P. Pfliegler \& E. Horváth).

Boubovia Svrček 
This genus and Coprotus Korf \& Kimbr. represents an independent clade of Pezizales, not included in Ascodesmidaceae, as shown by Perry et al. (2007) and also Hansen et al. (2013) (P. Alvarado).

Brachysporisporites R.T. Lange \& P.H. Sm. (fossil)

Granatisporites Elsik \& Janson. is a junior taxonomic synonym of Brachysporisporites (Kalgutkar \& Jansonius 2000). Spores of Brachysporisporites are usually compared to the conidia of the modern Brachysporium Sacc. (R.K. Saxena).

Bradymyces Hubka et al.

This genus currently includes with three species which form a monophyletic group within Trichomeriaceae (Réblová et al. 2016) (H. Madrid).

Brefeldiellites Dilcher (fossil)

The fossil form of this monotypic genus is similar to the Brefeldiella Speg. but cannot be placed in this extant genus because the spores are not known (R.K. Saxena).

Bresadolia Speg.

Motato-Vásquez et al. (2018) reinstated this genus and accepted as in Polyporaceae (V. Papp).

Brigantiaeaceae Hafellner \& Bellem.

Kraichak et al. (2018a) regarded Letrouitiaceae as a synonym of Brigantiaeaceae (N. Wijayawardene).

Brunneofusispora S.K. Huang \& K.D. Hyde

Brunneofusispora was introduced in Phookamsak et al. (2019) to accommodate massarinalike species and species identified as Massarina rubi (Fuckel) Sacc. Multi-gene phylogenetic analyses revealed that the genus formed a distinct clade within Occultibambusaceae (Phookamsak et al. 2019) (R. Phookamsak).

Brunneomurispora Phook. et al.

Phookamsak et al. (2019) introduced a monotypic genus Brunneomurispora to accommodate $B$. lonicerae. Multi-gene phylogenetic analyses showed that the genus formed a distinct clade basal to Neosetophoma in Phaeosphaeriaceae. However, the sexual morph of Neosetophoma has phragmosporous ascospores; whereas, Brunneomurispora has dictyosporous ascospores. Asexual morph of Brunneomurispora is undetermined (R. Phookamsak).

Bryobilimbia Fryday et al.

Fryday et al. (2014) introduced Bryobilimbia to accommodate six species, including Lecidea hypnorum and some related taxa based on morphological characters such as bacilliform conidia, slightly swollen paraphyses and thallus minutely squamulose without marginal lobes. Phylogenetic analyses revealed Bryobilimbia as a distinct monophyletic lineage within Lecideaceae, Lecideales, Lecanoromycetes (P. Rodriguez-Flakus).

Bulbomicroidium Marm. et al.

Marmolejo et al. (2018) introduced this monotypic genus and confirmed its placement in Erysiphaceae based on molecular phylogenetic analysis of the LSU (S. Takamatsu).

Byssonectria P. Karst.

The genus Kotlabaea Svrček has been reduced under Byssonectria by Lindemann et al. (2015) (P. Alvarado). 


\section{Callimothallus Dilcher (fossil)}

Callimothallus lacks any central dehiscence and is characterized by numerous pores. Elsik (1978) pointed out that the porate condition in Callimothallus is required for at least in a number of the cells to separate it from Phragmothyrites W.N. Edwards and that if the porate nature is well represented, even fragments of the fructification are recognizable. Kalgutkar \& Jansonius (2000) considered Pseudosphaerialites Venkatach. \& R.K. Kar and Siwalikiathyrites R.K. Saxena \& H.P. Singh as junior synonyms of Callimothallus (R.K. Saxena).

Calongea Healy, Bonito \& Trappe

This genus was erected to accommodate Pachyphloeus prieguensis (Healy et al. 2009), reflecting a separate lineage within Pezizaceae (I. Kušan, N. Matočec \& P. Alvarado).

\section{Calyptosphaeria Réblová \& A.N. Mill.}

Réblová et al. (2018) introduced this genus to accommodate two new species and two new combinations based on a multi-gene phylogeny and morphological characters (S. Fryar).

\section{Camptophora Réblová \& Unter.}

This genus, originally proposed to accommodate a 'Cyphellophora' species clustering outside the Cyphellophoraceae clade. It currently includes two species, viz. C. hylomeconis, the generic type and C. schimae (Réblová et al. 2013, Yang et al. 2018a) (H. Madrid).

\section{Candida Berkhout}

Transfer of several Candida species to various yeast genera (including novel genera) is expected. But Candida is expected to be the retained genus name for the $C$. tropicalis clade instead of Lodderomyces Van der Walt in Debaryomycetaceae to comply with the Melbourne Code. Transferring the hundreds of Candida species to monophyletic genera is one of the main current challenges in yeast taxonomy (Kurtzman \& Boekhout 2017) (W.P. Pfliegler \& E. Horváth).

Castanedospora G. Delgado \& A.N. Mill.

Delgado et al. (2018) introduced this hyphomycetous genus to accommodate Sporidesmium pachyanthicola R.F. Castañeda \& W.B. Kendr. In the phylogenetic analyses, it grouped in Extremaceae (Capnodiales, Dothideomycetes) distant from Sporidesmiaceae sensu stricto in Sordariomycetes (G. Delgado \& S. Fryar).

\section{Catinaria Vain.}

Kistenich et al. (2018) transferred this genus from Ramalinaceae to the Lecanorales genera incertae sedis (E. Timdal).

\section{Cenangiaceae Rehm}

In their 15-gene phylogeny, Johnston et al. (2019) found high support for a sclerotinioid clade including Cenangiaceae within the Helotiales. Here, we follow the emended concept of Pärtel et al. (2017) for the family Cenangiaceae, including the following genera: Cenangiopsis, Cenangium, Chlorencoelia, Crumenulopsis, Encoelia, Fabrella, Heyderia, Rhabdocline, Sarcotrochila, Trochila, and Velutarina (D. Haelewaters).

\section{Cephaliophora Thaxt.}

This genus is firmly nested as a separate lineage within Ascodesmidaceae, Pezizales (Kušan et al. 2018) (I. Kušan \& N. Matočec).

Cercosporites E.S. Salmon (fossil) 
Aspergillites Trivedi \& C.L. Verma ex Janson., Hills \& Hartk.-Fröd. is a taxonomic latter synonym of Cercosporites (Kalgutkar \& Jansonius 2000) (R.K. Saxena).

\section{Chaetosphaerites Félix (fossil)}

This genus is characterized by spores having two middle cells being dark brown and two end cells pale brown. The shape of the sporidia is strongly obtuse spindle-shaped, almost like that of a cylinder with rounded ends. Cannanorosporonites Ramanujam \& K.P. Rao is a latter taxonomic synonym of Chaetosphaerites (R.K. Saxena).

Cheirospora Moug. \& Fr.

Johnston et al (2019) accepted this genus in Mollisiaceae (D. Haelewaters).

\section{Chlorociboriaceae Baral \& P.R. Johnst.}

Based on Johnston et al.'s (2019) 15-gene phylogenetic analysis, Chlorobiboriaceae is part of the sclerotinioid clade within Helotiales (D. Haelewaters \& N. Wijayawardene).

Chroogomphus (Singer) O.K. Mill.

Scambler et al. (2018) revised the infrageneric classification of the genus, introduced three subgenera (Chroogomphus, Floccigomphus and Siccigomphus) and five sections/clades within subg. Chroogomphus (Chroogomphus, Confusi, Filiformis, Fulminei and the informal Vinicolores clade). Chroogomphus subfulmineus from Cyprus, Finland and the UK, and C. pakistanicus and C. pruinosus from Pakistan were recently described bringing the number of recognized species to 25 (Scambler et al. 2018, Kiran et al. 2020) (D. Haelewaters).

Chrysodiscaceae Baral \& Haelew.

Baral \& Polhorský (2019) introduced this family to accommodate Chrysodisca peziculoides, a broadly distributed European discomycete resembling Pezicula but phylogenetically separated from previously recognized families within Helotiales (D. Haelewaters).

Chrysonectria Lechat \& J. Fourn

Lechat et al (2018a) introduced this genus and showed that it is phylogenetically belongs to Bionectriaceae (N. Wijayawardene)

\section{Cinereomycetella Zmitr.}

Justo et al. (2017) showed that Diplomitoporus overholtsii (Pilát) Gilb. \& Ryvarden forms a distinct phylogenetic lineage in the family Polyporaceae. Hence, Zmitrovich (2018) introduced the monotypic genus Cinereomycetella in Gelatoporiaceae to accommodate Diplomitoporus overholtsii (V. Papp).

\section{Cladoniaceae Zenker}

Kraichak et al. (2018a) revised Lecanoromycetes and showed that Squamarinaceae Hafellner, Stereocaulaceae Chevall are synonyms of Cladoniaceae Zenker (in Lecanorales) based on temporal-based classification (N. Wijayawardene).

\section{Cladophialophora Borelli}

The genus Cladophialophora is polyphyletic within Chaetothyriales. The generic type, $C$. carrionii and most species of this genus belong in Herpotrichiellaceae, but a few species are related to other chaetothyrialean families, such as Epibryaceae and Trichomeriaceae (Madrid et al. 2016) (H. Madrid).

Cladorrhinum Sacc. \& Marchal 
Phylogenetic studies by Cai et al. (2006), Madrid et al. (2011) and Carmarán et al. (2015) proved that this genus belongs in Lasiosphaeriaceae. Nevertheless, Hyde et al. (2020) accepted this genus in Podosporaceae. The genus, however, is polyphyletic within this fungal family $(\mathrm{H}$. Madrid).

\section{Cladosporites Félix (fossil)}

The conidia greatly resemble those of the genera Cephalothecium Corda and Cladosporium Link. (R.K. Saxena).

\section{Clarireedia L.A. Beirn}

Salgado-Salazar et al. (2018) proposed this new genus with C. homoeocarpa as the type species (Rutstroemiaceae) based on molecular and morphological characters. The genus comprises three other species except the type species viz. C. bennettii, C. jacksonii, and $C$. monteithiana (Salgado-Salazar et al. 2018) (S. Fryar \& S. Somrithipol).

\section{Claussenomyces Kirschst.}

According to Quijada et al (2018b) and Species Fungorum (2020), the genus Claussenomyces currently contains 16 species. However, the genus is "heterogeneous" (Jaklitsch et al. 2016a) and polyphyletic. As a result, it is currently under taxonomic revision. "Claussenomyces" prasinulus is treated as Leotiales genera incertae sedis based on its placement in Johnston et al.'s (2019) 15-gene tree. (D. Haelewaters, I. Kušan \& N. Matočec).

\section{Cochlearomyces Crous}

Crous et al. (2017) established this genus to accommodate a synnematous fungus on leaf litter of Eucalyptus. The same authors also introduced a new family (Cochlearomycetaceae) for the genera Cochlearomyces and Satchmopsis, which was retrieved in Leotiales based on the phylogenies of both Ekanayaka et al. (2019) and Johnston et al. (2019) (D. Haelewaters).

\section{Coleophoma Hohn.}

Using a polyphasic approach, Crous \& Groenewald (2016) established the teleomorphanamorph connection between Coleophoma and Parafabraea Chen Chen, Verkley \& Crous, thus reducing Parafabraea to synonymy. Coleophoma is placed in Dermateaceae (Johnston et al. 2019) (D. Haelewaters).

Colligerites K.P. Jain \& R.K. Kar (fossil).

Involutisporonites Elsik broadly resembles Colligerites but in the former, coiling is not perfect and it has a hyaline cell at the tip (R.K. Saxena).

\section{Compsocladium I.M. Lamb}

Kistenich et al. (2018) transferred this genus from Ramalinaceae to the Lecanorales incertae sedis (E. Timdal).

Coprotus Korf \& Kimbr.

See under Boubovia Svrček (P. Alvarado).

Coprinites Poinar \& Singer (fossil)

The fossil mushroom has affinity with the present day genus Coprinus Pers (R.K. Saxena).

Corticifraga D. Hawksw. \& R. Sant.

The genus Corticifraga, comprising 7 species and has been recently transferred to Gomphilaceae (Pino-Bodas et al. 2017, Suija et aí. 2018), but so far only the type species has been sequenced (M. Kukwa \& A. Suija). 
Craspedodidimella F.R. Barbosa, R.F. Castañeda \& Gusmão

Barbosa et al. (2017) introduced this genus and showed that it belongs in Ascomycota genera incertae sedis (F.R. Barbosa).

Crassiclypeus A. Hashim. et al.

Hashimoto et al. (2018) proposed this genus with Crassiclypeus aquaticus as the type species, which was previously included in the Lophiostoma bipolare species complex (S. Fryar).

Crepatura C.L. Zhao

Zhao et al. (2019) proposed this new genus with Crepatura ellipsospora as the type species based on molecular and morphological characters. While, this genus phylogenetically closely related to Pirex concentricus but morphology is different (S. Tibpromma).

\section{Crustospathula Aptroot}

Kistenich et al. (2018) transferred this genus from the Ramalinaceae to the Malmideaceae (E. Timdal).

Cryptodidymosphaerites Currah, Stockey \& B.A. LePage (fossil)

This genus from the Princeton chert presents sufficient characters such as the presence of a pseudothecium, ascospores morphology, orientation and the mycoparasitic habit to place it close to Didymosphaeria Fuckel. (Aptroot 1995) (R.K. Saxena).

\section{Cryptodiscus Corda}

Pino-Bodas et al. (2017) regarded that Lettauia D. Hawksw. \& R. Sant. as a synonym of Cryptodiscus, however, 3 species are still orphaned under that name, but so far have not been relocated (M. Kukwa \& A. Suija).

\section{Cryptophyllachora L. Kiss, Kovács \& R.G. Shivas}

Kiss et al. (2018) proposed Cryptophyllachora to accommodate $C$. eurasiatica (the type species) collected from common ragweed (Ambrosia artemisiifolia) in Hungary, and C. ambrosiae that was transferred from Phyllachora ambrosiae (S. Somrithipol).

Cucitella Jaklitsch \& Voglmayr

Jaklitsch et al. (2018) proposed this new genus based on morphological and molecular data (S. Fryar).

Cucurbitariaceites R.K. Kar, R.Y. Singh \& S.C.D. Sah (fossil)

Cucurbitariaceites is easily distinguishable from all the fossil genera of Microthyriales $\mathrm{G}$. Arnaud. by its circular to subcircular shape, darker outer layer and thin inner layer, in the absence of true paraphyses and the presence of cylindrical asci. Cucurbitariaceites closely resembles the extant family Cucurbitariaceae G. Winter in all the characters and Cucurbitaria Gray is the widely known genus of this family (Bessey 1950) (R.K. Saxena).

\section{Culbersonia Essl.}

This genus is considered part of the Caliciaceae by Aptroot et al. (2019) (P. Alvarado).

\section{Cylindriaceae Crous \& L. Lombard}

Crous et al. (2018) introduced this new family to accommodate Cylindrium, which was previously in incertae sedis. Hyde et al. (2020) accepted this family in Amphisphaeriales (S. Fryar). 


\section{Cylindrosporium Grev.}

Baral (2016) and Ekanayaka et al. (2019) accepted this genus in Ploettnerulaceae (D. Haelewaters \& N. Wijayawardene).

\section{Cyphellophora G.A. de Vries}

Species of Cyphellophora are characterized by slow-growing colonies, dematiaceous mycelium and phialidic conidiogenous cells usually with conspicuous collarettes. Phylogenetically, they form a distinct clade in Chaetothyriales for which the family Cyphellophoraceae was erected (Réblová et al. 2013) (H. Madrid).

\section{Deccanodia Singhai (fossil)}

Deccanodia resembles Diplodia Fr. in its pycnidium and 2-celled, brown and mostly ellipsoid conidia (Barnett 1960, Gilman 1959). However, in Diplodia, black pycnidia and equal 2celled conidia are present thus showing a distinct difference from the fossil fungus which has slightly brown pycnidium and unequally divided 2-celled conidia. Deccanodia also resembles Apiocarpella Syd., belonging to Fungi Imperfecti (Barnett 1960) (R.K. Saxena).

\section{Dekkera Van der Walt}

Expected transfer of species to Brettanomyces Kufferath \& van Laer to comply with the Melbourne Code (Kurtzman \& Boekhout 2017) (W.P. Pfliegler and E. Horváth).

\section{Dendrostoma X.L. Fan \& C.M. Tian}

Fan et al. (2018a) placed this new genus in the family Erythrogloeaceae with three species viz. D. mali (the type species), D. quercinum, and D. osmanthi. The genus comprises 19 species associated wood canker disease (Jaklitsch et al. 2019, Jiang et al. 2019b, Zhu et al. 2019). (S. Fryar, X. Fan \& S. Somrithipol).

\section{Densocarpa Gilkey}

According to recently shown phylogenies (e.g. Kumar et al. 2017), this genus should not be considered as a synonymy of Stephensia as it represents separate lineage in Geopyxis clade (I. Kušan \& N. Matočec).

\section{Densospora McGee}

Single-locus and multigene phylogenetic reconstructions depict Densosporaceae as a distant sister clade of Endogonaceae within the order Endogonales (Desirò et al. 2017) (K. Bensch).

Dentocorticium (Parmasto) M.J. Larsen \& Gilb.

Liu et al. (2018b) treated Dendrodontia Hjortstam \& Ryvarden and Fuscocerrena Ryvarden as synonyms of Dentocorticium (V. Papp).

\section{Dextrinoporus H.S. Yuan}

Yuan \& Qin (2018) introduced this monotypic genus to accommodate the new species $D$. aquaticus H.S. Yuan and showed that it forms a distinct phylogenetic lineage in the family Polyporaceae (V. Papp).

Diademosa Shoemaker \& C.E. Babc.

This genus has been originally considered as a genus of Diademaceae (Shoemaker \& Babcock 1992). Later it was transferred to Pleosporaceae (Ariyawansa et al. 2014) but molecular phylogeny has not still used for its replacement (P.B. Gannibal). 
Yang et al. (2018b) introduced Diaporthosporellaceae for Diaporthosporella cercidicola, a new genus and species collected from diseased branches of Cercis chinensis in China (S. Somrithipol \& S.S.N. Maharachchikumbura).

Diaporthostoma X.L. Fan \& C.M. Tian

Diaporthostoma machili is the type species of recently introduced genus Diaporthostoma by Fan et al. (2018a) (S.S.N. Maharachchikumbura).

\section{Diaporthostomataceae X.L. Fan \& C.M. Tian}

This monotypic family was introduced by Fan et al. (2018a) based on morphology and the analysis of partial ITS, LSU, rpb2 and tef1- $\alpha$ gene sequences. This family belongs to the order Diaporthales (S.S.N. Maharachchikumbura and S. Fryar).

Dictyoceratosporella Y.R. Ma \& X.G. Zhang

Ma et al. (2016) established this hyphomycetous genus. Sequence data are lacking thus taxonomic placement is uncertain (J. Ma).

\section{Dictyosporites Félix (fossil)}

These spores are comparable to the conidia of some modern genera like Dictyosporium Corda, Stemphylium Wallr., Septosporium Corda and Alternaria Nees, and the ascospores of Pleospora Rabenh. ex Ces. \& De Not. Arbusculites Paradkar, Dactylosporites Paradkar, Pleosporonites R.T. Lange \& P.H. Sm. and Ravenelites Ramanujam \& Ramachar are latter taxonomic synonyms of Dictyosporites (Kalgutkar \& Jansonius 2000) (R.K. Saxena).

Digitiseta Gordillo \& Decock

Gordillo \& Decock (2018) proposed Digitiseta and transferred two Myrothecium (M. setiramosum and $M$. dimorphum) into the genus as $D$. setiramosa (the type species) and $D$. dimorpha. Digitiseta parvidigitata and D. multidigitata were described as new and also included. Digitiseta differs from the two closely related genera, Inaequalispora and Parvothecium, in having short apical branches of the setoid extension (S. Somrithipol).

\section{Diphymyces I.I. Tav.}

Diphymyces was introduced by Tavares (1985) to accommodate Laboulbeniales species with thalli that have a septum vertically separating cells II and VI, four tiers of perithecial wall cells, and (sub-) apical perithecial outgrowths. The separation of this genus from Corethromyces Thaxt. was primarily based on the position of cells II and VI, which is a variable character (discussed in De Kesel \& Haelewaters 2019). Also the genera Asaphomyces Thaxt. and Euphoriomyces Thaxt. might be synonymous. These four genera are placed in three different subtribes (Tavares 1985), but several higher taxa (subtribes and tribes) from the Tavares (1985) classification are polyphyletic following molecular phylogenetic treatments (Goldmann \& Weir 2018, Haelewaters et al. 2018b). Molecular phylogeny is needed to resolve these taxonomic and systematic problems (D. Haelewaters).

Diplodites D.N. Babajan \& Tasl. ex Kalgutkar, Nambudiri \& Tidwell (fossil)

Kalgutkar et al. (1993) validated the name Diplodites to encompass fossil taxa that are morphologically similar to the extant fungi Diplodia Fr., Botryodiplodia (Sacc.) Sacc. and other related genera such as Dothiorella Sacc. and Macrophoma (Sacc.) Berl. \& Voglino. Palaeodiplodites Kyoto Watanabe, H. Nishida \& Tak. Kobay. is a junior taxonomic synonym of Diplodites (Kalgutkar \& Jansonius 2000). (R.K. Saxena).

Diploneurospora K.P. Jain \& R.C. Gupta (fossil) 
This monotypic genus accommodates two-celled (cells unequal), uniseriate, elliptical ascospores with uneven margin; upper cell prominent, dark brown, thick-walled, wall sculptured with longitudinal ribs; lower cell hyaline, appendage-like, small in size, rib sculpture faint. It closely resembles to the single celled ascospores of extant genus Neurospora Shear \& B.O. Dodge (R.K. Saxena).

Diporisporites Hammen (fossil)

Kalgutkar \& Jansonius (2000) considered Scabradiporites Y.K. Mathur a latter taxonomic synonym of Diporisporites (R.K. Saxena).

Discinellaceae Ekanayaka \& K.D. Hyde

Ekanayaka et al. (2019) introduced this family within Helotiales. Johnston et al. (2019) found high support for what they referred to as the Discinella-Pezoloma lineage (sensu Baral 2016), placed as sister clade to Gelatinodiscaceae in the discinelloid clade of Helotiales. A number of genera that were previously placed elsewhere are now considered members of Discinellaceae: Cladochasiella, Fontanospora, Margaritispora, Naevala, Pseudopezicula and Tetrachaetum (D. Haelewaters).

\section{Dothiorella Sacc.}

Yang et al. (2017) considered that Spencermartinsia should no longer be considered a separate genus and placed it as a synonym of Dothiorella. Currently 389 names are listed in MycoBank, but only 38 are known from culture (A.J.L. Phillips).

\section{Drepanopezizaceae Baral}

Introduced in Johnston et al. (2019) as sister family to Ploettnerulaceae within Helotiales (D. Haelewaters).

\section{Ducatina Ertz \& Søchting}

Ertz et al. (2017b) introduced this genus which belongs in Trapeliaceae (N. Wijayawardene).

Durella Tul. \& C. Tul.

Baral (2016) treated this genus in his "Strossmayeria lineage", which was confirmed by the ITS tree of Johnston et al. (2019). However, two species, "Durella" macrospora and "D." melanochlora, are phylogenetically distinct from the type (D. connivens) and are here placed under Helotiales genera incertae sedis (D. Haelewaters).

\section{Durotheca Læssøe et al.}

De Long et al. (2019) described two new species of Durotheca from China and provided a new molecular phylogeny that proved the affinities of the genus to the Hypoxylaceae. This had already been indicated by morphological data, such as the presence of a nodulisporium-like asexual morph in some species (M. Stadler).

\section{Dyadosporites Hammen ex R.T. Clarke (fossil)}

Jansonius \& Hills (1976) remarked that "although van der Hammen (1954) gave a diagnosis and the name of the type species, the latter was never described (or illustrated)". Therefore, Dyadosporites was not a validly published name of a taxon, but as merely proposed in anticipation of future acceptance of the group. Clarke (1965) was the first to have validly published this genus name and also the first to assign a species (Dyadosporites ellipsus R.T. Clarke) to it. Dyadosporonites Elsik and Psidimobipiospora Sal.-Cheb. \& Locq. are latter taxonomic synonyms of Dyadosporites (Kalgutkar \& Jansonius 2000) (R.K. Saxena). 
Efibulella Zmitr.

Justo et al. (2017) showed that Phlebia deflectens (P. Karst.) Ryvarden forms a distinct phylogenetic lineage in the family Phanerochaetaceae. Hence, Zmitrovich (2018) introduced the monotypic genus Efibulella to accommodate Phlebia deflectens (V. Papp).

Elaphroporia Z.Q. Wu \& C.L. Zhao

$\mathrm{Wu}$ et al. (2018) introduced this monotypic genus to accommodate the new species $E$. ailaoshanensis Z.Q. Wu \& C.L. Zhao, and accepted it as a genus in the residual polyporoid clade based on phylogenetic analyses (V. Papp).

Emergomyces Dukik et al.

This genus of thermally dimorphic clinical fungi belongs in the family Ajellomycetaceae, according to multilocus phylogenetic studies by Dukik et al. (2017) (H. Madrid).

Endomelanconiopsis E.I. Rojas \& Samuels Tao

Yang et al. (2017) considered that this genus warrants a separate family and thus introduced Endomelanconiopsisaceae to accommodate it. Phillips et al. (2019) took into account phylogeny (ITS, LSU), morphology and evolutionary divergence times and concluded that Endomelanconiopsis resides within Botryosphaeriaceae. Hence, Phillips et al. (2019) regarded Endomelanconiopsisaceae as a synonym of Botryosphaeriaceae (A.J.L. Phillips).

Endophoma Tsuneda \& M.L Davey

This is a monotypic genus in Didymellaceae, typified by E. elongata. The fungus is an atypical coelomycete with endogenous conidiogenesis and its taxonomic position has been elucidated by DNA sequence analyses (Tsuneda et al. 2011a) (H. Madrid).

Enterodictyon Müll. Arg.

It is an autonomous genus in Wijayawardene et al. (2017a), but the type species, E. indicum Müll. Arg., was transferred to Diorygma (Joseph et al. 2018). However, some species still need to be studied and relocated (M. Kukwa).

Entrophospora Ames \& Schneider

The fungus was treated as an incertae sedis in the last classification (Wijayawardene et al. 2018). Nevertheless, all partial rDNA sequences published within the last years, suggest that their type fungus (E. infrequens) belongs to Claroideoglomus clade (Oehl et al 2011e,f), justifying the use of Entrophosporaceae instead of Claroideoglomeraceae.

Epicladonia D. Hawksw.

According to Pino-Bodas et al. (2017), the generic type, E. sandstedei (Zopf) D. Hawksw. belongs to Leotiomycetes while other species are placed in Ostropales (A. Suija).

Epithamnolia Zhurb.

Suija et al. (2017) showed that lichenicolous species of Hainesia form a distinct phylogenetic lineage within Phacidiales, and provisionally transferred lichenicolous species to the morphologically similar genus Epithamnolia. According to molecular analysis by Quijada et al. (2018a), the genus was placed in the poorly known Mniaecia lineage, which is now family Mniaeciaceae within Leotiales (Johnston et al. 2019). In contrast, Ekanayaka et al. (2019) regarded this genus as a member of the Epicladonia-Epithamnolia clade in Lichinodiales, however this placement had no statistical support (D. Haelewaters, A. Suija \& N. Wijayawardene).

\section{Eudarluca Speg.}

Rossman et al. (2015) treated Eudarluca as a synonym of Sphaerellopsis based on a study of Trakunyingcharoen et al. (2014). The congeneric status of Eudarluca and Sphaerellopsis was 
clarified based on Eudarluca caricis and Sphaerellopsis filum. However, Phookamsak et al. (2014) examined the isotype of Eudarluca australis and compared the morphology with E. caricis. Based on morphological examination, Phookamsak et al. (2014, 2019) mentioned that these two genera were not congeneric and suggested to instate Eudarluca in Phaeosphaeriaceae for pending further studies (R. Phookamsak).

\section{Eutiarosporella Crous}

Based on ITS and LSU sequence phylogeny, Crous et al. (2015a) introduced Eutiarosporella as a new genus for tiarosporella-like fungi with long-necked conidiomata and holoblastic conidiogenesis (A.J.L. Phillips).

\section{Exesisporites Elsik (fossil)}

The centrally located pore in Exesisporites is generally surrounded by a dark circular patch which is interpreted as a thickened wall. Ethridge Glass et al. (1986) cited possible affinity of Exesisporites to the extant fungus Nigrospora Zimm. (R.K. Saxena).

Exochalara W. Gams \& Hol.-Jech.

Crous et al (2018b) treat this genus as a member in Neolauriomycetaceae (K. Bensch).

\section{Exophiala J.W. Carmich.}

This is a species-rich genus of black yeast-like fungi, polyphyletic within Chaetothyriales. The type species, E. salmonis, and most members of this genus belong in Herpotrichiellaceae, but some species are related to other chaetothyrialean families, such as Trichomeriaceae (Madrid et al. 2016) (H. Madrid).

\section{Extremaceae Quaedvl. \& Crous}

Paradevriesiaceae was introduced by Crous et al. (2019) and comprises Paradevriesia compacta (CBS 118294), P. Americana (CBS 117726), and P. pseudoamericana (CPC 16174). However, Crous et al. (2019) did not include sequence of Extremaceae in their phylogenetic tree. In Hongsanan et al. (in prep), Paradevriesia strains form a distinct lineages within Extremaceae. Hence, Hongsanan et al. (in prep.) regarded Paradevriesiaceae as a synonym of Extremaceae (S. Hongsanan \& N. Wijayawardene).

Flabellascoma A. Hashim. et al.

Hashimoto et al. (2018) proposed this genus to accommodate two species which were previously in the Lophiostoma bipolare species complex (S. Fryar).

\section{Flagellospora Ingold}

Ekanayaka et al. (2019) retrieved this genus as a distinct phylogenetic lineage without support. We propose it here as Leotiales genera incertae sedis based on the placement of $F$. curvula, the type species, in the 15-gene tree of Johnston et al. (2019) (D. Haelewaters).

\section{Flavignomonia N. Jiang et al.}

Jiang et al. (2019c) proposed this new genus based on morphological and molecular data with synnemata similar to Synnemasporella but differ in orange synnematal tips and hyaline conidia (S. Tibpromma).

\section{Fomitopsidaceae Jülich}

Zmitrovich (2018) treated Adustoporiaceae Audet, Amyloporiaceae Audet, Fibroporiaceae Audet, Lentoporiaceae Audet, Pycnoporellaceae Audet, Rhodoniaceae Audet and Sarcoporiaceae Audet as synonyms of Fomitopsidaceae (V. Papp). 


\section{Fonsecaea Negroni}

This genus currently includes eight species, most of which are associated with infections in humans. Phylogenetically, Fonsecaea belongs in Herpotrichiellaceae (Arzanlou et al. 2007, Madrid et al. 2016, Dong et al. 2018) (H. Madrid).

Foveodiporites C.P. Varma \& Rawat emend. Kalgutkar \& Janson. (fossil)

Punctodiporites C.P. Varma \& Rawat is a later synonym of Foveodiporites as emended by Kalgutkar \& Jansonius (2000) (R.K. Saxena).

\section{Frasnacritetrus Taug. emend. R.K. Saxena \& S. Sarkar (fossil)}

Taugourdeau (1968) originally described this genus under Acritarcha incertae sedis from the Late Devonian (Frasnian) sediments of France. Kendrick \& Carmichael (1973) published a list of staurosporous genera and their illustrations which strongly suggests that Frasnacritetrus is a fossil representative of Tetraploa Berk. \& Broome, hence its placement under Acritarcha incertae sedis by Taugourdeau (1968) does not seem justified. All the seven assemblages studied by Saxena \& Sarkar (1986), wherefrom the present microfossils have been recovered, contain poaceous pollen as well. Since Tetraploa mainly grows on Poaceae, the association of Frasnacritetrus with poaceous pollen is considered a supporting evidence for the affinity of Frasnacritetrus with Tetraploa (R.K. Saxena).

\section{Frutidella Lalb}

Kistenich et al. (2018) transferred this genus from the Ramalinaceae to the Lecanoraceae (E. Timdal).

Fuscosclera Hern.-Restr. et al.

Johnston et al (2019) accepted this genus in Mollisiaceae (D. Haelewaters).

Fusichalara S. Hughes \& Nag Raj

Fusichalara minuta clustered in the family Sclerococcaceae (Réblová et al. 2016, Yu et al. 2018), but not type species (H. Zhang).

Fusiformisporites Rouse (fossil) Saxena).

Striadyadosporites Dueñas is a later taxonomic synonym of Fusiformisporites (R.K.

\section{Galbinothrix Frisch et al.}

This genus was recently introduced from Japan and Korea by Frish et al. (2018). Multi-gene phylogenetic analyses revealed that the monotypic genus formed a distinct clade together with Chrysothrix, Melarthonis, and Arthonia (P. Rodriguez-Flakus).

Geesterania Westphalen, Tomšovský \& Rajchenberg

Westphalen et al. (2018) introduced this genus in Meruliaceae to accommodate Junghuhnia carneola (Bres.) Rajchenb. and Geesterania davidii Westphalen \& Rajchenberg. (V. Papp).

\section{Gelatinoamylaria Prasher \& R. Sharma}

Prasher et al. (2016) introduced Gelatinoamylaria as a new genus to accommodate a species with gelatinous apothecia and amyloid ascospores. It is tentatively placed in Dermateaceae but no sequences are available. It should be analyzed phylogenetically to determine its true systematic position (I. Kušan \& N. Matočec).

\section{Gelatinosporium Peck}

This genus belongs to Tympanidaceae following the family concept of Baral (2016) and Quijada et al. (2020) (N. Wijayawardene). 
Geonectria Lechat \& J. Fourn.

This is newly described genus (Lechat et al. 2018b) that belongs to Bionectriaceae (I. Kušan \& N. Matočec).

Gloeoporellus Zmitr.

Justo et al. (2017) showed that Tyromyces merulinus (Berk.) G. Cunn. forms a distinct phylogenetic lineage in the family Incrustoporiaceae. Hence, Zmitrovich (2018) introduced the monotypic genus Gloeoporellus to accommodate Tyromyces merulinus (V. Papp).

Gloniales Jayasiri \& K.D. Hyde

Jayasiri et al. (2018) introduced this order as a distinct sister clade to the Mytinidiales (S. Fryar).

\section{Grovesiella M. Morelet}

Previously placed in Tympanidaceae. However, G. abieticola, the type species of the genus, was retrieved as a member of Godroniaceae in the ITS tree of Johnston et al. (2019) (D. Haelewaters).

Gymnostellatospora Udag. et al.

Previously regarded as a member of Myxotrichaceae (Baral 2016), this genus is now placed in Pseudeurotiaceae, Thelebolales based on the ITS phylogeny of Johnston et al. (2019). This placement agrees with the suggestion of Minnis \& Lindner (2013) to consider the genera Geomyces, Gymnostellatospora, Leuconeurospora, Pseudeurotium and Pseudogymnoascus as members of the family Pseudeurotiaceae (D. Haelewaters).

\section{Hanseniaspora Zikes}

This teleomorph genus has a priority over its anamorphic counterpart, Kloeckera. Kloeckera species have recently been transferred here (Kurtzman \& Boekhout 2017, Čadež et al. 2019) (W.P. Pfliegler \& E. Horváth).

\section{Harmoniella V.N. Boriss.}

This enigmatic genus currently includes two species, the generic type $H$. chrysocephala, and $H$. campanaensis. They have been collected on plant material in Ukraine and Chile, respectively. Both are apparently non-culturable fungi and so far it has been impossible to generate DNA sequence data of them. No sexual morph is known for Harmoniella (H. Madrid).

Hemigraphaceae D.Q. Dai \& K.D. Hyde

Dai et al. (2018) introduced this family in Asterinales to accommodate Hemigrapha (Müll. Arg.) D. Hawksw. (N. Wijayawardene).

\section{Hemiphacidiaceae Korf}

Ekanayaka et al. (2019) reinstated this family but careful analysis of previous work shows that this decision is flawed. Pärtel et al. (2017) included in their phylogenetic analysis the type species of the genus Cenangium, C. ferruginosum, to find that it is positioned in the clade that Ekanayaka et al. (2019) refer to as Hemiphacidiaceae but truly is Cenangiaceae. The clade named "Cenangiaceae" by Ekanayaka et al. (2019) is regarded as Helotiales genera incertae sedis by other studies, including Zhao et al. (2016) Pärtel et al. (2017), Johnston et al. (2019). This clade includes "Cenangium" acuum, Piceomphale bulgarioides, and P. pinicola (basionym Moellerodiscus pinicola). In this outline, we follow Pärtel et al.'s (2017) emended concept of Cenangiaceae including members of the previous Hemiphacidiaceae (see Cenangiaceae) (D. Haelewaters). 
Hemipholiota (Singer) Bon

Bon (1986) elevated Pholiota subgen. Hemipholiota to genus level and typified by Hemipholiota populnea (Singer) Bon as the type species. Molecular data analyses confirmed that Hemipholiota is a separate genus from Pholiota with a unique and uncertain phylogenetic position, distinct from Strophariaceae (Moncalvo et al. 2002, Gulden et al. 2005) (B. Dima).

Henssenia Ertz, R.S. Poulsen \& Søchting

Ertz et al. (2017a) introduced this genus and showed that it belongs in Koerberiaceae (N. Wijayawardene).

\section{Hermanssonia Zmitr.}

Justo et al. (2017) showed that Phlebia centrifuga P. Karst. forms a distinct phylogenetic lineage in the family Meruliaceae. Hence, Zmitrovich (2018) introduced the monotypic genus Hermanssonia to accommodate Phlebia centrifuga (V. Papp).

\section{Herpomycetales Haelew. \& Pfister}

Haelewaters et al. (2019b) introduced this new order to accommodate the genus Herpomyces, which was previously placed in Laboulbeniales. Blackwell et al. (2020) found high support for the polyphyly of the thallus-forming Laboulbeniomycetes, i.e. orders Herpomycetales and Laboulbeniales (D. Haelewaters).

\section{Hesperomyces Thaxt.}

Wijayawardene et al. (2017a) mentioned eleven species for this genus. This number will be much higher, after Haelewaters et al. (2018a) found that $H$. virescens Thaxt. is a complex of multiple species, each with their own host. At least 10 species can be recognized within $H$. virescens sensu lato (D. Haelewaters, unpubl. data) (D. Haelewaters).

\section{Heufleria Auersw.}

Baral (2016) and Ekanayaka et al. (2019) accepted this genus in Rhytismataceae (N. Wijayawardene \& D. Haelewaters).

Hilidicellites Kalgutkar \& Janson. (fossil)

Kalgutkar \& Jansonius (2000) opined that Dicellaesporites appendiculatus Sheffy \& Dilcher is a misfit in Dicellaesporites Elsik and therefore they proposed Hilidicellites to accommodate it (R.K. Saxena).

\section{Hoffmannoscypha Stielow, Göker \& Klenk}

This genus, erected for a single species (Geopora pellita), was recently shown to represent a separate phylogenetic lineage (Van Vooren et al. 2017) (I. Kušan \& N. Matočec).

\section{Hortaea Nishim. \& Miyaji}

This genus currently includes two species, $H$. werneckii and $H$. thailandica. Hortaea werneckii is a well-known clinically relevant fungus, causing superficial skin infections in humans (Marchetta et al. 2018) (H. Madrid).

\section{Hyaloscyphaceae Nannf.}

This family is currently polyphyletic. Based on a four-gene phylogenetic analysis, Han et al. (2014) found that Hyaloscyphaceae sensu lato is a heterogeneous assemblage of 10 hyaloscyphaceous taxa. In Johnston et al. (2019), three highly supported clades were retrieved, Han Clade 4 (Gamarada, Hyphodiscus, Hyphopeziza, Venturiocistella), Han Clade 7 (Amicodisca, Dematioscypha), and Hyaloscyphaceae ("Chalara" longipes, Hyalopeziza, type genus 
Hyaloscypha, Meliniomyces, Olla, Rhizoscyphus). More taxa and sequence data are needed to resolve the classification and taxonomy within Hyaloscyphaceae sensu lato (D. Haelewaters).

\section{Hydnocystis Tul.}

Stephensia Tul. \& C. Tul. has been treated as a synonym of Hydnocystis by Kumar et al. (2017) (P. Alvarado).

Hydnophanerochaete Sheng H. Wu \& C.C. Chen

Chen et al. (2018) introduced this monotypic genus in Meruliaceae to accommodate Phanerochaete odontoidea Sheng H. Wu (V. Papp).

\section{Hypomontagnella Sir et al.}

Lambert at al. (2019) erected the new genus Hypomontagnella Sir et al. (Hypoxylaceae) based on a comparison of molecular and morphological data for Hypoxylon monticulosum and similar species. Interestingly, all strains so far cultured produce the selective antifungal polyketides of the sporothiolide type, which can therefore be regarded as a chemotaxonomic marker (M. Stadler).

\section{Hypoxylonites Elsik. (fossil)}

Hypoxylonsporites P. Kumar is a later taxonomic synonym of Hypoxylonites Elsik (Elsik 1990) (R.K. Saxena).

\section{Immotthia M.E. Barr}

Hyde et al. (2017) accommodated Immotthia in Roussoellaceae based on morphological characteristics resembling the genus Roussoella. However, phylogenetic affinity of the genus needs to be confirmed by molecular data (R. Phookamsak).

\section{Inapertisporites Hammen (fossil)}

Triporisporonites Sheffy \& Dilcher is a later taxonomic synonym of Inapertisporites. Inapertisporites "Hammen ex Rouse" is illegitimate name being a later homonym (and later taxonomic synonym) of Inapertisporites Hammen (R.K. Saxena).

\section{Intralichen D. Hawksw. \& M.S. Cole}

This genus was introduced by Hawksworth \& Cole (2002) to accommodate dematiaceous hyphomycetes occurring in lichens, some of which were previously placed in Bispora or Trimmatostroma. The only available DNA sequence is labelled 'Bispora' christiansenii IMI 227584, a sequence of $18 \mathrm{~S}$ rDNA from a study by Sert et al. (2007), with the GenBank number AM279680. BLAST searches with this sequence, however, revealed that it shows $99 \%$ identity to yeasts of the genera Candida, Debaryomyces and Meyerozyma, indicating that probably a contaminant was sequenced (H. Madrid).

\section{Jansoniisporites Kalgutkar (fossil)}

Brachysporisporites endophragmia Kalgutkar \& Sigler is a misfit in Brachysporisporites R.T. Lange \& P.H. Sm. and therefore Kalgutkar (1997) proposed Jansoniisporites to accommodate it (R.K. Saxena).

\section{Japewia Tønsberg}

Kistenich et al. (2018) transferred this genus from the Ramalinaceae to the Lecanoraceae (E. Timdal)

Junewangiaceae J.W. Xia \& X.G. Zhang

See under Acrodictyaceae (J. Ma). 
Kellermania Ellis \& Everh.

Based on phylogenetic information and morphology, Minnis et al. (2012) placed Alpakesa, Piptarthron, Planistroma, and Planistromella as synonyms of Kellermania (A.J.L. Phillips).

Khaleijomyces Abdel-Wahab

Abdel-Wahab et al (2018) erected this new genus for a new marine species within Juncigenaceae (S. Fryar).

Kiliasia Hafellner

Kistenich et al. (2018) resurrected this genus in the Ramalinaceae (E. Timdal).

Knufia L.J. Hutchison \& Unter.

The molecular phylogeny and taxonomy of this genus has been studied by Tsuneda et al. (2011b), Isola et al. (2016) and Mehrabi et al. (2018) among others, revealing its placement in Trichomeriaceae (H. Madrid).

Kumarisporites Kalgutkar \& Janson. (fossil)

Imprimospora ramanujamii Kumar is a misfit in Imprimospora and therefore Kalgutkar \& Jansonius (2000) proposed Kumarisporites to accommodate it (R.K. Saxena).

Kusaghiporia J. Hussein et al.

Hussein et al. (2018) described this genus with K. usambarensis Hussein J., Tibell S. \& Tibuhwa as the type species and showed that it belongs to Laetiporaceae (V. Papp).

Kutchiathyrites R.K. Kar (fossil)

Kalgutkar \& Jansonius (2000) opined that Kutchiathyrites is a multicellular spore/ conidium showing a clear attachment area, scar or pore. Kutchiathyrites eccentricus R.K. Kar demonstrates close similarity to the conidia of the hyphomycetous fungus Mycocenterolobium platysporum Goos (R.K. Saxena).

\section{Laboulbenia Mont. \& C.P. Robin}

The most recent number of species in this genus is 633 (Song et al. 2019). This number will likely increase in the future; based on a preliminary concatenated ITS-LSU rDNA dataset, Haelewaters et al. (2019a) showed that one of the most cosmopolitan taxa in the genus, $L$. flagellata Peyr., is a complex of species. A number of taxa were recently lectotypified in Haelewaters et al. (2015, 2019a) (D. Haelewaters).

Lachnopsis Guatimosim et al.

Lachnopsis was introduced in Lachnaceae to accommodate two species that are only distinguishable from Lachnum based on DNA sequence data (Guatimosim et al. 2016) (D. Haelewaters).

Lasionectriella Lechat \& J. Fourn.

Lechat et al. (2016a) described this genus to accommodate two species within the family Bionectriaceae (I. Kušan \& N. Matočec).

Lecanoraceae Körb. (= Carbonicolaceae Bendiksby \& Timdal)

Kraichak et al. (2018a) regarded Carbonicolaceae as a synonym of Lecanoraceae (N. Wijayawardene).

Lentimurispora N.G. Liu et al. 
Liu et al. (2018a) introduced this genus and Lentimurisporaceae which has a distinct lineage in Pleosporales (S. Fryar).

\section{Lentistoma A. Hashim.}

Hashimoto et al. (2018) proposed this genus to accommodate Lentistoma bipolare, which was transferred from Lophiostoma based on molecular and morphological characters (S. Fryar).

Lentithecium K.D. Hyde et al.

Lentithecium aquaticum Yin. Zhang, J. Fourn. \& K.D. Hyde, L. arundinaceum (Sowerby) K.D. Hyde, J. Fourn. \& Yin. Zhang, and L. lineare (E. Müll. ex Dennis) K.D. Hyde, J. Fourn. \& Yin. Zhang do not group with L. fluviatile (Aptroot \& Van Ryck.) K.D. Hyde, J. Fourn. \& Yin. Zhang, the type species of the genus Lentithecium in molecular phylogenetic analysis (MycoBank: see under Lentithecium carbonneanum J. Fourn., Raja \& Oberlies) (H. Raja).

Leptoparies A. Hashim.

Hashimoto et al. (2018) proposed this genus to accommodate a single species within the Lophiostomataceae (S. Fryar).

\section{Leptosillia Höhn.}

Voglmayr et al (2019) demonstrated that the genus Leptosillia belongs to the Xylariales based on a multi locus DNA sequence analyses of SSU-ITS-LSU rDNA, rpb1, rpb2, tef1 and tub2. They also established the genera Cresporhaphis and Liberomyces are congeneric with Leptosillia and erected the new family Leptosilliaceae. A number of taxa were epi-or lectotypified, and the new genus Furfurella was erected in family Delonicicolaceae (M. Stadler).

\section{Lichinodium Nyl.}

Lichinodium was previously placed in class Lichinomycetes. However, Prieto et al. (2019) showed that this genus is placed within Leotiomycetes as a previously unrecognized lineage and introduced a new order (Lichinodiales) and family (Lichinodiaceae) (M. Prieto).

Lithophila Selbmann \& Isola

This monotypic genus, typified by L. guttulata, occurs on marble and was placed in Trichomeriaceae based on multilocus phylogenetic analyses (Isola et al. 2016) (H. Madrid).

Liua Phook. \& K.D. Hyde

Phookamsak et al. (2019) introduced a monotypic genus Liua to accommodate camarosporium-like species and is typified by L. muriformis Phookamsak, H.B. Jiang \& K.D. Hyde. Multi-gene phylogenetic analyses showed that Liua formed a sister lineage with Cycasicola in Thyridariaceae (R. Phookamsak).

Lodderomyces Van der Walt

Expected transfer of species to Candida Berkhout to comply with the Melbourne Code (Kurtzman \& Boekhout 2017) (W.P. Pfliegler and E. Horváth).

\section{Lonicericola Phook. et al.}

Phookamsak et al. (2019) introduced the new genus Lonicericola based on DNA sequence analyses. The genus formed a distinct clade closely related with Pseudomonodictys and Paratrimmatostroma in Parabambusicolaceae (R. Phookamsak).

\section{Lotinia Pérez-Butrón et al.}

This genus, erected for a single species (L. verna), represents a separate phylogenetic lineage (Van Vooren et 2017) (I. Kušan \& N. Matočec). 
Manglicola Kohlm. \& E. Kohlm.

Manglicola consists of two species, but molecular data supports only $M$. guatemalensis Kohlm. \& E. Kohlm. showing phylogenetic affiliations to Manglicolaceae, while M. samuelsii Huhndorf, was placed in the Hypsostromataceae based on morphological data (Huhndorf 1994). Sequence data from M. samuelsii is necessary to place it within a phylogenetic framework and assess if the genus is polyphyletic within the Dothideomycetes (H. Raja).

\section{Marasasiomyces Crous}

Based on ITS and LSU sequence phylogeny, Crous et al. (2015a) introduced Marasasiomyces as a new genus for tiarosporella-like fungi with long-necked conidiomata covered in brown setae (A.J.L. Phillips).

Marthamycetales P.R. Johnst. \& Baral

Introduced in Johnston et al. (2019) to accommodate the phylogenetically isolated family Marthamycetaceae within Leotiomycetes (D. Haelewaters).

\section{Masonhalea Kärnefelt}

Thell et al. (2018) proposed to resurrect the genus (A. Tsurykau).

\section{Massariosphaeria (E. Müll.) Crivelli}

This genus was firstly recognized as a section of Leptosphaeria (Müller 1950). However, Massariosphaeria was introduced by Crivelli (1983). Wang et al. (2007) showed that Massariosphaeria is polyphyletic. However, the type species $M$. phaeospora is closely related to the type species of Cyclothyriellaceae, Cyclothyriella rubronotata. Therefore, Massariosphaeria is placed in Cyclothyriellaceae (Jaklitsch et al. 2016a, Wijayawardene et al. 2018a) (S. Hongsanan).

Mathurisporites Kalgutkar \& Janson. (fossil)

Kalgutkar \& Jansonius (2000) opined that Pluricellaesporites ellipticus Y.K. Mathur \& K. Mathur is a misfit in Pluricellaesporites Hammen and therefore they proposed Mathurisporites to accommodate it (R.K. Saxena).

\section{Megalaria Hafellner}

Kistenich et al. (2018) regarded that Catillochroma Kalb and Lopezaria Kalb \& Hafellner as synonyms of this genus (E. Timdal).

Melaspileellaceae D.Q. Dai \& K.D. Hyde

Dai et al. (2018) introduced this family in Asterinales to accommodate Melaspileella (P. Karst.) Vain (N. Wijayawardene).

\section{Metschnikowiaceae T. Kamienski}

Family status is expected to change following the phylogenetic relationships of the genera of Debaryomycetaceae and Metschnikowiaceae (Shen et al. 2016) (W.P. Pfliegler \& E. Horváth).

Micraspidales Quijada \& Tanney

Previously, the genus Micraspis was placed in different families (Cryptomycetaceae, Helotiaceae, Phacidiaceae, Tympanidaceae). Because of its isolated phylogenetic position within Leotiomycetes, Quijada et al. (2020) established the family Micraspidaceae and order Micraspidales to accommodate the genus (D. Haelewaters). 
Kraichak et al. (2018a) accepted the family as in Pertusariales (N. Wijayawardene).

Microkamienskia Corazon-Guivin et al.

Corazon-Guivin et al. (2019) introduced Microkamienskia with two new combinations. This genus is an arbuscular mycorrhizal fungus and similar to Kamienskia in spore but differ in size (Błaszkowski et al. 2015) (S. Tibpromma).

\section{Minimelanolocus R.F. Castañeda \& Heredia}

This genus is currently considered a putative member of Herpotrichiellaceae, although no DNA sequence data is available for the type species, M. navicularis (H. Madrid).

\section{Mniaeciaceae Baral}

Introduced in Johnston et al. (2019), elevating the Mniaecia lineage sensu Baral (2016) to family level. Mniaeciaceae is sister to Leotiaceae within Leotiales (D. Haelewaters).

Monoporisporites Hammen (fossil)

Polyporisporites Hammen, Psiammopomopiospora Sal.-Cheb. \& Locq. and Reticulatisporonites Elsik are later taxonomic synonyms of Monoporisporites Hamme Dong $\mathrm{n}$ (Kalgutkar \& Jansonius 2000) (R.K. Saxena).

\section{Mortierella Coem.}

There are 247 records of Mortierella species in the Species Fungorum, but according to Yadav et al. (2015) nearly 100 of validated species have been described (A. L. C. M. de A. Santiago).

Mossopisporites Kalgutkar \& Janson. (fossil)

Kalgutkar \& Jansonius (2000) opined that Triporicellaesporites multicellulus Ke \& Shi is a misfit in Triporicellaesporites $\mathrm{Ke} \& \mathrm{Shi}$ and therefore they proposed Mossopisporites to accommodate it (R.K. Saxena).

Mucoharknessia Crous et al.

Based on ITS and LSU sequence phylogeny, Crous et al. (2015a) introduced Mucoharknessia as a new genus for tiarosporella-like fungi that resembles Harknessia (Harknessiaceae, Diaporthales), but are distinguished by having pycnidia that lack furfuraceous tissue around the ostiole and conidia with a mucoid apical appendage (A.J.L. Phillips).

\section{Mucor Fresen.}

The greatest number of mucoralean species described to date belongs to Mucor with more than 300 species cited in literature (Jacobs \& Botha 2008, Álvarez et al. 2011). Although the exact number of valid taxa is unknown, Gherbawy et al. (2010) stated the number of species may have ranged from 50 to 75 at the time of the study. Based on morphological characteristics, maximum growth temperature and mating experiments, Schipper (1973, 1975, 1976, 1978) monographed the genus and described 39 species, four varieties and 11 forms. Knowledge of the genus was subsequently expanded with a description of 26 new taxa [Mehrotra \& Mehrotra (1979), Mirza et al. (1979), Subrahamanyam (1983), Chen \& Zheng (1986), Schipper \& Samson (1994), Watanabe (1994), Zalar et al. (1997), Pei (2000), Alves et al. (2002), Jacobs \& Botha (2008), Hermet et al. (2012), Madden et al. (2012), Voglmayr \& Clémençon (2016), Li et al. (2016), Lima et al. (2017), Wanasinghe et al. (2018), De Souza et al. (2018), de Lima et al. (2018)]. (A. L. C. M. de A. Santiago)

Muellerella Müll. Arg. 
Muggia et al. (2015) hypothesized that the genus represents sexual stage of Lichenodiplis (A. Suija).

Multicellaesporites Elsik emend. P. Kumar (fossil)

Kumar (1990) emended the generic diagnosis. Warkallisporonites Ramanujam \& K.P. Rao is a later taxonomic synonym of Multicellaesporites Elsik (R. K. Saxena).

\section{Mycobilimbia Rehm}

Kistenich et al. (2018) transferred this genus from the Lecideaceae to the Ramalinaceae (E. Timdal).

Mycocarpon S.A. Hutch. (fossil)

Hutchinson (1955) opined that Sporocarpon pachydermum Will. is a misfit in Sporocarpon Will. and therefore he proposed Mycocarpon to accommodate it (R.K. Saxena).

Mycoceros D. Magyar \& Z. Merényi

This genus was recently described to accommodate a species parasitizing Pinaceae grain pollens. Based on an ITS+LSU phylogeny, it was clearly placed within Orbiliomycetes by Magyar et al. (2018) (I. Kušan \& N. Matočec).

\section{Mycomicrothelia Keissl.}

All the tropical species have been placed in Bogoriella (Trypetheliaceae, Trypetheliales), but type and other temperate taxa are still in Mycomicrothelia (Aptroot \& Lücking 2016) (A. Aptroot).

\section{Mycopappus Redhead \& G.P. White}

Baral (2016) and Ekanayaka et al. (2019) accepted this genus as in Sclerotiniaceae (N. Wijayawardene).

\section{Mycosphaerellaceae Lindau}

We accept only 111 genera which have been confirmed as well-established genera in Mycosphaerellaceae by phylogenetic analyses. Hongsanan et al. (2020) lists doubtful genera in Mycosphaerellaceae based on Videira et al. (2017) (N. Wijayawardene \& R. Phookamsak).

Myelorrhiza Verdon \& Elix

Kistenich et al. (2018) transferred this genus from the Cladoniaceae to the Ramalinaceae (E. Timdal).

\section{Myochroidea Printzen et al.}

Printzen et al. (2008) accommodate Myochroidea including four species of the Lecidea leprosula group such as M. leprosula (Arnold) Printzen, T. Sprib. \& Tønsberg, M. porphyrospoda (Anzi) Printzen, T. Sprib. \& Tønsberg, M. rufofusca (Anzi) Printzen, T. Sprib. \& Tønsberg and $M$. minutula Printzen, T. Sprib. \& Tønsberg based on morphological key characters. Although no phylogenetic analyses have been performed, the authors suggested to include this genus either in Psoraceae, Pilocarpaceae, or Ramalinaceae but to confirm the final placement of Myochroidea in the system, molecular analyses are needed (P. Rodriguez-Flakus).

\section{Myrmecocystis Harkn.}

This genus was recently resurrected by Alvarado et al. (2018) (P. Alvarado).

\section{Myxotrichaceae Currah}


Johnston et al. (2019) found high support for the placement of this family in the pezizelloid clade of Helotiales, placed sister to Amorphothecaceae. In the ITS tree of Johnston et al. (2019), Amorphothecaceaea consisting of Byssoascus, "Malbranchea" flavorosea, Myxotrichum and Oidiodendron was retrieved in a maximum supported branch sister to Amorphotheca resinae (D. Haelewaters).

\section{Neocelosporium Crous}

Crous et al. (2018) introduced a new family Neocelosporiaceae and new order Neocelosporiales to accommodate the genus Neocelosporium, which represents a distinct lineage in Dothideomycetes. However, Hongsanan et al. (in prep.) found that Neocelosporiales is placed within the order Dothideales. As a result, Neocelosporiales is here regarded as a synonym of Dothideales and Neocelosporiaceae is accommodated in Dothideales (N. Wijayawardene \& S. Hongsanan).

Neodendryphiella Iturrieta-González et al.

Iturrieta-González et al. (2018) introduced this new genus with three new species in Dictyosporiaceae. Currently, the genus comprises three species viz. N. mali, N. michoacanensis and $N$. tarraconensis (the type species). Neodendryphiella differs from Dendryphiella in lacking of nodulose conidiophores bearing conidiogenous cells with pores surrounded by a thickened and darkened wall (S. Fryar \& S. Somrithipol).

Neoeutypella M. Raza et al.

Neoeutypella was introduced as a monotypic genus in Phookamsak et al. (2019) to accommodate $N$. baoshanensis M. Raza et al. and the strains identified as "Eutypella caricae (strains EL51C and GL08362)". Based on phylogenetic analyses, Neoeutypella formed a distinct lineage, clustered with Diatrypella species but the genus differs from Diatrypella in having large entostromata, 8-spored, spindle-shaped asci and allantoid ascospores (Phookamsak et al. 2019) (R. Phookamsak).

\section{Neolauriomycetaceae Crous}

Neolauriomycetaceae was introduced within Helotiales by Crous et al. (2018) to accommodate three genera: Exochalara, Lareunionomyces, and Neolauriomyces (D. Haelewaters).

\section{Neomelanconiella Crous}

Crous et al. (2018) introduced this genus and showed that it has a distinct lineage in Diaporthales. A new family Neomelanconiellaceae is introduced (N. Wijayawardene).

\section{Neomelanconiella Crous}

Crous et al. (2018) introduced this genus and showed that it has a distinct lineage in Diaporthales. Hence introduced the new family Neomelanconiellaceae (N. Wijayawardene).

\section{Neoscytalidium Crous \& Slippers}

Two species of Neoscytalidium were frequently reported from opportunistic infections in humans, i.e. $N$. dimidiatum and $N$. hyalinum. The latter species, however, is currently considered a hyaline mutant of $N$. dimidiatum (Huang et al. 2016). Neoscytalidium currently includes three species (H. Madrid).

\section{Neoseptorioides Crous et al.}

This genus was introduced for species morphologically distinct from Septorioides (Crous et al. 2015a) (A.J.L. Phillips).

Neostagonosporella C.L. Yang et al. 
Yang et al. (2019) introduced a holomorph genus Neostagonosporella to accommodate massarina-like taxon collected from living bamboo culms from China. Multi-gene phylogenetic analyses revealed the genus in Phaeosphaeriaceae (R. Phookamsak).

Neptunomyces M. Gonçalves et al.

This monotypic genus was recently introduced by Gonçalves et al. (2019). Based on phylogenetic analysis this genus is closest to Xenocamarosporium but conidial morphology is distinct (Gonçalves et al. 2019) (S. Tibpromma).

Noosia Crous et al.

Recent phylogenetic analyses indicated that Noosia belongs to Periconiaceae (Tanaka et al. 2015, Thambugala et al. 2017) (D. Wanasinghe).

Nothomitra Maas Geest.

According to Hustad et al. (2013) and Hustad \& Miller (2015), this genus is phylogenetically close to Sarcoleotia in a basal clade of Geoglossomycetes (I. Kušan \& N. Matočec).

Oblongocollomyces Tao Yang \& Crous

This genus was introduced by Yang et al. (2017) to accommodate Sphaeropsis variablis (A.J.L. Phillips).

Odontoefibula C.C. Chen \& Sheng H. Wu

Chen et al. (2018) introduced this monotypic genus in Phanerochaetaceae to accommodate the new species Odontoefibula orientalis C.C. Chen \& Sheng H. Wu (V. Papp).

Odoria V. Papp \& Dima

Papp \& Dima (2018) introduced this new monotypic genus in Meruliaceae to accommodate the threatened old-growth forest polypore, Aurantiporus alborubescens (Bourdot \& Galzin) H. Jahn (V. Papp).

Ophiobolopsis Phook. et al.

Phookamsak et al. (2017) introduced Ophiobolopsis to accommodate ophiobolus-like species in Phaeosphaeriaceae based on multi-gene phylogenetic analyses (R. Phookamsak).

Opilionomyces Santam. et al.

Santamaria et al. (2017) introduced this monotypic genus and confirmed its placement in family Laboulbeniaceae, subfamily Laboulbenioideae, tribe Laboulbenieae based on morphological characters (D. Haelewaters).

Ornasporonites Ramanujam \& K.P. Rao (fossil)

This monotypic genus differs from Fusiformisporites because the latter possesses only a single septum and is longitudinally ribbed and inaperturate (R.K. Saxena).

Oscarbrefeldia Holterm.

Doubtful genus not treated by Kurtzman (2011) (W.P. Pfliegler \& E. Horváth).

Ovadendron Sigler \& J.W. Carmich.

This fungal genus is listed as a member of Onygenales in de Hoog et al. (2015), but its family placement needs to be thoroughly assessed (H. Madrid).

Pachydisca Boud. 
Dumont (1975) proposed to exclude the genus from Sclerotiniaceae and place it in Helotiales based on morphological study of the type species, P. guernisacii. Jaklitsch et al. (2016a) treated this genus as a synonym of Discinella Boud. However, Species Fungorum (2020) lists 32 accepted species. In this outline, Pachydisca is regarded as Helotiales genera incertae sedis (D. Haelewaters).

\section{Pachyphlodes Zobel}

Scabropezia Dissing \& Pfister has been regarded as a synonym of Plicariella or Pachyphlodes Zobel in Healy et al. (2018) (P. Alvarado).

Palaeoamphisphaerella Ramanujam \& Srisailam (fossil) Saxena).

Imprimospora Norris is considered as a later synonym of Palaeoamphisphaerella (R.K.

Palaeomycites Mesch. (fossil)

Phycomycites Ellis, Palaeomyces Renault ex Kidston \& Lang, Rhizophagites E.J. Butler ex Rosend., Propythium Elias, Aplanosporites R.K. Kar, Glomites T.N. Taylor, W. Remy, Hass \& Kerp are later taxonomic synonyms of Palaeomycites (R.K. Saxena).

Palaeopericonia Ibañez \& Zamuner (fossil)

The material is made up of only asexual structures represented by conidia produced on single conidiophores. The closely related genera are Periconia Tode, Torula Pers., Stachybotrys Corda, Humicola Traaen, Thermomyces Tsikl. and Chlamydomyces Bainier (Ibañez \& Zamuner 1996) (R.K. Saxena).

\section{Palaeophoma Singhai (fossil)}

One-celled hyaline, bent or curved or lunate conidia, and a spherical and brown pycnidium have been shared by the living genus Selenophoma Maire (Barnet 1960). But the fossil fungus also possesses spherical conidia which are not present in Selenophoma. Moreover, Palaeophoma has non-ostiolate pycnidium whereas Selenophoma possesses a definite ostiole (R.K. Saxena).

\section{Palaeosclerotium G.W. Rothwell (fossil)}

Dennis (1976) opined that Palaeosclerotium represents an intermediate evolutionary stage between Ascomycetes and Basidiomycetes. Pirozynski \& Weresub (1979) stated that Palaeosclerotium is neither an ascomycete nor a basidiomycete, but an early dikaryotic fungus and a representative of a group that links Basidiomycota with extinct, probably symbiotic, lichenlike nematophytes (R.K. Saxena).

\section{Paleoslimacomyces Kalgutkar \& Sigler (fossil)}

Conidia of this monotypic genus show some morphological similarity with those of extant Slimacomyces monospora (W.B. Kendr.) Minter, which was originally described by Kendrick (1958) in Helicoma Corda (R.K. Saxena).

\section{Pappia Zmitr.}

Papp \& Dima (2018) showed that Aurantiporus fissilis (Berk. \& M.A. Curtis) H. Jahn ex Ryvarden forms a distinct phylogenetic lineage in the family Meruliaceae. Hence, Zmitrovich (2018) introduced the new monotypic genus Pappia in Meruliaceae to accommodate Aurantiporus fissilis (V. Papp).

\section{Papulosporonites Schmied. \& A.J. Schwab (fossil)}

In Polyadosporites Hammen, the individual cells are less tightly appressed into a spherical mass than those in Papulosporonites (R.K. Saxena). 


\section{Paracladophialophora Crous}

This genus currently includes two species, $P$. carceris and $P$. cyperacearum. They form a distinct clade in Chaetothyriales for which the family Paracladophialophoraceae was recently proposed (H. Madrid).

Parafenestella Jaklitsch \& Voglmayr

Jaklitsch et al. (2018) proposed this new genus in the Cucurbitariaceae based on morphological and molecular characters. (S. Fryar)

Parallopsora Kistenich et al.

Kistenich et al. (2018) introduced this genus in Ramalinaceae (E. Timdal).

Paraophiobolus Phook. et al.

Phookamsak et al. (2017) introduced Paraophiobolus to accommodate ophiobolus-like species in Phaeosphaeriaceae based on multi-gene phylogenetic analyses (R. Phookamsak).

Pararoussoella Wanas. et al.

Wanasinghe et al. (2018) introduced a monotypic genus to accommodate roussoella-like species in Thyridariaceae. However, Phookamsak et al. (2019) accommodated the genus in Roussoellaceae based on multi-gene analyses and this concurred with Jiang et al. (2019a) and Karunarathna et al. (2019). (R. Phookamsak)

Paratrimmatostroma Jayasiri et al.

Phookamsak et al. (2019) introduced the new genus Paratrimmatostroma based on multigene phylogenetic analyses coupled with morphological characteristic. Paratrimmatostroma is sister to Pseudomonodictys in Parabambusicolaceae based on phylogenetic analyses of a combined SSU, ITS, LSU and TEF1- $\alpha$ sequence dataset (Phookamsak et al. 2019). Paratrimmatostroma can be distinguished from Pseudomonodictys in forming sporodochia on host substrate and having branched, straight or flexuous conidia, with variable conidial shape such as helicoid, cylindrical, sigmoid, or reniform (Phookamsak et al. 2019) (R. Phookamsak).

\section{Parazalerion Madrid et al.}

Phookamsak et al. (2019) introduced a monotypic conidial genus to accommodate zalerionlike taxon and is typified by $P$. indica Madrid, Gené, Cano \& Guarro. The genus was isolated from soil in India and is characterized by having irregularly coiled, dematiaceous, multiseptate conidia which often form knots of cells. Phylogenetic analysis revealed that the genus formed a sister lineage with Spirosphaera minuta in Microthyriales (R. Phookamsak \& H. Madrid).

Parmulariales D.Q. Dai \& K.D. Hyde

Dai et al. (2018) introduced this order to accommodate Parmulariaceae (N. Wijayawardene).

\section{Patinella Sacc.}

Patinella hyalophaea, the type species of the genus, was retrieved near Holwaya mucida and its anamorph Crinula caliciiformis in Johnston et al.'s (2019) ITS tree, as Leotiomycetes genera incertae sedis. In their 15-gene tree, the Holwaya-Crinula clade was placed sister to Thelebolales (Pseudeurotiaceae, Thelebolaceae) with high support (D. Haelewaters).

\section{Patinella Sacc.}

The type species of Patinella was nested near the order Thelebolales (Hyde et al. 2017) by two phylogenies (based on ITS and LSU) together with Ramgea Brumm. and Holwaya Sacc. as a weakly supported sister clade to the family Thelebolaceae. More phylogenetic information is 
needed to ascertain the true position of these three genera. It is best to place this genus into Phacidiales incertae sedis at the moment (I. Kušan \& N. Matočec).

\section{Peltigeraceae Dumort.}

Kraichak et al. (2018a) regarded that Lobariaceae Chevall. and Nephromataceae Wetm. ex J.C. David \& D. Hawksw. are synonyms of Peltigeraceae (N. Wijayawardene).

\section{Perennicordyceps Matočec \& I. Kušan}

The genus Perennicordyceps was erected by Matočec et al. (2014) to segregate a monophyletic clade of four species aside from Polycephalomyces based on both molecular and non-molecular evidence. According to Crous et al. (2017), this genus is phylogenetically placed within Ophiocordycipitaceae as a sister clade to Polycephalomyces (I. Kušan \& N. Matočec).

\section{Pertusaria DC.}

Several species were combined into other genera (Wei et al. 2017), but as no recent taxonomic revision of the genus is available, the exact number of species is obscure (M. Kukwa).

Petrophila de Hoog \& Quaedvl.

This monotypic genus, typified by $P$. incerta, was placed in the family Extremaceae in a phylogenetic study by Isola et al. (2016) (H. Madrid).

\section{Phacidiales Bessey}

The order Phacidiales includes two families, Helicogoniaceae and Phacidiaceae (Johnston et al. 2019). Two lineages that were previously recognized in Phacidiales (sensu Baral 2016), Mniaeciaceae (referred to as Mniaecia lineage in Baral (2016)) and Tympanidaceae, are now recognized within Leotiales (D. Haelewaters).

\section{Phaeopoacea Thambug. et al.}

Thambugala et al. (2017) introduced Phaeopoacea to accommodate phaeosphaeria-like taxa in Phaeosphaeriaceae and is typified by Phaeopoacea festucae. Thambugala et al. (2017) also transferred Phaeosphaeria phragmiticola Leuchtm. to Phaeopoacea as P. phragmiticola (Leuchtm.) Thambugala \& K.D. Hyde based on molecular data. The sexual and asexual morph connection of this genus is well-resolved (R. Phookamsak).

\section{Phialina Höhn.}

Baral (2016) and Ekanayaka et al. (2019) accepted this genus in Pezizellaceae (N. Wijayawardene).

\section{Phialocephala W.B. Kendr.}

Phialocephala is currently placed in Mollisiaceae based on the phylogenetic reconstruction of a 15-gene dataset. It should be noted that consensus remains unclear about the systematic position of several mollisioid genera, including Mollisia and Phialocephala (Tanney \& Seifert 2020) (D. Haelewaters).

\section{Phialophora Medlar}

This genus historically included a heterogeneous assemblage of phialidic dematiaceous hyphomycetes usually with poorly developed conidiophores, producing phialides with conspicuous collarettes. It comprised members of several families, orders and classes of ascomycetes (Gams 2000). The current concept of the genus, however, only includes phialidic members of Herpotrichiellaceae with or without a yeast phase in culture (Li et al. 2017) (H. Madrid). 


\section{Phragmonaevia Rehm}

Kirk et al. (2008) regarded this name as doubtful, but Baral (2016) listed it among Helotiales genera incertae sedis. No sequences are currently available for any member of this genus (N. Wijayawardene).

Phragmothyrites W.N. Edwards (fossil)

Microthallites Dilcher is a later taxonomic synonym of Phragmothyrites (R.K. Saxena).

\section{Phyllopsora Müll. Arg.}

Kistenich et al. (2018) regarded that Crocynia (Ach.) A. Massal. as a synonym of this genus (E. Timdal).

\section{Piricauda Bubák}

Da Silva et al. (2016) showed that Piricauda paraguayensis could be accommodated in Mycosphaerellaceae. However, it should be pointed out that Piricauda paraguayensis is not the type species, thus, we tentatively keep this genus in Mycosphaerellaceae (N. Wijayawardene, S. Hongsanan \& R. Phookamsak).

Picoa Vittad.

The genus Phaeangium Pat. Has been regarded as a synonym of Picoa, as discussed in Zitouni-Haouar et al. (2015) (P. Alvarado).

\section{Pilatotrama Zmitr.}

Zmitrovich (2018) introduced this new monotypic genus in Polyporaceae to accommodate Trametes ljubarskyi Pilát. (V. Papp).

\section{Placocrea Syd.}

Boonmee et al. (2017) treated this genus as a member in Teratosphaeriaceae (S. Boonmee).

\section{Plagiosphaera Petr.}

Voglmayr in Song et al. (2019) demonstrated that the taxon P. immersa Petr. belongs to the family Magnaporthaceae (Magnaporthales) based on a multi-locus phylogenetic study of ITSLSU-rpb1-tef1 DNA sequence analyses. The other members of the genus are listed as Sordariomycetes incertae sedis (Huhndorf et al. 2004, Index Fungorum 2019) (D. Haelewaters).

\section{Pleuromyces Dima, P.-A. Moreau \& V. Papp}

Crous et al. (2018) introduced this monotypic genus to accommodate the new species $P$. hungaricus V. Papp, Dima \& P.-A. Moreau, and accepted it as a genus in Tubariaceae based on phylogenetic analyses (V. Papp).

Plicariella (Sacc.) Rehm

See under Pachyphlodes Zobel (P. Alvarado).

Pluricellaesporites Hammen (fossil)

Piriurella Cookson \& Eisenack is a later taxonomic synonym of Pluricellaesporites Hammen (R.K. Saxena).

\section{Polycellaesporonites Anil Chandra et al. (fossil)}

Capsular, muriform fungal spores with a hilum, and distally with an elongated, knob-like or beaked, extension as that in the modern Alternaria (R.K. Saxena). 
After segregation of the genus Perennicordyceps (Matočec et al. 2014) and the description of several new species in the genus, Polycephalomyces currently includes 18 species (Xiao et al. 2018) (D. Haelewaters).

Prathoda Subram.

Simmons (2007) resurrected Prathoda which is distinct from Alternaria (Pleosporaceae). In MycoBank and Species Fungorum, Prathoda is mentioned as a synonym of Alternaria, but its molecular phylogeny has not been recovered and closest relationship with Alternaria has not been settled. Therefore we left Prathoda as a separate genus (P.B. Gannibal).

\section{Proliferophorum Wang et al.}

Phookamsak et al. (2019) introduced a monotypic genus Proliferophorum to accommodate hyphomycetous species, $P$. thailandicum G.N. Wang et al. in Diaporthomycetidae based on phylogenetic analysis. The genus is characterized by having mononematous, caespitose conidiophores, polyblastic, terminal, sympodial, pale brown or subhyaline, with minute, truncate conidiogenous cells, sometimes percurrently proliferating 1-2 times at broken ends of conidiogenous cells and fusiform to cylindrical, pigmented, septate conidia. The genus was collected from decaying submerged wood in Thailand (R. Phookamsak).

Protofenestella Jaklitsch \& Voglmayr

Jaklitsch et al. (2018) proposed this new genus in the Cucurbitariaceae based on morphological and molecular characters (S. Fryar).

Protothelenellaceae Vezda et al.

Kraichak et al. (2018a) regarded that Thrombiaceae Poelt \& Vezda ex J.C. David \& D. Hawksw. as a synonym of Protothelenellaceae. Further, the family has been transferred to Baeomycetales from Ostropales (N. Wijayawardene).

Pseudaegerita J.L. Crane \& Schokn.

This aero-aquatic hyphomycete genus was shown to be a member of Hyaloscyphaceae based on morphology of the associated sexual state (Abdullah et al. 2005) and DNA sequence data (Johnston et al. 2019, Vu et al. 2019) (H. Madrid).

\section{Pseudoanungitea Crous}

Crous et al. (2018) introduced this new genus in Venturiaceae (S. Fryar).

Pseudoastrosphaeriellopsis Devadatha et al.

Phookamsak et al. (2019) introduced Pseudoastrosphaeriellopsis as a monotypic genus in Pseudoastrosphaeriellaceae to accommodate trematosphaeria-like taxon. The genus is typified by Pseudoastrosphaeriellopsis kaveriana Devadatha et al. collected from decaying wood of Avicennia marina (Forssk.) Vierh. and Suaeda monoica Forssk. ex J.F.Gmel. in India. Based on multi-gene phylogenetic analyses, Pseudoastrosphaeriellopsis formed a distinct lineage basal to Pseudoastrosphaeriella (R. Phookamsak).

\section{Pseudobambusicola Hern.-Restr. \& Crous}

This new genus was placed in Sulcatisporaceae using multi-gene phylogenetics and morphological characters by Rupcic et al. (2018). It is closely related to Neobambusicola but differs in having cylindrical-necked conidiomata surrounded by dark brown, smooth to slightly verruculose hyphae (Rupcic et al. 2018) (S. Fryar \& S. Somrithipol).

\section{Pseudofusicoccum Mohali}

Yang et al. (2017) considered that this genus warrants a separate family and thus introduced Pseudofusicoccumaceae to accommodate it. Phillips et al. (2019) took into account phylogeny 
(ITS, LSU), morphology and evolutionary divergence times and concluded that Pseudofusicoccum resides within Phyllostictaceae. Hence, Phillips et al. (2019) synonymised Pseudofusicoccumaceae under Phyllostictaceae (A.J.L. Phillips).

\section{Pseudographis Nyl.}

Based on the phylogenetic analysis of a three-gene dataset (ITS, LSU, mtSSU), Karakehian et al. (2019) placed Pseudographis in Rhytismataceae, not Triblidiaceae. Because of the inclusion of Pseudographis in the family, the authors expanded the morphological description of Rhytismataceae to include "ascospore cell walls that produce a strong blue/purple reaction in iodine-based reagents" (D. Haelewaters).

\section{Pseudogymnoascus Raillo}

In Johnston et al.'s (2019) 15-gene tree, Pseudogymnoascus was strongly supported as sister genus to Leuconeurospora within Pseudeurotiaceae, Thelebolales. In a genomic-scale tree based on 3156 single-copy genes, Pseudogymnoascus destructans was sister to Thelebolus microsporus, confirming its position in the order Thelebolales (Johnston et al. 2019) (D. Haelewaters).

\section{Pseudolanzia Baral \& G. Marson}

Introduced to accommodate a species that bears morphological similarities to Lanzia but is phylogenetically distinct. It is placed on a long branch within Rutstroemiaceae based on an ITS+LSU phylogeny (Baral 2019) (D. Haelewaters).

\section{Pseudomelanconidaceae C.M. Tian \& X.L. Fan}

The asexual morph of the family Pseudomelanconidaceae is somewhat similar to members of Melanconiellaceae, and Juglanconidaceae. However, phylogenetic inferences resolved this family as an individual group with well-supported group from other families of Diaporthales (Fan et al. 2018a) (S.S.N. Maharachchikumbura \& S. Fryar).

\section{Pseudomelanconis C.M. Tian \& X.L. Fan}

Pseudomelanconis caryae is the type species of new genus Pseudomelanconis, and only occurs on Carya cathayensis in China (Fan et al. 2018a) (S.S.N. Maharachchikumbura).

\section{Pseudoneoconiothyrium Wanas. et al.}

Wanasinghe et al. (2018) introduced a monotypic genus to accommodate neoconiothyriumlike species in Thyridariaceae. However, Phookamsak et al. (2019) accommodated the genus in Roussoellaceae based on multi-gene analyses and this concurred with Jiang et al. (2019a) and Karunarathna et al. (2019) (R. Phookamsak).

\section{Pseudoophiobolus Phook. et al.}

Phookamsak et al. (2017) introduced Pseudoophiobolus to accommodate ophiobolus-like species in Phaeosphaeriaceae based on multi-gene phylogenetic analyses including $P$. achilleae, $P$. erythrosporus, $P$. galii, $P$. italicus, $P$. mathieui, $P$. rosae, $P$. subhyalinisporus and $P$. urticicola (R. Phookamsak).

\section{Pseudopaucispora A. Hashim.}

Hashimoto et al. (2018) introduced this monotypic genus in Lophiostomataceae based on molecular and morphological characters (S. Fryar).

\section{Ramalinaceae C. Agardh}

The taxonomy of family Ramalinaceae was recently revised by Kistenich et al. (2018). According to phylogenetic analysis genera Adelolecia, Catinaria, Compsocladium, Crustospathula, Frutidella, Japewia, Schadonia, Tasmidella do not belong this family (A. Suija). 
Ramasricellites Kalgutkar \& Janson. (fossil)

Kalgutkar \& Jansonius (2000) opined that Multicellaesporites differentialis Ramanujam \& Srisailam is a misfit in Multicellaesporites and therefore they proposed Ramasricellites to accommodate it. The sharp differentiation between the dark, broad central cells and the narrower, elongate hyaline terminal cells, as well as the lack of constriction at the median septum, differentiate this form from species in Multicellites (R.K. Saxena).

Ramomarthamyces P.R. Johnst.

Marthamyces was found to be polyphyletic and therefore Johnston \& Park (2019) described Ramomarthamyces within Marthamycetaceae (Marthamycetales) for species separated from Marthamyces sensu stricto. The four species in Ramomarthamyces have distinctly branched rather than propoloid paraphyses (D. Haelewaters).

Ramophialophora M. Calduch et al.

This genus is polyphyletic within Sordariales (Zhang et al. 2017), but its type species, $R$. vesiculosa is clearly phylogenetically placed in Lasiosphaeriaceae (Madrid et al. 2010) (H. Madrid)

\section{Ranadivia Zmitr.}

Zmitrovich (2018) introduced this new genus in Fomitopsidaceae to accommodate Daedalea allantoidea M.L. Han, B.K. Cui \& Y.C. Dai, D. africana I. Johans. \& Ryvarden, D. stereoides Fr., and Polyporus modestus Kunze ex Fr. Based on a multi-gene phylogeny, Han et al. (2016) accepted these species in the genus Daedalea Pers. (V. Papp).

Ratnagiriathyrites R.K. Saxena \& N.K. Misra (fossil)

This monotypic genus is characterized by its non-radiating, hexagonal porate cells (R.K. Saxena).

\section{Remersonia Samson \& Seifert}

Wang et al. (2018) showed that the genus belongs in the Chaetomiaceae (K. Bensch).

\section{Requienellaceae Boise}

This family was introduced by Boise (1986) in the class Dothideomycetes (as Loculoascomycetes) and she kept the family in Melanommatales (sensu Barr 1983) or Pyrenulales (sensu Eriksson 1984). However, Requienellaceae was not treated as a distinguished family by Hawksworth \& Eriksson (1986), who maintained it under Pyrenulaceae. Barr (1990) and Aptroot (1991) accepted Requienellaceae as a family of Melanommatales and again Kirk et al. (2008) as a family of the Pyrenulales. Based on the sequence data, Jaklitsch et al. (2016b) reinstated Requienellaceae as a family of Xylariales (S.S.N. Maharachchikumbura).

Resiniporus Zmitr.

Zmitrovich (2018) introduced this new genus in Irpicaceae to accommodate Ceriporiopsis resinascens (Romell) Domański and C. pseudogilvescens (Pilát) Niemelä \& Kinnunen (V. Papp).

Resinogalea Rikkinen \& A.R. Schmidt

Rikkinen et al. (2016) proposed Resinogalea for Resinogalea humboldtensis collected from resin of Araucaria humboldtensis in New Caledonia (S. Somrithipol).

Retihelicosporonites Ramanujam \& K.P. Rao (fossil) 
Helical spores (conidia) are found in various hyphomycetes, viz. Helicoma Corda, Helicomina L.S. Olive, Helicoon Morgan, Helicodendron Peyronel, Xenosporella Höhn, Hiospira R.T. Moore, etc. (Barnett 1956, Ellis 1971, Ainsworth et al. 1973) (R.K. Saxena).

Retroconis de Hoog \& Bat. Vegte

This genus belongs in Chaetomiaceae, Sordariales according to Crous et al. (2007) (H. Madrid).

\section{Rhamphoria Niessl}

Ramphoria is the type genus of the newly erected family Rhamphoriaceae (Réblová \& Štěpánek (2018) (K. Bensch).

\section{Rhamphoriaceae Réblová}

Rhamphoriaceae is a novel family introduced by Réblová \& Štěpánek (2018) to represent genera Rhamphoria, Rhamphoriopsis, Linkosia and Xylolentia (S.S.N. Maharachchikumbura).

Rhamphoriopsis Réblová \& Gardiennet

Genus in the Rhamphoriaceae with Rhamphoriopsis muriformis as the type species (Réblová \& Štěpánek 2018) (S.S.N. Maharachchikumbura).

Rhexoacrodictys W.A. Baker \& Morgan-Jones

Xia et al. (2017) treated this genus as a member in Savoryellales, Savoryellaceae. (J. Ma)

Rhodoveronaea Arzanlou, W. Gams \& Crous

Réblová \& Štěpánek (2108) referred this genus to the newly erected family Rhamphoriaceae (K. Bensch).

Rhizoglomus Sieverd. et al.

The genus Rhizophagus was not accepted in the Fungal Kingdom, as Rhizophagus populinus is not an arbuscular mycorrhizal fungi but a plant root pathogen originally attributed to the Peronosporales (Sieverding et al. 2014) which at time is attributed to the kingdom Chromista (Cavalier-Smith 2018). Glomus intraradices became the type species of the new genus Rhizoglomus with several new species described using Rhizoglomus as generic name (Sudová et al. 2015, Błaszkowski et al. 2018a, b, 2019a. b, Turrini et al. 2018).

\section{Rimularia Nyl.}

Four species were included in the phylogeny (Resl. et al. 2015), but 25 species still need molecular data for the correct genus placement (M. Kukwa).

Roesleria Thüm. \& Pass.

Baral (2016) maintained the family Roesleriaceae within his "Lineage B" (Helotiaceae sensu lato) to accommodate the genus Roesleria with its peculiar morphological characteristics. However, Johnston et al. (2019) found high support for the placement of this genus deep within the family Helotiaceae (D. Haelewaters).

Roselymyces Fiuza et al.

The monotypic genus Roselymyces was erected in the Xylariales based on morphological chacracters and a molecular phylogeny based on ITS and LSU data by Fiuza et al. (2018) with Roselymyces brasiliensis as the type species. The genus was not yet associated to one of the families of Xylariales, but shows morphological affinities to Cylindrium, Polyscytalum, Pseudoidriella and Tristratiperidium (M. Stadler). 
Rostania Trevis.

According to Košuthová et al. (2019) the genus is not monophyletic and 2 species were transferred to Leptogium and Scytinum. At present 3 species are known to belong to the genus in its strict sense, but the species delimitation within Rostania needs further studies (M. Kukwa).

\section{Rutstroemiaceae Holst-Jensen et al.}

The Rutstroemiaceae + Sclerotiniaceae clade was retrieved with high statistical support in the sclerotinioid clade of Helotiales in the 15-gene tree of Johnston et al. (2019). If Sclerotiniaceae is retained, Rutstroemiaceae as currently recognized is not monophyletic and would need to be split in four families. More multigene and genomic-scale work is needed to resolve this sclerotinioid clade. Ekanayaka et al. (2019) proposed that this family belongs to an informal clade named "Sclerotiniales". However, this placement was without support (D. Haelewaters \& N. Wijayawardene).

Saccharomycetes O.E. Erikss. \& Winka.

In the case of Saccharomycetes yeasts, the status of several families and the status and familial placement of several genera has not been unequivocally cleared (Daniel et al. 2014, Hittinger et al. 2015, Shen et al. 2016, 2018, Kurtzman \& Boekhout 2017) since the publication of the 5th edition of The Yeasts: A Taxonomic Study (Kurtzman 2011). Based on accumulating phylogenomic data, the status of some families are expected to change substantially (e.g. Shen et al. 2018). Thus, a comprehensive list of currently accepted sexual genera and non-synonymized asexual genera (that are expected to be retained following the Melbourne code, notably Candida Berkhout) are listed among the notes with additional notes on expected changes and protected names, following Kurtzman \& Boekhout (2017) (W.P. Pfliegler \& E. Horváth).

Saccisporonites Kalgutkar \& Janson. (fossil)

Kalgutkar \& Jansonius (2000) opined that Lacrimasporonites stoughiae Elsik is a misfit in Lacrimasporonites Elsik and therefore they proposed Saccisporonites to accommodate it (R.K. Saxena).

Sakireeta Subram. \& K. Ramakr.

Based on ITS and LSU sequence phylogeny, Crous et al. (2015a) transferred Sakireeta to Botryosphaeriaceae (A.J.L. Phillips).

Saprochaete Coker \& Shanor ex D.T.S. Wagner \& Dawes

Expected transfer of species to Magnusiomyces Zender (Kurtzman \& Boekhout 2017) (W.P. Pfliegler and E. Horváth).

Sarcoleotia S. Ito \& S. Imai

Phylogeny in Hustad et al. (2011) demonstrates that Sarcoleotia is sister species to Nothomitra, both in a separate clade than the rest of Geoglossomycetes (V.P. Hustad).

Sarcopeziza Loizides et al.

This genus was recently introduced by Agnello et al. (2018) (P. Alvarado).

Sardiniella Linaldeddu et al.

This genus was introduced by Linaldeddu et al. (2016) to accommodate a diplodia-like species from diseased Celtis africana trees in Sardinia. Morphologically similar to Diplodia and Dothiorella, but phylogenetically distinct (A.J.L. Phillips).

Saxophila Selbmann \& de Hoog 
This is a monotypic genus, typified by S. tyrrhenica, a dematiaceous microcolonial fungus obtained from marble and limestone in Europe. Its placement in Extremaceae is supported by multilocus DNA sequence data (Isola et al. 2016) (H. Madrid).

Schadonia Körb.

Kistenich et al. (2018) transferred this genus from Ramalinaceae to Pilocarpaceae (E. Timdal).

\section{Sclerencoelia Pärtel \& Baral}

This genus was introduced by Pärtel et al. (2017) to accommodate two species of Encoelia (E. fascicularis and E. pruinosa) that belonged to Sclerotiniaceae, whereas the type species E. furfuraceae was placed in Cenangiaceae. A third species of Sclerencoelia was also described by Pärtel et al. (2017) mostly based molecular data (D. Haelewaters).

\section{Sclerococcum Fr.}

Réblová et al. (2016) transferred it to Sclerococcaceae and this was supported by Yu et al. (2018). However, Sclerococcum has been transferred to Dactylosporaceae Bellem. \& Hafellner [= Sclerococcaceae Réblová, Unter. \& W. Gams] by Diederich et al. (2018) (H. Zhang \& J. Etayo).

Scolecolachnum Guatimosim et al.

Scolecolachnum was introduced in Hyaloscyphaceae (Guatimosim et al. 2016). Based on both ITS and LSU phylogenetic analyses, it is retrieved as sister to Hyphodiscus in Hyaloscyphaceae Han Clade 4 (sensu Han et al. 2014, Johnston et al. 2019) (D. Haelewaters).

\section{Scutula Tul.} Timdal).

Kistenich et al. (2018) transferred this genus from Pilocarpaceae to Ramalinaceae (E.

Seltsamia Jaklitsch \& Voglmayr

Jaklitsch et al. (2018) proposed this new genus in Cucurbitariaceae based on morphological and molecular characters (S. Fryar).

Septomelanconiella Samarak. \& K.D. Hyde

Phookamsak et al. (2019) introduced a monotypic genus Septomelanconiella to accommodate a single species S. thailandica Samarak. \& K.D. Hyde. Septomelanconiella can be distinguished from Melanconiella Sacc. in having finely verrucose brown mature conidia. Phylogenetic analysis revealed that the genus formed a distinct lineage with other genera in Melanconiellaceae (R. Phookamsak).

\section{Septoriella Oudem.}

Crous et al. (2015c) treated Wojnowicia as a synonym of Septoriella based on a neotypic study of Wojnowicia hirta Sacc. (R. Phookamsak).

\section{Septorioides Quaedvl. et al.}

This genus was introduced for species morphologically similar to Septoria but distinguishable on account of conidiomata that open by an irregular split, and paraphyses intermingled with the conidiogenous cells. Furthermore, they constitute a phylogenetic lineage in Botryosphaeriaceae and thus separate from Septoria and allied genera (Quaedvlieg et al. 2013). Wyka \& Broders (2016) introduced the family Septorioideaceae in Botryosphaeriales to accommodate Septorioides species. Phillips et al. (2019) took into account phylogeny (ITS, LSU), morphology and evolutionary divergence times and concluded that Septorioides resides within 
Saccharataceae. For this reason, Phillips et al. (2019) regarded Septorioideaceae as a synonym of Saccharataceae (A.J.L. Phillips).

Sepultariella Van Vooren et al.

This genus was erected to accommodate two species previously ascribed to Leucoscypha. It represents a separate phylogenetic lineage within Pyrenomataceae (Van Vooren et al. 2017) (I. Kušan \& N. Matočec).

\section{Sheathospora X.L. Fan}

Fan et al. (2018b) proposed this new genus based on unique pycnidial conidiomata and conidia with distinct hyaline sheath in Melanconiellaceae. Sheathospora cornuta is the type to accommodate Melanconiella cornuta and currently so far known from Cornus controversa and Juglans regia in China (X.L. Fan).

\section{Snippocia Ertz et al.}

Ertz et al. (2018) introduced this genus and placed it in Arthoniaceae based on phylogenetic analyses (N. Wijayawardene).

\section{Solanella Vaňha}

Ekanayaka et al. (2018) proposed that this genus should be transferred to Pezizomycotina incertae sedis (I. Kušan \& N. Matočec).

Solicorynespora R.F. Castañeda \& W.B. Kendr.

Hernández-Restrepo et al. (2014) showed that Solicorynespora insolita has a high affinity with members of Dothideomycetes, and more specifically with Astrosphaeriella livistonicola (J. $\mathrm{Ma})$

Spadicoides S. Hughes

Réblová et al. (2018) accommodated this genus in Xenospadicoidaceae based on phylogenetic analyses (S. Fryar).

\section{Spegazzinia Sacc.}

This genus was shown to belong in Didymosphaeriaceae by Tanaka et al. (2015) (P. Alvarado).

\section{Sphaerosporium Schwein.}

According to a multilocus phylogenetic study in Song et al. (2019), the generic type, $S$. lignatile Schwein., belongs to Pyronemataceae (Pezizomycetes, Pezizales), whereas S. equinum (Desm.) J.L. Crane \& Schokn. was placed among Onygenales based on LSU sequence data (Rokas et al. 2012) (D. Haelewaters).

\section{Sphaerosporium Schwein.}

According to a multilocus phylogenetic study by Haelewaters et al. in Song et al. (2019), the generic type, S. lignatile Schwein., belongs to Pyronemataceae (Pezizomycetes, Pezizales), whereas S. equinum (Desm.) J.L. Crane \& Schokn. was placed among Onygenales based on LSU sequence data (Rokas et al. 2012) (D. Haelewaters).

\section{Spinosporonites R.K. Saxena \& S. Khare (fossil)}

This monotypic genus includes circular to subcircular, inaperturate, multicellular spores, each cell giving rise to a robustly built spine. They readily resemble the setose pycnidia found in some Coelomycetes (R.K. Saxena). 
Spiromastigaceae Hirooka et al.

The family was first invalidly published in Rizzo et al (2014) (Arts 38.11 and 42.1) and later validated in Hirooka et al. (2015) (K. Bensch).

Spirotremesporites Dueñas (fossil)

This genus includes ellipsoidal to elongate, aseptate, psilate fungal spores having aperture in the form of a single furrow at an angle to the axis of the spore, straight or curved to S-shaped or sigmoidal in outline, or spiral around the spore axis. Varisulcosporites Rouse \& Mustard is a junior taxonomic synonym of Spirotremesporites (R.K. Saxena).

Sporacestra A. Massal.

Kistenich et al. (2018) resurrected this genus in Ramalinaceae (E. Timdal).

Sporastatiales Lumbsch \& Leavitt

Kraichak et al. (2018a) raised Sporastatiaceae to ordinal level as Sporastatiales (N. Wijayawardene).

\section{Sporocadaceae Corda}

Liu et al. (2019) provided a revision of the Sporocadaceae based on multi-locus phylogenetic analyses, using LSU, ITS, tef-1 $\alpha$, tub2 and rpb2 loci, in combination with morphological data. A total of 30 well-supported monophyletic clades were recognized, representing 23 known and seven new genera. Typifications for the type species Diploceras, Discosia, Monochaetia, Sporocadus and Truncatella) and emendations of various genera and species were also provided (M. Stadler).

Sporocarpon Will. (fossil)

Dubiocarpon S.A. Hutch. and Oidospora Will. are later taxonomic synonyms of Sporocarpon Will. (R.K. Saxena).

Sporormiella Ellis \& Everh.

The ostiolate Sporormiella has been recognized as a probable synonym of the earlier nonostiolate Preussia for several decades and, based on morphology and phylogeny, Zhang et al. (2012) and Hyde et al. (2013) adopted Preussia. However, the type species S. nigropurpurea has not been sequenced, and Sporormiella is widely used in the literature of coprophilous fungi (e.g. Doveri 2004, Bell 2005) and palaeoecology (Raper \& Bush 2009, Raczka et al. 2016) and contains many more species. If the genera are eventually proved to be congeneric molecularly, we consider that Sporormiella should be proposed for conservation over Preussia so both names are currently retained here (D.L. Hawksworth \& N.N. Wijayawardene).

Staphlosporonites Sheffy \& Dilcher (fossil) Saxena).

Transeptaesporites V.S. Ediger is a later taxonomic synonym of Staphlosporonites (R.K.

Steinera Zahlbr.

The genus, previously placed in Koerberiaceae, has been recently moved into Arctomiaceae (Ertz et al. 2017a). A new genus, Henssenia, was established for Steinera species remaining in Koerberiaceae (Ertz et al. 2017a) (M. Kukwa).

Stemphylium Wallr.

The major part of the family Pleosporaceae is represented by species of Pleospora, a genus that is considered paraphyletic (Kodsueb et al. 2006, Inderbitzin et al. 2009). The type species of Pleospora, P. herbarum, was synonymized with Stemphylium herbarum. At this time, however, 
several hundreds of Pleospora epithets still have not been assigned to Stemphylium or other genera and are not included in this outline (P.B. Gannibal).

Stereophlebia Zmitr.

Zmitrovich (2018) segregated this new monotypic genus from Lilaceophlebia (Parmasto) Spirin \& Zmitr. to accommodate Phlebia tuberculata (Berk. \& M.A. Curtis) Ţura, Zmitr., Wasser \& Spirinski (V. Papp).

Stictographaceae D.Q. Dai \& K.D. Hyde

Dai et al. (2018) introduced this family in Asterinales to accommodate Stictographa Mudd, Karschia Körb., Labrocarpon Etayo \& Pérez-Ortega and Melaspileopsis (Müll. Arg.) Ertz \& Diederich (N. Wijayawardene).

Stigmatodiscus Voglmayr \& Jaklitsch

Voglmayr \& Pintos (2018) synonymised Asterodiscus Voglmayr et al. with Stigmatodiscus (P. Alvarado).

Striadiporites C.P. Varma \& Rawat (fossil)

This genus includes unicellular, diporate fungal spores with striated spore wall. Stridiporosporites Ke \& Shi is a junior taxonomic synonym of Striadiporites (R.K. Saxena).

Symbiotaphrina Kühlw. \& Jurzitza ex W. Gams \& Arx

Baral et al. (2017) validated the order Symbiotaphrinales and introduced the new family Symbiotaphrinaceae (K. Bensch).

Symmetrospora Q.M. Wang et al.

Symmetrospora was recently introduced for species previously placed in the asexual genera Sporobolomyces and Rhodotorula in the "gracilis/marina clade" of Cystobasidiomycetes (Wang et al. 2015b). Haelewaters et al. (2020) recently proposed three new species and a new combination, making 10 recognized species (D. Haelewaters).

Synnemasporella X.L. Fan \& J.D.P. Bezerra

Synnemasporella was introduced by Fan et al. (2018a) to accommodate fungi with synnematous conidiomata. This genus is typified by Synnemasporella toxicodendri (S.S.N. Maharachchikumbura).

Synnemasporellaceae X.L. Fan \& J.D.P. Bezerra

Fan et al. (2018a) proposed this new family to accommodate one new genus, Synnemasporella (Type species: Synnemasporella toxicodendri). The new genus and species have been collected from Toxicodendron sylvestre in China, and S. aculeans was transferred from Cryptodiaporthe aculeans (basionym: Sphaeria aculeans) (S. Fryar \& S. Somrithipol).

Szczepkamyces Zmitr.

Zmitrovich (2018) introduced this new monotypic genus in Polyporaceae to accommodate Dichomitus campestris (Quél.) Domański \& Orlicz (V. Papp).

Taeniolella S. Hughes

Heuchert et al. (2018) showed that the genus is polyphyletic with type species belonging Kirschsteiniotheliaceae (Dothideomycetes) while saprobic species cluster within Sordariomycetes in different families. Lichenicolous species form a monophyletic clade within Asterotexiales, Dothideomycetes but many species are still not sequenced (A. Suija). 
Taitaia Suija et al.

Suija et al. (2018) introduced this lichenicolous genus and confirmed its placement in Gomphillaceae, Graphidales (N. Wijayawardene).

Tamsiniella S.W. Wong et al.

Phookamsak et al. (2019) treated Tamsiniella in Phyllachoraceae based on phylogenetic analysis (R. Phookamsak).

Tapesia (Pers.) Fuckel

Tapesia is considered a synonym of Mollisia (Hawksworth \& David 1989) but many species are still classified under Tapesia (Species Fungorum 2020, Tanney \& Seifert 2020). As a result, Tapesia is included in this outline (D. Haelewaters).

Tasmidella Kantvilas et al.

Kistenich et al. (2018) transferred this genus from Ramalinaceae to the Lecanorales incertae sedis (E. Timdal).

Thalloidima A. Massal.

Kistenich et al. (2018) resurrected this genus in the Ramalinaceae (E. Timdal).

Thecotheus Boud.

Placed in Ascobolaceae, Pezizales. This genus was treated by Kušan et al. (2015) who listed 23 known species, including the newly described T. platyapiculatus (I. Kušan \& N. Matočec).

Tenuitholiascaceae S.H. Jiang et al.

Jiang et al. (2020) introduced this family based on the new genus Tenuitholiascus, which resides in Strigulales (N. Wijayawardene).

Thysanorea Arzanlou et al.

According to phylogenetic studies by Arzanlou et al. (2007) and Dong et al. (2018), this genus is a member of Herpotrichiellaceae. Dong et al. (2018) introduced the second species Thysanorea aquatica W. Dong, H. Zhang \& K.D. Hyde. However, this species has been reported as a synonym with the type species Thysanorea papuana (Aptroot) Arzanlou, W. Gams \& Crous (Wang et al. 2018) (H. Madrid \& H. Zhang).

Toninia A. Massal.

Kistenich et al. (2018) placed Arthrosporum A. Massal. as a synonym of Toninia (E. Timdal)

Torrentispora K.D. Hyde et al.

Réblová et al. (2018) transferred Torrentispora from the Annulatascaceae to Xenospadicoidaceae based on multi-gene phylogenetic analyses (S. Fryar).

Tremellochaete Raitv.

Tremellochaete was reinstated in Auriculariaceae by Malysheva \& Spirin (2017) based on morphological characteristics and phylogenetic analyses. Phookamsak et al. (2019) updated a species number in this genus. Based on morphological characteristics and phylogenetic analysis, three species are accommodated in this genus (Malysheva \& Spirin 2017, Index Fungorum 2019, Phookamsak et al. 2019) (R. Phookamsak).

Triadelphia Shearer \& J.L. Crane 
Recent studies suggested that Triadelphia is polyphyletic, but no DNA sequence data is available for many of its members. The type species, T. heterospora, belongs in Microascales (Crous et al. 2015b) (H. Madrid).

Triblidiaceae Rehm

Triblidiaceae is considered a monophyletic family within Rhytismatales, including two genera, Huangshania and Triblidium. The previous order Triblidiales is synonymized under Rhytismatales (Karakehian et al. 2019) (D. Haelewaters).

Tribolites W.H. Bradley (fossil)

The fossil conidia resemble conidia of extant genera Tetrachaetum Ingold and Lemonniera

D. Wild. Trihyphaecites Peppers is a junior taxonomic synonym of Tribolites (R.K. Saxena).

Trichomonascaceae Kurtzman \& Robnett

Family status expected to change upon resolving Blastobotrys/Trichomonascus (W.P. Pfliegler and E. Horváth).

Trichomonascus H.S. Jackson emend. Kurtzman \& Robnett

Expected transfer of species to Blastobotrys Klopotek to comply with the Melbourne Code (Kurtzman \& Boekhout 2017) (W.P. Pfliegler \& E. Horváth).

Trichothyrites Rosend. (fossil)

Notothyrites Cookson and Sphaerialites Venkatach. \& R.K. Kar are later taxonomic synonyms of Trichothyrites (Kalgutkar \& Jansonius 2000) (R.K. Saxena).

Trihyphites Kalgutkar \& Janson. (fossil)

Trihyphaecites fractus Z.C. Song \& Liu Cao, in Song et al. (1989) belongs to Trihyphites. (R.K. Saxena).

Triporicellaesporites Ke \& Shi (fossil)

The spores of two species of Triporicellaesporites, viz. T. elongatus P. Ke \& Z.Y. Shi and $T$. simplex (Elsik \& Janson.) Kalgutkar \& Janson., are very similar to spores of the extant Ceratosporella bicornis (Morgan) Höhnel. (R.K. Saxena).

Trullella Zmitr.

Zmitrovich (2018) treated that the name Trulla Miettinen \& Ryvarden is illegitimate (non Trulla T.M. Harris), thus the new genus Trullella was proposed to accommodate Trulla dentipora (Ryvarden \& Iturr.) Miettinen \& Ryvarden and five other Trulla species (V. Papp).

Tubakiaceae U. Braun et al.

Braun et al. (2018) introduced Tubakiaceae (in Diaporthales) to accommodate Tubakia B. Sutton. and six other genera (viz. Apiognomonioides U. Braun, J.Z. Groenew. \& Crous, Involutscutellula U. Braun \& C. Nakash., Paratubakia U. Braun \& C. Nakash., Racheliella Crous \& U. Braun, Saprothyrium U. Braun, Crous \& J.Z. Groenew., Sphaerosporithyrium U. Braun, Crous, O. Moreno-Rico \& Marm.) (N. Wijayawardene).

Tylothallia P. James \& H. Kilias

Kistenich et al. (2018) transferred this genus from Lecanoraceae to Ramalinaceae (E. Timdal).

Umbilicaria Hoffm. 
Davydov et al. (2017) revised the Umbilicariaceae sensu stricto and accepted eight subgenera in the genus Umbilicaria: viz. Actinogyra (type: U. muehlenbergii), subg. Agyrophora (type: A. atropruinosa), subg. Floccularia subg. nov. (type: U. deusta), subg. Gyrophora (type: $U$. vellea), subg. Iwatakia subg. nov. (type: U. esculenta), subg. Lasallia (type: L. pustulata), subg. Umbilicaria (type: U. hyperborea), and subg. Umbilicariopsis subg. nov. (type: Umbilicaria polyrhiza) (G. Rambold).

Umthunziomyces Crous \& M.J. Wingf.

Umthunziomyces was introduced for a septoria-like species that resides in Planistromellaceae (Crous et al. 2016) (A.J.L. Phillips).

Uncinulites Pampal. (fossil) Saxena).

Graamspora Sal.-Cheb. \& Locq. is a later taxonomic synonym of Uncinulites (R.K.

\section{Unguicularia Hohn.}

Previously considered as a member of Hyaloscyphaceae (Baral 2016), but currently placed in Helotiales genera incertae sedis based on the ITS placement by Johnston et al. (2019). Additional sequence data are needed to resolve the placement of this genus (D. Haelewaters).

\section{Varicellaria Nyl.} Bensch).

In Kraichak et al. (2018b), the monotypic family Varicellariaceae was validated (K.

Varicosporellopsis Lechat \& J. Fourn.

Lechat \& Fournier (2016) described Varicosporellopsis and placed it in Nectriaceae (I. Kušan \& N. Matočec).

Varmasporites Kalgutkar \& Janson. (fossil)

Kalgutkar \& Jansonius (2000) opined that Fusiformisporites tonakkalensis Y.N.R. Varma \& R.S. Patil is a misfit in Fusiformisporites Rouse and therefore they proposed Varmasporites to accommodate it (R.K. Saxena).

Velebitea I. Kušan et al.

Velebitea was introduced in Phookamsak et al. (2019) to accommodate a single species, Velebitea chrysotexta I. Kušan, Matočec \& Jadan in Lachnaceae based on molecular data. The genus was collected from decorticated branches and stump base of Fagus sylvatica L. (Fagaceae) in Croatia and is characterized by having apothecial ascomata, elongated cylindrical-deltoid asci, protruding above paraphyses at maturity, with in Lugol's solution apical apparatus moderately euamyloid, of Calycina-type and hyaline, elongated fusoid ascospores (R. Phookamsak).

\section{Vermiconidia Egidi \& Onofri}

A multilocus phylogenetic study by Isola et al. (2016) placed all members of this genus in Extremaceae (H. Madrid).

Vitreoporus Zmitr.

Zmitrovich (2018) introduced this new genus to accommodate Gloeoporus dichrous (Fr.) Bres., G. africanus P.E. Jung \& Y.W. Lim, G. citrinoalbus Yuan Yuan \& Jia J. Chen, and G. orientalis P.E. Jung \& Y.W. Lim. However, phylogenetic analyses by Jung et al. (2018) revealed that these species belong to a monophyletic clade in Gloeoporus sensu stricto (V. Papp).

Vittaliana Devadatha et al. 
Devadatha et al. (2019) introduced this genus and showed that it is a member of Phaeosphaeriaceae. Its ascospores are similar to Acericola and Vagicola (S. Tibpromma).

Wheelerophlyctis P.M Letcher et al.

Letcher et al. (2018) introduced this genus, which comprises two species. Phylogenetic analyses confirmed its placement in Asterophlyctaceae (P. Letcher).

Xanthonectria Lechat et al.

Lechat et al. (2016b) described the monotypic genus Xanthonectria to accommodate Nectria pseudopeziza within Bionectriaceae (I. Kušan \& N. Matočec).

\section{Xenodactylaria Crous}

Crous et al. (2018) introduced this genus and showed that it has a distinct lineage in Myrmecridiales. As a result, the family Myrmecridiaceae was introduced (N. Wijayawardene).

Xenospadicoidaceae Hern.-Restr et al.

Réblová et al. (2018) accepted Calyptosphaeria, Lentomitella, Spadicoides and Torrentispora as members of the Xenospadicoidaceae, Xenospadicoidales. Furthermore, Réblová et al. (2018) reduced Xenospadicoides and Pseudodiplococcium under Spadicoides and synonymised Lentomitellaceae with Xenospadicoidaceae based on a multi-gene phylogeny (J. Ma \& S. Fryar).

Xyladictyochaetaceae Crous \& Hern.-Restr

Crous et al. (2018) introduced this family to accommodate the genus Xyladictyochaeta within the Xylariales (S. Fryar \& K. Bensch).

Xylolentia Réblová

Xylolentia is a newly introduced genus in the family Rhamphoriaceae, with the type species Xylolentia brunneola (Réblová \& Štěpánek 2018) (S.S.N. Maharachchikumbura).

Xylomyces Goos et al.

Nine species are currently reported within Xylomyces, but Xylomyces chlamydosporus Goos,

R.D. Brooks \& Lamore is the only species phylogenetically related to the Jahnulales (H. Raja).

Zopfochytrium M.J. Powell et al.

Powell et al. (2018) introduced this genus and confirmed its placement in Chytridiaceae (P. Letcher).

Zymochalara Guatimosim et al.

Zymochalara was introduced in Helotiales genera incertae sedis (Guatimosim et al. 2016) but in the ITS tree of Johnston et al. (2019) it was retrieved within Pezizellaceae (D. Haelewaters).

\section{Acknowledgements}

Nalin N. Wijayawardene thanks Mushroom Research Foundation and National Science Foundation of China (No. NSFC 31950410558) for financially supporting this project. Kevin D. Hyde acknowledges the Foreign Experts Bureau of Yunnan Province, Foreign Talents Program (2018; grant no. YNZ2018002), Thailand Research grants entitled Biodiversity, phylogeny and role of fungal endophytes on above parts of Rhizophora apiculata and Nypa fruticans (grant no: RSA5980068), the future of specialist fungi in a changing climate: baseline data for generalist and specialist fungi associated with ants, Rhododendron species and Dracaena species (grant no: DBG6080013), Impact of climate change on fungal diversity and biogeography in the Greater 
Mekong Subregion (grant no: RDG6130001). H.T. Lumbsch thanks support by the Grainger Bioinformatics Center. E. Malosso is grateful to CAPES for financial support (grant no. 88881.062172/2014-01). B.T. Goto, G.A. Silva and K. Jobim, L.C. Maia acknowledges CNPq (Brazilian Scientific Council, grants no. 465.420/2014-1, 307.129/2015-2 and 408011/2016-5) and CAPES for support. The study was partially supported by the National Science Centre, Poland, under Grants No. 2015/17/D/NZ8/00778 and 2017/25/B/NZ8/00473 to Julia Pawłowska. The research of Martin Kukwa received support from the National Science Centre (NCN) in Poland (project no 2015/17/B/NZ8/02441). Alan J.L. Phillips acknowledges the support from UID/MULTI/04046/2019 Research Unit grant from FCT, Portugal to BioISI. H. Zhang is financially supported by the National Natural Science Foundation of China (Project ID: NSF 31500017). S. Boonmee would like to thank the Thailand Research Fund (Project No. TRG6180001). Dong-Qin Dai and Li-Zhou Tang would like to thank the National Natural Science Foundation of China (No. NSFC 31760013, NSFC 31260087, NSFC 31460561), the Scientific Research Foundation of Yunnan Provincial Department of Education (2017ZZX186) and the Thousand Talents Plan, Youth Project of Yunnan Provinces for support. R. Phookamsak, M. Doilom, D. N. Wanasinghe, S.C. Karunarathna and J.C. Xu express sincere appreciations to Key Research Program of Frontier Sciences of the Chinese Academy of Sciences (grant no. QYZDYSSW-SMC014) for research financial support. R. Phookamsak thanks the Yunnan Provincial Department of Human Resources and Social Security (grant no. Y836181261), Chiang Mai University and National Science Foundation of China (NSFC) project code 31850410489 for research financial support. S.C. Kaunarathna thanks CAS President's International Fellowship Initiative (PIFI) for funding his postdoctoral research (No. 2018PC0006) and the National Science Foundation of China (NSFC) for funding this work under the project code 31851110759. S. Tibpromma would like to thank the International Postdoctoral Exchange Fellowship Program (number Y9180822S1), CAS President's International Fellowship Initiative (PIFI) (number 2020PC0009), China Postdoctoral Science Foundation and the Yunnan Human Resources, and Social Security Department Foundation for funding her postdoctoral research. Yuri S. Tokarev, Elena S. Nassonova and Irma V. Issi are indebtful to Yuliya Y. Sokolova (Institute of Cytology RAS, St. Petersbug, Russia) and Anastasia V. Simakova (Tomsk State University, Tomsk, Russia) for kind permission of reproduction of electron microscopy images of Metchnikovella incurvata and Crepidulospora beklemishevi, respectively. Yuri S. Tokarev and Irma V. Issi thank Russian Foundation of Basic Research, grant number 17-04-00871 (taxonomy of Rozellomycota). Elena S. Nassonova thank Russian Foundation of Basic Research, grant number 18-04-01359 (early evolution of Microsporidia, phylogeny of Metchnikovellida). Adam Flakus and Pamela Rodriguez-Flakus are greatly indebted to all staff of the Herbario Nacional de Bolivia, Instituto de Ecología, Universidad Mayor de San Andrés, La Paz and the SERNAP (http://sernap.gob.bo), for their generous cooperation providing permits, assistance and facilities support for scientific studies. The research of AF and PRF were financially supported by the National Science Centre (NCN) in Poland (DEC-2013/11/D/NZ8/03274). Adam Flakus and Pamela Rodriguez-Flakus received additional support under statutory funds from the W. Szafer Institute of Botany, Polish Academy of Sciences, Krakow, Poland. The authors would like to thank Yunnan Innovation Platform for Development and Utilization of Symbiotic Fungi Resources for finance support. LiFang Zhang would like to thank grant-in-aid from Science and Technology Department of Yunnan Province (2018FD080) for finance support. Chun-Ying Deng thanks the Biodiversity Survey and Assessment Project of the Ministry of Ecology and Environment, China (2019HJ2096001006). Yingqian Kang would like to thank Guizhou Scientific Plan Project [(2019) 2873]; Excellent Youth Talent Training Project of Guizhou Province [(2017) 5639]; Guiyang Science and Technology Project [(2017) No. 5-19]; Talent Base Project of Guizhou Province, China [FCJD2018-22]; Research Fund of Education Bureau of Guizhou Province, China [(2018) 481]. D. N. Wanasinghe would like to thank the CAS President's International Fellowship Initiative (PIFI) for funding his postdoctoral research (number 2019PC0008), the National Science Foundation of China and the Chinese Academy of Sciences for financial support under the 
following grants: 41761144055, 41771063 and Y4ZK111B01. Yuri K. Novozhilov and Oleg N. Shchepin acknowledge support from the Russian Foundation of Basic Research, project 18-0401232 A. Ivana Kušan, Neven Matočec, Armin Mešić and Zdenko Tkalčec are grateful to Croatian Science Foundation for their financial support under the project grant HRZZ-IP-201801-1736 (ForFungiDNA). K. Tanaka would like to thank the Japan Society for the Promotion of Science (JSPS 19K06802)

\section{References}

Abdel-Wahab MA, El-Samawaty AE, El Gorban AM, Yassin MA et al. 2018 - Khaleijomyces marinus gen. et sp. nov. (Juncigenaceae, Torpedosporales) a new lignicolous marine fungus from Saudi Arabia. Phytotaxa 340, 277-285.

Abdullah SK, Gené J, Guarro J. 2005 - A synopsis of the aero-aquatic genus Pseudaegerita and description of two new species. Mycological Research 109, 590-594.

Accioly T, Sousa JO, Moreau P-A, Lécuru C et al. 2019 - Hidden fungal diversity from the Neotropics: Geastrum hirsutum, G. schweinitzii (Basidiomycota, Geastrales) and their allies. Plos One 14, e0211388.

Adl SM, Bass D, Lane CE, Lukeš J et al. 2019 - Revisions to the classification nomenclature and diversity of Eukaryotes. Journal of Eukaryotic Microbiology 66, 4-119.

Agnello C, Alvarado P, Loizides M. 2018 - Sarcopeziza (Pezizceae, Ascomycota), a new monotypic genus for Inzenga's old taxon Peziza sicula. Ascomycete.org 10, 177-186.

Ainsworth GC, Sparrow FK, Sussman AS. (Eds.) 1973 - The fungi, an advanced treatise. I-IVB; Academic Press, New York, 3416 p.

Alvarado P, Healy RA, Moreno G, Cabero J et al. 2018 - Phylogenetic studies in Genabea, Myrmecocystis, and related genera. Mycologia 110, 401-418.

Álvarez E, Cano J, Stchigel AM, Sutton DA et al. 2011 - Two new species of Mucor from clinical samples. Medical Mycology 49, 62-72.

Alves MH, Trufem SFB, Milanez AI. 2002 - Táxons de Mucor Fresen. (Zygomycota) em fezes de herbívoros, Recife, PE, Brasil. Revista Brasileira de Biologia 25, 147-160.

Aptroot A, Lücking R. 2016 - A revisionary synopsis of the Trypetheliaceae (Ascomycota: Trypetheliales). Lichenologist 48, 763-982.

Aptroot A, Maphangwa KW, Zedda L, Tekere M et al. 2019 - The phylogenetic position of Culbersonia is in the Caliciaceae (lichenized ascomycetes). Lichenologist 51, 187-191.

Aptroot A, Sparrius LB, Alvarado P. 2018 - Aquacidia, a new genus to accommodate a group of skiophilous temperate Bacidia species that belong in the Pilocarpaceae (lichenized ascomycetes). Gorteria 40, 11-14.

Aptroot A. 1991 - Monograph of the Pyrenulaceae (excluding Anthracothecium and Pyrenula) and the Requienellaceae. J. Cramer.

Aptroot A. 1995 - A monograph of Didymosphaeria. Studies in Mycology 37, 1-16.

Ariyawansa HA, Phookamsak R, Tibpromma S, Kang JC et al. 2014 - A molecular and morphological reassessment of Diademaceae. The Scientific World Journal. Article ID 675348, http://dx.doi.org/10.1155/2014/675348

Arzanlou M, Groenewald JZ, Gams W, Braun U et al. 2007 - Phylogenetic and morphotaxonomic revision of Ramichloridium and allied genera. Studies in Mycology 58, 57-93.

Bader JA, Shotts EB Jr, Steffens WL, Lom J. 1998 - Occurrence of Loma cf. salmonae inbrook, brown and rainbow trout from Buford trout hatchery, Georgia, USA. DisAquat Organ 34, 211-216.

Balbiani G. 1882 - Sur les microsporidies ou psorospermies des articules. Comptes Rendus de l'Académie des Sciences 95, 1168-1171.

Baral HO, Polhorský A. 2019 - Chrysodisca peziculoides gen. et sp. nov. from xeric coniferous bark across Europe. Mycologia Montenegrina 20, 79-98. 
Baral HO, Weber E, Marson G, Quijada L. 2017 - A new connection between wood saprobism and beetle endosymbiosis: the rarely reported saprobic discomycete Tromeropsis is congeneric with the symbiotic yeast Symbiotaphrina (Symbiotaphrinales, Xylonomycetes) and two asexual morphs misplaced in Hyphozyma. Mycological Progress 17, 215-254.

Baral HO. 2016 - Inoperculate discomycetes. In: Jaklitsch W, Baral HO, Lücking R, Lumbsch HT, Frey W (eds) Syllabus of plant families: A. Engler's syllabus der Pflanzenfamilien part 1/2. Borntraeger, Stuttgart, 157-205.

Baral HO. 2019 - Pseudolanzia piceetorum gen. et sp. nov. (Rutstroemiaceae, Helotiales) from fallen Picea abies needles in Mecklenburg-Vorpommern (Germany). Mycologia Montenegrina 20, 151-166.

Barbosa FR, Gusmão LFP, Castañeda-Ruiz RF. 2017 - Craspedodidymella matogrossensis gen. \& sp. nov. from the Brazilian Amazon rainforest. Mycotaxon 132, 643-647.

Barnett HL. 1956 - Illustrated genera of imperfect fungi. Burgess Publishing Company, Minneapolis, $218 \mathrm{p}$.

Barnett HL. 1960 - Illustrated genera of imperfect fungi. Burgess Publishing Company, Minneapolis, $225 \mathrm{p}$.

Barr ME. 1983 - The ascomycete connection. Mycologia 75, 1-13.

Barr ME. 1990 - Melanommatales (Loculoascomycetes). North American Flora, Series 2, part 13. New York Botanical Garden, New York.

Bass D, Czech L, Williams BAP, Berney C et al. 2018 - Clarifying the relationships between Microsporidia and Cryptomycota. Journal of Eukaryotic Microbiology 65, 773-782.

Beakes GW, Thines M 2017 - Hyphochytriomycota and Oomycota. In: Archibald J, Simpson A, Slamovits C (eds) Handbook of the Protists, Second Edition. Springer International Publishing, Cham, 435-505.

Becnel JJ, Andreadis TG. 1999 - Microsporidia in insects. In: Wittner M (ed) The microsporidia and microsporidiosis. ASM Press, Washington DC, 447-501.

Becnel JJ, Takvorian PM, Cali A. 2014 - Checklist of available generic names for Microsporidia with type species and type hosts. In: Weiss LM, Becnel JJ (eds) Microsporidia: Pathogens of Opportunity. Wiley-Blackwell, pp 671-686.

Begerow D, McTaggart A, Agerer R, Frey W. 2018 - Syllabus of Plant Families-A. Engler's Syllabus der Pflanzenfamilien Part 1/3.

Bell A. 2005 - An illustrated guide to the coprophilous ascomycetes of Australia. CBS Biodiversity Series 3, 172 pp. Utrecht: Centraalbureau voor Schimmelcultures.

Bennett RM, Thines M 2019 - Revisiting Salisapiliaceae. Fungal Systematics and Evolution, 3, 353-366.

Benny GL, Smith ME, Kirk PM, Tretter ED et al. 2016 - Challenges and future perspectives in the systematics of Kickxellomycotina, Mortierellomycotina, Mucoromycotina, and Zoopagomycotina. In: Li D.-W (ed) Biology of Microfungi, Fungal Biology. Switzerland, Springer International Publishing, pp 65-126.

Bessey EA. 1950 - Morphology and taxonomy of fungi. Blakiston Company, Philadelphia, 791 p.

Beznoussenko GV, Dolgikh VV, Morzhina EV, Semenov PB et al. 2007 - Analogs of the Golgi complex in microsporidia: structure and avesicular mechanisms of function. Journal of Cell Science 120, 1288-1298.

Bigliardi E, Sacchi L. 2001 - Cell biology and invasion of the microsporidia. Microbes \& Infection 3, $373-379$.

Blackwell M, Haelewaters D, Pfister DH. 2020 - Laboulbeniomycetes: Evolution, natural history, and Thaxter's final word. Mycologia in press. doi:10.1080/00275514.2020.1718442.

Błaszkowski J, Chwat G, Góralska A, Ryszka P et al. 2015 - Two new genera, Dominikia and Kamienskia, and D. disticha sp. nov. in Glomeromycota. Nova Hedwigia 100, 225-238.

Błaszkowski J, Kozłowska A, Niezgoda P, Goto BT et al 2018a - A new genus, Oehlia with Oehlia diaphana comb. nov. and an emended description of Rhizoglomus vesiculiferum comb. nov. in the Glomeromycotina. Nova Hedwigia 107, 501-518. 
Błaszkowski J, Niezgoda P, Goto BT, Kozłowska A. 2018b - Halonatospora gen. nov. with H. pansihalos comb. nov. and Glomus bareae sp. nov. (Glomeromycota; Glomeraceae). Botany 96, 737-748.

Błaszkowski J, Niezgoda P, Paiva JN, Silva KJG et al. 2019a - Sieverdingia gen. nov., S. tortuosa comb. nov., and Diversispora peloponnesiaca sp. nov. in the Diversisporaceae (Glomeromycota). Mycological Progress 18, 1363-1382.

Błaszkowski J, Niezgoda P, Piatek M, Marguno F et al. 2019b - Rhizoglomus dalpae, R. maiae, and $R$. silesianum new species. Mycologia 1, 1-15.

Bohne W, Böttcher K, Gross U. 2011 - Theparasitophorous vacuole of Encephalitozooncuniculi: biogenesis and characteristics of the host cell-pathogen interface. International Journal of Medical Microbiology 301, 395 -399.

Boise J. 1986 - Requienellaceae, a new family of Loculoascomycetes. Mycologia 78, 37-41.

Bon M. 1986 - Novitates. Validations et taxons nouveaux. Documents Mycologiques 17, 51-56.

Boonmee S, Phookamsak R, Hongsanan S, Doilom M et al. 2017 - Mycosphere notes 51 - 101. Revision of genera in Perisporiopsidaceae and Pseudoperisporiaceae and other Ascomycota genera incertae sedis. Mycosphere 8, 1695-1801.

Braun U, Nakashima C, Crous PW, Groenewald JZ et al. 2018 -Phylogeny and taxonomy of the genus Tubakia s. lat. Fungal Systematics \& Evolution 1, 41-99.

Brefeld O. 1869 - Dictyosteliummucoroides. Einneuer Organismusaus der Verwandtschaft der Myxomyceten. Abhandl Senckenb Naturforsch Ges, 85-107.

Brown M, Silberman M. 2013 - The non-dictyostelidsorocarpic amoebae. Pages 219-242 in Dictyostelids: Evolution, Genomics and Cell Biology. Romeralo M, Baldauf S, Escalante R (Eds). Springer-Verlag, Berlin-Heidelberg.

Brown MW, Spiegel FW, Silberman JD. 2009 - Phylogeny of the "Forgotten" cellular slime mold Fonticula alba reveals a key evolutionary branch within Opisthokonta. Molecualr Biology \& Evolution 26, 2699-2709.

Buaya AT, Thines M 2020 - Diatomophthoraceae-a new family of olpidiopsis-like diatom parasitoids largely unrelated to Ectrogella. Fungal Systematics \& Evolution 5, 113-118.

Buaya AT, Ploch S, Hanic L, Nam B, Nigrelli L, Kraberg A, Thines M 2017 - Phylogeny of Miracula helgolandica gen. et sp. nov. and Olpidiopsis drebesii sp. nov., two basal oomycete parasitoids of marine diatoms, with notes on the taxonomy of Ectrogella-like species. Mycological Progress 16, 1041-1050.

Buaya AT, Ploch S, Inaba S, Thines M 2019 - Holocarpic oomycete parasitoids of red algae are not Olpidiopsis. Fungal Systematics \& Evolution 4, 21-31.

Buyck B, Duhem B, Das K, Jayawardena RS et al. 2017 - Fungal biodiversity profiles 21-30. Cryptogamie Mycologie 38, 101-146.

Čadež N, Bellora N, Ulloa R, Hittinger CT et al. 2019 - Genomic content of a novel yeast species Hanseniaspora gamundiae sp. nov. from fungal stromata (Cyttaria) associated with a unique fermented beverage in Andean Patagonia, Argentina. PLoS One 14, e0210792.

Cai L, Jeewon R, Hyde K. 2006 - Molecular systematics of Zopfiella and allied genera: evidence from multigene sequence analyses. Mycological Research 110, 359-368.

Cali A, Becnel JJ, Takvorian PM. 2017 - Microsporidia. In: Archibald JM, Simpson AGB, Slamovits CH (eds) Handbook of the Protists. Springer.pp 1569-1618.

Cali A, Takvorian PM. 2014 - Developmental morphology and life cycles of the Microsporidia. In: Weiss LM, Becnel JJ (eds) Microsporidia: pathogens of opportunity. Wiley-Blackwell Press, Ames, 71-133.

Cannon PF, Alcorn JL. 1994 - Omnidemptus affinis gen. et sp. nov., teleomorph of Mycoleptodiscus affinis sp. nov. Mycotaxon 51, 483-487

Carmarán CC, Berretta M, Martínez S, Barrera B et al. 2015 - Species diversity of Cladorrhinum in Argentina and description of a new species, Cladorrhinum australe. Mycological Progress 14, 94. 
Castellano MA, Elliott TF, Truong C, Séné O et al. 2016 - Kombocles bakaiana gen. sp. nov. (Boletaceae), a new sequestrate fungus from Cameroon. IMA fungus 7, 239-245.

Cavalier-Smith T. 1981 - Eukaryote kingdoms: seven or nine? Bio Systems. 14, 461-481.

Cavalier-Smith T. 1983 - A 6-kingdom classification and a unified phylogeny. In: Schenk HEA and Schwemmler WS (eds) Endocytobiology, W. S. Vol II. Walter de Gruyter \& Co, Berlin, pp. 1027-1034.

Cavalier-Smith T. 2018 - Kingdom Chromista and its eight phyla: a new synthesis emphasising periplastid protein targeting, cytoskeletal and periplastid evolution, and ancient divergences. Protoplasma 255, 297-357.

Cavender JC, Raper KB. 1965a - The Acrasieae in nature. I. Isolation. American Journal of Botany 52, 294-296.

Cavender JC, Raper KB. 1965b - The Acrasieae in nature. II. Forest soil as a primary habitat. American Journal of Botany 52, 297-302.

Chen CC, Wu SH, Chen CY. 2018 - Hydnophanerochaete and Odontoefibula, two new genera of phanerochaetoid fungi (Polyporales, Basidiomycota) from East Asia. MycoKeys 39, 75-96.

Chen GQ, Zheng RY 1986 - A new species of Mucor with giant spores. Acta Mycologica Sinica, Supplement I 1, 56-60.

Choi Y-J, Thines M 2015 - u Host jumps and radiation, not co - divergence drives diversification of obligate pathogens. A case study in downy mildews and Asteraceae. PloS ONE 10, $\mathrm{e} 0133655$.

Choudhary MM, Metcalfe MG, Arrambide K, Bern C et al. 2011 - Tubulinosema sp. microsporidian myositis in immunosuppressed patient. Emerging Infectious Diseases 17, 1727-1730.

Clarke RT 1965 - Fungal spores from Vermejo Formation coal beds (Upper Cretaceous) of central Colorado. Mountain Geologist 2, 85-93.

Cookson IC 1947 - Fossil fungi from Tertiary deposits in the southern hemisphere. Part I. Proceedings of the Linnean Society of New South Wales 72, 207-214.

Corazon-Guivin MA, Cerna-Mendoza A, Guerrero-Abad JC, Vallejos-Tapullima A et al. 2019 Microkamienskia gen. nov. and Microkamienskia peruviana, a new arbuscular mycorrhizal fungus from Western Amazonia. Nova Hedwigia 109, 355-368

Corazon-Guivin MA, Mendoza AC, Guerrero-Abad JC, Vallejos-Tapullima A et al. 2019a Funneliglomus, gen. nov., and Funneliglomus sanmartinensis, a new arbuscular mycorrhizal fungus from the Amazonia region in Peru. Sydowia 71 E-Book/S 17-24.

Corazon-Guivin MA, Mendoza AC, Guerrero-Abad JC, Vallejos-Tapullima A et al. 2019b Nanoglomus plukenetiae, a new fungus from Peru, and a key to small-spored Glomeraceae species, including three new genera in the "Dominikia complex/clades". Mycological Progress 18, 1395-1409.

Corsaro D, Michel R, Walochnik J, Venditti D et al. 2016 - Molecular identification of Nucleophaga terricolae sp. nov. (Rozellomycota), and new insights on the origin of the Microsporidia. Parasitology Research 115, 3003-3011.

Corsaro D, Walochnik J, Venditti D, Hauröder B et al. 2020 - Solving an old enigma: Morellospora saccamoebae gen. nov., sp. nov. (Rozellomycota), a Sphaerita-like parasite of free-living amoebae. Parasitology Research 119, 925-934.

Corsaro D, Walochnik J, Venditti D, Müller KD et al 2014a - Rediscovery of Nucleophaga amoebae, a novel member of the Rozellomycota. Parasitology Research 113, 4491-4498.

Corsaro D, Walochnik J, Venditti D, Steinmann J et al. 2014b - Microsporidia-like parasites of amoebae belong to the early fungal lineage Rozellomycota. Parasitology Research 113, 1909-1918.

Corsaro D, Wylezich C, Venditti D, Michel R et al. 2019 - Filling gaps in the microsporidian tree: rDNA phylogeny of Chytridiopsis typographi (Microsporidia: Chytridiopsida). Parasitology Research 118, 169-180.

Crivelli PG. 1983 - Über die heterogene Ascomycetengattung Pleospora Rabh. 1-213. 
Crivelli PG. 1983 - Ueber die heterogene Ascomycetengattung Pleospora Rabh. Vorschlag für eine Aufteilung, Zürich. Diss. ETH Nr. 7318. pp 1-215.

Crous PW, Braun U, Schubert K, Groenewald JZ. 2007 - Delimiting Cladosporium from morphologically similar genera. Studies in Mycology 58, 33-56.

Crous PW, Carris LM, Giraldo A, Groenewald JZ, Hawksworth DL et al. 2015c - The Genera of Fungi - fixing the application of the type species of generic names - G 2: Allantophomopsis, Latorua, Macrodiplodiopsis, Macrohilum, Milospium, Protostegia, Pyricularia, Robillarda, Rotula, Septoriella, Torula, and Wojnowicia. IMA fungus 1, 163-198.

Crous PW, Groenewald JZ 2016 - They seldom occur alone. Fungal Biology 120, 1392-1415.

Crous PW, Müller MM, Sánchez RM, Giordano L et al. 2015a - Resolving Tiarosporella spp. allied to Botryosphaeriaceae and Phacidiaceae. Phytotaxa. 202, 73-93.

Crous PW, Wingfield MJ, Burgess TI, Carnegie AJ et al. 2017 - Fungal Planet description sheets: 625 -715. Persoonia 39, 270-467.

Crous PW, Wingfield MJ, Burgess TI, Hardy GESt et al. 2016 - Fungal Planet description sheets: 469-557. Persoonia 37, 218-403.

Crous PW, Wingfield MJ, Burgess TI, Hardy GEStJ, Gené, J, Guarro J et al. 2018 - Fungal Planet description sheets: 716 - 784. Persoonia 40, 240-393.

Crous PW, Wingfield MJ, Guarro J, Hernández-Restrepo M et al. 2015b - Fungal Planet description sheets: 320-370. Persoonia 34, 167-266.

Cui BK, Li HJ, Dai YC. 2011b - Wood-rotting fungi in eastern China 6. Two new species of Antrodia (Basidiomycota) from Mt. Huangshan, Anhui Province. Mycotaxon 116, 13-20.

Cuomo CA, Desjardins CA, Bakowski MA, Goldberg J et al. 2012 - Microsporidian genome analysis reveals evolutionary strategies for obligate intracellular growth. Genome Research 22, 2478-2488.

da Silva M, Pinho DB, Pereira OL, Fernandes FM, Barreto RW. 2016 -Naming potentially endangered parasites: foliicolous mycobiota of Dimorphandra wilsonii, a highly threatened Brazilian tree species. PloS one 11, e0147895.

Dai DQ, Tang LZ, Liu C, Wang HB, Hyde KD. 2018 - Studies on Parmulariaceae I. A phylogeny based on available sequence data; introducing Parmulariales ord. nov., and Hemigraphaceae, Melaspileellaceae and Stictographaceae fam. nov. Phytotaxa 369, 63-79.

Daniel HM, Lachance MA, Kurtzman CP. 2014 - On the reclassification of species assigned to Candida and other anamorphic ascomycetous yeast genera based on phylogenetic circumscription. Antonie Leeuwenhoek 106, 67-84.

Daranagama DA, Hyde KD, Sir EB, Thambugala KM et al. 2018 - Towards a natural classification and backbone tree for Graphostromataceae, Hypoxylaceae, Lopadostomataceae and Xylariaceae. Fungal Diversity 88, 1-65.

Daru BH, Bowman EA, Pfister DH, Arnold AE. 2018 - A novel proof of concept for capturing the diversity of endophytic fungi preserved in herbarium specimens. Philosophical Transactions of the Royal Society of London Series B - Biological Sciences 374, 20170395.

Datlof EM, Amend AS, Earl K, Hayward J et al. 2017 - Uncovering unseen fungal diversity from plant DNA banks. PeerJ 5, e3730.

Davydov EA, Peršoh D, Rambold G. 2017 - Umbilicariaceae (lichenized Ascomycota)-Trait evolution and a new generic concept. Taxon 66, 1282-1303.

Dayarathne MC, Maharachchikumbura SSN, Jones EBG, Dong W et al. 2019 - Phylogenetic revision of Savoryellaceae and evidence for its ranking as a subclass. Frontiers in Microbiology 10, 840.

de Bary A. 1887 - Comparative morphology and biology of the fungi mycetozoa and bacteria Clarendon Press, Oxford.

de Hoog GS, Guarro J, Gené J, Figueras MJ. 2015 - Atlas of clinical fungi, $3^{\text {rd }}$ web edition. Utrecht, CBS-Fungal Biodiversity Centre.

De Kesel A, Haelewaters D. 2019 - Laboulbeniales (Fungi, Ascomycota) of cholevine beetles (Coleoptera, Leiodidae) in Belgium and the Netherlands. Sterbeeckia 35, 60-66. 
De Lima CLF, Lima DX, De Souza CAF, De Oliveira RJV et al. 2018 - Description of Mucor pernambucoensis (Mucorales, Mucoromycota), a new species isolated from the Brazilian Upland Rainforest. Phytotaxa 350, 274-282.

De Long Q, Liu LL, Zhang X, Wen TC et al. 2019 - Contributions to species of Xylariales in China-1. Durotheca species. Mycological Progress 18, 495-510.

De Souza CAF, Voig K, Gurgel LS, Cordeiro TRL et al 2018 - A new species of Mucor (Mucoromycotina, Mucorales) isolated from an enclave of Upland Atlantic Forest in the semi-arid region of Brazil. Phytotaxa 351, 53-62.

Delbac F, Polonais V. 2008 - The microsporidian polar tube and its role in invasion. Subcellular Biochemistry 47, 208-220.

Delgado G, Miller AN, Piepenbring M. 2018 - South Florida microfungi: Castanedospora, a new genus to accommodate Sporidesmium pachyanthicola (Capnodiales, Ascomycota). Cryptogamie, Mycologie 39, 109-128.

Desirò A, Rimington WR, Jacob A, Vande Pol N et al. 2017 - Multigene phylogeny of Endogonales, an early diverging lineage of fungi associated with plants. IMA fungus 8 , 245-257.

Desjardin D, Binder M, Roekring S, Flegel T. 2009 - Spongiforma, a new genus of gastroid boletes from Thailand. Fungal Diversity 37, 1-8.

Devadatha B, Mehta N, Wanasinghe DN, Baghela A et al. 2019 - Vittaliana mangrovei Devadatha, Nikita, A. Baghela \& VV Sarma, gen. nov, sp. nov. (Phaeosphaeriaceae), from mangroves near Pondicherry (India), based on morphology and multigene phylogeny. Cryptogamie Mycologie 40, 117-132

Dick MW 2001 - Straminipilous Fungi: Systematics of the Peronosporomycetes Including Accounts of the Marine Straminipilous Protists, the Plasmodiophorids and Similar Organisms. Dordrecht / Boston / London: Kluwer Academic Publishers.

Didier ES, Stovall ME, Green LC, Brindley PJ et al. 2004 - Epidemiology of microsporidiosis: sources and modes of transmission. Veterinary Parasitology 126, 145-166.

Diederich P, Lawrey JD, Ertz D. 2018 - The 2018 classification and checklist of lichenicolous fungi, with 2000 non-lichenized, obligately lichenicolous taxa. Bryologist 121, 340-425.

Dilcher DL. 1965 - Epiphyllous fungi from Eocene deposits in western Tennessee, U.S.A. Palaeontographica Abt B 116, 1-54.

Dong W, Hyde KD, Bhat DJ, Zhang H. 2018 - Introducing Aculeata aquatica, gen. et sp. nov., Minimelanolocus thailandensis sp. nov. and Thysanorea aquatica sp. nov. (Herpotrichiellaceae, Chaetothyriales) from freshwater in northern Thailand. Mycological Progress 17, 617-629.

Dougoud R. 2012 - Aeruginoscyphus, un nouveau genre pour Peziza sericea (Helotiales, Hyaloscyphaceae). Ascomycete.org 4, 1-4.

Doveri F. 2005 - Fungi Fimicoli Italici.1104 pp. Trento: Assozciazione Micoligica Bresadola.

Dukik K, Muñoz JF, Jiang Y, Feng P et al. 2017 - Novel taxa of thermally dimorphic systemic pathogens in the Ajellomycetaceae (Onygenales). Mycoses 60, 296-309.

Dumont KP. 1975 - Sclerotiniaceae VIII. The generic name Pachydisca, Mycologia 67, 161-167.

Dunn AM, Smith JE. 2001 - Microsporidian life cycles and diversity: the relationship between virulence and transmission. Microbes and Infection 3, 381-388.

Edwards WN. 1922 - An Eocene microthyriaceous fungus from Mull, Scotland. Transactions of the British Mycological Society 8, 66-72.

Ekanayaka AH, Hyde KD, Gentekaki E, McKenzie EHC et al. 2019 - Preliminary classification of Leotiomycetes. Mycosphere 10, 310-489.

Ekanayaka AH, Hyde KD, Jones EBG, Zhao QI. 2018 - Taxonomy and phylogeny of operculate discomycetes: Pezizomycetes. Fungal Diversity 90, 161-243.

Ellis MB. 1971 - Dematiaceous Hyphomycetes. Commonwealth Mycological Institute, Kew, England, 608 p. 
Elsik WC. 1968 - Palynology of a paleocene Rockdale lignite, Milam County, Texas. I. Morphology and taxonomy. Pollen Spores 10, 263-314.

Elsik WC. 1978 - Classification and geologic history of the microthyriaceous fungi. Proceedings of the Fourth International Palynological Conference, Lucknow 1976-77 volume 1, 331342.

Elsik WC. 1990 - Hypoxylonites and Spirotremesporites, form genera for Eocene to Pleistocene fungal spores bearing a single furrow. Palaeontographica Abt. B 216, 137-169.

Eriksson O. 1984 - Outline of the Ascomycetes 1983 - Systema Ascomycetum 3, 1-72.

Ertz D, Poulsen RS, Charrier M, Søchting U. 2017a - Taxonomy and phylogeny of the genus Steinera (Arctomiales, Arctomiaceae) in the subantarctic islands of Crozet and Kerguelen. Phytotaxa 324, 201-238.

Ertz D, Sanderson N, Łubek A, Kukwa M. 2018 - Two new species of Arthoniaceae from oldgrowth European forests, Arthonia thoriana and Inoderma sorediatum, and a new genus for Schismatomma niveum. Lichenologist 50, 161-72.

Ertz D, Søchting U, Gadea A, Charrier M et al. 2017b - Ducatina umbilicata gen. et sp. nov., a remarkable Trapeliaceae from the subantarctic islands in the Indian Ocean. Lichenologist 49, 127-40.

Ethridge Glass DL, Brown DD, Elsik WC. 1986 - Fungal spores from the Upper Eocene Manning Formation, Jackson Group, east and south-central Texas, U.S.A. Pollen Spores 28, 403420.

Fan XD, Bezerra JDP, Tian CM, Crous PW. 2018a - Families and genera of diaporthalean fungi associated with canker and dieback of tree hosts. Persoonia 40, 119-134.

Fan XD, Du Z, Bezerra JDP, Tian CM. 2018b - Taxonomic circumscription of melanconis-like fungi causing canker disease in China. Mycokeys 42, 89-124.

Fiore-Donno AM, Clissmann F, Meyer M, Schnittler M et al. 2013 - Two-gene phylogeny of bright-spored Myxomycetes (slime moulds superorder Lucisporidia). PLoS One 8, e62586.

Fiore-Donno AM, Kamono A, Meyer M, Schnittler M et al. 2012 - 18S rDNA phylogeny of Lamproderma and allied genera (Stemonitales Myxomycetes Amoebozoa). PLoS One 7, e35359.

Fiuza PO, Silva CR, Borges Santos TA, Raja H et al. 2018 - Roselymyces, a new asexual genus of the Xylariales (Ascomycota) from Brazil. Sydowia 70, 59-65.

Fokin SI, Giuseppe GDi, Erra F, Dini F. 2008 - Euplotespora binucleata n. gen., n. sp. (Protozoa: Microsporidia), a parasite infecting the hypotrichous ciliate Euplotes woodruffi, with observations on microsporidian infections in Ciliophora. Journal of Eukaryotic Microbiology 55, 214-228.

Franzen C, Nassonova ES, Scholmerich J, Issi IV. 2006 - Transfer of the members of the genus Brachiola (Microsporidia) to the genus Anncaliia based on ultrastructural and molecular data. Journal of Eukaryotic Microbiology 53, 26-35.

Franzen C. 2004 - Microsporidia: how can they invade other cells? Trends in Parasitology 20, 275-279.

Frisch A, Thor G, Moon KH, Ohmura Y. 2018 - Galbinothrix, a new monotypic genus of Chrysotrichaceae (Arthoniomycetes) lacking pulvinic acid derivatives. Plant \& Fungal Systematics 63, 31-37.

Fryar SC, Haelewaters D, Catcheside DEA. 2019 - Annabella australiensis gen. \& sp. nov. (Helotiales, Cordieritidaceae) from South Australian mangroves. Mycological Progress 18, 973-981.

Fryday AM, Printzen C, Ekman S. 2014 - Bryobilimbia, a new generic name for Lecidea hypnorum and closely related species. Lichenologist 46, 25-37.

Galindo LJ, Torruella G, Moreira D, Timpano H et al. 2018 - Evolutionary genomics of Metchnikovella incurvata (Metchnikovellidae): An Early Branching Microsporidium. Genome Biology \& Evolution 10, 2736-2748. 
Gams W. 2000 - Phialophora and some similar morphologically little-differentiated anamorphs of divergent ascomycetes. Studies in Mycology 45, 187-199.

Gannibal PhB, Lawrence DP. 2018 - Distribution of Alternaria species among sections. 6. Species formerly assigned to genus Ulocladium. Mycotaxon 133, 293-299.

Gannibal PhB. 2018 - Distribution of Alternaria species among sections. 4. Species formerly assigned to genus Nimbya. Mycotaxon 133, 37-43.

Gerdemann JW, Trappe JM. 1974 - The Endogonaceae of the Pacific Northwest. Micologia Memoir 5, 1-76

Ghani IA, Dieng H, Abu Hassan ZA, Ramli N et al. 2013 - Pathogenicity of a microsporidium isolate from the Diamondback moth against Noctuid moths: characterization and implications formicrobiological pest management. PLoS One 8, e81642.

Gherbawy Y, Kesselboth C, Elhariry H, Hoffmann K. 2010 - Molecular barcoding of microscopic fungi with emphasis on the mucoralean genera Mucor and Rhizopus. In: Gherbawy Y, Voight K (eds) Molecular identification of fungi. Springer-Verlag, Berlin Heidelberg, pp 225-265.

Gill EE, Fast NM. 2006 - Assessing the microsporidia-fungi relationship: Combined phylogenetic analysis of eight genes. Gene 375, 103-109.

Gilman JC. 1959 - A manual of soil fungi. Constable and Company Ltd, London.

Goldmann L, Weir A. 2018 - Molecular phylogeny of the Laboulbeniomycetes (Ascomycota). Fungal Biology 122, 87-100.

Gonçalves MF, Vicente TF, Esteves AC, Alves A. 2019 - Neptunomyces aureus gen. et sp. nov. (Didymosphaeriaceae, Pleosporales) isolated from algae in Ria de Aveiro, Portugal. MycoKeys 60, 31.

Gordillo A, Decock C. 2018 - Myrothecium-like (Ascomycota, Hypocreales) species from tropical areas: Digitiseta gen. nov. and additions to Inaequalispora and Parvothecium. Mycological Progress 17, 179-190.

Goto BT, Silva GA, Assis DMA, Silva DK et al. 2012 - Intraornatosporaceae (Gigasporales), a new family with two new genera and two new species. Mycotaxon 119, 117-132.

Gräfenhan T, Schroers H-J, Nirenberg HI, Seifert KA. 2011 - An overview of the taxonomy, phylogeny, and typification of nectriaceous fungi in Cosmospora, Acremonium, Fusarium, Stilbella, and Volutella. Studies in Mycology 68, 79-113.

Grube M, Matzer M, Hafellner J. 1995 - A preliminary account of the lichenicolous Arthonia species with reddish, K+ reactive pigments. Lichenologist 27, 25-42.

Grünig CR, Duo A, Sieber TN, Holdenrieder O. 2008 - Assignment of species rank to six reproductively isolated cryptic species of the Phialocephala fortinii s.l.-Acephala applanata species complex. Mycologia 100, 47-67.

Guatimosim E, Schwartsburd PB, Crous PW, Barreto RW. 2016 - Novel fungi from an ancient niche: lachnoid and chalara-like fungi on ferns. Mycological Progress 15, 1239-1267.

Gulden G, Stensrud Ø, Shalchian-Tabrizi K, Kauserud H. 2005 - Galerina Earle: A polyphyletic genus in the consortium of dark-spored agarics. Mycologia 97, 823-837.

Guswenrivo I, Tseng SP, Yang CCS, Yoshimura T. 2018 - Development of multiplex nested PCR for simultaneous detection of ectoparasitic fungi Laboulbeniopsis termitarius and Antennopsis gallica on Reticulitermes speratus (Blattodea: Rhinotermitidae). Journal of Economic Entomology 111, 1330-1336.

Haag KL, James TY, Pombert JF, Larsson R et al. 2014 - Evolution of a morphological novelty occurred before genome compaction in a lineage of extreme parasites. Proceedings of the National Academy of Sciences 111, 15480-15485.

Haelewaters D, De Kesel A, Gorczak M, Bao K et al. 2019a - Laboulbeniales (Ascomycota) of the Boston Harbor Islands II: species parasitizing Carabidae, and the Laboulbenia flagellata species complex. Northeastern Naturalist 25 (Special Issue 9), 110-149.

Haelewaters D, De Kesel A, Pfister DH. 2018a - Integrative taxonomy reveals hidden species within a common fungal parasite of ladybirds. Scientific Reports 8, 15966. 
Haelewaters D, Page RA, Pfister DH. 2018b - Laboulbeniales hyperparasites (Fungi, Ascomycota) of bat flies: Independent origins and host associations. Ecology and Evolution 8, 8396-8418.

Haelewaters D, Pfliegler WP, Gorczak M, Pfister DH. 2019b - Birth of an order: comprehensive molecular phylogenetic study reveals that Herpomyces (Fungi, Laboulbeniomycetes) is not part of Laboulbeniales. Molecular Phylogenetics \& Evolution 133, 286-301.

Haelewaters D, Rossi W. 2017 - Laboulbeniales parasitic on American small carrion beetles: new species of Corethromyces, Diphymyces, and Rodaucea. Mycologia 109, 655-666.

Haelewaters D, Toome-Heller M, Albu S, Aime MC. 2020 - Red yeasts from leaf surfaces and other habitats: three new species and a new combination of Symmetrospora (Pucciniomycotina, Cystobasidiomycetes). Fungal Systematics and Evolution 5, 187-196.

Haelewaters D, Zhao SY, Clusella-Trullas S, Cottrell TE et al. 2017 - Parasites of Harmonia axyridis: current research and perspectives. Biological Control 62, 355-371.

Haelewaters D, Zhao SY, De Kesel A, Royer IR et al. 2015 - Laboulbeniales (Ascomycota) of the Boston Harbor Islands I: species parasitizing Coccinellidae and Staphylinidae. Northeastern Naturalist 22, 459-477.

Hagiwara H. 1989 - The taxonomic study of Japanese dictyostelid cellular slime molds. National Science Museum, Tokyo.

Han JG, Hosoya T, Sung GH, Shin HD. 2014 - Phylogenetic reassessment of Hyaloscyphaceae sensu lato (Helotiales, Leotiomycetes) based on multigene analyses. Fungal Biology 118, 150-167.

Han ML, Chen YY, Shen LL, Song J et al. 2016 - Taxonomy and phylogeny of the brown-rot fungi: Fomitopsis and its related genera. Fungal Diversity 80, 343-373.

Hansen K, Perry BA, Dranginis AW, Pfister DH. 2013 - A phylogeny of the highly diverse cupfungus family Pyronemataceae (Pezizomycetes, Ascomycota) clarifies relationships and evolution of selected life history traits. Molecular Phylogenetics \& Evolution 67, 311-335.

Hao YJ, Qin J, Yang ZL. 2014 - Cibaomyces, a new genus of Physalacriaceae from East Asia. Phytotaxa 162, 198-210.

Hashimoto A, Hirayama K, Takahashi H, Matsumura M et al. 2018 - Resolving the Lophiostoma bipolare complex: Generic delimitations within Lophiostomataceae. Studies in Mycology 90, 161-189.

Hawksworth DL \& Lücking R. 2017 - Fungal diversity revisited: 2.2 to 3.8 million species. Microbiology Spectrum 5, doi: 10.1128/microbiolspec.FUNK-0052-2016.

Hawksworth DL, Cole MS. 2002 - Intralichen, a new genus for lichenicolous 'Bispora' and 'Trimmatostroma' species. Fungal Diversity11, 87-97.

Hawksworth DL, Eriksson OE. 1986 - The names of accepted orders of ascomycetes. Systema Ascomycetum 5, 175-184.

Hawksworth DL, Kirk PM, Sutton BC, Pegler DN, eds. 1983 - Ainsworth \& Bisby's Dictionary of the Fungi. $7^{\text {th }}$ ed. Commonwealth Mycol Institute, Kew.

He M Q, Zhao RL, Hyde KD, Begerow D et al. 2019 - Notes, outline and divergence times of Basidiomycota. Fungal Diversity 1-263.

Healy R, Bonito G, Trappe JM. 2009 - Calongea, a new genus of truffles in the Pezizaceae (Pezizales). Anales del Jardín Botánico de Madrid. Consejo Superior de Investigaciones Científicas 66, 25-32.

Helaly SE, Thongbai B, Stadler M. 2018 - Diversity of biologically active secondary metabolites from endophytic and saprotrophic fungi of the ascomycete order Xylariales. Natural Product Reports 35, 992-1014.

Henkel TW, Aime MC, Chin MM L, Miller SL et al. 2012 - Ectomycorrhizal fugal sporocarp diversity and discovery of new taxa in Dicymbe monodominant forests of the Guiana Shield. Biodiversity \& Conservation 21, 2195-2220.

Henkel TW, Obase K, Husbands D, Uehling JK et al. 2016 - New Boletaceae taxa from Guyana: Binderoboletus segoi gen. and sp. nov., Guyanaporus albipodus gen. and sp. nov., 
Singerocomus rubriflavus gen. and sp. nov., and a new combination for Xerocomus inundabilis. Mycologia 108, 157-173.

Hermet A, Méheust D, Monunier J, Barbier G et al. 2012 - Molecular systematics in the genus Mucor with special regards to species encountered in cheese. Fungal Biology 116, 692-705.

Hernández-Restrepo M, Castañeda-Ruíz RF, Gené J, Silvera-Simón C et al. 2014 - Two new species of Solicorynespora from Spain. Mycological Progress 13, 157-164.

Heuchert B, Braun U, Diederich P, Ertz D. 2018 - Taxonomic monograph of the genus Taeniolella s. lat. (Ascomycota). Fungal Systematics \& Evolution 2, 69-261.

Hibbett D, Abarenkov K, Kõljalg U, Öpik M et al. 2016 - Sequence-based classification and identification of Fungi. Mycologia 108, 1049-1068.

Hibbett DS, Binder M, Bischoff JF, Blackwell M et al. 2007 - A higher-level phylogenetic classification of the Fungi. Mycological Research 111, 509-547.

Hinney B, Sak B, Joachim A, Kváč M. 2016 - More than a rabbit's tale - Encephalitozoon spp. in wild mammals and birds. International Journal for Parasitology: Parasites \& Wildlife 5, 7687.

Hirooka Y, Tanney JB, Nguyen HDT, Seifert KA. 2015 - Xerotolerant fungi in house dust: Taxonomy of Spiromastix, Pseudospiromastix and Sigleria gen. nov. in Spiromastigaceae (Onygenales, Eurotiomycetes). Mycologia 108, 135-156.

Hittinger CT, Rokas A, Bai FY, Boekhout T et al. 2015 - Genomics and the making of yeast biodiversity. Current Opinion in Genetics \& Development 35, 100-109.

Hjortstam K, Ryvarden L. 2010 - Phaerodontia and Phaneroites two corticioid taxa (Basidiomycotina) proposed from tropical areas. Syn Fung 27, 26-33.

Hoek C van den DG, Mann HM, Jahns. 1995 - Algae: An Introduction to Phycology. Cambridge: Cambridge University Press. pp. 104, 124, 134, 166.

Hofstetter V, Redhead SA, Kauff F, Moncalvo JM et al. 2014 - Taxonomic revision and examination of ecological transitions of the Lyophyllaceae (Basidiomycota, Agaricales) based on a multigene phylogeny. Cryptogamie Mycologie 35, 399-425.

Hongsanan S, Maharachchikumbura SS, Hyde KD, Samarakoon MC et al. 2017 - An updated phylogeny of Sordariomycetes based on phylogenetic and molecular clock evidence. Fungal Diversity 84, 25-41.

Huang SK, Tangthirasunun N, Phillips AJL, Dai DQ et al. 2016 - Morphology and phylogeny of Neoscytalidium orchidacearum sp. nov. (Botryosphaeriaceae). Mycobiology 44, 79-84.

Huhndorf SM, Miller AN, Fernández FA. 2004 - Molecular systematics of the Sordariales: the order and the family Lasiosphaeriaceae redefined. Mycologia 96, 368-387.

Huhndorf SM. 1994 - Neotropical Ascomycetes 5. Hypsostromataceae, a new family of Loculoascomycetes and Manglicola samuelsii, a new species from Guyana. Mycologia 86, 266-269.

Humber RA. 2012 - Entomophthoromycota: a new phylum and reclassification for entomophthoroid fungi. Mycotaxon 120, 477-492.

Humber RA. 2016 - Entomophthoromycota: a new overview of some of the oldest terrestrial fungi. In: Li DW (ed) Biology of microfungi. Springer, Cham, pp 127-145.

Hussein JM, Tibuhwa DD, Tibell S. 2018 - Phylogenetic position and taxonomy of Kusaghiporia usambarensis gen. et sp. nov. (Polyporales). Mycology 9, 136-144.

Hustad VP, Miller AN, Dentinger BTM, Cannon PF. 2013 - Generic circumscriptions in Geoglossomycetes. Persoonia 31, 101-111.

Hustad VP, Miller AN, Moingeon JM, Priou JP. 2011 - Inclusion of Nothomitra in Geoglossomycetes. Mycosphere 2, 646-654.

Hustad VP, Miller AN. 2015 - Maasoglossum, a basal genus in Geoglossomycetes. Mycoscience $56,572-579$.

Hutchinson SA. 1955 - A review of the genus Sporocarpon Williamson. Annals of Botany 19, 425-435. 
Hyde KD, Chaiwan N, Norphanphoun C, Boonmee S et al. 2018a - Mycosphere notes 169-224. Mycosphere 9, 271-430.

Hyde KD, Jones EBG, Liu JK, Ariyawansa H et al. 2013 - Families of Dothideomycetes. Fungal Diversity 63, 1-313.

Hyde KD, Norphanphoun C, Bazzicalupo A, Karunarathna A et al. 2017 - Fungal diversity notes 603-708: Taxonomic and phylogenetic notes on genera and species. Fungal Diversity 87, $1-$ 235.

Hyde KD, Norphanphoun C, Chen J, Dissanayake AJ et al. 2018b - Thailand's amazing diversity: up to $96 \%$ of fungi in northern Thailand may be novel. Fungal Diversity 93, 215-239.

Hyde KD, Norphanphoun C, Maharachchikumbura SSN, Bhat DJ et al. 2020 - Refined families of Sordariomycetes. Mycosphere 11, 305-1059.

Ibañez CG \& Zamuner AB. 1996 - Hyphomycetes (Deuteromycetes) in cones of Araucaria mirabilis (Spegazzini) Windhausen, Middle Jurassic of Patagonia, Argentina; Mycotaxon 59, 137-143.

Inderbitzin P, Mehta YR, Berbee ML. 2009 - Pleospora species with Stemphylium anamorphs: a four locus phylogeny resolves new lineages yet does not distinguish among species in the Pleospora herbarum clade. Mycologia 101, 329-339.

Index Fungorum (2019) http://www.indexfungorum.org/Names/Names.asp

Ironside JE, Wilkinson TJ, Rock J. 2008 - Distribution and host range of the microsporidian Pleistophora mulleri. Journal of Eukaryotic Microbiology 55, 355-362.

Isola D, Zucconi L, Onofri S, Caneva G et al. 2016 - Extremotolerant rock-inhabiting black fungi from Italian monumental sites. Fungal Diversity 76, 75-96.

Issi IV 1980 - Modern situation in systematics of Microsporidia. Proceedings of Zoological Institute 94, 75-84 (In Russian)

Issi IV, Tokarev YS, Seliverstova EV, Voronin VN. 2012a - Taxonomy of Neopereziachironomi and Neoperezia semenovaiae comb. nov. (Microsporidia, Aquasporidia): lessons from ultrastructure and ribosomal DNA sequence data. European Journal of Protistology 48, 17 29.

Issi IV, Tokarev YS, Seliverstova EV, Voronin VN. 2012b - The parasite-host interface between Crispospora chironomi (Microsporidia, Terresporidia) and Chironomus plumosus (Diptera, Chironomidae) enterocytes. Euroasian Entomological Journal 11, 395-400.

Issi IV, Tokarev YS, Voronin VN, Seliverstova EV et al. 2010 - Ultrastructure and molecular phylogeny of Mrazekia macrocyclopis sp. n. (Microsporidia, Mrazekiidae), a microsporidian parasite of Macrocyclops albidus (Jur.) (Crustacea, Copepoda). Acta Protozoologica 49, 7584.

Issi IV. 1986 - Microsporidia as a phylum of parasitic protozoa. In: Beyer TV and Issi IV (eds.), Protozoology 10. Leningrad: Nauka, pp 1-136.

Iturrieta-González I, Gené J, Guarro J, Castañeda-Ruiz RF, García D. 2018 - Neodendryphiella, a novel genus of the Dictyosporiaceae (Pleosporales). MycoKeys 37, 19-38.

Jacobs K, Botha A. 2008 - Mucor renisporus sp. nov., a new coprophilous species from Southern Africa. Fungal Diversity 29, 27-35.

Jain KP, Gupta RC. 1970 - Some fungal remains from the Tertiaries of Kerala Coast. The Palaeobotanist 18, 177-182.

Jaklitsch WM, Baral HO, Lücking R, Lumbsch HT. 2016a - Ascomycota. In: Frey W (ed) Syllabus of Plant Families - Adolf Engler's Syllabus der Pflanzenfamilien, 13th ed. Borntraeger, Stuttgart

Jaklitsch WM, Checa J, Blanco MN, Olariaga I et al. 2018 - A preliminary account of the Cucurbitariaceae. Studies in Mycology 90, 71-118.

Jaklitsch WM, Gardiennet A, Voglmayr H. 2016b - Resolution of morphology-based taxonomic delusions: Acrocordiella, Basiseptospora, Blogiascospora, Clypeosphaeria, Hymenopleella, Lepteutypa, Pseudapiospora, Requienella, Seiridium and Strickeria. Persoonia 37, 82-105. 
Jaklitsch WM, Voglmayr H. 2019 -European species of Dendrostoma (Diaporthales). MycoKeys 59, 1-26.

James TY, Kauff F, Schoch CL, Matheny P et al. 2006 - Reconstructing the early evolution of Fungi using a six-gene phylogeny. Nature 443, 818-822.

James TY, Pelin A, Bonen L, Ahrendt S et al. 2013 - Shared signatures of parasitism and phylogenomics unite Cryptomycota and microsporidia. Current Biology 23, 1548-1553.

Jansonius J, Hills LV. 1976 - Genera file of fossil spores. Special Publication, Department of Geology, University of Calgary.

Jiang HB, Hyde KD, Jayawardena RS, Doilom M et al. 2019a - Taxonomic and phylogenetic characterizations reveal two new species and two new records of Roussoella (Roussoellaceae, Pleosporales) from Yunnan, China. Mycol Prog 18, 577-591.

Jiang N, Fan XL, Crous PW, Tian CM. 2019b - Species of Dendrostoma (Erythrogloeaceae, Diaporthales) associated with chestnut and oak canker diseases in China. MycoKeys 48, 67-96.

Jiang N, Yang Q, Liang YM, Tian CM. 2019c - Taxonomy of two synnematal fungal species from Rhus chinensis, with Flavignomonia gen. nov. described. MycoKeys 60, 17-29.

Jiang SH, Hawksworth DL, Lücking R, Wei JC 2020 - A new genus and species of foliicolous lichen in a new family of Strigulales (Ascomycota: Dothideomycetes) reveals remarkable class-level homoplasy. IMA fungus 11, 1-13.

Jobim K, Błaszkowski J, Niezgoda P, Kowsloska A et al. 2019 - New sporocarpic taxa in the phylum Glomeromycota: Sclerocarpum amazonicum gen. et sp. nov. in the family Glomeraceae (Glomerales) and Diversispora sporocarpia sp. nov. in Diversisporaceae (Diversisporales). Mycological Progress 18, 369-384.

Johnston PR, Park D. 2019 - New species of Marthamyces and Ramomarthamyces gen. nov. from New Zealand and the Cook Islands. Mycotaxon 134, 489-516.

Johnston PR, Quijada L, Smith CA, Baral HO, Hosoya T et al. 2019 - A multigene phylogeny toward a new phylogenetic classification for the Leotiomycetes. IMA Fungus 10, 1.

Jones EBG, Sakayaroj J, Suetrong S, Somrithipol S et al. 2009 - Classification of marine Ascomycota, anamorphic taxa and Basidiomycota. Fungal Diversity 35, 1-187.

Jones MDM, Forn L, Gadelha C, Egan MJ et al. 2011 - Discovery of novel intermediate forms redefines the fungal tree of life. Nature 474, 200-203.

Joseph S, Sinha GP, Ramachandran VS. 2018 - Taxonomic revision of the lichen genus Opegrapha sense lato (Roccellaceae) in India. Indian Journal of Forestry Additional Series $6,1-172$.

Jung PE, Lee H, Wu SH, Hattori T et al. 2018 - Revision of the taxonomic status of the genus Gloeoporus (Polyporales, Basidiomycota) reveals two new species. Mycological Progress 17, 855-863.

Justo A, Miettinen O, Floudas D, Ortiz-Santana B et al. 2017 - A revised family-level classification of the Polyporales (Basidiomycota). Fungal Biology 121, 798-824.

Kalgutkar RM, Jansonius J. 2000 - Synopsis of fungal spores, mycelia and fructifications. AASP Contribution Series 39, 1-423.

Kalgutkar RM, Nambudiri EMV, Tidwell WD. 1993 - Diplodites sweetii sp. nov. from the Late Cretaceous (Maastrichtian) Deccan Intertrappean beds of India. Review of Palaeobotany and Palynology 77, 107-118.

Kalgutkar RM. 1997 - Fossil fungi from the lower Tertiary Iceberg Bay Formation, Eureka Sound Group, Axel Heiberg Island, Northwest Territories, Canada. Review of Palaeobotany and Palynology 97, 197-226.

Kang S, Tice AK, Spiegel FW, Silberman JD et al. 2017 - Between a pod and a hard test: The deep evolution of Amoebae. Molecular Biology and Evolution 34, 2258-2270.

Karakehian JM, Quijada L, Friebes G, Tanney JB et al. 2019 - Placement of Triblidiaceae in Rhytismatales and comments on unique ascospore morphologies in Leotiomycetes (Fungi, Ascomycota). Mycokeys 54, 99-133. 
Karpov SA, Mamkaeva MA, Aleoshin VV, Nassonova E et al. 2014 - Morphology, phylogeny, and ecology of the aphelids (Aphelidea, Opisthokonta) and proposal for the new superphylum Opisthosporidia. Frontiers in Microbiology 5, 112.

Karunarathna A, Phookamsak R, Jayawardena RS, Cheewangkoon R et al. 2019 - Neoroussoella alishanense sp. nov on Pennisetum purpureum (Poaceae) with an asexual/sexual morph connection. Phytotaxa 406, 218-236.

Keane R, Berlemann J. 2016 - The predatory life cycle of Myxococcusxanthus. Microbiol 162, 111.

Keeling PJ, Fast NM, Corradi N. 2014 - Microsporidian genome structure and function. In: Weiss LM, Becnel JJ (eds) Microsporidia: pathogens of opportunity. Wiley-Blackwell Press, Ames, pp 221-229

Keeling PJ, Luker MA, Palmer JD. 2000 - Evidence from beta-tubulin phylogeny that microsporidia evolved from within the fungi. Mol Biol Evol 17, 23-31.

Kendrick WB, Carmichael JW. 1973 - Hyphomycetes. in Ainsworth GC, Sparrow FK \& Sussman AA (eds.) The Fungi. An Advanced Treatise, volume 4A, Academic Press, New York: 323509.

Kendrick WB. 1958 - Helicoma monospora sp. nov. from pine litter. Transactions of the British Mycological Society 41, 446-448.

Kiran M, Sattar A, Zamir K, Haelewaters D et al. 2020 - Additions to the genus Chroogomphus (Boletales, Gomphidiaceae) from Pakistan. MycoKeys 66, 23-38.

Kirk PM, Cannon PF, Minter DW, Stalpers JA. 2008 - Dictionary of the Fungi. $\left(10^{\text {th }}\right.$ edn). Wallingford, UK.

Kirk PM, Stalpers JA, Braun U, Crous PW et al. 2013 - A without prejudice list of generic names of fungi for protection under the International Code of Nomenclature for algae, fungi, and plants. IMA Fungus 4, 381-443.

Kiss L, Kovács GM, Bóka K, Bohár G et al. 2018 - Deciphering the biology of Cryptophyllachora eurasiatica gen. et sp. nov., an often cryptic pathogen of an allergenic weed, Ambrosia artemisiifolia. Scientific Reports 8, 10806.

Kistenich S, Timdal E, Bendiksby M, Ekman S. 2018 - Molecular systematics and character evolution in the lichen family Ramalinaceae (Ascomycota: Lecanorales). Taxon 67, 871904.

Kodsueb R, Dhanasekaran V, Aptroot A, Lumyong S et al. 2006 - The family Pleosporaceae: intergeneric relationships and phylogenetic perspectives based on sequence analyses of partial 28S rDNA. Mycologia 98, 571-583.

Kohout P, Sudová R, Janoušková M, Čtvrtlíková M et al. 2014 - Comparison of commonly used primer sets for evaluating arbuscular mycorrhizal fungal communities: Is there a universal solution? Soil Biology \& Biochemistry 68, 482-493.

Košuthová A, Westberg M, Otálora MAG, Wedin M. 2019 - Rostania revised: testing generic delimitations in Collemataceae (Peltigerales, Lecanoromycetes). MycoKeys 47, 17-33.

Kraichak E, Huang JP, Nelsen M, Leavitt SD et al. 2018a - A revised classification of orders and families in the two major subclasses of Lecanoromycetes (Ascomycota) based on a temporal approach. Botanical Journal of the Linnean Society 188, 233-249.

Kraichak E, Huang JP, Nelsen M, Leavitt SD et al. 2018b - Nomenclatural novelties. Index Fungorum 375, 1-1.

Kumar LM, Smith ME, Nouhra ER, Orihara T et al. 2017 - A molecular and morphological reexamination of the generic limits of truffles in the tarzetta-geopyxis lineage - Densocarpa, Hydnocystis, and Paurocotylis. Fungal Biology 121, 264-284.

Kumar P. 1990 - Fungal remains from the Miocene Quilon beds of Kerala State, South India. Review of Palaeobotany and Palynology 63, 13-28.

Kurtzman CP, Boekhout T. 2017 - Yeasts as distinct life forms of fungi. In: Buzzini P, Lachance MA, Yurkov A (eds) Yeasts in natural ecosystems: Ecology. Springer International, Cham, pp 1-37. 
Kurtzman CP. 2011 - Discussion of teleomorphic and anamorphic ascomycetous yeasts and yeast-like Taxa. In: Kurtzman CP, Fell JW, Boekhout T (eds) The yeasts - A taxonomic study, $5^{\text {th }}$ edn. Elsevier Science, Burlington, pp 293-307.

Kušan I, Matočec N, Jadan M, Tkalčec Z et al. 2018 - An overview of the genus Coprotus (Pezizales, Ascomycota) with notes on the type species and description of $C$. epithecioides sp, nov. Mycokeys 29, 15-47.

Kušan I, Matočec N, Mešić A, Tkalčec Z. 2015 - A new species of Thecotheus from Croatia with a key to the known species with apiculate spores. Sydowia 67, 51-63.

Labbé A. 1899 - Microsporidiida. In: Bütschli O, Ed Das Tierreich Vol 5 Sporozoa, Berlin, Germany: Friedländer und Sohn, pp. 104-112.

Lakhanpal RN, Maheshwari HK, Awasthi N. 1976 - A Catalogue of Indian Fossil Plants. Birbal Sahni Institute of Palaeobotany, Lucknow, India. pp. 1-318.

Lambert C, Wendt L, Hladki AI, Stadler M et al. 2019 - Hypomontagnella (Hypoxylaceae): a new genus segregated from Hypoxylon by a polyphasic taxonomic approach. Mycological Progress 18, 187-201.

Lang PL, Willems FM, Scheepens JF, Burbano HA et al. 2019 - Using herbaria to study global environmental change. New Phytologist 221, 110-122.

Larsson JIR 2014 - The primitive microsporidia. In: Weiss LM and Becnel JJ (eds.), Microsporidia pathogens of opportunity. John Wiley \& Sons, Inc., Ames, Iowa, pp 605-634.

Lechat C, Fournier J, Moreau PA. 2016b - Xanthonectria, a new genus for the nectrioid fungus Nectria pseudopeziza. Ascomycete.org 8, 172-178.

Lechat C, Fournier J, Priou JP. 2018a - Chrysonectria, a new genus in the Nectriaceae with the new species $C$. finisterrensis from France. Ascomycete.org 10, 121-125.

Lechat C, Fournier J, Vega M, Priou J-P 2018b - Geonectria, a new genus in the Bionectriaceae from France. Ascomycete.org 10, 81-85.

Lechat C, Fournier J. 2016 - Varicosporellopsis, a new aquatic genus from southern France. Ascomycete.org 8, 96-100.

Lechat C, Fournier J. 2016a - Lasionectriella, a new genus in the Bionectriaceae, with two new species from France and Spain. Ascomycete.org 8, 59-65.

Leedale GF 1974 - How many are the kingdoms of organisms? Taxon 23, 261-270.

Lee SC, Heitman J, Ironside JE. 2014 - Sex and the Microsporidia In: Weiss LM, Becnel JJ (eds) Microsporidia: pathogens of opportunity. Wiley-Blackwell Press, Ames, 231-243.

Leontyev DV, Schnittler M, Stephenson S, NovozhilovYK et al. 2019 - Towards a phylogenetic classification of Myxomycetes. Phytotaxa 399, 209-238.

Leontyev DV, Schnittler M, Stephenson SL. 2014 - Pseudocapillitium or true capillitium? A study of capillitial structures in Alwisia bombarda (Myxomycetes). Nova Hegwigia 99, 441451.

Leontyev DV, Schnittler M. 2017 - The phylogeny of myxomycetes. Pages 83-105 in: Stephenson SL, Rojas C (Eds.) Myxomycetes. Biology, Systematics, Biogeography and Ecology. Elsevier Academic Press.

Letcher PM, Powell MJ, Davis WJ. 2018 - Morphology, zoospore ultrastructure, and molecular position of taxa in the Asterophlyctis lineage (Chytridiales, Chytridiomycota). Fungal Biology 122, 1109-1123.

Letcher PM, Powell MJ. 2018 - A taxonomic summary and revision of Rozella (Cryptomycota). IMA fungus 9, 383-399.

Li GJ, Hyde KD, Zhao RL, Hongsanan S et al. 2016 - Fungal diversity notes 253-366: taxonomic and phylogenetic contributions to fungal taxa. Fungal Diversity77, 1-237.

Li Y, Xiao J, de Hoog GS, Wang X et al. 2017 - Biodiversity and human pathogenicity of Phialophora verrucosa and relatives in Chaetothyriales. Persoonia 38, 1-19.

Li YM, Shivas RG, Cai L. 2017 - Cryptic diversity in Tranzscheliella spp. (Ustilaginales) is driven by host switches. Scientific Reports 7, 43549. 
Lima DX, Souza-Motta CM, Wagner L, Voigt K et al. 2017 - Circinella simplex - a misapplied name of Mucor circinatus sp. nov. Phytotaxa 329, 269-276.

Lin CC, Aronson JM 1970 - Chitin and cellulose in the cell walls of the oomycete, Apodachlya sp. Archiv für Mikrobiologie, 72, 111-114.

Linaldeddu BT, Alves A, Phillips AJL. 2016 - Sardiniella urbana gen. et sp. nov., a new member of the Botryosphaeriaceae isolated from declining Celtis australis trees in Sardinian streetscapes. Mycosphere 7, 893-905.

Lindemann U, Vega M, Alvarado P. 2015 - Revision der Gattung Kotlabaea: K. deformis, K. delectans und K. Benkertii. Zeitschrift für Mykologie 81, 373-402.

Lister A 1894 - A monograph of the Mycetozoa. British Museum London.

Liu F, Bonthond G, Groenewald JZ, Cai L et al. 2019 - Sporocadaceae, a family of coelomycetous fungi with appendage-bearing conidia. Studies in Mycology 92, 287-415.

Liu JK, Hyde KD, Jeewon R, Phillips AJL et al. 2017 - Ranking higher taxa using divergence times: a case study in Dothideomycetes. Fungal Diversity 84, 75-99.

Liu NG, Hyde KD, Bhat DJ, Jumpathong J, Liu JK. 2019 - Morphological and phylogenetic studies of Pleopunctum gen. nov. (Phaeoseptaceae, Pleosporales) from China. Mycosphere 10, 757-775.

Liu NG, Lin CG, Liu JK, Samarakoon MC et al. 2018a - Lentimurisporaceae, a new Pleosporalean family with divergence times estimates. Cryptogamie, Mycologie 39, 259283.

Liu SL, Nakasone KK, Wu SH, He SH, Dai YC. 2018b - Taxonomy and phylogeny of Lopharia s.s., Dendrodontia, Dentocorticium and Fuscocerrena (Basidiomycota, Polyporales). MycoKeys 32, 25-48.

Liu XZ, Wang Q M, Göker M et al. 2015 - Towards an integrated phylogenetic classification of the Tremellomycetes. Studies in Mycology 81, 85-147.

Lücking R, Dal-Forn, M, Sikaroodi M, Gillevet PM et al. 2014 - A single macrolichen constitutes hundreds of unrecognized species. Proceedings of the National Academy of Sciences of the USA 111, 11091-11096.

Lücking R, Hodkinson BP, Leavitt SD. 2017 - The 2016 classification of lichenized fungi in the Ascomycota and Basidiomycota-Approaching one thousand genera. Bryologist 119, 361417.

Lücking R, Kalb K. 2018 - Formal instatement of Allographa (Graphidaceae): How to deal with a hyperdiverse genus complex with cryptic differentiation and paucity of molecular data. Herzogia 31, 535-561.

Lücking R, Moncada B, Hawksworth DL. 2019 - Gone with the wind: sequencing its type species supports inclusion of Cryptolechia in Gyalecta (Ostropales: Gyalectaceae). Lichenologist 51, 287-299.

Lücking R, Tehler A, Bungartz F, Rivas Plata E et al. 2013 - Journey from the West: did tropical Graphidaceae (lichenized Ascomycota: Ostropales) evolve from a saxicolous ancestor along the American Pacific coast?. American Journal of Botany 100, 844-56.

Lumbsch HT, Huhndorf SM 2010 - Outline of Ascomycota - 2009. Myconet 14, 1-64.

Ma X, Zhao CL. 2019 - Crepatura ellipsospora gen. et sp. nov. in Phanerochaetaceae (Polyporales, Basidiomycota) bearing a tuberculate hymenial surface. Mycological Progress $18,785-793$

Ma YR, Xia JW, Gao JM, Li Z et al. 2016 - Dictyoceratosporella gen. nov. with the description of two new species collected from Hainan, China. Sydowia 68, 57-61.

Madden AA, Stchigel AM, Guarro J, Sutton D et al. 2012 - Mucor nidicola sp. nov., a novel fungal species isolated from an invasive paper wasp nest. International Journal of Systematic \& Evolutionary Microbiology 62, 1710-1714.

Madrid H, Cano J, Gené J, Guarro J. 2011 - Two new species of Cladorrhinum. Mycologia 103, 795-805. 
Madrid H, Gené J, Cano J, Stchigel A et al. 2010 - Ramophialophora humicola and Fibulochlamys chilensis, two new microfungi from soil. Mycologia 102, 605-612.

Madrid H, Hernández-Restrepo M, Gené J, Cano J et al. 2016 - New and interesting chaetothyrialean fungi from Spain. Mycological Progress 15, 1179-1201.

Magyar D, Merényi Z, Udvardy O, Kajtor-Apatini D et al. 2018 - Mycoceros antennatissimus gen. et sp. nov.: a mitosporic fungus capturing pollen grains. Mycological Progress 17, 3343.

Malysh JM, Ignatieva AN, Artokhin KS, Frolov AN et al. 2018b - Natural infection of the beet webworm Loxostegesticticalis L. (Lepidoptera: Crambidae) with three Microsporidia and host switching in Nosema ceranae. Parasitology Research 117, 3039-3044.

Malysh JM, Kononchuk AG, Frolov AN 2019 - Detection of microsporidia infecting beet webworm Loxostege sticticalis (Pyraloidea: Crambidae) in European part of Russia in 2006-2008. Plant Protection News [Vestnik zashchity rasteniy] 2, 45-51.

Malysh JM, Vorontsova YL, Glupov VV, Tsarev AA, Tokarev YS. 2018a - Vairimorpha ephestiae is a synonym of Vairimorpha necatrix (Opisthosporidia: Microsporidia) based on multilocus sequence analysis. European Journal of Protistology 66, 63-67.

Malysheva V, Spirin V. 2017 - Taxonomy and phylogeny of the Auriculariales (Agaricomycetes, Basidiomycota) with stereoid basidiocarps. Fungal Biology 121, 689-715.

Mapook A, Hyde KD, McKenzie EHC, Gareth Jones EBG et al. 2020 - Taxonomic and phylogenetic contributions to fungi associated with the invasive weed Chromolaena odorata (Siam weed). Fungal Diversity https://doi.org/10.1007/s13225-020-00444-8

Marano AV, Jesus AL, de Souza JI, Jeronimo GH, Gonçalves DR, Boro MC, Rocha SCO, PiresZottarelli CLA 2016 - Ecological roles of saprotrophic Peronosporales (Oomycetes, Straminipila) in natural environments. Fungal Ecology 19, 77-88.

Marchetta A, Gerrits van den Ende B, Al-Hatmi AMS, Hagen F et al. 2018 - Global molecular diversity of the halotolerant fungus Hortaea werneckii. Life 8, 31 .

Marinho F, Silva GA, Ferreira ACA, Veras JSN et al. 2014 - Bulbospora minima, new genus and new species in the Glomeromycetes from semi-arid Northeast Brazil. Sydowia 66, 313-323.

Marmolejo J, Siahaan SAS, Takamatsu S, Braun U. 2018 - Three new records of powdery mildews found in Mexico with one genus and one new species proposed. Mycoscience 59, $1-7$.

Martin GW, Alexopoulos CJ. 1969 - The Myxomycetes. Iowa Univ Press, Iowa City.

Massee G. 1892 - A monograph of the Myxogasteres. Methuen Co, London.

Matočec N, Kušan I, Ozimec R. 2014 - The genus Polycephalomyces (Hypocreales) in the frame of monitoring Veternica cave (Croatia) with the new segregate genus Perennicordyceps. Ascomycete.org 6, 125-133.

Mehrabi M, Asgari B, Hemmati R. 2018 - Knufia perfecta, a new black yeast from Iran, and a key to Knufia species. Nova Hedwigia 106, 519-534.

Mehrotra BS, Mehrotra BM. 1979 [1978] - Another azygosporic species of Mucor from India. Sydowia 31, 94-96.

Meissner EG, Bennett JE, Qvarnstrom Y, da Silva A et al. 2012 - Disseminated microsporidiosis in an immunosupplpressed patient. Emerging Infectious Diseases 18, 1155-1158.

Mikhailov KV, Simdyanov TG, Aleoshin VV. 2016 - Genomic survey of a hyperparasitic microsporidian Amphiamblys sp. (Metchnikovellidae). Genome Biology and Evolution 9, 454-467.

Minnis AM, Kennedy AH, Grenier DB, Palm ME, Rossman AY. 2012 - Phylogeny and taxonomic revision of the Planistromellaceae including its coelomycetous anamorphs: contributions towards a monograph of the genus Kellermania. Persoonia 29, 11-28.

Minnis AM, Lindner DL 2013 - Phylogenetic evaluation of Geomyces and allies reveals no close relatives of Pseudogymnoascus destructans, comb. nov., in bat hibernacula of eastern North America. Fungal Biology 117, 638-649. 
Mirza JH, Khan SM, Begum S, Shagufta S. 1979 - Mucorales of Pakistan. University of Agriculture, Faisalabad, Pakistan.

Mishra B, Choi Y-J, Thines M 2018 - Phylogenomics of Bartheletia paradoxa reveals its basal position in Agaricomycotina and that the early evolutionary history of basidiomycetes was rapid and probably not strictly bifurcating. Mycological Progress 17, 333-341.

Moncalvo JM, Vilgalys R, Redhead SA, Johnson JE et al. 2002 - One hundred and seventeen clades of euagarics. Molecular Phylogenetics and Evolution 23, 357-400.

Morton JB, Benny GL. 1990 - Revised classification of arbuscular mycorrhizal fungi (Zygomycetes). A new order, Glomales, two new suborders, Glomineae and Gigasporineae, and two new families, Acaulosporaceae and Gigasporaceae, with an emendation of Glomaceae. Mycotaxon 37, 471-491.

Morton JB, Msiska Z. 2010 - Phylogenies from genetic and morphological characters do not support a revision of Gigasporaceae (Glomeromycota) into four families and five genera. Mycorrhiza 20, 483-496.

Motato-Vásquez V, Grassi E, Gugliotta AM, Robledo GL. 2018 - Evolutionary relationships of Bresadolia (Basidiomycota, Polyporales) based on molecular and morphological evidence. Mycological Progress 17, 1031-1048.

Moussa TA, Gerrits van den Ende BH, Al Zahrani HS, Kadasa NM et al. 2017 - The genus Anthopsis and its phylogenetic position in Chaetothyriales. Mycoses 60, 254-259.

Muggia L, Kopun T, Ertz D. 2015 - Phylogenetic placement of the lichenicolous, anamorphic genus Lichenodiplis and its connection to Muellerella-like teleomorphs. Fungal Biology 119, 1115-1128.

Müller E. 1950 - Die schweizerischen Arten der Gattung Leptosphaeria und ihrer Verwandten. Sydowia 4, 185-319

Nägeli C. 1857 - Über die neue Krankheit der Seidenraupe und verwandte Organismen. Bot Zeitung 15, 760-761.

Nascimento MM, Selbmann L, Sharifynia S, Al-Hatmi AM et al. 2016 - Arthrocladium, an unexpected human opportunist in Trichomeriaceae (Chaetothyriales). Fungal Biology 120, 207-218.

Nasr S, Soudi MR, Fazeli SAS, Nguyen HD et al. 2014 - Expanding evolutionary diversity in the Ustilaginomycotina: Fereydouniaceae fam. nov. and Fereydounia gen. nov., the first urocystidalean yeast lineage. Mycological Progress 13, 1012.

Nylund S, Nylund A, Watanabe K, Arnesen CE et al. 2010 - Paranucleospora theridion n. gen., n. sp. (Microsporidia, Enterocytozoonidae) with a Life Cycle in the Salmon Louse (Lepeophtheirussalmonis, Copepoda) and Atlantic Salmon (Salmosalar). Journal of Eukaryotic Microbiology 57, 95-114.

Oehl F, da Silva DKA, Maia LC, de Sousa NMF, da Silva GA et al. 2011d - Orbispora gen. nov., ancestral in the Scutellosporaceae (Glomeromycetes). Mycotaxon 116, 161-169.

Oehl F, da Silva GA, Goto BT, Sieverding E. 2011e - New recombinations in Glomeromycota. Mycotaxon 117, 429-434.

Oehl F, da Silva GA, Sánchez-Castro I, Goto BT et al. 2011f - Revision of Glomeromycetes with entrophosporoid and glomoid spore formation with three new genera. Mycotaxon 117, 297 316.

Oehl F, de Souza FA, Sieverding E. 2008 - Revision of Scutellospora and description of five new genera and three new families in the arbuscular mycorrhiza-forming Glomeromycetes. Mycotaxon 106, 311-360.

Oehl F, Sánchez-Castro I, Palenzuela J, da Silva GA. 2015 - Palaeospora spainii, a new arbuscular mycorrhizal fungus from Swiss agricultural soils. Nova Hedwig 101, 89-102.

Oehl F, Sieverding E, Palenzuela J, Ineichen K et al. 2011c - Advances in Glomeromycota taxonomy and classification. IMA fungus 2, 191-199.

Oehl F, Silva GA, Goto BT, Maia LC et al. 2011b - Glomeromycota: two new classes and a new order. Mycotaxon 116, 75-120. 
Oehl F, Silva GA, Goto BT, Sieverding E. 2011a - Glomeromycetes: three new genera and glomoid species reorganized. Mycotaxon 116, 75-120.

Olive LS, Stoianovitch C. 1975 - The Mycetozoans. Academic Press, New York.

Orihara T, Smith ME. 2017 - Unique phylogenetic position of the African truffle-like fungus, Octaviania ivoryana (Boletaceae, Boletales), and the proposal of a new genus, Afrocastellanoa. Mycologia 109, 323-332.

Ovcharenko M, Wróblewski P, Kvach Y, Drobiniak O. 2017 - Study of Loma acerinae (Microsporidia) detected from three Ponto-Caspian gobies (Gobiidae) in Ukraine. Parasitology Research 116, 1453-1462.

Papp V, Dima B. 2018 - New systematic position of Aurantiporus alborubescens (Meruliaceae, Basidiomycota), a threatened old-growth forest polypore. Mycological Progress 17, 319332.

Pärtel K, Baral HO, Tamm H, Põldmaa K. 2017 - Evidence for the polyphyly of Encoelia and Encoelioideae with reconsideration of respective families in Leotiomycetes. Fungal Diversity 82, 183-219.

Pei KQ. 2000 - A new variety of Mucor variosporus and the validation of M. luteus Linnemann and $M$. variosporus Schipper. Mycosystema 19, 10-12.

Pérez-Ortega S, Suija A, de los Rios A. 2011 - The connection between Abrothallus and its anamorph state Vouauxiomyces established by Denaturing Gradient Gel Electrophoresis (DGGE). Lichenologist 43, 277-279.

Perry BA, Hansen K, Pfister DH. 2007 - A phylogenetic overview of the family Pyronemataceae (Ascomycota, Pezizales). Mycological Research 111, 549-571.

Petersen HE 1905 - Contributions a la connaissance des Phycomycetes marins (Chytridineae Fischer). Oversigt over det Kongelige Danske videnskabernes selskabs forhandlinger 5, 439-188.

Petersen RH, Hughes KW. 2010 - The Xerulal Oudemansiella complex (Agaricales). Nova Hedwigia 137, 1-165.

Peyretaillade E, Boucher D, Parisot N, Gasc C et al. 2015 - Exploiting the architecture and the features of the microsporidian genomes to investigate diversity and impact of these parasites on ecosystems. Heredity 114, 441-449.

Phillips AJL, Hyde KD, Alves A, Liu JK. 2018 - Families in Botryosphaeriales: a phylogenetic, morphological and evolutionary perspective. Fungal Diversity 94, 1-22.

Phookamsak R, Boonmee S, Norphanphoun C, Wanasinghe DN et al. 2016 - Schizothyriaceae. Mycosphere 7, 154-189.

Phookamsak R, Hyde KD, Jeewon R, Bhat DJ et al. 2019 - Fungal Diversity notes 929-1035: taxonomic and phylogenetic contributions on genera and species of fungal taxa. Fungal Diversity 95, 1-273.

Phookamsak R, Liu JK, McKenzie EH, Manamgoda DS et al. 2014 - Revision of Phaeosphaeriaceae. Fungal Diversity 68, 159-238.

Phookamsak R, Wanasinghe DN, Hongsanan S, Phukhamsakda C et al. 2017 - Towards a natural classification of Ophiobolus and ophiobolus-like taxa; introducing three novel genera Ophiobolopsis, Paraophiobolus and Pseudoophiobolus in Phaeosphaeriaceae (Pleosporales). Fungal Diversity 87, 299-339.

Pino-Bodas R, Zhurbenko MP, Stenroos S. 2017 - Phylogenetic placement within Lecanoromycetes of lichenicolous fungi associated with Cladonia and some other genera. Persoonia 39, 91-117.

Pirozynski KA, Weresub LK. 1979 - The classification and nomenclature of fossil fungi. in Kendrick, B. (ed.), The whole fungus, the sexual-asexual synthesis. Proceedings of the 2nd International Mycological Conference, University of Calgary, Kananaskis, Alberta (published by National Museum of Natural Sciences, National Museums of Canada and the Kananaskis Foundation) volume 2, 653-688. 
Pirozynski KA. 1978 - Fungal spores through the ages - a mycologist's view. Proceedings of the Fourth International Palynological Conference, Lucknow 1976-77, volume 1, 327-330.

Poulain M, Meyer M, Bozonnet J. 2011 - Les Myxomycètes. FédMycol Bot Dauphiné-Savoie, Delémont.

Powell MJ, Letcher PM, Longcore JE, Blackwell WH. 2018 - Zopfochytrium is a new genus in the Chytridiales with distinct zoospore ultrastructure. Fungal Biology 122, 1041-1049.

Prasher IB, Sharma R, Singh G 2016 - Gelatinoamylaria gen. nov. (Dermateaceae, Helotiales) from Bhutan. Kavaka 46, 35-36.

Prieto M, Schultz M, Olariaga I, Wedin M. 2018 - Lichinodium is a new lichenized lineage in the Leotiomycetes. Fungal Diversity 94, 23-39.

Pringle A, Baker DM, Platt JL, Wares JP et al. 2005 - Cryptic speciation in the cosmopolitan and clonal human pathogenic fungus Aspergillus fumigatus. Evolution 59, 1886-1899.

Printzen C, Spribille T, Tønsberg T. 2008 - Myochroidea, a new genus of corticolous, crustose lichens to accommodate the Lecidea leprosula group. Lichenologist 40, 195-207.

Purrini K, Weiser J. 1985 - Ultrastructural study of the microsporidian Chytridiopsis typographi (Chytridiopsida: Microspora) infecting the bark beetle, Ips typographus (Scolytidae: Coleoptera), with new data on spore dimorphism. Journal of Invertebrate Pathology 45, 6674.

Puytorac P de, Grain J, Mignot JP. 1987 - Précis de protistologie. Lubrecht \& Cramer Ltd, 1987, $581 \mathrm{p}$.

Quaedvlieg W, Verkley GJM, Shin HD, Barreto RW et al. 2013 - Sizing up Septoria. Studies in Mycology 75, 307-390.

Quandt CA, Beaudet D, Corsaro D, Walochnik J et al. 2017 - The genome of an intranuclear parasite, Paramicrosporidium saccamoebae, reveals alternative adaptations to obligate intracellular parasitism. Elife 24; 6, e29594.

Quijada L, Johnston PR, Cooper JA \& Pfister D. 2018a - Overview of Phacidiales, including Aotearoamyces gen. nov. on Nothofagus. IMA fungus 9, 371-38.

Quijada L, Matočec N, Kušan I, Baral HO et al. 2018b - Insights into Claussenomyces Kirschst.: Past, Present and Future. 11th International mycological congress. Mycological discoveries for a better world. Abstract Book 273, San Juan, Puerto Rico

Quijada L, Tanney JB, Popov E, Johnston PR et al. 2020 - Cones, needles and wood: Micraspis (Micraspidaceae, Micraspidales fam. et ord. nov.) speciation segregates by host plant tissues. Fungal Systematics and Evolution 5, 99-112.

Raczka MF, Bush MB, Folcik AM, McMichael CH. 2016 - Sporormiella as a tool for detecting the presence of large herbivores in the Neotropics. Biota Neotropica 16(1).

Rao AR. 1959 - Fungal remains from some Tertiary deposits of India. Palaeobotanist 7, 43-46.

Raper D, Bush M. 2009 - A test of Sporormiella representation as a predictor of megaherbivore presence and abundance. Quaternary Research 71, 490-496.

Raper KB, Smith NR. 1939 - The growth of Dictyostelium discoideum on pathogentic bacteria. Journal of Microbiology 38, 431-445.

Raper KB. 1984 - The Dictyostelids. Princeton University Press, United States.

Réblová M, Hubka V, Thureborn O, Lundberg J et al. 2016 - From the tunnels into de treetops: new lineages of black yeasts from biofilm in the Stockholm Metro system and their relatives among ant - associated fungi in the Chaetothyriales. PloS One 11, e0163396.

Réblová M, Miller AN, Réblová K, Štěpánek V 2018 - Phylogenetic classification and generic delineation of Calyptosphaeria gen. nov., Lentomitella, Spadicoides and Torrentispora (Sordariomycetes). Studies in Mycology 89, 1-62.

Réblová M, Štěpánek V. 2018 - Introducing the Rhamphoriaceae fam. nov. (Sordariomycetes), two new genera and new life histories for taxa with Phaeoisaria-and Idriella-like anamorphs. Mycologia 110, 750-770. 
Réblová M, Untereiner WA, Réblová K. 2013 - Novel evolutionary lineages revealed in the Chaetothyriales (Fungi) based on multigene phylogenetic analyses and comparison of ITS secondary structure. PloS ONE 8, e63547, 1-28.

Réblová M, Untereiner WA, Štěpánek V, Gams W. 2016 - Disentangling Phialophora section Catenulatae: disposition of taxa with pigmented conidiophores and recognition of a new subclass, Sclerococcomycetidae (Eurotiomycetes). Mycological Progress 16, 27-46.

Resl P, Schneider K, Westberg M, Printzen C et al. 2015 - Diagnostics for a troubled backbone: testing topological hypotheses of trapelioid lichenized fungi in a large-scale phylogeny of Ostropomycetidae (Lecanoromycetes). Fungal Diversity 73, 239-258.

Ride WDL, Cogger HG, Dupuis C, Kraus O et al. 1999 - International code of zoological nomenclature. Fourth Edition. The Natural History Museum, London.

Riess K, Schön ME, Lutz M, Butin H et al. 2016 - On the evolutionary history of Uleiella chilensis, a smut fungus parasite of Araucaria araucana in South America: Uleiellales ord. nov. in Ustilaginomycetes. PloS one 11, e0147107.

Rikkinen J, Beimforde C, Seyfullah LJ, Perrichot V et al. 2016 - Resinogalea humboldtensis gen. et sp. nov., a new resinicolous fungus from New Caledonia, placed in Bruceomycetaceae fam. nova (Ascomycota). Annales Botanici Fennici 53, 205-215.

Rizzo L, Sutton DA, Wiederhold NP, Thompson EH et al. 2014 - Isolation and characterisation of the fungus Spiromastix asexualis sp. nov. from discospondylitis in a German Shepherd dog, and review of Spiromastix with the proposal of the new order Spiromastixales (Ascomycota). Mycoses 57, 419-428.

Rollins AW, Landolt JC, Stephenson SL. 2010 - Dictyostelid cellular slime molds associated with grasslands of the central and western United States. Mycologia 102, 996-1003.

Romeralo M, Baldauf S, Escalante R 2013 - Dictyostelids Evolution, Genomics and Cell Biology. Springer, Berlin Heidelberg.

Romeralo M, Cavender JC, Landolt JC, Stephenson SL, Baldauf SL. 2011 - An expanded phylogeny of social amoebas (Dictyostelia) shows increasing diversity and new morphological patterns. BMC Evolutionary Biology 11, 84.

Romeralo M, Escalante R, Baldauf SL. 2012 - Evolution and diversity of dictyostelid social Amoebae. Protist 63, 327-343.

Ronikier A, Halamski AT. 2018 - Is Myxomycetes (Amoebozoa) a truly ambiregnal group? A major issue in protist nomenclature. Protist 169, 484-493.

Ropars J, Cruaud C, Lacoste S, Dupont J. 2012 - A taxonomic and ecological overview of cheese fungi. International Journal of Food Microbiology 155, 199-210.

Rosendahl CO 1943 - Some fossil fungi from Minnesota. Bulletin of the Torrey Botanical Club 70, 126-138.

Rosling A, Cox F, Cruz-Martinez K, Ihrmark K et al. 2011 - Archaeorhizomycetes: unearthing an ancient class of ubiquitous soil fungi. Science 333, 876-879.

Rossman AY, Cavan AW, Braun U, Castlebury LA et al. 2016 - Overlooked competing asexual and sexually typifed generic names of Ascomycota with recommendations for their use or protection. IMA fungus 7, 289-308.

Rossman AY, Crous PW, Hyde KD, Hawksworth DL et al. 2015 - Recommended names for pleomorphic genera in Dothideomycetes. IMA fungus 6, 507-523.

Rupcic Z, Chepkirui C, Hernández-Restrepo M, Crous PW et al. 2018 - New nematicidal and antimicrobial secondary metabolites from a new species in the new genus, Pseudobambusicola thailandica. MycoKeys 33, 1-23.

Salgado-Salazar C, Beirn LA, Ismaiel A, Boehm MJ et al. 2018 - Clarireedia: A new fungal genus comprising four pathogenic species responsible for dollar spot disease of turfgrass. Fungal Biology 122, 761-773.

Samarakoon MC, Thongbai B, Hyde KD, Brönstrup M et al. 2020 - Elucidation of the life cycle of the endophytic genus Muscodor and its transfer into the genus Induratia in Induratiaceae 
fam. nov., based on a polyphasic taxonomic approach. Fungal Diversity DOI: 10.1007/s13225-020-00443-9.

Sanders D, Borys KD, Kisa F, Rakowski SA et al. 2017 - Multiple dictyostelid species destroy biofilms of Klebsiellaoxytoca and other gram negative species. Protist 168, 311-325. Santamaria S, Enghoff H, Gruber J, Reboleira ASPS 2017 - First Laboulbeniales from harvestmen: the new genus Opilionomyces. Phytotaxa 305, 285-292.

Santamaria S, Enghoff H, Reboleira ASPS. 2016 - Hidden biodiversity revealed by collectionsbased research - Laboulbeniales in millipedes: genus Rickia. Phytotaxa 243, 101-127.

Saxena RK, Sarkar S. 1986 - Morphological study of Frasnacritetrus taugourdeau emend. from the tertiary sediments of Himachal Pradesh, India. Review of Palaeobotany \& Palynology 46, 209-225.

Saxena RK, Tripathi SKM. 2011 - Indian Fossil Fungi. Palaeobotanist 60, 1-208.

Saxena RK. 1991 - A catalogue of fossil plants from India - Part 5B. Tertiary fungi. Special Publication, Birbal Sahni Institute of Palaeobotany, Lucknow. pp. 1-19.

Saxena RK. 2006 - A Catalogue of Tertiary Fungi from India (1989-2005). Special Publication, Birbal Sahni Institute of Palaeobotany, Lucknow. pp. 1-37.

Scambler R, Niskanen T, Assyov B, Ainsworth AM et al. 2018 - Diversity of Chroogomphus (Gomphidiaceae, Boletales) in Europe, and typification of C. rutilus. IMA fungus 9, 271290.

Schaap P, Winckler T, Nelson M, Alvarez-Curto E et al. 2006 - Molecular phylogeny and evolution of morphology in the social amoebas. Science 14(5799), 661-3.

Schenck NC, Pérez Y. 1990 - Manual for identification of VA mycorrhizal fungi. Synergistic Publications, Gainesville.

Schipper MAA, Samson RA. 1994 - Miscellaneous notes on Mucoraceae. Mycotaxon 50, 475491.

Schipper MAA. 1973 - A study on variability in Mucor hiemalis and related species. Studies in Mycology 4, 1-40.

Schipper MAA. 1975 - Мисоr mисеdo, Mucor flavus and related species. Studies in Mycology 10, $1-33$.

Schipper MAA. 1976 - On Mucor circinelloides, Mucor racemosus and related species. Studies in Mycology 12, 1-40.

Schipper MAA. 1978 - On certain species of Mucor with a key to all accepted species. Studies in Mycology 17, 1-69.

Schnepf E, Deichgräber G, Drebes G 1977 - Development and ultrastructure of the marine, parasitic oomcete, Lagenisma coscinodisci (Lagenidiales): sexual reproduction. Canadian Journal of Botany, 56, 1315-1325.

Schoch CL, Sung GH, López-Giráldez F, Townsend JP et al. 2009 - The Ascomycota Tree of Life: A phylum-wide phylogeny clarifies the origin and evolution of fundamental reproductive and ecological traits. Systematic Biology 58, 224-239

Schuld M, Madel G, Schmuck R. 1999 - Impact of Vairimorpha sp. (Microsporidia: Burenellidae) on Trichogramma chilonis (Hymenoptera, Trichogrammatidae), a hymenopteran parasitoid of the cabbage moth, Plutella xylostella (Lepidoptera, Yponomeutidae). Journal of Invertebrate Pathology 74, 120-126.

Schüßler A, Schwarzott D, Walker C. 2001 - A new fungal phylum, the Glomeromycota: phylogeny and evolution. Mycological Research 105, 1413-1421

Seifert K, Morgan-Jones G, Gams W, Kendrick B 2011 - The genera of hyphomycetes. CBS Biodiversity Series 9, 1-997.

Senderskiy IV, Timofeev SA, Seliverstova EV, Pavlova OA et al. 2014 - Secretion of Antonospora (Paranosema) locustae proteins into infected cells suggests an active role of Microsporidia in the control of host programs and metabolic processes. PLoS ONE 9, e93585. 
Sert HB, Sumbul H, Sterflinger K. 2007 - Microcolonial fungi from antique marbles in Perge/Side/Termessos (Antalya/Turkey). Antonie van Leewenhoek 91, 217-227.

Shadwick LL, Spiegel FW, Shadwick JD, Brown MW et al. 2009 - Eumycetozoa = Amoebozoa? SSU rDNA phylogeny of protosteloid slime molds and its significance for the amoebozoan supergroup. PLoS One 4, e6754.

Sharma R, Kulkarni G, Sonawane MS. 2017 - Alanomyces, a new genus of Aplosporellaceae based on four loci phylogeny. Phytotaxa 297, 168-175.

Sharma R, Xia X, Cano LM, Evangelisti E, Kemen E et al (2015) Genome analyses of the sunflower pathogen Plasmopara halstedii provide insights into effector evolution in downy mildews and Phytophthora. BMC Genomics 16, 741.

Shchepin O, Schnittler M, Dagamac N, Leontyev D et al. 2019 - Unexplored diversity of microscopic myxomycetes: evidence from environmental DNA. Plant Ecology \& Evolution, 152, 499-506.

Sheikh S, Thulin M, Cavender JC, Escalante R et al. 2018 - A new classification of the Dictyostelids. Protist 169, 1-28.

Shen XX, Opulente DA, Kominek J, Zhou X et al. 2018 - Tempo and mode of genome evolution in the budding yeast subphylum. Cell 75, 1533-1545.e20

Shen XX, Zhou X, Kominek J, Kurtzman CP et al. 2016 - Reconstructing the backbone of the Saccharomycotina yeast phylogeny using genome-scale data. G3 (Bethesda) 6, 3927-3939.

Shi WP, Zheng X, Jia WT, Li AM et al. 2018 - Horizontal transmission of Paranosema locustae (Microsporidia) in grass hopper populations via predatory natural enemies. Pest Management Science 74, 2589-2593.

Shoemaker RA, Babcock CE. 1992 - Applanodictyosporous Pleosporales: Clathrospora, Comoclathris, Graphyllium, Macrospora, and Platysporoides. Canadian Journal of Botany 70, 1617-1658.

Sieverding E, Silva GA, Berndt R, Oehl F. 2014 - Rhizoglomus, a new genus in the Glomeraceae. Mycotaxon 129, 373-386.

Silva GA, Maia LC, Oehl F. 2012 - Phylogenetic systematics of the Gigasporales. Mycotaxon 122, 207-220.

Silva RM, Oliveira RJ, Bezerra JD, Bezerra JL et al. 2019 - Bifusisporella sorghi gen. et sp. nov. (Magnaporthaceae) to accommodate an endophytic fungus from Brazil. Mycological Progress 18, 847-854.

Simakova AV, Pankova TF, Tokarev YS, Issi IV. 2005 - New genus of microsporidia Senoma gen. n. with type species Senoma globulifera comb. n. (syn. Issia globulifera Issi, Pankova, 1983 from malaria mosquito Anopheles messae Fall. Protistology 4, 134-145.

Simmons EG. 2007 - Alternaria: an identification manual. CBS Biodiversity Series 6. 775 pp.

Singh BN. 1947 - Studies on soil Acrasieae. 1. Distribution of species of Dictyostelium in soils of Great Britain and the effects of bacteria on their development. Journal of General Microbiology 1.

Singh G, Dal Grande F, Divakar PK, Otte J et al. 2015 - Coalescent-based species delimitation approach uncovers high cryptic diversity in the cosmopolitan lichen-forming fungal genus Protoparmelia (Lecanorales, Ascomycota). Plos One 10, e0124625.

Slippers B, Boissin E, Phillips AJL, Groenewald JZ et al. 2013 - Phylogenetic lineages in the Botryosphaeriales: A systematic and evolutionary framework. Studies in Mycology 76, 3149.

Smith ME, Amses KR, Elliott TF, Obase K et al. 2015 - New sequestrate fungi from guyana: Jimtrappea guyanensis gen. sp. nov., Castellanea pakaraimophila gen. sp. nov, and Costatisporus cyanescens gen. sp. nov. (Boletaceae, Boletales). IMA fungus 6, 297-317.

Sokolova Y, Pelin A, Hawke J, Corradi N. 2015 - Morphology and phylogeny of Agmasomapenaei (Microsporidia) from the type host, Litopenaeus setiferus, and the typelocality, Louisiana, USA. International Journal for Parasitology 45, 1-16. 
Sokolova YK, Issi IV, Voronin VN. 2018 - Annotated list of species of the Microsporidia described in the Former Soviet Union and Russia in 20th century 1967-2000. Protistology $12,12-37$.

Sokolova YY, Bowers LC, Alvarez X, Didier ES. 2019 - Encephalitozoon cuniculi and Vittaforma corneae (Phylum Microsporidia) inhibit staurosporine-induced apoptosisin human THP-1 macrophages in vitro. Parasitology 146, 569-579.

Sokolova YY, Paskerova GG, Rotari YM, Nassonova ES et al. 2013 - Fine structure of Metchnikovella incurvata Caullery \& Mesnil 1914 (Microsporidia), a hyperparasite of gregarines Polyrhabdina sp. from the polychaete Pygospioelegans. Parasitology 140, 855867.

Song J, Liang JF, Mehrabi-Koushki M, Krisai-Greilhuber I, Ali B et al. 2019 - Fungal Systematics and Evolution 5. Sydowia 71, 141-245.

Song Z, Li G, Cao L, Luo H et al. 1989 - Early Tertiary sporo-pollen assemblages from the Dongpu region. Edited by Research Institute of Exploration and Development, Zhongyuan Petroleum Exploration Bureau, Nanjing Institute of Geology and Palaeontology, Academia Sinica, $192 \mathrm{p}$.

Spatafora JW, Chang Y, Benny GL, Lazarus K et al. 2016 - A phylum-level phylogenetic classification of zygomycete fungi based on genome-scale data. Mycologia 108, 1028-1046.

Species Fungorum 2019 http://www.speciesfungorum.org/Names/SynSpecies.asp

Stefani FOP, Jones RH, May TW. 2014 - Concordance of seven gene genealogies compared to phenotypic data reveals multiple cryptic species in Australian dermocyboid Cortinarius (Agaricales). Molecular Phylogenetics \& Evolution 71, 249-260.

Stentiford GD, Feist SW, Stone DM, Bateman KS et al. 2013 - Microsporidia: diverse, dynamic, and emergent pathogens in aquatic systems. Trends in Parasitology 29, 567-578.

Stentiford GD, Ramilo A, Abollo E, Kerr R et al. 2017 - Hypersporaaquatica n. gn., n. sp. (microsporidia), hyperparasitic in Marteiliacochillia (Paramyxida), is closely related to crustacean-infectingmicrospordian taxa. Parasitology 144, 186-199.

Stentiford GD, Ross S, Minardi D, Feist SW et al. 2018 - Evidence for trophic transfer of Inodosporus octospora and Ovipleistophora arlo n. sp. (microsporidia) between crustacean and fish hosts. Parasitology 145, 1105-1117.

Stephenson SL, Landolt JC. 1992 - Vertebrates as vectors of cellular slime moulds in temperate forests. Mycological Research 96, 670-672.

Stephenson SL, Landolt JC. 1998 - Dictyostelid cellular slime molds in canopy soils of tropical forests. Biotropica 30, 657-661.

Stephenson SL, Landolt JC. 2011 - Dictyostelids from aerial "canopy soil" microhabitats. Fungal Ecology 4, 191-195.

Stephenson SL, Rajguru SN. 2010 - Dictyostelid cellular slime moulds in agricultural soils. Mycosphere 1, 333-336.

Subrahamanyam A. 1983 - Studies on themomycology. Mucor thermo-hyalospora sp. nov. Bibliotheca Mycologica 91, 421-423.

Subramanian CV. 1971 - Hyphomycetes, an account of Indian species, except Cercosporae. Indian Council of Agricultural Research, New Delhi, 930 p.

Sudová R, Sýkorová Z, Rydlová J, Čtvrtlíková M, Oehl F. 2015 - Rhizoglomus melanum, a new arbuscular mycorrhizal fungal species associated with submerged plants in freshwater lake Avsjøen in Norway. Mycologial Progress 14, 9

Suija A, de los Ríos A, Pérez-Ortega S. 2015 - A molecular reappraisal of Abrothallus species growing on lichens of the order Peltigerales. Phytotaxa, 195, 201-226.

Suija A, Kaasalainen U, Kirika PM, Rikkinen J. 2018 - Taitaia, a novel lichenicolous fungus in tropical montane forests in Kenya (East Africa). Lichenologist 50, 173-184.

Suija A, van den Boom PPG, Zimmermann E, Zhurbenko MP et al. 2017 - Lichenicolous species of Hainesia belong to Phacidiales (Leotiomycetes) and are included in an extended concept of Epithamnolia. Mycologia 109, 882-889. 
Sumbali G. (ed. Johri BM) 2005 - The fungi. ISBN 978-1-84265-153-7

Suthers HB. 1985 - Ground-feeding migratory songbirds as cellular slime mold distribution vectors. Oecologia 65, 526-530.

Tanaka K, Hirayama K, Yonezawa H, Sato G et al. 2015 - Revision of the Massarineae (Pleosporales, Dothideomycetes). Studies in Mycology 82, 75-136.

Tanney JB, Douglas B, Seifert KA. 2016 - Sexual and asexual states of some endophytic Phialocephala species of Picea. Mycologia 108, 255-280.

Tanney JB, Seifert KA. 2020 - Mollisiaceae: An overlooked lineage of diverse endophytes, Studies in Mycology, https://doi.org/10.1016/j.simyco.2020.02.005.

Taugourdeau P. 1968 - Sur un curieux microfossile incertae sedis du Frasnien du Boulonnais, Frasnacritetrus nov. gen. (Acritarche). Cahiers de Micropaléontologie, série 1, no. 10 (Archives originales du Centre de Documentation du C.N.R.S. no. 452, 1-4.

Tavares II. 1985 - Laboulbeniales (Fungi, Ascomycetes). Mycologia Memoir 9, 1-627.

Tedersoo L, Sánchez-Ramírez S, Koljalg U et al. 2018 - High-level classification of the Fungi and a tool for evolutionary ecological analyses. Fungal Diversity 90, 135-159.

Teterevnikova-Babaian DN, Taslakhchian MG. 1973 - New data on fossil fungal spores in Armenia. Mycology and Phytology 4, 159-164.

Thambugala KM, Wanasinghe DN, Phillips AJ, Camporesi E et al. 2017 - Mycosphere notes 150: grass (Poaceae) inhabiting Dothideomycetes. Mycosphere 8, 697-796.

Thines M, Nam B, Nigrelli L, Beakes G, Kraberg A. 2015 - The diatom parasite Lagenisma coscinodisci (Lagenismatales, Oomycota) is an early diverging lineage of the Saprolegniomycetes. Mycological Progress 14, 75.

Thines M, Crous PW, Aime MC, Aoki T et al. 2018 - Ten reasons why a sequence-based nomenclature is not useful for fungi anytime soon. IMA fungus 9, 177-183.

Thines M. 2019 - An evolutionary framework for host shifts-jumping ships for survival. New Phytologist 224, 605-617.

Tice AK, Shadwick LL, Fiore-Donno AM, Geisen S et al. 2016 - Expansion of the molecular and morphological diversity of Acanthamoebidae (Centramoebida, Amoebozoa) and identification of a novel life cycle type within the group. Biology Direct 11, 69-90.

Tokarev YS, Voronin VN, Seliverstova EV, Dolgikh VV et al. 2010 - Ultrastructure and molecular phylogeny of Anisofilariata chironomi sp. n. g. n. (Microsporidia: Terresporidia), a microsporidian parasite of Chironomus plumosus L. (Diptera: Chironomidae). Parasitology Research 106, 39-46.

Tokarev YS, Voronin VN, Seliverstova EV, Grushetskaya TA et al. 2012 - Ultrastructure and molecular phylogenetics of Helmichialacustris, a microsporidium with an uncoiled isofilar polar filament. Parasitology Research 110, 1201-1208.

Tonka T, Weiser JJr, Weiser J. 2010 - Budding: a new stage in the development of Chytridiopsis typographi (Zygomycetes: Microsporidia). Journal of Invertebrate Pathology 104, 17-22.

Torruella G, Grau-Bové X, Moreira D, Karpov SA et al. 2018 - Global transcriptome analysis of the aphelid Paraphelidium tribonemae supports the phagotrophic origin of fungi. Communications Biology 1, 231.

Trakunyingcharoen T, Lombard L, Groenewald JZ, Cheewangkoon R et al. 2014 - Mycoparasitic species of Sphaerellopsis, and allied lichenicolous and other genera. IMA fungus 5, 391414.

Truong C, Mujic AB, Healy R, Kuhar F et al. 2017 - How to know the fungi: combining field inventories and DNA-barcoding to document fungal diversity. New Phytologist 214, 913919.

Tsaousis AD, Kunji ER, Goldberg AV et al. 2008 - A novel route for ATP acquisition by the remnant mitochondria of Encephalitozoon cuniculi. Nature 453(7194), 553-556.

Tsuneda A, Davey ML, Tsuneda I, Hudgins A et al. 2011a - Endophoma, a new didymellaceous endoconidial genus from bat - cave soil. Mycologia 103, 1146-1155. 
Tsuneda A, Hambleton S, Currah RS. 2011b - The anamorph genus Knufia and its phylogenetically allied species in Coniosporium, Sarcinomyces and Phaeococcomyces. Botany 89, 523-536.

Turland NJ, Wiersema JH, Barrie FR et al. 2018 - International Code of Nomenclature for algae fungi and plants (Shenzhen Code) adopted by the Nineteenth International Botanical Congress Shenzhen, China, July 2017. Regnum Vegetabile, 159 Koeltz Botanical Books Glashütten.

Turrini A, Saran M, Giovannetti M, Oehl F. 2018 - Rhizoglomus venetianum, a new arbuscular mycorrhizal fungal species from a heavy metal contaminated site, downtown Venice in Italy. Mycological Progress 17, 1213-1224.

Van der Hammen T. 1954 - El desarrollo de la flora Colombiana en los periodos geológicos. I. Maestrichtiano hasta Terciario más inferior. Boletín Geológico 2, 49-106.

Van Vooren N, Lindemann, Healy R. 2017 - Emendation of the genus Tricharina (Pezizales) based on phylogenetic, morphological and ecological data. Ascomycete.org 9, 101-123.

Vavra J, Larsson JIR. 2014 - Structure of Microsporidia. In: Weiss LM, Becnel JJ (eds) Microsporidia: Pathogens of Opportunity. Wiley-Blackwell, pp 1-70

Vavra J, Lukes J. 2013 - Microsporidia and 'the art of living together'. Advances in Parasitology $82,253-319$.

Vellinga EC, Sysouphanthong P, Hyde KD. 2011 - The family Agaricaceae: phylogenies and two new white-spored genera. Mycologia 103, 494-509.

Venkatachala BS, Kar RK 1969 - Palynology of the Tertiary sediments in Kutch-2. Epiphyllous fungal remains from the borehole no. 14. Palaeobotanist 17, 179-183.

Vivier E 1975 - The microsporidia of the protozoa. Protistologica 9, 345-361.

Vizzini A, Contu M, Ercole E. 2011 - Musumecia gen. nov. in the Tricholomatoid clade (Basidiomycota, Agaricales) related to Pseudoclitocybe. Nordic Journal of Botany 29, 734740.

Vlk W 1939 - Über die Geißelstruktur der Saprolegniaceenschwärmer. Archiv für Protistenkunde 92, 157-160.

Voglmayr H, Aguirre-Hudson MB, Wagner HG, Tello S et al. 2019 - Lichens or endophytes? The enigmatic genus Leptosillia in the Leptosilliaceae fam. nov. (Xylariales), and Furfurella gen. nov. (Delonicicolaceae). Persoonia 42, 228-260.

Voglmayr H, Clémençon H. 2016 - Identification and taxonomic position of two mucoralean endoparasites of Hysterangium (Basidiomycota) based on molecular and morphological data. Mycological Progress 15, 1-17.

Voglmayr H, Friebes G, Gardiennet A, Jaklitsch WM. 2018 - Barrmaelia and Entosordaria in Barrmaeliaceae (fam. nov., Xylariales) and critical notes on Anthostomella-like genera based on multigene phylogenies. Mycological Progress 17, 155-177.

Voglmayr H, Pintos A. 2018 - Three new species of Stigmatodiscus from Mallorca (Spain). Mycological Progress 17, 1189-1201.

Vossbrinck CR, Debrunner-Vossbrinck BA, Weiss LM. 2014 - Molecular phylogeny of the Microsporidia. In: Weiss LM, Becnel JJ (eds) Microsporidia: Pathogens of Opportunity. Wiley-Blackwell, pp 203-220.

Vossbrinck CR, Debrunner-Vossbrinck BA. 2005 - Molecular phylogeny of the Microsporidia: ecological, ultrastructural and taxonomic considerations. Folia Parasitologica 52, 131-142.

Vu D, Groenewald M, de Vries M, Gehrmann T et al. 2019 - Large-scale generation and analysis of filamentous fungal DNA barcodes boosts coverage for kingdom Fungi and reveals thresholds for fungal species and higher taxon delimitation. Studies in Mycology 92, 135154.

Wanasinghe DN, Phukhamsakda C, Hyde KD, Jeewon R et al. 2018 - Fungal diversity notes 709839: taxonomic and phylogenetic contributions to fungal taxa with an emphasis on fungi on Rosaceae. Fungal Diversity 89, 1-236. 
Wang HK, Aptroot A, Crous PW, Hyde KD et al. 2007 -. The polyphyletic nature of Pleosporales: an example from Massariosphaeria based on rDNA and RBP2 gene phylogenies. Mycological Research 111, 1268-1276.

Wang QM, Groenewald M, Takashima M, Theelen B et al. 2015a - Phylogeny of yeasts and related filamentous fungi within Pucciniomycotina determined from multigene sequence analyses. Studies in Mycology 8127-53.

Wang QM, Theelen B, Groenewald M, Bai FY et al. 2014a - Moniliellomycetes and Malasseziomycetes, two new classes in Ustilaginomycotina. Persoonia 33, 41-47.

Wang QM, Yurkov AM, Göker M, Lumbsch HT et al. 2015b - Phylogenetic classification of yeasts and related taxa within Pucciniomycotina. Studies in Mycology 81, 149-189.

Wang XW, Yang FY, Meijer M, Kraak B et al. 2018 - Redefining Humicola sensu stricto and related genera in the Chaetomiaceae. Studies in Mycology 93, 65-153.

Watanabe T. 1994 - Two new species of homothallic Mucor in Japan. Mycologia 86, 691-695.

Wei X, Schmitt I, Hodkinson BP, Flakus A et al. 2017 - Circumscription of the genus Lepra, a recently resurrected genus to accommodate the "Variolaria" - group of Pertusaria sensu lato (Pertusariales, Ascomycota). PLoS ONE 12, e0180284.

Weir A, Blackwell M. 2001 - Molecular data support the Laboulbeniales as a separate class of Ascomycota, Laboulbeniomycetes. Mycological Research 105, 715-722.

Weir A, Hammond PM. 1997 - Laboulbeniales on beetles: host utilization patterns and species richness of the parasites. Biodiversity \& Conservation 6, 701-719.

Wendt L, Sir EB, Kuhnert E, Heitkämper S et al. 2018 - Resurrection and emendation of the Hypoxylaceae, recognised from a multi-gene phylogeny of the Xylariales. Mycological Progress 17, 115-154.

Westphalen MC, Rajchenberg M, Tomšovský M, Gugliotta AM. 2018 - A re-evaluation of Neotropical Junghuhnia s. lat. (Polyporales, Basidiomycota) based on morphological and multigene analyses. Persoonia 41, 130-141.

Wijayawardene DNN, McKenzie EHC, Hyde KD. 2012 - Towards incorporating anamorphic fungi in a natural classification checklist and notes for 2011. Mycosphere 3, 157-228.

Wijayawardene NN, Hyde KD, Lumbsch HT, Liu JK et al. 2018a - Outline of Ascomycota: 2017 Fungal Diversity 88, 167-263.

Wijayawardene NN, Hyde KD, Rajeshkumar KC, Hawksworth DL et al. 2017a - Notes for genera: Ascomycota. Fungal Diversity 86, 1-594.

Wijayawardene NN, Hyde KD, Tibpromma S, Wanasinghe DN et al. 2017b - Towards incorporating asexual fungi in a natural classification: checklist and notes 2012-2016. Mycosphere 8, 1457-1554.

Wijayawardene NN, Hyde KD, Wanasinghe DN, Papizadeh M et al. 2016 - Taxonomy and phylogeny of dematiaceous coelomycetes. Fungal Diversity 77, 1-316.

Wijayawardene NN, Pawłowska J, Letcher PM, Kirk PM et al. 2018b - Notes for genera: basal clades of Fungi (including Aphelidiomycota, Basidiobolomycota, Blastocladiomycota, Calcarisporiellomycota, Caulochytriomycota, Chytridiomycota, Entomophthoromycota, Glomeromycota, Kickxellomycota, Monoblepharomycota, Mortierellomycota, Mucoromycota, Neocallimastigomycota, Olpidiomycota, Rozellomycota and Zoopagomycota). Fungal Diversity 92, 43-129.

Williams BA, Hirt RP, Lucocq JM, Embley TM. 2002 - A mitochondrial remnant in the microsporidian Trachipleistophor hominis. Nature 418(6900), 865-869.

Wittstein K, Cordsmeier A, Lambert C, Wendt L et al. 2020 - Identification of Rosellinia species as producers of cyclodepsipeptide PF1022 A and resurrection of the genus Dematophora as inferred from polythetic taxonomy. Studies in Mycology 96, 1-16.

Woudenberg JHC, Seidl MF, Groenewald JZ, De Vries M et al. 2015 - Alternaria section Alternaria: Species, formae speciales or pathotypes? Studies in Mycology 82, 1-21.

Woudenberg JHC, Truter M, Groenewald JZ, Crous PW. 2014 - Large-spored Alternaria pathogens in section Porri disentangled. Studies in Mycology 79, 1-47. 
Wu G, Zhao K, Li YC, Zeng NK et al. 2016 - Four new genera of the fungal family Boletaceae. Fungal Diversity 81, 1-24

Wu ZQ, Xu TM, Shen S, Liu XF et al. 2018 - Elaphroporia ailaoshanensis gen. et sp. nov. in Polyporales (Basidiomycota). MycoKeys 29, 81-95.

Wyka SA, Broders KD. 2016 - The new family Septorioideaceae, within the Botryosphaeriales and Septorioides strobi as a new species associated with needle defoliation of Pinus strobus in the United States. Fungal Biology 120, 1030-1040.

Xia JW, Ma YR, Li Z, Zhang XG. 2017 - Acrodictys-like wood decay fungi from southern China, with two new families Acrodictyaceae and Junewangiaceae. Scientific Reports 7 (no. 7888), 1-21.

Xiao Y, Wen T, Hongsanan S, Jeewon R et al. 2018 - Multigene phylogenetics of Polycephalomyces (Ophiocordycipitaceae, Hypocreales), with two new species from Thailand. Scientific Reports 8, 18087.

$\mathrm{Xu}$ Y, Weiss LM. 2005 - The microsporidian polar tube: a highly specialised invasionorganelle. International Journal for Parasitology 35, 941-953.

Yadav DR, Kim SW, Adhikari M, Um YH et al. 2015 - Three new records of Mortierella species isolated from crop field soil in Korea. Mycobiology 43, 203-209.

Yang CL, Xu XL, Wanasinghe DN, Jeewon R et al. 2019 - Neostagonosporella sichuanensis gen. et sp. nov. (Phaeosphaeriaceae, Pleosporales) on Phyllostachys heteroclada (Poaceae) from Sichuan Province, China. MycoKeys 46, 119-150.

Yang H, Hyde KD, Karunarathna SC, Deng C et al. 2018a - New species of Camptophora and Cyphellophora from China, and first report of sexual morphs for these genera. Phytotaxa 343, 149-159.

Yang J, Liu JK, Hyde KD, Jones EG et al. 2019 - Aquimonospora tratensis gen. et sp. nov. (Diaporthomycetidae, Sordariomycetes), a new lineage from a freshwater habitat in Thailand. Phytotaxa 397, 146-158.

Yang Q, Fan XL, Du Z, Tian CM. 2018b - Diaporthosporellaceae, a novel family of Diaporthales (Sordariomycetes, Ascomycota). Mycoscience 59, 229-235.

Yang T, Groenewald JZ, Cheewangkoon R, Jami F et al. 2017 - Families, genera, and species of Botryosphaeriales. Fungal Biology 121, 322-346.

Yoshida K, Schuenemann VJ, Cano LM, Pais M et al. 2013 - The rise and fall of the Phytophthora infestans lineage that triggered the Irish potato famine. eLife 2, e00731.

Yu X, Hoyle RL, Guo F, Ratliff CM et al. 2019 - A Vavraia-like microsporidium as the cause of deadly infection in threatenedand endangered Eurycea salamanders in the United States. Parasites \& Vectors 12, 108.

Yu XD, Dong W, Bhat DJ, Boonmee S et al. 2018 - Cylindroconidiis aquaticus gen. et sp nov., a new lineage of aquatic hyphomycetes in Sclerococcaceae (Eurotiomycetes). Phytotaxa 372, 79-87.

Yuan HS, Qin WM. 2018 - Multiple genes phylogeny and morphological characters reveal Dextrinoporus aquaticus gen. et sp. nov. (Polyporales, Basidiomycota) from southern China. Mycological Progress 17, 773-780.

Zalar P, Hennebert GL, Gunde-Cimerman N, Cimerman A. 1997 - Mucor troglophilus, a new species from cave crickets. Mycotaxon 65, 507-516.

Zamora JC, Svensson M, Kirschner R, Olariaga I et al. 2018 - Considerations and consequences of allowing DNA sequence data as types of fungal taxa. IMA fungus 9, 167-175.

Zhang ZF, Liu F, Zhou X, Liu XZ et al. 2017 - Culturable mycobiota from Karst caves in China, with descriptions of 20 new species. Persoonia 39, 1-31.

Zhao RL, Li GJ, Sanchez-Ramirez S, Stata M et al. 2017c - A six-gene phylogenetic overview of Basidiomycota and allied phyla with estimated divergence times of higher taxa and a phyloproteomics perspective. Fungal Diversity 84, 43-74. 
Zhao YJ, Hosaka K, Hosoya T. 2016 - Taxonomic re-evaluation of the genus Lambertella (Rutstroemiaceae, Helotiales) and allied stroma-forming fungi. Mycological Progress 15, 1215-1228.

Zhu HY, Pan M, Bonthond G, Tian CM, Fan XL. 2019 - Diaporthalean fungi associated with canker and dieback of trees from Mount Dongling in Beijing, China. Mycokeys 59, 67-94.

Zitouni-Haouar FEH, Alvarado P, Sbissi I, Boudabous A et al. 2015 - Contrasted genetic diversity, relevance of climate and host plants, and comments on the taxonomic problems of the genus Picoa (Pyronemataceae, Pezizales). PLoS ONE 10, e0138513.

Zmitrovich IV. 2018 - Conspectus systematis Polyporacearum v. 1.0. Folia Cryptogamica Petropolitana 6, 3-145. 